\title{
Topological and non-equilibrium superconductivity in low-dimensional strongly correlated quantum systems
}

\author{
Dissertation \\ zur Erlangung des mathematisch-naturwissenschaftlichen Doktorgrades \\ "Doctor rerum naturalium" \\ der Georg-August-Universität Göttingen \\ im Promotionsprogramm Gauss \\ der Georg-August University School of Science (GAUSS ProPhys)
}

vorgelegt von

Sebastian Paeckel

aus Meißen

Göttingen, 2020 


\section{Betreuungsausschuss:}

Referent: Priv.-Doz. Dr. Salvatore R. Manmana

Institut für Theoretische Physik, Georg-August-Universität Göttingen

Korreferent: Prof. Dr. Stefan Kehrein

Institut für Theoretische Physik, Georg-August-Universität Göttingen

\section{Mitglieder der Prüfungskommission:}

Referent: Priv.-Doz. Dr. Salvatore R. Manmana

Institut für Theoretische Physik, Georg-August-Universität Göttingen

Korreferent: Prof. Dr. Stefan Kehrein

Institut für Theoretische Physik, Georg-August-Universität Göttingen

Zweiter Korreferent: Prof. Dr. Frank Pollmann (kein Mitglied der Prüfungskommission)

Fakultät für Physik, Technische Universität München

\section{Weitere Mitglieder der Prüfungskommission:}

Prof. Dr. Fabian Heidrich-Meisner

Institut für Theoretische Physik, Georg-August-Universität Göttingen

Prof. Dr. Stefan Mathias

I. Physikalisches Institut, Georg-August-Universität Göttingen

Prof. Dr. Matthias Krüger

Institut für Theoretische Physik, Georg-August-Universität Göttingen

Prof. Dr. Michael Seibt

IV. Physikalisches Institut, Georg-August-Universität Göttingen

Tag der mündlichen Prüfung: 05.02.2020 


\section{Abstract}

Superconductivity in its various manifestations has been stimulating both experimental and theoretical progress in condensed-matter physics for more than a hundred years. The remarkable property of electrons to pair up and form quasi-particles gives rise to a plethora of phenomena featuring important practical applications not only in science, but, for instance, also in medicine and metrology. Recently, new directions in investigating this fascinating subject emerged, such as superconductivity out-of equilibrium and topological superconductors. Providing experimental evidence for enhanced superconducting correlations in optically pumped copper oxides at temperatures far above the equilibrium transition temperature, the first issue caused considerable excitement. On the other hand, topological superconductors are believed to provide realizations of highly fault-tolerant qubits by means of hosting non-Abelian quasi-particles, which can be the building blocks of scalable quantum computers. Experimentally verifying the emergence of these Majorana edge modes, exotic quasi-particles in heterostructures consisting of a conventional superconductor and semiconductors or topological insulators, is one of the most urgent questions to be answered right now. Both subjects cannot be accounted for with analytically solvable approximations only, and also provide very challenging numerical problems.

We implemented a matrix-product state (MPS) based toolkit exploiting $U(1)$ symmetries, providing a flexible and efficient platform to study these complex systems. In order to efficiently simulate out-of equilibrium setups we studied, compared, and developed time-evolution algorithms for MPS enabling us to choose the most suitable method for a given task. We also developed a new framework to represent operators in an enlarged Hilbert space so that benefits from conserving $U(1)$ symmetries can also be exploited in systems that originally break such symmetries (projected purification). Using this method we could efficiently model mesoscopic phenomena such as a charging energy controlled by a gate electrode without further approximations.

Equipped with this techniques we studied out-of equilibrium spectral functions to explore how to identify superconducting correlations more reliably on ultra-short timescales. We found conclusive evidence that in particular two-particle spectral functions yield excellent probes for the formation of a (quasi-)condensate out-of equilibrium. Furthermore, we also investigated the question whether in a particular model system there is the possibility of true long-range order out-of equilibrium by studying correlation matrices and the scaling of their eigenvalues. Here, we observe a change in the algebraic decay of the correlations, even though the extrapolated order parameter is still zero within the error bounds.

Furthermore, we also investigated the effects of coupling a superconductor-semiconductor heterostructure, which is subject to an in-plane magnetic field and a charging energy controlled by a gate voltage, to normal leads. In the context of experimentally verifying the existence of Majorana edge modes, such systems are believed to be the most promising and recent studies seem to underline this expectation. However, in order to consistently analyze the experimental data, the effects of quantum fluctuations caused by hybridization of the heterostructure with the leads have to be understood. Here, only perturbative limits are available so far, i.e., the weak and strong tunneling limit, while the experimentally relevant regime is expected to be somewhere inbetween. We aimed to fill this gap using the projected purification method to calculate the ground state phase diagram over a wide parameter regime. Our results indicate that the 
iv

experimental situation is much more involved than what is predicted from perturbative analysis. 


\section{Acknowledgments}

Being a PhD student in Göttingen for four years, I was blessed to get to know a lot of wonderful people: Collaborators, colleagues, students, and friends. To all of you, I would like to say thank you for creating an inspiring, exciting, and also (very important) well-functioning environment. You helped me immensely to proceed, learn, and eventually finish this thesis.

My deepest gratitude is devoted to my supervisor Salvatore R. Manmana. After being out of academia for more than a year, he offered me the possibility to become his student and return to university. Even more, he always gave me the opportunity to explore and pursue ideas, was interested, open-minded and patient, and thereby created a work environment that was both motivating and enriching for all this time. I admire his endurance when we were spending the nights debugging code and, not to forget, having pizza and coffee, until we finally solved this very problem.

I also want to thank Stefan Kehrein and Fabian Heidrich-Meisner for enlightening discussions and always having an open door. Additionally, I am very thankful to Fabian Heidrich-Meisner and his student Jan Stolpp for providing me benchmark results from their DMRG3S+LBO code. Furthermore, I want to say thank you to all the faculty members that helped me to solve the mysteries of administration. Moreover, I want to thank Peter Blöchl for providing me access to his cluster at the TU Clausthal, where half of the results in this thesis were produced and for forgiving me if I underestimated the data-production rates of certain simulations. Of course, I also want to thank the FOR 1807 which financed most of my projects throughout this thesis and provided a great foundation for collaborations and inspiring discussions during the anual retreats.

I am very grateful to Bela Bauer for giving me the possibility of having an internship at Station $\mathrm{Q}$ in Santa Barbara. He did an amazing job in being both, a scientifc supervisor introducing me into the fascinating topic of topological insulators, and a guide unveiling beautiful and exciting places in a wonderful piece of earth.

At this point, I want to thank Thomas Köhler. He was the best office mate I can imagine. Most of this work would have not been possible without his helpfulness, patience, fruitful discussions in front of every blackboard we could get our hands on, and his talent to organize our coffee rounds. I also owe a big thank you to his wife Isabel for proofreading an uncountable number of pages. Furthermore, I like to thank Lorenzo Cevolani and Nils Abeling for all these interesting, insightful, and always entertaining discussions (not only at the institute). And also, very important, to all of my colleagues that made the institute a lively and joyful place, in particular, Michael ten-Brink, Florian Sohn, Laura Endter, Jan Stolpp, Kristof Harms, Benjamin Lenz, Alexander Osterkorn, Constantin Meier, Trisha Nath, Markus Schmitt, Fabian (FRA-)Biebl, Ebad Kamil, Robert Schade: Thank you so much!

I want to thank my family for supporting me from the very beginning even though they never knew what I was doing. Finally, I want to say thank you to my life-mate and girlfriend Eva who joined me 11 years ago and went with me through the good and also the hard times. Thank you, for motivating, inspiring and also distracting me in the most fulfilling way! 


\section{List of publications}

Parts of this thesis have been published in a peer-reviewed journal. The corresponding references are listed below. While the first two manuscripts have already been published in a peer-reviewed journal, the last item refers to a preprint.

$\left[\mathrm{PKS}^{+} 19\right]$ Sebastian Paeckel, Thomas Köhler, Andreas Swoboda, Salvatore R. Manmana, Ulrich Schollwöck, and Claudius Hubig. Time-evolution methods for matrix-product states. Annals of Physics, 411:167998, Dec 2019

[PKM17] Sebastian Paeckel, Thomas Köhler, and Salvatore R. Manmana. Automated construction of $u(1)$-invariant matrix-product operators from graph representations. SciPost Phys., 3:035, 2017

$\left[\mathrm{PFO}^{+} 19\right]$ Sebastian Paeckel, Benedikt Fauseweh, Alexander Osterkorn, Thomas Köhler, Dirk Manske, and Salvatore R. Manmana. Detecting superconductivity out-of-equilibrium. arXiv e-prints, 1905.08638:arXiv:1905.08638, May 2019

Additionally, while working on this thesis the author contributed to the publications listed below.

$\left[\mathrm{SBP}^{+} 19\right]$ Oskar Schnaack, Niklas Bölter, Sebastian Paeckel, Salvatore R. Manmana, Stefan Kehrein, and Markus Schmitt. Tripartite information, scrambling, and the role of hilbert space partitioning in quantum lattice models. Physical Review B, 100:224302, Dec 2019

[KRS $\left.{ }^{+} 18\right]$ Thomas Köhler, Sangeeta Rajpurohit, Ole Schumann, Sebastian Paeckel, Fabian R. A. Biebl, Mohsen Sotoudeh, Stephan C. Kramer, Peter E. Blöchl, Stefan Kehrein, and Salvatore R. Manmana. Relaxation of photoexcitations in polaron-induced magnetic microstructures. Physical Review B, 97:235120, Jun 2018

[KPM18] Thomas Köhler, Sebastian Paeckel, and Salvatore R. Manmana. Charge density patterns in spin-selectively photoexcited interacting fermions. arXiv e-prints, page arXiv:1808.02138, Aug 2018 
To Giulia 



\section{Table of contents}

\section{Theory}

2 Quantum many-body systems $\quad 7$

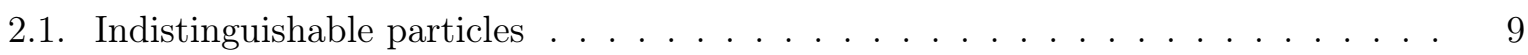

2.2. Second quantization . . . . . . . . . . . . . . . . . . . 12

2.3. Tight-binding approximation and the Hubbard model . . . . . . . . . . . . . 14

3 Order in quantum many-body systems $\quad 19$

3.1. Phase transitions . . . . . . . . . . . . . . . . . . . . 20 20

3.1.1. Spontaneous symmetry breaking . . . . . . . . . . . . . 22

3.1.2. Correlation functions and off-diagonal long-range order . . . . . . . . . 25

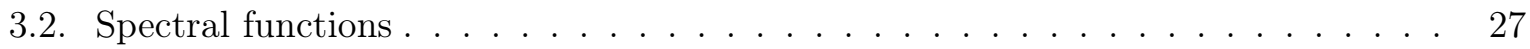

3.2.1. Spectral functions from Greens function . . . . . . . . . . . . . . 28

3.2.2. Spectral function of non-interacting electrons . . . . . . . . . . . 32

3.3. Superconductivity . . . . . . . . . . . . . . . . . . 33

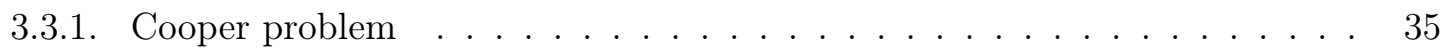

$3.3 .2 . \quad$ BCS theory . . . . . . . . . . . . . . . . . . . 38

3.3.3. Beyond BCS and non-equilibrium superconductivity . . . . . . . . . . . 40

3.4. Topological superconductivity . . . . . . . . . . . . . . . . . . . . 42

3.4.1. Bogoliubov de-Gennes representation . . . . . . . . . . . . . . . . . . . . . . . 42

3.4.2. Majorana zero modes . . . . . . . . . . . . . . . . . . . . 44

3.4.3. Edge states and topological phase . . . . . . . . . . . . . . . 46

3.4.4. Majorana number and topological index . . . . . . . . . . . . . . . 49

3.4.5. Topological superconductors beyond the Kitaev chain . . . . . . . . . . 51

3.4.6. Majorana oscillations and the Albrecht experiment . . . . . . . . . . . 52

\section{Methods}

4 Matrix-product states $\quad \mathbf{5 7}$

4.1. Notation . . . . . . . . . . . . . . . . . . . . . . . . 59

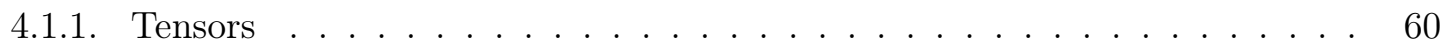

4.1.2. Matrix-product states (MPS) _ . . . . . . . . . . . . . . 60

4.1.3. Matrix-product operators $(\mathrm{MPO}) \ldots \ldots \ldots$. . . . . . . . . . 62

4.2. MPS manipulations . . . . . . . . . . . . . . . . . . . . 63 
4.2.1. Canonical form . . . . . . . . . . . . . . . . . . . . . 63

4.2.2. Normalizing an MPS . . . . . . . . . . . . . . . . . . . 64

4.2.3. Truncating an MPS . . . . . . . . . . . . . . . . 64

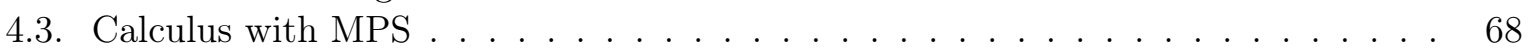

4.3.1. Application of an MPO to an MPS . . . . . . . . . . . . . 68

4.3.2. Expectation values . . . . . . . . . . . . . . . . . . . 71

4.4. Ground-state search . . . . . . . . . . . . . . . . . . . 72

4.4.1. Formulation as local optimization-problem . . . . . . . . . . . . . 72

4.4.2. Algorithm . . . . . . . . . . . . . . . . . . . . . . . . . . 75

4.4.3. Convergence and extensions . . . . . . . . . . . . . . . . 81

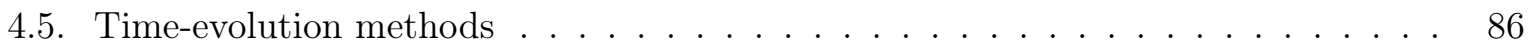

4.5.1. The MPO $W^{\mathrm{I} I \mathrm{II}} \operatorname{method} \ldots \ldots \ldots \ldots \ldots$. . . . . . . . . . 87

4.5.2. Approximating the action of $\hat{U}(\delta) \ldots \ldots \ldots \ldots$. . . . . . . . . . 95

4.5.3. Local Krylov . . . . . . . . . . . . . . . . . . . . . . . . . 97

4.5.4. Time-dependent variational principle (TDVP) . . . . . . . . . . . . 111

4.6. Exploiting $U(1)$ symmetries . . . . . . . . . . . . . . . . . . . . . . . . . . . . 119

4.6.1. Reducing computational complexity exploiting symmetries . . . . . . . . 120

4.6.2. Decomposition of general tensor networks . . . . . . . . . . . . . . 121

4.6.3. Implementation . . . . . . . . . . . . . . . . . . . . . . . 124

$5 U(1)$ symmetric matrix-product operators and finite-state machines $\quad \mathbf{1 2 7}$

5.1. MPO construction from FSMs . . . . . . . . . . . . . . . . . 127

5.2. Two-site gates as building blocks for $U(1)$ symmetric MPOs . . . . . . . . . . 130

5.3. $U(1)$-invariant MPO representation from FSMs . . . . . . . . . . . . . 133

5.4. MPO compression, graph arithmetics and implementation . . . . . . . . . 135

6 Projected purifications to restore $U(1)$ symmetries $\quad 137$

6.1. Projected purified operators . . . . . . . . . . . . . . . . . 138

6.2. Projected purified MPS . . . . . . . . . . . . . . . . . 140

6.2.1. Characterization of numerical expenses . . . . . . . . . . . . . . 143

6.2.2. Numerical testcase: Groundstate of the Holstein model . . . . . . . . . . . 147

\section{Systems}

7 Coulomb blockaded topological superconductivity 153

7.1. Proximity-coupled isolated superconducting island . . . . . . . . . . . . . 154

7.1.1. Gauge transformation . . . . . . . . . . . . . . . . 155

7.1.2. Topological and trivial phases . . . . . . . . . . . . . . . . 157

7.1.3. Charging energy and Coulomb blockade . . . . . . . . . . . . . . 158

7.2. Proximity-coupled superconducting island coupled to a normal lead . . . . . . . . 161

7.2.1. Charge fluctuations . . . . . . . . . . . . . . . . . 162

7.2.2. Charge-degeneracy points oscillations . . . . . . . . . . . . . . . 169

7.3. Proximity-coupled superconducting island coupled to two normal leads . . . . . . 173

7.3.1. Mapping to a ladder geometry . . . . . . . . . . . . . . . . . . . 174

7.3.2. Charge-degeneracy points oscillations . . . . . . . . . . . . . . 175

7.3.3. Permanent ring current and conductance . . . . . . . . . . . . . . 178

7.4. Summary and Outlook . . . . . . . . . . . . . . . . . . 180 


\section{Detecting Superconductivity out-of Equilibrium}

8.1. Introduction . . . . . . . . . . . . . . . . . . . . . . . . 183

8.2. Model . . . . . . . . . . . . . . . . . . . . . . . . . . . 184

8.3. Signatures of superconductivity out-of equilibrium _ . . . . . . . . . . 185

8.3.1. Time-dependent optical conductivity . . . . . . . . . . . . . . . 185

8.3.2. Spectral Functions . . . . . . . . . . . . . . . . . . . . . . . . . . 189

8.3.3. Convergence . . . . . . . . . . . . . . . . . . . . 193

8.4. Correlation matrices . . . . . . . . . . . . . . . . . . . . . 195

8.5. Comparison to finite temperature states . . . . . . . . . . . . 196

8.6. Summary and Outlook . . . . . . . . . . . . . . . . . . . . 197

9 Conclusion $\quad 199$

References in Alphabetical Order of Labels 203 



\section{\begin{tabular}{l|l} 
& \\
Introduction & 1
\end{tabular}}

Superconductors, semiconductors, Lasers, LEDs, magnets: The world we are used to would not be the same if there were no electrons correlating and interacting with each other, governed by the laws of quantum mechanics. As an abstract theory developed to describe atomic spectra, the quantum mechanics of electrons eventually sneaked into our every-day life with the invention of the transistor in 1947 by Shockley, Bardeen, and Brattein and the advent of commercial microelectronics in the 1970s. Notably, Bohr already realized in 1911 that without quantum mechanics, there would be no permanent magnetism in solids [Ros72]. Today we know that the vast majority of spontaneous magnetic order is an outcome of strong electron-electron interactions.

Of the listed phenomena, surely, one of the most intriguing is superconductivity, which was discovered in 1911 by Heike Kamerlingh Onnes in solid mercury. ${ }^{1}$ The property to have electric transport with zero resistance is exciting by itself. But the discovery of Meissner in 1933 that superconductors expell magnetic flux upon cooling below their critical temperature [MO33] had a deep theoretical meaning. In fact, the Meissner effect is not a result of the vanishing resistance but an independent property of the superconducting state. It paved the way to formulate phenomenologic and eventually microscopic theories for superconductivity by the important conclusion that superconductivity is not just a curious transport phenomenon but a thermodynamic phase.

However, it took another 20 years and the development of a language to quantum-mechanically describe many-body systems before the microscopic sources of superconductivity became tangible. The discovery by Leon Cooper in 1956 that the Fermi sea is unstable against an arbitrary weak attractive interaction causing the formation of bound electron pairs (Cooper pairs) may have been the last piece in the puzzle to be solved. Consequently, in 1957, Bardeen, Cooper, and Schrieffer (BCS) came up with their famous theory yielding not only a microscopic model but also the corresponding ground state [BCS57]. They introduced an effective interaction between electrons in a solid that is generated by lattice distortions caused by the electrons themselves, moving through the ionic lattice. Being conceptually simple, the BCS model is in fact independent of the underlying mechanism that causes the attractive interaction between the electrons. This stimulated both theoretical and experimental efforts to look for alternative pairing mechanisms of electrons, the so-called unconventional superconductivity, lasting until today. Here, one goal is to understand the origin of the high-temperature superconductivity in copper oxides [Dag94], which was discovered by Bednorz and Müller in 1986 [BM86].

In fact, Zhang and Rice [ZR88] only shortly after realized that a possible effective microscopic model for the copper oxides is the strongly interacting limit of the Hubbard model [Hub63, Gut63, Kan63, $\mathrm{EFG}^{+} 05$ ] describing two-dimensional $\mathrm{CuO}$ planes. While the latter was introduced originally in 1963 to describe ferromagnetism in itinerant electron systems, the strong correlations between electrons give rise to a much richer phase diagram. A significant amount of effort has been invested until today to understand this phase diagram in two dimensions, in particular at low temperatures. Here, a major obstacle is the fact that in lower spatial dimensions

\footnotetext{
${ }^{1}$ Dutch experimentalists seem to have a good hand for breakthrough discoveries related to superconductivity, one may look for instance at $\left[\mathrm{MZF}^{+} 12\right]$.
} 
quantum fluctuations are more relevant suspending a mean-field treatment. In turn, numerical techniques become more important enabling the study of low-energy excitations such as dynamical mean-field theory (DMFT) [GKKR96], variational cluster approaches [PAD03, Sén03], or the density-matrix renormalization group (DMRG) [Whi92].

Recently, the search for unconventional superconductivity gained another twist from the extremly high level of control allowing to design ultra-short terahertz $(\mathrm{THz})$ laser pulses on the subpicosecond time-scale [Cav18]. Targeting single phonon modes of certain copper oxides, transient states are created that exhibit signatures of enhanced superconductivity far above the equilibrium critical temperature $\left[\mathrm{KHN}^{+} 14\right]$. However, due to the extremly short time scales experimental measures probing superconducting signatures directly are somewhat rare.

We take up exactly this point by studying spectral functions of pair-excitations probing the formation of a superconducting condensate. The corresponding experimental technique, timeand angle-resolved photo-emission spectroscopy, is a standard tool for measuring single-particle excitation. However, since there is no direct microscopic interaction term in the usually employed semi-classical approach coupling two electrons to one photon, corresponding experimental techniques require different analysis schemes. Recent suggestions to evaluate the momentum correlations between photo-excited electrons [SE19] may be an important step forward. We believe that according to our results this will yield a unique tool to study the emergence of superconductivity out-of equilibrium.

Another striking feature of superconductors is the fact that in BCS theory the elementary excitations are superpositions of electrons and holes. Such quasi-particles have the potential to feature exotic properties. It was due to Kitaev to come-up with a model that demonstrated that this potential can turn into real exotic physics. He observed that in a simple one-dimensional chain of spinless fermions a nearest-neighbor $p$-wave pairing term can give rise to a topological phase, which features unpaired Majorana edge modes [Kit01]. Originally proposed as a real solution to the Dirac equation by Ettore Majorana in 1937, Majorana fermions appeared in different contexts since then, most prominently as a candidate for describing the neutrino. The defining property of Majorana fermions is the fact that they are their own antiparticles, i.e., their creation and annihilation operators are identical. From a pragmatic point of view, there is nothing too special about this, since every pair of fermionic ladder operators can be decomposed into its real and imaginary parts, which are exactly given by Majorana operators. However, Kitaev's insight was that in a topological superconductor such a pair of Majorana fermions constituting one normal fermion can be spatially separated, for instance to the ends of a one-dimensional superconducting wire. Upon separating Majorana fermions sufficiently they can become stable if the solid in between has a finite bulk gap, so that they do not interact and annihilate each other. However, they are still representing a single fermion but this is now delocalized over the whole system. This non-local structure renders the resulting single-particle state very robust against local perturbations. Additionally, these so-called Majorana edge modes obey a non-Abelean statistics (which is typical for topological phases). Therefore, exchanging two Majoranas the wavefunction picks up a complex phase factor that can be exploited to construct unitary operations (braiding), which implement quantum logical gates [SFN15]. In summary, the topological protection combined with the possibility of separate manipulations renders Majorana edge modes a promising candidate for a highly fault-tolerant qubit, the elementary building block of a quantum computer.

Recently, there has been a lot of effort to create samples which feature such Majorana edge modes. A key observation by Fu and Kane [FK08] was that proximitizing an $s$-wave superconductor with a topological insulator featuring strong spin-orbit coupling, the resulting heterostructure can become a topological superconductor if time-reversal symmetry is broken by an external magnetic field. However, creating a well-behaved two-dimensional interface comes with several practical 
problems. Nevertheless, the crucial insight was the necessity of strong spin-orbit coupling and it turned out that the same physics can be realized in one-dimensional heterostructures made-up of a semiconducting nanowire proximity-coupled to an $s$-wave superconductor [LSDS10].

The following experimental works indeed found indications for Majorana edge modes by studying transport properties where the presence of Majoranas can give rise to zero-bias conductance peaks. However, such peaks can also be generated by other mid-gap bound states and in fact, heterostructures are prone to exhibit a lot of them. A more direct measure would be to probe the exponential localization of the Majorana edge modes as suggest by das Sarma [DSSS12]. A corresponding experiment was realized by Albrecht et al. in 2016 where they reported the observation of exactly such signatures $\left[\mathrm{AHM}^{+} 16\right]$. Exploiting tunneling contacts on a floating nanowire in the Coulomb blockade regime enables them to study characteristic oscillations in conductance peaks whose amplitude yields a measure for the localization length of the Majorana edge modes. Naturally, such transport experiments require a finite tunneling amplitude between the leads and the proximity-coupled nanowire. However, the effects of quantum fluctuations induced by the hybridization between the Majorana edge modes and the leads have to be taken into account when analyzing the conductance-peak oscillations.

At this point, the analysis of the experimental data by Albrecht et al. has to be treated with care, since they used an isolated nanowire to draw their conclusions. Therefore, we studied the behavior of such proximitized nanowires in the Coulomb blockade regime coupled to normal leads for a broad range of tunneling amplitudes. Since perturbative results are available only for the weak- and strong-tunneling limit we aim to fill this gap with our studies trying to cover exactly the intermediate tunneling regime, which should be the experimentally most relevant.

Throughout this thesis, we used state-of-the-art numerical methods based on matrix-product state (MPS) representations to tackle current problems of unconventional superconductivity inand out-of equilibrium. In equilibrium, we studied a mesoscopic model system which is a promising candidate for the experimental verification of Majorana edge modes. We treated this very challenging numerical problem using a newly developed variant of a DMRG groundstate algorithm (Projected Purified DMRG, Chap. 6) which allows us to efficiently model the charging energy, the latter being represented by large bosonic Hilbert spaces. Out-of equilibrium we investigated the effect of optically pumped phonon modes on electron correlations by means of a global quantum quench. By analyzing in detail state-of-the-art MPS algorithms for nonequilibrium situations and for the calculation of dynamical properties, we were able to calculate time-dependent dynamical spectral functions for this challenging non-equilibrium problem. Motivated by our findings, we propose pairing spectral functions as indicator for the emergence of non-equilibrium superconductivity, which we hope will be measurable in future experiments. 

Theory 



\section{Quantum many-body systems 2}

Often, quantum mechanics is introduced as the theory that describes the motion and interaction of small particles on the atomic and sub-atomic scale (see for example [Sak94, Gri04]). Putting aside the question of what is meant by saying atomic and sub-atomic scale, typical questions that led to the formulation of quantum mechanics are: What are the trajectories of an electron in an electrostatic potential? What are the bound states? How does an electron interact with an electromagnetic field? One may notice that these problemes are mostly dealing with only one particle (here the electron). This is not by accident. The non-relativistic Schrödinger equation was proposed to describe the stationary states and motions of an electron in the hydrogen atom [Sch26]

$$
\mathrm{i} \hbar \frac{d}{d t} \psi(t)=\hat{H}(t) \psi(t) .
$$

Here, $\hat{H}(t)$ is the Hamilton operator, which in case of the hydrogen atom is that of an electron in the electrostatic potential generated by a positive point charge at the origin

$$
\hat{H}=\frac{\hat{\mathbf{p}}^{2}}{2 m_{e}}-\frac{e^{2}}{4 \pi \varepsilon_{0}|\hat{\mathbf{r}}|},
$$

where $m_{e}$ is the electron mass, $e$ the elementary charge, and $\varepsilon_{0}$ the permitivity of the free vacuum. $\hat{\mathbf{p}}, \hat{\mathbf{r}}$ are canonically conjugate operators, which are given by

$$
\hat{\mathbf{p}}=-\mathrm{i} \hbar \nabla_{\mathbf{r}}, \quad \hat{\mathbf{r}}=\mathbf{r}
$$

in the position representation, and which obey the commutation relation

$$
\left[\hat{r}_{\alpha}, \hat{p}_{\beta}\right]=\mathrm{i} \hbar \delta_{\alpha, \beta}
$$

Note that here and in the following we use Greek indices to label spatial dimensions and Latin indices to enumerate different particles or lattice sites. The wave function $\psi(t)$ contains the information about the state of the electron and in the position representation $\psi(\mathbf{r}, t)$ can be intepreted as probability amplitude so that $|\psi(\mathbf{r}, t)|^{2}$ gives the probability density to detect the electron at time $t$ in the region $[\mathbf{r}, \mathbf{r}+d \mathbf{r}]$. Additionally, the probability-density interpretation imposes a normalization constraint on $\psi(t)$, which has to be square-integrable and normalized. But how can we then attempt to describe mesoscopic or even macroscopic systems with $10^{23}$ particles in it? The rescue is that the Hamilton operator can be written more generally in terms of the operators for kinetic and potential energy

$$
\hat{H}(t)=\sum_{j=1}^{N}\left(\hat{T}_{j}+\hat{V}\left(\mathbf{r}_{j}, t\right)\right)+\hat{V}_{I}(t) .
$$

Each operator $\hat{T}_{j}=\frac{\hat{\mathbf{p}}_{j}^{2}}{2 m}$ captures the kinetic energy of a single particle with mass $m$ and $\hat{V}\left(\mathbf{r}_{j}, t\right)$ describes the potential energy of the $j$ th particle, while $\hat{V}_{I}(t)$ contains interactions between the particles. The representations of the potential energy operators can be obtained by taking the 
classically expected potential and replacing $\mathbf{r}_{j} \rightarrow \hat{\mathbf{r}}_{j}, \mathbf{p}_{j} \rightarrow \hat{\mathbf{p}}_{j}$. Looking at Eq. (2.5), the whole dilemma becomes apparent. In this representation, the Hamilton operator for $N$ particles is a partial differential equation of $3 N$ spatial coordinates, which are coupled by the interaction potential $\hat{V}_{I}(t)$. For any given potential containing at least pairwise interactions there is no way to solve this problem exactly. One way out of this problem is to ignore interactions between the particles for a moment. Assuming that $\hat{V}\left(\mathbf{r}_{j}, t\right) \equiv \hat{V}\left(\mathbf{r}_{j}\right)$, i.e., there is no time-dependence, we can look for stationary solutions $\psi\left(\mathbf{r}_{j}\right)$ for each particle independently

$$
\hat{H}_{j} \psi\left(\mathbf{r}_{j}\right)=\left(\frac{\hat{\mathbf{p}}_{j}^{2}}{2 m}+\hat{V}\left(\mathbf{r}_{j}\right)\right) \psi\left(\mathbf{r}_{j}\right)=\varepsilon \psi\left(\mathbf{r}_{j}\right) .
$$

The last equality follows from the fact that for time-independent Hamiltonians inserting the ansatz $\psi\left(\mathbf{r}_{1}, \ldots, \mathbf{r}_{N}, t\right)=\psi\left(\mathbf{r}_{1}, \ldots, \mathbf{r}_{N}\right) \chi(t)$ and $\psi\left(\mathbf{r}_{1}, \ldots, \mathbf{r}_{N}\right)=\psi\left(\mathbf{r}_{1}\right) \cdots \psi\left(\mathbf{r}_{N}\right)$ into the left and right side of the time-dependent Schrödinger equation Eq. (2.1) they have to equal each other independent on the choice of $\left(t, \mathbf{r}_{j}\right)$; that is, they are constants. We set this constant to $\varepsilon$ as it corresponds to the expectation value of $\hat{H}_{j}$ being the energy of the corresponding singleparticle state $\psi\left(\mathbf{r}_{j}\right)$. Usually, there are boundary conditions so that we obtain a discrete set of solutions $\varphi_{k}\left(\mathbf{r}_{j}\right)(k \in \mathbb{N})$ fulfilling

$$
\hat{H}_{j} \varphi_{k}\left(\mathbf{r}_{j}\right)=\varepsilon_{k} \varphi_{k}\left(\mathbf{r}_{j}\right), \quad \text { and } \quad \int_{\mathbb{R}^{3}} d^{3} \mathbf{r}_{j} \varphi_{k}^{*}\left(\mathbf{r}_{j}\right) \varphi_{l}\left(\mathbf{r}_{j}\right)=\delta_{k, l} .
$$

Using multilinearity of the Hamilton operator we can construct stationary solutions, i.e., eigenstates of $\hat{H}$ by forming products from the individual eigenstates of each particle

$$
\varphi_{k_{1}, \ldots, k_{N}}\left(\mathbf{r}_{1}, \ldots, \mathbf{r}_{N}\right)=\varphi_{k_{1}}\left(\mathbf{r}_{1}\right) \cdots \varphi_{k_{N}}\left(\mathbf{r}_{N}\right)
$$

The constructed many-body states have energies

$$
\varepsilon=\int_{\mathbb{R}^{3 N}} d^{3 N} \mathbf{r} \varphi_{k_{1}, \ldots, k_{N}}^{*}\left(\mathbf{r}_{1}, \ldots, \mathbf{r}_{N}\right)\left[\sum_{j=1}^{N} \hat{H}_{j}\right] \varphi_{k_{1}, \ldots, k_{N}}\left(\mathbf{r}_{1}, \ldots, \mathbf{r}_{N}\right)=\sum_{j=1}^{N} \varepsilon_{k_{j}}
$$

There is a degeneracy in the eigenstates constructed this way. Exchanging for instance the single-particle states $k_{i} \leftrightarrow k_{j}$ that is, transforming the many-body states

$$
\varphi_{k_{1}}\left(\mathbf{r}_{1}\right) \cdots \varphi_{k_{i}}\left(\mathbf{r}_{i}\right) \cdots \varphi_{k_{j}}\left(\mathbf{r}_{j}\right) \cdots \varphi_{k_{N}}\left(\mathbf{r}_{N}\right) \rightarrow \varphi_{k_{1}}\left(\mathbf{r}_{1}\right) \cdots \varphi_{k_{j}}\left(\mathbf{r}_{i}\right) \cdots \varphi_{k_{i}}\left(\mathbf{r}_{j}\right) \cdots \varphi_{k_{N}}\left(\mathbf{r}_{N}\right),
$$

we obtain the same energy. Reordering then reveals that this is equivalent to an exchange of the particle's coordinates $\mathbf{r}_{i} \leftrightarrow \mathbf{r}_{j}$. Obviously, the Hamilton operator $\hat{H}$ for the many-particle problem is invariant under such an exchange of the coordinates. However, the product states created by a particle exchange are orthogonal to each other. Intuitively, we would expect that the eigenstates of $\hat{H}$ should somehow transform under particle exchange, too. This intuition turns out to touch one of the fundamental differences between quantum and classical mechanics. And as if that were not enough it gives rise to a reformulation putting the emphasis away from the probabilistic wavefuntions to an operator representation, yielding a convenient way to describe quantum many-body systems (see for instance [TMB61]). 


\section{\begin{tabular}{l|l} 
Indistinguishable particles & 2.1
\end{tabular}}

In quantum mechanics, the observation that if particles have the same observable properties (e.g., charge, mass, etc.), then they are indistinguishable, has some deep consequences. At first, this means that exchanging two particles, for instance by swapping their labels in the many-body wavefunction, leaves the overall state invariant. Therefore, the expectation value for an arbitrary hermitian operator $\hat{O}$ has to fulfill

$$
\begin{aligned}
& \int_{\mathbb{R}^{3 N}} d^{3 N} \mathbf{r} \varphi_{k_{1}, \ldots, k_{N}}^{*}\left(\mathbf{r}_{1}, \ldots, \mathbf{r}_{i}, \ldots, \mathbf{r}_{j}, \ldots, \mathbf{r}_{N}\right) \hat{O} \varphi_{k_{1}, \ldots, k_{N}}\left(\mathbf{r}_{1}, \ldots, \mathbf{r}_{i}, \ldots, \mathbf{r}_{j}, \ldots, \mathbf{r}_{N}\right) \\
= & \int_{\mathbb{R}^{3 N}} d^{3 N} \mathbf{r} \varphi_{k_{1}, \ldots, k_{N}}^{*}\left(\mathbf{r}_{1}, \ldots, \mathbf{r}_{j}, \ldots, \mathbf{r}_{i}, \ldots, \mathbf{r}_{N}\right) \hat{O} \varphi_{k_{1}, \ldots, k_{N}}\left(\mathbf{r}_{1}, \ldots, \mathbf{r}_{j}, \ldots, \mathbf{r}_{i}, \ldots, \mathbf{r}_{N}\right) .
\end{aligned}
$$

It is easy to see that the previously constructed eigenstates of $\hat{H}$ violate this condition if we set $\hat{O}=\hat{H}_{j}$. In fact, the condition can only be satisfied if the states transform as

$$
\varphi_{k_{1}, \ldots, k_{N}}\left(\mathbf{r}_{1}, \ldots, \mathbf{r}_{j}, \ldots, \mathbf{r}_{i}, \ldots, \mathbf{r}_{N}\right)=e^{\mathrm{i} \vartheta} \varphi_{k_{1}, \ldots, k_{N}}\left(\mathbf{r}_{1}, \ldots, \mathbf{r}_{i}, \ldots, \mathbf{r}_{j}, \ldots, \mathbf{r}_{N}\right) .
$$

The phase $\vartheta$ has an important meaning: in $d \geq 3$ spatial dimensions it can be shown that it can only posses values $\vartheta=0, \pi$. This is related to the spin of the particles under consideration, ${ }^{1}$ where $\vartheta=0$ corresponds to bosons with integer spin and $\vartheta=\pi$ to fermions with half integer spin. Importantly, in $d=2$ spatial dimensions $\vartheta$ can take any value giving rise to (quasi-)particles which are therefore called anyons, which is important in Sec. 3.4 and Chap. 7.

Returning back to the problem of finding physical eigenstates of $\hat{H}$, we therefore have to construct superpositions of all possible eigenstates belonging to one and the same many-particle energy $\varepsilon$ that obey the correct exchange behavior. This can be done by defining unitary symmetrization operators $\hat{S}_{\vartheta}$, which for the case of bosons and fermions are given by

$$
\begin{aligned}
& \hat{S}_{0}=\frac{1}{N !} \sum_{\pi \in \mathcal{P}_{N}} \hat{P}_{\pi}, \\
& \hat{S}_{\pi}=\frac{1}{N !} \sum_{\pi \in \mathcal{P}_{N}} \operatorname{sgn}(\pi) \hat{P}_{\pi} .
\end{aligned}
$$

Here, $\mathcal{P}_{N}$ is the group of permutations of $N$ elements, $\hat{P}_{\pi}$ the operator that applies the permutation $\left(\mathbf{r}_{1}, \ldots, \mathbf{r}_{N}\right) \rightarrow\left(\pi\left(\mathbf{r}_{1}\right), \ldots, \pi\left(\mathbf{r}_{N}\right)\right)$, and $\operatorname{sgn}(\pi)= \pm 1$ the signum of the permutation. The physically realizable eigenstates of $\hat{H}$ with energy $\varepsilon$ are obtained from the application of the proper sysmmetrization operator to one representant from the set of degenerated eigenstates with energy $\varepsilon$

$$
\begin{aligned}
\varphi_{0, \varepsilon}\left(\mathbf{r}_{1}, \ldots, \mathbf{r}_{N}\right) & =\sqrt{\frac{N !}{n\left(k_{1}\right) ! \cdots n\left(k_{N}\right) !}} \hat{S}_{0} \varphi_{k_{1}, \ldots, k_{N}}\left(\mathbf{r}_{1}, \ldots, \mathbf{r}_{1}\right) \\
\varphi_{\pi, \varepsilon}\left(\mathbf{r}_{1}, \ldots, \mathbf{r}_{N}\right) & =\sqrt{N ! \hat{S}_{\pi} \varphi_{k_{1}, \ldots, k_{N}}\left(\mathbf{r}_{1}, \ldots, \mathbf{r}_{1}\right)}
\end{aligned}
$$

\footnotetext{
${ }^{1}$ The spin-statistics theorem connects the particle spin and their exchange properties [SW78] but it can only be proven rigorously in quantum field theory [AM03].
} 
where $n\left(k_{j}\right)$ counts the number of appearances of the single-particle eigenstate with energy $\varepsilon_{k_{j}}$. Note, that the antisymmetrized wavefunction can be written as a Slater determinant if we arrange the single-particle states as a matrix

$$
\varphi_{\pi, \varepsilon}\left(\mathbf{r}_{1}, \ldots, \mathbf{r}_{N}\right)=\frac{1}{\sqrt{N !}}\left|\begin{array}{cccc}
\varphi_{k_{1}}\left(\mathbf{r}_{1}\right) & \varphi_{k_{1}}\left(\mathbf{r}_{2}\right) & \cdots & \varphi_{k_{1}}\left(\mathbf{r}_{N}\right) \\
\varphi_{k_{2}}\left(\mathbf{r}_{1}\right) & \varphi_{k_{2}}\left(\mathbf{r}_{2}\right) & \cdots & \varphi_{k_{2}}\left(\mathbf{r}_{N}\right) \\
\vdots & \vdots & \cdots & \vdots \\
\varphi_{k_{N}}\left(\mathbf{r}_{1}\right) & \varphi_{k_{N}}\left(\mathbf{r}_{2}\right) & \cdots & \varphi_{k_{N}}\left(\mathbf{r}_{N}\right)
\end{array}\right| .
$$

It is important to realize that for fermions no single-particle eigenstate can appear twice (that is $k_{j}=k_{l}$ for some $j \neq l$ ) because the Slater determinant vanishes in these cases. Now it is evident that the notation in terms of wavefunctions becomes increasingly tedious if we consider systems with many particles. However, having a look at the above normalization factors, we find an elegant way to resolve this problem. For this purpose, from now on we use the Dirac bra-ket notation. Therein, states $\psi$ are elements of an abstract Hilbert space $|\psi\rangle \in \mathcal{H}$ (called ket) or its dual space $\langle\psi| \in \mathcal{H}^{*}$ (called bra). Scalar products are denoted by forming a bra-ket $\langle\psi \mid \phi\rangle \in \mathbb{C}$. The many-body Hilbert space $\mathcal{H}^{N}$ of $N$ identical particles is spanned by the tensor product of $N$ Hilbert spaces $\mathcal{H}^{N}=\mathcal{H} \otimes \cdots \otimes \mathcal{H}$. Elements of such tensor product Hilbert spaces can then be expanded in terms of the tensor product of the basis states $|\sigma\rangle \in \mathcal{H}$ of each individual Hilbert space

$$
|\psi\rangle=\sum_{\sigma_{1}, \ldots, \sigma_{N}} c_{\sigma_{1}, \ldots, \sigma_{N}}\left|\sigma_{1}\right\rangle \otimes \cdots \otimes\left|\sigma_{N}\right\rangle \quad \sum_{\sigma_{1}, \ldots, \sigma_{N}}\left|c_{\sigma_{1}, \ldots, \sigma_{N}}\right|^{2}=1 .
$$

Note that in this example we assumed a discrete basis set $\left|\sigma_{n}\right\rangle$ but the notation can be generalized to continuous basis sets by simply replacing the sums with integrals and ensuring integrability of the basis coefficients $c_{\sigma_{1}, \ldots, \sigma_{N}}$. Most of the time we also drop the $\otimes$ between the basis-kets and group the labels into one large ket for convenience. Finally, we can write basis transformations by inserting a sum over the projectors onto all basis states of the (many-body tensor product) Hilbert space. We will denote such projectors by $|\sigma\rangle\langle\sigma|$ so that, for instance, we can formally expand any state $|\psi\rangle \in \mathcal{H}^{N}$ in terms of a given basis by writing

$$
|\psi\rangle=\sum_{\sigma_{1}, \ldots, \sigma_{N}}\left|\sigma_{1}, \ldots, \sigma_{N}\right\rangle \underbrace{\left\langle\sigma_{1}, \ldots, \sigma_{N} \mid \psi\right\rangle}_{\equiv c_{\sigma_{1}}, \ldots, \sigma_{N}}=\sum_{\sigma_{1}, \ldots, \sigma_{N}} c_{\sigma_{1}, \ldots, \sigma_{N}}\left|\sigma_{1}, \ldots, \sigma_{N}\right\rangle .
$$

Note that the scalar product $\left\langle\sigma_{1}, \ldots, \sigma_{N} \mid \psi\right\rangle$ evaluates to a $\mathbb{C}$-number, which we can pull in front of the basis-kets. Using this formalism the wavefunction in the position representation can be written formally as

$$
|\psi\rangle=\int_{\mathbb{R}^{3 N}} d^{3 N} \mathbf{r} \underbrace{\left\langle\mathbf{r}_{1}, \ldots, \mathbf{r}_{N} \mid \psi\right\rangle}_{\equiv \psi\left(\mathbf{r}_{1}, \ldots, \mathbf{r}_{N}\right)}\left|\mathbf{r}_{1}, \ldots, \mathbf{r}_{N}\right\rangle=\int_{\mathbb{R}^{3 N}} d^{3 N} \mathbf{r} \psi\left(\mathbf{r}_{1}, \ldots, \mathbf{r}_{N}\right)\left|\mathbf{r}_{1}, \ldots, \mathbf{r}_{N}\right\rangle .
$$

Here, the basis-kets $\left|\mathbf{r}_{j}\right\rangle$ are eigenstates of the position operator ${ }^{2} \hat{\mathbf{r}}_{j}\left|\mathbf{r}_{j}\right\rangle=\mathbf{r}_{j}\left|\mathbf{r}_{j}\right\rangle$ and thus form an orthonormal basis of the single-particle Hilbert space with $\left\langle\mathbf{r}_{j}^{\prime} \mid \mathbf{r}_{j}\right\rangle=\delta^{3}\left(\mathbf{r}_{j}-\mathbf{r}_{j}^{\prime}\right)$.

We can now build on this framework and write the eigenstates of the single-particle Hamiltonians $\hat{H}_{j}$ in the single-particle Hilbert space as $\left|\varepsilon_{k_{j}}\right\rangle \in \mathcal{H}$. Since $\hat{H}_{j}$ is hermitian and (bounded

\footnotetext{
${ }^{2}$ This is actually the reason for the representation of the momentum and position operators in the Hamiltonian Eq. (2.2). We already worked implicitely in the position basis and $\hat{H}$ is actually defined by its matrix elements $\left\langle\mathbf{r}_{1}^{\prime}, \ldots, \mathbf{r}_{N}^{\prime}|\hat{H}| \mathbf{r}_{1}, \ldots, \mathbf{r}_{N}\right\rangle$ in this basis.
} 
from below) these states form a complete orthonormal basis of $\mathcal{H}$ and we get the single-particle wavefunctions from the formal scalar product $\varphi_{k_{j}}\left(\mathbf{r}_{j}\right)=\left\langle\mathbf{r}_{j} \mid \varepsilon_{k_{j}}\right\rangle$. The physical eigenstates in the many-particle Hilbert space are then simply given by correctly symmetrized tensor-product states of the basis-kets

$$
\begin{aligned}
|\varepsilon\rangle_{0} & =\sqrt{\frac{N !}{n\left(k_{1}\right) ! \cdots n\left(k_{N}\right) !}} \hat{S}_{0}\left|\varepsilon_{k_{1}}, \ldots, \varepsilon_{k_{N}}\right\rangle, \\
|\varepsilon\rangle_{\pi} & =\sqrt{N ! \hat{S}_{\pi}\left|\varepsilon_{k_{1}}, \ldots, \varepsilon_{k_{N}}\right\rangle}
\end{aligned}
$$

where $\sum_{j=1}^{N} \varepsilon_{k_{j}}=\varepsilon$. Note that here the symmetrization operators $\hat{S}_{\vartheta}$ are permuting singleparticle eigenstates $\left(k_{1}, \ldots, k_{N}\right) \rightarrow\left(\pi\left(k_{1}\right), \ldots, \pi\left(k_{N}\right)\right)$. We can simplify the notation even more by writing the states in a way in which the indistinguishable nature of the particles becomes manifest. Therefore note that counting the number of occupied single-particle states $n\left(k_{j}\right)$ already specifies the whole state. We can hence define a set of states

$$
\left|n_{1}, n_{2}, \ldots\right\rangle_{\vartheta}=N_{\vartheta} \hat{S}_{\vartheta}\left|\varepsilon_{k_{1}}, \ldots, \varepsilon_{k_{N}}\right\rangle, \quad \sum_{k} n_{k}=N
$$

for all possible distributions of the single-particle states' occupations $n_{k}$ and we have abbreviated the normalization factor $N_{\vartheta}$ for bosons and fermions. It can be shown that these states form an orthonormal basis of a subset of $\mathcal{H}^{N}$ containing only the correctly symmetrized, physical states. We can therefore define the symmetrized $N$-particle Hilbert spaces

$$
\mathcal{H}_{\vartheta}^{N}=\operatorname{span}\left\{\left|n_{1}, n_{2}, \ldots\right\rangle_{\vartheta}\right\} \quad \text { with } \quad \sum_{k} n_{k}=N
$$

where the sum runs over all possible single-particle eigenstates.

Using this representation, we can easily find the ground states of non-interacting systems of fermions or bosons. At first, we have to solve the single-particle problem $\hat{H}_{j} \varphi_{j}=\varepsilon_{k_{j}} \varphi_{j}$ and sort the single-particle energies $\varepsilon_{k_{j}}$ so that $k_{j}<k_{j}^{\prime} \Leftrightarrow \varepsilon_{k_{j}}<\varepsilon_{k_{j}^{\prime}}$. Then, for $N$ bosons or fermions the ground state $|G S\rangle_{\vartheta}$ and ground-state energy $\varepsilon$ of the many-body problem $\hat{H}$ is simply given by

$$
\begin{aligned}
|G S\rangle_{0} & =\left|n_{1}=N, n_{2}=0, \ldots\right\rangle_{0}, \quad \varepsilon=N \varepsilon_{1}, \\
|G S\rangle_{\pi} & =\left|n_{1}=1, n_{2}=1, \ldots, n_{N}=1, n_{N+1}=0, \ldots\right\rangle, \quad \varepsilon=\sum_{k=1}^{N} \varepsilon_{k} .
\end{aligned}
$$

Here, the antisymmetrization for fermions restricts the occupations of the single-particle eigenstates to be $n_{j}=0,1$, i.e., the Pauli principle. A drastic consequence of these findings is that if we want to excite $M$ fermions out of the ground state, we have to pay an energy $\sum_{m=1}^{M} \sum_{k=0}^{m-1} \varepsilon_{N-k}$, i.e., for equally spaced single-particle energies the cost grows quadratically in $M$ ! This is the reason why even at room temperature in metals only a small number of electrons can be excited above the ground state and thereby contribute to transport processes. In contrast, for bosons the single-particle ground state can be occupied macroscopically. In the presence of interactions there can be a gap opening above the lowest single-particle energy level. This gives rise to Bose-Einstein condensation at finite temperatures. 


\section{Second quantization}

Exploiting the previously, introduced representation we can define occupation-number operators $\hat{n}_{k}$ measuring the number of appearances of the $k$ th single-particle eigenstate in the wavefunction (dropping the label to distinguish bosons and fermions for now)

$$
\hat{n}_{k}\left|n_{1}, \ldots, n_{k}, \ldots\right\rangle=n_{k}\left|n_{1}, \ldots, n_{k}, \ldots\right\rangle .
$$

The states $\left|n_{1}, n_{2}, \ldots\right\rangle$ are then eigenstates of the total particle-number operator $\hat{N}=\sum_{j} \hat{n}_{j}$

$$
\hat{N}\left|n_{1}, n_{2}, \ldots\right\rangle=N\left|n_{1}, n_{2}, \ldots\right\rangle \text {. }
$$

Clearly, there should be some way to write the Hamiltonian $\hat{H}$ in this occupation number representation even in the presence of interactions. For that purpose we introduce the symmetrized Fock space as the infinite direct sum of symmetrized $N$-particle Hilbert spaces ${ }^{3}$

$$
\mathcal{F}_{\vartheta}=\mathcal{H}_{\vartheta}^{0} \oplus \mathcal{H}_{\vartheta}^{1} \oplus \mathcal{H}_{\vartheta}^{2} \oplus \cdots,
$$

where $\mathcal{H}_{\vartheta}^{0}$ is the Hilbert space with 0 particles, which contains only the vacuum state. We can now introduce creation and annihilation operators as maps $\mathcal{H}_{\vartheta}^{N} \rightarrow \mathcal{H}_{\vartheta}^{N+1}$ and $\mathcal{H}_{\vartheta}^{N} \rightarrow \mathcal{H}_{\vartheta}^{N-1}$, respectively. Specifically for the creation operators we define

$$
\begin{aligned}
\hat{b}_{k}^{\dagger}\left|n_{1}, n_{2}, \ldots, n_{k}, \ldots\right\rangle & =\sqrt{N+1} \hat{S}_{0}\left(\left|\varepsilon_{k}\right\rangle \otimes\left|n_{1}, n_{2}, \ldots, n_{k}, \ldots\right\rangle\right) \\
& =\sqrt{n_{k}+1}\left|n_{1}, n_{2}, \ldots, n_{k}+1, \ldots\right\rangle, \\
\hat{c}_{k}^{\dagger}\left|n_{1}, n_{2}, \ldots, n_{k}, \ldots\right\rangle & =\sqrt{N+1} \hat{S}_{\pi}\left(\left|\varepsilon_{k}\right\rangle \otimes\left|n_{1}, n_{2}, \ldots, n_{k}, \ldots\right\rangle\right) \\
& =\left\{\begin{array}{ll}
e^{\mathrm{i} \pi \sum_{l<k} n_{l}}\left|n_{1}, n_{2}, \ldots, n_{k}+1, \ldots\right\rangle & \text { if } n_{k}=0 \\
0 & \text { if } n_{k}=1
\end{array},\right.
\end{aligned}
$$

where the normalization for the bosonic creation operators follows from the normalization factors in $\hat{S}_{0}$. Accordingly, the annihilation operators follow from taking the adjoints

$$
\begin{aligned}
& \hat{b}_{k}\left|n_{1}, n_{2}, \ldots, n_{k}, \ldots\right\rangle=\sqrt{n_{k}}\left|n_{1}, n_{2}, \ldots, n_{k}-1, \ldots\right\rangle, \\
& \hat{c}_{k}\left|n_{1}, n_{2}, \ldots, n_{k}, \ldots\right\rangle=\left\{\begin{array}{ll}
e^{\mathrm{i} \pi \sum_{l<k} n_{l}}\left|n_{1}, n_{2}, \ldots, n_{k}-1, \ldots\right\rangle & \text { if } n_{k}=1 \\
0 & \text { if } n_{k}=0
\end{array} .\right.
\end{aligned}
$$

Note that we have to account for the ordering of the single-particle eigenstates in the Slater determinant Eq. (2.17) by the phase factor $e^{\mathrm{i} \pi \sum_{l<k} n_{l}}$ when defining the fermionic creation/annihilation operators. It can be verified that they obey the (anti-)commutation relations

$$
\left[\hat{b}_{k}, \hat{b}_{l}^{\dagger}\right]=\delta_{k, l}, \quad\left\{\hat{c}_{k}, \hat{c}_{l}^{\dagger}\right\}=\delta_{k, l},
$$

where the curly brackets indicate the anticommutator. The occupation number operators can now be represented in terms of the creation and annihiliation operators

$$
\hat{n}_{k}^{(b)}=\hat{b}_{k}^{\dagger} \hat{b}_{k}, \quad \hat{n}_{k}^{(c)}=\hat{c}_{k}^{\dagger} \hat{c}_{k}
$$

\footnotetext{
${ }^{3}$ We keep the definitions also applicable to the anyonic case, which is indicated by carrying around the subscript $\vartheta$. That said, speaking about symmetrized Hilbert spaces and Fock spaces we always refer to correctly symmetrized spaces, which can be symmetric or antisymmetric under particle exchange, or even pick up an arbitrary complex phase.
} 
and we drop the superscript for bosons or fermions if the context in which they appear is clear. An important observation here is that employing the vacuum state $|\Omega\rangle_{\vartheta} \in \mathcal{H}_{\vartheta}^{0}$ a correctly symmetrized many-body state can be written as

$$
\begin{aligned}
\left|n_{1}, n_{2}, \ldots\right\rangle_{0} & =\frac{1}{\sqrt{n_{1} ! n_{2} ! \cdots}}\left[b_{1}^{\dagger}\right]^{n_{1}}\left[b_{2}^{\dagger}\right]^{n_{2}} \cdots|\Omega\rangle_{0}, \\
\left|n_{1}, n_{2}, \ldots\right\rangle_{\pi} & =\left[c_{1}^{\dagger}\right]^{n_{1}}\left[c_{2}^{\dagger}\right]^{n_{2}} \cdots|\Omega\rangle_{\pi} .
\end{aligned}
$$

This way, the correct symmetrization is completely mapped to the algebra of creation and annihilation operators and in case of fermions the ordering of the creation operators fixes the ordering of the single-particle states in the generated Slater determinant.

We can now express operators acting on the correctly symmetrized many-particle Hilbert spaces $\mathcal{H}_{\vartheta}^{N}$ in terms of the defined creation and annihilation operators (which are also called ladder operators) acting on the Fock space. A single-particle operator is expressed in terms of ladder operators by expansion in terms of the single-particle states $\left|\varepsilon_{k}\right\rangle_{j}$ at site $j$ and we sketch the derivation of the representation exemplary

$$
\begin{aligned}
\sum_{j=1}^{N} \hat{O}_{j}\left|n_{1}, n_{2}, \ldots\right\rangle_{\vartheta} & =\sum_{j=1}^{N} \sum_{k, l}[\left|\varepsilon_{k}\right\rangle_{j} \underbrace{\left\langle\varepsilon_{k}\left|\hat{O}_{j}\right| \varepsilon_{l}\right\rangle_{j}}_{:=O_{k, l}} j\left\langle\varepsilon_{l}\right|]\left|n_{1}, n_{2}, \ldots\right\rangle_{\vartheta} \\
& =\sum_{k, l} \sum_{j=1}^{N}\left[O_{k, l}\left|\varepsilon_{k}\right\rangle_{j j}\left\langle\varepsilon_{l}\right|\right] \sqrt{N !} \hat{S}_{\vartheta}\left|\varepsilon_{l}\right\rangle_{j} \otimes\left|n_{1}, n_{2}, \ldots, n_{l}-1, \ldots\right\rangle_{\vartheta} \\
& =\sum_{k, l} O_{k, l} \sqrt{n_{k}+1} \sqrt{n_{l}}\left|n_{1}, n_{2}, \ldots, n_{k}+1, \ldots, n_{l}-1, \ldots\right\rangle
\end{aligned}
$$

where we utilized that the symmetrization operator commutes with $\sum \hat{O}_{j}$. Comparing the last line to the definition of the creation and annihilation operators for bosons and fermions we obtain

$$
\begin{aligned}
& \sum_{j=1}^{N} \hat{O}_{j}\left|n_{1}, n_{2}, \ldots\right\rangle_{0}=\sum_{k, l} O_{k, l} \hat{b}_{k}^{\dagger} \hat{b}_{l}\left|n_{1}, n_{2}, \ldots\right\rangle_{0}, \\
& \sum_{j=1}^{N} \hat{O}_{j}\left|n_{1}, n_{2}, \ldots\right\rangle_{\pi}=\sum_{k, l} O_{k, l} \hat{c}_{k}^{\dagger} \hat{c}_{l}\left|n_{1}, n_{2}, \ldots\right\rangle_{0} .
\end{aligned}
$$

A completely analog derivation yields for two-particle operators

$$
\begin{aligned}
\sum_{i \neq j=1}^{N} \hat{O}_{i, j}\left|n_{1}, n_{2}, \ldots\right\rangle_{0} & =\sum_{k, l, r, s} O_{k l, r s} \hat{b}_{k}^{\dagger} \hat{b}_{l}^{\dagger} \hat{b}_{r} \hat{b}_{s}\left|n_{1}, n_{2}, \ldots\right\rangle_{0}, \\
\sum_{i \neq j=1}^{N} \hat{O}_{i, j}\left|n_{1}, n_{2}, \ldots\right\rangle_{\pi} & =\sum_{k, l, r, s} O_{k l, r s} \hat{c}_{k}^{\dagger} \hat{c}_{l}^{\dagger} \hat{c}_{s} \hat{c}_{r}\left|n_{1}, n_{2}, \ldots\right\rangle_{\pi},
\end{aligned}
$$

with $O_{k l, r s}={ }_{i, j}\left\langle\varepsilon_{k}, \varepsilon_{l}\left|\hat{O}_{i, j}\right| \varepsilon_{r}, \varepsilon_{s}\right\rangle_{i, j}$. Note the interchanged ordering of the single-particle annihilation operators in the fermionic case. The generalization to $n$-particle interactions is straightforward. Additionally, we have to take into consideration vacuum contributions, which are simply given by evaluating $\langle\Omega|\hat{O}| \Omega\rangle$ for operators $\hat{O}$ acting independently on the particle's coordinates. 
In the above described formalism, the Hamilton operator for non-interacting fermions is already diagonal because the single-particle states used to construct the many-body wavefunctions are the eigenstates of single-particle Hamiltonians. For a finite two-particle interaction $V\left(\mathbf{r}_{i}, \mathbf{r}_{j}\right)$ we therefore obtain the fermionic Hamiltonian $\hat{H}$ in the Fock-space representation, i.e., second quantization

$$
\hat{H}=\sum_{k} \varepsilon_{k} \hat{n}_{k}+\sum_{k, l, r, s} V_{k l, r s} \hat{c}_{k}^{\dagger} \hat{c}_{l}^{\dagger} \hat{c}_{s} \hat{c}_{r}
$$

This reformulation promotes the probabilistic state representation in terms of wavefunction depending on $3 N$ continuous variables to an operator representation, which motivated the naming second quantization. On the one hand, we can now proceed and introduce field operators by transforming the ladder operators for single-particle eigenstates of $\hat{H}_{j}$ into the position representation. For instance, for fermions we can define

$$
\hat{\psi}^{\dagger}(\mathbf{r})=\sum_{k} \varphi_{k}^{*}(\mathbf{r}) \hat{c}_{k}^{\dagger}, \quad \text { and } \quad \hat{\psi}(\mathbf{r})=\sum_{k} \varphi_{k}(\mathbf{r}) \hat{c}_{k} .
$$

From orthonormalization of the single-particle eigenstates and the anticommutation relations of the fermionic ladder operators, the field operators obey canonical anticommutation relations $\left\{\hat{\psi}(\mathbf{r}), \hat{\psi}\left(\mathbf{r}^{\prime}\right)\right\}=\delta^{3}\left(\mathbf{r}-\mathbf{r}^{\prime}\right)$ and can be interpreted to create/annihilate a fermion at position $\mathbf{r}$. Constructing a density operator $\hat{\rho}(\mathbf{r})=\hat{\psi}^{\dagger}(\mathbf{r}) \hat{\psi}(\mathbf{r})$ is then a starting point to the formulation of the density-functional theory [HK64, KS65], which is one of the most powerful ab-initio methods to calculate groundstate properties of many-electron systems. On the other hand, writing the Hamilton operator in second quantization maps the problem of solving coupled differential equations in $3 N$ variables to a discrete one. Using the matrix representation of $\hat{H}$ in the occupation number basis enables us to use tensor-network states as an ansatz class for the coefficients of the wavefunctions. This is the fundament of the MPS description employed throughout this thesis to study quantum-many body systems. ${ }^{4}$

\section{Tight-binding approximation and the Hubbard model}

So far we have not further specified the single-particle potential $\hat{V}\left(\mathbf{r}_{j}\right)$ in Eq. (2.5). In the scope of this thesis we studied the electronic properties of crystals. It is therefore of major importance to account for the potential created by the atomic nucleus. In the following, we briefly discuss the ideas leading to the so-called tight-binding approximation, which is the lattice representation we used to model the systems under consideration.

In crystals, atoms form a periodic lattice that is characterized by a unit cell and invariant under point-group symmetry-transformations [BZ06]. In a first approximation, we decouple the heavy atomic nucleus from the electrons (Born-Oppenheimer approximation [BO27]). This also means that displacements of atoms and their relaxations in form of quantized lattice oscillations (phonons) are neglected. On the one hand, this is not much of an issue since we are interested in the electronic properties of solids. On the other hand, the effects of electron-phonon interactions can be incorporated later by expanding the basis set of single-particle states to also account for

\footnotetext{
${ }^{4}$ It should be noted that in the previous discussion we neglected the electron spin. However, it is straightforward to introduce the spin by only expanding the position labels $\mathbf{r}_{j} \rightarrow\left(\mathbf{r}_{j}, \sigma_{j}\right)$, where $\sigma_{j}=\uparrow / \downarrow$ labels the spin projection along a chosen quantization axis. The whole discussion is then pursued completely equivalent with only the singleparticle basis states being expanded by an additional label for the spin projection so that the ladder operators are modified as $\hat{c}_{k}^{[\dagger]} \rightarrow \hat{c}_{k, \sigma}^{[\dagger]}$.
} 
small fluctuations of the atomic nucleus around their equilibrium positions. We further simplify the problem by neglecting atomic core electrons. This can be motivated by imagining the crystal being formed from infinitely separated atoms that are adiabatically moved towards each other. The initial configuration is described by calculating the ground-state configurations for each atom independently, yielding a set of atomic orbitals for each atomic species. Even though this is a challenging problem by itself, we are not going into the details, here. While moving the atoms closer to each other, those electronic orbitals that are most delocalized start to hybridize once the distance between the atoms is small enough. Due to the hybridization the overall energy can be lowered where the energy gain is a function of the distance between the atoms. Minimizing this function yields the lattice structure of the crystal. Usually, the distances between the atoms are comparably large so that only a few atomic orbitals are hybridizing significantly and we can neglect most of the core electrons that, however, screen the nucleus' positive charge.

The resulting atomic positions $\mathbf{R}_{\mathbf{n}}^{p}$ can be decomposed into a set of basis vectors $\mathbf{R}^{p}(p \in \mathbb{N})$ defining the crystal's unit cell, and a set of primitive vectors $\mathbf{a}_{\alpha=1,2,3}$ spanning the Bravais lattice of the crystal

$$
\mathbf{R}_{\mathbf{n}}^{p}=n_{1} \mathbf{a}_{1}+n_{2} \mathbf{a}_{2}+n_{3} \mathbf{a}_{3}+\mathbf{R}^{p}, \quad n_{i} \in \mathbb{Z} .
$$

At each Bravais lattice site defined by the integers $n_{i}$ (which we abbreviate by the vector $\mathbf{n} \in \mathbb{Z}^{3}$ ), there is a set of atoms whose positions relative to the lattice site are given by $\mathbf{R}^{p}$. Thus, a generic ansatz for $\hat{V}\left(\mathbf{r}_{j}\right)$ is an electrostatic potential (written in the position representation)

$$
\hat{V}\left(\mathbf{r}_{j}\right)=-\frac{e^{2}}{4 \pi \varepsilon_{0}} \sum_{\mathbf{n}} \sum_{p} \frac{\eta^{p}}{\left|\mathbf{r}_{j}-\mathbf{R}_{\mathbf{n}}^{p}\right|},
$$

where $\eta^{p}$ is the number of valence electrons of the $p$ th unit-cell's basis atom contributing to the bonding, i.e., the unbound charge. Note that in this ansatz we are assuming a homogeneous screening by the core electrons. If we formally define the crystal as the set of infinitely many lattice translations generated by the Bravais vectors $\mathbf{a}_{\alpha}$, then the potential $\hat{V}\left(\mathbf{r}_{j}\right)$ is invariant under arbitrary translations by the $\mathbf{a}_{\alpha}$ 's.

The periodicity of the lattice has an important consequence on the structure of the solutions of the single-particle Hamiltonian $\hat{H}_{j}$ in the position representation. By Blochs' theorem [Blo29], the eigenfunctions of $\hat{H}_{j}$ with a periodic single-particle potential fulfilling $\hat{V}\left(\mathbf{r}_{j}+\mathbf{a}_{n}\right)=\hat{V}\left(\mathbf{r}_{j}\right)$ have to satisfy

$$
\varphi_{\mathbf{k}}\left(\mathbf{r}_{j}+\mathbf{a}_{n}, \sigma_{j}\right)=e^{\mathrm{i} \mathbf{k} \cdot \mathbf{a}_{\alpha}} \varphi_{\mathbf{k}}\left(\mathbf{r}_{j}, \sigma_{j}\right) \quad \text { with } \alpha=1,2,3 .
$$

Here, $\mathbf{k} \in \mathbb{R}^{3}$ is a vector that defines a crystal momentum and classifies the eigenfunctions. The plane-wave factor is insensitive to a translation by a reciprocal lattice vector $\mathbf{G}_{\beta}(\nu=1,2,3)$, which is defined via $\mathbf{a}_{\alpha} \cdot \mathbf{G}_{\beta}=2 \pi \nu \delta_{\alpha, \beta}$ for some $\nu \in \mathbb{Z}$. Thus, the eigenfunctions are rather classified in bands distinguished by the band-index $\nu$. Then $\mathbf{k}$ is an element of the first Brillouin zone with $k_{\alpha} \in\left[-\frac{\pi}{a_{\alpha}}, \frac{\pi}{a_{\alpha}}\right]$, where $a_{\alpha}$ is the lattice constant along the direction defined by $\mathbf{a}_{\alpha}$. For the $p$ th atom in the Bravais lattice unit cell this suggests linear combination of atomic orbitals (LCAO) as ansatz

$$
\varphi_{\mathbf{k}, \nu}^{p}\left(\mathbf{r}_{j}, \sigma_{j}\right)=\frac{1}{\sqrt{N}} \sum_{\mathbf{n}} e^{\mathbf{i k} \cdot \mathbf{R}_{\mathbf{n}}^{p}} \sum_{n_{p}} c_{n_{p}} u_{n_{p}, \nu}^{p}\left(\mathbf{r}_{j}-\mathbf{R}_{\mathbf{n}}^{p}, \sigma_{j}\right)
$$

where the $u_{n_{p}, \nu}^{p}\left(\mathbf{r}_{j}-\mathbf{R}_{\mathbf{n}}^{p}, \sigma_{j}\right)$ are the atomic orbitals that hybridize and $c_{n_{p}} \in \mathbb{C}$. It can be checked easily that these states satisfy the Bloch theorem. Note that strictly speaking this ansatz is only a good approximation to the single-particle eigenstates of $\hat{H}_{j}$ if the atomic orbitals $u_{\nu}\left(r_{j}\right)$ are 
not too delocalized over the lattice. While we can expect this to give a good description for, for example, transition metals where the localized $3 d, 4 d, 5 d$ orbitals are mainly hybridizing, alkali metals on the other hand cannot be expected to be well-approximated because they exhibit very delocalized outermost $s$-orbitals.

In this thesis we are only dealing with isotropic, one-dimensional systems with a homonuclear unit cell. Therefore, from now on we simplify the discussion by setting $p \equiv 1$ and considering only one orbital per lattice site

$$
\varphi_{k}\left(r_{j}, \sigma_{j}\right)=\frac{1}{\sqrt{N}} \sum_{n} e^{\mathrm{i} k R_{n}} u\left(r_{j}-R_{n}, \sigma_{j}\right) .
$$

These so-called Bloch states are delocalized in real space, which seems counter-intuitive if we are exploiting tightly bound atomic orbitals as the building blocks. It is therefore convenient to take the Fourier transformation as single-particle basis states

$$
w_{\sigma_{j}}\left(r_{j}-R_{n}\right)=\frac{1}{\sqrt{N}} \sum_{k} e^{-\mathrm{i} k R_{n}} \varphi_{k}\left(r_{j}, \sigma_{j}\right) .
$$

Note that here the Bravais lattice vectors $R_{n}$ are taken as parameters. Now, the Wannier functions $w_{\sigma_{j}}\left(r_{j}-R_{n}\right)$ are localized at the lattice site $r_{j}$. The Hamiltonian for tightly bound electrons in a periodic potential in second quantization then reads

$$
\hat{H}=\sum_{m, n} \sum_{\sigma_{m}, \sigma_{n}} T_{m, n}^{\sigma_{m}, \sigma_{n}} \hat{c}_{m, \sigma_{m}}^{\dagger} \hat{c}_{n, \sigma_{n}}+\sum_{m, n, r, s} \sum_{\sigma_{m}, \sigma_{n}} V_{m n, \sigma_{s}}^{\sigma_{m} \sigma_{n}, \sigma_{r} \sigma_{s}} \hat{c}_{m, \sigma_{m}}^{\dagger} \hat{c}_{n, \sigma_{n}}^{\dagger} \hat{c}_{s, \sigma_{s}} \hat{c}_{r, \sigma_{r}} .
$$

Here, the single- and two-particle matrix elements are given by

$$
\begin{aligned}
T_{m, n}^{\sigma_{m}, \sigma_{n}} & =\left\langle m, \sigma_{m}\left|\frac{\hat{p}^{2}}{2 m_{e}}+\hat{V}(r)\right| n, \sigma_{n}\right\rangle \\
& =\int_{\mathbb{R}} d r w_{\sigma_{m}}^{*}\left(r-R_{m}\right)\left(-\frac{\hbar^{2}}{2 m_{e}} \frac{\partial^{2}}{\partial r^{2}}+V(r)\right) w_{\sigma_{n}}\left(r-R_{n}\right), \\
V_{m n, r s}^{\sigma_{m} \sigma_{n}, \sigma_{r} \sigma_{s}} & =\left\langle m, \sigma_{m}\left|\left\langle n, \sigma_{n}\left|\hat{V}_{I}\right| r, \sigma_{r}\right\rangle\right| s, \sigma_{s}\right\rangle \\
& =\int_{\mathbb{R}^{2}} d r d r^{\prime} w_{\sigma_{m}}^{*}\left(r-R_{m}\right) w_{\sigma_{n}}^{*}\left(r^{\prime}-R_{n}\right) \frac{e^{2}}{4 \pi \varepsilon_{0}\left|r-r^{\prime}\right|} w_{\sigma_{r}}\left(r-R_{r}\right) w_{\sigma_{s}}\left(r^{\prime}-R_{s}\right),
\end{aligned}
$$

where we dropped the electron's position labels for convenience. Since the Wannier functions are strongly localized around $r$, the first integral usually is assumed to be non-vanishing only for $|m-n| \leq 1$. The case $m=n$ contributes an on-site energy, which by translation invariance is independent on the value of $n$

$$
\int_{\mathbb{R}} d r w_{\sigma_{m}}^{*}(r)\left(-\frac{\hbar^{2}}{2 m_{e}} \frac{\partial^{2}}{\partial r^{2}}+V(r)\right) w_{\sigma_{n}}(r) \equiv \mu \delta_{\sigma_{m}, \sigma_{n}}
$$

The second contribution is called hopping amplitude and using the above assumption of an isotropic system it is set to

$$
\int_{\mathbb{R}} d r w_{\sigma_{m}}^{*}(r)\left(-\frac{\hbar^{2}}{2 m_{e}} \frac{\partial^{2}}{\partial r^{2}}+V(r)\right) w_{\sigma_{n}}(r-R) \equiv-t_{h} \delta_{\sigma_{m}, \sigma_{n}}
$$

The minus sign is not by accident but typically arises when calculating the overlap integrals, for instance, for adjacent $s$-orbitals. The second integral describes the Coulomb interaction 
between two electrons. In solids, Thomas-Fermi screening typically reduces the region in which one electron feels the potential created by the remaining electrons to a few neighoring sites, only. Being somewhat dramatic and neglecting all contributions that are not on-site in the second integral we obtain a contact-interaction

$$
\int_{\mathbb{R}^{2}} d r d r^{\prime} w_{\sigma_{m}}^{*}\left(r-R_{m}\right) w_{\sigma_{n}}^{*}\left(r^{\prime}-R_{n}\right) \frac{e^{2} \delta_{m, r} \delta_{n, s}}{4 \pi \varepsilon_{0}\left|r-r^{\prime}\right|} w_{\sigma_{r}}\left(r-R_{r}\right) w_{\sigma_{s}}\left(r^{\prime}-R_{s}\right) \equiv U \delta_{m, r} \delta_{n, s} \delta_{\sigma_{m}, \sigma_{r}} \delta_{\sigma_{n}, \sigma_{s}} .
$$

The resulting Hamiltonian is the paradigmatic Hubbard model [Hub63]

$$
\hat{H}_{\mathrm{Hub}}=-t_{h} \sum_{j, \sigma}\left(\hat{c}_{j, \sigma}^{\dagger} \hat{c}_{j+1, \sigma}+\text { h.c. }\right)+\mu \sum_{j, \sigma} \hat{n}_{j, \sigma}+U \sum_{j} \hat{n}_{j, \uparrow} \hat{n}_{j, \downarrow} .
$$

In this representation the tight-binding approximation reveals its beauty. The Hubbard model describes atoms with two valence electrons that can hop through the lattice and experience a (repulsive) contact interaction when two electrons share the same site. Despite its simplicity it exhibits important physics such as a transition between a conducting phase and a Mott-insulator in one dimension $\left[\mathrm{EFG}^{+} 05\right]$. In two dimensions it is supposed to model unconventional hightemperature superconductivity in cuprates [SLMT05]. Replacing the fermionic with bosonic ladder operators yields the Bose-Hubbard model, which has been realized experimentally in ultra-cold atoms $\left[\mathrm{GME}^{+} 02\right]$ verifying the transition from the superfluid into a Mott insulating phase.

In the limit $U=0$ the Hubbard model reduces to a simple tight-binding chain which we used to model normal leads in Chap. 7. If $U \gg t$ and at half filling (i.e., one electron per site $\left\langle\hat{n}_{j}\right\rangle=1$ ) the electronic degrees of freedom are frozen out and one obtains the isotropic Heisenberg model with exchange coupling $J=\frac{4 t^{2}}{U}$. Finally, in Chap. 8 we extend the Hubbard model by an effective nearest-neighbor density-density interaction. The resulting extended Hubbard model in one dimension exhibits an even richer phase diagram with singlet- and triplet superconducting phases. There, we studied the dynamics of the electronic system after an optical excitation in a chargedensity wave (CDW) ground state, which showed indications of an enhanced superconductivity in experiments $\left[\mathrm{FFK}^{+} 14, \mathrm{MSF}^{+} 14, \mathrm{HKN}^{+} 14, \mathrm{HNK}^{+} 16, \mathrm{MCN}^{+} 16\right]$. 



\section{Order in quantum many-body systems}

There are things in our every-day life we are used to so much that we recognize their value only if they are missing. In the following, we discuss such a subject which, if absent, would leave us in a listless world. Clearly, we are not thinking of coffee $^{1}$, even though it may serve as an illustrative example. Here, we are speaking about the property of matter to arrange itself in various kinds of ordered states that is, various kinds of phases.

Systematically classifying and distinguishing different phases of matter has been a driving force for theoretical physics over centuries. The question how to phenomenologically characterize phase transitions between solids, liquids, and gases was one of the central problems of classical thermodynamics. Exploring its theoretical foundation then led to a variety of paradoxa such as the Gibbs paradoxon or the ultraviolet catastophe eventually stimulating the development of quantum mechanics. Therein, the notion of a classical phase transition in which thermal fluctuations are the driving force had to be rethought. Surprisingly, quantum systems can undergo phase transitions even at zero temperature with quantum fluctuations being the driving force [Car10]. A unifying framework was formulated by Landau [Lan37a, Lan37b], who argued that phase transitions from a disordered into an ordered state are characterized by the breaking of a continuous symmetry accompanied with the emergence of a local order-parameter.

However, with the theoretical description of chiral spin states [WWZ89, Wil90] and the experimental discovery of the fractional quantum Hall state [TSG82, Lau83], even this turned out not to be the complete story. These states cannot be described by a local order-parameter. In turn they are characterized by topological invariants that are protected by the conservation of a symmetry. Their non-local nature also features exotic quasi-particles exhibiting fractional exchange statistics. Recently, even breaking of discrete time-translational symmetry [KLMS16, EBN16, EBN17] has been observed experimentally $\left[\mathrm{ZHK}^{+} 17\right]$ in periodically driven Floquet systems. Therein, a discrete symmetry generated by the peridioc Floquet-drive is broken accompanied by the emergence of spatio-temporal long-ranged correlations. This may open a new approach to characterize ordered states of matter breaking discrete symmetries [KVKS17] out-of equilibrium.

It has been a long way since the early phenomenological approaches to describe ordered states of matter and there is no chance to give a complete overview. We therefore focus on the necessary ingredients to describe the systems being considered in this thesis. These cover unconventional superconductivity where we investigate the proposed enhancement of superconducting correlations out-of equilibrium. We also studied systems combining superconductivity and topological order, where the emergence and stability of Majorana fermions is one of the central questions. To introduce the basic concepts required for this program, we briefly introduce the notion of a phase transition and discuss spontaneous symmetry breaking. Therefrom we distill the necessary notions of local order-parameters, correlation functions, and long-range order and describe numerical quantities that can be exploited to distinguish ordered states. Finally, we introduce superconductivity and topological superconductivity in Secs. 3.3 and 3.4, the phenomena studied in this thesis and dicussed in Chaps. 7 and 8. As the main focus of our studies was on systems at zero temperature, we take into account finite temperatures only when required for methodical

\footnotetext{
${ }^{1}$ However, there certainly is an analogy to the author's constitution without the existence of coffee.
} 
and conceptional considerations. In these situations, we explicitely label states and expectation values with their (inverse) temperature.

\section{Phase transitions $\mid 3.1$}

Identifying phase transitions [Sch06, Sac99, Car10] is something most of us do intuitively in every day life. Deciding if the water on the boiling plate is transitioning into the gas phase can be done by listening to the sound of the boiling kettle. Even though, such an experimental procedure is not the method of choice to study the phase diagram of a new sample of a high- $T_{\mathrm{c}}$ superconductor, it can serve as a guide for which questions we may reasonably ask. From elementary thermodynamics we know that in a stable phase varying external, macroscopic parameters the free energy of the system responds with smooth changes, too. In the same way, observables derived from this thermodynamic potential behave in a continuous manner.

However, passing a phase transition some observables change in a non-analytic way. In case of boiling water we observe a discontinuous jump in the density upon varying the pressure. We can parametrize the free energy as function of temperature, pressure, and particle number, i.e., the Gibbs free energy $G(T, p, N)$. The volume is then obtained from the isothermal derivative $V=$ $\left.\frac{\partial G}{\partial p}\right|_{T}$. This means that for the density $\rho \propto \frac{1}{V}$ being discontinuous, the Gibbs free energy must be non-analytic at the phase transition. In general, thermodynamic observables are calculated from taking a proper number of derivatives of the free energy with respect to its variables. The Ehrenfest classification characterizes phase transitions by the lowest order derivative of the free energy, which shows a discontinuity. For instance, the liquid gas transition described above is a first-order phase transition. However, the free energy is given by the partition function, which for finite systems is always analytic. Therefore, phase transitions characterized by discontinuities of observables can only occur in the thermodynamic limit, which leaves us with a problem. Usually the partition function cannot be evaluated analytically, in particular for interacting systems. Then the question arises, how can we identify phase transitions numerically having access only to a finite number of constitutents (e.g., particles, lattice sites, etc.) as in DMRG?

One way to deal with this problem is to study the system in a finite region around the critical parameters and in the following we restrict the discussion to continuous phase transitions, i.e., those of second order or higher. Such phase transitions are characterized by continuous first derivatives of the free energy, while discontinuities and divergences are appearing in higher orders. Close to the phase transition the partition function is an analytic function. However, intuitively there should be some precursor regime indicating the non-analycity right at the phase transition. Experimental observations of critical opalescence in binary mixtures close to the liquid gas phase transition, for instance, are an indicator for the so-called critical behavior, and already found in 1823. Its origin are density-fluctuations that are correlated over length-scales comparable to the wavelength of the incident light enabling scattering processes as first noted by Smoluchowski in 1908. These are the critical phenomena one may look for also in finite systems, i.e., diverging susceptibilities and fluctuations of thermodynamic observables.

Surprisingly, these critical behaviors show universality, i.e., systems with completely different microscopic constituents and interactions exhibit the same properties in the vicinity of the phase transition. A scaling analysis of the free energy connects universality to the large, experimentally observed length scales. In fact, naive extensivity of the free energy at the phase transition cannot hold as can be seen, for instance, by evaluating the scaling of susceptibilities, which are extensive on the one hand and infinite on the other at the phase transition. However, the free energy 
has to be a homogeneous function. Therefore, upon rescaling the extensive variables (e.g., the system size $L$ ) there has to be a rescaling of the intensive variables (e.g., $\frac{T-T_{\mathrm{c}}}{T_{\mathrm{c}}}$ with the critical temperature $T_{\mathrm{c}}$ ). To illustrate this important observation we may consider a canonical ensemble $F(T, L)$ on a $d$-dimensional hypercube with $N=L^{d}$ lattice sites ${ }^{2}$ and only vary temperature across the phase transition at $T_{\mathrm{c}}$. Defining the reduced temperature $t=\frac{T-T_{\mathrm{c}}}{T_{\mathrm{c}}}$ a generalized scaling relation has to be imposed for $F$

$$
F(t, L)=\frac{b^{y_{f}}}{b^{d}} F\left(b^{-y_{t}} t, b L\right) \stackrel{b=L^{-1}}{\Rightarrow} F(t, L)=N L^{-y_{f}} F\left(\frac{t}{L^{-y_{t}}}, 1\right),
$$

with scaling exponents $y_{f}, y_{t}>0$ that are introduced to restore homogenity of $F$. Near $t=0$ we consider the leading functional dependence $\frac{F(x, 1)}{N} \sim|x|^{q}$ and determine the exponent $q$ so that the free energy is extensive (linear in $N$ ) for $t \neq 0$

$$
\frac{F(t, L)}{N}=L^{-y_{f}} F\left(\frac{t}{L^{-y_{t}}}, 1\right) \sim L^{-y_{f}+q \cdot y_{t}}|t|^{q} \Rightarrow q=\frac{y_{f}}{y_{t}} .
$$

This is an example of a scaling relation involving critical exponents $y_{f}, y_{t}$. More importantly, we have encountered a length scale that can be seen when rescaling by $b=|t|^{1 / y_{t}} \equiv 1 / \xi$

$$
F(t, L)=\xi^{-y_{f} / d} F\left(1, \frac{L}{\xi}\right)
$$

$\xi$ diverges as $t \rightarrow 0$ and can be shown to set the length scale for correlations, thereby being responsible for the experimentally observed critical fluctuations. The important conclusion is that close to the phase transition the microscopic details are not important any more. This can be made more precise in context of a renormalization-group analysis, in which also relations between the various critical exponents for the different observables and susceptibilites can be derived [Kad66]. The overall finding is that close to the phase transition the microscopic degrees of freedom can be integrated out by renormalizing length scales until in the end an effective, scale-invariant theory for the macroscopic system is obtained determining the critical exponents. Thereby, phase transitions can be grouped into universality classes that exhibit the same critical behavior and are distinguished by dimensionality and symmetry of the system under consideration.

From the numerical point of view, critical behavior such as diverging length scales can be modeled as tensor network, for instance in the multi-scale entanglement-renormalization-group Ansatz (MERA) [Vid08]. Therein, the real-space renormalization incorporating increasing length scales is achieved by a hierarchy of layers of entangler and disentangler gates. Crucially, this permits to extract critical exponents in the thermodynamic limit [MRGF09, RMS $\left.{ }^{+} 10\right]$.

Another important observation is the dependency of the critical behavior on the symmetries of the system. Even more, if a classical system undergoes a continuous phase transition, then the state in the low-temperature phase typically breaks a symmetry, which is conserved in the overall Hamiltonian. This is the starting point for Landau's theory of phase transitions, where the spontaneous breaking of a system's symmetry is connected to the emergence of an order parameter acquiring a finite value in the ordered phase, only.

Before continuing, we want to comment on how to carry over the described frameworks to quantum systems. Doing so, the framework of second quantization described in Sec. 2.2 is inevitable in particular when describing systems with variable particle number. In classical systems, phase transitions are driven by critical fluctuations at finite temperature. Quantum systems extend

\footnotetext{
${ }^{2}$ Being on a hypercubic lattice we can express $V$ through $N=L^{d}$ so that $F(T, V, N)=F(T, L)$.
} 
this formalism by quantum fluctuations, which may cause phase transitions at zero temperature while varying system parameters, only. Again, continuous phase transitions can be classified by critical exponents and categorized into universality classes. For instance, the Ising universality class contains liquid gas transitions as well as the transition between para- and ferromagnetic phase in the Ising model. Importantly, close to a quantum phase transition there is a quantum critical regime, which also extends to finite temperatures. Finally, the partition function of classical systems at a finite temperature in $d$ dimensions can be mapped to the partition function of a corresponding quantum system in $d+1$ dimensions. This so-called quantum-classical mapping is based on the non-commutivity of operators describing the quantum mechanical degrees of freedom. Thereby, the kinetic and potential energy do not factorize in the exponential as in the classical case. Recasting the quantum many-body partition function into the form of a classical one, decomposition schemes need to be employed, which evaluate to additional terms in the Hamiltonian that act like an extra dimension.

\section{\begin{tabular}{l|l} 
Spontaneous symmetry breaking & 3.1 .1
\end{tabular}}

We already pointed out the role of symmetries under which the system under consideration is invariant. However, we are not satisfied by only classifying the critical behavior close to a (quantum) phase transition by symmetries. What we are looking for is an answer to the question: How can we distinguish different phases in a numerical computation on a finite system. We can approach this problem by looking at a physical system before and after a phase transition only in terms of the symmetries it respects. A prototype is the Ising model with discrete lattice degrees of freedom $\hat{S}_{j}^{z}$ (in the following we consider $S=1 / 2$ spins) and the transition from the disordered high-temperature paramagnetic into the ordered ferromagnetic phase

$$
\hat{H}_{I}=-J \sum_{\langle i, j\rangle} \hat{S}_{i}^{z} \hat{S}_{j}^{z}, \quad J>0
$$

where the sum is over nearest neighbors and we are not imposing a restriction on the spatial dimensions of the problem, yet. The Hamiltonian is invariant under a discrete transformation flipping all spins simultaneously $\hat{S}_{j}^{z} \rightarrow-\hat{S}_{j}^{z}$ that is it exhibits a global $\mathbb{Z}_{2}$ symmetry. The ground state at zero temperature $k_{\mathrm{B}} T=0$ is doubly degenerate and fully polarized so that it can be in an arbitrary superposition

$$
|G S\rangle_{\infty}=\alpha|\uparrow, \uparrow, \ldots\rangle+\beta|\downarrow, \downarrow, \ldots\rangle, \quad|\alpha|^{2}+|\beta|^{2}=1,
$$

where the subscript $\infty$ refers to the inverse temperature $\beta=\frac{1}{k_{\mathrm{B}} T}$ at $T=0$. The elementary bulk excitations are spin flips at the cost of an energy $\Delta E=\frac{p J}{2}$, where $p \in \mathbb{N}$ counts the nearest neighbors for the considered lattice in the bulk, i.e., the coordination number. Note that if there are already flipped spins in the vicinity, then the energy cost reduces, for instance, in a one-dimensional system, once a spin flip occured, further flips come with no energy cost growing a domain of opposite spin direction

$$
|\ldots, \uparrow, \uparrow, \uparrow, \uparrow, \ldots\rangle \stackrel{\Delta E=J}{\rightarrow}|\ldots, \uparrow, \downarrow, \uparrow, \uparrow, \ldots\rangle \stackrel{\Delta E=0}{\rightarrow}|\ldots, \uparrow, \downarrow, \downarrow, \uparrow, \ldots\rangle .
$$

At finite temperatures such excited states have a finite probability $\propto e^{-\beta \Delta E}$. We therefore expect a critical temperature $T_{\mathrm{c}} \geq 0$ at which the fluctuation-induced excitations dominate and 
the magnetization averages to zero $\left\langle\sum_{j} \hat{S}_{j}^{z}\right\rangle_{\beta<\beta_{\mathrm{c}}}=0$. Note that employing the notation $\langle\ldots\rangle_{\beta}$ we refer to the thermodynamical average of the quantum mechanical expectation values

$$
\langle\hat{O}\rangle_{\beta}=\operatorname{tr}\left\{\hat{O} e^{-\beta \hat{H}}\right\} .
$$

This high temperature state is manifestly invariant under the global $\mathbb{Z}_{2}$ symmetry. On the other hand, even an infinitesimally small magnetic field $\hat{h}=h \sum_{j} \hat{S}_{j}^{z}$ can lift the degeneracy of the ground state in the low temperature phase. Such perturbations are always present in real materials. One may, for instance, think of magnetic impurities that can create an infinitesimally small mean field or perturbations from an external magnetic field. Spontaneous symmetry breaking can then be defined more rigorously by carefully taking the limits to obtain the magnetization density in the thermodynamic limit

$$
m(\beta):=\lim _{h \rightarrow 0} \lim _{N \rightarrow \infty} \frac{1}{N}\left\langle\sum_{j} \hat{S}_{j}^{z}\right\rangle_{\beta} .
$$

If we have $m\left(\beta>\beta_{\mathrm{c}}\right) \neq 0$, then in thermodynamic limit an infinitesimally small perturbation breaks the global $\mathbb{Z}_{2}$ symmetry as the system chooses one of the two possible degenerated ground states. The order of the limits matters in this definition. Interchanging the order will always result in a vanishing magnetization $m \equiv 0$.

In general, if there is a global symmetry under which the Hamilton operator is invariant, then the eigenstates of the Hamiltonian are eigenstates of the corresponding operator $\hat{S}$ representing the global symmetry. At infinite temperature the system is in a mixed state, which is composed of all states in the Hilbert space having equal weights. Therefore, the state transforms trivially under the global symmetry preserved by the Hamilton operator. Upon reducing the temperature (increasing the inverse temperature) infinitesimal perturbations $\hat{h}$ may yield a supression of some eigenstates, which span subspaces belonging to particular eigenvalues of $\hat{S}$. If this happens, then the system breaks the global symmetry on the one hand. On the other hand, only the remaining subspaces contribute with their eigenvalues to the expectation value $\langle\hat{S}\rangle_{\beta}$ of the symmetry operator. We can now define an order parameter that uniquely identifies breaking of such a symmetry via

$$
s(\beta):=\frac{1}{N}\left(\langle\hat{S}\rangle_{\beta}-\langle\hat{S}\rangle_{0}\right) .
$$

Taking the limits $h \rightarrow 0, N \rightarrow \infty$ in the correct order, in the disordered high-temperature phase $s\left(\beta<\beta_{\mathrm{c}}\right)$ vanishes identically, while in the ordered low-temperature phase $s\left(\beta>\beta_{\mathrm{c}}\right)$ acquires a finite value.

Let us return to the ferromagnetic Ising model $(J>0)$ introduced above and discuss the question under which conditions spontaneous symmetry breaking can occur at this particular example. At zero temperature the ground state is given by a superposition of the two fully polarized states Eq. (3.5). Landau argued beautifully why there can be no spontaneous symmetry breaking in one dimension [Lan37b], so we may recapitulate it in the following. As already mentioned, in the ground state at zero temperature we can flip a spin at cost $\Delta E=J$ and then add arbitrary spin flips as long as we are not hitting a boundary. We can thus grow a region of opposite magnetization domains, i.e., there is a huge degeneracy in the excited states. We can estimate this degeneracy by noting that there are always two boundaries of such a domain, i.e., we can create two domain walls at cost $\Delta E=J$. Since these domain walls are free to move around, there are $(N-2)(N-3)$ possibilities do distribute the domain walls so their number scales as $N^{2}$ and therefore the entropy contribution is $\propto k_{\mathrm{B}} \log N$ in the thermodynamic limit. For the free energy $F=U-T S$ this implies that for temperatures $T>0$ we can lower the free energy 
by creating two domain walls at arbitrary places in the chain if $J<k_{B} T \log N$. In the limit $N \rightarrow \infty$ this is the case for any finite temperature so there will always be domain walls being equally distributed over the lattice with equal probability $e^{-\beta J}$. But this also means that at finite temperature all symmetry sectors of the total spin operator $\sum_{j} \hat{S}_{j}^{z}$ are equally populated yielding an overall vanishing magnetization density $m=0$. Hence, in one dimension at finite temperature there can be no spontaneous symmetry breaking in the Ising model. This argument does not necessarily hold in spatial dimensions $d>1$. To see this, note that we exploited the fact that the cost of flipping domains of $L^{d}$ spins with area $A \sim L^{d}$ is proportional to the surface area of the domain $\partial A$. In one dimension this is a constant and thereby the energy costs are overcompensated by the entropy contribution. For $d>1$ the energy costs scale as $\partial A \sim L^{d-1}$. Noting that asymptotically $\log x$ grows slower than any power of $x$, we would expect that in the thermodynamic limit the entropy growth at small temperatures can be compensated by the energy costs for large flipped domains. In fact, it was shown by Peierls [Pei36, Gri64] that there is spontaneous symmetry breaking in $d=2$. The critical temperature can be determined by the Kramers-Wannier duality [KW41] and is given by $T_{\mathrm{c}}=\frac{2}{\ln (1+\sqrt{2})} \frac{J}{k_{\mathrm{B}}}$.

The connection between the dimensionality and the possible existence of spontaneous symmetry breaking was further explored by Mermin, Wagner, and Hohenberg [MW66a, Hoh67]. They proved that in the classical $X Y$-Heisenberg model in $d \leq 2$ spatial dimensions, there cannot be any finite magnetization at temperatures $T>0$ for sufficiently short-ranged interactions. The proof relies on the Bogoliubov inequality [Bog62] and some assumptions on the decay of the interactions. Thereby, the theorem can be generalized into its present form stating that in spatial dimensions $d \leq 2$ there can be no spontaneous breaking of a contiuous symmetry for sufficiently short-ranged interactions. ${ }^{3}$ Importantly, by the quantum-classical mapping these findings apply to quantum systems at zero temperature, too. Therefore, in one-dimensional quantum systems at zero temperature there can be no breaking of a continuous symmetry.

However, the theorem neither makes statements about discrete symmetries, nor the possible existence of phase transitions in systems with continuous symmetries in general. The first remark immediately applies to the Ising model in two dimensions above discussed. The second remark, however, is a lot more delicate. Already during the time Mermin and Wagner published their theorem, there was evidence for critical behavior in two-dimensional systems with continuous symmetries, for instance by numerical simulations on hard disks [AW62]. The apparantly contradicting results motivated Kosterlitz and Thouless to come up with a new notion of a phase transition into an ordered state as we discuss further below. It is also worth to note that the above formulation of the Mermin-Wagner-Hohenberg theorem strictly applies only in thermal equilibrium. The question, whether spontaneous breaking of a continuous symmetry of a quantum mechanical system in one spatial dimension can happen out-of equilibrium is still under debate and we studied this problem in the context of optically enhanced superconductivity in Chap. 8.

The concepts of spontaneous symmetry breaking and induced order parameters are powerful tools in studying ordered phases analytically. However, evaluating the expectation value of the order parameter can also be done numerically in a finite system when applying a finite perturbation. Then, a finite-size scaling is required to extrapolate into the thermodynamic limit, which ideally is repeated for different values of the perturbation to also extract the limit $h \rightarrow 0$. This, in turn, yields a numerically very costly procedure and may not be applicable for complicated systems, in which a single calculation already requires significant computational effort. Therefore, we discus related approaches, which come with less numerical effort and are used throughout this thesis,

\footnotetext{
${ }^{3}$ Here, we are a bit too generous, in fact, the theorem has to be proven for each observable separately, which has been done for the systems we are interested in [Hal18].
} 
next.

\section{Correlation functions and off-diagonal long-range order}

The emergence of an order parameter can be related to the existence of long-range correlations. The foundation of this observation is the cluster decomposition theorem, which states that measurements of observables being spatially separated sufficiently should be independent of each other (see for instance [SW78]). This means that given two local observables $\hat{A}_{i}, \hat{B}_{j}$ the connected correlation function constructed out of them has to vanish in the limit of infinite separation

$$
\lim _{|i-j| \rightarrow \infty}\left\langle\hat{A}_{i} \hat{B}_{j}\right\rangle-\left\langle\hat{A}_{i}\right\rangle\left\langle\hat{B}_{j}\right\rangle=0 .
$$

We may now assume that the $\hat{A}_{j}=\hat{B}_{j} \equiv \hat{O}_{j}$ constitute a global order-parameter via $\hat{O}=$ $\frac{1}{N} \sum_{j} \hat{O}_{j}$. In an ordered phase with $\langle O\rangle \neq 0$ this gives rise to a finite value $\left\langle\hat{O}_{j}\right\rangle \equiv \bar{O}=\frac{\langle O\rangle}{N}$ by translational invariance so that

$$
\lim _{|i-j| \rightarrow \infty}\left\langle\hat{O}_{i} \hat{O}_{j}\right\rangle-\left\langle\hat{O}_{i}\right\rangle\left\langle\hat{O}_{j}\right\rangle=0 \Rightarrow \lim _{|i-j| \rightarrow \infty}\left\langle\hat{O}_{i} \hat{O}_{j}\right\rangle=\bar{O}^{2} \neq 0
$$

Therefore, instead of studying the order parameter we can also investigate the corresponding correlation function, which should saturate at a finite value in an ordered phase. In contrast, in a disordered phase correlations decay exponentially $\left\langle\hat{O}_{i} \hat{O}_{j}\right\rangle \sim e^{-\frac{|i-j|}{\xi}}$. The importance of this connection is that evaluating the correlation function, we are able to detect spontaneous symmetry breaking without the requirement of doing a scaling analysis in the infinitesimally small perturbation ( $h$ in case of the Ising model discussed above). Additionally we may note that in the thermodynamic limit the expectation value $\left\langle\hat{O}_{j}\right\rangle=\left\langle\psi\left|\hat{O}_{j}\right| \psi\right\rangle$ can only have a finite value if $|\psi\rangle$ is an eigenstate of $\hat{O}_{j}$. Otherwise the so-called orthogonality catastrophe would cause the overlap to vanish

$$
\left\langle\psi\left|\hat{O}_{j}\right| \psi\right\rangle \equiv\langle\psi \mid \phi\rangle \stackrel{N \rightarrow \infty}{\longrightarrow} 0
$$

We may now set $\hat{A}_{j}=\hat{a}_{j}^{\dagger}$ and $\hat{B}_{j}=\hat{a}_{j}$, where $\hat{a}_{j}^{[\dagger]}$ are annihilation (creation) operators obeying canonical commutation or anticommutation relations and $\left[\hat{a}_{j}\right]^{2}=\left[\hat{a}_{j}^{\dagger}\right]^{2}=0$. If their correlation function approaches a finite value in the limit $|i-j| \rightarrow \infty$, the corresponding order is usually called off-diagonal long-range order (LRO). As this is one tool we used to study superconductivity, we are going to discuss it in more detail.

Determining the asymptotics of the correlation function can be done by analyzing the correlation matrix

$$
\chi_{\hat{a}}(i, j)=\left\langle\psi\left|\hat{a}_{i}^{\dagger} \hat{a}_{j}\right| \psi\right\rangle,
$$

and was discussed first by Penrose and Onsager [PO56, Yan62]. Then, off-diagonal LRO in the thermodynamic limit occurs if

$$
\lim _{|i-j| \rightarrow \infty} \chi_{\hat{a}}(i, j) \neq 0
$$

For a finite system with $L$ lattice sites, $\chi_{\hat{a}}(i, j)$ is a Hermitian $L \times L$ matrix, which we can formally diagonalize to obtain real eigenvalues $\lambda_{\nu}$ and corresponding eigenvectors $\mathbf{v}_{\nu}$. Expanding $\chi_{\hat{a}}(i, j)$ 
as matrix $\chi_{\hat{a}}$ in its eigenbasis, we can write

$$
\boldsymbol{\chi}_{\hat{a}}=\sum_{\nu} \mathbf{v}_{\nu} \underbrace{\left\langle\psi\left|\left(\sum_{i} v_{\nu, i}^{*} \hat{a}_{i}^{\dagger}\right)\left(\sum_{j} v_{\nu, j} \hat{a}_{j}\right)\right| \psi\right\rangle}_{\lambda_{\nu}} \mathbf{v}_{\nu}^{\dagger} .
$$

Introducing operator fields $\hat{\eta}_{\nu}=\sum_{i} v_{\nu, i} \hat{a}_{i}$, their squared expectation values are related to the eigenvalues $\lambda_{\nu}$ of $\chi_{\hat{a}}$

$$
\mathbf{v}_{\nu}^{\dagger} \chi_{\hat{a}} \mathbf{v}_{\nu}=\left\langle\psi\left|\hat{\eta}_{\nu}^{\dagger} \hat{\eta}_{\nu}\right| \psi\right\rangle=\lambda_{\nu} \geq 0
$$

i.e., the eigenvalues are strictly positive. The eigenvectors $\mathbf{v}_{\nu}$ provide a natural basis of singleparticle wavefunctions $\left|\phi_{\nu}\right\rangle=\sum_{j} v_{\nu, j} \hat{a}_{j}|\psi\rangle$. Therefore, they are referred to as natural orbitals and the eigenvalues are called natural-orbital occupations $\lambda_{\nu}$ of the correlation matrix $\chi_{\hat{a}}$. The existence of off-diagonal LRO can now be related to properties of the natural orbitals and their occupations. To see this we use that if the system is ordered and $\hat{a}_{j}$ is the local order-parameter, then in the thermodynamic limit $\left\langle\psi\left|\hat{a}_{j}\right| \psi\right\rangle \neq 0$ is possible only if $|\psi\rangle$ is an eigenstate of $\hat{a}_{j}$. Thus we have by the cluster decomposition theorem

$$
\begin{aligned}
\lim _{|i-j| \rightarrow \infty} \chi_{\hat{a}}(i, j) & =\lim _{|i-j| \rightarrow \infty} \sum_{\nu} v_{\nu, i} \underbrace{\left\langle\psi\left|\left(\sum_{k} v_{\nu, k}^{*} \hat{a}_{k}^{\dagger}\right)\right| \psi\right\rangle}_{\sqrt{\lambda_{\nu}}{ }^{*}} \underbrace{\left\langle\psi\left|\left(\sum_{l} v_{\nu, l} \hat{a}_{l}\right)\right| \psi\right\rangle}_{\sqrt{\lambda_{\nu}}} v_{\nu, j}^{\dagger} \\
& =\lim _{|i-j| \rightarrow \infty}\left\langle\hat{a}_{i}^{\dagger}\right\rangle\left\langle\hat{a}_{j}\right\rangle .
\end{aligned}
$$

On the one hand, the last equality can only be valid if there is only one eigenvalue $\lambda_{\nu} \equiv \lambda_{\max }$ that is non-vanishing. On the other hand, we can read-off the order parameter $\left\langle\hat{a}_{j}\right\rangle=\sqrt{\lambda_{\max }}$. For finite systems these relations do not hold exactly. In turn, asymptotically there has to be one dominating eigenvalue $\lambda_{L}$ (sorting the eigenvalues in ascending order) so that we can approximate for large systems

$$
\lim _{|i-j| \rightarrow \infty} \chi_{\hat{a}}(i, j) \approx \lambda_{L} v_{L, i} v_{L, j}^{*}
$$

However, the eigenvectors $\mathbf{v}_{\nu}$ are normalized so that their coefficients in general scale as $\sim \frac{1}{\sqrt{L}}$. Therefore, in order to have a finite value in the thermodynamic limit, the dominating eigenvalue has to scale as $\lambda_{L} \sim L$. This yields a condition on the scaling of the largest eigenvalue of $\boldsymbol{\chi}_{\hat{a}}$, which can be tested numerically.

There are some remarks concerning the above considerations. Most importantly, the asymptotic behavior of the dominating eigenvalue can be shown rigorously [PO56]. Second, the coefficients $v_{L, j}=\left\langle\Omega\left|\hat{c}_{j}^{\dagger}\right| \phi_{\nu}\right\rangle$ determine the particle density at site $j$ in state $|\psi\rangle$. Therefore, if there is only one natural orbital occupied, $|\psi\rangle=\hat{\eta}_{L}^{\dagger}|\Omega\rangle$ is the wavefunction of the ordered state. Also note that this single-particle state is macroscopically occupied, i.e., $|\psi\rangle$ describes a condensate with $\frac{\lambda_{L}}{L}$ being the condensate fraction. Finally, by Mermin-Wagner-Hohenberg in two spatial dimensions ( $1+1$ for quantum systems) there can be no spontaneous breaking of a continuous symmetry. Even though this means that following the discussion in Sec. 3.1.1 we cannot expect a finite local order-parameter in the thermodynamic limit, there can be critical behavior and nonanalytic behavior in thermodynamic observables. Berezinsky [Ber72] as well as Kosterlitz and Thouless [KT73] described such features in the two-dimensional XY model of classical spins. 
They argued that in the ordered phase there are algebraically decaying correlation functions. As a consequence, particles can be correlated over large distances motivating the notion of quasi-long-range order (qLRO). Importantly, this was the first description of a topologically ordered state for which no local order-parameter can be defined. QLRO can also be found in one-dimensional quantum systems, for instance in a system of non-interacting hard-core bosons where the dominating natural orbital occupation scales as $\lambda_{L} \sim L^{1 / 2}$. These signatures also carry over to the experimentally relevant realizations of hard-core bosons being confined by a harmonic lattice [RM05].

\section{\begin{tabular}{l|l} 
Spectral functions & 3.2
\end{tabular}}

So far we have discussed time-independent correlation functions and how they are related to physical properties of a system. However, some of the most powerful experimental tools rely on a procedure that is generally characterized by exciting the system and measuring its response. Describing such a process theoretically involves the evaluation of time-dependent correlation functions. A physical picture one may keep in mind is that of an incoming particle traversing the probed material for a certain time and being ejected and measured in a detector, eventually. While travelling through the system the probe particle may be scattered by low-energy excitations. Properties of these excitations, such as their excitation energies, life times, etc., determine the finally detected state of the probe particle. It is, hence, not too surprising that we can gain many insights into the spectral properties of the probed system by studying their time-dependent correlation functions, which determine the outcome of such scattering experiments.

In fact, the relation between the spectral properties of the material and time-dependent correlation functions is very fruitful and goes way beyond the scattering experiments described above. The response of a system to a weak external perturbation can be evaluated by means of linear-response theory. Again, the central quantity is the time-dependent correlation function constructed from the operators modelling the perturbation and the response. Therefrom, susceptibilities can be derived as well as their fundamental connection to thermal fluctuations that is, the celebrated fluctuation-dissipation theorem [CW51, Kub66].

Apart from deep theoretical insights, the established connections between outcomes of scattering experiments and spectral properties stimulated the development of experimental tools that can not be overestimated. For instance, in angle-resolved photoemission spectroscopy (ARPES) experiments the so-called lesser Greens function can be probed directly close to the materials surface, which enables experimentalists to determine the electronic band structure [Dam04]. This way, the existence of a Dirac cone in $\mathrm{Bi}_{2} \mathrm{Te}_{3}$ could be measured proving this material class to be a realization of a three-dimensional topological insulator $\left[\mathrm{CAC}^{+} 09\right]$. Moreover, having direct access to the band structure, ARPES experiments can be exploited to study superconducting gaps giving insight into the underlying pairing mechanisms [DRN $\left.{ }^{+} 08\right]$. Finally, extending the setup by an additional pump excitation ARPES measurements can be performed time-resolved (trARPES). This opens the door to study ultrafast electronic processes with a resolution on the femtosecond time scale. By employing this advanced technique it is possible to follow the dynamics of the band structure, for instance, while the system undergoes a phase transition [PLL $\left.{ }^{+} 06, \mathrm{RHW}^{+} 11\right]$. The theoretical quantities characterizing ARPES spectra measured in experiments are the socalled spectral functions. In the following, we set up the theory of spectral functions utilized in this thesis. We work out the important aspects when calculating this quantity from timedependent correlation functions, i.e., Greens functions, via time-evolution methods for MPS. But before, we briefly introduce the basic principle of ARPES experiments. 
Our starting point is a system composed of electrons described by the Hamilton operator $\hat{H}_{0}$. Photoemission spectroscopy is modeled by the absorption of an incident photon with energy $\hbar \omega$ and momentum $\mathbf{k}$

$$
\hat{H}=\hat{H}_{0}+\hat{H}_{\text {int }}(\mathbf{k}, \omega),
$$

where $\hat{H}_{\text {int }}(\mathbf{k}, \omega)$ contains the light-matter interaction. In ARPES a photo current is measured generated from emitted electrons after exciting the system. This photo current is proportional to the transition rate for the absorption and emission process. The transition rate $w_{i \rightarrow f}$ for exciting an electron by $\hat{H}_{\text {int }}(\mathbf{k}, \omega)$ is obtained from time-dependent perturbation theory and given by Fermi's golden rule

$$
w_{i \rightarrow f}=\frac{2 \pi}{\hbar}\left|\left\langle f\left|\hat{H}_{\mathrm{int}}(\mathbf{k}, \omega)\right| i\right\rangle\right|^{2} \delta\left(E_{f}-E_{i}-\hbar \omega\right),
$$

where $|i\rangle,|f\rangle$ are the initial and final states of the absorption and emission process. Here, we are not going into the conceptional details on how to model the interaction Hamiltonian but recapitulate the usual procedure (at $T=0$ ), only. Typically, the incident light field is introduced by a semi-classical approximation in terms of the minimal coupling $\hat{\mathbf{p}}_{j} \rightarrow \hat{\mathbf{p}}-e \hat{\mathbf{A}}\left(\mathbf{r}_{j}, t\right)$ and described as a plane wave $\hat{\mathbf{A}}(\mathbf{r}, t)=\mathbf{A}_{0} e^{\mathrm{i}(\mathbf{q} \cdot \mathbf{r}-\omega t)}$ in position representation. The initial state $|i\rangle$ is the many-body ground state $\left|N, E_{0}\right\rangle$ for $N$ electrons with ground-state energy $E_{0}$. After the photoemission the system is in a final state given by a superposition of the many-body wavefunctions $\left|N-1, E(\mathbf{q}), \mathbf{k}^{\prime}\right\rangle$, where $\mathbf{q}$ labels excited states with energies $E(\mathbf{q})$ and $\mathbf{k}^{\prime}$ is the label of the excited and emitted electron. The final many-body state is factorized employing the sudden approximation, i.e., the emitted electron is described by a plane wave and decouples from the remaining $N-1$ electron system immediately after the excitation

$$
\left|N-1, E(\mathbf{q}), \mathbf{k}^{\prime}\right\rangle=\hat{c}_{k^{\prime}, \sigma}^{\dagger}|N-1, E(\mathbf{q})\rangle \text {. }
$$

Expressing the light-matter interaction Hamiltonian in second quantization

$$
\hat{H}_{\mathrm{int}}(\mathbf{k}, \omega)=\sum_{n, m} \sum_{\sigma, \sigma^{\prime}} M_{(n \sigma),\left(m \sigma^{\prime}\right)} \hat{c}_{n, \sigma}^{\dagger} \hat{c}_{m, \sigma^{\prime}},
$$

one typically assumes $M_{(n \sigma),\left(m \sigma^{\prime}\right)} \approx$ const. in the energy and momentum-range considered. Then, the overall transition rate can be written as

$$
\begin{aligned}
w_{i \rightarrow f}\left(\mathbf{k}^{\prime}, \varepsilon\right) & \propto \sum_{\mathbf{q}}\left|\left\langle N-1, E(\mathbf{q})\left|\hat{c}_{\mathbf{k}^{\prime}, \sigma}\right| N, E_{0}\right\rangle\right|^{2} \delta\left(E(\mathbf{k})+\varepsilon-E_{0}-\hbar \omega\right) \\
& \equiv A(\mathbf{k}, \varepsilon-\hbar \omega) f_{T=0}(\varepsilon-\hbar \omega)
\end{aligned}
$$

where $\varepsilon$ is the energy of the emitted electron. In the last line we introduced the spectral function $A(\mathbf{k}, \varepsilon-\hbar \omega) \equiv A(\mathbf{k}, \omega)$ as well as the Fermi-Dirac distribution $f_{T}(\varepsilon-\hbar \omega)$ at zero temperature. Now, the goal is to obtain an expression for $A(\mathbf{k}, \omega)$, which can be evaluated numerically.

\section{Spectral functions from Greens function}

We begin by defining the lesser/greater Greens functions $G_{\hat{A}, \hat{B}}^{>/<}\left(t_{1}, t_{2}\right)$ for operators $\hat{A}, \hat{B}$

$$
\begin{array}{lll}
G_{\hat{A}, \hat{B}}^{>}\left(t_{1}, t_{2}\right) & =\left\langle\psi\left|\hat{A}\left(t_{1}\right) \hat{B}\left(t_{2}\right)\right| \psi\right\rangle \quad \text { with } \quad t_{1}>t_{2} \\
G_{\hat{A}, \hat{B}}^{<}\left(t_{1}, t_{2}\right) & =\left\langle\psi\left|\hat{A}\left(t_{2}\right) \hat{B}\left(t_{1}\right)\right| \psi\right\rangle \quad \text { with } \quad t_{1}<t_{2},
\end{array}
$$


and the retarded Greens function $G_{\hat{A}, \hat{B}}^{r}\left(t_{1}, t_{2}\right)$, which can be defined in terms of $G_{\hat{A}, \hat{B}}^{>/<}\left(t_{1}, t_{2}\right)$ as

$$
\begin{aligned}
G_{\hat{A}, \hat{B}}^{r}\left(t_{1}, t_{2}\right) & =-\mathrm{i} \theta\left(t_{1}-t_{2}\right)\left(G_{\hat{A}, \hat{B}}^{>}\left(t_{1}, t_{2}\right)+G_{\hat{B}, \hat{A}}^{<}\left(t_{1}, t_{2}\right)\right) \\
& =-\mathrm{i} \theta\left(t_{1}-t_{2}\right)\left(\left\langle\psi\left|\hat{A}\left(t_{1}\right) \hat{B}\left(t_{2}\right)\right| \psi\right\rangle+\left\langle\psi\left|\hat{B}\left(t_{2}\right) \hat{A}\left(t_{1}\right)\right| \psi\right\rangle\right),
\end{aligned}
$$

with the heaviside step function $\theta(t)$. The time evolution of the operators is generated by a Hamiltonian $\hat{H}$ which we require to be time independent. Using the Schrödinger picture, we can collect some valuable information about these functions. At first we note that using $\hat{U}\left(t_{1}\right) \hat{U}\left(t_{2}\right)=\hat{U}\left(t_{1}+t_{2}\right)$ as well as $\hat{U}^{\dagger}(t)=\hat{U}(-t)$, the time dependencies of $G_{\hat{A}, \hat{B}}^{>/<}\left(t_{1}, t_{2}\right)$ can be recast into a time-translationally invariant operator time evolution and a time-dependency of the state:

$$
\begin{aligned}
G_{\hat{A}, \hat{B}}^{>}\left(t_{1}, t_{2}\right) & =\left\langle\psi\left|\hat{U}^{\dagger}\left(t_{1}\right) \hat{A} \hat{U}\left(t_{1}\right) \hat{U}^{\dagger}\left(t_{2}\right) B \hat{U}\left(t_{2}\right)\right| \psi\right\rangle \\
& =\left\langle\psi\left|\hat{U}^{\dagger}\left(t_{2}\right) \hat{U}\left(t_{2}\right) \hat{U}^{\dagger}\left(t_{1}\right) \hat{A} \hat{U}\left(t_{1}\right) \hat{U}^{\dagger}\left(t_{2}\right) B \hat{U}\left(t_{2}\right)\right| \psi\right\rangle \\
& =\left\langle\psi\left(t_{2}\right)\left|\hat{U}^{\dagger}\left(t_{1}-t_{2}\right) \hat{A} \hat{U}\left(t_{1}-t_{2}\right) \hat{B}\right| \psi\left(t_{2}\right)\right\rangle \equiv G_{\hat{A}, \hat{B}}^{>}\left(t_{1}-t_{2}, t_{2}\right)
\end{aligned}
$$

where in the second line we inserted an identity $\hat{U}^{\dagger}\left(t_{2}\right) \hat{U}\left(t_{2}\right)$ and used $\hat{U}\left(t_{2}\right) \hat{U}^{\dagger}\left(t_{1}\right)=\left(\hat{U}\left(t_{1}\right) \hat{U}^{\dagger}\left(t_{2}\right)\right)^{\dagger}$ in the third equality. By the same reasoning we obtain

$$
G_{\hat{A}, \hat{B}}^{<}\left(t_{1}, t_{2}\right)=\left\langle\psi\left(t_{2}\right)\left|\hat{A} \hat{U}^{\dagger}\left(t_{1}-t_{2}\right) \hat{B} \hat{U}\left(t_{1}-t_{2}\right)\right| \psi\left(t_{2}\right)\right\rangle \equiv G_{\hat{A}, \hat{B}}^{<}\left(t_{1}-t_{2}, t_{2}\right) .
$$

Inserting these expressions into $G_{\hat{A}, \hat{B}}^{r}\left(t_{1}, t_{2}\right)$ yields

$$
G_{\hat{A}, \hat{B}}^{r}\left(t_{1}, t_{2}\right)=-\mathrm{i} \theta\left(t_{1}-t_{2}\right)\left(G_{\hat{A}, \hat{B}}^{>}\left(t_{1}-t_{2}, t_{2}\right)+G_{\hat{B}, \hat{A}}^{<}\left(t_{1}-t_{2}, t_{2}\right)\right) .
$$

Note that in general we can move the operator time evolution from $\hat{A}$ to $\hat{B}$ :

$$
\begin{aligned}
\left\langle\psi\left(t_{2}\right)\left|\hat{A}\left(t_{1}-t_{2}\right) \hat{B}(0)\right| \psi\left(t_{2}\right)\right\rangle & =\left\langle\psi\left|\hat{U}^{\dagger}\left(t_{1}\right) \hat{A} \hat{U}\left(t_{1}\right) \hat{U}^{\dagger}\left(t_{2}\right) \hat{B} \hat{U}\left(t_{2}\right)\right| \psi\right\rangle \\
& =\left\langle\psi\left|\hat{U}^{\dagger}\left(t_{1}\right) \hat{A} \hat{U}\left(t_{1}\right) \hat{U}^{\dagger}\left(t_{2}\right) \hat{B} \hat{U}\left(t_{2}\right) \hat{U}^{\dagger}\left(t_{1}\right) \hat{U}\left(t_{1}\right)\right| \psi\right\rangle \\
& =\left\langle\psi\left(t_{1}\right)\left|\hat{A}(0) \hat{B}\left(t_{2}-t_{1}\right)\right| \psi\left(t_{1}\right)\right\rangle
\end{aligned}
$$

Let us assume for a moment that the reference state $|\psi\rangle$ is an eigenstate of $\hat{H}$ with $\hat{H}|\psi\rangle=E|\psi\rangle$ so that we can evaluate its time dependency: $|\psi(t)\rangle=e^{-\frac{i}{\hbar} E t}|\psi\rangle$. Then, in Eqs. (3.27) and (3.28) the time dependency of the reference state cancels, and setting $\tau \equiv t_{1}-t_{2}$ we can write in a compact notation

$$
G_{\hat{A}, \hat{B}}^{r}\left(t_{1}, t_{2}\right)=-\mathrm{i} \theta(\tau)\left(G_{\hat{A}, \hat{B}}^{>}(\tau)+G_{\hat{B}, \hat{A}}^{<}(\tau)\right) \equiv G_{\hat{A}, \hat{B}}^{r}(\tau) .
$$

We are interested in the frequency space representation of $G_{\hat{A}, \hat{B}}^{r}(\tau)$. Thus, we perform a Fourier transformation

$$
\begin{aligned}
G_{\hat{A}, \hat{B}}^{r}(\omega) & =\int_{-\infty}^{\infty} d \tau G_{\hat{A}, \hat{B}}^{r}(\tau) e^{i \omega \tau} \\
& =-\mathrm{i} \int_{0}^{\infty} d \tau\left(G_{\hat{A}, \hat{B}}^{>}(\tau) e^{i \omega \tau}+G_{\hat{B}, \hat{A}}^{<}(\tau) e^{i \omega \tau}\right) \equiv-\mathrm{i}\left(G_{\hat{A}, \hat{B}}^{+}(\omega)+G_{\hat{B}, \hat{A}}^{-}(\omega)\right),
\end{aligned}
$$


where we have introduced frequency dependent helper functions $G_{\hat{A}, \hat{B}}^{ \pm}(\omega)$. Let the operators $\hat{A}, \hat{B}$ fulfill $\hat{A}^{\dagger}=\hat{B}$ so that

$$
\begin{aligned}
& \langle\psi|\hat{A}| n\rangle\langle n|\hat{B}| \psi\rangle=\langle\psi|\hat{A}| n\rangle\left\langle n\left|\hat{A}^{\dagger}\right| \psi\right\rangle=|\langle\psi|\hat{A}| n\rangle|^{2} \\
& \langle\psi|\hat{B}| n\rangle\langle n|\hat{A}| \psi\rangle=\langle\psi|\hat{B}| n\rangle\left\langle n\left|\hat{B}^{\dagger}\right| \psi\right\rangle=|\langle\psi|\hat{B}| n\rangle|^{2}
\end{aligned}
$$

Writing out explicitely the time-evolution operator and using time-independency of the Hamiltonian we find for $G_{\hat{A}, \hat{B}}^{+}(\omega)$

$$
\begin{aligned}
G_{\hat{A}, \hat{B}}^{+}(\omega) & =\int_{0}^{\infty} d \tau\langle\psi|\hat{A}(\tau) \hat{B}(0)| \psi\rangle e^{i \omega \tau}=\int_{0}^{\infty} d \tau\left\langle\psi\left|\hat{A} e^{-\frac{\mathrm{i}}{\hbar} \hat{H} \tau} \hat{B}\right| \psi\right\rangle e^{i(E / \hbar+\omega) \tau} \\
& =\int_{0}^{\infty} d \tau \sum_{m, n}\langle\psi|\hat{A}| m\rangle\left\langle m\left|e^{\mathrm{i}(E / \hbar+\omega-\hat{H} / \hbar) \tau}\right| n\right\rangle\langle n|\hat{B}| \psi\rangle \\
& =\sum_{n}|\langle\psi|\hat{A}| n\rangle|^{2} \int_{0}^{\infty} d \tau e^{\mathrm{i}\left(E / \hbar+\omega-\omega_{n}\right) \tau}
\end{aligned}
$$

where we have inserted two sets of energy eigenstates $\{|m\rangle\},\{|n\rangle\}$ and defined $\omega_{n}=\langle n|\hat{H}| n\rangle / \hbar$. In case of $G_{\hat{B}, \hat{A}}^{-}$we move the time dependency from $\hat{A}$ to $\hat{B}$ employing Eq. (3.30) and transform the integration variable as $\tau \longrightarrow-\tau$ to obtain

$$
\begin{aligned}
G_{\hat{B}, \hat{A}}^{-}(\omega) & =\int_{0}^{\infty} d \tau\langle\psi|\hat{B}(0) \hat{A}(\tau)| \psi\rangle e^{\mathrm{i} \omega \tau}=\int_{-\infty}^{0} d \tau\langle\psi|\hat{B}(\tau) \hat{A}(0)| \psi\rangle e^{-\mathrm{i} \omega \tau} \\
& =\int_{-\infty}^{0} d \tau\left\langle\psi\left|\hat{B} e^{-\frac{\mathrm{i}}{\hbar} \hat{H} \tau} \hat{A}\right| \psi\right\rangle e^{\mathrm{i}(E / \hbar-\omega) \tau} \\
& =\int_{-\infty}^{0} d \tau \sum_{m, n}\langle\psi|\hat{B}| m\rangle\left\langle m\left|e^{\mathrm{i}(E / \hbar-\omega-\hat{H} / \hbar) \tau}\right| n\right\rangle\langle n|\hat{A}| \psi\rangle \\
& =\sum_{n}|\langle\psi|\hat{B}| n\rangle|^{2} \int_{-\infty}^{0} d \tau e^{\mathrm{i}\left(E / \hbar-\omega-\omega_{n}\right) \tau}
\end{aligned}
$$

These representations imply an important connection between $G_{\hat{A}, \hat{B}}^{+}(\omega)$ and $G_{\hat{A}, \hat{B}}^{-}(\omega)$ :

$$
\begin{aligned}
\left(G_{\hat{A}, \hat{B}}^{+}(\omega)\right)^{*} & =\sum_{n}\left|\left\langle\psi\left|\hat{A}^{\dagger}\right| n\right\rangle\right|^{2} \int_{0}^{\infty} d \tau e^{-\mathrm{i}\left(E / \hbar+\omega-\omega_{n}\right) \tau} \\
& =\sum_{n}|\langle\psi|\hat{A}| n\rangle|^{2} \int_{-\infty}^{0} d \tau e^{\mathrm{i}\left(E / \hbar+\omega-\omega_{n}\right) \tau}=G_{\hat{A}, \hat{B}}^{-}(-\omega)
\end{aligned}
$$

Now, we can write for the real and imaginary part of $G_{\hat{A}, \hat{B}}^{r}(\omega)$

$$
\begin{aligned}
\Re\left[G_{\hat{A}, \hat{B}}^{r}(\omega)\right] & =-\frac{\mathrm{i}}{2}\left(G_{\hat{A}, \hat{B}}^{+}(\omega)+G_{\hat{B}, \hat{A}}^{-}(\omega)-G_{\hat{A}, \hat{B}}^{-}(-\omega)-G_{\hat{B}, \hat{A}}^{+}(-\omega)\right) \\
& =\frac{1}{2 \mathrm{i}}\left(G_{\hat{A}, \hat{B}}^{+}(\omega)-G_{\hat{A}, \hat{B}}^{-}(-\omega)\right)-\frac{1}{2 \mathrm{i}}\left(G_{\hat{B}, \hat{A}}^{+}(-\omega)-G_{\hat{B}, \hat{A}}^{-}(\omega)\right) \\
\Im\left[G_{\hat{A}, \hat{B}}^{r}(\omega)\right] & =-\frac{1}{2}\left(G_{\hat{A}, \hat{B}}^{+}(\omega)+G_{\hat{B}, \hat{A}}^{-}(\omega)+G_{\hat{A}, \hat{B}}^{-}(-\omega)+G_{\hat{B}, \hat{A}}^{+}(-\omega)\right) \\
& =-\frac{1}{2}\left(G_{\hat{A}, \hat{B}}^{+}(\omega)+G_{\hat{A}, \hat{B}}^{-}(-\omega)\right)-\frac{1}{2}\left(G_{\hat{B}, \hat{A}}^{+}(-\omega)+G_{\hat{B}, \hat{A}}^{-}(\omega)\right) .
\end{aligned}
$$


These relations are particularly important since they permit to extend the integration range from $\int_{0}^{\infty} d \tau \longrightarrow \int_{-\infty}^{\infty} d \tau$ in the imaginary part of $G_{\hat{A}, \hat{B}}^{r}(\omega)$. Furthermore, we can write more compactly

$$
\begin{aligned}
& \Re\left[G_{\hat{A}, \hat{B}}^{r}(\omega)\right]=\Im\left[G_{\hat{A}, \hat{B}}^{+}(\omega)\right]-\Im\left[G_{\hat{B}, \hat{A}}^{+}(-\omega)\right] \\
& \Im\left[G_{\hat{A}, \hat{B}}^{r}(\omega)\right]=-\left(\Re\left[G_{\hat{A}, \hat{B}}^{+}(\omega)\right]+\Re\left[G_{\hat{B}, \hat{A}}^{+}(-\omega)\right]\right) .
\end{aligned}
$$

Now we are in the position to connect the helper function $G_{\hat{A}, \hat{B}}^{+}(\omega)$ to the Fourier transformation of the lesser and greater Greens functions. Explicitly replacing $\hat{B}^{\dagger}=\hat{A}$ we find for the real part of $G_{\hat{A}, \hat{B}}^{+}( \pm \omega)$

$$
\begin{aligned}
\Re\left[G_{\hat{A}, \hat{B}}^{+}(\omega)\right] & =\frac{1}{2} \sum_{n}|\langle\psi|\hat{A}| n\rangle|^{2}\left(\int_{0}^{\infty} d \tau e^{\mathrm{i}\left(E / \hbar+\omega-\omega_{n}\right) \tau}+\int_{-\infty}^{0} d \tau e^{\mathrm{i}\left(E / \hbar+\omega-\omega_{n}\right) \tau}\right) \\
& =\frac{1}{2} \sum_{n}|\langle\psi|\hat{A}| n\rangle|^{2} \int_{-\infty}^{\infty} d \tau e^{\mathrm{i}\left(E / \hbar+\omega-\omega_{n}\right) \tau}=\frac{1}{2} \int_{-\infty}^{\infty} d \tau G_{\hat{A}, \hat{B}}^{>}(\tau) e^{\mathrm{i} \omega \tau} \\
& \equiv \frac{1}{2} G_{\hat{A}, \hat{B}}^{>}(\omega),
\end{aligned}
$$

and similarly

$$
\begin{aligned}
\Re\left[G_{\hat{B}, \hat{A}}^{+}(-\omega)\right] & =\frac{1}{2} \sum_{n}|\langle\psi|\hat{B}| n\rangle|^{2} \int_{-\infty}^{\infty} d \tau e^{\mathrm{i}\left(E / \hbar-\omega-\omega_{n}\right) \tau}=\frac{1}{2} \int_{-\infty}^{\infty} d \tau G_{\hat{B}, \hat{A}}^{<}(\tau) e^{\mathrm{i} \omega \tau} \\
& \equiv \frac{1}{2} G_{\hat{B}, \hat{A}}^{<}(\omega) .
\end{aligned}
$$

Using these equalities we can connect to Eq. (3.23), i.e., the photo current measured in ARPES by noting that the integrals are representations of the Dirac $\delta$-function

$$
\begin{aligned}
& \frac{1}{2} G_{\hat{A}, \hat{B}}^{>}(\omega)=\pi \sum_{n}|\langle\psi|\hat{A}| n\rangle|^{2} \delta\left(E / \hbar-\omega_{n}+\omega\right), \\
& \frac{1}{2} G_{\hat{B}, \hat{A}}^{<}(\omega)=\pi \sum_{n}|\langle\psi|\hat{B}| n\rangle|^{2} \delta\left(E / \hbar-\omega_{n}-\omega\right) .
\end{aligned}
$$

Behind these relations, there is a very intuitive physical picture. The quantities $\frac{1}{2} G_{\hat{A}, \hat{B}}^{>/<}(\omega)$ constitute a measure for the energy level structure for they create a $\delta$-pulse signal whenever the frequency $\pm \omega$ matches an energy difference between an energy eigenstate $|n\rangle$ and the reference state $|\psi\rangle$, where the transition $|\psi\rangle \leftrightarrow|n\rangle$ is caused by $\hat{A}$ or $\hat{B}$. In particular, we can identify two distinct types of processes captured by these equations independently if we restrict the frequency to $\omega>0$. Refering to Eq. (3.23) the frequency $\omega$ describes the energy difference between the emitted electron and the incident photon. Denoting for a moment by $\omega$ the frequency of the incident photon we have:

$$
\begin{array}{ll}
G_{\hat{A}, \hat{B}}^{>}(\omega) \neq 0 \Rightarrow\left(E / \hbar-\omega_{n}\right)<0, & \text { Emission of electron with energy } \varepsilon-\hbar \omega>0, \\
G_{\hat{B}, \hat{A}}^{<}(\omega) \neq 0 \Rightarrow\left(E / \hbar-\omega_{n}\right)>0, & \text { Emission of electron with energy } \varepsilon-\hbar \omega<0 .
\end{array}
$$

In ARPES typically emision processes with energies $\varepsilon<\hbar \omega$ are captured ${ }^{4}$ so the relevant quantity is $G_{\hat{B}, \hat{A}}^{<}(\omega)$.

\footnotetext{
${ }^{4}$ The other process would mean that the emitted electron has a larger energy than the incident photon.
} 


\section{Spectral function of non-interacting electrons}

To illustrate these ideas let us consider a chain of $L \in \mathbb{N}$ spinless fermions with periodic boundary conditions and lattice constant $a$

$$
\hat{H}=-t \sum_{i} \hat{c}_{i}^{\dagger} \hat{c}_{i+1}+\hat{c}_{i+1}^{\dagger} \hat{c}_{i}, \quad t>0 \quad \text { with }\left\{\hat{c}_{i}, \hat{c}_{j}^{\dagger}\right\}=\delta_{i, j}
$$

We introduce momentum space operators

$$
\hat{c}_{q_{n}}=\frac{1}{\sqrt{L}} \sum_{i=1}^{L} \hat{c}_{i} e^{-\mathrm{i} q_{n} r_{i}}, \quad q_{n}=\frac{2 \pi}{L}(n-1), \quad n=1, \cdots, L
$$

with $r_{i}=a \cdot i$. Using the relation $\sum_{i} e^{-\mathrm{i} q_{n} r_{i}}=L \delta_{q_{n}, 0}$ and

$$
\sum_{i} \hat{c}_{i}^{\dagger} \hat{c}_{i+1}=\frac{1}{L} \sum_{i} \sum_{n, m} c_{q_{n}}^{\dagger} \hat{c}_{q_{m}} e^{-\mathrm{i}\left(q_{n}-q_{m}\right) r_{i}+\mathrm{i} q_{m} a}=\sum_{q} c_{q}^{\dagger} \hat{c}_{q} e^{\mathrm{i} q a}
$$

the Hamiltonian is diagonalized

$$
\begin{aligned}
\hat{H} & =-t \sum_{i} \hat{c}_{i}^{\dagger} \hat{c}_{i+1}+\hat{c}_{i+1}^{\dagger} \hat{c}_{i}=-t \sum_{q} c_{q}^{\dagger} \hat{c}_{q}\left(e^{\mathrm{i} q a}+e^{-\mathrm{i} q a}\right) \\
& =-2 t \sum_{q} c_{q}^{\dagger} \hat{c}_{q} \cos (q a) \equiv \sum_{q} c_{q}^{\dagger} \hat{c}_{q} \varepsilon_{q}
\end{aligned}
$$

$\varepsilon_{q}=-2 t \cos (q a)$ is the dispersion relation for a system of non-interacting fermions in one dimension. It follows that the eigenstates of $\hat{H}$ can be labeled by their occupations of momentum eigenstates $\hat{c}_{q}^{\dagger} \hat{c}_{q}|q\rangle=q|q\rangle$ and any product state of the form $\left|n(q=-\pi / a), \cdots n\left(q=\pi / a \frac{L-1}{L}\right)\right\rangle$ with $n(q)=0,1$ is an eigenstate of $\hat{H}$. Conveniently, a short notation is given by counting the number of occupied modes $N=\sum_{q} n(q)$ and the eigenstates are written as $\mid N$, $\left.\mathbf{q}\right\rangle$, where $\mathbf{q} \in[0,1]^{\otimes L}$ is a vector labeling the occupied modes. They obey the orthogonality relation $\left\langle N, \mathbf{q} \mid N^{\prime}, \mathbf{q}^{\prime}\right\rangle=\delta_{N, N^{\prime}} \delta_{\mathbf{q}, \mathbf{q}^{\prime}}$. The energy of an eigenstate of $\hat{H}$ is

$$
E(N, \mathbf{q})=\left\langle N, \mathbf{q}\left|\sum_{q} \varepsilon_{q} \hat{c}_{q}^{\dagger} \hat{c}_{q}\right| N, \mathbf{q}\right\rangle=\sum_{q \in \mathbf{q}} \varepsilon_{q}
$$

Then, the ground state for a fixed particle number $N \leq L$ is obtained by filling up modes beginning with the state with lowest energy

$$
\left|N, \mathbf{q}_{0}\right\rangle=\prod_{q=-N / 2}^{N / 2-1} \hat{c}_{q}^{\dagger}|\Omega\rangle
$$

where $\mathbf{q}_{0}$ is the vector with only modes $n \in\left[-\frac{N}{2}, \frac{N}{2}-1\right]$ occupied. We can now evaluate the spectral function measured by ARPES. Using Eq. (3.45) with $\hat{A}=\hat{c}_{q}, \hat{B}=\hat{c}_{q}^{\dagger}$ and setting $\hbar \equiv 1$ it is

$$
\frac{1}{2 \pi} G_{\hat{c}_{q}^{\dagger}, \hat{c}_{q}}^{<}(\omega)=\sum_{\mathbf{q}^{\prime}}\left|\left\langle N, \mathbf{q}_{0}\left|\hat{c}_{q}^{\dagger}\right| N-1, \mathbf{q}^{\prime}\right\rangle\right|^{2} \delta\left(E\left(N, \mathbf{q}_{0}\right)-E\left(N-1, \mathbf{q}^{\prime}\right)-\omega\right) .
$$


Expanding the transition amplitude $|\langle\cdots\rangle|^{2}$, the sum over all possible combinations $\mathbf{q}^{\prime}$ with $N-1$ modes occupied collapses yielding

$$
\begin{aligned}
\frac{1}{2 \pi} G_{\hat{c}_{q}^{\dagger}, \hat{c}_{q}}^{<}(\omega) & =\sum_{\mathbf{q}^{\prime}}\left|\left\langle N-1, \mathbf{q}^{\prime}\left|\hat{c}_{q} \prod_{n=N / 2}^{(N-1) / 2} \hat{c}_{q_{n}}^{\dagger}\right| \Omega\right\rangle\right|^{2} \delta\left(E\left(N, \mathbf{q}_{0}\right)-E\left(N-1, \mathbf{q}^{\prime}\right)-\omega\right) \\
& =\theta\left(q_{N-1}-q\right) \delta\left(\sum_{q^{\prime}=0}^{N-1} \varepsilon_{q}^{\prime}-\sum_{q^{\prime} \neq q} \varepsilon_{q^{\prime}}-\omega\right)=n\left(q_{F}-q\right) \delta\left(\varepsilon_{q}-\omega\right),
\end{aligned}
$$

where we introduced the Fermi momentum $q_{F}=q_{N / 2}$ and identified the Fermi-Dirac distribution $n\left(q_{F}-q\right)=\theta\left(q_{F}-q\right)$ (at zero temperature). The spectral function as measured in ARPES is therefore

$$
A(k, \omega)=\delta\left(\varepsilon_{q}-\omega\right)
$$

i.e., it resembles the electron distribution in the occupied single-particle states at zero temperature. Correspondingly, we find for the greater Greens function

$$
\begin{aligned}
\frac{1}{2 \pi} G_{\hat{c}_{q}, \hat{c}_{q}^{\dagger}}^{>}(\omega) & =\sum_{\mathbf{q}^{\prime}}\left|\left\langle N, \mathbf{q}_{0}\left|\hat{c}_{q}\right| N+1, \mathbf{q}^{\prime}\right\rangle\right|^{2} \delta\left(E\left(N, \mathbf{q}_{0}\right)-E\left(N+1, \mathbf{q}^{\prime}\right)+\omega\right) \\
& =\left[1-n\left(q_{F}-q\right)\right] \delta\left(\sum_{q^{\prime}=0}^{N-1} \varepsilon_{q}^{\prime}-\sum_{q^{\prime} \neq q} \varepsilon_{q^{\prime}}+\omega\right)=\left[1-n\left(q_{F}-q\right)\right] \delta\left(\varepsilon_{q}+\omega\right) .
\end{aligned}
$$

Equations (3.53) and (3.55) demonstrate the numerical relevance of the Fourier transformations of the lesser and greater Greens functions. We have access to the single-particle spectrum of the tight-binding chain where the intensity is given by the Fermi-Dirac distribution. Importantly, evaluating the spectral function numerically we can study the dispersion or gaps of excitations with respect to $n$-particle Greens functions. This is crucial since it permits to detect the condensation of Cooper pairs in the two-particle channel at $k=0$ in superconductors.

\section{\begin{tabular}{l|l} 
Superconductivity & 3.3
\end{tabular}}

Superconductivity (SC) is one of the hallmarks of condensed-matter systems and since its discovery in solid mercury in 1911 by Onnes it is a topic of ongoing research. Accompanied by the effort to develop theories to understand the underlying mechanisms of superconductivity, it also has inspired many concepts of modern physics. For instance, the Higgs mechanism in elementary-particle physics was motivated by the finite penetration depths of magnetic fields into superconductors, i.e., the Meissner effect [MO33]. The Meissner effect itself turned out to be one of the key elements in understanding superconductivity. It enabled a phenomenological theory by Fritz and Heinz London who constructed an expression for the free energy of superconductors, which upon minimization yields the celebrated London equation [LLL35]. For the latter correctly describing the Meissner effect they demonstrated that superconductivity in fact is a thermodynamic phase. Based on this observation, Ginzburg and Landau formulated a phenomenological theory [GL50] founded on Landau's theory of spontaneaous symmetry breaking in continuous phase transitions introducing a complex order parameter field $\Psi$.

One of the main advances due to their formulation is that it gives rise to two types of superconductivity that can be distinguished by their breakdown behavior upon increasing the external 
magnetic field. In particular, in type-II superconductors subject to a strong magnetic field, there are vortices of the magnetic induction forming a regular lattice, each of which carries exactly one flux quantum [A.A57]. At that time it also became clear that the complex order parameter field corresponds to a macroscopic wavefunction for electrons. Nevertheless, the reason for the formation of such an extended state was unclear. An important contribution was the study of the isotope effect in elemental superconductors. Experiments revealed that the transition temperature and thereby the relevant energy scale depends on the isotopic mass as $T_{\mathrm{c}} \propto \frac{1}{\sqrt{M}}$ suggesting that vibrations of the ionic lattice play a crucial role [SRN50, RSN51]. It was due to Bardeen, Cooper and Shrieffer (BCS) to come up with a microscopic theory identifying electron-phonon interactions as the driving force for the formation of bosonic quasi-particles (Cooper pairs), which then condensate into a macroscopically occupied ground state [BCS57]. Astonishingly, they did not only provide a microscopic model but also explicitly constructed the ground-state wave function being a product state of bosonic creation operators. Eventually it was Gor'kov who explicitly derived the Ginzburg-Landau free energy from the BCS theory [Gor59] and to some extend finished the theoretical effort to understand the origin of conventional superconductivity. Interestingly, BCS theory is in principle independent on the underlying mechanism to cause attractive electron-electron interactions. However, the pair-binding energies created by electronphonon interactions are limited to $\sim 1 \mathrm{meV}$ and thereby the largest possible transition temperature to roughly $T_{\mathrm{c}} \approx 30 \mathrm{~K}$. So the question arises, could there be other mechanism for effectively attracting electrons in a solid and if so, can they give rise to higher transition temperatures? The second question has been answered impressively by the discovery of high- $T_{\mathrm{c}}$ superconductivity in the copper oxide $\mathrm{Ba}_{x} \mathrm{La}_{5-x} \mathrm{Cu}_{5} \mathrm{O}_{5(3-y)}$ by Bednorz and Müller in 1986 [BM86]. Their discovery stimulated intense research on unconventional superconductors in cuprate materials [WAT ${ }^{+} 87$, Dag94, LNW06].

The first question has been subject to intense, ongoing research for more than 30 years and there have been various approaches. Asking for symmetry breaking, which goes beyond the homogeneous $s$-wave superconductivity described by BCS theory, is one route. Indeed, there has been experimental verification of $p$ and $d$-wave superconductivity [ORR $\left.{ }^{+} 84, \mathrm{FNP}^{+} 14\right]$. Explicitely investigating alternative pairing mechanisms, for instance mediated by spin fluctuations [JHA99], contributes another fruitful direction. However, the underlying mechanism for the high transition temperatures observed in copper oxides is still under debate.

Recently, another exciting discovery shifted the focus away from studying superconductivity in thermal equilibrium towards non-equilibrium setups. Experiments (e.g., [FFK ${ }^{+} 14, \mathrm{MSF}^{+} 14$, $\left.\mathrm{HKN}^{+} 14, \mathrm{HNK}^{+} 16, \mathrm{MCN}^{+} 16\right]$ on copper oxides, or on $\mathrm{K}_{3} \mathrm{C}_{60}$ ) report the observation of possible photo-induced transient $\mathrm{SC}$ phases, which can exist at elevated temperatures, even above the equilibrium critical temperature $\mathrm{T}_{c}\left[\mathrm{FMK}^{+} 11, \mathrm{FTD}^{+} 11, \mathrm{KHN}^{+} 14\right]$. In these investigations, ultrashort $\mathrm{THz}$ pulses excite single phonon modes, which decay very slowly compared to the typical time scale of the electron dynamics and thereby offer the possibility to control the interaction parameters of the electronic system $\left[\mathrm{SCK}^{+} 15\right]$. Subsequently, the $\omega$-dependent optical conductivity is determined as a function of time via reflectivity measurements using a probe pulse, and $\mathrm{SC}$ correlations are identified by the emergence or enhancement of a signal at $\omega \rightarrow 0$. This has become now a standard experimental procedure, which, however, leaves many questions open, in particular concerning the characterization of the state induced by the pump excitation (see, e.g., Refs. EKW10, KWRM17, WCMD17, BTKM19). We connected to this point studying the two-particle spectral function as an alternative measure for superconductivity in an out-of equilibrium setup in Chap. 8. Additionally, we addressed the question, whether in a minimal model there is the emergence of superconductivity out-of equilibrium modelling the photo excitation by a global quench. Therefore, in the following we recapitulate the basic ingredients of BCS theory 
and the signatures of superconductivity employed in our numerical simulations.

\section{Cooper problem}

The overwhelming success of BCS theory is founded, besides others, on an observation by Cooper that seems paradoxical ${ }^{5}$ [Coo56]. We consider a lattice with non-interacting spin $S=1 / 2$ fermions with Fermi energy $\varepsilon_{F}\left(k_{F}\right)$ given by the highest occupied single-particle mode with Fermi momentum $\mathbf{k}_{F}$ in its ground state (assuming an even number of particles)

$$
|0\rangle=\prod_{|\mathbf{k}|<\mathbf{k}_{F}} \hat{c}_{\mathbf{k}, \uparrow}^{\dagger} \hat{c}_{\mathbf{k}, \downarrow}^{\dagger}|\Omega\rangle
$$

By non-interacting we refer to any system that can be brought into a representation with a Fermi sea as ground state. This also includes weakly interacting systems, i.e., Fermi liquids. The Hamiltonian for the fermions shall be given by $\hat{\tilde{H}}$ and contains a spin-independent two-particle interaction

$$
\begin{aligned}
\hat{\tilde{H}}=\sum_{\mathbf{k}, \sigma}\left(\varepsilon_{k}-\varepsilon_{F}\right) \hat{c}_{\mathbf{k}, \sigma}^{\dagger} \hat{c}_{\mathbf{k}, \sigma} & +\frac{1}{2} \sum_{(\mathbf{k} \sigma),\left(\mathbf{k}^{\prime} \sigma^{\prime}\right), \mathbf{q}}^{k>k_{F}, k \mid>k_{F}} V_{\mathbf{k}, \mathbf{k}^{\prime}, \mathbf{q}} \hat{c}_{\mathbf{k}+\mathbf{q}, \sigma}^{\dagger} \hat{c}_{\mathbf{k}^{\prime}-\mathbf{q}, \sigma^{\prime}}^{\dagger} \hat{c}_{\mathbf{k}^{\prime}, \sigma^{\prime}} \hat{c}_{\mathbf{k}, \sigma}+ \\
& +\sum_{(\mathbf{k} \sigma),\left(\mathbf{k}^{\prime} \sigma^{\prime}\right), \mathbf{q}}^{k>k_{F}, k<k_{F}} V_{\mathbf{k}, \mathbf{k}^{\prime}, \mathbf{q}} \hat{c}_{\mathbf{k}+\mathbf{q}, \sigma}^{\dagger} \hat{c}_{\mathbf{k}^{\prime}-\mathbf{q}, \sigma^{\prime}} \hat{c}_{\mathbf{k}^{\prime}, \sigma^{\prime}}^{\dagger} \hat{c}_{\mathbf{k}, \sigma}+ \\
& +\frac{1}{2} \sum_{(\mathbf{k} \sigma),\left(\mathbf{k}^{\prime} \sigma^{\prime}\right), \mathbf{q}}^{k<k_{F}, k<k_{F}} V_{\mathbf{k}, \mathbf{k}^{\prime}, \mathbf{q}} \hat{c}_{\mathbf{k}+\mathbf{q}, \sigma} \hat{c}_{\mathbf{k}^{\prime}-\mathbf{q}, \sigma^{\prime}} \hat{c}_{\mathbf{k}^{\prime}, \sigma^{\prime}}^{\dagger} \hat{c}_{\mathbf{k}, \sigma}^{\dagger}
\end{aligned}
$$

Note that we introduced the matrix elements $V_{\mathbf{k}, \mathbf{k}^{\prime}, \mathbf{q}}$ of the interaction potential very generally so that only momentum conservation is imposed. Additionally, the sum over the quasi-particle scattering momenta was split and the summation fixed in a way that only excitations above the ground state interact. This reflects the fact that the creation/annihilation operators are already defined in a way accounting for interactions in the ground state. The ground-state energy is denoted by $E_{0}=2 \sum_{k<k_{F}} \varepsilon_{k}$ and we shift the energy scale by $E_{0}$

$$
\hat{H}=\hat{\tilde{H}}-E_{0} \Rightarrow \hat{H}|0\rangle=0 \text {. }
$$

Then, single-particle excitations are eigenstates of $\hat{H}$

$$
\hat{H} \hat{c}_{\mathbf{k}, \sigma}^{\dagger}|0\rangle \equiv \hat{H}|\mathbf{k}, \sigma\rangle= \begin{cases}\left(\varepsilon_{k}-\varepsilon_{F}\right) \hat{H}|\mathbf{k}, \sigma\rangle & \text { if } k>k_{F} \\ -\left(\varepsilon_{k}-\varepsilon_{F}\right) \hat{H}|\mathbf{k}, \sigma\rangle & \text { if } k<k_{F}\end{cases}
$$

with energy $\langle\mathbf{k}, \sigma|\hat{H}| \mathbf{k}, \sigma\rangle=\left|\varepsilon_{k}-\varepsilon_{F}\right| \equiv \eta_{k}$. We now ask for the effect of adding two-particle excitations $\hat{c}_{\mathbf{p}, \sigma}^{\dagger} \hat{c}_{\mathbf{p}^{\prime}, \sigma^{\prime}}^{\dagger}$ above the Fermi sea $\left(|\mathbf{p}|,\left|\mathbf{p}^{\prime}\right|>k_{F}\right)$

$$
\hat{H} \hat{c}_{\mathbf{p}, \sigma^{\prime}}^{\dagger} \hat{c}_{\mathbf{p}^{\prime}, \sigma^{\prime}}^{\dagger}|0\rangle=\left(\eta_{p}+\eta_{p^{\prime}}\right) \hat{c}_{\mathbf{p}, \sigma^{\prime}}^{\dagger} \hat{c}_{\mathbf{p}^{\prime}, \sigma^{\prime}}^{\dagger}|0\rangle+\sum_{\mathbf{q}} \sum_{\sigma, \sigma^{\prime}} V_{\mathbf{p}, \mathbf{p}^{\prime}, \mathbf{q}} \hat{c}_{\mathbf{p}+\mathbf{q}, \sigma}^{\dagger} \hat{c}_{\mathbf{p}-\mathbf{q}, \sigma^{\prime}}^{\dagger}|0\rangle .
$$

\footnotetext{
${ }^{5}$ The following discussion is motivated by chapter two in [Par69]
} 


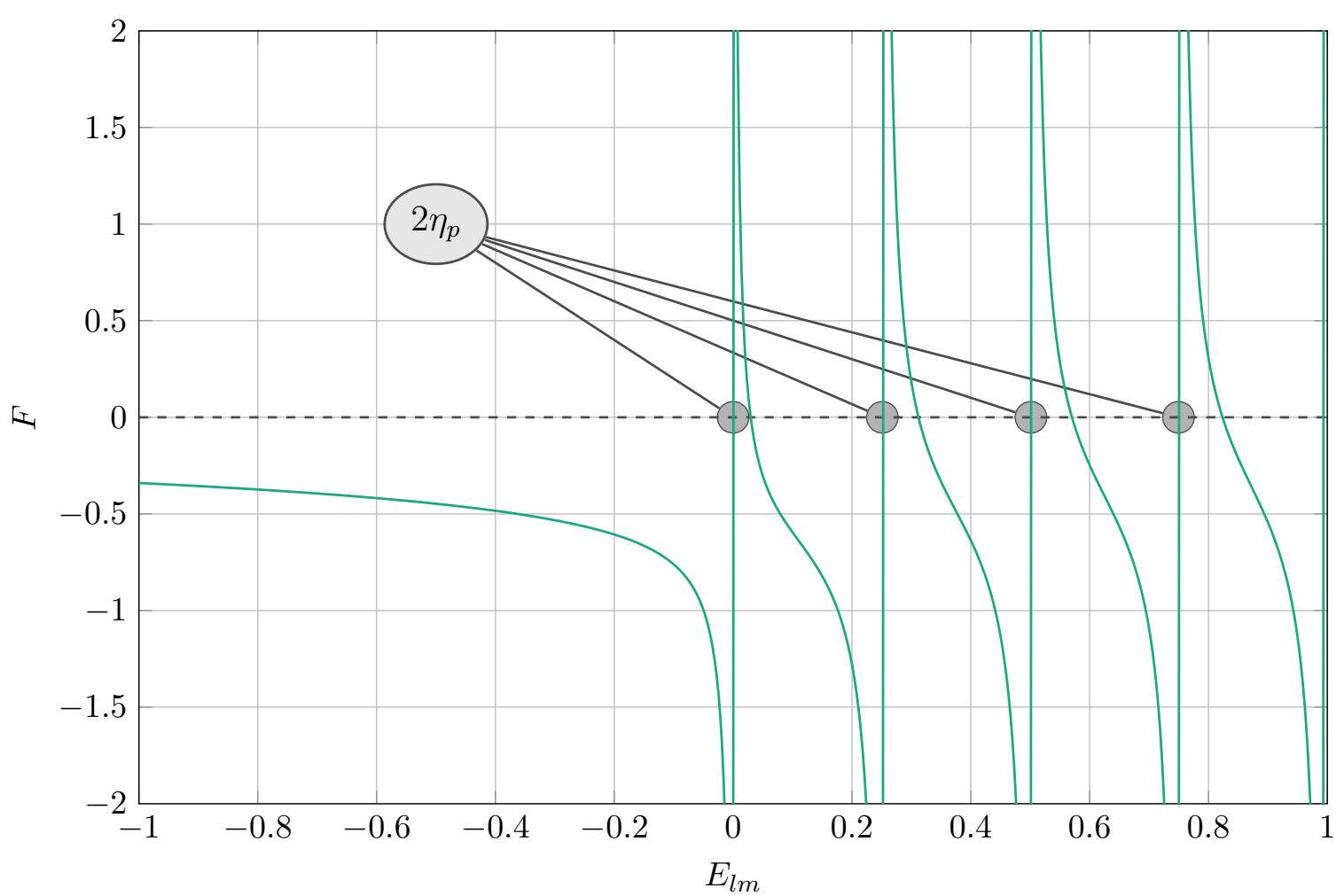

Figure 3.1: Function $F\left(E_{l m}\right)$ (defined in the main text in Eq. (3.67)) determining the possible solutions with negative energy calculated for constant moments of the potential $v_{p}^{l} \equiv 0.1$. Energies are obtained from a tight-binding chain of non-interacting fermions with $t \equiv 1$. The poles of $F\left(E_{l m}\right)$ are marked and correspond to the points where $E_{l m} / 2$ approaches the single-particle excitation energies $\eta_{p}$.

The particles interact and scatter via the potential, and thus this is not an eigenstate of $\hat{H}$ in general but maybe a superposition could do the job. Cooper investigated what the two-particle state with lowest energy might be (and thereby the one with the longest quasi-particle lifetime) by using a general superposition of two-particle excitations. One would expect such a pair-state to have zero momentum. Suppressing the spin degrees of freedom for a moment we have the eigenvalue equation

$$
\begin{aligned}
\hat{H} \sum_{|\mathbf{p}|>k_{F}} g_{\mathbf{p}} \hat{c}_{\mathbf{p}}^{\dagger} \hat{c}_{-\mathbf{p}}^{\dagger}|0\rangle & =2 \sum_{|\mathbf{p}|>k_{F}} g_{\mathbf{p}} \eta_{p} \hat{c}_{\mathbf{p}}^{\dagger} \hat{c}_{-\mathbf{p}}^{\dagger}|0\rangle+\sum_{\mathbf{q}} \sum_{|\mathbf{p}|>k_{F}} g_{\mathbf{p}} V_{\mathbf{p},-\mathbf{p}, \mathbf{q}} \hat{c}_{\mathbf{p}+\mathbf{q}}^{\dagger} \hat{c}_{-\mathbf{p}-\mathbf{q}}^{\dagger}|0\rangle \\
& =E \sum_{|\mathbf{p}|>k_{F}} g_{\mathbf{p}} \hat{c}_{\mathbf{p}}^{\dagger} \hat{c}_{-\mathbf{p}}^{\dagger}|0\rangle \equiv E|\psi\rangle .
\end{aligned}
$$

Multiplication with $\langle 0| \hat{c}_{-\mathbf{p}^{\prime}, \bar{\sigma}^{\prime}} \hat{c}_{\mathbf{p}^{\prime}, \sigma^{\prime}}$ then after a bit of algebra yields

$$
\left(E-2 \eta_{p^{\prime}}\right)\left(g_{\mathbf{p}^{\prime}}-g_{-\mathbf{p}^{\prime}} \delta_{\sigma, \sigma \prime}\right)=\sum_{\mathbf{q}} V_{\mathbf{p}^{\prime}-\mathbf{q},-\mathbf{p}^{\prime}+\mathbf{q}, \mathbf{q}}\left(g_{\mathbf{p}^{\prime}-\mathbf{q}}-g_{-\mathbf{p}^{\prime}+\mathbf{q}} \delta_{\sigma, \sigma^{\prime}}\right) .
$$

At this point the potential has not been specified further. However, if we assume an isotropic system, then $|\psi\rangle$ needs to be an angular momentum eigenstate. Consequently, the state has to have a well-defined parity, i.e., the coeffients have to transform as $g_{-\mathbf{p}} \rightarrow \pm g_{\mathbf{p}}$. In case of an even parity the above equation becomes

$$
\left(E-2 \eta_{p^{\prime}}\right) g_{\mathbf{p}^{\prime}}\left(1-\delta_{\sigma, \sigma \prime}\right)=\sum_{\mathbf{q}} V_{\mathbf{p}^{\prime}-\mathbf{q},-\mathbf{p}^{\prime}+\mathbf{q}, \mathbf{q}} g_{\mathbf{p}^{\prime}-\mathbf{q}}\left(1-\delta_{\sigma, \sigma^{\prime}}\right),
$$


and, hence, can have non-trivial solutions only if $\delta_{\sigma, \sigma^{\prime}}=0$, i.e., the resulting pair is in a singlet state. Therefore, it is reasonable to express the interaction coefficients in spherical harmonics. For that purpose, we relabel $\mathbf{p}^{\prime} \rightarrow \mathbf{p}$ and $\mathbf{p}^{\prime}-\mathbf{q} \rightarrow \mathbf{p}^{\prime}$ and expand

$$
V_{\mathbf{p}^{\prime},-\mathbf{p}^{\prime}, \mathbf{p}-\mathbf{p}^{\prime}}=\sum_{l} \sum_{m=-l}^{l} \lambda_{l} v_{p}^{l}\left[v_{p^{\prime}}^{l}\right]^{*} Y_{l m}(\mathbf{p} / p) Y_{l m}^{*}\left(\mathbf{p}^{\prime} / p^{\prime}\right),
$$

where $l$ is chosen even to ensure correct parity. For the coefficients we make the ansatz $g_{\mathbf{p}}=$ $g_{p} Y_{l m}(\mathbf{p} / p)$ to obtain

$$
\begin{gathered}
\left(E_{l m}-2 \eta_{p}\right) g_{p} Y_{l m}(\mathbf{p} / p)=\sum_{\mathbf{p}^{\prime}} g_{p^{\prime}} Y_{l m}\left(\mathbf{p}^{\prime} / p^{\prime}\right) \sum_{l^{\prime}} \sum_{m^{\prime}=-l^{\prime}}^{l^{\prime}} \lambda_{l^{\prime}} v_{p}^{l^{\prime}}\left[v_{p^{\prime}}^{l^{\prime}}\right]^{*} Y_{l^{\prime} m^{\prime}}(\mathbf{p} / p) Y_{l^{\prime} m^{\prime}}^{*}\left(\mathbf{p}^{\prime} / p^{\prime}\right) \\
\Leftrightarrow g_{p}=\frac{\lambda_{l} v_{p}^{l}}{E_{l m}-2 \eta_{p}} K_{l}, \quad K_{l}=\sum_{p^{\prime}} g_{p^{\prime}}\left[v_{p^{\prime}}^{l}\right]^{*},
\end{gathered}
$$

where we used the orthogonality of the spherical harmonics and replaced $E \rightarrow E_{l m}$. The coefficients $g_{p}$ can be eliminated by substituting the left into the right equation so that we find a condition for the pair energies $E_{l m}$

$$
1=\lambda_{l} \sum_{p^{\prime}} \frac{\left|v_{p^{\prime}}^{l}\right|^{2}}{E_{l m}-2 \eta_{p^{\prime}}} \equiv \lambda_{l} F\left(E_{l m}\right) .
$$

The function $F\left(E_{l m}\right)$ has poles at $E_{l m}=2 \eta_{p}$ where it changes sign as shown in Fig. 3.1. Importantly, if $E_{l m}<\min _{p} 2 \eta_{p}$ then $F\left(E_{l m}\right)$ is always negative and $F\left(E_{l m}<\min _{p} 2 \eta_{p}\right) \in(0,-\infty)$. Therefore, if there is only one negative interaction coefficient $\lambda_{l}$, then there is always a pair state with energy $E_{l m}<\eta_{F}$, no matter how small $\left|\lambda_{l}\right|$ might be. It follows that the Fermi sea is instable against an arbitrary small attractive interaction. The drastic consequence is that as long as the quasi-particle description remains approximately valid, i.e., there is a Fermi surface, there will always be the formation of pairs with opposite momentum condensing into a new ground state.

Now, we simplify the problem and assume that there is a cut-off energy so that

$$
v_{p}^{l}= \begin{cases}1 & 0<\eta_{p}<\omega_{0} \\ 0 & \text { else } .\end{cases}
$$

Note that for an attractive interaction mediated by phonon scattering such a cut-off energy naturally arises by the Debye frequency of the solid. We can write $F\left(E_{l m}\right)$ for negative $E_{l m}$ as

$$
\begin{aligned}
F\left(E_{l m}\right) & =-\int_{0}^{\omega_{0}} d \eta N(\eta) \frac{1}{\left|E_{l m}\right|+2 \eta} \approx-N(0) \int_{0}^{\omega_{0}} d \eta \frac{1}{\left|E_{l m}\right|+2 \eta} \\
& =-\frac{N(0)}{2} \ln \left(\frac{\left|E_{l m}\right|+\omega_{0}}{\left|E_{l m}\right|}\right),
\end{aligned}
$$

where we approximated the density of states $N(\eta)$ to be constant in the energy interval $\left[0, \omega_{0}\right]$ above the Fermi level and absorbed a normalization factor arising from setting $v_{p}^{l} \equiv 1$. Reinserting into Eq. (3.67) we can solve for the pair energy

$$
\left|E_{l m}\right|=2 \omega_{0} \frac{1}{e^{\frac{2}{N(0) \mid \lambda_{l} l}}-1} .
$$


The limit of weak attractive interaction $N(0) \lambda_{l} \ll 1$ is of particular interest and we find

$$
\left|E_{l m}\right| \approx 2 \omega_{0} e^{\frac{-2}{N(0)\left|\lambda_{l}\right|}}
$$

It is important to realize that the binding energy cannot be obtained by a perturbation theory in the two-particle interaction potential $V_{\mathbf{k}, \mathbf{k}^{\prime}, \mathbf{q}} \sim \lambda_{l}$ since the exponent is singular at $\lambda_{l}=0$. The limit of strong attractive interaction leads to tightly bound electron pairs. However, they do not give rise to a superconducting ground state but in turn yield a Bose-Einstein condensate, which can be characterized by pairs being localized in real space whereas Cooper pairs are localized in momentum space. Note that the same analysis can be carried out assuming the parity of the pair to be odd. In this situation it follows that non-trivial solutions of Eq. (3.63) only exist for triplet pairing.

It is a remarkable finding that the Fermi sea can collapse under the influence of an arbitrary weak attractive interaction. The only assumption in the above reasoning was the existence of a Fermi surface separating the unccoupied from the occupied fermionic quasi-particle states. Therefore, even though these considerations did not yield the new ground state after the collapse, the practical insight that an arbitrary weak attractive interaction completely changes the game is worth the work.

\section{\begin{tabular}{l|l} 
BCS theory & 3.3 .2
\end{tabular}}

Motivated by the instability of the Fermi sea, Cooper, Bardeen, and Schrieffer proposed a simplified model extracting the most important lessons learned from the Cooper problem [BCS57]. Therein, a weak interaction is assumed acting only in the case of vanishing total momentum. Additionally, they restricted their model to the isotropic case that is $l=0$ and therefore we expect singlet pairing. The resulting model is the famous BCS Hamiltonian

$$
\hat{H}_{\mathrm{BCS}}=\sum_{\mathbf{k}, \sigma}\left(\varepsilon_{k}-\varepsilon_{F}\right) \hat{c}_{\mathbf{k}, \sigma}^{\dagger} \hat{c}_{\mathbf{k}, \sigma}-\frac{g}{V} \sum_{\mathbf{k}, \mathbf{k}^{\prime} \in \Omega_{0}} \hat{c}_{\mathbf{k}^{\prime}, \uparrow}^{\dagger} \hat{c}_{-\mathbf{k}^{\prime}, \downarrow}^{\dagger} \hat{c}_{-\mathbf{k}, \downarrow} \hat{c}_{\mathbf{k}, \uparrow},
$$

where $\Omega_{0}=\left\{\mathbf{k} \in \mathbb{R}^{d} \mid \varepsilon_{F}-\omega_{0}<\varepsilon_{k}<\varepsilon_{F}+\omega_{0}\right\}$. Therein, $g>0$ is the strength of the attractive interaction and $\Omega_{0}$ the volume in momentum space in which it is active. The prefactor $1 / V$ is a normalization that is required because the double sum $\sum_{\mathbf{k}, \mathbf{k}^{\prime}}$ scales as $V^{2}$ but the Hamiltonian should scale extensively in the system's volume $V$.

The model can be solved by employing the Bogoliubov-Valatin transformation [Bog58, VB58]. The physical picture on which it is founded is to assume that the ground state contains pairs of quasi-particles. A single-particle excitation on top of such a ground state cannot be made of an isolated quasi-particle excitation. This can be deduced easily from the previous consideration in Sec. 3.3.1: Exciting a single quasi-particle leaves behind a corresponding hole, which is an excitation, too. An ansatz to account for this observation is to assume elementary excitations being constructed from superpositions of quasi-particles and their corresponding holes

$$
\hat{b}_{\mathbf{k}, \uparrow}=u_{k} \hat{c}_{\mathbf{k}, \uparrow}-v_{k} \hat{c}_{-\mathbf{k}, \downarrow}^{\dagger}, \quad \hat{b}_{\mathbf{k}, \downarrow}=u_{k} \hat{c}_{\mathbf{k}, \downarrow}+v_{k} \hat{c}_{-\mathbf{k}, \uparrow}^{\dagger},
$$

with real coefficients $u_{k}, v_{k}$. The ansatz should canonically transform fermionic quasi-particles into each other so that canonical commutator relations are required. This means in particular that

$$
\begin{aligned}
\left\{\hat{b}_{\mathbf{k}, \sigma}, \hat{b}_{\mathbf{k}^{\prime}, \sigma^{\prime}}^{\dagger}\right\} & =\left(u_{\mathbf{k}, \sigma}^{2}+v_{\mathbf{k}, \sigma}^{2}\right) \delta_{\mathbf{k}, \mathbf{k}^{\prime}} \delta_{\sigma, \sigma^{\prime}} \stackrel{!}{=} \delta_{\mathbf{k}, \mathbf{k}^{\prime}} \delta_{\sigma, \sigma^{\prime}} \\
\Rightarrow 1 & =u_{\mathbf{k}, \sigma}^{2}+v_{\mathbf{k}, \sigma}^{2} .
\end{aligned}
$$


It is easy to verify that $\left\{\hat{b}_{\mathbf{k}, \sigma}, \hat{b}_{\mathbf{k}^{\prime}, \sigma^{\prime}}\right\}=\left\{\hat{b}_{\mathbf{k}, \sigma}^{\dagger}, \hat{b}_{\mathbf{k}^{\prime}, \sigma^{\prime}}^{\dagger}\right\}=0$, and therefore the $\hat{b}_{\mathbf{k}, \sigma}^{[\dagger]}$ 's are indeed fermionic operators. Using the normalization condition the inverse transformation is given by

$$
\hat{c}_{\mathbf{k}, \uparrow}^{\dagger}=u_{k} \hat{b}_{\mathbf{k}, \uparrow}+v_{k} \hat{b}_{-\mathbf{k}, \downarrow}^{\dagger}, \quad \hat{c}_{\mathbf{k}, \downarrow}^{\dagger}=u_{k} \hat{b}_{\mathbf{k}, \downarrow}+v_{k} \hat{b}_{-\mathbf{k}, \uparrow}^{\dagger} .
$$

The BCS Hamiltonian can now be written in terms of the $\hat{b}_{\mathbf{k}, \sigma}^{[\dagger]}$ 's

$$
\begin{aligned}
\hat{H}_{\mathrm{BCS}}=2 \sum_{\mathbf{k}}\left(\varepsilon_{k}-\varepsilon_{F}\right) v_{k}^{2} & +\sum_{\mathbf{k}, \sigma}\left(\varepsilon_{k}-\varepsilon_{F}\right)\left(u_{k}^{2}-v_{k}^{2}\right) \hat{b}_{\mathbf{k}, \sigma}^{\dagger} \hat{b}_{\mathbf{k}, \sigma}+ \\
& 2 \sum_{\mathbf{k}, \sigma}\left(\varepsilon_{k}-\varepsilon_{F}\right) u_{k} v_{k}\left(\hat{b}_{\mathbf{k}, \uparrow}^{\dagger} \hat{b}_{-\mathbf{k}, \downarrow}^{\dagger}+\hat{b}_{-\mathbf{k}, \downarrow} \hat{b}_{\mathbf{k}, \uparrow}\right)-\frac{g}{V} \sum_{\mathbf{k}, \mathbf{k}^{\prime} \in \Omega_{0}} \hat{\Delta}_{\mathbf{k}}^{\dagger} \hat{\Delta}_{\mathbf{k}^{\prime}},
\end{aligned}
$$

with the definition

$$
\hat{\Delta}_{\mathbf{k}}=\hat{c}_{-\mathbf{k}, \downarrow} \hat{c}_{\mathbf{k}, \uparrow}=u_{k}^{2} \hat{b}_{-\mathbf{k}, \downarrow} \hat{b}_{\mathbf{k}, \uparrow}-v_{k}^{2} \hat{b}_{\mathbf{k}, \uparrow}^{\dagger} \hat{b}_{-\mathbf{k}, \downarrow}^{\dagger}+u_{k} v_{k}\left(\hat{b}_{-\mathbf{k}, \downarrow} \hat{b}_{-\mathbf{k},-\uparrow}^{\dagger}-\hat{b}_{\mathbf{k}, \uparrow}^{\dagger} \hat{b}_{\mathbf{k}, \uparrow}\right) .
$$

The transformed Hamiltonian contains anomalous terms, for instance, $\hat{b}_{\mathbf{k}, \uparrow}^{\dagger} \hat{b}_{-\mathbf{k}, \downarrow}^{\dagger}$. Defining the quasi-particle number operators $\hat{n}_{\mathbf{k}, \sigma}^{b}=\hat{b}_{\mathbf{k}, \sigma}^{\dagger} \hat{b}_{\mathbf{k}, \sigma}$ the Hamiltonian does not conserve the global quasi-particle number $\hat{N}^{b}=\sum_{\mathbf{k}, \sigma} \hat{n}_{\mathbf{k}, \sigma}^{b}$. However, due to the large number of degrees of freedom, we can assume that in the ground state the fluctuations $\delta \hat{n}_{\mathbf{k}, \sigma}^{b}=\hat{n}_{\mathbf{k}, \sigma}^{b}-\left\langle\hat{n}_{\mathbf{k}, \sigma}^{b}\right\rangle$ are small. Thus we can ignore the effects of these anomalous contributions. Our motivation was to construct operators consituting the elementary excitations above the ground state. We thus assume that the ground state is the vacuum of these excitations, i.e., an eigenstate of the occupation-number operators. Minimizing the expectation value of $E=\left\langle\hat{H}_{\mathrm{BCS}}\right\rangle$ with respect to $u_{k}$ for a general eigenstate of the $\hat{n}_{\mathbf{k}, \sigma}^{b}$ 's yields

$$
0 \stackrel{!}{=} \frac{\partial E}{\partial u_{k}}=\left[-4\left(\varepsilon_{k}-\varepsilon_{F}\right) u_{k}+2 \Delta \frac{u_{k}^{2}-v_{k}^{2}}{v_{k}}\right]\left(1-n_{\mathbf{k}, \uparrow}-n_{\mathbf{k}, \downarrow}\right)
$$

where

$$
\Delta=\frac{g}{V} \sum_{\mathbf{k} \in \Omega_{0}}\left\langle\Delta_{\mathbf{k}}\right\rangle=\frac{g}{V} \sum_{\mathbf{k} \in \Omega_{0}} u_{k} v_{k}\left(1-n_{\mathbf{k}, \uparrow}-n_{\mathbf{k}, \downarrow}\right) .
$$

Employing normalization $u_{k}^{2}+v_{k}^{2}=1$ we can solve for $u_{k}, v_{k}$ as a function of $\Delta$

$$
2 u_{k} v_{k}=\frac{\Delta}{\sqrt{\left(\varepsilon_{k}-\varepsilon_{F}\right)^{2}+\Delta^{2}}},
$$

and upon inserting into the expression for $\Delta$ we obtain a self-consistency condition

$$
1=\frac{g}{2 V} \sum_{\mathbf{k} \in \Omega_{0}} \frac{1-n_{\mathbf{k}, \uparrow}-n_{\mathbf{k}, \downarrow}}{\sqrt{\left(\varepsilon_{k}-\varepsilon_{F}\right)^{2}+\Delta^{2}}}
$$

This is the gap equation for general occupation-number eigenstates of the quasi-particle excitations $\hat{b}_{\mathbf{k}, \sigma}^{[\dagger]}$. It determines $\Delta$ as a function of the attractive interaction $g$. According to our intuition the ground state should be obtained when setting $n_{\mathbf{k}, \sigma} \equiv 0$ and in fact, using Eq. (3.80) it is easy to see that this is indeed the case when calculating the matrix element $\left\langle\hat{H}_{\mathrm{BCS}}\right\rangle$ with the occupation number eigenstates. Therefore, in the ground state the self-consistency condition becomes

$$
1=\frac{g}{2 V} \sum_{\mathbf{k} \in \Omega_{0}} \frac{1}{\sqrt{\left(\varepsilon_{k}-\varepsilon_{F}\right)^{2}+\Delta^{2}}} .
$$


Assuming a constant density of states near the Fermi level we can replace the sum by an integral, again, and solve for $\Delta$

$$
\begin{gathered}
1 \approx \frac{g N(0)}{2} \int_{-\omega_{0}}^{\omega_{0}} d E \frac{1}{\sqrt{E^{2}-\Delta^{2}}}=\frac{g N(0)}{2} \ln \left(\frac{\sqrt{\omega_{0}^{2}+\Delta^{2}}+\omega_{0}}{\sqrt{\omega_{0}^{2}+\Delta}-\omega_{0}}\right) \approx \frac{g N(0)}{2} \ln \left(\frac{4 \omega_{0}^{2}}{\Delta^{2}}\right) \\
\Rightarrow \Delta=2 \omega_{0} e^{-\frac{1}{g N(0)}} .
\end{gathered}
$$

In the approximation of vanishing fluctuations of the quasi-particle excitations $\hat{b}_{\mathbf{k}, \sigma}^{\dagger}$ the ground state exhibits a finite value of $\Delta$. It is then straightforward to use this approximation and expand the transformed BCS Hamiltonian $\hat{H}_{\mathrm{BCS}}$ in a mean-field decoupling scheme

$$
\frac{g}{V} \sum_{\mathbf{k}, \mathbf{k}^{\prime} \in \Omega_{0}} \hat{\Delta}_{\mathbf{k}}^{\dagger} \hat{\Delta}_{\mathbf{k}^{\prime}} \approx \Delta \sum_{\mathbf{k} \in \Omega_{0}}\left(\hat{\Delta}_{\mathbf{k}}^{\dagger}+\hat{\Delta}_{\mathbf{k}}\right)
$$

As expected, the obtained Hamiltonian is diagonal in the quasi-particle excitations

$$
\hat{H}_{\mathrm{BCS}}=\sum_{\mathbf{k}, \sigma} \eta_{k} \hat{b}_{\mathbf{k}, \sigma}^{\dagger} \hat{b}_{\mathbf{k}, \sigma}+\text { const. }, \quad \eta_{k}=\sqrt{\left(\varepsilon_{k}-\varepsilon_{F}\right)^{2}+\Delta^{2}},
$$

with dispersion relation $\eta_{k}$. Here, we can see that the BCS ground state is separated from the excitations by the finite gap $\Delta$. Upon closing the gap the single-particle dispersion relation for the non-interacting Fermi sea is recovered. Note that a finite gap also implies finite expectation values $\left\langle\hat{\Delta}_{\mathbf{k}}\right\rangle=\left\langle\hat{c}_{-\mathbf{k}, \downarrow} \hat{c}_{\mathbf{k}, \uparrow}\right\rangle \neq 0$ in the ground state. This is a remarkable observation since it implies that the ground state breaks the $U(1)$ gauge symmetry $\hat{c}_{\mathbf{k}, \sigma} \rightarrow e^{\mathrm{i} \varphi} \hat{c}_{\mathbf{k}, \sigma}$ under which the BCS Hamiltonian is invariant. Therefore, $\left\langle\hat{\Delta}_{\mathbf{k}}\right\rangle$ constitutes a local order-parameter in momentum space and from Sec. 3.1.2 the correlation function $\left\langle\hat{\Delta}_{\mathbf{k}} \hat{\Delta}_{\mathbf{k}^{\prime}}\right\rangle$ should acquire a finite value in the thermodynamic limit. Additionally, note that the pair operators $\hat{c}_{-\mathbf{k}, \downarrow} \hat{c}_{\mathbf{k}, \uparrow}$ have zero total momentum $\mathbf{K}$. We can, hence, expect the ground state wavefunction to be a condensate of such Cooper pairs exhibiting an overall wave vector $\mathbf{K}=0$, i.e., a state with zero center of mass motion. The corresponding real space wavefunction is then expected to be constant $\psi\left(\mathbf{r}_{1}, \ldots\right)=\Psi e^{\mathrm{i} \varphi}$ where $|\Psi|^{2}$ is the density of electrons in the condensate. Moreover the ground state expectation value $\left\langle\hat{c}_{i, \uparrow} \hat{c}_{i, \downarrow}\right\rangle$ has to be finite in the superconducting state, though not constant. Numerically, this enables us to use the framework of natural orbitals and occupations (see Sec. 3.1.2) and use a finite-size scaling to extrapolate into the thermodynamic limit. From the considerations about natural orbital occupations in the thermodynamic limit we also know that these expectation values should scale extensively. Thus, a macroscopic fraction of such Cooper pairs occupies the ground state, which should translate into a dominating spectral weight at $\mathbf{K}=0$ in the spectral function of such pair operators.

\section{Beyond BCS and non-equilibrium superconductivity}

The discussion so far was based on a mean-field decoupling of the pair interaction and isotropic $s$-wave pairing. Clearly, after the formulation of BCS theory there were many extensions to overcome these restrictions (and also those that have not been mentioned yet; consider multiband systems, for instance). Studying anisotropic potentials/gap functions for instance gave rise to $p$ - and $d$-wave superconductors. Therein, the dominating expansion coefficient for the attractive potential has $l \neq 0$. As a consequence, the gap function exhibits nodes allowing for 
low-energy excitations, which in turn can modify thermodynamic quantities such as the specific heat $\left[\mathrm{ORR}^{+} 84\right]$. Triplet pairing is also of great interest. On the one hand it is this pairing mechanism that drives $\mathrm{He}_{3}$ into its superfluid phase and thus can be studied experimentally in great detail. On the other hand it has been shown that spin fluctuations in heavy fermion systems can give rise to triplet pairing [MSRV86] and were found in $\mathrm{Sr}_{2} \mathrm{RuO}_{4}$ [RS95, NMML04]. Finally, the origin of high- $T_{\mathrm{c}}$ superconductivity in underdoped cuprates is still an open problem, more than 30 years after their experimental discovery. For the latter Zhang and Rice [ZR88] argued that an effective microscopic model for the $\mathrm{Cu}-\mathrm{O}$ planes is given by the single-band Hubbard model Eq. (2.57) in two dimensions and in the limit of strong repulsion $U / t \gg 1$, yielding the so-called $t-J$ model

$$
\hat{H}_{t-J}=-t_{h} \sum_{j, \sigma}\left(\hat{c}_{j, \sigma}^{\dagger} \hat{c}_{j+1, \sigma}+\text { H. c. }\right)+J \sum_{j}\left(\hat{\mathbf{S}}_{j} \hat{\mathbf{S}}_{j+1}-\frac{\hat{n}_{i} \hat{n}_{j}}{4}\right) .
$$

Here, the fermionic operators $\hat{c}_{j, \sigma}^{[\dagger]}$ are subject to projections that restrict the local basis states to $|0\rangle,|\uparrow\rangle,|\downarrow\rangle$, i.e., double occupancies are forbidden. Understanding the properties of this model in two dimensions is still an open problem.

However, the Hubbard model comes only with a contact interaction. Therefore, in order to study unconventional pairing it seems reasonable to include a finite interaction range between electrons, too. Taking into account the nearest-neighbor interactions only motivates the extended Hubbard model

$$
\hat{H}=-t_{h} \sum_{j, \sigma}\left(\hat{c}_{j, \sigma}^{\dagger} \hat{c}_{j+1, \sigma}+\text { H. c. }\right)+U \sum_{j} \hat{n}_{j, \uparrow} \hat{n}_{j, \downarrow}+V \sum_{j} \hat{n}_{j} \hat{n}_{j+1} \cdot
$$

Already in one dimension and at zero temperature this model exhibits a rich ground state phase diagram and the ground state in the $U-V$ plane at half filling has been subject to intense studies [TF02, Jec02, SBC04]. In particular, there are singlet and triplet superconducting as well as charge- and spin-density wave ground states. Conceptually, it is tempting to assume that the nearest-neighbor density-density interactions can be modulated by vibrations of the underlying lattice degrees of freedom. The basic idea behind this is that the lifetime of phonon excitations are much longer than the time scales on which electronic dynamics happens. This way, the overlaps between single-particle orbitals can be modulated and consequently the derived effective coupling constants. In fact, there are experimental indications strengthening this picture $\left[\mathrm{FMK}^{+} 11, \mathrm{SCK}^{+} 15\right]$.

This guides us to a very recent set of experiments in which superconductivity was studied out-of equilibrium by photo-exciting a sample using THz pulses to address single phonon modes $\left[\mathrm{FFK}^{+} 14\right.$, $\left.\mathrm{MSF}^{+} 14, \mathrm{HKN}^{+} 14, \mathrm{HNK}^{+} 16, \mathrm{MCN}^{+} 16\right]$. Experimentally, copper oxides as well as $\mathrm{K}_{3} \mathrm{C}_{60}$ were studied and indications for enhanced superconducting correlations in a transient state reported. Most strikingly, the enhancement was found above the equilibrium critical temperature $T>T_{\mathrm{c}}$. Pushing systems out-of equilibrium seems to render the problem even more complicated. However, there are also nice features, for instance the Mermin-Wagner-Hohenberg theorem explicitly relies on thermal equilibrium. This may open loopholes to have local order-parameters acquiring a finite value also in one-dimensional quantum systems. We studied this question in Chap. 8, where we used the concept of off-diagonal long-range order to ask for the possible emergence of superconductivity out-of equilibrium. 


\section{Topological superconductivity

Topological superconductivity is a fascinating subject that recently experienced rapidly growing interest [LSDS10, $\mathrm{AOR}^{+} 11, \mathrm{SASF} 11, \mathrm{LF} 12, \mathrm{KMB}_{12}, \mathrm{AHM}^{+} 16, \mathrm{SA17}, \mathrm{XB18}$ ]. Combining two intriguing phenomena of condensed-matter physics is by itself a convincing argument for intense studies. However, apart from the academic interest, materials exhibiting topological superconductivity are also promising candidates for an experimental realization of low-decoherence qubits, the building blocks of quantum computers. But what is the connection between toplogical superconductivity and quantum computers?

Let us sketch the general idea. On the one hand, topological superconductors break global charge conservation down to a global $\mathbb{Z}_{2}$ symmetry, i.e., parity. Here, keeping parity conserved is crucial because it allows for eigenstates with zero energy. On the other hand, by the bulkboundary correspondence [MS11, EG11] they feature helical edge modes. Applying an external magnetic field, i.e., breaking time-reversal symmetry, energetically separates the edge modes so that the low-energy physics of the system can be described by an effective theory for only one spin direction. The clue is that in superconductors the excitations are superpositions of electron and hole states rendering these edge modes Majorana fermions that can appear spatially separated. They are topologically protected by particle-hole symmetry, i.e., resistent against external perturbations and in principle can be manipulated individually while encoding their information non-locally. ${ }^{6}$ Finally, exhibiting a non-Abelean statistics makes them a seemingly ideal candidate for a highly fault-tolerant qubit [Kit03, NSS $\left.{ }^{+} 08\right]$. However, despite the large interest finding unique experimental signatures for Majorana fermions turned out to be a very challenging task and there are a variety of suggestions (see [Bee13] for an overview).

The basic idea how to obtain spatially separated and localized Majorana modes can be formulated in terms of a very simple model first discussed in that context by Kitaev [Kit01]. In order to clarify the connection between topological superconductivity and localized Majorana modes, we are going to summarize the important parts of Kitaev's analysis in the following. Thereby we can collect the formalism and important ingredients to motivate more realistic models exhibiting the desired physics.

\section{Bogoliubov de-Gennes representation}

Let us stick to the case of spinless fermions with creation/annihilation operators $\hat{c}_{j}^{\dagger}, \hat{c}_{j}$ fulfilling the usual anticommutation relations $\left\{\hat{c}_{j}, \hat{c}_{k}^{\dagger}\right\}=\delta_{j, k}$. Now we consider an arbitrary Hamiltonian $\hat{H}$, which is quadratic in the fermions, i.e., in its most general form it can be written in a Bogoljubov de-Gennes (BdG) representation

$$
\hat{H}=-\frac{1}{2} \sum_{j, k=1}^{L}\left(\begin{array}{ll}
\hat{c}_{j}^{\dagger} & \hat{c}_{j}
\end{array}\right) \underbrace{\left(\begin{array}{cc}
A_{j, k} & -\bar{B}_{j, k} \\
B_{j, k} & -\bar{A}_{j, k}
\end{array}\right)}_{:=\mathbf{H}_{j, k}}\left(\begin{array}{c}
\hat{c}_{k} \\
\hat{c}_{k}^{\dagger}
\end{array}\right),
$$

where the prefactor is just for convention and $\mathbf{H}_{j, k}$ is a $2 \times 2$ matrix which fulfills $\mathbf{H}_{j, k}=-\mathbf{H}_{k, j}$ for $k \neq j$. The $\mathrm{BdG}$ representation yields insight into important properties of quadratic fermion

\footnotetext{
${ }^{6}$ Note that Majorana fermions have to come in pairs but the information encoded is inscribed in the parity of the state, which can be very non-local.
} 
models. First of all note that the matrix $\mathbf{H}$ created from $\mathbf{H}_{j, k}$ has the dimensions $2 L \times 2 L$, so it may seem we have complicated the problem. In fact we have not, and the doubling of the representation comes with some nice symmetry properties of the eigenvalues and eigenstates. Clearly, we can immediately ask for the effect of a particle-hole transformation $\hat{c}_{j} \longrightarrow \hat{c}_{j}^{\dagger} \hat{c}_{j}^{\dagger} \longrightarrow$ $-\hat{c}_{j}$. In the BdG representation this is conveniently written in terms of the Pauli matrix $\tau^{x}$ and the operator of complex conjugation $\hat{K}$

$$
\begin{aligned}
\mathbf{C}=\boldsymbol{\tau}^{x} \hat{K} \Rightarrow \mathbf{C H}_{j, k} \mathbf{C}^{-1} & =\left(\begin{array}{cc}
0 & 1 \\
1 & 0
\end{array}\right) \hat{K}\left(\begin{array}{cc}
A_{j, k} & -\bar{B}_{j, k} \\
B_{j, k} & -\bar{A}_{j, k}
\end{array}\right) \hat{K}^{\dagger}\left(\begin{array}{ll}
0 & 1 \\
1 & 0
\end{array}\right) \\
& =\left(\begin{array}{cc}
-A_{j, k} & \bar{B}_{j, k} \\
-B_{j, k} & \bar{A}_{j, k}
\end{array}\right)=-\mathbf{H}_{j, k} .
\end{aligned}
$$

On the other hand we also have

$$
\left(\begin{array}{ll}
-\hat{c}_{j} & \hat{c}_{j}^{\dagger}
\end{array}\right)\left(\begin{array}{cc}
A_{j, k} & -\bar{B}_{j, k} \\
B_{j, k} & -\bar{A}_{j, k}
\end{array}\right)\left(\begin{array}{c}
\hat{c}_{k}^{\dagger} \\
-\hat{c}_{k}
\end{array}\right)=\left(\begin{array}{cc}
\hat{c}_{j}^{\dagger} & \hat{c}_{j}
\end{array}\right) \mathbf{C}\left(\begin{array}{cc}
A_{j, k} & -\bar{B}_{j, k} \\
B_{j, k} & -\bar{A}_{j, k}
\end{array}\right) \mathbf{C}^{-1}\left(\begin{array}{c}
\hat{c}_{k} \\
\hat{c}_{k}^{\dagger}
\end{array}\right) .
$$

Hence, invariance under particle-hole symmetry requires $\mathbf{C H}_{j, k} \mathbf{C}^{-1}=-\mathbf{H}_{j, k}$ which is manifestly ensured by the above definition of the matrix elements of $\mathbf{H}_{j, k}$. Now from invariance under particle-hole symmetry of the BdG representation one can immediately conclude that the eigenvalues of $\mathbf{H}$ come in pairs $\pm \varepsilon_{\nu}$. This can be seen if we define $\hat{\mathbf{c}}^{[\dagger]}=\left(\begin{array}{ccc}\hat{c}_{1}^{[\dagger]} & \cdots & \hat{c}_{L}^{[\dagger]}\end{array}\right)$ and reorder the columns and rows of $\mathbf{H}$ in a proper way so that it can be represented in an equivalent BdG form

$$
\hat{H}=-\frac{1}{2} \underbrace{\left(\begin{array}{ll}
\hat{\mathbf{c}}^{\dagger} & \hat{\mathbf{c}}
\end{array}\right)}_{:=\boldsymbol{\psi}^{\dagger}} \underbrace{\left(\begin{array}{cc}
\mathbf{H}_{\hat{\mathbf{c}}^{\dagger}, \hat{\mathbf{c}}} & \mathbf{H}_{\hat{\mathbf{c}}^{\dagger}, \hat{\mathbf{c}}^{\dagger}} \\
\mathbf{H}_{\hat{\mathbf{c}}, \hat{\mathbf{c}}} & \mathbf{H}_{\hat{\mathbf{c}}, \hat{\mathbf{c}}^{\dagger}}
\end{array}\right)}_{:=\mathbf{H}_{B d G}} \underbrace{\left(\begin{array}{c}
\hat{\mathbf{c}} \\
\hat{\mathbf{c}}^{\dagger}
\end{array}\right)}_{:=\boldsymbol{\psi}} .
$$

Particle-hole symmetry of the $\mathbf{H}_{j, k}$ enforces $\overline{\mathbf{H}}_{\hat{\mathbf{c}}^{\dagger}, \hat{\mathbf{c}}}=-\mathbf{H}_{\hat{\mathbf{c}}, \hat{\mathbf{c}}^{\dagger}}$ and $\overline{\mathbf{H}}_{\hat{\mathbf{c}}^{\dagger}, \hat{\mathbf{c}}^{\dagger}}=-\mathbf{H}_{\hat{\mathbf{c}}, \hat{\mathbf{c}}}$. Thus, $\mathbf{H}_{B d G}$ transforms under the symmetry operation $\left(\tau^{x} \otimes \hat{\mathbf{1}}_{L \times L}\right) \hat{K}$ and can be brought into blockdiagonal form

$$
\mathbf{H}_{B d G}=\mathbf{H}_{B d G}^{+} \oplus \mathbf{H}_{B d G}^{-},
$$

and the eigenvalues of $\mathbf{H}_{B d G}^{ \pm}$fulfill $\varepsilon_{\nu}^{+}=-\varepsilon_{\nu}^{-} \equiv-\varepsilon_{\nu}$.

The ground state of $\hat{H}$ is obtained by constructing the eigenstate with the lowest energy. Diagonalizing $\mathbf{H}_{B d G}$ in the BdG representation by means of a unitary transformation $\chi$ we obtain

$$
\hat{H}=-\frac{1}{2} \boldsymbol{\psi}^{\dagger} \boldsymbol{\chi}^{\dagger} \boldsymbol{\chi} \mathbf{H}_{B d G} \boldsymbol{\chi}^{\dagger} \boldsymbol{\chi} \boldsymbol{\psi}=-\frac{1}{2} \sum_{\nu=1}^{L} \varepsilon_{\nu}^{+} \phi_{+, \nu}^{\dagger} \phi_{+, \nu}+\varepsilon_{\nu}^{-} \phi_{-, \nu}^{\dagger} \phi_{-, \nu},
$$

where we have already labeled the eigenmodes $\hat{\phi}_{\nu}=\sum_{j=1}^{L} \chi_{\nu, j} \hat{c}_{j}+\chi_{\nu, j+L} \hat{c}_{j}^{\dagger}$ by their eigenvalues \pm 1 with respect to particle-hole symmetry. Since the eigenmodes $\hat{\phi}_{ \pm, \nu}$ inherit the anticommutation relations of the fermionic ladder operators, the ground state of $\hat{H}$ is obtained by adding all modes $\phi_{\nu}^{+}$to the fermionic vacuum so that the ground state has the minimal energy $-\frac{1}{2} \sum_{\nu=1}^{L} \varepsilon_{\nu}$. Here we can make an important observation, which is related to the fact that eigenvalues of $\mathbf{H}_{B d G}$ always come in pairs. When adding a mode $\hat{\phi}_{\nu}^{-}$to the ground state it cancels the energy contribution of the corresponding mode $\hat{\phi}_{\nu}^{+}$in the ground state, i.e., the quasi-particle created by $\hat{\phi}_{\nu}^{+}$ 
(a)

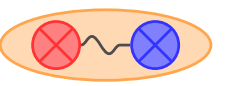

(b)
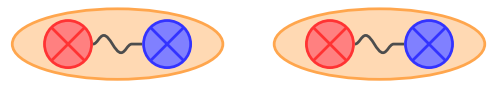

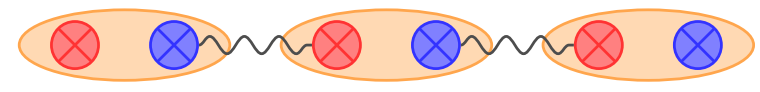

Figure 3.2: Tight-binding chains with fermionic degrees of freedom at each site (orange circles) decomposed into Majorana fermions (red and blue circles), here at the example of $L=3$ lattice sites. (a) illustrates the typical on-site pairing of Majorana fermions while (b) shows the situation suggested by Kitaev [Kit01], where neighboring Majorana fermions are paired across bonds only.

is annihilated by $\hat{\phi}_{\nu}^{-}$. This means that if under a change of the parameters of $\hat{H}$ a quasi-particle created by $\hat{\phi}_{\nu}^{+}$crosses $E=0$, then its partner $\hat{\phi}_{\nu}^{-}$also has to cross $E=0$, i.e., there is a level crossing at $E=0$. Note that if the ground state has a well-defined parity, then this level-crossing causes a change in the parity of the ground state as it removes a quasi-particle created by $\hat{\phi}_{\nu}^{+}$.

\section{\begin{tabular}{l|l} 
Majorana zero modes & 3.4 .2
\end{tabular}}

In any model that can be mapped to $L$ fermionic degrees of freedoms, the latter can be decomposed in terms of $2 L$ Majorana fermions. Using the defining property of Majorana fermions: $\hat{\gamma}^{\dagger}=\hat{\gamma}$ we can construct two Majorana fermions from the real and imaginary part of the fermionic ladder operators

$$
\hat{\gamma}_{2 j-1}=\hat{c}_{j}+\hat{c}_{j}^{\dagger}, \quad \hat{\gamma}_{2 j}=-\mathrm{i}\left(\hat{c}_{j}-\hat{c}_{j}^{\dagger}\right)
$$

and it is easy to check the anticommutation relations of the so-defined operators

$$
\left\{\hat{\gamma}_{j}, \hat{\gamma}_{k}\right\}=2 \delta_{j, k}, \quad j, k \in\{1, \ldots, 2 L\}
$$

In the $\mathrm{BdG}$ representation we can transform the vector of ladder operators by defining a unitary transformation $\mathbf{U}$

$$
\mathbf{U}=\frac{1}{\sqrt{2}} \mathbf{Q} \quad \text { with } \quad \mathbf{Q}=\left(\begin{array}{cc}
1 & 1 \\
-\mathrm{i} & \mathrm{i}
\end{array}\right)
$$

so that the Hamiltonian becomes

$$
\hat{H}=-\frac{1}{4} \sum_{j, k=1}^{L}\left(\begin{array}{ll}
\hat{\gamma}_{2 j-1} & \hat{\gamma}_{2 j}
\end{array}\right) \mathbf{Q} \mathbf{H}_{j, k} \mathbf{Q}^{\dagger}\left(\begin{array}{c}
\hat{\gamma}_{2 k-1} \\
\hat{\gamma}_{2 k}
\end{array}\right) \equiv-\frac{1}{2} \sum_{j, k=1}^{2 L} \hat{\gamma}_{j} \tilde{H}_{j, k} \hat{\gamma}_{k} .
$$

Thus, any quadratic fermionic Hamiltonian can be written in terms of Majorana fermions. In general tight-binding models the parameters $A_{j, k}, B_{j, k}$ in Eq. (3.89) are pairing up Majorana fermions on the same site and thus there are no isolated Majoranas as shown in Fig. 3.2a. However, Kitaev noted that Eq. (3.98) enables us to design pairings in a way that can leave unpaired Majorana operators. The simplest way to do so would be a Hamiltonian that pairs two Majoranas across a bond and not on the same site

$$
\hat{H}_{M}=-\mathrm{i} \Gamma \sum_{j=1}^{L-1} \hat{\gamma}_{2 j} \hat{\gamma}_{2 j+1}
$$


as also shown in Fig. 3.2b. In order to obtain such a Hamiltonian we need to impose conditions on the matrix elements $\tilde{\mathbf{H}}_{j, k}$. Let us write out the transformed matrix first

$$
\begin{aligned}
\tilde{\mathbf{H}}_{j, k} & =-\frac{1}{2}\left(\begin{array}{cc}
1 & 1 \\
-\mathrm{i} & \mathrm{i}
\end{array}\right)\left(\begin{array}{cc}
A_{j, k} & -\bar{B}_{j, k} \\
B_{j, k} & -\bar{A}_{j, k}
\end{array}\right)\left(\begin{array}{cc}
1 & \mathrm{i} \\
1 & -\mathrm{i}
\end{array}\right) \\
& =\left(\begin{array}{cc}
\mathrm{i} \Im\left[A_{j, k}+B_{j, k}\right] & -\mathrm{i} \Re\left[A_{j, k}-B_{j, k}\right] \\
\mathrm{i} \Re\left[A_{j, k}+B_{j, k}\right] & \mathrm{i} \Im\left[A_{j, k}-B_{j, k}\right]
\end{array}\right) \equiv\left(\begin{array}{cc}
\tilde{H}_{2 j-1,2 k-1} & \tilde{H}_{2 j-1,2 k} \\
\tilde{H}_{2 j, 2 k-1} & \tilde{H}_{2 j, 2 k}
\end{array}\right) .
\end{aligned}
$$

The diagonal elements are generated by the imaginary parts of the coefficients $A_{j, k}, B_{j, k}$ and we restrict our considerations to $\Im\left[A_{j, k}\right]=\Im\left[B_{j, k}\right] \equiv 0$. Note that possible complex phases in $B_{j, k}$ can be wrapped conveniently into a redefinition of the Majorana operators $\hat{\gamma}_{j} \rightarrow e^{\mathrm{i} \phi_{j}} \hat{\gamma}_{j}$. Comparing to Eq. (3.99) and using Eq. (3.100) we then find that only the nearest-neighbor coefficients can be non-vanishing. They generate the contributions

$$
\begin{aligned}
& k=j+1: \quad-\frac{1}{2}\left(\begin{array}{ll}
\hat{\gamma}_{2 j-1} & \hat{\gamma}_{2 j}
\end{array}\right)\left(\begin{array}{cc}
0 & \tilde{H}_{2 j-1,2 j+2} \\
\tilde{H}_{2 j, 2 j+1} & 0
\end{array}\right)\left(\begin{array}{l}
\hat{\gamma}_{2 j+1} \\
\hat{\gamma}_{2 j+2}
\end{array}\right) \\
& k=j-1: \quad-\frac{1}{2}\left(\begin{array}{ll}
\hat{\gamma}_{2 j-1} & \hat{\gamma}_{2 j}
\end{array}\right)\left(\begin{array}{cc}
0 & \tilde{H}_{2 j-1,2 j-2} \\
\tilde{H}_{2 j, 2 j-3} & 0
\end{array}\right)\left(\begin{array}{l}
\hat{\gamma}_{2 j-3} \\
\hat{\gamma}_{2 j-2}
\end{array}\right) .
\end{aligned}
$$

In this representation we read off the conditions

$$
\left.\begin{array}{ll}
k=j+1: & \Re\left[A_{j, j+1}\right]-\Re\left[B_{j, j+1}\right] \stackrel{!}{=} 0 \\
k=j-1: & \Re\left[A_{j, j-1}\right]+\Re\left[B_{j, j-1}\right] \stackrel{!}{=} 0
\end{array}\right\} \Rightarrow \Re\left[A_{j, j \pm 1}\right]= \pm \Re\left[B_{j, j \pm 1}\right]= \pm \Gamma .
$$

Reinserting this parameter into the tight-binding Hamiltonian we obtain Kitaev's result for a chain of spinless fermions with unpaired Majorana operators at the boundaries

$$
\hat{H}_{M}=\sum_{j=1}^{L-1}-\Gamma\left(\hat{c}_{j}^{\dagger} \hat{c}_{j+1}+\text { h.c. }\right)+\Gamma\left(\hat{c}_{j} \hat{c}_{j+1}+\text { h.c. }\right) .
$$

Notably, there has to be a nearest-neighbor pairing term $\hat{c}_{j} \hat{c}_{j+1}+$ h.c. in order to have unpaired Majorana fermions. The existence of a zero-mode can be checked easily by returning to Eq. (3.99) and defining new ladder operators, which act on the bonds $(j, j+1)$

$$
\hat{\tilde{c}}_{j}=\frac{1}{2}\left(\hat{\gamma}_{2 j}+\mathrm{i} \hat{\gamma}_{2 j+1}\right), \quad \hat{\tilde{c}}_{j}^{\dagger}=\frac{1}{2}\left(\hat{\gamma}_{2 j}-\mathrm{i} \hat{\gamma}_{2 j+1}\right) .
$$

They yield commutation relations with the Hamiltonian

$$
\left[\hat{\tilde{c}}_{j}, \hat{H}_{M}\right]=-2 \Gamma \hat{\tilde{c}}_{j}, \quad\left[\hat{\tilde{c}}_{j}^{\dagger}, \hat{H}_{M}\right]=2 \Gamma \hat{\tilde{c}}_{j}^{\dagger},
$$

and therefore

$$
\hat{H}_{M}=2 \Gamma \sum_{j=1}^{L-1}\left(\hat{\tilde{c}}_{j}^{\dagger} \hat{\tilde{c}}_{j}-\frac{1}{2}\right)
$$

We have found $\hat{H}_{M}$ to be diagonal in the modes created by the bond operators $\hat{\tilde{c}}_{j}^{\dagger}$. But these operators are completely decoupled from the left and right Majorana edge states $\hat{\gamma}_{0,2 L}$ so that

$$
\left[\hat{\gamma}_{0,2 L}, \hat{H}_{M}\right]=0
$$


Thus, if $\left|\varepsilon_{0}\right\rangle$ is a ground state of $\hat{H}_{M}$, then $\left(c_{0} \hat{\gamma}_{0}+c_{2 L} \hat{\gamma}_{2 L}\right)\left|\varepsilon_{0}\right\rangle$ is another ground state and it is easy to see that they can be distinguished by their parity. Therefore, the ground state is doubly degenerated. Additionally, since the left and right Majorana operators do not appear in $\hat{H}_{M}$ at all, the eigenstate of $\left(c_{0} \hat{\gamma}_{0}+c_{2 L} \hat{\gamma}_{2 L}\right)\left|\varepsilon_{0}\right\rangle$ must have the same energy as $\left|\varepsilon_{0}\right\rangle$. By particle-hole symmetry this is only possible if these are zero-energy states, i.e., $\varepsilon_{0}=0$.

Next, we are going to elaborate on these states in more detail, convince ourselves that they correspond to localized Majorana fermions and that their appearance is not restricted to a particular point in parameter space. But to do so we have to study the phase diagram in more detail.

\section{Edge states and topological phase}

To treat the more general situation of generic hopping and pairing, we reintroduce a hopping parameter $t$ and pairing energy $\Delta$ while also taking into account an on-site chemical potential $\mu$. To diagonalize $\hat{H}_{M}$ in the bulk we perform a Fourier transformation $\hat{c}_{j}=\frac{1}{\sqrt{L}} \sum_{q} e^{-\mathrm{i} q r_{j}} \hat{c}_{q}$ with $r_{j}=j \cdot a$, lattice constant $a$, and $q=-\pi a / L, \ldots, \pi a / L$, imposing periodic boundary conditions. We then find

$$
\begin{aligned}
\hat{H}_{M} & =\sum_{j}-t\left(\hat{c}_{j}^{\dagger} \hat{c}_{j+1}+\text { h.c. }\right)+\Delta\left(\hat{c}_{j} \hat{c}_{j+1}+\text { h.c. }\right)-\mu \hat{c}_{j}^{\dagger} \hat{c}_{j} \\
& =-\frac{1}{L} \sum_{q, k} t\left(\hat{c}_{q}^{\dagger} \hat{c}_{k} e^{\mathrm{i}(q-k) r_{j}} e^{-\mathrm{i} k a}+\text { h.c. }\right)-\left(\Delta \hat{c}_{q} \hat{c}_{k} e^{-\mathrm{i}(q+k) r_{j}} e^{-\mathrm{i} k a}+\text { h.c. }\right)-\mu \sum_{k} \hat{c}_{k}^{\dagger} \hat{c}_{k} \\
& =-\sum_{k}\left(\left(t e^{-i k a}+\frac{\mu}{2}\right) \hat{c}_{k}^{\dagger} \hat{c}_{k}+\text { h.c. }\right)-\left(\Delta \hat{c}_{k} \hat{c}_{-k} e^{-i k a}+\text { h.c. }\right) \\
& =\frac{1}{2} \sum_{k}\left(\begin{array}{ll}
\hat{c}_{k}^{\dagger} & \hat{c}_{-k}
\end{array}\right)\left(\begin{array}{cc}
-(2 t \cos (k a)+\mu) & 2 \mathrm{i} \Delta \sin (k a) \\
-2 \mathrm{i} \Delta \sin (k a) & 2 t \cos (k a)+\mu
\end{array}\right)\left(\begin{array}{c}
\hat{c}_{k} \\
\hat{c}_{-k}^{\dagger}
\end{array}\right)
\end{aligned}
$$

which can be diagonalized in each block labeled by the momentum $k$ using a unitary transformation $\mathbf{U}(k)=\left(\begin{array}{ll}\mathbf{u}^{+}(k) & \mathbf{u}^{-}(k)\end{array}\right)^{\dagger}$. The eigenvalues are

$$
E^{ \pm}(k)= \pm \frac{1}{2} \sqrt{(2 t \cos (k a)+\mu)^{2}+4 \Delta^{2} \sin ^{2}(k a)}
$$

and the ground state is obtained by populating all eigenstates with $E(k)<0$

$$
|G S\rangle=\prod_{k}\left(u_{1}^{-}(k) \hat{c}_{k}^{\dagger}+u_{2}^{-}(k) \hat{c}_{-k}\right)|0\rangle .
$$

The situation $\Delta=0$ corresponds to a tight-binding chain and finite $|\Delta|$ opens a gap with the ground state being superconducting if $|\mu|<2 t$. Since the spectrum is invariant under a sign change in $\mu$, this means that there are three phases: two specified by $|\mu|>2 t$ and one with $|\mu|<2 t$. The single-particle dispersion relations $E^{ \pm}(k)$ are shown exemplary in Fig. 3.3 for both phases as well as the dispersion at the phase transitions $|\mu|=2 t$. Note the gap closing and reopening at the phase boundaries. The phase regions $|\mu|>2 t$ correspond to trivial superconducting phases as can be seen by taking the limit $\mu \rightarrow \mp \infty$ yielding either a fully occupied or empty chain. To gain some further insight into the phase inbetween, it is instructive 
(a)

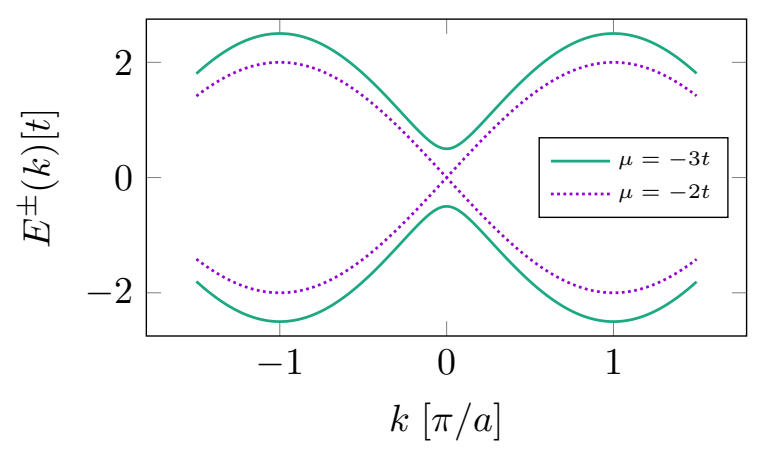

(b)

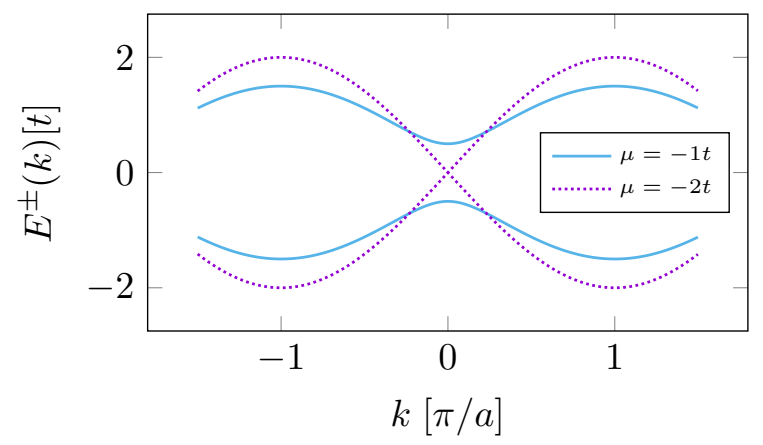

Figure 3.3: Positive and negative eigenvalues $E^{ \pm}(k)$ of Kitaev chain in trivial (a) and topological (b) phase. Parameters are set to $t=\Delta=1.0$ with $\mu$ varying between trivial $(\mu=-3 t)$ and topological phase $(\mu=-1 t)$ as well as at the phase transition $\mu=-2 t$.

to rewrite Eq. (3.109) in terms of Pauli matrices

$$
\begin{gathered}
\boldsymbol{\tau}^{y}=\left(\begin{array}{cc}
0 & -\mathrm{i} \\
\mathrm{i} & 0
\end{array}\right) \quad \text { and } \boldsymbol{\tau}^{z}=\left(\begin{array}{cc}
1 & 0 \\
0 & -1
\end{array}\right), \\
\Rightarrow \hat{H}_{M}=\frac{1}{2} \sum_{k}\left(\begin{array}{cc}
\hat{c}_{k}^{\dagger} & \hat{c}_{-k}
\end{array}\right) \underbrace{\left(-(2 t \cos (k a)+\mu) \boldsymbol{\tau}^{z}-2 \Delta \sin (k a) \boldsymbol{\tau}^{y}\right)}_{:=\mathbf{H}(k)}\left(\begin{array}{c}
\hat{c}_{k} \\
\hat{c}_{-k}^{\dagger}
\end{array}\right) .
\end{gathered}
$$

In this representation we can formally take $k \in[-\pi, \pi]$ to be a continuous variable to study the continuum limit. We model a boundary by cutting the system at the origin, for instance, imposing the boundary conditions $\psi(x \leq 0) \equiv 0$ and $\psi(x \rightarrow \infty)=0$. We now ask for the existence of eigenstates $|\phi\rangle$ with $E(k)=0$ that is, $\left\langle\hat{H}_{M} \phi \mid \hat{H}_{M} \phi\right\rangle=0$ and make the (somewhat clever) ansatz

$$
\mathbf{v}_{\phi}^{\dagger}(x)=\left(\begin{array}{l}
u \\
v
\end{array}\right)\left(e^{-q_{1} x}-e^{-q_{2} x}\right) .
$$

Note that we need two independent eigenvectors specified by $q_{1,2}$ to fulfill the boundary condition $\mathbf{v}_{\phi}^{\dagger}(0)=0$. Let us determine these $q$ 's by performing a Fourier transformation of $\mathbf{v}_{\phi}^{\dagger}$ and searching for eigenstates of $\mathbf{H}^{2}=0$. We obtain the condition

$$
\begin{aligned}
\mathbf{H}^{2}(\mathrm{i} q) & =\left(-(2 t \cosh (q)+\mu) \boldsymbol{\tau}^{z}-2 \Delta \sinh (q) \boldsymbol{\tau}^{y}\right)^{2} \stackrel{!}{=} 0, \\
\Leftrightarrow 0 & =(2 t \cosh (q)+\mu)^{2}-4 \Delta^{2} \sinh ^{2}(q) .
\end{aligned}
$$

Here, the adavantage of searching eigenstates of $\hat{H}_{M}^{2}$ becomes evident, enabling us to use orthogonality of the Pauli matrices to get the equation in the last line. The last equation can have four solutions, which are determined by

$$
2 t \cosh (q)+\mu= \pm 2 \Delta \sinh (q) .
$$

However, the signs are due to the fact that there are two orthogonal eigenstates of the matrix $\alpha \boldsymbol{\tau}^{z}+\beta \boldsymbol{\tau}^{y}$ and we absorb them in the pairing term for the moment $\Delta_{\alpha}=\alpha \Delta, \alpha= \pm 1$. Solving 
this last equation for $q$ we obtain two solutions

$$
e^{-q_{1,2}^{\alpha}}=\frac{-\mu \pm \sqrt{\mu^{2}-4 t^{2}+4 \Delta_{\alpha}^{2}}}{2\left(t+\Delta_{\alpha}\right)}
$$

Now we can return to determining the coefficients $u, v$ by solving the eigenvalue equation

$$
\begin{gathered}
\left(\begin{array}{cc}
-2 t \cosh \left(q_{1,2}^{\alpha}\right)+\mu & 2 \mathrm{i} \Delta \sinh \left(q_{1,2}^{\alpha}\right) \\
-2 \mathrm{i} \Delta \sinh \left(q_{1,2}^{\alpha}\right) & 2 t \cosh \left(q_{1,2}^{\alpha}\right)+\mu
\end{array}\right)\left(\begin{array}{c}
u_{\alpha} \\
v_{\alpha}
\end{array}\right)=0 \cdot v_{\phi}^{\dagger}\left(q_{1,2}^{\alpha}\right) \\
\Rightarrow\left(\begin{array}{c}
u_{+} \\
v_{+}
\end{array}\right)=\left(\begin{array}{c}
1 \\
1
\end{array}\right), \quad\left(\begin{array}{c}
u_{-} \\
v_{-}
\end{array}\right)=-\mathrm{i}\left(\begin{array}{c}
1 \\
-1
\end{array}\right) .
\end{gathered}
$$

We found two Majorana modes and we can choose $\alpha=1$ for the left boundary, i.e., being localized at $x=0$. Clearly, we can choose $\alpha=-1$ for a Majorana mode being localized at the right boundary $x=L$ in a finite system so that we arrive at the representation of Majorana zero modes being localized at the boundaries

$$
\begin{aligned}
& \hat{\phi}_{l}=\sum_{j}\left(\hat{c}_{j}+\hat{c}_{j}^{\dagger}\right)\left(e^{-i q_{1}^{+} x_{j}}+e^{-i q_{2}^{+} x_{j}}\right), \\
& \hat{\phi}_{r}=-\mathrm{i} \sum_{j}\left(\hat{c}_{j}-\hat{c}_{j}^{\dagger}\right)\left(e^{-i q_{1}^{-}\left(L+1-x_{j}\right)}+e^{-i q_{2}^{-}\left(L+1-x_{j}\right)}\right) .
\end{aligned}
$$

The existence of these zero modes in the whole phase region $|\mu|<2 t$ is caused by the boundaries and can be shown to be realized also at topological defects such as domain walls between the topological and trivial phases. The requirement for these modes to be stable is the bulk gap supressing hybridization due to tunneling of the Majorana edge modes, which in this case is given by the effective interaction Hamiltonian

$$
\hat{H}_{l, r}=\frac{\mathrm{i}}{2} \hat{\phi}_{l} \hat{\phi}_{r} t_{l, r}, \quad t_{l, r} \propto e^{-\frac{L}{\xi}}
$$

where $1 / \xi=\min \left(\ln \left(q_{1}^{+}\right), \ln \left(q_{2}^{+}\right)\right)$.

As already mentioned for the special case $\mu=0, \Delta=t=1$ in Sec. 3.4.2, the appearance of Majorana zero modes is accompanied by a ground state degeneracy. This observation can be generalized by noting that, somewhat hidden, $\hat{H}_{M}$ commutes with the parity operator

$$
\hat{P}_{f}=\prod_{j=1}^{L} e^{\mathrm{i} \pi \hat{n}_{j}}
$$

To see this we write $\hat{P}_{f}$ in terms of Majorana fermions

$$
\hat{P}_{f}=\prod_{j}\left(1-2 \hat{c}_{j}^{\dagger} \hat{c}_{j}\right)=\prod_{j}-\mathrm{i} \hat{\gamma}_{2 j-1} \hat{\gamma}_{2 j} .
$$

Then we make use of the BdG representation of Eq. (3.89) expressed in terms of Majorana 
operators (c.f. Eq. (3.100))

$$
\begin{aligned}
& \hat{P}_{f} \hat{H}_{M}=-\frac{(-\mathrm{i})^{N}}{2} \prod_{j} \hat{\gamma}_{2 j-1} \hat{\gamma}_{2 j} \sum_{j, k}\left(\begin{array}{ll}
\hat{\gamma}_{2 j-1} & \hat{\gamma}_{2 j}
\end{array}\right)\left(\begin{array}{cc}
\tilde{H}_{2 j-1,2 k-1} & \tilde{H}_{2 j-1,2 k} \\
\tilde{H}_{2 j, 2 k-1} & \tilde{H}_{2 j, 2 k}
\end{array}\right)\left(\begin{array}{c}
\hat{\gamma}_{2 k-1} \\
\hat{\gamma}_{2 k}
\end{array}\right) \\
& =-\frac{(-\mathrm{i})^{N}}{2} \sum_{j, k} \prod_{l \neq j} \hat{\gamma}_{2 l-1} \hat{\gamma}_{2 l}\left(\begin{array}{ll}
-\hat{\gamma}_{2 j} & \hat{\gamma}_{2 j-1}
\end{array}\right)\left(\begin{array}{cc}
\tilde{H}_{2 j-1,2 k-1} & \tilde{H}_{2 j-1,2 k} \\
\tilde{H}_{2 j, 2 k-1} & \tilde{H}_{2 j, 2 k}
\end{array}\right)\left(\begin{array}{c}
\hat{\gamma}_{2 k-1} \\
\hat{\gamma}_{2 k}
\end{array}\right) \\
& =-\frac{(-\mathrm{i})^{N}}{2} \sum_{j, k}\left(\begin{array}{ll}
\hat{\gamma}_{2 k-1} & \hat{\gamma}_{2 k}
\end{array}\right) \underbrace{\left(\begin{array}{cc}
\tilde{H}_{2 j-1,2 k-1} & \tilde{H}_{2 j-1,2 k} \\
\tilde{H}_{2 j, 2 k-1} & \tilde{H}_{2 j, 2 k}
\end{array}\right)^{T}}_{=-\tilde{\mathbf{H}}_{k, j}}\left(\begin{array}{c}
-\hat{\gamma}_{2 j} \\
\hat{\gamma}_{2 j-1}
\end{array}\right) \prod_{l \neq j} \hat{\gamma}_{2 l-1} \hat{\gamma}_{2 l} \\
& =-\frac{(-\mathrm{i})^{N}}{2} \sum_{j, k}\left(\begin{array}{ll}
\hat{\gamma}_{2 k-1} & \hat{\gamma}_{2 k}
\end{array}\right) \tilde{\mathbf{H}}_{k, j}\left(\begin{array}{c}
\hat{\gamma}_{2 j-1} \hat{\gamma}_{2 j-1} \hat{\gamma}_{2 j} \\
-\hat{\gamma}_{2 j-1} \hat{\gamma}_{2 j} \hat{\gamma}_{2 j}
\end{array}\right) \prod_{l \neq j} \hat{\gamma}_{2 l-1} \hat{\gamma}_{2 l}=\hat{H}_{M} \hat{P}_{f}
\end{aligned}
$$

where we used $\hat{\gamma}_{j} \hat{\gamma}_{j}=1$ and the fact that $\tilde{\mathbf{H}}_{j, k}$ is skew-symmetric, which follows from $A_{j, k}=$ $-A_{k, j}$ and $B_{j, k}=-B_{k, j}$ for $j \neq k$. The important conclusion is therefore that ground states of $\hat{H}_{M}$ have a well-defined fermion parity. It is easy to see that in the trivial phases $|\mu|>2 t$ the parity is always even. A simple argument can be constructed by looking at two distinct points in parameter space where parity can be easily evaluated. Denote the ground state by $|G S\rangle$ and choose $t=\Delta=0$ for the trivial phase so that

$$
\hat{c}_{j}|G S\rangle=0 \Rightarrow \hat{P}_{f}|G S\rangle=|G S\rangle .
$$

In the topological phase we already found that the ground state is doubly degenerate. This degeneracy is related to the parity of the ground state. Let again $|G S\rangle$ be the ground state, then (with normalization constants $\left|c_{l}\right|^{2}+\left|c_{r}\right|^{2}=1$ )

$$
\left(c_{l} \hat{\phi}_{l}+c_{r} \hat{\phi}_{r}\right) \hat{P}_{f}|G S\rangle=-\hat{P}_{f}\left(c_{l} \hat{\phi}_{l}+c_{r} \hat{\phi}_{r}\right)|G S\rangle,
$$

that is in the topological phase the two ground states differ by parity. Both phases cannot be connected without closing the gap. In fact, particle-hole symmetry protects the ground state degeneracy due to the pairwise appearance of the single-particle states. As already discussed in the end of Sec. 3.4.1, the degeneracy of the ground state in the parity sectors can only be lifted by a level crossing of the single-particle states, which occurs only at two points in the parameter space $|\mu|=2 t$. Therefore, in the topological phase localized Majorana edge modes are robust against continuous deformations of the Hamiltonian as long as there is no gap-closing.

\section{Majorana number and topological index}

It was shown by Kitaev [Kit01] that a global property of the ground state of $\hat{H}_{M}$ called Majorana number $\mathcal{M}$ can be calculated by the Pfaffian of $\hat{H}_{M}$ in the BdG representation in terms of Majorana operators

$$
\mathcal{M}:=\operatorname{sgn} \operatorname{Pf}(\tilde{\mathbf{H}}) .
$$

Using the property that for any skew-symmetric $2 n \times 2 n$ matrix $A$ the Pfaffian fulfills $\operatorname{Pf}^{2}(\mathbf{A})=$ $\operatorname{det}(\mathbf{A})$, then for any unitary transformation $\mathbf{U}$ it is

$$
\operatorname{Pf}^{2}\left(\mathbf{U} \mathbf{A} \mathbf{U}^{\dagger}\right)=\operatorname{det}\left(\mathbf{U} \mathbf{A} \mathbf{U}^{\dagger}\right)=\operatorname{det}(A) \operatorname{det}(\mathbf{U})^{2} \Rightarrow \operatorname{Pf}\left(\mathbf{U} \mathbf{A} \mathbf{U}^{\dagger}\right)=\operatorname{Pf}(\mathbf{A}) \operatorname{det}(\mathbf{U}) .
$$


Now the idea is to diagonalize the BdG representation of the Hamiltonian in the Majorana basis

$$
\hat{H}_{M}=-\frac{1}{2} \sum_{j} \hat{\gamma}_{j} \tilde{H}_{j, k} \hat{\gamma}_{k}=-\frac{1}{2} \sum_{\nu=1}^{2 L} \varepsilon_{\nu} \hat{\phi}_{\nu}^{\dagger} \hat{\phi}_{\nu}
$$

where again the eigenvalues $\varepsilon_{\nu}$ come in pairs $\pm \varepsilon_{\nu}$ and $\hat{\phi}_{\nu}=\sum_{j=1}^{2 L} R_{\nu, j} \hat{\gamma}_{j}$ with $\mathbf{R}$ being the matrix that diagonalizes $\tilde{\mathbf{H}}$. Since the eigenvalues come in pairs we can always transform the matrix $\mathbf{R}$ by means of a transformation with determinant +1 so that $\varepsilon$ is a blockdiagonal Jordan matrix

$$
\varepsilon=\bigoplus_{\nu=1}^{L}\left(\begin{array}{cc}
0 & \varepsilon_{\nu} \\
-\varepsilon & 0
\end{array}\right) \text {. }
$$

Kitaev noted [Kit01] that one can now use the transformation rule for the Pfaffian to conclude

$$
\operatorname{Pf}(\tilde{\mathbf{H}})=\underbrace{\operatorname{Pf}(\varepsilon)}_{\prod_{\nu=1}^{L} \varepsilon_{2 \nu-1}} \operatorname{det}(\mathbf{R}) \Rightarrow \operatorname{sgn}(\operatorname{Pf}(\tilde{\mathbf{H}}))=\operatorname{sgn}(\operatorname{det}(\mathbf{R}))=\mathcal{M} .
$$

The Majorana number can now be evaluated using the block-diagonal decomposition of $\tilde{\mathbf{H}}$ in $2 \times 2$ matrices $\tilde{\mathbf{H}}(k)$ in its Fourier representation. Then the Fourier transformation of the matrix $\mathbf{R}$ is also blockdiagonal so that

$$
\operatorname{Pf}\left(\hat{H}_{M}\right)=\prod_{k} \operatorname{det}(\mathbf{R}(k))=\prod_{k=0, \pi} \operatorname{det}(\mathbf{R}(k)) .
$$

The second equality follows from the fact that the eigenvalues with $k \neq 0, \pm \pi$ are real and come in pairs and due to particle-hole symmetry fulfill $E(k)=-E(-k)$, which implies $\mathbf{R}^{\dagger}(k)=\mathbf{R}(-k)$ so that we can use $\operatorname{det}\left(\mathbf{R}^{\dagger}(k)\right)=\operatorname{det}(\mathbf{R}(k))^{*}=\operatorname{det}(\mathbf{R}(-k))$. This finally yields

$$
\mathcal{M}=\operatorname{sgn}(\operatorname{Pf}(\tilde{\mathbf{H}}(0))) \operatorname{sgn}(\operatorname{Pf}(\tilde{\mathbf{H}}(\pi))) .
$$

The usefulness of this relation cannot be overestimated. For instance, it is related to the appearance of level crossings of the single-particle states of $\hat{H}_{M}$. This is seen immediately by substituting $k=0, \pi$ into Eq. (3.109) and transforming into the Majorana representation. The Pfaffians $\operatorname{Pf}\left(\tilde{\mathbf{H}}(k=0, \pi)\right.$ are then given by the eigenvalues $E(0, \pi)$ of $\hat{H}_{M}$, i.e., they change sign exactly when the gap closes at $|\mu|=2 t$. Thereby $\mathcal{M}$ also constitutes a measure for the parity change of the ground state. In fact, using the above formalism it is easy to see that the Pfaffian of the block-Hamiltonian $\mathbf{H}(k)$ in the Bogoliubov de-Gennes representation directly yields the parity of the corresponding eigenstate. The above considerations are remarkable in the sense that they connect properties of the bulk Hamiltonian to the existence of topologically protected edge modes. It is hence not too surprising that by studying the topological properties of $\hat{H}_{M}$ the corresponding topological index is given by $\mathcal{M}$. A simple argument can be formulated by returning to the $\mathrm{BdG}$ representation in the basis of the $\boldsymbol{\tau}^{\alpha}$ matrices. For that purpose we rewrite $\mathbf{H}(k)$ (c.f. Eq. (3.113)) in the following way

$$
\mathbf{H}(k)=E(k) \mathbf{x}(k) \boldsymbol{\tau}=\left(-(2 t \cos (k a)+\mu) \boldsymbol{\tau}^{z}-2 \Delta \sin (k a) \boldsymbol{\tau}^{y}\right)
$$

with the definitions

$$
\begin{aligned}
& \mathbf{x}(k)=\left(\begin{array}{lll}
\frac{\Re[d(k)]}{E(k)} & -\frac{\Im[d(k)]}{E(k)} & \frac{h(k)}{E(k)}
\end{array}\right)^{T}, \\
& d(k)=-2 \mathrm{i} \Delta \sin (k a) \text { and } h(k)=-2 t \cos (k a)-\mu \text {. }
\end{aligned}
$$


With this definition it is $\mathbf{x}(k)^{2}=1$ so that $\mathbf{x}(k)$ constitutes a map from the unit circle $\mathcal{S}^{1}$ to the unit sphere $\mathcal{S}^{2}$. Particle-hole symmetry imposes $d(k)=-d(-k)$ and thereby $d(0)=d(\pi)=0$. The image of $\mathbf{x}(k)$, hence, has to pass through a pole at $k=0, \pi$ independent of the model parameters:

$$
0, \pi \longmapsto\left(\begin{array}{ccc}
0 & 0 & \pm 1
\end{array}\right)^{T} \forall t, \mu, \Delta .
$$

We can now vary $t, \mu, \Delta$ to create different images of $\mathbf{x}(k)$ but they all have to correspond to closed paths on $\mathcal{S}^{2}$. If we only consider paths generated with $\operatorname{sgn}(h(0))=\operatorname{sgn}(h(\pi))$, then the paths can be deformed continuously to a point, i.e., they are topologically trivial. If on the other hand we restrict the images to those that have $\operatorname{sgn}(h(0)) \neq \operatorname{sgn}(h(\pi))$, then the paths have to traverse opposite poles of $\mathcal{S}^{2}$ and can not be shrunk to a point by any smooth transformation, i.e., they are topologically non-trivial. But this is just the definition of the Majorana number $\mathcal{M}$ and the classification between trivial and non-trivial phase we found by evaluating the Pfaffian of $\tilde{\mathbf{H}}$. The derived index $\mathcal{M}$ distinguishing the trivial from the topological phases is a $\mathbb{Z}_{2}$ index. We can have a non-trivial topological number in one dimension because quadratic fermionic models are always conserving particle-hole symmetry. In fact, the anti-symmetric connections between the coefficients $A_{j, k}, B_{j, k}$ of the BdG Hamiltonian in Eq. (3.89) are indispensable for $\hat{H}$ being hermitian. Being equipped with this $\mathbb{Z}_{2}$ symmetry there can be topological phases in one dimension and in particular not only in the simplest case of a quadratic spinless fermion Hamiltonian that can be recast into BdG representation [QHZ08]. At this point we should mention that the phase transition between the trivial and the topological phase cannot be described by Landau theory. There is no global symmetry, which is broken in the one and conserved in the other phase and thereby also no local order-parameter can distinguish between both. This seems a bit bizarre since it is well-known that the Kitaev chain and the transverse field Ising model (TFI) are linked to each other by a duality transformation [Chi18]. Clearly, the TFI does not feature topologically protected degeneracy in its ground states and the phase transition between the ordered and disordered phase is well-captured by Landau's theory. However, this puzzle can be resolved by noting that the duality transformation is given by the Jordan-Wigner transformation, which in particular maps the local observables of the TFI to non-local observables in the Kitaev chain [WK17].

\section{Topological superconductors beyond the Kitaev chain}

The discussion so far was guided by the properties of the Kitaev chain and restricted to spinless fermions in one dimension. Unfortunately, spinless fermions with $p$-wave pairing as described by Eq. (3.104) are not something nature provides us with firsthand. Solely asking for $p$-wave superconductivity in real materials is a challenging problem with, for instance, experimental evidence to be found only recently in iron-based superconductors $\left[\mathrm{TIO}^{+} 19\right]$ or hybrid graphene structures $\left[\mathrm{DBMB}^{+} 17\right]$. Therefore, we start asking where to find topological superconductivity in one and higher dimensions and then try to identify systems in which "spinless" superconductivity can be realized. Searching for topological superconductivity is equivalent to classifying superconductors by discrete symmetries they can preserve and the topological invariants they give rise to [HHZ05, SRFL08, Kit09]. The situation is that as along as there is a mean-field-like description of the pairing potential with perturbations smaller than the gap the Hamiltonian always preserves particle-hole symmetry. We already saw that this gives rise to a $\mathbb{Z}_{2}$ index in one dimension and the situation is comparable in two dimensions. Additionally, there can be a conservation of time-reversal symmetry which gives rise to another $\mathbb{Z}_{2}$ index in one, two, and three 
dimensions. In 2D this so-called spin Chern number is related to a block-diagonal decomposition of the Hamiltonian in spin space if the total $S^{z}$ spin is conserved [SWSH06]. Notably, even in the presence of spin-orbit coupling, and thus breaking of spin conservation, time-reversal symmetry can give rise to a $\mathbb{Z}_{2}$ index by using the Kramers degeneracy to decompose the Hamiltonian into a block-diagonal form [KM05]. In three dimensions conservation of both particle-hole and timereversal symmetry can give rise to a combined symmetry, the so-called chiral symmetry. Then a $3 \mathrm{D}$ winding number can be defined inducing a $\mathbb{Z}$ index [SRFL08]. Finally, there can be discrete conserved space-group symmetries [TH13, UYTS13, SS14]. In that case the Brillouin zone can be decomposed into planes in which the BdG representation commutes with some symmetry operator. Eigenstates of the Hamiltonian on such symmetry-invariant planes are also eigenstates of the corresponding symmetry operator. Defining a Chern number in each such plane gives rise to various topological indices, which can even exhibit spinless superconductivity.

It can be seen that the class of Hamiltonians that can feature topological superconductivity is large and we may now resort to such systems exhibiting spinless superconductivity. Promising candidates are spin-triplet or odd-parity superconductors, which can be obtained from doping topological insulators and have been confirmed experimentally on $\mathrm{Cu}_{x} \mathrm{Bi}_{2} \mathrm{Se}_{3}\left[\mathrm{HWC}^{+} 10\right]$. Another approach was suggested by Fu and Kane [FK08], in which a topological insulator with strong spin-orbit coupling is interfaced with an $s$-wave superconductor. The proximity induced superconductivity at the surface can become topological if time-reversal symmetry is broken by means of an applied external magnetic field. The crucial ingredient is the strong spin-orbit coupling and it has been shown by Lutchyn et al. [LSDS10] that this can also be exploited in one dimension. Their suggested setup of a nanowire with strong spin-orbit coupling proximitycoupled to an $s$-wave superconductor can be mapped to the Kitaev chain when a sufficiently strong Zeeman splitting gaps out one spin-degree of freedom. Experimentally, such heterostructures have been investigated and transport measurements point towards the existence of a spinless superconducting topologically ordered state hosting localized Majorana fermions at the boundaries $\left[\mathrm{MZF}^{+} 12, \mathrm{DYH}^{+} 12, \mathrm{DRM}^{+} 12\right]$.

\section{Majorana oscillations and the Albrecht experiment $\mid 3.4 .6$}

As described above, a promising candidate for the experimental realization of Majorana edge states are nanowires proximity-coupled to an s-wave superconductor with strong spin-orbit coupling. The question remains how to show the existence of Majorana zero modes in such devices. The most obvious experimental proof surely is the confirmation of non-Abelian statistics of Majorana edge modes [AOR $\left.{ }^{+} 11\right]$ or a fractional Josephson effect [KSY03] but the level of experimental control seems not reachable in the near future. Here, we discuss a method that is only a slight modification of the zero-bias conductance measurements $\left[\mathrm{MZF}^{+} 12, \mathrm{DYH}^{+} 12, \mathrm{DRM}^{+} 12\right]$ and, hence, is experimentally more conveniently realized. In [DSSS12] Das Sarma et al. proposed to use the finite hybridization of Majorana edge modes in mesoscopic proximity-coupled nanowires. They showed that the resulting splitting generates unique oscillations in the position of zero-bias conductance peaks when tuning the magnetic field. The idea is based on the exact form of the Majorana wave functions, which in the limit $v_{S O} \ll B$ fulfill

$$
\phi(x) \propto e^{\mathrm{i} k_{F, e f f} x-x / \xi},
$$

where $\xi$ is the coherence length and $k_{F, e f f}$ the Fermi wave vector, which depends on the magnetic field $B$. The finite hybridization causes an energy splitting depending on whether the mode is 


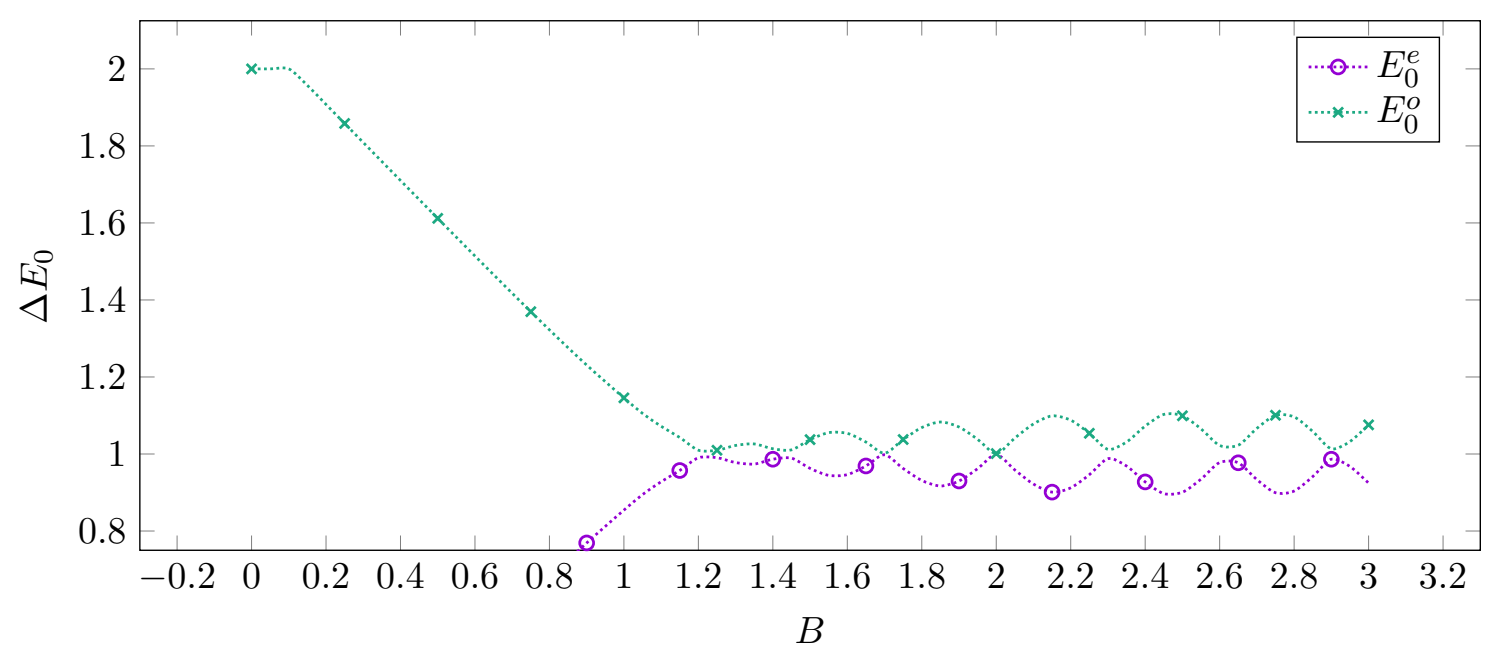

Figure 3.4: Ground state energies in even $\left(E_{0}^{e}\right)$ and odd $\left(E_{0}^{o}\right)$ parity sectors for isolated nanowire proximity coupled to $s$-wave superconductor. Results are obtained from exact diagonalization and show a generic behavior. More specific data with actual parameter sets is discussed in Sec. 7.2.2.

occupied or not. The splitting is found to be

$$
\Delta E \propto k_{F, e f f} e^{-2 L / \xi} \cos \left(k_{F, e f f} L\right) .
$$

Therefore, upon tuning the magnetic field, one can observe oscillations in the hybridization energy. Also note that the amplitude of the oscillations is field-dependent, too. In Fig. 3.4 the ground-state energies for the even and odd parity sector obtained from exact diagonalization (for details see Sec. 7.1) of a chain with $L=16$ sites are shown. For the case of an isolated nanowire without leads these oscillations are in one-to-one correspondence to zero-bias conductance peaks when measuring in the Coulomb blockade regime [Fu10] (c.f. Sec. 7.2.2). An experimental realization has been published by Albrecht et al. in $\left[\mathrm{AHM}^{+} 16\right]$ where they report the finding of exactly such oscillations. However, despite the huge experimental success in realizing and measuring such a system at all, there are many questions left. One of the most intriguing is the effect of the hybridization of the nanowire, with states in the leads which are required to perform zero-biased conductance measurements. In particular they assumed the one-to-one correspondence between energy oscillations and conductance peaks to hold as in the isolated case. Therefore, we took up to this question and studied the effects when coupling the nanowire to one or two leads numerically in great detail to shed light on this problem in Chap. 7. 

Methods 



\section{Matrix-product states 4}

MPS have a long history in mathematics and physics being rediscoverd independently not only once. In 1941 Kramers and Wannier explored an MPS representation as an ansatz class for approximating the partition function of the two-dimensional Ising model [KW41]. Before the advent of modern computers they evaluated the resulting set of linear equations and established the best estimate for the Curie point at that time. In statistical mechanics, MPS re-appeared in the 1960s in the context of monomer-dimer systems. In [Bax68] Baxter demonstrated how MPS could be employed to solve an eigenvalue problem and numerically approximate a partition function. Despite the different context, the developed algorithm has a striking similarity with the modern formulation of ground-state searches using MPS. In particular, a scaling analysis of the obtained results depending on the matrix dimensions permitted for a sensible extrapolation to the thermodynamic limit. In the context of quantum many-body systems, MPS came into appearance as finitely correlated states [FNW92] or valence bond states [AKLT87, AKLT88]. The probably most appealing emergence of MPS was in studies of systems with valence bond ground-state (VBGS) by Affleck, Kennedy, Lieb and Takagi in 1987 [AKLT87] on a spin-1 system. They constructed a Hamiltonian whose ground-state exhibits fractionalization of the degrees of freedom from spin 1 to spin $1 / 2$ and has an exact representation in terms of an MPS. Describing a topogically ordered quantum state, the power of MPS in capturing non-trivial quantum manybody physics yielded a first glimpse onto the developments leading to the modern formulation of DMRG.

From a mathematical point of view an important reason for their success in describing physical systems is buried in the fact that MPS form a manifold which is embeded into a high-dimensional vector space [HOV13, HMOV14] as well as being able to systematically approximate long-ranged correlations within this manifold. Combining both properties allow for the formulation of variational principles to obtain both ground-states and the dynamics of quantum many-body systems $\left[\mathrm{HCO}^{+} 11, \mathrm{Sch} 11, \mathrm{HLO}^{+} 16\right]$. Notably, it is not only their mathematical structure that renders MPS a unique tool for succesfully treating quantum many-body systems. Studying the properties of one-dimensional quantum systems from an information point of view, it was realized that a wide class of physically relevant models have ground states which share a ubiquitous property. In [VC06] Verstaete and Cirac proved that the entanglement entropy $S_{N}$ of gapped,

(a)

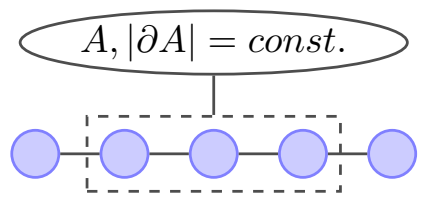

(b)

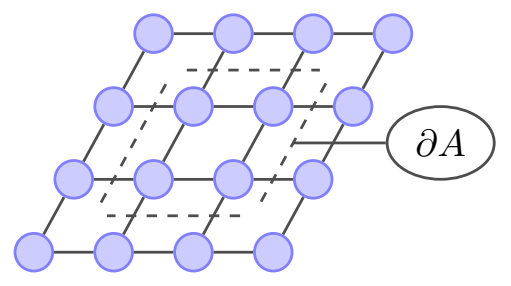

Figure 4.1: One- and two-dimensional lattices with subsystem $A$ and surface area $\partial A$. Note that for the one-dimensional system $\partial A$ consists only of a set of two points independent of the chosen bipartition. 
one-dimensional spin systems is captured within an MPS representation to a certain accuracy threshold $\epsilon(m)$ as a function of the matrix dimension which scales only polynomial in the system size. On the other hand, it was shown by Hastings in a seminal work that $S_{N}$ exhibits an area law for gapped one-dimensional quantum systems [Has07a], that is $S_{N} \sim \mathcal{O}(|\partial A|)=$ const. independent of the system $\operatorname{size}^{1}$ (c.f. Fig. 4.1a). As a consequence, one-dimensional quantum systems with a finite gap can be represented in terms of MPS with high accuracy but with only a polynomial growth of the matrix dimension with respect to the system size as the ground states of these systems are located within the so-called physical corner of the Hilbert space [Eis13] exhibiting an area law $S_{N} \sim \mathcal{O}(|\partial A|)$ of the entanglement entropy. It is worth mentioning at this point that the area law is not restricted to one-dimensional quantum systems only. In fact, there is a wide class of systems known in higher dimensions which also fulfill an area law [PEDC05, VWPGC06, CEP07]. Furthermore, the property of capturing such an area law with a proper ansatz class is not restricted to MPS, only. A natural generalization of MPS are so-called projected entangled pair-states (PEPS) which have been shown to describe area-law states in two dimensions [VWPGC06, SWVC07]. However, the computational complexity of PEPS and further tensor-network state (TNS) ansatz classes (e.g. tree-tensor networks [SDV06], fork tensor networks [BZT $\left.{ }^{+} 17\right]$, multi-scale entanglement-renormalization networks [Vid08]) in general scales worse in terms of the matrix contractions which need to be performed. Additionally, if the underlying tensor network contains loops, efficient contraction schemes are still under investigation where a promising scheme to convert PEPS into a computationally advantageous (canonical) form has been proposed only recently [HOC19].

As it happened often in history of science, the developement of MPS towards becoming one of the most important languages to formulate numerical algorithms in low-dimensional quantum many-body systems was not only pursued by isolated, pivoting works. In fact, the density matrix renormalization group (DMRG) already exploited the outstanding numerical properties of this ansatz class in a different formulation even before the mathematically and physically advantageous properties which are at the heart of its success merged into our today's comprehension. This alternative formulation is guided from an intuitive physical picture and fertilized the creation of new algorithms until the present day. The idea of renormalization to keep the number of required states describing a quantum system manageable that is identifying a suitable subspace of the overall Hilbert space (if it exists!), which can be parametrized efficiently, at first was successfully applied in the context of condensed matter physics by Wilson in 1975 [Wil75] to numerically solve the Kondo problem [Kon64, YA70, AFL83]. Renormalizing the energy spectrum of the system under consideration by discarding high-energy contributions so that an efficient representation of the Hamiltonian in a low-energy subspace is obtained, Wilson was able to prove that the ground state of the Kondo model is a singlet state. Even though selecting the parametrized subspace by discarding high-energy contributions turned out to yield efficient algorithms mainly in the case of impurity models, an important ingredient of this procedure paved the way towards the developement of DMRG, namely the mapping of quantum many-body problems to a chain geometry which is constructed iteratively. Following this idea, in 1992 White argued that in order to faithfully represent states of quantum lattice-models an optimal approximation scheme is obtained when partitioning the system $\hat{H} \rightarrow \hat{H}_{A A}$ and truncating the eigenvalue spectrum of the corresponding reduced density matrix $\hat{\rho}_{A}$ [Whi92]. A renormalization procedure is then introduced by subsequently adding lattice sites between the blocks of the previous partition $\hat{H}_{A A} \rightarrow \hat{H}_{A \bullet \bullet A}$ and diagonalizing the resulting Hamiltonian. Absorbing the lattice sites into a new biparition the corresponding density matrix $\hat{\rho}_{A}$ is diagonalized and truncated keeping only the dominating eigenvalues up to a threshold matrix dimension $m$. The corresponding transfor-

\footnotetext{
${ }^{1}$ The proof has been extended until today, see for instance [AKLV13]
} 
mation of the partitioned Hamiltonian $\hat{H}_{A \bullet \bullet A}$ is obtained from the truncated transformations diagonalizing $\hat{\rho}_{A}$. This renormalization scheme connects the ideas of iteratively finding a global approximation of a system's ground state while representing the states (and operators) in a basis which is locally optimized. Both concepts are at the heart of modern MPS ground-state algorithms and in fact, it has been realized very quickly that the DMRG algorithm is searching for the ground state in the MPS manifold [DMDNS98, McC07, Sch11]. Given the power of DMRG in describing ground states of strongly correlated quantum systems it is only natural to extend this framework to also study dynamics and various formulations of time-dependent DMRG have been developed [WF04, DKSV04, FW05, MMN05]. Even though these approaches were successfully extending the applicability of DMRG to study also quantities that are directly accessible in experiment $\left[\mathrm{RMR}^{+} 06\right.$, WA08, BSW09] the fact that operators need to undergo basis transformations rendered the implementation technically more involved. It is this point where a reformulation of DMRG in terms of MPS becomes more powerful as it permits for a direct and straight-forward representation of the required basic linear algebra operations enabling highly flexible algorithms. Additionally, exploiting directly the underlying manifold structure new algorithms to perform time evolution could be developed $\left[\mathrm{HCO}^{+} 11, \mathrm{HLO}^{+} 16, \mathrm{ZMK}^{+} 15\right]$ which would be very hard to realize in the language of reduced density matrices. In particular, the formulation of an algorithm exploiting a time-dependent variational principle by Haegeman et al. $\left[\mathrm{HLO}^{+} 16\right]$ for MPS is an important developement in performing time evolution of strongly correlated quantum many-body systems.

In this chapter, we will setup the notation of TNS and MPS, introduce the canonical form as well as elementary MPS manipulations and operations and give an introduction into the formulation of DMRG in terms of MPS. Then, we will discuss different time-evolution schemes used throughout this thesis including a reformulation of time-dependent density-matrix renormalization group (tDMRG) in terms of MPS. These sections are based on [PKS $\left.{ }^{+} 19\right]$ which was written and published in collaboration by the author and Thomas Köhler, Andreas Swoboda, Salvatore Manmana, Ulrich Schollwöck and Claudius Hubig. The major contributions to these sections were written by the author and are imported into this thesis with only minor changes. Pictures and diagrams which are adopted unaltered are marked as being part of this publication, originally. Subsequently, we will discuss in a compact form the exploitation of global $U(1)$ symmetries in MPS implementations. Finally, we will present a reformulation of systems which break $U(1)$-symmetry in terms of $U(1)$-invariant Hamiltonians by means of a purification scheme and discuss the adoption and consequencecs of this scheme to MPS and to what extend this can speed-up calculations. We used this approach to perform calculations on superconducting islands in the Coulomb blockaded regime as discussed later in this thesis in Chap. 7 demonstrating the applicability of our ideas.

\section{\begin{tabular}{l|l} 
Notation & 4.1
\end{tabular}}

In literature, there is a variety of notations in use to describe and formulate MPS algorithms, which come with different symbols, connotations and even representations of states. For the sake of clarity, here we will setup the notation used throughout this thesis, which is merely based on $\left[\mathrm{PKS}^{+} 19\right]$. It is the author's hope that the consecutive use of this notation (which for instance is also used in [Köh19]) also simplifies the accessibility of further literature to the reader. The following is based on the sections Tensor-notation, Matrix-product state (MPS) and Matrix-product operators (MPO) of the author's publication $\left[\mathrm{PKS}^{+} 19\right]$. 

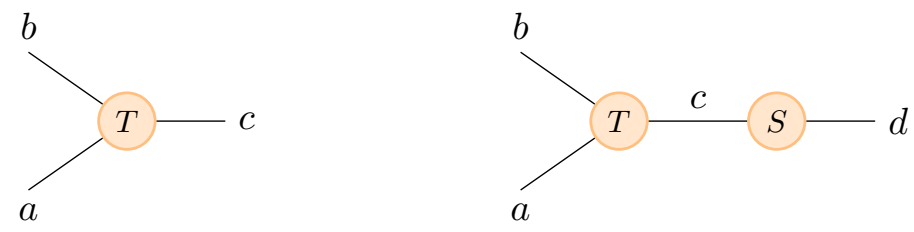

Figure 4.2: Left: Graphical representation of the example tensor $T_{a, b, c}$ with three indices (legs) $a, b$ and $c$. Right: Graphical representation of the tensor contraction $\sum_{c} T_{a, b, c} S_{c, d}=T \cdot S$. This graphic is adopted from the author's publication $\left[\mathrm{PKS}^{+} 19\right]$.

\section{Tensors $\quad 4.1 .1$}

The fundamental objects of MPS algorithms are tensors. In our context, tensors are multidimensional collections of complex or real numbers constituting multi-linear maps between vector spaces. Each index of a tensor corresponds to one of its dimensions; graphically, tensors are represented as shapes (circles, triangles, squares) with one leg per tensor index (cf. Fig. 4.2). A tensor $T$ on e.g. three associated vector spaces $A, B$ and $C$ has three indices $a=[1, \ldots, \operatorname{dim}(A)]$, $b=[1, \ldots, \operatorname{dim}(B)]$ and $c=[1, \ldots, \operatorname{dim}(C)]$. The tensor then has scalar entries $T_{a, b, c}$. We explicitly do not consider global symmetries ${ }^{2}$ in this section and hence do not associate a direction to our tensor legs (or, conversely, differentiate between vector and dual spaces). As such, the upstairs/downstairs location of tensor indices is meaningless and $T_{a, b, c}=T_{b, c}^{a}=T^{a, b, c}$. By complex-conjugating every element of a tensor $T$, we obtain a new tensor $\bar{T}$ with elements $\bar{T}_{\bar{a}, \bar{b}, \bar{c}}=T_{a, b, c}^{\star}$. We will use $\bar{a}$ to denote indices of conjugated tensors. Contracting $\bar{T}$ and $T$ over the index $a$ and $b$, we write:

$$
\sum_{a, b} \bar{T}_{a, b, \bar{c}} T_{a, b, c}=X_{\bar{c}, c}
$$

which is equivalent to

$$
\sum_{\bar{a}, a, \bar{b}, b} \delta_{\bar{a}, a} \delta_{\bar{b}, b} \bar{T}_{\bar{a}, \bar{b}, \bar{c}} T_{a, b, c}
$$

Note that we may drop overbars indicating complex conjugated indices if it is clear from the context that they are contracted over. Given two tensors $A_{a, b, c}$ and $B_{b, d, c}$, the shorthand $A \cdot B$ denotes the contraction over all shared indices:

$$
A \cdot B=\sum_{b, c} A_{a, b, c} B_{b, d, c}=Y_{a, d} .
$$

Tensor contractions can also be represented graphically by drawing tensors with connected legs, cf. Fig. 4.2. Note that we will, where possible without confusion, also use $a, b, c, \ldots$ to refer to the $\operatorname{dimension} \operatorname{dim}(A)$ etc. respectively.

\section{Matrix-product states (MPS)}

As already mentioned, MPS are efficient representations for one-dimensional weakly-entangled quantum states on a tensor-product Hilbert space $\mathcal{H}=\mathcal{H}_{\sigma}^{\otimes L}$ spanned by $L \in \mathbb{N}$ local, $\sigma$ dimensional Hilbert spaces $\mathcal{H}_{\sigma}$. The main idea is to represent the coefficient tensor $c_{\sigma_{1} \ldots \sigma_{L}}$

\footnotetext{
${ }^{2}$ The use of global symmetries is certainly crucial for efficient implementations and will be discussed in Sec. 4.6.
} 


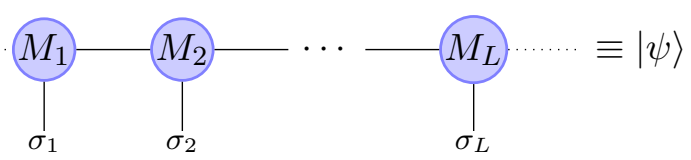

Figure 4.3: Schematic of the tensor network of a matrix-product state (MPS). Horizontal lines denote the internal indices with bond dimension $m$, whereas the vertical lines denote physical indices with dimension $\sigma$. Dotted lines to the left and right indicate the dummy indices $m_{0}$ and $m_{L}$. This graphic is adopted from the author's publication $\left[\mathrm{PKS}^{+} 19\right]$.

of a general quantum state on a discrete lattice

$$
|\psi\rangle=\sum_{\sigma_{1}, \ldots, \sigma_{L}} c_{\sigma_{1} \ldots \sigma_{L}}\left|\sigma_{1} \cdots \sigma_{L}\right\rangle
$$

as a product of $L$ rank-3 tensors $M_{i}$ (cf. Fig. 4.3)

$$
|\psi\rangle=\sum_{\substack{\sigma_{1}, \ldots, \sigma_{L}, m_{0}, \ldots, m_{L}}} M_{1 ; m_{0}, m_{1}}^{\sigma_{1}} \cdots M_{L ; m_{L-1}, m_{L}}^{\sigma_{L}}\left|\sigma_{1} \cdots \sigma_{L}\right\rangle
$$

where $m_{0}$ and $m_{L}$ are 1-dimensional dummy indices introduced for consistency. For a specific set of local states $\left\{\sigma_{1} \ldots \sigma_{L}\right\}$ characterizing an element $\left|\sigma_{1} \cdots \sigma_{L}\right\rangle \in \mathcal{H}$, we use a single matrix $M_{j}^{\sigma_{j}}$ per site. We hence evaluate a matrix product to give a single entry $c_{\sigma_{1} \ldots \sigma_{L}}$, resulting in the name "matrix-product" states. We will use $\sigma_{j}$ to index the local physical states on site $j$ associated to the basis states $\left|\sigma_{j}\right\rangle$ of the $j$ th local Hilbert space $\mathcal{H}_{\sigma}$. Depending on the bond dimensions $m_{j}$ of the tensors $M_{j}$ (also called virtual or auxiliary dimension), different quantum states can be represented exactly. If we let $m_{j}$ grow exponentially by a factor of $\sigma$ per site towards the center of the system, any quantum state can be written as an MPS. The entanglement entropy $S_{N}$ between the part of an MPS to the left of bond $(j, j+1)$ and to the right of this bond is bounded by $\log m_{j}$. This can be seen immediately by contracting all tensors to the left (right) of bond $(j, j+1)$ and forming the reduced density matrix of the left (right) partition:

$$
\rho_{\underline{j}}^{L}=\operatorname{Tr}_{k>j}|\psi\rangle\langle\psi|=\bar{M}_{j} \cdots \bar{M}_{1} M_{1} \cdots M_{j}
$$

where we introduced underlined site-indices to denote the bond $(j, j+1)$. The matrix dimension of $\rho_{j}^{L}$ is given by the bond dimension of the tensor $M_{j}$ and due to normalization of the reduceddensity matrix the von-Neumann entropy is bounded by

$$
S_{N}=-\sum_{\mu=1}^{m_{j}} \rho_{\underline{j} ; \mu, \mu}^{L} \log \rho_{\underline{j} ; \mu, \mu}^{L} \leq-\sum_{\mu=1}^{m_{j}} \frac{1}{m_{j}} \log \frac{1}{m_{j}}=\log m_{j}
$$

a similar estimate holds if the right partition is considered. The required bond dimension ${ }^{3} m=$ $\max _{j} m_{j}$ to represent a quantum state exactly hence grows exponentially with its entanglement along left/right partitions. On the other hand, lowly-entangled states require only a small bond dimension, leading to an efficient representation of the quantum state [Sch05, VC06, Has07a, Has07b, ECP10, Sch11]. 


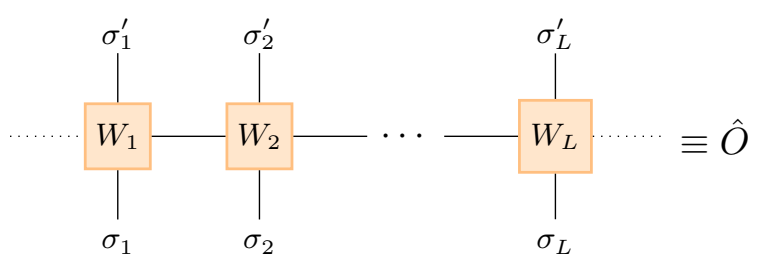

Figure 4.4: Schematic of the tensor network of a matrix-product operator (MPO). Horizontal lines denote the internal indices with bond dimension $w$, whereas the vertical lines denote physical indices with dimension $\sigma$. This graphic is adopted from the author's publication $\left[\mathrm{PKS}^{+} 19\right]$.

\section{\begin{tabular}{l|l} 
Matrix-product operators (MPO) & 4.1 .3
\end{tabular}}

By analogy to quantum states, we can express every operator as a matrix-product operator (MPO), i.e., a contraction of $L$ rank- 4 tensors $W_{j}$ :

$$
\begin{aligned}
\hat{O} & =\sum_{\substack{\sigma_{1}, \ldots, \sigma_{L}, \sigma_{1}^{\prime}, \ldots, \sigma_{L}^{\prime}}} c_{\sigma_{1} \ldots \sigma_{L}, \sigma_{1}^{\prime} \ldots \sigma_{L}^{\prime}}\left|\sigma_{1} \cdots \sigma_{L}\right\rangle\left\langle\sigma_{1}^{\prime} \cdots \sigma_{L}^{\prime}\right| \\
& =\sum_{\substack{\sigma_{1}, \ldots, \sigma_{L}, \sigma_{1}^{\prime}, \ldots, \sigma_{L}^{\prime}, w_{0}, \ldots, w_{L}}} W_{1 ; w_{0}, w_{1}}^{\sigma_{1}, \sigma_{1}^{\prime}} \ldots W_{L ; w_{L-1}, w_{L}}^{\sigma_{L}, \sigma_{L}^{\prime}}\left|\sigma_{1} \cdots \sigma_{L}\right\rangle\left\langle\sigma_{1}^{\prime} \cdots \sigma_{L}^{\prime}\right| .
\end{aligned}
$$

The only difference is that the tensor components $W_{j}$ are now rank- 4 tensors to account for the domain and image Hilbert spaces. There are different avenues to construct matrix-product operators $\left[\mathrm{CKN}^{+} 16, \mathrm{HMS17}\right.$, PKM17]. When used to represent the Hamiltonian $\hat{H}$ or other operators which are sums of local terms, the construction can be understood by splitting the system at bond $j$ (connecting sites $j$ and $j+1$ ). We then separate terms of the operator that act only within their partition $\hat{H}_{j-1}^{L}, \hat{H}_{j+1}^{R}$ and those that connect the partitions ${ }^{4}$

$$
\hat{H}=\hat{H}_{j-1}^{L} \otimes \hat{\mathbf{1}}_{j}^{R}+\hat{\mathbf{1}}_{j}^{L} \otimes \hat{H}_{j+1}^{R}+\sum_{a_{j}=1}^{N_{j}} \hat{h}_{j ; a_{j}}^{L} \otimes \hat{h}_{j ; a_{j}}^{R} .
$$

Based on the tensor product structure there is an operator-valued matrix $\hat{W}_{j}$ which relates the partitioned representations between the bonds $j-1$ and $j$; e.g., for the right partition we have

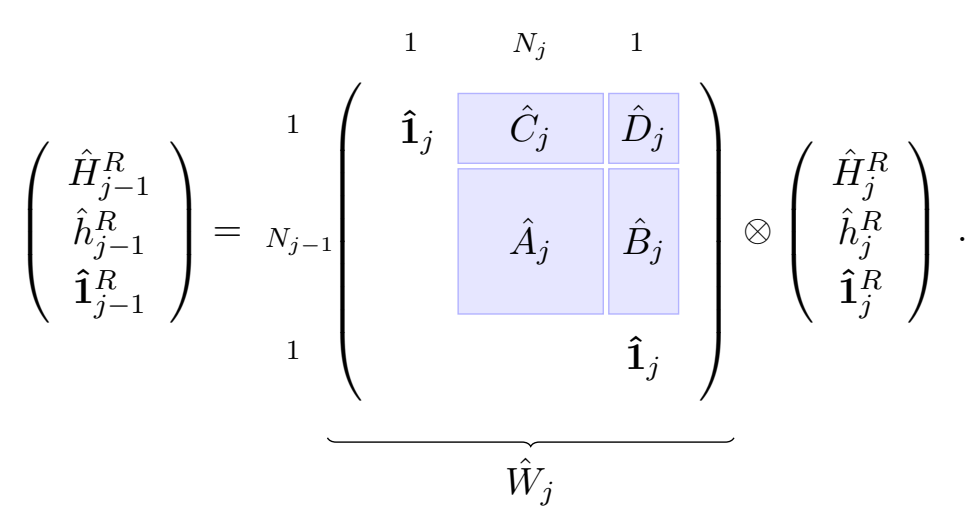

\footnotetext{
${ }^{3}$ In the following, we will use this notation in a more general context, i.e. by dropping the indices we denote the maximal matrix dimension of these particular indices.

${ }^{4}$ This is actually what is called superblock Hamiltonian in the standard DMRG[PWKH99].
} 
The operator-valued matrices $\hat{A}, \hat{B}, \hat{C}$ and $\hat{D}$ then define the recursion relations to iteratively build the complete operator $\hat{H}$. This picture directly leads to a construction scheme for an MPO based on a finite-state machine (FSM) [CB08, MZMP16, EWN17, PKM17] and we discuss this scheme and the derived MPO-arithmetics in Chap. 5, also taking into account a global $U(1)$ symmetry $^{5}$. In analogy to MPS with bonds $m_{j}$ and a maximal bond dimension $m$, the bonds of matrix-product operators are labelled by $w_{j}$ with the maximal bond dimension denoted by $w$.

\section{\begin{tabular}{l|l} 
MPS manipulations & 4.2
\end{tabular}}

Having introduced the basic notation, we turn to essential manipulations of MPS. In fact, the formulation of numerically efficient and stable algorithms is only possible, if the gauge-degree of freedom of MPS is exploited in a particular manner. Here, we are going to describe this procedure called canonization and discuss compression schemes derived from it where we put a special emphasis on the formulation of numerically efficient implementations. The following is based on the sections Canonical form, Normalizing an MPS and Truncating an MPS of the author's publication $\left[\mathrm{PKS}^{+} 19\right]$.

\section{Canonical form $\mid 4.2 .1$}

Considering Eq. (4.5), we can clearly insert resolutions of the identity $X X^{-1}$ in between any two MPS tensors $M_{j}$ and $M_{j+1}$. Multiplying $X$ into $M_{j}$ and $X^{-1}$ into $M_{j+1}$ changes the numerical content of each tensor while keeping the state invariant. This gauge freedom can be exploited to increase the numerical stability of algorithms and simplify many tensor contractions. Two possible choices to fix the gauge are enforcing the left or right normalization of the tensors $M_{j}$. We write $A_{j}$ for a left-normalized tensor and $B_{j}$ for a right-normalized tensor with the defining property that

$$
\begin{aligned}
\sum_{\sigma_{j}, m_{j-1}} \bar{A}_{j ; m_{j-1}, \bar{m}_{j}}^{\sigma_{j}} A_{j ; m_{j-1}, m_{j}}^{\sigma_{j}} & =\mathbf{1}_{\bar{m}_{j}, m_{j}} \\
\sum_{\sigma_{j}, m_{j}} B_{j ; m_{j-1}, m_{j}}^{\sigma_{j}} \bar{B}_{j ; \bar{m}_{j-1}, m_{j}}^{\sigma_{j}} & =\mathbf{1}_{m_{j-1}, \bar{m}_{j-1}} .
\end{aligned}
$$

Graphically they are represented by red (left-normalized) or green (right-normalized) triangles (see Fig. 4.5) where the orientation of the triangles also indicates the normalization. Left-/Rightcanonical MPS are now defined by requiring that they consist of left-/right-normalized tensors only. Furthermore, a mixed-canonical MPS is defined by fixing a site $j$ with unnormalized tensor $M_{j}$ and demanding all site tensors to the left/right to be left-/right-normalized tensors (see Fig. 4.6). The unnormalized site is often called active site or orthogonality center and we can generalize this idea to also have more than just a single site-tensor being not in a canonical form. We will refer to this as MPS representation with $k \geq 1$ active sites where $k$ counts the number of site tensors that are not in a canonical form.

\footnotetext{
${ }^{5}$ An alternative construction scheme is discussed in [HMS17].
} 


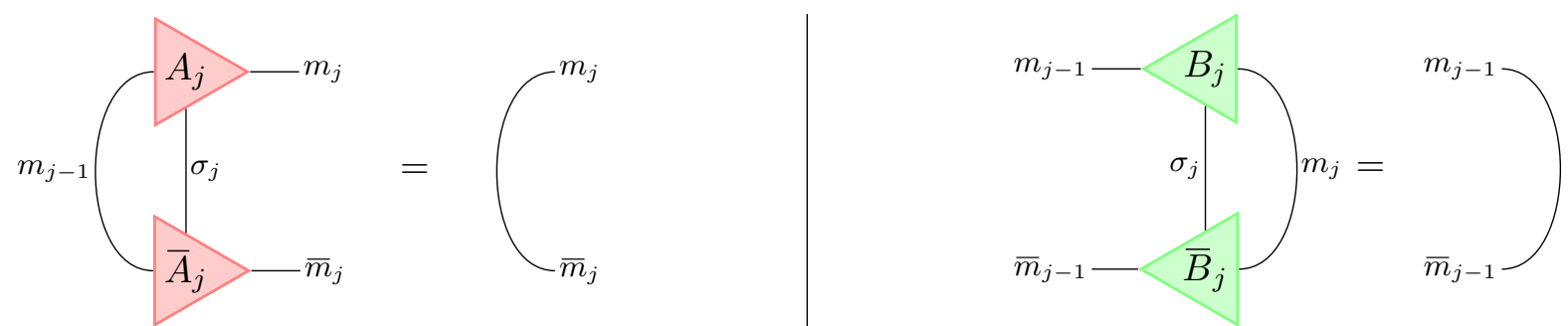

Figure 4.5: Left (Right) normalized tensor $A_{j}$ (red, right-pointing triangle) ( $B_{j}$ (green, left-pointing triangle)) contracted with its adjoint resulting in an identity. This graphic is adopted from the author's publication $\left[\mathrm{PKS}^{+} 19\right]$.

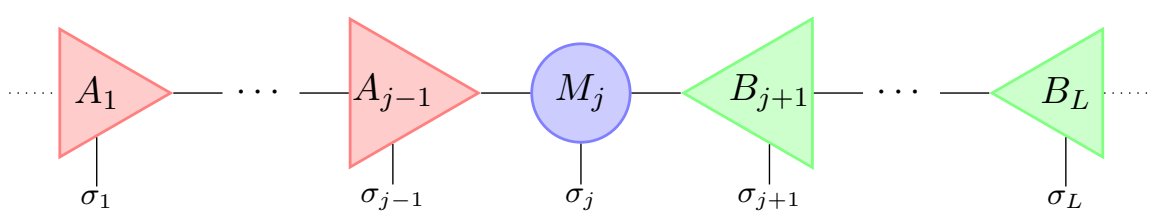

Figure 4.6: MPS with active site $j$ and consequently left (right) normalized site tensor left (right) of site $j$ as defined in Eq. (4.12). This graphic is adopted from the author's publication [PKS $\left.{ }^{+} 19\right]$.

\section{Normalizing an MPS $\quad 4.2 .2$}

Given an unnormalized MPS with site tensors $M_{j}$, the first step is often to bring it into a left or right-canonical form. This can be done by a series of $\mathrm{QR}$ decompositions: The tensor $M_{j}$ is reshaped into a matrix $\tilde{M}$ with the left and physical tensor legs forming the rows and the right tensor leg the columns of the matrix, that is $\tilde{M}_{\left(\sigma_{j} m_{j-1}\right), m_{j}}=M_{j ; m_{j-1}, m_{j}}^{\sigma_{j}}$. Applying a QR decomposition to this matrix, one obtains two new matrices $Q_{j}$ and $R_{j}$. The reshaping operation on $M_{j}$ is done in reverse on $Q$ to give the new left-normalized site tensor $A_{j}$ while the transfer tensor $R_{j}$ is multiplied into the next site tensor $M_{j+1}$. Likewise reshaping the $M_{j}$ with right and physical legs as rows and left tensor leg as columns results in a right-normalized tensor $B_{j}$ (with the transfer tensor multiplied into the previous site tensor $M_{j-1}$ ).

Starting on the left edge of the system and subsequently performing left-normalizations on each MPS tensor results in a complete left-normalized state. Equivalently, starting on the right edge of the system and moving to the left results in a right-normalized state.

\section{\begin{tabular}{l|l} 
Truncating an MPS & 4.2 .3
\end{tabular}}

Operations on matrix-product states typically increase the bond dimension of the state (e.g. MPO-MPS applications or the addition of two MPS). Finding an optimal approximation to such a quantum state for a smaller bond dimension is the purpose of this section. This is of particular relevance to time-evolution methods, as entanglement generically grows during realtime evolution and time-evolved states hence per se already need a larger bond dimension than e.g. ground states. Hence finding good approximation methods is crucial.

Let us consider a state $|\psi\rangle$ which is represented by an MPS with an initial large bond dimension $m$. We wish to find another state $\left|\psi^{\prime}\right\rangle$ with smaller bond dimension $m^{\prime}$ which approximates $|\psi\rangle$ well in the sense that it minimizes the Hilbert space distance

$$
\||\psi\rangle-\left|\psi^{\prime}\right\rangle \| .
$$




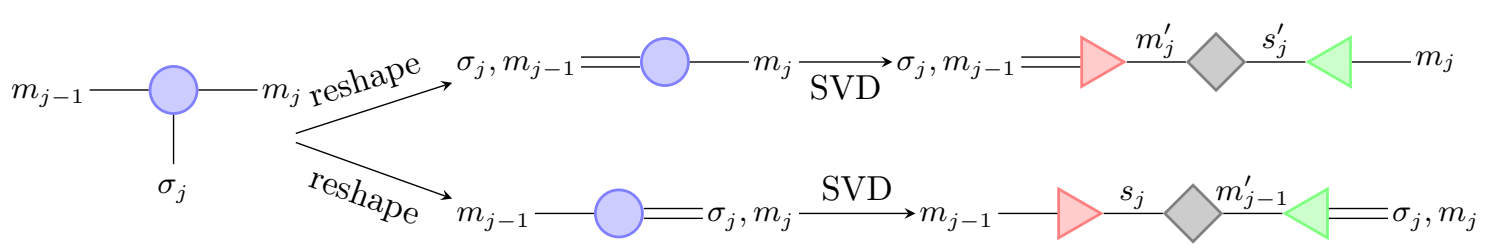

Figure 4.7: The singular value decomposition of a rank-three tensor $M$ into $U S V$ within a truncation sweep to the right (top) or to the left (bottom). This graphic is adopted from the author's publication $\left[\mathrm{PKS}^{+} 19\right]$.

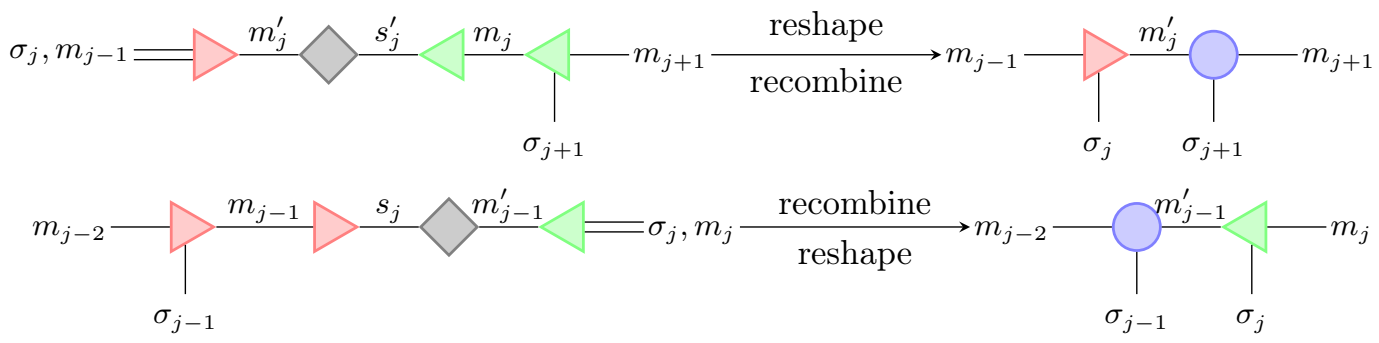

Figure 4.8: Assignment of the result of the singular value decomposition (Sec. 4.2.3) into the new left (right) normalized rank three tensor $A_{j}\left(B_{j}\right)$ and the new active site $M_{j+1}\left(M_{j-1}\right)$ at the top (bottom). This graphic is adopted from the author's publication $\left[\mathrm{PKS}^{+} 19\right]$.

The most direct way to proceed is to use a series of singular value decompositions to successively truncate each bond of the MPS. On each individual bond, the optimal choice is made, but this does not have to result in the globally optimal state $\left|\psi^{\prime}\right\rangle$. It is also possible to optimize each site tensor of $\left|\psi^{\prime}\right\rangle$ sequentially to maximize the overlap between $|\psi\rangle$ and $\left|\psi^{\prime}\right\rangle$. Multiple sweeps of this variational optimization can be done to approach the global optimum as well as possible.

\section{Direct truncation via SVD}

Consider a cut of the MPS on one bond $(j, j+1)$ into a left and right half such that the state $|\psi\rangle$ can be represented in effective left and right basis sets as

$$
|\psi\rangle=\sum_{l, r=1}^{m_{j}} \Psi_{\underline{j} l, l, r}|l\rangle_{L} \otimes|r\rangle_{R} .
$$

The coefficient tensor $\Psi_{j ; l, r}$ then occupies the bond between sites $j$ and $j+1$, such rank-2 tensors are called bond tensors in the following. For orthonormal left and right basis sets as realized in an MPS with orthogonality center on bond $(j, j+1)$, we can use a singular-value decomposition (SVD) (cf. Ref. [Sch11] Sec. 4.5) of the $\Psi$ tensor to obtain the approximation which is optimal under the condition that all other site tensors are kept fixed. That is, we decompose

$$
\Psi_{\underline{j} ; l, r}=\sum_{s=1}^{m} U_{l, s} S_{s, s} V_{s, r}^{\dagger}
$$

with $U$ and $V^{\dagger}$ left-/right-unitary and $S$ diagonal and non-negative. An approximation is then given by

$$
\Psi_{\underline{j} ; l, r}^{\prime}=\sum_{s=1}^{m^{\prime} \leq m} U_{l, s}^{\prime} S_{s, s}^{\prime} V_{s, r}^{\prime \dagger}
$$




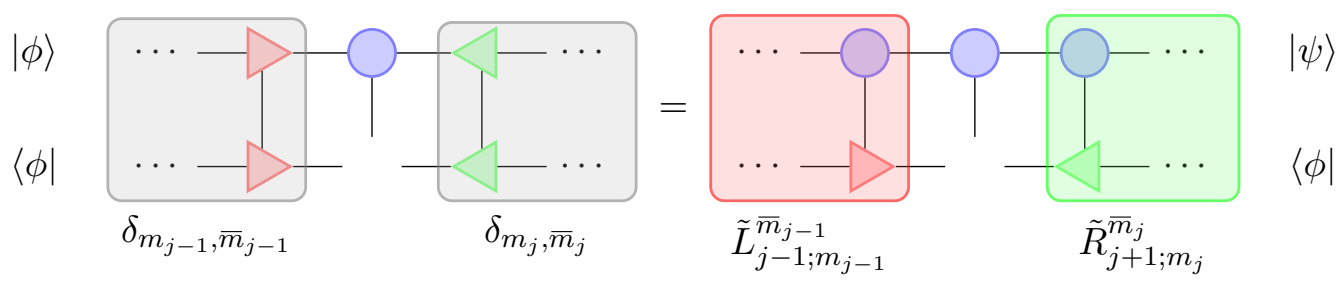

Figure 4.9: Iterative truncation considering the truncated state to be in a mixed canonical form. The left hand side can then be reduced to the new optimal site tensor $M_{j}^{\prime}$. The right hand side, which needs to be considered completely, can nevertheless be calculated iteratively via the bond tensors $\tilde{L}$ and $\tilde{R}$. This graphic is adopted from the author's publication $\left[\mathrm{PKS}^{+} 19\right]$.

where we only keep the $m^{\prime}$ largest singular values and also correspondingly truncate the rows and columns of $U$ and $V^{\dagger}$. The error of this approximation of $|\psi\rangle$ is given by

$$
\epsilon \approx \sqrt{\sum_{k=m^{\prime}+1}^{m} S_{k, k}^{2}}
$$

where the argument to the square root is also known as the discarded weight

$$
\delta\left(m^{\prime}\right)=\sum_{k=m^{\prime}+1}^{m} S_{k, k}^{2} .
$$

In practice, one selects $m^{\prime}$ such that some target discarded weight is obtained, for instance

$$
\delta\left(m^{\prime}\right)=10^{-10} \Rightarrow \epsilon \approx 10^{-5} \cdot L,
$$

where the additional factor $L$ is to account for the accumulation of errors when truncating every site tensor. Instead of working on the bond tensor $\Psi_{j ; l, r}$ we can also apply the decomposition directly to the MPS tensor $M_{j}$ by a suitable reshaping (cf. Secs. 4.2.3 and 4.2.3). Starting e.g. on the left end of a right-normalized MPS, we can sweep left-to-right and sequentially truncate every bond, each time obtaining the locally optimal state.

Due to this sweeping through the system, the truncation of site $L-1$ becomes dependent on the truncation of site 1 but not vice versa. In the case of small truncations the error resulting from this asymmetry is small and can be ignored. If truncation errors are large, however, this asymmetry leads to a series of optimal approximations which together do not constitute a globally optimal approximation, as each of the singular value decompositions always only produces the optimal approximation on a particular bond. To increase accuracy in this case, a subsequent variational optimization of the state may be necessary.

\section{Variational truncation}

To overcome the problems of the direct truncation by SVD, an iterative sweeping mechanism is often employed. By sweeping multiple times through the system and finding on each site the locally optimal tensor, it is more likely that one obtains the globally optimal MPS. We start from an initial guess state $|\phi\rangle$ with tensors $M_{j}^{\prime}$ and a chosen bond dimension $m^{\prime}$ and variationally minimize the distance to the un-truncated state $|\psi\rangle$ with tensors $M_{j}$. We must stress that the convergence of the variational optimization algorithm strongly depends on the inital guess state. Being unlucky and starting from an unsuitable initial state, the variational 
optimization may take a long time to converge or even may get completely stuck in a locally optimal but globally suboptimal state. Typically, a good choice for the initial state is produced by the direct truncation using the SVD. Once we have an initial state, the distance to minimize is given by

$$
\||\psi\rangle-|\phi\rangle \|^{2}=\langle\psi \mid \psi\rangle-\langle\psi \mid \phi\rangle-\langle\phi \mid \psi\rangle+\langle\phi \mid \phi\rangle
$$

We now keep all tensors but $M_{j}^{\prime}$ fixed and hence only minimize the single site tensor $M_{j}^{\prime}$, differentiating with respect to $\bar{M}_{j}^{\prime}$. Because this tensor only occurs in the second half of Eq. (4.21), the new (optimized) $M_{j}^{\prime}$ can be obtained via

$$
\frac{\partial}{\partial{\overline{M_{j}}}^{\prime}}(\langle\phi \mid \phi\rangle-\langle\phi \mid \psi\rangle)=0
$$

Let us now consider that the truncated state is in a mixed canonical form with the active site at position $j$. Then the new tensor is given by

$$
M_{j ; m_{j-1}, m_{j}}^{\prime \sigma_{j}}=\sum_{\bar{m}_{j-1}} \tilde{L}_{j-1 ; m_{j-1}}^{\bar{m}_{j-1}}\left(\sum_{\bar{m}_{j}} \tilde{R}_{j+1 ; m_{j}}^{\bar{m}_{j}} M_{j ; m_{j-1}, m_{j}}^{\sigma_{j}}\right),
$$

in which the left (right) parts of the tensor network (Fig. 4.9, right hand side) are contracted into $\tilde{L}(\tilde{R})$,

$$
\begin{aligned}
& \tilde{L}_{j-1 ; m_{j-1}}^{\bar{m}_{j-1}}=\sum_{\substack{\sigma_{j-1}, \bar{m}_{j-2}, m_{j-2}}} \bar{A}_{j-1 ; \bar{m}_{j-2}, \bar{m}_{j-1}}^{\sigma_{j-1}}\left(\cdots\left(\sum_{\substack{\sigma_{1}, \bar{m}_{0}, m_{0}}} \bar{A}_{1 ; \bar{m}_{0}, \bar{m}_{1}}^{\prime \sigma_{1}} M_{1 ; m_{0}, m_{1}}^{\sigma_{1}}\right) \cdots\right) M_{j-1 ; m_{j-2}, m_{j-1}}^{\sigma_{j-1}} \\
& \tilde{R}_{j+1 ; m_{j}}^{\bar{m}_{j}}=\sum_{\substack{\sigma_{j+1}, \bar{m}_{j+1}, m_{j+1}}} \bar{B}_{j+1 ; \bar{m}_{j}, \bar{m}_{j+1}}^{\prime \sigma_{j+1}}\left(\cdots\left(\sum_{\substack{\sigma_{L}, \bar{m}_{L}, m_{L}}} \bar{B}_{L ; \bar{m}_{L-1}, \bar{m}_{L}}^{\sigma_{L}} M_{L ; m_{L-1}, m_{L}}^{\sigma_{L}}\right) \ldots\right) M_{j+1 ; m_{j}, m_{j+1}}^{\sigma_{j+1}} .
\end{aligned}
$$

It is never necessary to calculate the complete contraction of the boundary tensors Eq. (4.24), because the next tensor in sweep direction was already calculated in the sweep before and the other tensor is obtained by reusing the one from the previous sweep step.

In practice, one should consider a two-site variational optimization in which neighboring site tensors $M_{j}^{\prime}, M_{j+1}^{\prime}$ are optimized at the same time. This allows for flexibility in the bond dimension and distribution of quantum number sectors on the bond $m_{j}$. For convenience we will demonstrate the necessity of a two-site update to permit for an increased bond dimension. Consider the reshaped updated tensor

$$
\Theta_{\sigma_{j+1} m_{j+1}}^{\sigma_{j} m_{j-1}}=M_{j ; m_{j-1}, m_{j}}^{\prime \sigma_{j}} \cdot M_{j+1 ; m_{j}, m_{j+1}}^{\prime \sigma_{j+1}} .
$$

Any matrix factorization $\Theta=X \cdot Y$ may then create an index with intermediate bond dimension which is bounded by $\min \left\{m_{j-1} \sigma_{j}, \sigma_{j+1} m_{j+1}\right\}$ and potentially larger than $m_{j}$, which is possible iff at least two MPS sites are considered at the same time. 


\section{Calculus with MPS}

In the previous sections we have set-up the notation and canonization procedure which are the elementary ingredients of any MPS implementations. In order to perform actual calculations we now turn to the realization of algebraic operations which can be implemented in a very natural way in the framework of MPS. We will show, how to efficiently apply Operators in terms of MPO to a state represented by an MPS and present a numerically cheap evaluation scheme for observables by means of realizing a scalar product between MPS. The following is based on the sections Application of an MPO to an MPS and Expectation values of the author's publication $\left[\mathrm{PKS}^{+} 19\right]$.

\section{Application of an MPO to an MPS}

One of the most important operations within the framework of matrix-product states is the application of an MPO to an MPS. In theory this can be done by a straightforward tensor multiplication of the corresponding site tensors of the MPS and MPO. In practice this is not the method of choice as in most applications the resulting target state $\hat{O}|\psi\rangle$ has a much higher bond dimension $m^{\prime}=m \cdot w$, which, however, in general is not required to represent the target state efficiently $^{6}$. It is therefore helpful to look at different approaches - nevertheless, for pedagogical reasons we will begin with the direct application before we turn to more elaborate application schemes.

\section{Direct application}

The direct application of an MPO to an MPS is obtained by regrouping the contractions such that the target MPS tensor can be obtained as a tensor product of the individual site tensors

$$
\begin{aligned}
\hat{O}|\psi\rangle= & \sum_{\substack{\sigma_{1}, \ldots, \sigma_{L}, m_{0}, \ldots, m_{L}, \sigma_{1}^{\prime}, \ldots, \sigma_{L}^{\prime}, w_{0}, \ldots, w_{L} \\
\sigma_{1}^{\prime \prime}, \ldots, \sigma_{L}^{\prime \prime}}}\left(W_{1 ; w_{0}, w_{1}}^{\sigma_{1} \sigma_{1}^{\prime}} \cdots W_{L ; w_{L-1}, w_{L}}^{\sigma_{L} \sigma_{L}^{\prime}}\right)\left|\sigma_{1} \ldots \sigma_{L}\right\rangle\left\langle\sigma_{1}^{\prime} \ldots \sigma_{L}^{\prime}\right| \\
& \times\left(M_{1 ; m_{0}, m_{1}}^{\sigma_{1}^{\prime \prime}} \cdots M_{L ; m_{L-1}, m_{L}}^{\sigma_{L}^{\prime \prime}}\right)\left|\sigma_{1}^{\prime \prime} \ldots \sigma_{L}^{\prime \prime}\right\rangle \\
= & \sum_{\substack{\sigma_{1} \ldots \sigma_{L}, m_{0}, \ldots, m_{L}, w_{0}, \ldots, w_{L}}} M_{1 ;\left(w_{0} m_{0}\right),\left(w_{1} m_{1}\right)}^{\prime \sigma_{1}} \cdots M_{L ;\left(w_{L-1} m_{L-1}\right),\left(w_{L} m_{L}\right)}^{\prime \sigma_{L}}\left|\sigma_{1} \ldots \sigma_{L}\right\rangle=|\phi\rangle,
\end{aligned}
$$

with the tensors $M_{j}^{\prime}$ given by

$$
M_{j ;\left(w_{j-1} m_{j-1}\right),\left(w_{j} m_{j}\right)}^{\prime \sigma_{j}}=\sum_{\sigma_{j}^{\prime}} W_{j ; w_{j-1}, w_{j}}^{\sigma_{j} \sigma_{j}^{\prime}} M_{j ; m_{j-1}, m_{j}}^{\sigma^{\prime}} .
$$

\footnotetext{
${ }^{6}$ Exceptions are pathological cases for instance an MPO with uniform Schmidt coefficients.
} 


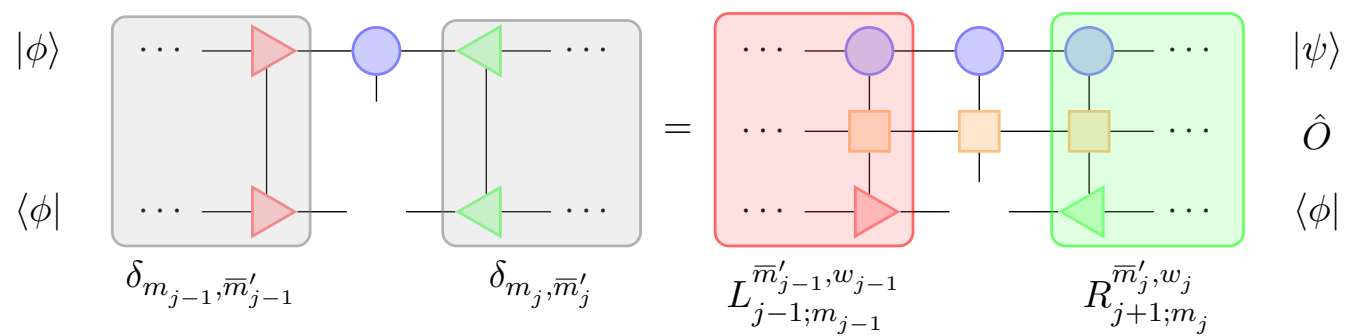

Figure 4.10: The variational application of an MPO to an MPS considering the truncated guess state $|\phi\rangle$ to be in a mixed canonical form. The left hand side can then be reduced to the active site $j$ that we want to optimize. The right hand side, which needs to be evaluated completely, can nevertheless be calculated iteratively via the bond tensors $L_{j-1}$ and $R_{j+1}$. This graphic is adopted from the author's publication $\left[\mathrm{PKS}^{+} 19\right]$.

The resulting state $|\phi\rangle$ is therefore again an MPS, but with a larger dimension $m^{\prime}=m \cdot w$ with $w$ being the matrix dimension of the MPO site tensors $W_{j}$. Repeated application of an operator onto a state in this manner hence quickly increases the dimension of the state and truncation becomes necessary with computational costs scaling as $m^{3} w^{3} d$ per site if we do an SVD truncation or $m^{\prime 2} m w d$ per site for the variational compression.

\section{Variational application}

In the spirit of variationally compressing a state towards a target state we can try to apply the same considerations to the application of an MPO to a source state $|\psi\rangle$ with the subsequent compression of the state in one optimization step. Therefore we seek to minimize the distance between a guess state $|\phi\rangle$ with tensors $M_{j}^{\prime}$ and bond dimension $m^{\prime}$ and the source state with the MPO applied to it, which we denote as $|\hat{O} \psi\rangle$,

$$
\begin{gathered}
|\phi\rangle=\min _{|\phi\rangle} \||\phi\rangle-|\hat{O} \psi\rangle \|^{2}=\min _{|\phi\rangle}(\langle\phi \mid \phi\rangle-\langle\phi \mid \hat{O} \psi\rangle-\langle\hat{O} \psi \mid \phi\rangle+\langle\hat{O} \psi \mid \hat{O} \psi\rangle) \\
\Rightarrow \frac{\partial}{\partial \bar{M}_{j}^{\prime}}(\langle\phi \mid \phi\rangle-\langle\phi \mid \hat{O} \psi\rangle) \stackrel{!}{=} 0
\end{gathered}
$$

for all guess site tensors $\bar{M}_{j}^{\prime}$. If we keep the current guess state in a mixed canonical form, the above set of coupled equations again reduces to a local update scheme for the target tensors

$$
M_{j ; \bar{m}_{j-1}^{\prime}, \bar{m}_{j}^{\prime}}^{\prime \sigma_{j}}=\sum_{m_{j-1}, w_{j-1}} L_{j-1 ; m_{j-1}}^{\bar{m}_{j-1}^{\prime}, w_{j-1}}\left(\sum_{\sigma_{j}^{\prime}, m_{j}, w_{j}} W_{j ; w_{j-1}, w_{j}}^{\sigma_{j} \sigma_{j}^{\prime}} M_{j ; m_{j-1}, m_{j}}^{\sigma_{j}^{\prime}} R_{j+1 ; m_{j}}^{\bar{m}_{j}^{\prime}, w_{j}}\right),
$$

where the boundary tensors $L_{j-1}, R_{j+1}$ can be built recursively by sweeping through the system and evaluating the contractions

$$
\begin{aligned}
L_{j-1 ; m_{j-1}}^{\bar{m}_{j-1}^{\prime}, w_{j-1}}= & \sum_{\substack{\sigma_{j-1}, \sigma_{j-1}^{\prime}, \bar{m}_{j-2}^{\prime}, m_{j-2}, w_{j-2}}} \bar{A}_{j-1 ; \bar{m}_{j-2}^{\prime}, \bar{m}_{j-1}^{\prime}}^{\sigma_{j-1}} L_{j-2 ; m_{j-2}}^{\bar{m}_{j-2}^{\prime}, w_{j-2}} W_{j-1 ; w_{j-2}, w_{j-1}}^{\sigma_{j-1} \sigma_{j-1}^{\prime}} M_{j-1 ; m_{j-2}, m_{j-1}}^{\sigma_{j-1}^{\prime}} \\
R_{j+1 ; m_{j}}^{\bar{m}_{j}^{\prime}, w_{j}}= & \sum_{\substack{\sigma_{j+1}, \sigma_{j+1}^{\prime}, w_{j+1}, \bar{m}_{j+1}^{\prime}, m_{j+1}}} W_{j+1 ; w_{j}, w_{j+1}}^{\sigma_{j+1} \sigma_{j+1}^{\prime}} M_{j+1 ; m_{j}, m_{j+1}}^{\sigma_{j+1}^{\prime}} R_{j+2 ; m_{j+1}}^{\bar{m}_{j+1}^{\prime}, w_{j+1} \bar{B}_{j+1 ; \bar{m}_{j}^{\prime}, \bar{m}_{j+1}^{\prime}}^{\sigma_{j+1}}} .
\end{aligned}
$$

The overall onsite contractions are depicted in Fig. 4.10. Care must be taken to use the (typically) optimal contraction order $\left(\left(L_{j-1} \cdot A_{j}\right) \cdot W_{j}\right) \cdot \bar{A}_{j}$. 

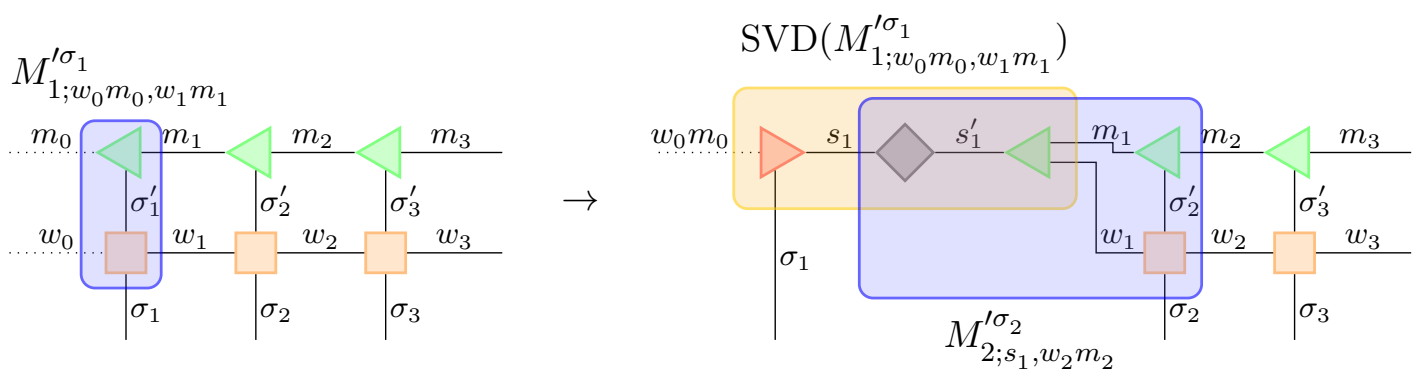

Figure 4.11: The essential steps of the zip-up method proposed in Ref. [SW10]. (left) Initial tensor network that shall be contracted, consisting of a right-canonical MPS and an MPO that only slightly destroys the canonical form. The first step is to interpret the combination of the first MPS tensor and the first MPO tensor as a new tensor $M_{1 ; w_{0} m_{0}, w_{1} m_{1}}^{\prime \sigma_{1}}$, which is slightly non-normalized and therefore framed in blue. (right) The next step is to apply an SVD (including a relaxed truncation) on the tensor $M_{1}^{\prime}$ and finally build the next tensor $M_{2}^{\prime}$ leaving the contracted, left-normalized MPS tensor $A_{1 ; m_{0} w_{0}, s_{1}}^{\prime \sigma_{1}}$ on the left side. This graphic is adopted from the author's publication $\left[\mathrm{PKS}^{+} 19\right]$.

\section{The zip-up method}

An alternative to the direct application of an MPO to an MPS is the zip-up method described in Ref. [SW10]. The central assumption is that the operator, such as a time-evolution operator, only slightly destroys the canonical form of the MPS. Hence, a modest truncation is already possible during the contraction process without too much loss of information because there is only a small loss of orthogonality in the used left and right basis sets.

The first step is similar to Eq. (4.29), but we work with a right-normalized initial tensor $B$,

$$
M_{1 ; w_{0} m_{0}, w_{1} m_{1}}^{\sigma_{1}}=\sum_{\sigma_{1}^{\prime}} W_{1 ; w_{0}, w_{1}}^{\sigma_{1} \sigma_{1}^{\prime}} B_{1 ; m_{0}, m_{1}}^{\sigma_{1}^{\prime}} .
$$

Note that $m_{0}$ and $w_{0}$ are dummy indices and can therefore easily be fused into a new dummy index. Applying the SVD with a relaxed truncation criterion we obtain the left-normalized tensor $A_{1}$, with a single right index $m_{1}^{\prime}$

$$
\operatorname{SVD}\left(M_{1 ; w_{0} m_{0}, w_{1} m_{1}}^{\prime \sigma_{1}}\right) \approx \sum_{s_{1}, s_{1}^{\prime}} A_{1 ; m_{0} w_{0}, s_{1}}^{\prime \sigma_{1}} S_{\underline{1} ; s_{1}, s_{1}^{\prime}}^{\prime} V_{\underline{1} ; s_{1}^{\prime}, w_{1} m_{1}}^{\prime}
$$

In the next step, the remaining parts of the result of the SVD are incorporated as before, now also including the MPO tensor, to obtain the next slightly unnormalized tensor

$$
M_{2 ; s_{1}, w_{2} m_{2}}^{\prime \sigma_{2}}=\sum_{\sigma_{2}^{\prime}, s_{1}^{\prime}, m_{1}, w_{1}} S_{\underline{1} ; s_{1}, s_{1}^{\prime}}^{\prime} V_{\underline{1} ; s_{1}^{\prime}, w_{1} m_{1}}^{\prime} W_{2 ; w_{1}, w_{2}}^{\sigma_{2} \sigma_{2}^{\prime}} B_{2 ; m_{1}, m_{2}}^{\sigma_{2}^{\prime}}
$$

This procedure is depicted in Fig. 4.11. It is repeated until the right end of the system is reached and hence the complete operator is applied. Note that even though the SVD decomposition of the tensor $M_{j ; s_{j-1}, w_{j}, m_{j}}^{\prime \sigma_{j}}$ is a well-defined operation, we do not obtain an optimal approximation to $\hat{O}|\psi\rangle$ in the sense of Sec. 4.2.3. Sweeping from the left to the right, the left basis states form an orthonormal basis, which is optimized to approximate the target state. However, the right system of the combined MPS and MPO tensors can be evaluated formally by means of the exact MPO application. Then it is easy to see that the overall target state is not normalized 


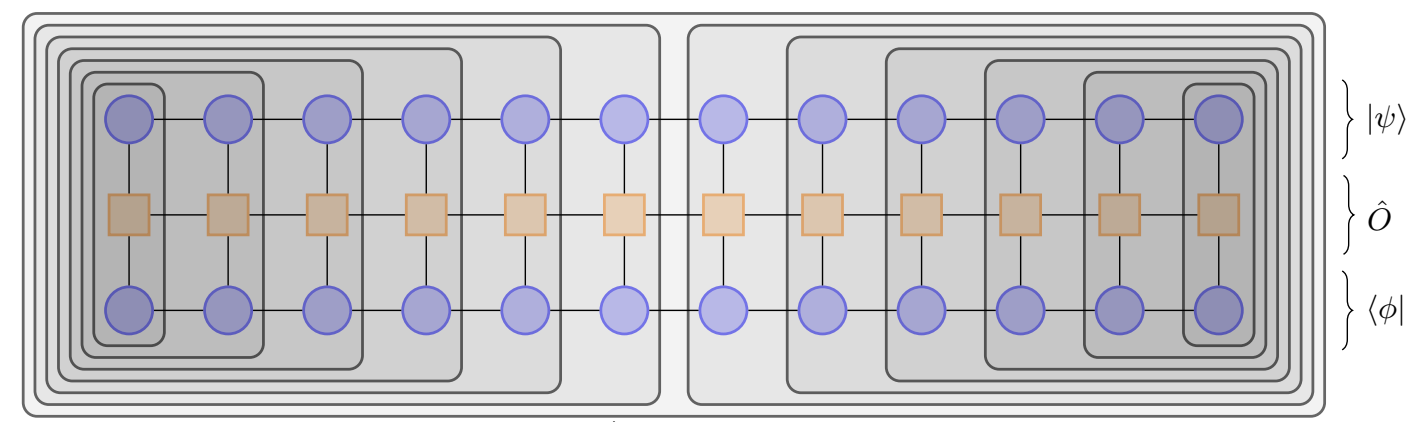

Figure 4.12: The expectation value $\langle\phi|\hat{O}| \psi\rangle$ of an operator between two (possibly different) states represented as MPO and MPS respectively. The optimal contraction order is sideways, e.g. from left to right, as indicated by the shading. To add an additional column, the optimal evaluation order is $L_{j-1} \cdot M_{j} \cdot W_{j} \cdot M_{j}^{\dagger}$. Here and in all following diagrams, we leave off the dummy left/right indices indicated by dotted lines earlier. This graphic is adopted from the author's publication [PKS $\left.{ }^{+} 19\right]$.

anymore. Hence, the truncated weight occuring during the SVD does not constitute a measure of the Froebenius norm for the distance between the exact and the approximated target state in the current bipartition any longer.

For the relaxed truncation scheme a maximal growth factor of the MPS bond dimension $m^{\prime}=2 m$ and a truncated weight of $1 / 10$ of the target weight turns out to be a suitable choice, as long as $\hat{O}$ is close to a unitary operator. The contraction then has complexity $2 m^{3} w \sigma+2 m^{2} w^{2} \sigma^{2}$. The main cost is in the SVD of a $2 m \sigma \times m w$ matrix at cost $\mathcal{O}\left(m^{3} \sigma w\right)$, i.e., linear in $w$. The MPS is in left canonical form now. A subsequent compression as described in Sec. 4.2.3 should be applied to obtain the resulting MPS with the target bond dimension.

\section{Expectation values}

In standard dense numerical linear algebra, to evaluate the expectation value $\langle\phi|\hat{O}| \psi\rangle$, it is necessary to evaluate $\hat{O}|\psi\rangle$ and subsequently the overlap between $\langle\phi|$ and $\hat{O}|\psi\rangle$. As we have seen in the previous section, MPO-MPS products are relatively costly to evaluate. Luckily, the tensor network representing $\langle\phi|\hat{O}| \psi\rangle$ (Fig. 4.12) allows many different contraction orders. Contracting it from left-to-right (using iteratively updated tensors $L_{j}$ as defined in Eq. (4.33)) or right-to-left (using $R_{j}$ as defined in Eq. (4.34) respectively) leads to an asymptotic contraction cost of $O\left(L\left(m^{3} w \sigma+m^{2} w^{2} \sigma^{2}\right)\right)$. The optimal contraction sequence is also indicated by the shading in Fig. 4.12. Note that we can further optimize these contractions if we distinguish local and global observables where the former are defined by operators that act only on a single site $\hat{O} \equiv \hat{o}_{j}$. Assuming the MPS representations of the states $|\phi\rangle,|\psi\rangle$ are in a mixed-canonical form with the center of orthogonality at site $j$, expectation values $\left\langle\phi\left|\hat{o}_{j}\right| \psi\right\rangle$ can be obtained very cheaply. We only have to evaluate a single contraction

$$
\left\langle\phi\left|\hat{o}_{j}\right| \psi\right\rangle=\operatorname{Tr} \bar{M}_{j}^{\prime} W_{j} M_{j}
$$

where $M_{j}^{\prime}$ and $M_{j}$ are the site tensors of the MPS representations of $|\phi\rangle$ and $|\psi\rangle$, respectively and $W_{j}$ is the MPO site-tensor generated from $\hat{o}_{j}$. 


\section{\begin{tabular}{l|l} 
Ground-state search & 4.4
\end{tabular}}

The formalism introduced in the previous sections allows for a natural formulation of a variational ground-state search algorithm exploiting the tensor-network structure of MPS. Optimizing the site tensors locally while ensuring a mixed-canonical state representation, the high-dimensional global minimization is decomposed into local eigenvalue problems. These correspond to finding the ground states of the Hamiltonian projected to the active site spaces ${ }^{7}$ with left and right environments, which are obtained from the left-/right-canonical site tensors of previous optimization steps. The connection to the old-style DMRG is exactly this identification of the environment system with the basis transformations generated from the canonization procedure of the previously updated site tensors. In the following, we recapitulate this ground-state search as it serves as a prototypical variational MPS algorithm and its formulation is of crucial importance for understanding and implementing further methods such as time-evolution schemes. Compared to the previous sections, the considerations are carried out in more detail at points the author wants to put a special emphasis on, and which can be read as completion of potentially left-open questions.

\section{Formulation as local optimization-problem $\mid 4.4 .1$}

The ground state $\left|\psi_{0}\right\rangle$ with eigenvalue $E_{0}$ of a system's Hamiltonian $\hat{H}$ acting on a Hilbert space $\mathcal{H}$ can be found by global minimization of the Rayleigh quotient ${ }^{8} E[\psi]$

$$
\left|\psi_{0}\right\rangle=\min _{|\psi\rangle \in \mathcal{H}} E[\psi]=\min _{|\psi\rangle} \frac{\langle\psi|\hat{H}| \psi\rangle}{\langle\psi \mid \psi\rangle} \Rightarrow \frac{\delta E[\psi]}{\delta \psi} \stackrel{!}{=} 0 .
$$

In case of a discrete system described by a tensor-product Hilbert space a representation of the trial state $|\psi\rangle$ as MPS allows for an evaluation of the variation

$$
\frac{\delta E[\psi]}{\delta \psi}=\sum_{j=1}^{L} \frac{\delta}{\delta M_{j}} \frac{\left\langle\psi\left[M_{j}\right]|\hat{H}| \psi\left[M_{j}\right]\right\rangle}{\left\langle\psi\left[M_{j}\right] \mid \psi\left[M_{j}\right]\right\rangle}
$$

where we used the multilinearity of MPS and introduced the notation $\left|\psi\left[M_{j}\right]\right\rangle$ to indicate that the components of the site tensor $M_{j}$ are varied while all other site tensors are kept fixed. Enforcing normalization of the trial state $|\psi\rangle$, we introduce a Lagrange multiplicator $\lambda$ yielding the optimization problem

$$
0=\sum_{j=1}^{L} \frac{\delta}{\delta M_{j}}\left(\left\langle\psi\left[M_{j}\right]|\hat{H}| \psi\left[M_{j}\right]\right\rangle-\lambda\left\langle\psi\left[M_{j}\right] \mid \psi\left[M_{j}\right]\right\rangle\right) .
$$

Solving this equation for the global minimum can be done by diagonalizing $\hat{H}$ but would ignore the local structure of the MPS ansatz. However, we can make use of the decomposition of the global optimization problem into $L$ local problems that are obtained by independently varying the site tensors $M_{j}$

$$
0=\frac{\delta}{\delta M_{j}}\left(\left\langle\psi\left[M_{j}\right]|\hat{H}| \psi\left[M_{j}\right]\right\rangle-\lambda\left\langle\psi\left[M_{j}\right] \mid \psi\left[M_{j}\right]\right\rangle\right) .
$$

\footnotetext{
${ }^{7}$ Typically the local problems are solved with a single or two active site tensors.

${ }^{8}$ We assume the Hamiltonian to be bounded from below.
} 
It should be mentioned that here we have to be careful with the notation separating the meanings of a variation and an optimization. Given an initial set of site tensors $M_{1}, \ldots M_{L}$ the optimizations must not be done independently for each $M_{j}$ (while the variations can). In turn, an optimization of $M_{j}$ should take into account previous results, for instance, by forming the scalar product with site tensors $\tilde{M}_{k<j}$, which were already subject to preceeding optimizations. We will come to this point in the next section when we describe the algorithm in more detail. Instead of varying the elements of $\mathcal{H}$ we can also vary elements of the dual space $\mathcal{H}^{*}$. Similar considerations then yield

$$
0=\frac{\delta}{\delta \bar{M}_{j}}\left(\left\langle\psi\left[M_{j}\right]|\hat{H}| \psi\left[M_{j}\right]\right\rangle-\lambda\left\langle\psi\left[M_{j}\right] \mid \psi\left[M_{j}\right]\right\rangle\right)
$$

which is the commonly used representation (e.g., in [Sch11]). For pedagocial reasons we evaluate the scalar products for the general MPS representation

$$
\begin{gathered}
\left\langle\psi\left[M_{j}\right]|\hat{H}| \psi\left[M_{j}\right]\right\rangle=\operatorname{Tr}\left[\sum_{\left\{\sigma_{k}\right\},\left\{\sigma_{k}^{\prime}\right\}} \bar{M}^{\sigma_{L}} \cdots \bar{M}^{\sigma_{j}} \cdots \bar{M}^{\sigma_{1}} \cdot W^{\sigma_{1}, \sigma_{1}^{\prime}} \cdot M^{\sigma_{1}^{\prime}} \cdots W^{\sigma_{j}, \sigma_{j}^{\prime}} \cdot M^{\sigma_{j}^{\prime}} \ldots\right. \\
\left.\cdots W^{\sigma_{L}, \sigma_{L}^{\prime}} \cdot M^{\sigma_{L}^{\prime}}\right] \\
\left\langle\psi\left[M_{j}\right] \mid \psi\left[M_{j}\right]\right\rangle=\operatorname{Tr}\left[\sum_{\left\{\sigma_{k}\right\}} \bar{M}^{\sigma_{L}} \cdots \bar{M}^{\sigma_{j}} \cdots \bar{M}^{\sigma_{1}} \cdot M^{\sigma_{1}} \cdots M^{\sigma_{j}} \cdots M^{\sigma_{L}}\right]
\end{gathered}
$$

where we introduced a formal trace over the dummy indices $m_{0}$ and $m_{L}$. As already described in Sec. 4.2, we can reorder the matrix contractions in the traces and construct left- and rightboundary tensors (see Eqs. (4.33) and (4.34)) to simplify the expressions keeping the focus only on the site tensors of interest

$$
\begin{aligned}
\left\langle\psi\left[M_{j}\right]|\hat{H}| \psi\left[M_{j}\right]\right\rangle & =\operatorname{Tr}\left[\sum_{\sigma_{j}, \sigma_{j}^{\prime}} L_{j-1}^{W} \bar{M}^{\sigma_{j}} \cdot W^{\sigma_{j}, \sigma_{j}^{\prime}} \cdot M^{\sigma_{j}^{\prime}} R_{j+1}^{W}\right] \\
\left\langle\psi\left[M_{j}\right] \mid \psi\left[M_{j}\right]\right\rangle & =\operatorname{Tr}\left[\sum_{\sigma_{j}} L_{j-1} \bar{M}^{\sigma_{j}} \cdot M^{\sigma_{j}} R_{j+1}\right]
\end{aligned}
$$

and we put a subscript $W$ to boundary tensors that are created from contractions involving MPO site-tensors. In this compact notation it is easy to see how to perform the variations w.r.t. $\bar{M}_{j}$, namely by exploiting the linearity of the matrix product they translate directly into componentwise derivatives $\sum_{\sigma_{j}} \delta / \delta \bar{M}^{\bar{\sigma}_{j}} \rightarrow \sum_{\sigma_{j}} \sum_{m_{j-1}, m_{j}} \partial / \partial \bar{M}_{j ; \bar{m}_{j-1}, \bar{m}_{j}}^{\bar{\sigma}_{j}}$. Carrying out the derivatives yields

$$
\begin{aligned}
& \frac{\delta}{\delta M_{j}}\left\langle\psi\left[M_{j}\right]|\hat{H}| \psi\left[M_{j}\right]\right\rangle=\sum_{\sigma_{j}^{\prime}} \sum_{\substack{w_{j}, w_{j-1} \\
m_{j-1}^{\prime}, m_{j}^{\prime}}} L_{j-1 ; \bar{m}_{j-1}}^{W ; m_{-1}^{\prime}, w_{j-1}} W_{j ; w_{j-1}, w_{j}}^{\sigma_{j}, \sigma_{j}^{\prime}} M_{j ; m_{j-1}^{\prime}, m_{j}^{\prime}}^{\sigma_{j}^{\prime}} R_{j+1 ; \bar{m}_{j}}^{W ; m_{j}^{\prime}, w_{j}} \\
& \frac{\delta}{\delta M_{j}}\left\langle\psi\left[M_{j}\right] \mid \psi\left[M_{j}\right]\right\rangle=\sum_{\sigma_{j}} \sum_{m_{j-1}^{\prime}, m_{j}^{\prime}} L_{j-1 ; \bar{m}_{j-1}}^{m_{j-1}^{\prime}} M_{j ; m_{j-1}^{\prime}, m_{j}^{\prime}}^{\sigma_{j}} R_{j+1 ; \bar{m}_{j}}^{m_{j}^{\prime}} .
\end{aligned}
$$



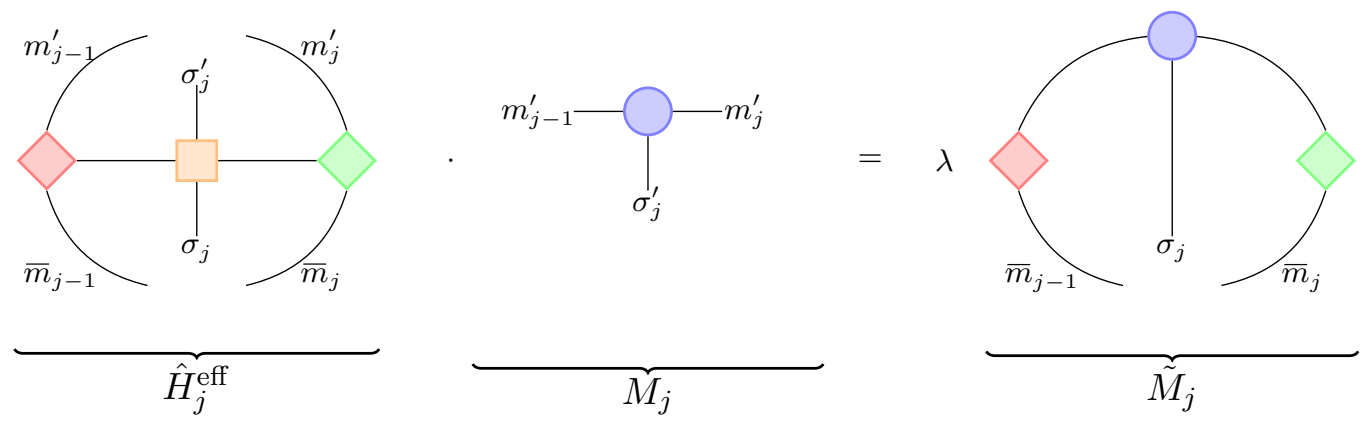

Figure 4.13: Tensor-network representation of the local eigenvalue problem Eq. (4.51). The canonical form which should be exploited to construct $\tilde{M}_{j}$ is indicated by color-encoding the boundary tensors. For notational clarity, indices derived from contractions with adjoint tensors carry overbars.

Equation (4.48) has an interesting structure, which is revealed by performing the contractions over the auxiliary bonds $w_{j-1}, w_{j}$

$$
\sum_{w_{j-1}, w_{j}} L_{j-1 ; \bar{m}_{j-1}}^{W ; m_{j-1}^{\prime}, w_{j-1}} W_{j ; w_{j-1}, w_{j}}^{\sigma_{j}, \sigma_{j}^{\prime}} R_{j+1 ; \bar{m}_{j}}^{W ; m_{j}^{\prime}, w_{j}} \equiv h_{j ; \sigma_{j}, \bar{m}_{j-1}, \bar{m}_{j}}^{\sigma_{j}^{\prime}, m_{j-1}^{\prime}, m_{j}^{\prime}}
$$

where we have defined the effective single-site Hamiltonian $\hat{H}_{j}^{\text {eff }} \equiv h_{j ; \sigma_{j}, \bar{m}_{j-1}, \bar{m}_{j}}^{\sigma_{j}^{\prime}, m^{\prime}}$ and slightly reordered the indices. Ordering the indices in the same way in Eq. (4.49) and interpreting $\hat{H}_{j}^{\text {eff }}$ as matrix with upstairs indices enumerating the columns and downstairs indices the rows we can write Eq. (4.43) as eigenvalue equation

$$
\hat{H}_{j}^{\mathrm{eff}} \cdot M_{j}=\lambda \tilde{M}_{j}
$$

for the local site tensors $\tilde{M}_{j}=\sum_{\sigma_{j}} \sum_{m_{j-1}^{\prime}, m_{j}^{\prime}} L_{j-1 ; \bar{m}_{j-1}}^{m_{j-1}^{\prime}} M_{j ; m_{j-1}^{\prime}, m_{j}^{\prime}}^{\sigma_{j}} R_{j+1 ; \bar{m}_{j}}^{m_{j}^{\prime}}$. Here, $\lambda$ plays the role of the approximation to the global eigenvalue in $\hat{H}|\psi\rangle=\lambda|\psi\rangle$ since it is introduced as Lagrange multiplicator in the global minimization problem. Equation (4.51) yields a reduction of the global optimization problem to $L$ local eigenvalue problems, which can be treated numerically very efficiently. To achieve this goal we now make use of the canonization procedure described in Sec. 4.2.1 and enforce the MPS representation to be in a mixed canonical representation with center of orthogonality at site $j$. Ensuring this representation the boundary tensors $L_{j-1} / R_{j+1}$ collapse to identities yielding $\tilde{M}_{j}=M_{j}$ and we save contractions, which scale as $\sigma m_{j-1}^{2} m_{j}+$ $\sigma m_{j-1} m_{j}^{2}$, while performing only an additional matrix factorization. ${ }^{9}$

From the computational point of view a direct solution of Eq. (4.51) is not recommended because $\hat{H}_{j}^{\text {eff }}$ is a matrix with dimensions $\sigma w_{j-1} m_{j-1} \times \sigma w_{j} w_{j}$ and hence the diagonalization asymptotically scales as $\mathcal{O}\left(\sigma^{3} m^{3} w^{3}\right)$. Instead, it is more practical to use recursive methods to construct a subspace, which has increasing overlap with the ground state of $\hat{H}_{j}^{\text {eff }}$ such as the Lanczos [L50] or (Jacobi)-Davidson [Dav93, SvdV96] procedures. The matrix to be diagonalized then only acts on this subspace and typically matrix dimensions of $\mathcal{O}(10)$ are sufficient to achieve numerical precision. ${ }^{10}$ These methods share the property that they require repeating evaluations of $\hat{H}_{j}^{\text {eff }} \cdot M_{j}$, which hence dominates the numerical expenses and scales as $\mathcal{O}\left(m^{3} w \sigma\right)$ for large maximal bond

\footnotetext{
${ }^{9}$ The choice of a proper factorization method should depend on whether the bond dimensions $m_{j}$ can be increased during the ground-state search. If this is not the case, a cheap QR decomposition is sufficient, otherwise truncation may be desired and rank-revealing factorizations become the methods of choice, such as the SVD.

${ }^{10}$ The reason for this fast convergence of the local eigenvalue problems is hidden in the truncation of the site
} 
dimensions $m$. In the code developed by the author in collaboration with Thomas Köhler, a Lanczos solver was implemented to treat the local eigenvalue problem.

\section{\begin{tabular}{l|l} 
Algorithm & 4.4 .2
\end{tabular}}

The described decomposition of the global minimization of the Rayleigh quotient $E[\psi]$ into $L$ local eigenvalue problems can be used to iteratively determine the ground state of a Hamiltonian $\hat{H}$ motivated by the method of alternating least squares (ALS). In the following, we will describe a possible algorithmic realization that was also implemented by the author in collaboration with Thomas Köhler and mainly followed the ideas in [Sch11]. The steps are formulated for a singlesite realization but are easy to generalize to $k>1$ active site-tensors. In fact, the ground-state searches done in the context of this thesis were all performed with $k=2$ active sites.

For simplicity, we assume that there is a good initial guess $\left|\psi_{0}\right\rangle$ and postpone the discussion on how to find such a state for a moment. In what follows, we put a lower label to the state $\left|\psi_{n}\right\rangle$ and the corresponding energy $\lambda_{n}=\left\langle\psi_{n}|\hat{H}| \psi_{n}\right\rangle$ to indicate the sweep index ( $n=0$ corresponds to the initial guess). In a first preliminary step we bring the state $\left|\psi_{0}\right\rangle$ into a right canonical representation. Starting from the site $j=L$ we apply the canonization procedure described in Sec. 4.2 .1 (i.e. $M_{j} \leftrightarrow B_{j}$ ) to all site tensors except for the site $j=1$, which defines the orthogonality center. Additionally, while sweeping from the right to the left, at each site we construct boundary tensors $R_{j}^{W}$ similar to Eq. (4.34) using the shorthand notation for tensor contractions

$$
R_{j}^{W}=\left(\left(\bar{B}_{j} \cdot R_{j+1}^{W}\right) \cdot W_{j}\right) \cdot B_{j}
$$

where the brackets indicate the usually most efficient contraction order. It is crucial to construct these boundary tensors recursively reusing previously constructed tensors and store them for later purposes. Schematically, this sweep can be represented as operations on lists $\mathcal{T}, \mathcal{B}$ providing the site and boundary tensors, respectively; as shown in the diagram Fig. 4.14. Note that for convenience we introduced a boundary tensor $R_{L+1}^{W}$, which is just the identity acting on the dummy indices $m_{L}, \bar{m}_{L}$ of the site tensors $M_{L}, \bar{M}_{L}$ and on the dummy index $w_{L}$ of the MPO site-tensor $W_{L}$. Furthermore, in the last step we add another tensor $L_{0}^{W}$ to the set of boundary tensors: $\mathcal{B}=\left\{L_{0}^{W}, R_{2}^{W}, \ldots, R_{L+1}^{W}\right\}$, which acts as identity on the dummy indices $m_{0}, \bar{m}_{0}$ of the site tensors $M_{0}, \bar{M}_{0}$ and on the dummy index $w_{0}$ of the MPO site-tensor $W_{0}$. Having finished this initial sweep, all the subsequent operations are going to alter only the sets $\mathcal{T}, \mathcal{B}$.

The general procedure now is to sweep from the left to the right through the system (and back) solving the local eigenvalue problems $\hat{H}_{j}^{\text {eff }} \cdot M_{j}=\lambda M_{j}$ at each site. Thereby, we employ an iterative solver (e.g. the Lanczos algoritm [Lan50]) to construct a subspace $\mathcal{K}=$ $\left\{M_{j}, \hat{H}_{j}^{\text {eff }} \cdot M_{j}, \hat{H}_{j}^{\text {eff }} \cdot \hat{H}_{j}^{\text {eff }} \cdot M_{j}, \cdots\right\},{ }^{11}$ in which an effective matrix representation of $\hat{H}_{j}^{\text {eff }}$ is generated, ${ }^{12}$ which can be diagonalized numerically using common linear algebra libraries (e.g. BLAS [Don02] or MKL [WZS $\left.\left.{ }^{+} 14\right]\right)$. Sweeping through the system, we obtain a sequence of states $\left|\psi_{1, j}\right\rangle$ that approximate the Hamiltonian's overall ground state with increasing precision ${ }^{13}$.

\footnotetext{
tensors to a maximal bond dimension $m_{\max }$ which introduces a cutoff in the length scale of the treated correlations. In fact, using sparse solver without truncation the number of iterations until convergence typically is much larger $\sim \mathcal{O}(100)$.

${ }^{11}$ Such a subspace is called Krylov subspace.

${ }^{12}$ We will not discuss these iterative eigensolver for the ground state in the scope of this thesis.

${ }^{13}$ Actually, we are approximating the eigenstate with the lowest energy accessible to the variational method. We will cover this point in more detail in Sec. 4.4.3.
} 


\begin{tabular}{|l|l|}
\hline $\mathcal{T}=\left\{M_{1}, \ldots, M_{L}\right\}$ & \multicolumn{1}{|c|}{$\mathcal{B}=\left\{R_{L+1}^{W}\right\}$} \\
\hline $\mathcal{T}=\left\{M_{1}, \ldots, M_{L-1}, B_{L}\right\}$ & $\mathcal{B}=\left\{R_{L}^{W}, R_{L+1}^{W}\right\}$ \\
\hline & $M_{L-1} \leftarrow B_{L-1}$ \\
& \\
\hline $\mathcal{T}=\left\{M_{1}, B_{2}, \ldots B_{L}\right\}$ & $\mathcal{B}=\left\{L_{0}^{W}, R_{2}^{W}, \ldots, R_{L+1}^{W}\right\}$ \\
\hline
\end{tabular}

Figure 4.14: Schematic representation of initial sweep to prepare the guess state $\left|\psi_{0}\right\rangle$ for a numerically efficient realization of the ALS ground-state search algorithm. Boxes are representing the status of the lists $\mathcal{T}, \mathcal{B}$ on which the algorithm acts while the transitions indicate the corresponding operations, which are described in detail in the main text.

Let the state $\left|\psi_{1, j}\right\rangle$ be in a mixed canonical representation with orthogonality center at site $j$ where we add another lower index $j$ for clarity. As already mentioned, the subspace in which we solve the eigenproblem is generated by repeatedly contracting the effective hamiltonian $\hat{H}_{j}^{\text {eff }}$ with site tensors $M_{j}^{k}$

$$
\tilde{M}_{j}^{k}=\hat{H}_{j}^{\mathrm{eff}} \cdot \tilde{M}_{j}^{k-1}, \quad \tilde{M}_{j}^{0} \equiv M_{j} .
$$

In general, the specific construction scheme may involve further arithmetic operations on the site tensors

$$
M_{j}^{k}=f\left(\hat{H}_{j}^{\mathrm{eff}} \cdot \tilde{M}_{j}^{k-1}, \tilde{M}_{j}^{k-1}, \tilde{M}_{j}^{k-2}, \ldots\right),
$$

depending on the chosen solver. However, for the current purpose they are of minor importance because the operation dominating the numerical costs is the application of the local Hamiltonian. Diagonalizing the low-dimensional matrix-representation $h_{j ; m, n}^{k}=\left\langle M_{j}^{m}\left|\hat{H}_{j}^{\text {eff }}\right| M_{j}^{n}\right\rangle$ in $\mathcal{K}(m, n=$ $0 \ldots k)$ we obtain a sequence of approximations of the ground-state energy by picking the lowest eigenstate $\lambda^{k}$ and we can use the change $\delta \lambda^{k}=\left|\lambda^{k}-\lambda^{k-1}\right|$ as convergence criterion. Note that typically the matrix coefficients $h_{j ; m, n}^{k}$ are generated during the construction of the $M_{j}^{k}$ 's, which saves additional calculations of expectation values. Once the solver has converged below a certain threshold $\delta>0$ we can update the value of the global ground state energy and the corresponding site tensor approximating the new ground-state candidate

$$
\lambda \hookleftarrow \lambda^{k_{0}}, \quad M_{j} \leftrightarrow \sum_{m=0}^{k_{0}} v_{j ; m}^{k_{0}} M_{j}^{m}, \quad k_{0}=\max _{k} \delta \lambda^{k}<\delta,
$$

where $v_{j ; m}^{k_{0}}$ are the coefficients of the lowest eigenstate in the subspace $\mathcal{K}$ of dimension $k_{0}$. The evaluation of the tensor contractions $\hat{H}_{j}^{\text {eff }} \cdot \tilde{M}_{j}^{k}$ itself is the crucial point and the (in general) optimal contraction order is a good starting point for the implementation of an efficient algorithm

$$
\tilde{M}_{j}^{k}=L_{j-1}^{W} \cdot\left(W_{j} \cdot\left(\tilde{M}_{j}^{k-1} \cdot R_{j+1}^{W}\right)\right) .
$$




\begin{tabular}{|c|c|}
\hline $\mathcal{T}=\left\{M_{1}, B_{2}, \ldots, B_{L}\right\}$ & $\mathcal{B}=\left\{L_{0}^{W}, R_{2}^{W}, \ldots, R_{L+1}^{W}\right\}$ \\
\hline$M_{1} \hookleftarrow \sum_{m=0}^{k_{0}} v_{1 ; m}^{k_{0}} M_{1}^{m}$ & $\lambda \hookleftarrow \lambda^{k_{0}}, \quad M_{1} \hookleftarrow A_{1}$ \\
\hline \multicolumn{2}{|c|}{$\mathcal{T}=\left\{A_{1}, M_{2}, B_{3}, \ldots, B_{L}\right\} \quad \mathcal{B}=\left\{L_{0}^{W}, L_{1}^{W}, R_{3}^{W}, \ldots, R_{L+1}^{W}\right\}$} \\
\hline$M_{2} \hookleftarrow \sum_{m=0}^{k_{0}} v_{2 ; m}^{k_{0}} M_{2}^{m}$ & $\lambda \hookleftarrow \lambda^{k_{0}}, \quad M_{2} \hookleftarrow A_{2}$ \\
\hline$M_{L} \leftrightarrow \sum_{m=0}^{k_{0}} v_{L ; m}^{k_{0}} M_{L}^{m}$ & $\lambda \hookleftarrow \lambda^{k_{0}}, \quad M_{L} \hookleftarrow \frac{M_{L}}{\left|M_{L}\right|}$ \\
\hline $\mathcal{T}=\left\{A_{1}, \ldots A_{L-1}, M_{L}\right\}$ & $\mathcal{B}=\left\{L_{0}^{W}, \ldots, L_{L-1}^{W}, R_{L+1}^{W}\right\}$ \\
\hline
\end{tabular}

Figure 4.15: Schematic representation of a single sweep for a numerically efficient realization of the ALS ground-state search algorithm. Boxes are representing the status of the lists $\mathcal{T}, \mathcal{B}$ on which the algorithm acts while the transitions indicate the corresponding operations, which are described in detail in the main text.

Having updated the site tensor $M_{j}$ we continue optimizing the next site. Here, it is important to keep the site tensors in a mixed canonical representation by shifting the center of orthogonality to the next site $j+1(j-1$ updating the list $\mathcal{T}$ (c.f. Sec. 4.2.1). Also, we update the list of boundary tensors $\mathcal{B}$ to prepare the next evaluation of the tensor contractions including $\hat{H}_{j+1}^{\text {eff }}$. These operations can be represented diagramatically as shown in Fig. 4.15 for a right sweep. Note that in the last step we do not need to employ another canonization procedure, instead we only normalize the site tensor by dividing it with its norm $\left|M_{L}\right|=\sqrt{\bar{M}_{L} \cdot M_{L}}$ (which actually is identical to applying the canonization scheme Sec. 4.2.1 and drop the remaining singular value, which is just the norm of the state itself). Sweeping through the system this way we obtain a new set of site tensors representing a new guess for the ground state $\left|\psi_{1}\right\rangle$ with orthogonality center at site $L$ and energy $\lambda_{1}$. Therefore, we can repeat the whole procedure by changing the sweep direction, which yields the ground-state search algorithm. Clearly, we have to adjust the update steps of the lists $\mathcal{T}, \mathcal{B}$ when sweeping to the left by shifting the center of ortogonality in $\mathcal{T}$ properly and also construct right boundary tensors $R_{j}^{W}$ instead of left boundary tensors $L_{j}^{W}$. A reasonable escape criterion would be to monitor the energy gain per sweep $\delta \lambda=\lambda_{n-1}-\lambda_{n}$ and stop the algorithm if $\delta \lambda \leq \varepsilon$ with the desired target precision $\varepsilon$. Another possibility is to evaluate the variance

$$
\operatorname{Var}(|\psi\rangle)=\left\langle\psi\left|(\hat{H}-E)^{2}\right| \psi\right\rangle=\left\langle\psi\left|\hat{H}^{2}\right| \psi\right\rangle-\langle\psi|\hat{H}| \psi\rangle^{2}
$$

during the sweeps, which obeys the usefull property that there is an eigenstate $|\tilde{\psi}\rangle$ of $\hat{H}$ with energy $\tilde{E}$ such that $E \geq \tilde{E} \geq E-\sqrt{\operatorname{Var}(|\psi\rangle)}$. Therefore, if $|\tilde{\psi}\rangle$ is the ground state of $\hat{H}$ then $\sqrt{\operatorname{Var}(|\psi\rangle)}$ gives an error bound on the approximation of the ground state by means of $|\psi\rangle$. However, there are good reason not to do this after every sweep. The naive evaluation of the expectation value $\left\langle\psi_{n}\left|\hat{H}^{2}\right| \psi_{n}\right\rangle$ usually is very costly as it scales with $m^{3} w^{2}$. Furthermore, calculating the difference $\left\langle\psi_{n}\left|\hat{H}^{2}\right| \psi_{n}\right\rangle-\left\langle\psi_{n}|\hat{H}| \psi_{n}\right\rangle^{2}$ may suffer from catastrophic cancellation, which reduces the precision of its outcome by several magnitudes. Even though there are ways to either reduce the numerical costs [HHS18] or minimize the effects of numerical artefacts such as catastrophic cancellation [PKM17] a general solution to overcome both restrictions at the same 
time has not been formulated so far, to the best of the author's knowledge.

\section{Initial states}

At this point we return to the initially postponed question about how to construct a good initial guess $\left|\psi_{0}\right\rangle$. Clearly, the convergence of the described algorithm will crucially depend on the choice of this state. In order to realize an efficient algorithm it is therefore necessary to find a reasonable trade-off between a well-conditioned initial guess that already has a large overlap with the targeted ground state on the one hand and a numerically efficient construction scheme on the other.

Random matrices The simplest we can do of course is to create a random initial state by initializing all site tensors with random matrices where the entries are drawn from a uniform distribution. It is important to ensure the matrices to have a norm of order one, which can be achieved, for instance, by rescaling the elements with the Frobenius norm. Unfortunately, the convergence properties of random states are very poor. First of all, the overlap of a randomly drawn state with the targeted ground state scales as $1 / \operatorname{dim} \mathcal{H} \stackrel{L \gg 1}{\longrightarrow} 0$. Another problem is that randomly drawn states do not obey the area law $S_{N} \sim|\partial A|$ of entanglement but follow a volume law $S_{N} \sim|A|$ instead. This causes the first sweeps to be particularly costly because the site tensors cannot be truncated efficiently until it (slowly) approaches the ground state obeying an area law. The reasoning behind this statement is a bit counterintuitive since MPS obey an area law by construction. Nevertheless, there is a straight forward way to equip MPS with a measure for distinguishing area law states from such following a volume law. Consider for instance a state whose reduced density matrix has nearly equal eigenvalues $\lambda_{\mu}=\sigma^{-|A|}+\delta \lambda_{\mu}$ where $\delta \lambda_{\mu} \ll \sigma^{-|A|}$. Then obviously, the entanglement entropy satisfies a volume law and one way to quantify this is to compare the decay rate of the singular values over the range they are spanning.

Random superpositions Another possibility is to construct a random initial state exploiting local ladder operators spanning the algebra of operators on $\mathcal{H}_{\sigma}$. Consider for example a Hamiltonian describing a chain of $L$ Spin-1/2 degrees of freedom. We can independently draw coeffcients $c_{j}$ from a probability distribution $w(x)$ and construct an operator being a superposition of Spin-raising operators $\hat{S}_{j}^{+}$with weights $c_{j}$

$$
\hat{C}=\sum_{j=1}^{L} c_{j} \hat{S}_{j}^{+}, \quad\left|\psi_{0,\left\{c_{j}\right\}}\right\rangle=\frac{\hat{C}}{\sqrt{\sum_{j} c_{j}^{2}}}|\downarrow, \ldots, \downarrow\rangle .
$$

In the same spirit we can construct guess states for magnetization sectors $\hat{S}^{z}=(L-N) / 2, N=$ $1, \ldots, L$ from repeatedly applying operators $\hat{C}^{p}$ for independently drawn random coefficients $c_{j}^{p}$ (here, $p$ labels a set of coefficients $\left\{c_{j}^{p}\right\}$ independently drawn from $w(x)$ for the whole lattice)

$$
\left|\psi_{0,\left\{c_{j}^{1}\right\}, \ldots,\left\{c_{j}^{N}\right\}}\right\rangle=\frac{1}{\mathcal{N}} \prod_{p=1}^{N} \hat{C}^{p}|\downarrow, \ldots, \downarrow\rangle,
$$

with normalization $\mathcal{N}^{2}=\left\langle\psi_{0,\left\{c_{j}^{1}\right\}, \ldots,\left\{c_{j}^{N}\right\}} \mid \psi_{0,\left\{c_{j}^{1}\right\}, \ldots,\left\{c_{j}^{N}\right\}}\right\rangle$. The state $\left|\psi_{0,\left\{c_{j}^{1}\right\}, \ldots,\left\{c_{j}^{N}\right\}}\right\rangle$ constructed this way is generated from a superposition of spin-flip excitations and we would expect intuitively that in this case it provides a good starting point for a ground-state search for short-ranged interacting Spin-1/2 Hamiltonians. In fact, the described construction implies that $\left|\psi_{0,\left\{c_{j}^{1}\right\}, \ldots,\left\{c_{j}^{N}\right\}}\right\rangle$ 


\begin{tabular}{|c|c|}
\hline $\mathcal{T}=\{\}$ & $\mathcal{B}=\left\{L_{0}^{W}, R_{3}^{W}\right\}$ \\
\hline random $\Theta_{1,2}$ & $W_{2} \hookleftarrow W_{2} P_{L=2}$ \\
\hline$\Theta_{1,2} \leftrightarrow \sum_{m=0}^{k_{0}} v_{1 ; m}^{k_{0}} \Theta_{1,2}^{m}$, & $\lambda \hookleftarrow \lambda^{k_{0}},\left(A_{1}, M_{2}\right) \hookleftarrow$ split $\Theta_{1,2}$ \\
\hline $\mathcal{T}=\left\{A_{1}, M_{2}\right\}$ & $\mathcal{B}=\left\{L_{0}^{W}, L_{1}^{W}, R_{4}^{W}\right\}$ \\
\hline random $T_{3}, \Theta_{2,3} \leftarrow M_{2} \cdot T_{3}$, & $W_{3} \leftrightarrow W_{3} P_{L=3}$ \\
\hline$\Theta_{2,3} \leftrightarrow \sum_{m=0}^{k_{0}} v_{2 ; m}^{k_{0}} \Theta_{2,3}^{m}$, & $\lambda \hookleftarrow \lambda^{k_{0}},\left(A_{2}, M_{3}\right) \hookleftarrow \operatorname{split} \Theta_{2,3}$ \\
\hline random $T_{L}, \Theta_{L-1, L} \leftrightarrow M_{L-1} \cdot T_{L}$ & $\Theta_{L-1, L} \leftrightarrow \sum_{m=0}^{k_{0}} v_{L ; m}^{k_{0}} \Theta_{L-1, L}^{m}$ \\
\hline$\lambda \hookleftarrow \lambda^{k_{0}},\left(A_{L-1}, M_{L}\right) \hookleftarrow$ split $\Theta_{L-1, L}$ & $M_{L} \leftrightarrow \frac{M_{L}}{\left|M_{L}\right|}$ \\
\hline $\mathcal{T}=\left\{A_{1}, \ldots A_{L-1}, M_{L}\right\}$ & $\mathcal{B}=\left\{L_{0}^{W}, \ldots, L_{L-1}^{W}, R_{L+1}^{W}\right\}$ \\
\hline
\end{tabular}

Figure 4.16: Schematic representation of the developed warm-up sweep for a numerically efficient construction of an initial guess state for the ground-state search algorithm. Boxes are representing the status of the lists $\mathcal{T}, \mathcal{B}$ on which the algorithm acts while the transitions indicate the corresponding operations, which are described in detail in the main text.

has no notion of locality in the sense that correlations are induced at all length scales. It is therefore natural to expect the resulting state to exhibit a logarithmic scaling of the entanglement entropy $S_{N} \sim \log |A|$ at least. In most cases states obeying such a scaling relation belong to critical systems [ECP10] strengthening the expectation that such guess states serve as good starting point to find ground states of local Hamiltonians.

Warmup A completely different approach follows the ideas of inifinite-size density-matrix renormalization group (iDMRG) that was already used as preconditioner in the original DMRG algorithm on finite systems [Whi92, Whi93, NW99]. Here, we present a variant of this warm-up procedure inspired by iDMRG that can be used to construct initial guess states, which turned out to be already very close to the ground states of most of the systems studied in this thesis. The construction scheme is based on solving local problems in a two-site representation (c.f. Sec. 4.4.2) while subsequently adding new site tensors to build up the overall system. We begin with a system consisting of two sites, for which we initialize a tensor $\Theta_{1,2} \equiv \Theta_{1,2 ; m_{0}, m_{2}}^{\sigma_{1}, \sigma_{2}}$ with random values with auxiliary bond dimensions $m_{0}=m_{2}=1$, i.e., we built up the system in a sweep from the left to the right. This tensor is optimized to represent the ground state of a system with size $L=2$ by constructing boundary tensors $L_{0}^{W}, R_{3}^{W}$ acting as identities on the bond spaces of both the site tensor and the MPO tensors $W_{1}, W_{2}$ and solving the effective problem $h_{1,2} \cdot \Theta_{1,2}=\lambda \Theta_{1,2}$. Note that in order to have an MPO bond dimension $w_{2}=1$ we chose $W_{2}$ to be the MPO site-tensor corresponding to the last site of the overall system. To achieve this, we can always apply a projection to the tensor $W_{2} P_{L=2}$ in order to render it the 
last physical tensor, that is, truncating the sum of interaction terms in the overall Hamiltonian $\hat{H}$ at site 2 (as discussed in detail in Sec. 5.1). Performing a SVD on the optimized site tensor (c.f. Sec. 4.2.1) we obtain a left-canonical site tensor $A_{1}$ as well as a site tensor $M_{2}$, which is not in a canonical form. Following the prescription in Sec. 4.4.2 we use the tensors $L_{0}^{W}, A_{1}, W_{1}$ to construct a new boundary tensor $L_{1}^{W}$. Now, we increase the system size by adding a new site tensor $T_{3 ; m_{2}, m_{3}}^{\sigma_{3}}$ with random entries that again has bond dimensions $m_{2}=m_{3}=1$; and construct a boundary tensor $R_{4}^{W}$ that acts as identity. Using the full MPO site tensor $W_{2}$ and projecting the site tensor $W_{3}$ so that all interactions are terminated at site 3 we solve the reduced problem $\Theta_{2,3} \cdot h_{2,3}=\lambda \Theta_{2,3}$ where we contracted $M_{2} \cdot T_{3}=\Theta_{2,3}$. Having obtained the optimized site tensor a subsequent SVD decomposition prepares the chain for adding the next site and we continue the procedure until the desired system size is reached. The described scheme yields the algorithm shown in Fig. 4.16, which constructs sets of site tensors $\mathcal{T}$ and boundary tensors $\mathcal{B}$ that are then used as input for the ground-state algorithm Fig. 4.15.

\section{Connection to DMRG}

Finally, we would like to comment on the connection between the presented formulation of the ground-state search algorithm and DMRG. In its original formulation [Whi92, Whi93] the algorithm corresponds to a ground-state search with active sites $k=2$. The left and right enviroments $A_{j-1}, A_{j+2}$ can be identified with the boundary tensors $L_{j-1}^{W}$ and $R_{j+2}^{W}$, respectively. Then, the effective Hamiltonian $H_{A_{j-1} \bullet A_{j+2}}{ }^{14}$ is constructed by collecting all interactions connecting the left and the right environment, which corresponds to creating the effective Hamiltonian $h_{j, j+1}$ for the two active site-tensors $M_{j}, M_{j+1}$. Finding the ground state of $H_{A_{j-1} \bullet A_{j+2}}$ yields a state $\left|\psi_{j, j+1}\right\rangle$ in the combined basis of the environment and the two active sites. In the bond basis of the left and right boundary tensors this state can be written as

$$
\left|\psi_{j, j+1}\right\rangle=\sum_{l_{j-1}=1}^{m_{j-1}} \sum_{r_{j+2}=1}^{m_{j+1}} \sum_{\sigma_{j}, \sigma_{j+1}} \Psi_{j, j+1 ; l_{j-1}, r_{j+2}}^{\sigma_{j}, \sigma_{j+1}}\left|l_{j-1}\right\rangle\left|\sigma_{j}\right\rangle\left|\sigma_{j+1}\right\rangle\left|r_{j+2}\right\rangle
$$

Assuming that the basis states of the right boundary-tensors are right orthogonal we can construct the reduced density matrix of the left part of the system

$$
\rho_{j, j+1 ; \sigma_{j}^{\prime}, l_{j-1}^{\prime}}^{\sigma_{j}, l_{j-1}}=\sum_{\sigma_{j+1}, r_{j+2}} \Psi_{j, j+1 ; l_{j-1}, r_{j+2}}^{\sigma_{j}, \sigma_{j+1}} \bar{\Psi}_{j, j+1 ; l_{j-1}^{\prime}, r_{j+2}}^{\sigma_{j}^{\prime}, \sigma_{j+1}},
$$

where we have reordered the indices in the density matrix so that upper indices correspond to row indices and lower indices correspond to column indices. In DMRG this density matrix is diagonalized

$$
\rho_{j, j+1 ; \sigma_{j}^{\prime}, l_{j-1}^{\prime}}^{\sigma_{j}, l_{j-1}}=\sum_{m_{j}} U_{j ; m_{j}}^{\sigma_{j}, l_{j-1}} W_{m_{j}} \bar{U}_{j ; \sigma_{j}^{\prime}, l_{j-1}^{\prime}}^{m_{j}}
$$

and only the $m$ largest eigenstates and eigenvalues are kept. Finally, the truncated transformation matrices $U_{j}, \bar{U}_{j}$ are contracted into the environment generating a new environment matrix $A_{j} \leftarrow$ $\bar{U}_{j} A_{j-1} U_{j}$. The identification of this step with the splitting of the optimized two-site tensor $M_{j, j+1 ; m_{j-1}, m_{j+1}}^{\sigma_{j}, \sigma_{j+1}}$ in the MPS ground-state search algorithm can be done by regrouping the indices

\footnotetext{
${ }^{14}$ Which is also called superblock Hamiltonian
} 
according to $M_{j, j+1 ; m_{j-1}, m_{j+1}}^{\sigma_{j}, \sigma_{j+1}} \rightarrow M_{j+1 ; \sigma_{j+1}, m_{j+1}}^{j ; \sigma_{j}, m_{j-1}}$ and identifying the bond basis indices $l_{j-1}=$ $m_{j-1}$ and $r_{j+2}=m_{j+1}$. A SVD can separate the upper and lower indices

$$
M_{j+1 ; \sigma_{j+1}, m_{j+1}}^{j ; \sigma_{j}, m_{j-1}}=\sum_{m_{j}} U_{m_{j}}^{j ; \sigma_{j}, m_{j-1}} \Sigma_{m_{j}} V_{j+1 ; \sigma_{j+1}, m_{j+1}}^{m_{j}}
$$

and after truncating to the maximal bond dimension $m$ the tensor $U_{j}$ is set to the new site tensor $A_{j} \leftrightarrow U_{j}$. Ensuring a mixed-canonical representation we see immediately that determining the transformation matrix $U_{j}$ to diagonalize $\rho_{j, j+1}$ is equivalent to finding the left-orthogonal transformation matrix when decomposing $M_{j, j+1}$ using a SVD. The construction of the new left boundary tensor $L_{j}^{W}$ is then completely equivalent to create the new environment matrix $A_{j}$ in the DMRG algorithm.

The equivalence of the DMRG algorithm to the MPS ground-state search reveals an interesting interpretation of the site tensors in the mixed-canonical representation. Apart from the center of orthogonality the left-/right-orthogonal site tensors $A_{j}, B_{j}$ can be interpreted as basis transformations mapping the physical Hilbert space of the left/right part of the system to bond spaces, which are optimized to faithfully represent the state by means of the site tensor(s) at the orthogonality center. In the context of time-evolution algorithms we will pursue this interpretation constructing projectors onto the bond spaces that allow us to formulate efficient time-evolution schemes.

\section{Convergence and extensions}

The algorithm presented in Sec. 4.4.2 is a non-linear Gauss-Seidel method [OR00] and can be formulated mathematically as iterative solution strategy for a tensor representation of a quadratic optimization problem in a high-dimensional vector space $\mathcal{H}$. In our situation the tensor representation of elements of $\mathcal{H}$ is defined by parametrizing the states $|\psi\rangle \in \mathcal{H}$ in terms of site tensors $M_{j}$ with a maximal bond dimension $m_{\max }$. The space spanned by all such tensor representations can be introduced as the space of multilinear maps $\mathcal{U}: \mathcal{P}_{1} \times \cdots \times \mathcal{P}_{L} \longrightarrow \mathcal{H}$ where $\mathcal{P}_{j}$ is the set of rank-3 tensors with dimensions $\left(\sigma, m_{\max }, m_{\max }\right)$. Then, finding the groundstate of a Hamiltonian $\hat{H}$ by minimizing the Rayleigh quotient within $\mathcal{U}$ can be written as

$$
\left|\psi_{0}\left[M_{1}, \ldots, M_{L}\right]\right\rangle=\min _{\left\{M_{j} \in \mathcal{P}_{j}\right\}_{j}} E\left[\left|\psi\left[M_{1}, \ldots, M_{L}\right]\right\rangle\right] \equiv \min _{\left\{M_{j} \in \mathcal{P}_{j}\right\}_{j}} E\left[M_{1}, \ldots, M_{L}\right] .
$$

A sweep step as described above is then equivalent to find the optimal site tensor $\tilde{M}_{j}$ taking into account the previous solutions $\tilde{M}_{k<j}$ (in case of a left-to-right sweep)

$$
\tilde{M}_{j}=\min _{M_{j} \in \mathcal{P}_{j}} E\left[\tilde{M}_{1}, \ldots, \tilde{M}_{j-1}, M_{j}, M_{j+1}, \ldots, M_{L}\right] .
$$

The convergence of this iterative solution strategy has been discussed in literature [Moh13, EHK15] and the crucial insight is that solving the local problems while maintaining a mixedcanonical form of the site-tensors, the algorithm converges towards the groundstate of $\hat{H}$ if the latter is an isolated point in the spectrum of $\hat{H}$ restricted to $\mathcal{U}$. Therefore the ground states of gapped Hamiltonians usually can be found very efficiently while gapless systems can prevent the algorithm from converging. In particular, systems featuring long-ranged interactions can cause the algorithm to get stuck in excited states if the maximal dimension of the tensor representation, that is, the bond dimension $m_{\max }$ is to small too resolve the respective energy differences. 
The previous considerations imply that convergence of the ground-state algorithm can depend crucially on the different realizations of the algorithm with $k=1$ and $k>1$ active sites. In the following, we restrict the discussion to $k=1$ (single-site density-matrix renormalization group (1DMRG)) and $k=2$ (two-site density-matrix renormalization group (2DMRG)). The most prominent difference between these methods concerning the convergence of the groundstate search algorithm is the reduced local search space $\mathcal{P}_{j}$ for the eigensolver. This can be seen immediately by counting the dimensions of the effective Hamiltonians in 1DMRG and 2DMRG interpreted as matrices acting on the linearized active site tensors. At each step of the sweep we have to solve the following eigenvalue equations:

$$
\begin{aligned}
& \hat{H}_{j}^{\text {eff }} \cdot M_{j}=\lambda M_{j}, \quad M_{j} \in \mathbb{V}^{N_{1} \times 1}, \hat{H}_{j}^{\mathrm{eff}} \in \mathbb{V}^{N_{1} \times N_{1}}, \quad N_{1}=\sigma \cdot m_{j-1} \cdot m_{j}, \\
& h_{j, j+1} \cdot M_{j, j+1}=\lambda M_{j, j+1}, \quad M_{j, j+1} \in \mathbb{V}^{N_{2} \times 1}, h_{j, j+1} \in \mathbb{V}^{N_{2} \times N_{2}}, \quad N_{2}=\sigma^{2} \cdot m_{j-1} \cdot m_{j+1} \text {, }
\end{aligned}
$$

so that in 2DMRG the available local state space to approximate the ground state is larger by a factor of $\sigma$ than the one in 1DMRG. Another drawback of 1DMRG is that the bond dimension of the site tensors cannot be increased in the course of the algorithm and hence the choice of the tensor representation $\mathcal{U}$ can not be adjusted during the algorithm. This stems from the fact that during the canonization step of the updated site tensor the matrices $M_{j ;\left(\sigma_{j}, m_{j-1}\right), m_{j}}$ we need to factorize have maximal rank $\min \left(\sigma \cdot m_{j-1}, m_{j}\right)$. Thus, states having maximal bond dimension $m$ before a sweep can maximally maintain this bond dimension. In contrast, in 2DMRG the matrices $M_{j ;\left(\sigma_{j}, m_{j-1}\right),\left(\sigma_{j+1}, m_{j+1}\right)}$ we need to factorize have maximal rank $\min \left(\sigma \cdot m_{j-1}, \sigma \cdot m_{j+1}\right)$ so that after a sweep with initial maximal bond dimension $m$ the state can exhibit a maximal bond dimension $\sigma \cdot m$. Additionally, having a system with conserved quantum numbers that are exploited via block-diagonal tensors a redistribution of initially occupied quantum number sectors is also not possible in 1DMRG but is automatically achieved during the factorization step of 2DMRG. Therefore, without further modifications $2 \mathrm{DMRG}$ is less prone to get stuck in local minima during each iteration step than 1DMRG.

Convergence for different initial guess states As already pointed out before, the specific choice of the initial state can also be an important factor. We discussed three construction schemes for obtaining initial guess states $\left|\psi_{0}\right\rangle$ whose impact on the convergence of the groundstate search algorithm for a paradigmatic model will be presented in the following. We chose the antiferromagnetic Spin-1/2 $X X Z$ Heisenberg-chain

$$
\hat{H}_{X X Z}=-\sum_{j} \frac{1}{2}\left(\hat{S}_{j}^{+} \hat{S}_{j+1}^{-}+\hat{S}_{j}^{-} \hat{S}_{j+1}^{+}\right)+J_{z} \hat{S}_{j}^{z} \hat{S}_{j+1}^{z}
$$

with varying Ising interaction $J_{z} \in[0,1]$ and performed ground-state searches using the described algorithm in Sec. 4.4.2 with $k=2$ active sites, i.e., 2DMRG. We constructed initial guess states for systems with up to 200 lattice sites where for the method of random superpositions we used two different probability distributions to generate the weights $c_{j}^{p}$

random superpositions :

$$
\begin{aligned}
w(x) & =\frac{\delta(x)+\delta(1-x)}{2}, \\
w_{s}(x) & =\frac{\delta(1+x)+\delta(x)+\delta(1-x)}{3} .
\end{aligned}
$$

The initial states served as input parameter for the 2DMRG algorithm and we converged each ground state until after the $n$th sweep a relative precision $\delta \lambda / \lambda_{n}<10^{-12}$ was obtained. We fixed the truncated weight to $\delta=10^{-12}$ and set the maximal bond dimension to $m_{\max }=$ 


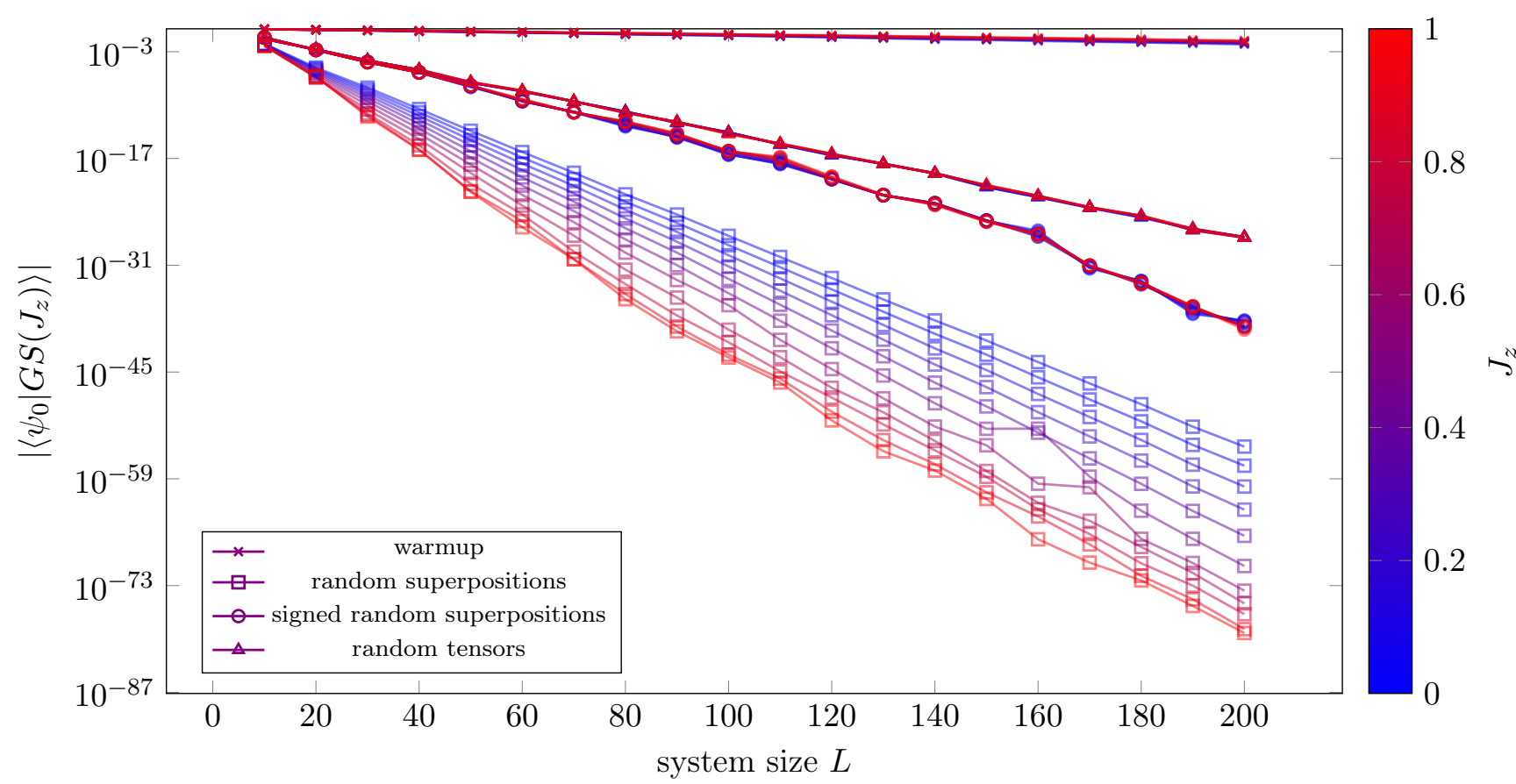

Figure 4.17: Overlap of initial guess states for the 2DMRG ground-state search algorithm constructed from the methods described in the main text with ground states $\left|G S\left(J_{z}\right)\right\rangle$ of the $X X Z$-chain for varying values of the Ising interaction $J_{z}$ and as a function of the system size $L$.

200. For each system size and Ising interaction we initialized 21 different realizations of initial state configurations and averaged the evaluated observables. In Fig. 4.17 we show the absolute values of the overlaps between the converged ground states $\left|\psi_{\text {ref }}\right\rangle$ and the initial states. The initial state generated by the warmup procedure is very close to the converged ground state with a scaling $\sim 2^{-\frac{L}{100}}$ which is nearly independent on the Ising interaction. All the other methods generate initial guess states with a scaling $\sim 2^{-\frac{L}{\mathcal{O}(1)}}$, which is the expected scaling for states whose coefficients are drawn uniformly and independently from the many-body Hilbert space $\mathcal{H}$. Notably, the initial guess generated from random superpositions exhibits the worst scaling and also a strong dependency on the Ising interaction strength. Both properties can be understood very easily by general considerations about the Hamiltonian and demonstrate how a good intuition can significantly improve the quality of the constructed intial guess state by choosing a good probability distribution $w(x)$. Inspecting the first two summands in Eq. (4.68), we find that for neighboring sites these are minimized by singlet configurations $\frac{|\uparrow \downarrow\rangle-|\downarrow \uparrow\rangle}{\sqrt{2}}$. We also note that the random superposition sampled from $w(x)$ does not incorporate a sign change in the applied spin-raising operators. While the ground state features an antisymmetric exchange between neighboring sites the guess state created from sampling coefficients according to $w(x)$ has no antisymmetric components at all, which explains the poor scaling in Fig. 4.17 compared to random superpositions generated from sampling w.r.t. $w_{s}(x)$. Approaching the phase transition at $J_{z}=1$ Ising interactions become dominant and a gap opens, eventually. This opening of a gap is acompanied with a localization of the Spin degree of freedoms when tuning $J_{z} \rightarrow 1$. Clearly, the construction of the random superpositions generated from $w(x)$ cannot account for any type of localization of Ising moments and therefore an increase of $J_{z}$ naturally comes with a decrease of the overlap of the constructed initial guess with the ground state.

In Fig. 4.18 we show the runtimes obtained when performing 2DMRG ground state searches 


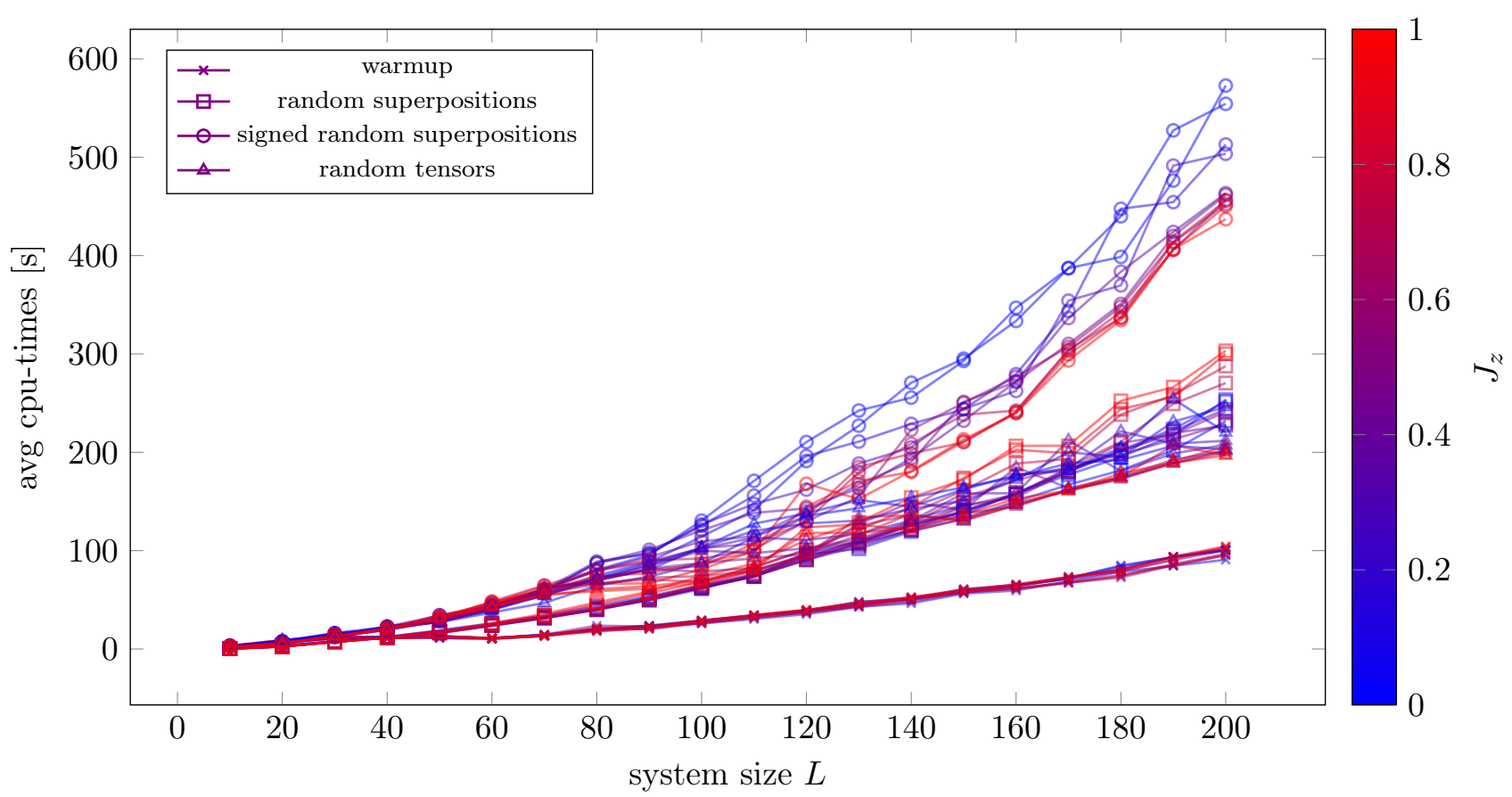

Figure 4.18: CPU times for the 2DMRG ground-state search algorithm initialized with the different guess states constructed from the methods described in the main text for varying values of the Ising interaction $J_{z}$ and as a function of the system size $L$.

starting from the different initial guess states. The runtimes were measured until after the $n$th sweep the ground-state algorithm converged to a relative energy gain $\delta \lambda / \lambda_{n}<10^{-12}$. As expected from the calculated overlaps, the warmup procedure generates the most efficient initial guess states. For larger system sizes these are converging towards the ground state with a speedup of at least a factor of two, compared to the runs with initial guess states created by the other methods. Interestingly, we also find that the worse scaling of the guess states obtained by random superpositions according to $w(x)$ does not severely affect the runtimes as long as $J_{z}$ is not to close to the phase transition. On the other hand, the random superpositions sampled from $w_{s}(x)$ exhibit a much slower convergence. The reason for this somewhat unexpected behavior is unclear at this point but maybe worth a careful investigation in a future work.

Figure 4.19 displays the half-chain entanglement entropy obtained for the different construction methods. We find that the warmup procedure already creates initial guess states obeying an area law, which is consistent with the previous findings and adds another reason for the quick convergence of the subsequent ground-state search. The randomly initialized states on the other hand show an interesting variety of dependencies on the system size. We observe a logarithmic correction $S_{N} \sim \log L$ of the half-chain entanglement entropy for states generated by a random superposition sampled from $w(x)$. In contrast, states obtained from random superpositions with coefficients $c_{j}^{p}$ sampled from $w_{s}(x)$ are saturating quickly at a value close to $S_{N} \approx 6$. States sampled from randomly initialized tensors exhibit a similar rapid growth but a saturation value that is even larger: $S_{N} \approx 8.25$. Both behaviors point towards a volume law, which is expected for the case of randomly drawn tensors and reasonable for states generated from random superpositions. Surprisingly, changing the distributions from $w_{s}(x)$ to $w(x)$ in case of the random superpositions the volume law transforms into an area law with logarithmic corrections. It is not completely understood what drives this change but it would be reasonable to begin with a 


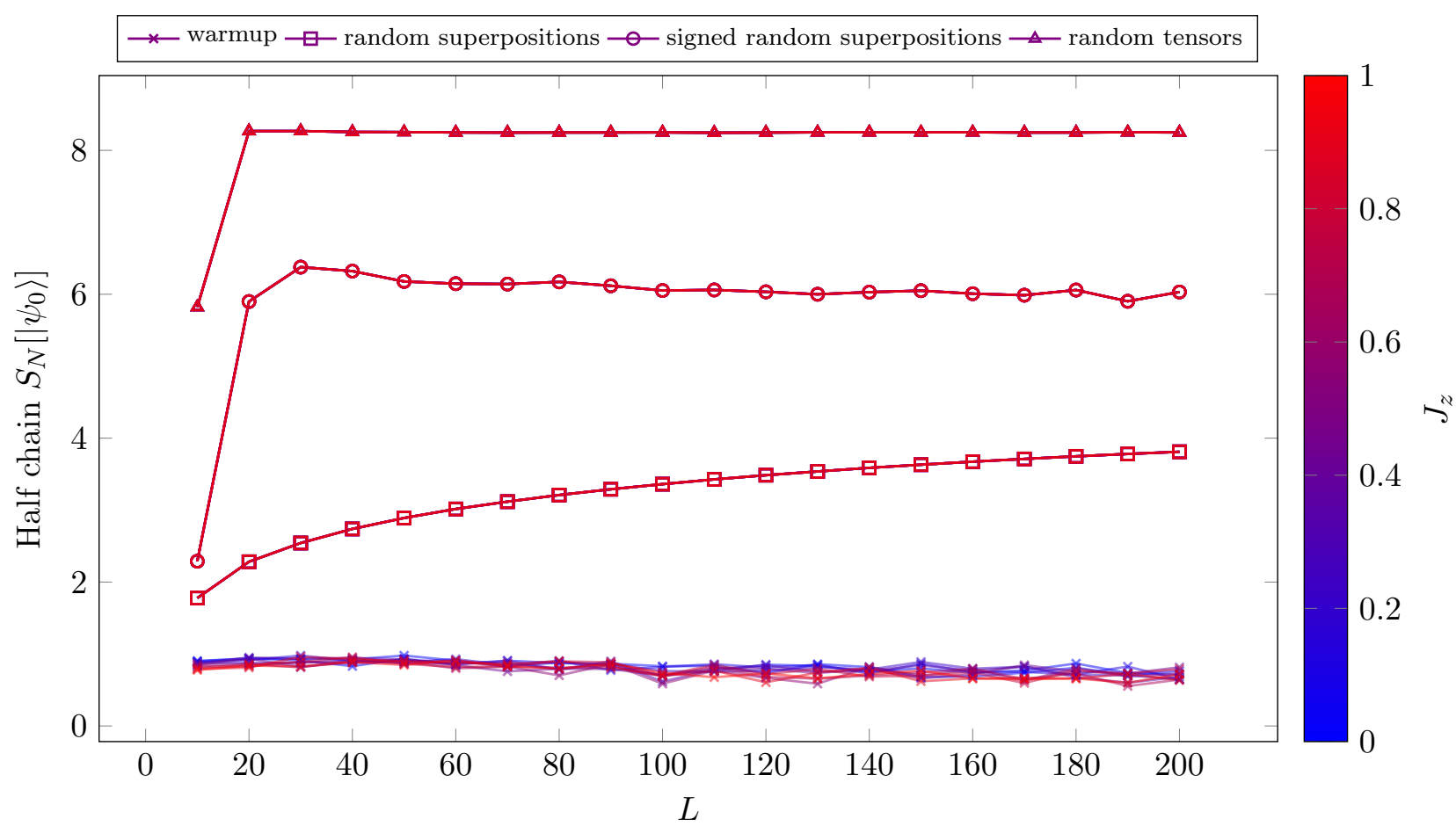

Figure 4.19: Half-chain entanglement entropy for the different guess states constructed from the methods described in the main text as a function of the system size $L$.

modified sampling probability distribution

$$
w_{\tau}(x)=\frac{1}{3} \delta(x)+\frac{2 \tau}{3} \delta(1-x)+\frac{2(1-\tau)}{3} \delta(1+x),
$$

and see how $S_{N}$ behaves when $\tau$ is tuned from $\tau=1$ (corresponding to a sampling from $w(x)$ ) to $\tau=1 / 2$ (corresponding to a sampling from $w_{s}(x)$ ). However, we leave this question open for investigations of a motivated reader.

Strictly single-site DMRG The better convergence properties of 2DMRG are paid with more expensive tensor contractions compared to 1DMRG. The asymptotic scalings are $\mathcal{O}\left(m^{3} w \sigma^{k}\right)$ for $k$ active site-tensors. In particular when considering local Hilbert spaces with "larger" local dimension such as $\sigma=4$ in case of Spin- $1 / 2$ fermions, it would be highly desirable to avoid the additional factor of $\sigma$ in 2DMRG. An elegant solution is the so-called subspace expansion by Hubig et al. [HMSW15], which was inspired by an extension of 1DMRG introduced by White [Whi05]. In this approach the obstacle of a static bond dimension during the sweep steps in 1DMRG is overcome by increasing the bond space dimension artificially. For this purpose, in case of a sweep from the left to the right the optimized site tensor $M_{j}$ is replaced by an expanded tensor

$$
M_{j}^{\sigma_{j}} \rightarrow \tilde{M}_{j}^{\sigma_{j}}=\left(\begin{array}{cc}
M_{j}^{\sigma_{j}} & P_{j}^{\sigma_{j}}
\end{array}\right),
$$

with a perturbation tensor $P_{j}$ that has dimensions $\left(\sigma, m_{j-1}, m_{j}^{p}\right)$. Accordingly, the next site tensor to the right is expanded properly so that the overall state content remains unchanged

$$
B_{j+1}^{\sigma_{j+1}} \rightarrow \tilde{B}_{j+1}^{\sigma_{j+1}}=\left(\begin{array}{c}
B_{j+1}^{\sigma_{j+1}} \\
0
\end{array}\right)
$$


where the block dimension of the tensor 0 is given by $\left(m_{j}^{p}, m_{j+1}\right)$. The coice of the perturbation is crucial for the convergence properties of this so- called enrichment step. Hubig et al. suggested to construct $P_{j}=\alpha L_{j-1} M_{j} W_{j}$ merging the auxiliary bonds: $P_{j}=P_{j ; m_{j-1},\left(w_{j}, m_{j}\right)}^{\sigma_{j}}$. Here, $\alpha$ is a convergence parameter that controls the degree of the perturbation induced by $P_{j}$. Large values of $\alpha \sim \mathcal{O}(1)$ are strongly perturbing the optimized site tensor $M_{j}$ reducing convergence speed of the overall algorithm. Therefore, a careful adaption of $\alpha$ throughout the sweep is necessary to obtain optimal convergence towards the ground state that requires $\alpha \rightarrow 0$, eventually. A subsequent canonization of the expanded site tensor $\tilde{M}_{j}$ truncates the increased auxiliary bond dimension and completes the expansion procedure. The convergence properties of the strictly single-site densitymatrix renormalization group (DMRG3S) are superior to the simple implementation of 1DMRG. In particular, the chance that the algorithm gets stuck in a local minimum is reduced drastically compared to $1 \mathrm{DMRG}$ while the overall computation time can be reduced asymptotically by a factor of $\sigma$ compared to 2DMRG.

\section{\begin{tabular}{l|l} 
Time-evolution methods & 4.5
\end{tabular}}

We already saw the power of MPS representations in the previous sections in which we described equilibrium algorithms for quantum many-body systems. But MPS would have not become the de-facto standard in low-dimensional condensed matter physics if their applicability was restricted to equilibrium setups only. In fact, their mathematical structure allows to formulate a number of different approaches to perform time evolutions. They can be distinguished by either i) constructing an MPO representation of the time-evolution operator $\hat{U}(\delta)$ or ii) evaluating its action and directly obtain an approximation of a state $|\psi(t)\rangle$ being evolved by a time step $\delta$ : $|\psi(t+\delta)\rangle=\hat{U}(\delta)|\psi(t)\rangle$. Even though we preferably used a method of the second kind for the calculations done in the context of this thesis, in the following we will also present a method of the first kind. However, this is not for pedagogical reasons only. Each toolbox providing time-evolution methods for MPS should contain implementations of both methods since they complement each other and thus can be used to check consistency of obtained results.

Historically, the first approaches were formulated in the framework of the old-style DMRG [WF04, FW05, MMN05, GR06] around 2004/2005. Another important contribution was made by Vidal [Vid03] formulating a time-evolution scheme by means of a Suzuki-Trotter decomposition [Suz90] of the time-evolution operator already exploiting a particular MPS representation. This so-called time-evolution block-decimation (TEBD) algorithm was the first time-evolution scheme for MPS and adapted to a DMRG formulation by Daley et al. [DKSV04]. However, in its original formulation TEBD was presented for systems with couplings between nearest neighbors only. And even though in the MPS representation a generalization to longer-ranged couplings is straight forward by introducing swap gates, it became clear very quickly that the method is technically much more involved in its DMRG formulation. Conceptually TEBD belongs to the time-evolution methods of the first kind, that is it constructs an MPO representation of $\hat{U}(\delta)$. Remembering the DMRG representation of wavefunction sketched in Sec. 4.4.2 it becomes clear that such operator-based approximations of the time-evolution operator are naturally harder to realize within the old-style DMRG for couplings ranging beyong nearest neighbors. Therefore, time-evolution schemes of the second kind, i.e., based on approximating the action of $\hat{U}(\delta)$ on the wavefunctions directly, typically were the methods of choice. One such developement was the adaptive tDMRG based on the time-step targeting DMRG [FW05, MMN05, GR06, RMR ${ }^{+} 06$, RLJHC17]. We have reformulated this method in terms of a MPS representation $\left[\mathrm{PKS}^{+} 19\right]$ and surprisingly found an intimite relation to one of the most successful time-evolution schemes developed for MPS: the 
time-dependent variational principle (TDVP) algorithm $\left[\mathrm{HCO}^{+} 11, \mathrm{HLO}^{+} 16\right]$.

In the following we will introduce and discuss three methods to perform time-evolution of quantum-many body systems out-of equilibrium: The $W^{\mathrm{I}, \mathrm{II}}$ method (first kind), the local Krylov method and TDVP (both of the second kind). We will restrict ourselves to discussing situations in which the Hamiltonian is time-independent. However, time-dependency can be incorporated into these schemes by disretizing the time axis over the desired interval $t \in[0, T]$ into $N \in \mathbb{N}$ steps and choosing the step size $\delta=T / N$ in such a way that the time-dependent Hamiltonian $\hat{H}(t)$ is nearly constant throughout the interval $[t, t+\delta]$. The particular choice of the methods described is based on the authors contribution to the publication $\left[\mathrm{PKS}^{+} 19\right]$ and the fact that for the numerical calculations mostly a hybrid TDVP time-evolution scheme was used. The content is based on the sections The MPO $W^{\mathrm{I}, \mathrm{II}}$ method, The local Krylov method and The time-dependent variational principle (TDVP) of the author's publication [ $\left.\mathrm{PKS}^{+} 19\right]$.

\section{The MPO $W^{\mathrm{I}, \mathrm{II}}$ method $\mid 4.5 .1$}

Approximations of the operator exponential $\hat{U}(\delta)=e^{-\mathrm{i} \hat{\delta} H}$ have been under investigation for a long time. One of the first approaches to obtain a MPO representation was exploiting a SuzukiTrotter decomposition [Suz76, Suz90], which to first and second order is given via

$$
\begin{aligned}
\text { First order: } & e^{-\mathrm{i} \delta \hat{H}}=e^{-\mathrm{i} \delta \hat{H}_{1}} e^{-\mathrm{i} \delta \hat{H}_{2}}+\mathcal{O}\left(\delta^{2}\right) \\
\text { Second order: } & e^{-\mathrm{i} \delta \hat{H}}=e^{-\mathrm{i} \delta / 2 \hat{H}_{2}} e^{-\mathrm{i} \delta / 2 \hat{H}_{1}} e^{-\mathrm{i} \delta / 2 \hat{H}_{2}}+\mathcal{O}\left(\delta^{3}\right),
\end{aligned}
$$

with a decomposition $\hat{H}=\hat{H}_{1}+\hat{H}_{2}$ of the system's Hamiltonian. Note that the order of the expansion refers to the overall error accumulated after evolving $T=N \delta$ time steps. For instance, after propagating the initial state $|\psi(t)\rangle N$ times towards the approximated final state $|\psi(t+T)\rangle$ by means of the first order Suzuki-Trotter decomposition, the overall error scales as $\mathcal{O}\left(\delta^{2}\right)^{T} / \delta \sim \mathcal{O}(\delta)$. Before we discuss the $W^{\mathrm{I}, \mathrm{II}}$ representation, which also exploits a Suzuki-Trotter decomposition it is instructive to recapitulate the TEBD construction scheme as it creates a good intuition on how to deal with the problem of decomposing operators on the many-body Hilbert space so that operator exponentials can be constructed. It has been noted by Vidal [Vid03] that for a Hamiltonian containing only nearest neighbor couplings, i.e., $\hat{H}$ can be decomposed into terms $\hat{H}=\sum_{j} \hat{h}_{j}$ acting on no more then a single bond $(j, j+1)$, there is a decomposition of $\hat{H}$ that creates an easily accessible MPO representation. The idea is to chose the decomposition in such a way that $\hat{H}_{1}$ and $\hat{H}_{2}$ consist only of summands that commute with each other. For the case of only nearest neighbors coupling this is achieved by defining $\hat{h}_{e, j}, \hat{h}_{o, j}$ to be the operators acting only on the even/odd bond $(j, j+1)$ so that we decompose the Hamiltonian as

$$
\hat{H}_{1}=\sum_{j \text { even }} \hat{h}_{e, j}, \quad \hat{H}_{2}=\sum_{j \text { odd }} \hat{h}_{o, j}
$$

Since $\left[\hat{h}_{e, j}, \hat{h}_{e, k}\right]=\left[\hat{h}_{o, j}, \hat{h}_{o, k}\right]=0$ the MPO bond dimensions fulfill $w_{j+1}=1$ for the operators $\hat{h}_{e, j}, \hat{h}_{o, j}$ and thus the exponentials are factorizing

$$
e^{-\mathrm{i} \delta \hat{H}_{1}}=\prod_{j \text { even }} e^{-\mathrm{i} \delta \hat{h}_{e, j}}, \quad e^{-\mathrm{i} \delta \hat{H}_{2}}=\prod_{j \text { odd }} e^{-\mathrm{i} \delta \hat{h}_{o, j}}
$$


This way, each exponential $e^{-\mathrm{i} \delta \hat{h}_{e / o, j}}$ is given by a matrix of dimension $\sigma^{2} \times \sigma^{2}$, which can be calculated easily. A factorization in the basis of adjacent sites

$$
\left\langle\sigma_{j} \sigma_{j+1}\left|e^{-\mathrm{i} \delta \hat{h}_{e / o, j}}\right| \sigma_{j}^{\prime} \sigma_{j+1}^{\prime}\right\rangle=\sum_{w_{j}} W_{j ; 1, w_{j}}^{\sigma_{j}, \sigma_{j}^{\prime}} W_{j+1 ; w_{j}, 1}^{\sigma_{j+1}, \sigma_{j+1}^{\prime}}
$$

then yields the desired MPO representation. Pursuing this idea we can also approximate longerranged couplings by either extending the decomposition scheme to include more sites and thereby decompose the Hamiltonian into more summands, or applying swap gates to deform the lattice so that couplings are local. But it should be clear by now that both approaches come with either an increased implementational or computational effort so that for generic and efficient codes alternatives to TEBD are desired.

In Ref. $\left[\mathrm{ZMK}^{+} 15\right]$ Zaletel et al. proposed a generalization of the Euler approximation of the operator exponential $e^{-\mathrm{i} \delta \hat{H}}$, which can be implemented efficiently using MPOs. In this scheme, the error per site is independent of the system size. Furthermore, the construction is capable of dealing with long-ranged interaction terms making it suitable for two-dimensional systems, too. In this section, we will sketch the derivation of the MPO representations $W^{\mathrm{I}, \mathrm{II}}$ and discuss how to construct these operators as well as the numerical errors and the stability of the overall method.

\section{Motivation and construction}

The general idea is to exploit the intrinsic factorization of MPO representations of operators. We consider operators that have a local structure in the sense that they are given by a sum of terms $\hat{H}_{j}$ acting on a subset of the lattice, starting at site $j: \hat{H}=\sum_{j} \hat{H}_{j}$. The Euler approximation of the operator exponential is $e^{-\mathrm{i} \delta \hat{H}}=1-\mathrm{i} \delta \sum_{j} \hat{H}_{j}+\mathcal{O}\left(\delta^{2}\right)$ and since there are $\sim L^{2}$ contributions from all possible combinations of local terms with finite support the error per site is $\sim L \delta^{2}$; hence the approximation becomes more and more unstable with increasing system size. Ref. [ZMK $\left.{ }^{+} 15\right]$ introduced the following local version of the Euler stepper

$$
\begin{aligned}
e^{-\mathrm{i} \hat{H} \delta} & =1-\mathrm{i} \delta \sum_{j} \hat{H}_{j}-\delta^{2} \sum_{j<k}^{\prime} \hat{H}_{j} \hat{H}_{k}+\mathcal{O}\left(L \delta^{2}\right)+\mathcal{O}\left(\delta^{3}\right) \\
& \approx \prod_{j}\left(1-\mathrm{i} \delta \hat{H}_{j}\right) \equiv \hat{U}^{\mathrm{I}}(\delta)
\end{aligned}
$$

where the primed sum indicates that the local operator terms $\hat{H}_{j}, \hat{H}_{k}$ do not act on a common subset of the lattice, i.e., they do not overlap. Even though the error of $\hat{U}^{\mathrm{I}}$ is still of order $\delta^{2}$ in the step size there are only $\mathcal{O}(L)$ contributions that are missed, namely those combinations of local terms with overlapping support. Hence, the overall error is bounded by $\mathcal{O}\left(L \delta^{2}\right)$, and thus the error per site is constant in the system size.

Recall now the decomposition of an MPO into a left, right and local part

$$
\hat{H}=\hat{H}_{j-1}^{L} \otimes \hat{\mathbf{1}}_{j}^{R}+\hat{\mathbf{1}}_{j}^{L} \otimes \hat{H}_{j+1}^{R}+\sum_{a_{j}=1}^{N_{j}} \hat{h}_{j ; a_{j}}^{L} \otimes \hat{h}_{j ; a_{j}}^{R} .
$$


with $N_{j}$ interaction terms crossing bond $j$, or equivalently:

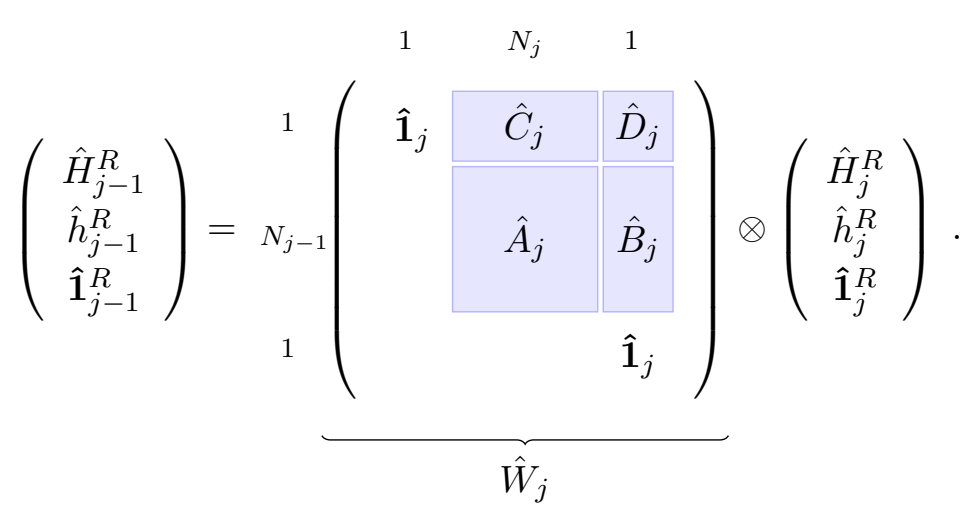

The operator-valued matrices $\hat{A}_{j}, \hat{B}_{j}, \hat{C}_{j}, \hat{D}_{j}$ specify the local structure of the interactions described by $\hat{H}$ and the set of all matrices $\hat{W}_{1} \ldots \hat{W}_{L}$ define the MPO representation of $\hat{H}$. The MPO representation $W^{\mathrm{I}}$ of $\hat{U}^{\mathrm{I}}$ is given by

$$
\hat{W}_{j}^{\mathrm{I}}=\left(\begin{array}{c|c|}
\hline \hat{\mathbf{1}}_{j}+\delta \hat{D}_{j} & \sqrt{\delta} \hat{C}_{j} \\
\hline \sqrt{\delta} \hat{B}_{j} & \hat{A}_{j} \\
&
\end{array}\right) .
$$

In this case the representation of $\hat{H}$ as finite state machine is particularly useful since it permits to directly deduce the MPO representation for $W^{\mathrm{I}}$. The MPO bond dimension of $W^{\mathrm{I}}$ is $w-1$ with $w$ the bond dimension of $\hat{H}$. Hence, this MPO can be applied numerically very efficiently. However, the restriction of $W^{\mathrm{I}}$ to treat only non-overlapping local operator terms $\hat{H}_{j}$ is very strong and fails to even reproduce the correct time evolution of a purely on-site Hamiltonian. For example, $W^{\mathrm{I}}$ for $\hat{H}=\sum_{j} \hat{s}_{j}^{z}$ generates only operator strings $\hat{s}_{k}^{z} \cdots \hat{s}_{k+n}^{z}$ but not $\hat{s}_{k}^{z} \hat{s}_{k}^{z}$. An improvement is to permit operator strings that overlap on one site:

$$
\hat{U}^{\mathrm{II}}(\delta)=1-\mathrm{i} \delta \sum_{j} \hat{H}_{j}-\frac{\delta^{2}}{2} \sum_{j, k}^{\prime \prime} \hat{H}_{j} \hat{H}_{k}+\cdots,
$$

where the double-primed sum only excludes terms $\hat{H}_{j}, \hat{H}_{k}$ overlapping at more than one site, sharing a bond. For instance, consider the expansion of the time-evolution operator for the $S=1 / 2$-Heisenberg chain: expressions of the form $\left(\hat{s}_{j}^{+} \hat{s}_{j+1}^{-}\right)\left(\hat{s}_{j+1}^{z}\right)$ are kept, while those of the form $\left(\hat{s}_{j}^{+} \hat{s}_{j+1}^{-}\right)\left(\hat{s}_{j}^{z} \hat{s}_{j+1}^{z}\right)$ are discarded. Hence, the error is again of order $\delta^{2}$ but contributions with arbitrary powers of single-site terms are treated exactly. There is no closed general MPO representation for $\hat{U}^{\mathrm{II}}$ but we can give an approximation that has an error $\mathcal{O}\left(\delta^{3}\right)$ and hence does not affect the second-order approximation of $\hat{U}^{\mathrm{II}}$. In the following, we will first explicitly demonstrate how to numerically construct the MPO representation $W^{\mathrm{II}}$ from the block-triangular structure of the MPO representation of $\hat{H}$ and subsequently motivate the used formalism. As for $\hat{U}^{\mathrm{I}}$, the MPO representation of $\hat{U}^{\mathrm{II}}$ is of the form

$$
\hat{W}_{j}^{\mathrm{II}}=\left(\begin{array}{cc}
\hat{W}_{D_{j}}^{\mathrm{II}} & \hat{W}_{C_{j}}^{\mathrm{II}} \\
\hat{W}_{B_{j}}^{\mathrm{II}} & \hat{W}_{A_{j}}^{\mathrm{II}}
\end{array}\right) .
$$


In order to construct the matrices $W_{\left\{A_{j}, B_{j}, C_{j}, D_{j}\right\}}^{\mathrm{II}}$ we employ transition amplitudes between hardcore bosonic states following the notation in Ref. [ZMK $\left.{ }^{+} 15\right]$. Let $\mathcal{H}_{2, a_{j}}$ denote the $a_{j}$-th hardcore bosonic Hilbert space. $N_{j}$ of these spaces form $\mathcal{H}_{c}=\bigotimes_{a_{j}=1}^{N_{j}} \mathcal{H}_{2, a_{j}}$ and we work in the joint Hilbert space $\mathcal{H}_{c} \otimes \mathcal{H}_{\bar{c}}$, which spans $2 N_{j}$ individual hard-core bosonic Hilbert spaces. The ladder operators acting on $\mathcal{H}_{c}$ and $\mathcal{H}_{\bar{c}}$ are $\hat{c}_{a_{j}}^{\dagger}, \hat{c}_{a_{j}}, \hat{\bar{c}}_{\bar{a}_{j}}^{\dagger}$ and $\hat{\bar{c}}_{\bar{a}_{j}}$ respectively.

The generator of the matrix elements is a map on the joint bosonic and physical Hilbert spaces $\mathcal{H}_{c} \otimes \mathcal{H}_{\bar{c}} \otimes \mathcal{H}_{\text {phys }}$ and given by the operator-valued exponentials

$$
\hat{\Phi}_{j ; a_{j}, \bar{a}_{j}}=e^{\hat{F}_{j ; a_{j}, \bar{a}_{j}}}=e^{\hat{c}_{a_{j}}^{\dagger} \hat{c}_{\bar{a}_{j}}^{\dagger} \hat{A}_{j ; a_{j}, \bar{a}_{j}}+\sqrt{\delta}\left(\hat{c}_{a_{j}}^{\dagger} \hat{B}_{j ; a_{j}}+\hat{\bar{c}}_{\bar{a}_{j}}^{\dagger} \hat{C}_{j ; \bar{a}_{j}}\right)+\delta \hat{D}_{j}}
$$

Denoting the combined bosonic vacuum state by $|0\rangle \otimes|\overline{0}\rangle \equiv|0, \overline{0}\rangle$ the following transition amplitudes determine the operator-valued entries of the MPO representation $\hat{W}_{j}^{\mathrm{II}}$ :

$$
\begin{aligned}
\hat{W}_{A_{j} ; a_{j}, \bar{a}_{j}}^{\mathrm{II}} & =\left\langle 0, \overline{0}\left|\hat{c}_{a_{j}} \hat{\bar{c}}_{\bar{a}_{j}} \hat{\Phi}_{j ; a_{j}, \bar{a}_{j}}\right| 0, \overline{0}\right\rangle=\left\langle 0\left|\hat{c}_{a_{j}} \hat{\bar{c}}_{\bar{a}_{j}} e^{\hat{c}_{a_{j}}^{\dagger} \hat{\bar{c}}_{\bar{a}_{j}}^{\dagger} \hat{A}_{j ; a_{j}, \bar{a}_{j}}+\sqrt{\delta}\left(\hat{c}_{a_{j}}^{\dagger} \hat{B}_{j ; a_{j}}+\hat{\bar{c}}_{\bar{a}_{j}}^{\dagger} \hat{c}_{j ; \bar{a}_{j}}\right)+\delta \hat{D}_{j}}\right| 0\right\rangle \\
\hat{W}_{B_{j} ; a_{j}}^{\mathrm{II}} & =\left\langle 0, \overline{0}\left|\hat{c}_{a_{j}} \hat{\Phi}_{j ; a_{j}, \bar{a}_{j}}\right| 0, \overline{0}\right\rangle=\left\langle 0\left|\hat{c}_{a_{j}} e^{\sqrt{\delta} \hat{c}_{a_{j}}^{\dagger} \hat{B}_{j ; a_{j}}+\delta \hat{D}_{j}}\right| 0\right\rangle \\
\hat{W}_{C_{j} ; \bar{a}_{j}}^{\mathrm{II}} & =\left\langle 0, \overline{0}\left|\hat{\bar{c}}_{\bar{a}_{j}} \hat{\Phi}_{j ; a_{j}, \bar{a}_{j}}\right| 0, \overline{0}\right\rangle=\left\langle\overline{0}\left|\hat{\bar{c}}_{\bar{a}_{j}} e^{\sqrt{\delta} \hat{\bar{c}}_{\bar{c}_{j}}^{\dagger} \hat{c}_{j ; \bar{a}_{j}}+\delta \hat{D}_{j}}\right| \overline{0}\right\rangle \\
\hat{W}_{D_{j}}^{\mathrm{II}} & =\left\langle 0, \overline{0}\left|\hat{\Phi}_{j ; a_{j}, \bar{a}_{j}}\right| 0, \overline{0}\right\rangle=e^{\delta \hat{D}_{j}}
\end{aligned}
$$

For clarity, in the following we will explicitly demonstrate the calculation of the elements of $W_{A_{j} ; a_{j}, \bar{a}_{j}}^{\mathrm{II}} \cdot$ We use

$$
|0\rangle_{a_{j}}=\left(\begin{array}{l}
1 \\
0
\end{array}\right) \in \mathcal{H}_{2, a_{j}}, \quad \hat{c}_{a_{j}}=\left(\begin{array}{ll}
0 & 1 \\
0 & 0
\end{array}\right), \quad \hat{c}_{a_{j}}^{\dagger}=\left(\begin{array}{ll}
0 & 0 \\
1 & 0
\end{array}\right), \quad \hat{\mathbf{1}}_{a_{j}}=\left(\begin{array}{ll}
1 & 0 \\
0 & 1
\end{array}\right),
$$

to represent the hard-core bosonic states and operators. Since $\hat{F}_{j ; a_{j}, \bar{a}_{j}}$ only contains creation operators for the modes $a_{j}, \bar{a}_{j}$ the vacuum expectation value over all $2 N_{j}$ bosonic modes can be simplified to the relevant modes, i.e., we can replace $|0, \overline{0}\rangle \rightarrow\left|0_{a_{j}}, \overline{0}_{\bar{a}_{j}}\right\rangle$. Therefore, the generator is obtained by exponentiating the matrix

$$
\begin{aligned}
\hat{F}_{j ; a_{j}, \bar{a}_{j}}= & \hat{c}_{a_{j}}^{\dagger} \otimes \hat{\bar{c}}_{\bar{a}_{j}}^{\dagger} \hat{A}_{j ; a_{j}, \bar{a}_{j}}+\sqrt{\delta}\left(\hat{c}_{a_{j}}^{\dagger} \otimes \hat{\mathbf{1}}_{\bar{a}_{j}} \hat{B}_{j ; a_{j}}+\hat{\mathbf{1}}_{a_{j}} \otimes \hat{\bar{c}}_{\bar{a}_{j}}^{\dagger} \hat{C}_{j ; \bar{a}_{j}}\right)+\hat{\mathbf{1}}_{a_{j}} \otimes \hat{\mathbf{1}}_{\bar{a}_{j}} \delta \hat{D}_{j} \\
= & \left(\begin{array}{cccc}
\delta \hat{D}_{j} & 0 & 0 & 0 \\
\sqrt{\delta} \hat{C}_{j ; \bar{a}_{j}} & \delta \hat{D}_{j} & 0 & 0 \\
\sqrt{\delta} \hat{B}_{j ; a_{j}} & 0 & \delta \hat{D}_{j} & 0 \\
\hat{A}_{j ; a_{j}, \bar{a}_{j}} & \sqrt{\delta} \hat{B}_{j ; a_{j}} & \sqrt{\delta} \hat{C}_{j ; \bar{a}_{j}} & \delta \hat{D}_{j}
\end{array}\right),
\end{aligned}
$$

which has been truncated to the relevant bosonic Hilbert spaces $\mathcal{H}_{2, a_{j}}, \mathcal{H}_{2, \bar{a}_{j}}$. The entries of $\hat{W}_{A_{j} ; a_{j}, \bar{a}_{j}}^{\mathrm{II}}$ are then obtained by evaluating the power series of the matrix exponential and calculating the vacuum expectation value

$$
\begin{aligned}
\hat{W}_{A_{j} ; a_{j}, \bar{a}_{j}}^{\mathrm{II}} & =\left\langle 0, \overline{0}\left|\hat{c}_{a_{j}} \hat{\bar{c}}_{\bar{a}_{j}} \hat{\Phi}_{j ; a_{j}, \bar{a}_{j}}\right| 0, \overline{0}\right\rangle=\left\langle 1_{a_{j}}, \overline{1}_{\bar{a}_{j}}\left|\hat{\Phi}_{j ; a_{j}, \bar{a}_{j}}\right| 0, \overline{0}\right\rangle \\
& =\left(\begin{array}{cccc}
0 & 0 & 0 & 1
\end{array}\right) \exp \left\{\left(\begin{array}{cccc}
\delta \hat{D}_{j} & 0 & 0 & 0 \\
\sqrt{\delta} \hat{C}_{j ; \bar{a}_{j}} & \delta \hat{D}_{j} & 0 & 0 \\
\sqrt{\delta} \hat{B}_{j ; a_{j}} & 0 & \delta \hat{D}_{j} & 0 \\
\hat{A}_{j ; a_{j}, \bar{a}_{j}} & \sqrt{\delta} \hat{B}_{j ; a_{j}} & \sqrt{\delta} \hat{C}_{j ; \bar{a}_{j}} & \delta \hat{D}_{j}
\end{array}\right)\right\}\left(\begin{array}{l}
1 \\
0 \\
0 \\
0
\end{array}\right) .
\end{aligned}
$$


A compact notation for all matrix elements of $W^{\mathrm{II}}$ can be obtained if we let the annihilation operators $\hat{c}_{a_{j}}, \hat{c}_{\bar{a}_{j}}$ in Eqs. (4.87) to (4.90) act on the bra $\langle 0, \overline{0}|$ and define the formal symbol $\mathcal{S}_{j} \in\left\{A_{j}, B_{j}, C_{j}, D_{j}\right\}$

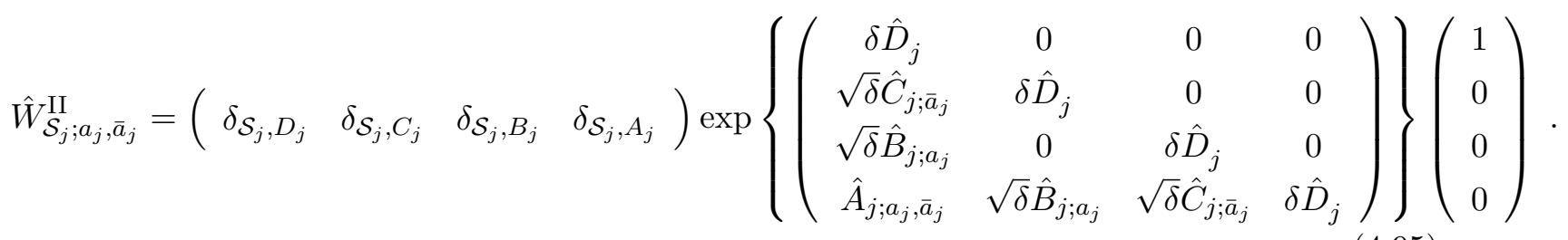

Hence, the $N_{j}^{2}$ exponentials $\exp \left\{\hat{F}_{j ; a_{j}, \bar{a}_{j}}\right\}$ already contain all relevant information to construct the stepper $W^{\mathrm{II}}$ and in particular there is no need to calculate different exponentials as suggested by Eq. (4.87) - Eq. (4.90).

\section{Detailed derivation of the $W^{\mathrm{II}}$ representation}

We will present the construction of $W^{\mathrm{II}}$ in more detail, following Ref. [ZMK $\left.{ }^{+} 15\right]$, in this section. Before digging into the details, let us briefly sketch the derivation: The goal is to find an MPO representation of the time stepper $\hat{U}^{\mathrm{II}}(\delta)$ capturing interaction terms in the series expansion as described above. The corresponding MPO site tensors $W^{\mathrm{II}}$ are obtained by making use of the MPO recursion Eq. (4.82) to factorize the exponential $e^{-\mathrm{i} \delta \hat{H}}$. The factorization itself is performed exploiting complex Gaussian integrals via the introduction of auxiliary fields $\phi_{j}, \bar{\phi}_{j}$ on each bond that is integrated over. The resulting MPO is of the form

$$
W^{\mathrm{II}}=\int D\left[\phi_{1}, \bar{\phi}_{1}\right] U_{\bar{\phi}_{1}} e^{-\bar{\phi}_{1} \phi_{1}} \int D\left[\phi_{2}, \bar{\phi}_{2}\right] U_{\phi_{1}, \bar{\phi}_{2}} e^{-\bar{\phi}_{2} \phi_{2}} U_{\phi_{2}, \bar{\phi}_{3}} \cdots
$$

that is, the bond indices $\phi_{j}, \bar{\phi}_{j}$ are continuous degrees of freedom. In a final step, these bond indices are discretized using bosonic coherent state path integrals yielding the desired expression for the MPO site tensors.

We begin by employing the bond representation of $\hat{H}$ (compare Eq. (4.81)) and write the operator as a formal scalar product over auxiliary degrees of freedom $a_{j}=1 \ldots N_{j}$, where $N_{j}$ again is the number of interaction terms crossing the bond $j$,

$$
\begin{aligned}
\hat{H} & =\hat{H}_{j-1}^{L} \otimes \hat{\mathbf{1}}_{j}^{R}+\hat{\mathbf{1}}_{j}^{L} \otimes \hat{H}_{j+1}^{R}+\left(\begin{array}{lll}
\hat{h}_{j ; 1}^{L} & \ldots & \hat{h}_{j ; N_{j}}^{L}
\end{array}\right)\left(\begin{array}{c}
\hat{h}_{j ; 1}^{R} \\
\vdots \\
\hat{h}_{j ; N_{j}}^{R}
\end{array}\right) \\
& \equiv \hat{H}_{j-1}^{L} \otimes \hat{\mathbf{1}}_{j}^{R}+\hat{\mathbf{1}}_{j}^{L} \otimes \hat{H}_{j+1}^{R}+\hat{J}_{j}^{t} \hat{\bar{J}}_{j}
\end{aligned}
$$

and the product between the entries of the operator-valued "vectors" $\hat{J}_{j}$ and $\hat{\bar{J}}_{j}$ are tensor products between operators acting on partitioned Hilbert spaces to the left and right of the bond $j$, respectively. Next, we introduce complex vector fields $\phi_{j ; a_{j}}$ and their complex conjugate $\bar{\phi}_{j ; a_{j}}$ 
and define a mapping from the auxiliary indices into the full Hilbert space

$$
\begin{aligned}
\hat{J}_{j}^{t} \cdot \bar{\phi}_{j}: \quad\left(\mathcal{H}_{j}^{L} \rightarrow \mathcal{H}_{j}^{L}\right)^{\otimes N_{j}} \times \mathbb{C}^{N_{j}} & \longrightarrow(\mathcal{H} \rightarrow \mathcal{H}) \\
\left(\hat{J}_{j}, \bar{\phi}_{j}\right) & \longmapsto \hat{J}_{j}^{t} \cdot \bar{\phi}_{j}:=\hat{h}_{j ; 1}^{L} \bar{\phi}_{j ; 1} \hat{\mathbf{1}}_{j}^{R}+\cdots+\hat{h}_{j ; N_{j}}^{L} \bar{\phi}_{j ; N_{j}} \hat{\mathbf{1}}_{j}^{R} \\
\phi_{j} \cdot \hat{\bar{J}}_{j}: \quad \mathbb{C}^{N_{j}} \times\left(\mathcal{H}_{j}^{R} \rightarrow \mathcal{H}_{j}^{R}\right)^{\otimes N_{j}} & \longrightarrow(\mathcal{H} \rightarrow \mathcal{H}) \\
\left(\phi_{j}, \hat{\bar{J}}_{j}\right) & \longmapsto \phi_{j} \cdot \hat{\bar{J}}_{j}:=\hat{\mathbf{1}}_{j}^{L} \phi_{j ; 1} \hat{h}_{j ; 1}^{R}+\cdots+\hat{\mathbf{1}}_{j}^{L} \phi_{j ; N_{j}} \hat{h}_{j ; N_{j}}^{R},
\end{aligned}
$$

where we will suppress the identities acting on the left and right partitions of the Hilbert space in the following. We then use complex Gaussian integrals to decouple each left and right interaction term

$$
e^{\hat{J}_{j ; a_{j}} \hat{\bar{J}}_{j ; a_{j}}}=\frac{1}{\pi} \int d \phi_{j ; a_{j}} d \bar{\phi}_{j ; a_{j}} e^{-\bar{\phi}_{j ; a_{j}} \cdot \phi_{j ; a_{j}}+\hat{J}_{j ; a_{j}} \cdot \bar{\phi}_{j ; a_{j}}+\phi_{j ; a_{j}} \cdot \hat{\bar{J}}_{j ; a_{j}}}
$$

Furthermore, we factorize the operator exponential $e^{-\mathrm{i} \delta \hat{H}}(\operatorname{setting} \tau=-\mathrm{i} \delta$ )

$$
\begin{aligned}
e^{\tau \hat{H}} & =e^{\tau\left(\hat{H}_{j-1}^{L} \otimes \hat{\mathbf{1}}_{j}^{R}+\hat{\mathbf{1}}_{j}^{L} \otimes \hat{H}_{j+1}^{R}+\hat{J}_{j}^{t} \hat{\bar{J}}_{j}\right)} \\
& =e^{\tau \hat{H}_{j-1}^{L} \otimes \hat{\mathbf{1}}_{j}^{R}} e^{\tau \hat{\mathbf{1}}_{j}^{L} \otimes \hat{H}_{j+1}^{R}} e^{\tau \hat{J}_{j}^{t} \hat{\bar{J}}_{j}}+\mathcal{O}\left(\tau^{2}\right)
\end{aligned}
$$

where the error occurs at second order in $\tau$ if the operators $\hat{J}_{j}, \hat{\bar{J}}_{j}, \hat{H}_{j-1}^{L}, \hat{H}_{j+1}^{R}$ do not commute. ${ }^{15}$ Exploiting $N_{j}$ complex Gaussian integrals and defining $D\left[\phi_{j}, \bar{\phi}_{j}\right]=\prod_{a_{j}} \frac{d \phi_{j ; a_{j}}}{\sqrt{\pi}} \frac{d \bar{\phi}_{j ; a_{j}}}{\sqrt{\pi}}$ we obtain

$$
\begin{aligned}
e^{\tau \hat{H}} & =\int D\left[\phi_{j}, \bar{\phi}_{j}\right] e^{\tau \hat{H}_{j-1}^{L} \otimes \hat{\mathbf{1}}_{j}^{R}} e^{\tau \hat{\mathbf{1}}_{j}^{L} \otimes \hat{H}_{j+1}^{R}} e^{-\bar{\phi}_{j} \cdot \phi_{j}+\sqrt{\tau} \hat{J}_{j} \cdot \bar{\phi}_{j}+\sqrt{\tau} \phi_{j} \cdot \hat{\bar{J}}_{j}}+\mathcal{O}\left(\tau^{2}\right) \\
& =\int D\left[\phi_{j}, \bar{\phi}_{j}\right] e^{\tau \hat{H}_{j-1}^{L} \cdot \hat{\mathbf{1}}_{j}^{R}+\sqrt{\tau} \hat{J}_{j} \cdot \bar{\phi}_{j}} e^{-\bar{\phi}_{j} \cdot \phi_{j}} e^{\tau \hat{\mathbf{1}}_{j}^{L} \cdot \hat{H}_{j+1}^{R}+\sqrt{\tau} \phi_{j} \cdot \hat{J}_{j}}+\mathcal{O}\left(\tau^{2}\right) .
\end{aligned}
$$

Here we used that the identities $\hat{\mathbf{1}}_{j}^{L}$ and $\hat{\mathbf{1}}_{j}^{R}$ can also be interpreted as maps from an auxiliary index into the full Hilbert space.

Now the MPO recursion Eq. (4.82) can be applied to, e.g., shift the active bond $j \rightarrow j+1$ in the third exponential

$$
e^{\tau \hat{\mathbf{1}}_{j}^{L} \cdot \hat{H}_{j+1}^{R}+\sqrt{\tau} \phi_{j} \hat{\bar{J}}_{j}}=e^{\tau \hat{\mathbf{1}}_{j} \cdot \hat{H}_{j+1}^{R}+\sqrt{\tau} \phi_{j} \cdot \hat{A}_{j} \hat{\bar{J}}_{j+1}+\sqrt{\tau} \phi_{j} \cdot \hat{B}_{j} \hat{\mathbf{1}}_{j+1}^{R}+\tau \hat{C}_{j} \hat{\bar{J}}_{j+1}+\tau \hat{D}_{j} \cdot \hat{\mathbf{1}}_{j+1}^{R}}
$$

From now on we replace the formal dot product $\hat{D}_{j} \cdot \hat{\mathbf{1}}_{j+1}^{R}$ by its action on the full Hilbert space, which reduces for the case of $\hat{D}_{j}$ to on-site terms only. The same holds for the dot product $\phi_{j} \cdot \hat{B}_{j} \hat{\mathbf{1}}_{j+1}^{R} \equiv \phi_{j} \cdot \hat{B}_{j}$, which only connects sites to the left of $j$. In the exponent the summands $\sqrt{\tau} \phi_{j} \cdot \hat{B}_{j} \cdot \hat{\mathbf{1}}_{j+1}^{R}, \tau \hat{D}_{j} \cdot \hat{\mathbf{1}}_{j+1}^{R}$ and $\tau \hat{\mathbf{1}}_{j} \cdot \hat{H}_{j+1}^{R}$ already act separately on site $j$ and the right partition of the Hilbert space $\mathcal{H}_{j+1}^{R}$. In order to separate the remaining summands, too, we introduce another set of auxiliary fields $\phi_{j+1}, \bar{\phi}_{j+1}$ by insertion of more complex Gaussian integrals

$$
\begin{aligned}
& e^{\sqrt{\tau} \phi_{j} \cdot \hat{A}_{j} \hat{\bar{J}}_{j+1}+\tau \hat{C}_{j} \cdot \hat{\bar{J}}_{j+1}}=\int D\left[\phi_{j+1}, \bar{\phi}_{j+1}\right] e^{-\bar{\phi}_{j+1} \phi_{j+1}} e^{\phi_{j} \cdot \hat{A}_{j} \cdot \bar{\phi}_{j+1}+\sqrt{\tau} \hat{C}_{j} \cdot \bar{\phi}_{j+1}+\sqrt{\tau} \phi_{j+1} \cdot \hat{\bar{J}}_{j+1}} \\
\Rightarrow & e^{\tau \hat{\mathbf{1}}_{j}^{L} \cdot \hat{H}_{j+1}^{R}+\sqrt{\tau} \phi_{j} \cdot \hat{\bar{J}}_{j}}=\int D\left[\phi_{j+1}, \bar{\phi}_{j+1}\right] U_{\phi_{j}, \bar{\phi}_{j+1}} e^{-\bar{\phi}_{j+1} \phi_{j+1}} e^{\tau \hat{\mathbf{1}}_{j+1}^{L} \cdot \hat{H}_{j+2}^{R}+\sqrt{\tau} \phi_{j+1} \cdot \hat{\bar{J}}_{j+1}}
\end{aligned}
$$

\footnotetext{
${ }^{15}$ The commutators need to be evaluated on the full Hilbert space by completing the partitioned operators $\hat{J}_{j}, \hat{\bar{J}}_{j}, \hat{H}_{j-1}^{L}, \hat{H}_{j+1}^{R}$, which is achieved by taking the appropriate tensor products with $\hat{\mathbf{1}}_{j}^{L}$ and $\hat{\mathbf{1}}_{j}^{R}$.
} 
with $U_{\phi_{j}, \bar{\phi}_{j+1}}=e^{\phi_{j} \cdot \hat{A}_{j} \cdot \bar{\phi}_{j+1}+\sqrt{\tau} \phi_{j} \cdot \hat{B}_{j}+\sqrt{\tau} \hat{C}_{j} \cdot \bar{\phi}_{j+1}+\tau \hat{D}_{j}}$. The last equation defines a recursion

$$
e^{\tau \hat{\mathbf{1}}_{j}^{L} \cdot \hat{H}_{j+1}^{R}+\sqrt{\tau} \phi_{j} \cdot \hat{\bar{J}}_{j}}=\mathcal{F}_{j, j+1}\left[e^{\tau \hat{\mathbf{1}}_{j+1}^{L} \cdot \hat{H}_{j+2}^{R}+\sqrt{\tau} \phi_{j+1} \cdot \hat{\bar{J}}_{j+1}}\right]
$$

generated by the integration of $U_{\phi_{j}, \bar{\phi}_{j+1}} e^{-\bar{\phi}_{j+1} \phi_{j+1}}$ over the continuous bond degrees of freedom $\phi_{j}, \bar{\phi}_{j+1}$. Iterating through the whole system we finally obtain

$$
e^{\tau \hat{H}}=\int D\left[\phi_{1}, \bar{\phi}_{1}\right] U_{\bar{\phi}_{1}} e^{-\bar{\phi}_{1} \phi_{1}} \int D\left[\phi_{2}, \bar{\phi}_{2}\right] U_{\phi_{1}, \bar{\phi}_{2}} e^{-\bar{\phi}_{2} \phi_{2}} U_{\phi_{2}, \bar{\phi}_{3}} \cdots+\mathcal{O}\left(\tau^{2}\right),
$$

which is a first-order approximation in $\tau$ of $e^{\tau \hat{H}}$ in terms of an MPO with continuous bond labels $\phi_{j}=\left(\phi_{1}, \cdots, \phi_{N_{j}}\right)$.

In a final step the continuous bond labels $\phi_{a_{j}}$ are transformed into discrete ones. We note that for a set of $N_{j}$ bosonic ladder operators $\hat{c}_{a_{j}}, \hat{c}_{a_{j}}^{\dagger}$ we can rewrite the tensors at site $j$ as expectation values for coherent states

$$
\left|\phi_{a_{j}}\right\rangle=\sum_{n} \frac{\phi_{a_{j}}^{n}}{n !}\left(\hat{c}_{a_{j}}^{\dagger}\right)^{n}|0\rangle
$$

which have the properties

$$
\hat{c}_{a_{j}}\left|\phi_{a_{j}}\right\rangle=\phi_{a_{j}}\left|\phi_{a_{j}}\right\rangle, \quad\left\langle\phi_{a_{j}}\right| \hat{c}_{a_{j}}^{\dagger}=\left\langle\phi_{a_{j}}\right| \bar{\phi}_{a_{j}}, \quad\left\langle\phi_{a_{j}} \mid \phi_{a_{j}}\right\rangle=e^{\bar{\phi}_{a_{j}} \phi_{a_{j}}} .
$$

For instance, we may consider the case when $N_{j} \equiv 1$. Then a single bond label $\phi_{j ; a_{j}} \equiv z_{j}$ is sufficient and (abbreviating $\hat{c}_{a_{j}} \equiv \hat{c}_{j}, \hat{c}_{a_{j}}^{\dagger} \equiv \hat{c}_{j}^{\dagger}$ ) we have at the bond $(j, j+1)$

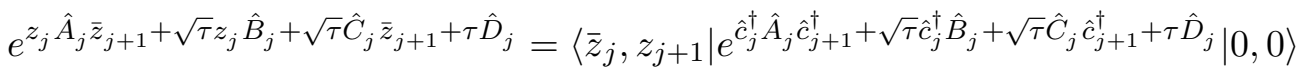

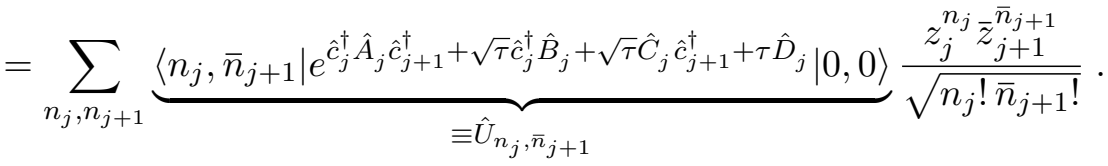

In the last equality we expanded the coherent states $\left|z_{j}\right\rangle,\left|z_{j+1}\right\rangle$ in the bosonic occupations number basis $\left|n_{j}\right\rangle,\left|\bar{n}_{j+1}\right\rangle$. Now, we turn to the integral over one pair of bond labels $z_{j}, \bar{z}_{j}$, considering only those factors $\frac{z_{j}^{k_{j}}}{\sqrt{k_{j} !}}$ that contribute to the integration,

$$
\begin{aligned}
\int \frac{d z_{j} d \bar{z}_{j}}{\pi} \hat{U}_{z_{j-1}, \bar{z}_{j}} e^{-\bar{z}_{j} z_{j}} \hat{U}_{z_{j}, \bar{z}_{j+1}} & =\sum_{n_{j}, \bar{n}_{j}} \int \frac{d z_{j} d \bar{z}_{j}}{\pi} \hat{U}_{n_{j-1}, \bar{n}_{j}} e^{-\bar{z}_{j} z_{j}} \hat{U}_{n_{j}, \bar{n}_{j+1}} \frac{\bar{z}_{j}^{\bar{n}_{j}} z_{j}^{n_{j}}}{\sqrt{\bar{n}_{j} ! n_{j} !}} \\
& =\sum_{n_{j}, \bar{n}_{j}} \hat{U}_{n_{j-1}, \bar{n}_{j}} \hat{U}_{n_{j}, \bar{n}_{j+1}} \int_{0}^{\infty} d \rho_{j} \frac{\rho_{j}^{\bar{n}_{j}+n_{j}+1}}{\sqrt{\bar{n}_{j} ! n_{j} !}} e^{-\rho_{j}^{2}} \int_{0}^{2 \pi} \frac{d \varphi_{j}}{\pi} e^{\mathrm{i} \varphi_{j}\left(n_{j}-\bar{n}_{j}\right)} \\
& =\sum_{n_{j}} \hat{U}_{n_{j-1}, n_{j}} \hat{U}_{n_{j}, \bar{n}_{j+1}} \frac{2}{n_{j} !} \underbrace{\int_{0}^{\infty} d \rho \rho^{2 n_{j}+1} e^{-\rho^{2}}}_{=\frac{1}{2} \Gamma\left(n_{j}\right)} \\
& =\sum_{n_{j}} \hat{U}_{n_{j-1}, n_{j}} \hat{U}_{n_{j}, \bar{n}_{j+1}} .
\end{aligned}
$$


We parametrized the complex integration in polar coordinates in the second identity and exploited the representation of the $\Gamma$ function in the last line. For the general case $N_{j}>1$ we define the boson occupation number $\mathbf{n}_{j}=\left(n_{1} \cdots n_{N_{j}}\right)$ for each bond. Since we can always reorder the $\phi_{a_{j}}$, we can perform the same integrals for each continuous bond label so that we finally find

$$
\hat{U}(\delta)=\sum_{\mathbf{n}_{1} \cdots \mathbf{n}_{L}} \hat{U}_{\overline{\mathbf{n}}_{1}} \hat{U}_{\mathbf{n}_{1}, \overline{\mathbf{n}}_{2}} \cdots+\mathcal{O}\left(\delta^{2}\right),
$$

yielding the desired MPO representation.

A pedestrian way to obtain a compact MPO representation from this very formal derivation is to analyze $\hat{U}_{\mathbf{n}_{j-1}, \overline{\mathbf{n}}_{j}}$ when truncating the bosonic Hilbert spaces to a maximal boson occupation $b$, i.e., $n_{j ; a_{j}} \in\{0, \ldots, b\}$. We define bosonic field operators $\hat{\varphi}_{j}^{\dagger}=\left(\hat{c}_{1}^{\dagger} \cdots \hat{c}_{L}^{\dagger}\right)$ and denote the maximal occupation number by an upper index $(b)$. Then, for each $b$ we have operator-valued bosonic matrix elements

$$
\hat{U}_{j ; \mathbf{n}_{j}, \overline{\mathbf{n}}_{j+1}}^{(b)}=\left\langle\mathbf{n}_{j}, \overline{\mathbf{n}}_{j+1}|\underbrace{\underbrace{\hat{\varphi}_{j}^{\dagger} \cdot \hat{A}_{j} \cdot \hat{\bar{\varphi}}_{j+1}^{\dagger}+\sqrt{\tau} \hat{\varphi}_{j}^{\dagger} \cdot \hat{B}_{j}+\sqrt{\tau} \hat{C}_{j} \cdot \hat{\varphi}_{j+1}^{\dagger}+\tau \hat{D}_{j}}}_{\equiv \hat{\Phi}_{j}}| \mathbf{0}, \overline{\mathbf{0}}\right\rangle
$$

of maps $\hat{\Phi}_{j}$ on the joint Hilbert spaces of $b$ bosons $\mathcal{H}_{b ; a_{j}}$ for every $a_{j} \in\left\{1, \ldots, N_{j}\right\}$ at each bond and the physical Hilbert space $\mathcal{H}_{\text {phys }}$ at each site

$$
\hat{\Phi}_{j}: \bigotimes_{a_{j}} \mathcal{H}_{b ; a_{j}} \otimes \bigotimes_{\bar{a}_{j}} \mathcal{H}_{\bar{b} ; \bar{a}_{j}} \otimes \mathcal{H}_{\mathrm{phys}} \longrightarrow \bigotimes_{a_{j}} \mathcal{H}_{b ; a_{j}} \otimes \bigotimes_{\bar{a}_{j}} \mathcal{H}_{\bar{b} ; \bar{a}_{j}} \otimes \mathcal{H}_{\mathrm{phys}}
$$

Let us consider $b \equiv 0$, then the matrix elements collapse to pure on-site terms $\hat{U}_{j ; \mathbf{0}, \overline{\mathbf{0}}}^{(0)}=\left\langle\mathbf{0}, \overline{\mathbf{0}}\left|\hat{\Phi}_{j}\right| \mathbf{0}, \overline{\mathbf{0}}\right\rangle=$ $e^{\tau \hat{D}_{j}}$. The next order $b \equiv 1$ additionally collects contributions from all interactions crossing either the bond $(j-1, j)$ or $(j, j+1)$

$\hat{U}_{j ; \mathbf{0}, \overline{\mathbf{0}}}^{(1)}=\left\langle\mathbf{0}, \overline{\mathbf{0}}\left|\hat{\Phi}_{j}\right| \mathbf{0}, \overline{\mathbf{0}}\right\rangle, \quad \hat{U}_{j ; \mathbf{0}, \overline{\mathbf{1}}}^{(1)}=\left\langle\mathbf{0}, \overline{\mathbf{1}}\left|\hat{\Phi}_{j}\right| \mathbf{0}, \overline{\mathbf{0}}\right\rangle, \quad \hat{U}_{j ; \mathbf{1}, \overline{\mathbf{0}}}^{(1)}=\left\langle\mathbf{1}, \overline{\mathbf{0}}\left|\hat{\Phi}_{j}\right| \mathbf{0}, \overline{\mathbf{0}}\right\rangle, \quad \hat{U}_{j ; \mathbf{1}, \overline{\mathbf{1}}}^{(1)}=\left\langle\mathbf{1}, \overline{\mathbf{1}}\left|\hat{\Phi}_{j}\right| \mathbf{0}, \overline{\mathbf{0}}\right\rangle$,

which can be arranged as an operator-valued matrix

$$
\hat{U}_{j}^{(1)}=\left(\begin{array}{cc}
\left\langle\mathbf{0}, \overline{\mathbf{0}}\left|\hat{\Phi}_{j}\right| \mathbf{0}, \overline{\mathbf{0}}\right\rangle & \left\langle\mathbf{0}, \overline{\mathbf{1}}\left|\hat{\Phi}_{j}\right| \mathbf{0}, \overline{\mathbf{0}}\right\rangle \\
\left\langle\mathbf{1}, \overline{\mathbf{0}}\left|\hat{\Phi}_{j}\right| \mathbf{0}, \overline{\mathbf{0}}\right\rangle & \left\langle\mathbf{1}, \overline{\mathbf{1}}\left|\hat{\Phi}_{j}\right| \mathbf{0}, \overline{\mathbf{0}}\right\rangle
\end{array}\right) \equiv\left(\begin{array}{cc}
\hat{W}_{D_{j}}^{\mathrm{II}} & \hat{W}_{C_{j}}^{\mathrm{II}} \\
\hat{W}_{B_{j}}^{\mathrm{II}} & \hat{W}_{A_{j}}^{\mathrm{II}}
\end{array}\right) \equiv \hat{W}_{j}^{\mathrm{II}}
$$

and yields the suggested form of $W^{\mathrm{II}}$. The lower right matrix element $\hat{W}_{A_{j}}^{\mathrm{II}}$ contains two bosons. When formally contracting $\hat{U}_{j}^{(1)}$ with the neighboring matrix $\hat{U}_{j+1}^{(1)}$, these bosons connect, for instance, all local operators from $\hat{A}_{j}$ to local operators from $\hat{B}_{j+1}$. Following this procedure, we find that in this way truncating the MPO approximation to $b=1$ we keep all local operator strings that overlap at most at one site and hence the error is $\mathcal{O}\left(\tau^{2}\right)$.

\section{Errors}

The MPO $W^{\mathrm{II}}$ approximation to the time-evolution operator $\hat{U}(\delta)$ primarly exhibits an error $\mathcal{O}\left(\delta^{2}\right)$ due to the truncation of the auxiliary degrees to hard-core bosons with maximal occupation $b \equiv 1$. The Trotter error created by Eq. (4.102) was shown in Ref. [ZMK $\left.{ }^{+} 15\right]$ to be $\mathcal{O}\left(\tau^{3}\right)$ so that it is subleading compared to the auxiliary boson field truncation error. However, the $W^{\text {II }}$ 
MPO representation is not invariant under the particular choice of the decomposition of the Hamiltonian into local terms $\hat{H}=\sum_{j} \hat{H}_{j}$. This degree of freedom can be used to reduce the truncation error and is discussed in detail in Ref. [ZMK $\left.{ }^{+} 15\right]$. A further possibility to reduce the error per time step that is based on the fact that here we have an MPO representation of $\hat{U}(\delta)$ at hand is discussed in the next section.

\section{Complex time steps}

Let us assume that we have an approximation of the time-evolution operator that is exact to first order

$$
\hat{U}^{\prime}(\delta)=\hat{\mathbf{1}}-\mathrm{i} \delta \hat{H}+\sum_{k=2}^{\infty} \frac{\delta^{k}}{k !} \hat{U}_{k}^{\prime} \quad \text { with } \quad \hat{U}_{k}^{\prime}=\left.\frac{\partial^{k}}{\partial t^{k}} \hat{U}^{\prime}(t)\right|_{t=0},
$$

where we replaced the Taylor expansion coefficients $\hat{U}_{k}^{\prime}$ at orders $k=0,1$ with their exact expressions. Applying this operator to a state will result in an error $O\left(\delta^{2}\right)$ compared to the exact evolution with the operator $\hat{U}(\delta)=e^{-\mathrm{i} \delta \hat{H}}$. Repeating the process $T / \delta$ times to obtain a state at final time $T$, we incur an error $O\left(\delta^{2}\right)^{T} / \delta=O(\delta)$. However, if we allow complex intermediate steps $\delta_{1}$ and $\delta_{2}$, we can expand $\hat{U}(\delta)$ to second order and demand

$$
\hat{U}^{\prime}\left(\delta_{1}\right) \hat{U}^{\prime}\left(\delta_{2}\right)-\left(\hat{\mathbf{1}}-\mathrm{i} \delta \hat{H}-\frac{\delta^{2}}{2} \hat{H}^{2}\right) \stackrel{!}{\sim} \mathcal{O}\left(\delta^{3}\right) .
$$

Expanding the left-hand side:

$$
\left(\hat{\mathbf{1}}-\mathrm{i}\left(\delta_{1}+\delta_{2}\right) \hat{H}-\delta_{1} \delta_{2} \hat{H}^{2}-\frac{\delta_{1}^{2}+\delta_{2}^{2}}{2} \hat{U}_{2}^{\prime}\right)-\left(\hat{\mathbf{1}}-\mathrm{i} \delta \hat{H}-\frac{\delta^{2}}{2} \hat{H}^{2}\right)+\mathcal{O}\left(\delta^{3}\right) \sim \mathcal{O}\left(\delta^{3}\right)
$$

we can compare terms with the same order of the expansion to obtain conditions on $\delta_{1,2}$

$$
\delta=\delta_{1}+\delta_{2}, \quad \frac{\delta^{2}}{2}=\delta_{1} \delta_{2}, \quad 0=\delta_{1}^{2}+\delta_{2}^{2},
$$

so that Eq. (4.118) is fulfilled. Solving for $\delta_{1,2}$ we find

$$
\delta_{1}=\frac{1-\mathrm{i}}{2} \delta \wedge \delta_{2}=\frac{1+\mathrm{i}}{2} \delta .
$$

Choosing these values for $\delta_{1,2}$ then results in a third-order error per time step and a second-order error overall. The cost of the method only grows linearly with the number of evolution operators and, e.g., four operators $\hat{U}^{\prime}\left(\delta_{1,2,3,4}\right)$ are required for a third-order error [ZMK $\left.{ }^{+} 15\right]$. An improved stepper is hence available without too much numerical effort.

The drawback is the loss of unitarity at each individual time step, which may be disadvantageous. Furthermore, if the time evolution is purely imaginary (e.g., for finite-temperature calculations) and the Hamiltonian does not contain complex coefficients, one may avoid complex arithmetic entirely and only use real floating-point scalars for $50 \%$ less memory usage and an approximately four-fold speed-up on matrix multiplications. Unfortunately, it is then impossible to use this trick to reduce the time-step error.

\section{Approximating the action of $\hat{U}(\delta)$}

Given a Hamiltonian $\hat{H}$ and a state $|\psi(t)\rangle$ describing a system at time $t$ we can approximate the solution of the time-dependent Schrödinger equation only in a small time interval $[t, t+\delta]$. 
The goal is to find such an approximation in an optimal way, where there are different notions of optimal, without explicitely constructing the time-evolution operator $\hat{U}(\delta)$. For instance, let us denote a set of states parametrized by $t \in[t, t+\delta]$ as $|\tilde{\psi}(t)\rangle$. Then, we can formulate a condition for a $k$ th order approximation

$$
|\psi(t+\delta)\rangle-|\tilde{\psi}(t+\delta)\rangle \mid \stackrel{!}{\sim} \mathcal{O}\left(\delta^{k+1}\right),
$$

and by expanding both states

$$
\begin{aligned}
|\psi(t+\delta)\rangle & =|\psi(t)\rangle-\left.\mathrm{i} \delta \frac{\partial}{\partial \tau}|\psi(\tau)\rangle\right|_{\tau=t}+\cdots \\
|\tilde{\psi}(t+\delta)\rangle & =|\tilde{\psi}(t)\rangle-\left.\mathrm{i} \delta \frac{\partial}{\partial \tau}|\tilde{\psi}(\tau)\rangle\right|_{\tau=t}+\cdots
\end{aligned}
$$

it follows from inserting the Schrödinger equation in the first line that we need to find a parametrization $|\tilde{\psi}(t)\rangle$ in such a way that the first $k$ derivatives fulfill

$$
\left.\left|\hat{H}^{n}\right| \psi(t)\right\rangle-\left.\frac{\partial^{n}}{\partial \tau^{n}}|\tilde{\psi}(\tau)\rangle\right|_{\tau=t} \mid \stackrel{!}{=} 0, \quad n \in\{1, \cdots k\} .
$$

These considerations imply that projecting the Schrödinger equation into a Krylov space of dimension $k+1$

$$
\mathcal{K}=\operatorname{span}\left\{|\psi(t)\rangle, \hat{H}|\psi(t)\rangle, \ldots, \hat{H}^{k}|\psi(t)\rangle\right\},
$$

the desired approximation $|\tilde{\psi}(t+\delta)\rangle$ of order $k$ [HL97] is obtained by exponentiating only a $k \times k$ sparse matrix, which can be done numerically very easily. In fact, there is a straight forward way to implement an algorithm in terms of MPS/MPO representations, which basically consists of repeated applications of $\hat{H}$ and subsequent reorthogonalizations to construct $|\tilde{\psi}(t+\delta)\rangle\left[\mathrm{PKS}^{+} 19\right]$ (see Alg. 1 for a possible implementation). However, this approach comes with severe drawbacks when the states are represented as MPS with a fixed maximal bond dimension. This can be understood by noting that typically each application of $\hat{H}$ increases the entanglement of the resulting state by building up non-local correlations. Therefore, large truncation errors can occur while constructing the basis of the Krylov space. On the other hand, the exact timeevolved state $|\psi(t+\delta)\rangle$ typically exhibits only a very moderate growth of entanglement as long as the Hamiltonian governing the dynamics features only local interactions and $\delta \ll 1$. As a consequence, the approximated time-evolved state $|\tilde{\psi}(t+\delta)\rangle$ that is constructed by a global superposition of basis vectors in the Krylov space $\mathcal{K}$ inherits their truncation errors rendering the quality of the approximation much worse and also way less controlled than expected if exact arithmetics is used.

It is, hence, desirable to exploit the local structure of the MPS representation when approximating $|\psi(t+\delta)\rangle$. One way to proceed is to return to the notion of DMRG in which boundary tensors, appearing during the partial contraction of MPS-(MPO-)MPS networks, are interpreted as basis transformations. In a mixed-canonical representation with center of orthogonality at site $j$, these map a partition of the physical Hilbert space onto bond spaces of the auxiliary indices of the site tensor $M_{j}$. Pursuing this idea we can define projectors similar to the construction of these maps and decompose the Schrödinger equation under the action of these projectors to obtain LieTrotter decompositions. In the following, we discuss two such decomposition schemes: the local Krylov method and the time-dependent variational principle (TDVP). The local Krylov method merely is a reformulation of time-step targeting tDMRG in terms of MPS and can be seen as 


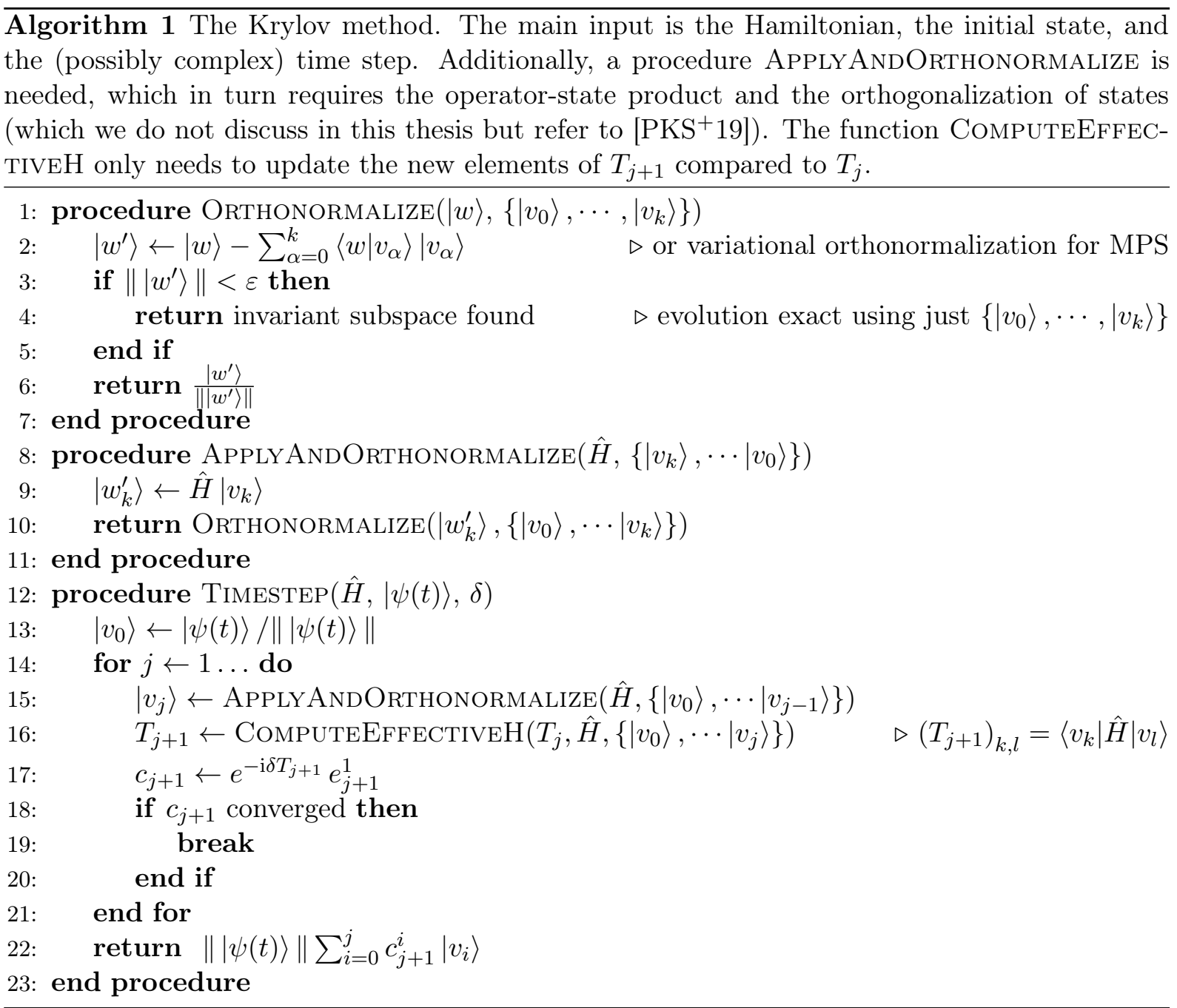

a precursor to TDVP introducing the idea of decomposing the global Schrödinger equation by means of projections into differential equations acting only on the local site-tensors. To the best of the authors knowledge, this reformulation of tDMRG was first introduced in $\left[\mathrm{PKS}^{+} 19\right]$ by the author so that it is going to be presented here. Thereafter, we discuss TDVP that can be motivated very naturally by analyzing and resolving a technical issue of the local Krylov method.

\section{Local Krylov $\mid 4.5 .3$}

As already mentioned, deriving a Lie-Trotter integration scheme while working in the local reduced spaces is equivalent to translating the time-step targeting DMRG [GR06, FW05, MMN05, $\mathrm{RMR}^{+}$06, RLJHC17] into the MPS framework. Crucially, the MPS framework makes it possible to precisely analyze the errors made, something, which would be very difficult in the standard environment-system DMRG picture. To integrate the local time-dependent Schrödinger equations resulting from the Lie-Trotter decomposition, we will use a Krylov-based approach [MMN05, $\mathrm{RMR}^{+}$06, GR06]. This approach has the advantage of a very precise solution of the local equations and a large degree of similarity with both ground-state search DMRG and the timedependent variational principle (cf. Sec. 4.5.4). Alternatively, Runge-Kutta integrators have also 
been used extensively with only minor changes to the overall method [FW05, RLJHC17]. In particular, the error analysis presented here is also valid for the Runge-Kutta integrator, though one of course also has to include the additional time-step error of this integrator.

This section introduces several important concepts in detail as they are also at the heart of the TDVP algorithm presented later. They are discussed before formulating the algorithm so that readers who are only interested in the actual implementation can skip the very technical considerations. Nevertheless, digging deep into the rabbit hole is worth it in this situation and yields a profound unterstanding of the important ideas and consequences of projections into the bond basis, which sets the fundaments to introduce new concepts such as the tangent space in the context of TDVP.

\section{Lie-Trotter decomposition}

Motivated by the numerically efficient recursive solution strategy in the ground-state search algorithm (c.f. Sec. 4.4.2) we seek a similar approach to iteratively integrate the time-dependent Schrödinger equation. Thus, the natural starting point is to look at the general Lie-Trotter decomposition of this first-order differential equation

$$
\mathrm{i} \frac{d}{d t}|\psi\rangle=\hat{H}|\psi\rangle \equiv \sum_{\nu=1}^{K} \hat{H}_{\nu}|\psi\rangle,
$$

for some $K>1$ Let us assume we have found such a decomposition of the Hamiltonian ${ }^{16}$. Then, a first order integration scheme can be formulated by integrating each differential equation in the interval $\left[t_{0}, t_{0}+\delta\right]$ separately by means of the following recursively connected initial value problems:

$$
\begin{aligned}
-\mathrm{i} \frac{d}{d t}\left|\psi^{\nu}(t)\right\rangle & =\hat{H}_{\nu}\left|\psi^{\nu}(t)\right\rangle \\
\text { and } \quad\left|\psi^{\nu}\left(t_{0}\right)\right\rangle & =\left|\psi^{\nu-1}\left(t_{0}+\delta\right)\right\rangle,
\end{aligned}
$$

with $\left|\psi^{1}\left(t_{0}\right)\right\rangle \equiv\left|\psi\left(t_{0}\right)\right\rangle$ and the approximated overall solution $\left|\psi\left(t_{0}+\delta\right)\right\rangle \equiv\left|\psi^{K}\left(t_{0}+\delta\right)\right\rangle$. It can be shown easily that this construction generates a first order approximation to the solution of the time-dependent Schrödinger equation by comparing to the formal Taylor expansion of the exact solution $|\psi(t+\delta)\rangle_{\text {exact }}$ (note that we replace $t_{0} \equiv t$ )

$$
\begin{aligned}
|\psi(t+\delta)\rangle_{\text {exact }} & =|\psi(t)\rangle-\mathrm{i} \delta \hat{H}|\psi(t)\rangle-\frac{\delta^{2}}{2} \hat{H}^{2}|\psi(t)\rangle+\cdots \\
\left|\psi^{\nu}(t+\delta)\right\rangle & =\left|\psi^{\nu}(t)\right\rangle+\delta \frac{d}{d t}\left|\psi^{\nu}(t)\right\rangle+\frac{\delta^{2}}{2} \frac{d^{2}}{d t^{2}}\left|\psi^{\nu}(t)\right\rangle+\cdots \\
& =\left|\psi^{\nu-1}(t+\delta)\right\rangle-\mathrm{i} \delta \hat{H}_{\nu}\left|\psi^{\nu}(t)\right\rangle-\frac{\delta^{2}}{2} \hat{H}_{\nu} \hat{H}_{\nu}\left|\psi^{\nu}(t)\right\rangle+\cdots \\
\Rightarrow|\psi(t+\delta)\rangle & =|\psi(t)\rangle-\mathrm{i} \delta \hat{H}|\psi(t)\rangle-\frac{\delta^{2}}{2} \sum_{\mu \leq \nu} \hat{H}_{\mu} \hat{H}_{\nu}|\psi(t)\rangle \cdots
\end{aligned}
$$

where we have inserted Eq. (4.128) into the expansion of the partially time-evolved intermediate state $\left|\psi^{\nu}(t+\delta)\right\rangle$. Replacing $\left|\psi^{\nu-1}(t+\delta)\right\rangle$ by its Taylor expansion generates a recursion for the partially time-evolved states. Applying this recursion $K$ times and using the initial condition

\footnotetext{
${ }^{16}$ In fact, in Sec. 4.5.1 we have already used such a decomposition by splitting the Hamiltonian into terms acting on even and odd bonds only
} 


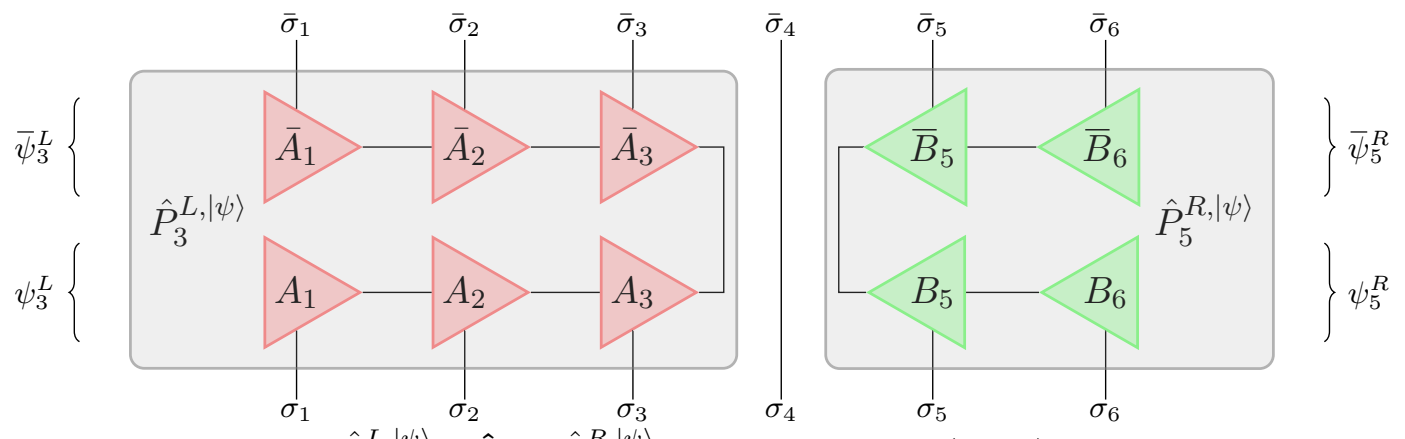

Figure 4.20: Projector $\hat{P}_{3}^{L,|\psi\rangle} \otimes \hat{\mathbf{1}}_{4} \otimes \hat{P}_{5}^{R,|\psi\rangle}$ as defined in Eq. (4.137) and also used in the TDVP projector Eq. (4.180), here at the example of a six-site system. The other terms in Eq. (4.180) are constructed correspondingly. This graphic is adopted from the author's publication [PKS $\left.{ }^{+} 19\right]$.

$\left|\psi^{1}(t)\right\rangle=|\psi(t)\rangle$ yields the last equation. As expected, $\left|\psi^{K}(t+\delta)\right\rangle$ coincides with the exact solution up to the first order of the expansion. The first errors occur at second-order terms in the expansion and are given by

$$
|\psi(t+\delta)\rangle_{\text {exact }}-|\psi(t+\delta)\rangle=\frac{\delta^{2}}{2} \sum_{\mu<\nu}\left[\hat{H}_{\mu}, \hat{H}_{\nu}\right]|\psi(t)\rangle
$$

and further commutators at arbitrary high orders. Clearly, solving the $K$ independent problems iteratively is already reminiscent of the procedure we have seen in the ground-state search algorithm. The question remains how to choose the decomposition in a way such that each individual problem takes benefit of the MPS representation. In order to answer this we need to learn a bit more about the gauge invariance of MPS and how the different representations can be mapped into each other by means of projection operators.

\section{Projection operators}

We define orthogonal projectors $\hat{P}_{j}^{L,|\psi\rangle}$ and $\hat{P}_{j}^{R,|\psi\rangle}$ acting on the physical degrees of freedom in a partition of the Hilbert space. For that purpose we introduce bipartitions $\mathcal{H}=\mathcal{H}_{j}^{L} \otimes \mathcal{H}_{j+1}^{R}$ where $\mathcal{H}_{j}^{L}=\mathcal{H}_{1} \otimes \cdots \otimes \mathcal{H}_{j}$ and $\mathcal{H}_{j+1}^{R}=\mathcal{H}_{j+1} \otimes \cdots \otimes \mathcal{H}_{L}$ and declare

$$
\begin{aligned}
& \hat{P}_{j}^{L,|\psi\rangle}: \mathcal{H}_{j}^{L} \otimes \mathcal{H}_{j+1}^{R} \longrightarrow \mathcal{H}_{j}^{L} \otimes \mathcal{H}_{j+1}^{R} \\
& \hat{P}_{j}^{L,|\psi\rangle}=\sum_{\substack{\sigma_{1}, \ldots, \sigma_{1}, \ldots, \bar{\sigma}_{j}, m_{j}}} \underbrace{A_{1} \cdots A_{j}}_{\equiv \psi_{j ; m_{j}}^{L}} \underbrace{\bar{A}_{j} \cdots \bar{A}_{1}}_{\equiv \bar{\psi}_{j ; m_{j}}^{L}}\left|\sigma_{1} \cdots \sigma_{j}\right\rangle\left\langle\bar{\sigma}_{1} \cdots \bar{\sigma}_{j}\right| \otimes \hat{\mathbf{1}}_{j+1}^{R} \\
& \hat{P}_{j}^{R,|\psi\rangle}: \mathcal{H}_{j-1}^{L} \otimes \mathcal{H}_{j}^{R} \longrightarrow \mathcal{H}_{j-1}^{L} \otimes \mathcal{H}_{j}^{R} \\
& \hat{P}_{j}^{R,|\psi\rangle}=\hat{\mathbf{1}}_{j-1}^{L} \otimes \sum_{\substack{\sigma_{j}, \ldots, \sigma_{L} \\
\bar{\sigma}_{j}, \ldots, \bar{\sigma}_{L}, \sum_{j}, \bar{\psi}_{j ; m_{j-1}}^{R}}}^{\mathcal{B}_{m_{j-1}}^{L} \cdots \bar{B}_{j}} \underbrace{B_{j} \cdots B_{L}}_{\equiv \psi_{j ; m_{j-1}}^{R}}\left|\sigma_{j} \cdots \sigma_{L}\right\rangle\left\langle\bar{\sigma}_{j} \cdots \bar{\sigma}_{L}\right|
\end{aligned}
$$

with mappings $\psi_{j ; m_{j}}^{L}$ from a part of the physical Hilbert space into the bond space $m_{j}$. By construction, these operators fulfill $\left(\hat{P}_{j}^{L / R,|\psi\rangle}\right)^{2}=\hat{P}_{j}^{L / R,|\psi\rangle}$ and $\left(\hat{P}_{j}^{L / R,|\psi\rangle}\right)^{\dagger}=\hat{P}_{j}^{L / R,|\psi\rangle}$, i.e., they are projectors. They are explicitly constructed from left-/right orthogonalized MPS site 


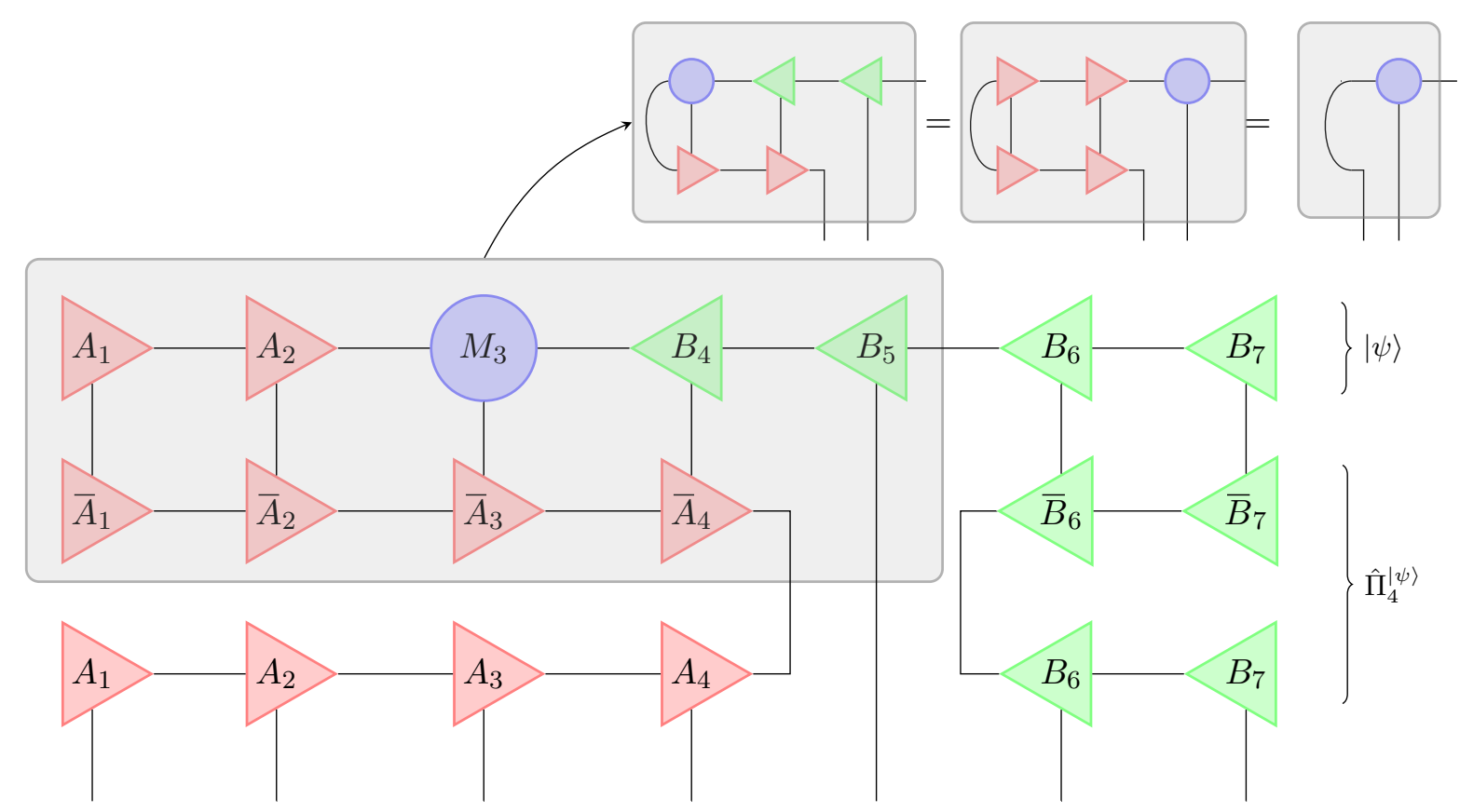

Figure 4.21: Shifting the center of orthogonality from $3 \rightarrow 5$ under the action of $\hat{\Pi}_{5}^{|\psi\rangle}|\psi\rangle=\hat{P}_{4}^{L,|\psi\rangle} \otimes$ $\hat{\mathbf{1}}_{5} \otimes \hat{P}_{6}^{R,|\psi\rangle}|\psi\rangle$. The orthogonality center is implicitly shifted to site 5 (without changing the state content, c.f. gray boxes), which gives identities on sites 1 through 4 and sites 6 and 7 and a new orthogonality center tensor $M_{5}$. The completely contracted upper two rows then define the new tensor $M_{5}$. This graphic is adopted from the author's publication $\left[\mathrm{PKS}^{+} 19\right]$.

tensors. The action of such projectors onto an MPS representation of $|\psi\rangle$ in canonical form with orthogonality center at site $j+1(j-1)$ is then given via

$$
\begin{aligned}
& \hat{P}_{j}^{L,|\psi\rangle}|\psi\rangle=\sum_{\substack{\sigma_{1}, \ldots, \sigma_{j}, \sigma_{1}^{\prime}, \ldots, \sigma_{j}^{\prime}, \bar{\sigma}_{1}^{\prime}, \ldots, \bar{\sigma}_{j}^{\prime}, m_{1}, \ldots, m_{j}, \bar{m}_{j}^{\prime}}} \psi_{j ; m_{j}^{\prime}}^{L ; \sigma_{1}^{\prime}, \ldots, \sigma_{j}^{\prime}} \underbrace{\bar{\psi}_{j ; \bar{m}_{j}^{\prime}, \ldots, \bar{\sigma}_{j}^{\prime}}^{L ;} A_{1 ; m_{1}}^{\sigma_{1}} \cdots A_{j ; m_{j-1}, m_{j}}^{\sigma_{j}}}_{=\delta_{\bar{m}_{j}^{\prime}, m_{j}} \delta_{\bar{\sigma}_{1}^{\prime}, \sigma_{1}} \cdots \delta_{\bar{\sigma}_{j}^{\prime}, \sigma_{j}}} \sum_{\sigma_{j+1}, \ldots, \sigma_{L}} M_{j+1} B_{j+2} \cdots B_{L}\left|\sigma_{1} \cdots \sigma_{L}\right\rangle \\
& =\sum_{\sigma_{1}, \ldots, \sigma_{L}} A_{1} \cdots A_{j} M_{j+1} B_{j+2} \cdots B_{L}\left|\sigma_{1} \cdots \sigma_{L}\right\rangle=|\psi\rangle
\end{aligned}
$$

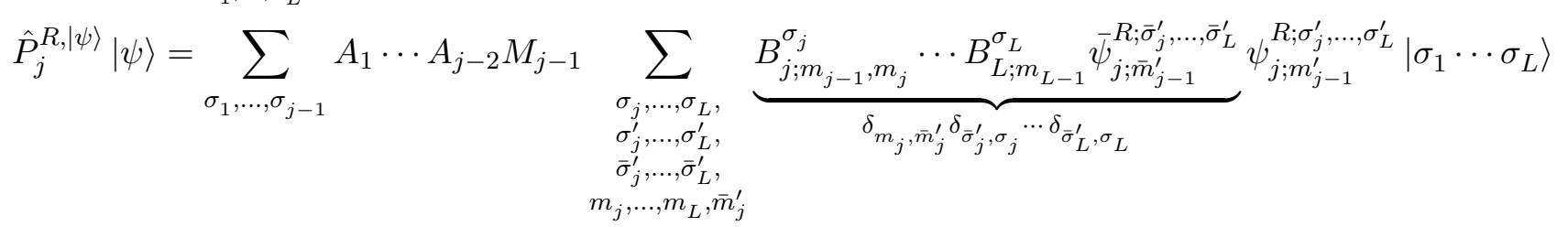

$$
\begin{aligned}
& =\sum_{\sigma_{1} \cdots \sigma_{L}} A_{1} \cdots A_{j-2} M_{j-1} B_{j} \cdots B_{L}\left|\sigma_{1} \cdots \sigma_{L}\right\rangle=|\psi\rangle .
\end{aligned}
$$

That is, if the state $|\psi\rangle$ has orthogonality center to the right (left) of the target index $j$, the projectors constructed from it act as an identity on their Hilbert space partition. Next we define the projector on the reduced site-space at site $j$ (cf. Fig. 4.20) via

$$
\hat{\Pi}_{j}^{|\psi\rangle} \equiv \hat{P}_{j-1}^{L,|\psi\rangle} \otimes \hat{\mathbf{1}}_{j} \otimes \hat{P}_{j+1}^{R,|\psi\rangle}
$$


The action of such a projector $\hat{\Pi}_{j}^{|\psi\rangle}$ on a state $|\psi\rangle$ is to shift the orthogonality center of $|\psi\rangle$ to the site $j$, which can be shown by applying the manipulation depicted in Fig. 4.21 on to $|\psi\rangle$ repeatedly. Therein gauge invariance is employed so that the action of the projector is trivial on the site tensors $A_{k} / B_{k}, k \neq j$ rendering $j$ the center of orthogonality. Thus, the quantum state $|\psi\rangle$ remains unchanged under the action of $\hat{\Pi}_{j}^{|\psi\rangle}$ and therefore

$$
\left\langle\phi\left|\hat{\Pi}_{j}^{|\psi\rangle}\right| \psi\right\rangle=\langle\phi \mid \psi\rangle
$$

The last expression implies an instructive way to think of $\hat{\Pi}_{j}^{|\psi\rangle}$ as an operator acting on both the physical and gauge degrees of freedom of the MPS representation. In the physical system it acts as a projector on the physical indices $\sigma_{j}$. In the gauge degrees of freedom of the MPS representation, $\hat{\Pi}_{j}^{|\psi\rangle}|\phi\rangle$ fixes the orthogonality center to site $j$. As the physical content of the state is independent of the location of its orthogonality center, we must have

$$
\langle\psi \mid \psi\rangle=\left(\langle\psi| \hat{\Pi}_{j}^{|\psi\rangle}\right)\left(\hat{\Pi}_{i}^{|\psi\rangle}|\psi\rangle\right)
$$

as also immediately follows from Eq. (4.138). Next, we evaluate the transformation of operators under such a projection and for convenience we define a compact notation for quantum states in terms of the bond maps defined in Eqs. (4.133) and (4.134)

$$
\left|\psi_{j}^{L}\right\rangle_{m_{j}}=\sum_{\sigma_{1}, \ldots, \sigma_{j}} \psi_{j ; m_{j}}^{L ; \sigma_{1}, \ldots, \sigma_{j}}\left|\sigma_{1} \cdots \sigma_{j}\right\rangle, \quad\left|\psi_{j}^{R}\right\rangle_{m_{j-1}}=\sum_{\sigma_{j}, \ldots, \sigma_{L}} \psi_{j ; m_{j-1}}^{R ; \sigma_{j}, \ldots, \sigma_{L}}\left|\sigma_{j} \cdots \sigma_{L}\right\rangle
$$

Without truncation these bond maps can in principle be constructed so that they constitute a complete mapping from the left/right partition of the Hilbert space into the bond spaces $m_{j} / m_{j-1}$. Now we express an operator subject to a projection $\hat{\Pi}_{j}^{|\psi\rangle}$ in this notation

$$
\hat{\Pi}_{j}^{|\psi\rangle} \hat{O} \hat{\Pi}_{j}^{|\psi\rangle}=\sum_{\substack{m_{j-1}, m_{j} \\ m_{j-1}^{\prime}, m_{j}^{\prime}}} \sum_{\sigma_{j}, \sigma_{j}^{\prime}}\left|\psi_{j-1}^{L}, \psi_{j+1}^{R}\right\rangle_{m_{j-1}, m_{j}}\left|\sigma_{j}\right\rangle O_{\sigma_{j}^{\prime}, m_{j-1}^{\prime}, m_{j}^{\prime}}^{\sigma_{j}, m_{j-1}, m_{j}}\left\langle\left.\sigma_{j}^{\prime}\right|_{m_{j-1}^{\prime}, m_{j}^{\prime}}\left\langle\psi_{j-1}^{L}, \psi_{j+1}^{R}\right|\right.
$$

where we defined

$$
O_{\sigma_{j}^{\prime}, m_{j-1}^{\prime}, m_{j}^{\prime}}^{\sigma_{j}, m_{j-1}, m_{j}}={ }_{m_{j-1}, m_{j}}\left\langle\psi_{j-1}^{L}, \psi_{j+1}^{R}\left|\left\langle\sigma_{j}|\hat{O}| \sigma_{j}^{\prime}\right\rangle\right| \psi_{j-1}^{L}, \psi_{j+1}^{R}\right\rangle_{m_{j-1}^{\prime}, m_{j}^{\prime}} .
$$

Here, $O_{\sigma_{j}^{\prime}, m_{j-1}^{\prime}, m_{j}^{\prime}}^{\sigma_{j}, m_{j-1}, m_{j}}$ is essentially the single-site representation of the Hamiltonian in Fig. 4.13. The action of the projected operator on a state $|\phi\rangle$ in a mixed-canonical MPS representation with orthogonality center at site $j$ is then given by

$$
\begin{aligned}
\hat{\Pi}_{j}^{|\psi\rangle} \hat{O} \hat{\Pi}_{j}^{|\psi\rangle}|\phi\rangle= & \sum_{\substack{m_{j-1}, m_{j} \\
m_{j-1}^{\prime}, m_{j}^{\prime}}} \sum_{\sigma_{j}, \sigma_{j}^{\prime}}\left|\psi_{j-1}^{L}, \psi_{j+1}^{R}\right\rangle_{m_{j-1}, m_{j}}\left|\sigma_{j}\right\rangle O_{\sigma_{j}^{\prime}, m_{j-1}^{\prime}, m_{j}^{\prime}}^{\sigma_{j}, m_{j-1}, m_{j}} \times \\
& \sum_{\substack{m_{j-1}^{\prime \prime}, m_{j}^{\prime \prime} \\
m_{j-1}^{\prime}}}\left\langle\psi_{j-1}^{L} \mid \phi_{j-1}^{L}\right\rangle_{m_{j-1}^{\prime \prime}} M_{j ; m_{j-1}^{\prime \prime}, m_{j}^{\prime \prime} m_{j-1}^{\prime}}^{\sigma_{j}^{\prime}}\left\langle\psi_{j+1}^{R} \mid \phi_{j+1}^{R}\right\rangle_{m_{j}^{\prime \prime}} \\
= & \sum_{\substack{m_{j-1}, m_{j} \\
m_{j-1}^{\prime}, m_{j}^{\prime}}} \sum_{\sigma_{j}, \sigma_{j}^{\prime}}\left|\psi_{j-1}^{L}\right\rangle_{m_{j-1}} O_{\sigma_{j}^{\prime}, m_{j-1}^{\prime}, m_{j}^{\prime}}^{\sigma_{j}, m_{j-1}, m_{j}} \tilde{M}_{j ; m_{j-1}^{\prime}, m_{j}^{\prime}}^{\sigma^{\prime}}\left|\sigma_{j}^{\prime}\right\rangle\left|\psi_{j+1}^{R}\right\rangle_{m_{j}},
\end{aligned}
$$




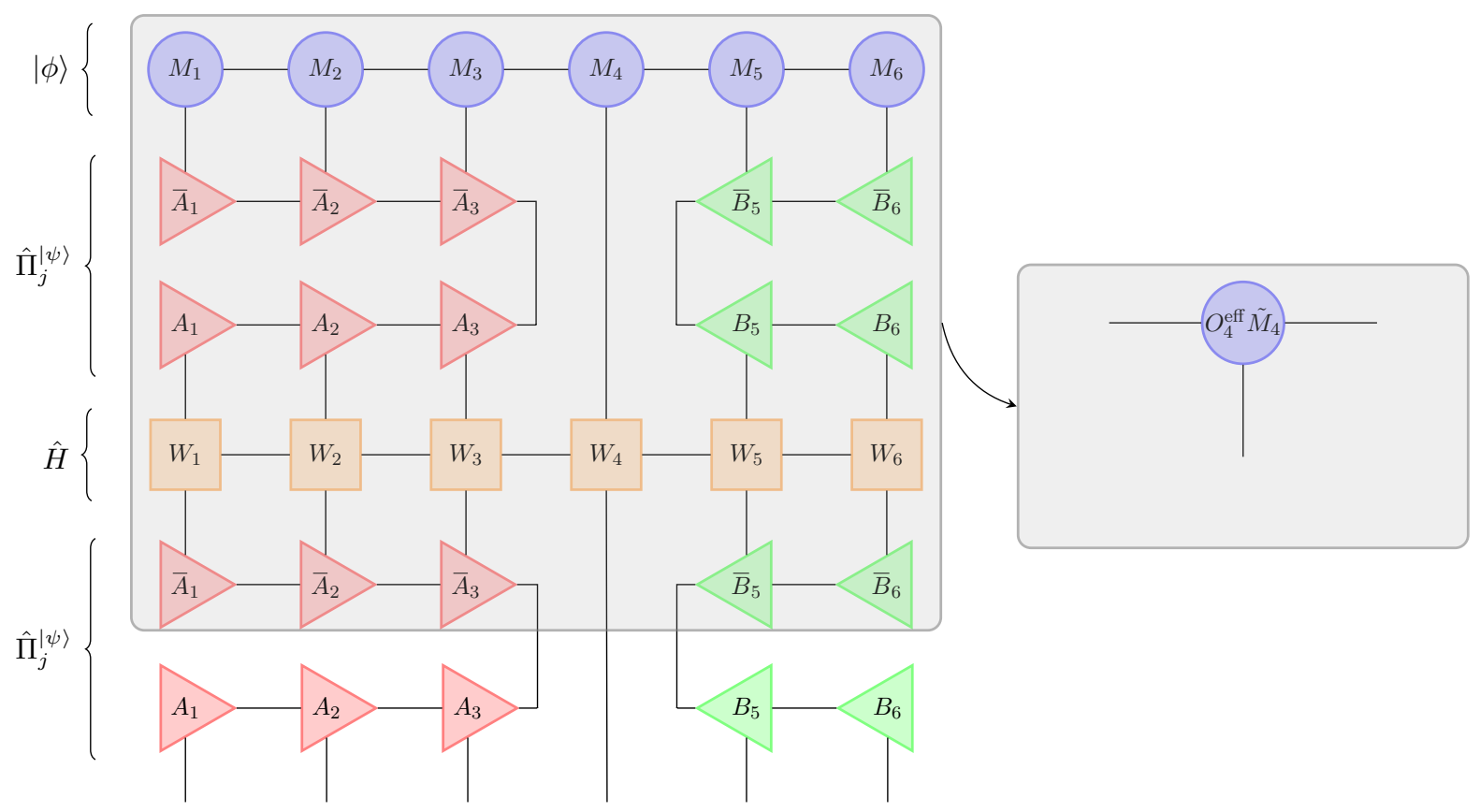

Figure 4.22: Action of projected Hamiltonian $\hat{H}^{\hat{\Pi}_{j}^{|\psi\rangle}}$ on arbitrary state $|\phi\rangle$ at the example of a system with $L=6$ and center of orthogonality at site $j=4$. Contracting the gray shaded box on the left yields a new site-tensor $O_{4}^{\text {eff }} \tilde{M}_{4}$ at site 4 . The overall state $\hat{H}^{\hat{\Pi}_{4}^{|\psi\rangle}}|\phi\rangle$ is in a mixed-canonical form with orthogonality center at site 4 and the $A / B$-tensors to the left/right are identical to those from the state $|\psi\rangle$ from which the projector $\hat{\Pi}_{4}^{|\psi\rangle}$ is constructed.

where we have defined

$$
\tilde{M}_{j ; m_{j-1}^{\prime}, m_{j}^{\prime}}^{\sigma_{j}^{\prime}}=\sum_{m_{j-1}^{\prime \prime}, m_{j}^{\prime \prime}} \underbrace{\sigma_{j ; m_{j-1}^{\prime \prime}, m_{j}^{\prime \prime}}^{\sigma_{j}^{\prime}} \underbrace{m_{j}^{\prime}}_{:=Q_{\underline{j} ; m_{j}^{\prime}, m_{j}^{\prime \prime}}}\left\langle\psi_{j+1}^{R} \mid \phi_{j+1}^{R}\right\rangle_{m_{j}^{\prime \prime}}}_{:=Q_{\underline{j-1 ; m_{j-1}^{\prime}, m_{j-1}^{\prime \prime}}}^{m_{j-1}^{\prime}}\left\langle\psi_{j-1}^{L} \mid \phi_{j-1}^{L}\right\rangle_{m_{j-1}^{\prime \prime}}}=Q_{\underline{j-1}}^{L} M_{j} Q_{\underline{j}}^{R} .
$$

In the last equation we have defined transformations $Q_{j}^{L / R}$, which act on the bond spaces only and are obtained by contracting bond maps from the left/right partition of the Hilbert space. Note that $Q_{\underline{j}}^{L / R}$ are complete basis transformations between the bond basis sets of the reference state $|\psi\rangle$ and the target state $|\phi\rangle$ iff we do not allow for truncation. If this is not the case the error is hard to quantify, but we would expect it to scale with the discarded weights $\epsilon_{\phi, \psi}^{2}$ of the states $|\phi\rangle,|\psi\rangle$ yielding an estimation $\sim \sqrt{\epsilon_{\phi}^{2}\left(1-\epsilon_{\psi}^{2}\right)+\epsilon_{\psi}^{2}\left(1-\epsilon_{\phi}^{2}\right)}$. Inspecting Eq. (4.143) we note that we can perform all contractions over the primed indices, which we write compactly as

$$
\sum_{\substack{m_{j-1}^{\prime}, m_{j}^{\prime} \\ \sigma \prime_{j}}} O_{\sigma_{j}^{\prime}, m_{j-1}^{\prime}, m_{j}^{\prime}}^{\sigma_{j}, m_{j-1}, m_{j}} \tilde{M}_{j ; m_{j-1}^{\prime}, m_{j}^{\prime}}^{\sigma^{\prime}}=O_{j}^{\mathrm{eff}} \tilde{M}_{j}
$$

as is also shown in Fig. 4.22. Therefore, the action of the projected operator yields a state in a mixed-canonical representation with orthogonality center at site $j$ and the left-/right-canonical 
site-tensors of the source state $|\psi\rangle$ :

$$
\hat{\Pi}_{j}^{|\psi\rangle} \hat{O} \hat{\Pi}_{j}^{|\psi\rangle}|\phi\rangle=A_{1} \cdots A_{j-1}\left(O_{j}^{\mathrm{eff}} Q_{\underline{j-1}}^{L} M_{j} Q_{\underline{j}}^{R}\right) B_{j} \cdots B_{L}
$$

This yields an important observation: if the target state $|\phi\rangle$ is in a mixed-canonical representation with center of orthogonality at site $j$ and left-/right-canonical site-tensors being equivalent to those of the source state $|\psi\rangle$, the action of the projected operator is non-trivial only on the physical index $\sigma_{j}$ and the bond-spaces $m_{j-1}, m_{j}$.

\section{Decomposing the time-dependent Schrödinger equation}

Combining the statements of the previous sections we can reformulate the action of the Hamiltonian by decomposing it into representations $\hat{H}_{j}^{\hat{\Pi}_{j}^{|\psi\rangle}}$ acting only onto reduced site-spaces:

$$
\begin{aligned}
\hat{H} & \approx \frac{1}{L \||\psi\rangle \|} \sum_{j} \hat{\Pi}_{j}^{\left|\psi^{j}\right\rangle} \hat{H} \hat{\Pi}_{j}^{\left|\psi^{j}\right\rangle} \equiv \frac{1}{L \||\psi\rangle \|} \sum_{j} \hat{H}^{\hat{\Pi}_{j}^{|\psi\rangle}} \\
\hat{H}|\psi\rangle & \approx \frac{1}{L \||\psi\rangle \|} \sum_{j} \hat{\Pi}_{j}^{\left|\psi^{j}\right\rangle} \hat{H} \hat{\Pi}_{j}^{\left|\psi^{j}\right\rangle}|\psi\rangle=\frac{1}{L \||\psi\rangle \|} \sum_{j} \hat{H}^{\hat{\Pi}_{j}^{\left|\psi^{j}\right\rangle}}|\psi\rangle,
\end{aligned}
$$

which indeed yields a Lie-Trotter decomposition of the time-dependent Schrödinger equation

$$
-\mathrm{i} \frac{d}{d t}|\psi(t)\rangle=\frac{1}{L \||\psi\rangle \|} \sum_{j} \hat{H}^{\hat{\Pi}_{j}^{\left|\psi^{j}\right\rangle}}|\psi(t)\rangle
$$

Note that without loss of generality the projection operators $\hat{\Pi}_{j}^{\left|\psi^{j}\right\rangle}$ are constructed from sitedependent source states $\left|\psi^{j}\right\rangle$ that, hence, can differ from site to site. We obtain the following set of $L$ coupled initial value problems approximating the overall solution in the interval $\left[t_{0}, t_{0}+\delta\right]$

$$
\begin{aligned}
-\mathrm{i} \frac{d}{d t}\left|\psi^{j}(t)\right\rangle & =\hat{H}^{\hat{\Pi}_{j}^{\left|\psi^{j}\right\rangle}}\left|\psi^{j}(t)\right\rangle \\
\text { and } \quad\left|\psi^{j}\left(t_{0}\right)\right\rangle & =\left|\psi^{j-1}\left(t_{0}+\delta\right)\right\rangle,
\end{aligned}
$$

with $\left|\psi^{1}\left(t_{0}\right)\right\rangle=\left|\psi\left(t_{0}\right)\right\rangle$. In order to take benefit of the MPS representation, in the $j$ th problem we construct projection operators from the previous solution (identifying again $t_{0} \equiv t$ )

$$
\hat{\Pi}_{j}^{\left|\psi^{j-1}\right\rangle}=\hat{P}_{j-1}^{L,\left|\psi^{j-1}(t+\delta)\right\rangle} \otimes \hat{\mathbf{1}}_{j} \otimes \hat{P}_{j+1}^{R,\left|\psi^{j-1}(t+\delta)\right\rangle} .
$$

Now note that the initial value of the $j$ th solution is just given by $\left|\psi^{j-1}(t+\delta)\right\rangle$ so that from Eq. (4.146) it follows that for each infinitesimal time step $\hat{H}^{\hat{\Pi}_{j}^{\left|\psi^{j}\right\rangle}}$ only evolves the $j$ th site tensor. Therefore, the search space for the solution of the $j$ th initial value problem Eq. (4.150) is restricted to states in a mixed canonical representation with orthogonality center at site $j$ and left-/right-canonical site tensors obtained from $\left|\psi^{j-1}(t+\delta)\right\rangle$ with only the active-site tensor $M_{j}$ being modified. On the other hand, expanding the derivative in the $j$ th local problem we find

$$
-\mathrm{i} \frac{d}{d t}\left|\psi^{j}(t)\right\rangle=-\mathrm{i}\left(\left[\frac{\partial}{\partial t} M_{j}(t)\right] \frac{\partial}{\partial M_{j}}+\sum_{k \neq j}\left[\frac{\partial}{\partial t} M_{k}(t)\right] \frac{\partial}{\partial M_{k}}\right)\left|\psi^{j}(t)\right\rangle .
$$




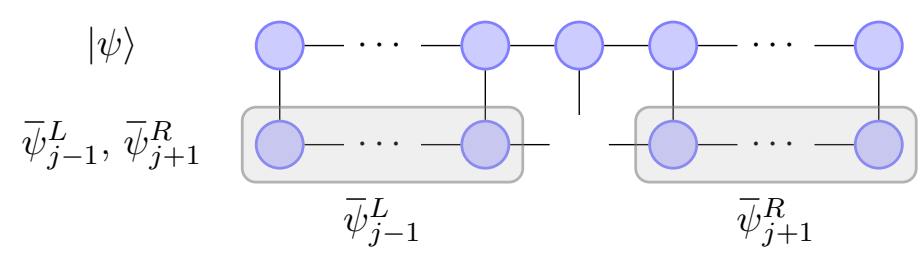

Figure 4.23: The effective state $\left|\psi_{j}^{\text {eff }}\right\rangle$ is obtained by projecting the MPS with itself. In case of a mixed-orthogonal MPS with orthogonality center on site $j,\left|\psi_{j}^{\text {eff }}\right\rangle$ is simply the local site tensor $M_{j}$ and the left and right projectors are identity matrices. This graphic is adopted from the author's publication $\left[\mathrm{PKS}^{+} 19\right]$.

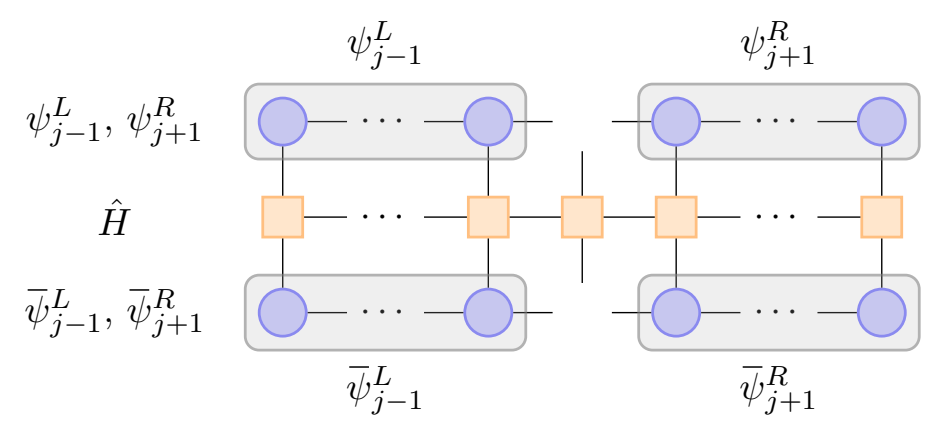

Figure 4.24: The effective Hamiltonian $\hat{H}_{j}^{\text {eff }}$ obtained by projecting the MPO for $\hat{H}$ using $\psi_{j-1}^{L}, \psi_{j+1}^{R}$, $\bar{\psi}_{j-1}^{L}, \bar{\psi}_{j+1}^{R}$. Note that this tensor is never explicitly constructed! Only its action on the state tensor $M_{j}$ is evaluated. This graphic is adopted from the author's publication [PKS $\left.{ }^{+} 19\right]$.

The restricted search space immediately implies that all derivatives $\frac{d}{d t} M_{k}(t)$ with $k \neq j$ can be discarded so that the $j$ th projected differential equation is given by

$$
-\mathrm{i}\left[\frac{\partial}{\partial t} M_{j}(t)\right] \frac{\partial}{\partial M_{j}}\left|\psi^{j}(t)\right\rangle=\hat{H}^{\hat{\Pi}_{j}^{\left|\psi^{j}\right\rangle}}\left|\psi^{j}(t)\right\rangle
$$

We can, hence, project out the unimportant site tensors by multiplying both sites with the bond maps $\bar{\psi}_{j-1 ; m_{j-1}}^{L} \otimes \hat{\mathbf{1}}_{j} \otimes \bar{\psi}_{j+1 ; m_{j}}^{R}\left(\right.$ constructed from $\left.\left|\psi^{j-1}(t+\delta)\right\rangle\right)$

$$
-\mathrm{i} \frac{\partial}{\partial t} M_{j}=\hat{H}_{j}^{\mathrm{eff}} Q_{\underline{j-1}}^{L} M_{j} Q_{\underline{j}}^{R} .
$$

This is the desired local representation of Eq. (4.150) expressed in terms of an effective Hamiltonian and a projected site tensor (see also Figs. 4.23 and 4.24). Observing that $Q_{\underline{j-1}}^{L}$ and $Q_{\underline{j}}^{R}$ are constructed from the above introduced bond maps $\bar{\psi}_{j-1}^{L}$ and $\psi_{j+1}^{R}$, which themselves should be build recursively, the transformation can be updated and applied before solving the $j$-th problem. Additionally, solving Eq. (4.150) from the left to the right the transformation $Q_{j}^{R}$ is always given by an identity since the constituting right-canonical site-tensors are not modified and the same holds for $Q_{j-1}^{L}$ when sweeping from the left to the right. Therefore, in the actual implementation always only one transformation has to be applied, dependening on the current sweep direction. Note that as already mentioned, if we allow for truncation the error incurred by this transformation depends on the discarded weight. Since for small time steps $\delta$ the time-evolved state is expected to be relatively close to the unevolved state the overall deviation after solving the whole set of equations is also small $\propto L \epsilon^{2}$.

Instead of mapping onto the space of a single site, in practice we map onto the space of two sites. The two-site local TDSE is solved using the time-dependent Lanczos approach to obtain 
$A_{j}(t+\delta)$. The original orthogonality center MPS tensor $M_{j+1}(t)$ is then projected onto the new left basis as described above. This allows for a flexible adaptation of not only the tensor $A_{j}$ itself but also of the bond basis and - if necessary - MPS bond dimension between sites $j$ and $j+1$. Historically, only this two-site variant was used; but in analogy to the 2TDVP method presented later, it may well make sense to initially use the two-site local Krylov method until the desired maximal bond dimension has been obtained and then switch to the single-site integrator to save computational effort.

\section{Algorithm}

The actual implementation of the local Krylov method can be obtained from an existing DMRG ground-state search algorithm with only minor modifications. The main difference is the approximation of an operator exponential $e^{-\mathrm{i} \hat{\delta} H_{j, j+1}^{\text {eff }}}$ in the reduced site space (here we use a two-site realization of the algorithm) and the construction and application of the transformations $Q_{j}^{L / R}$. The two-site local Krylov method is described in detail in Alg. 3. It relies on some basic initialization functions familiar from the DMRG algorithm, which are summarized in Alg. 2. Additionally, the exponential in the local Krylov spaces can be adopted from Alg. 1 where we only need to replace the global states by the active site-tensors and use contraction methods to compute the action of $\hat{H}_{j, j+1}^{\mathrm{eff}}$ as described in Sec. 4.4.2.

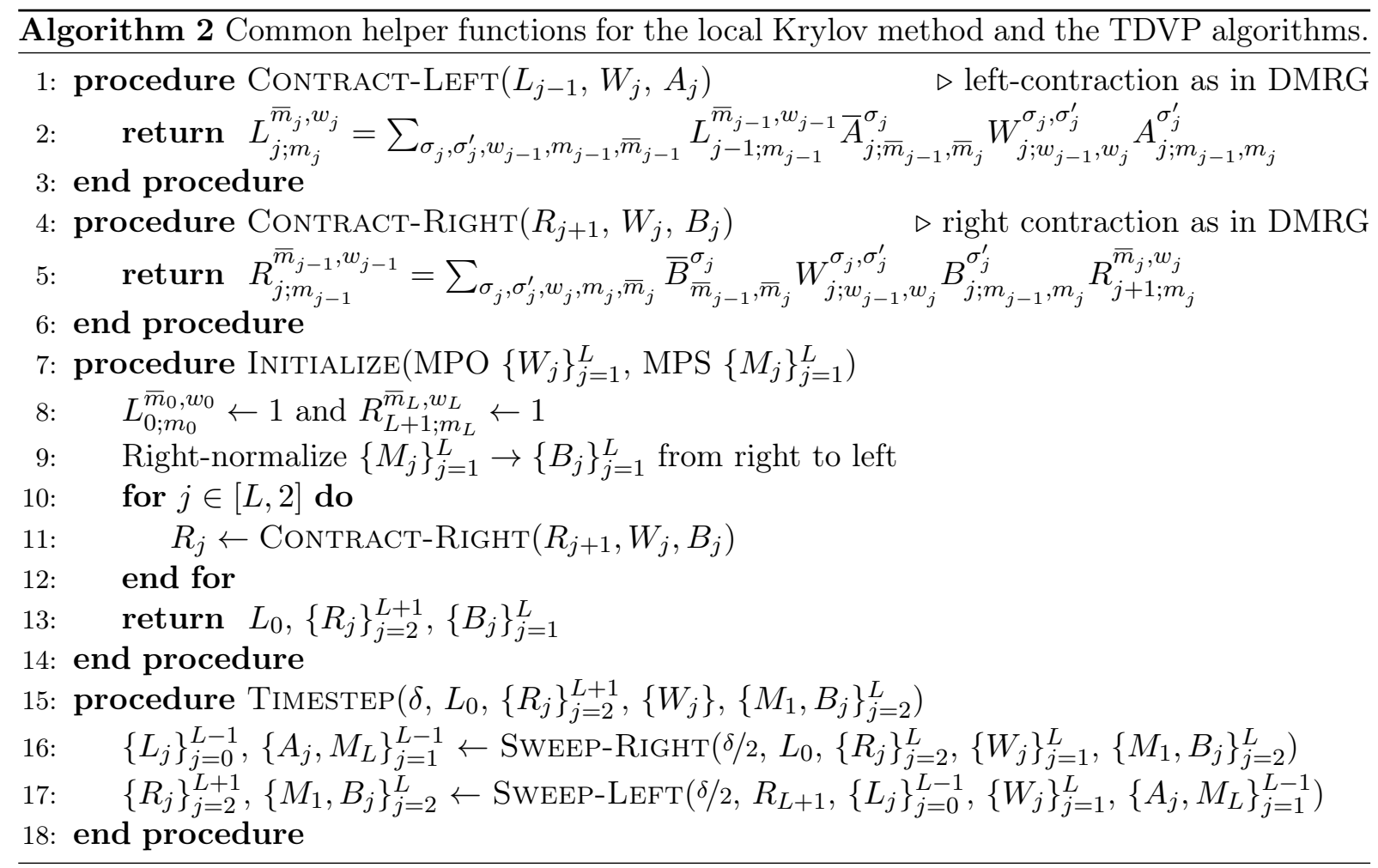

\section{Errors}

Four errors are present in the local Krylov method when used in its (standard) two-site variant. The smallest of those stems from the inexact solution of the local TDSE Eq. (4.154). This error can be made very small using a precise solver; in practice, a Krylov-solver as already described in Sec. 4.5.2 with only a small dimension of the Krylov space (5-10) is sufficient. The second error 


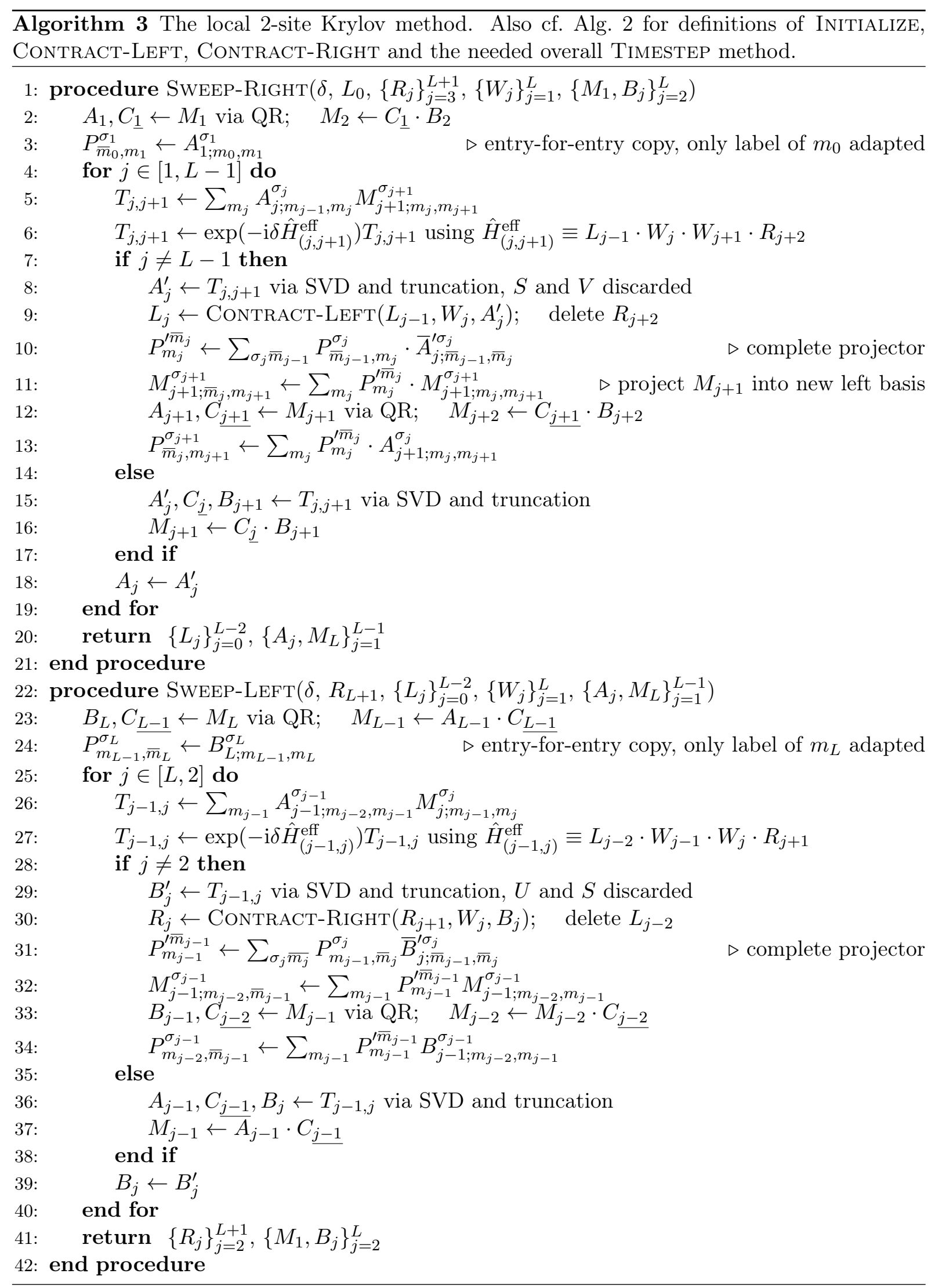



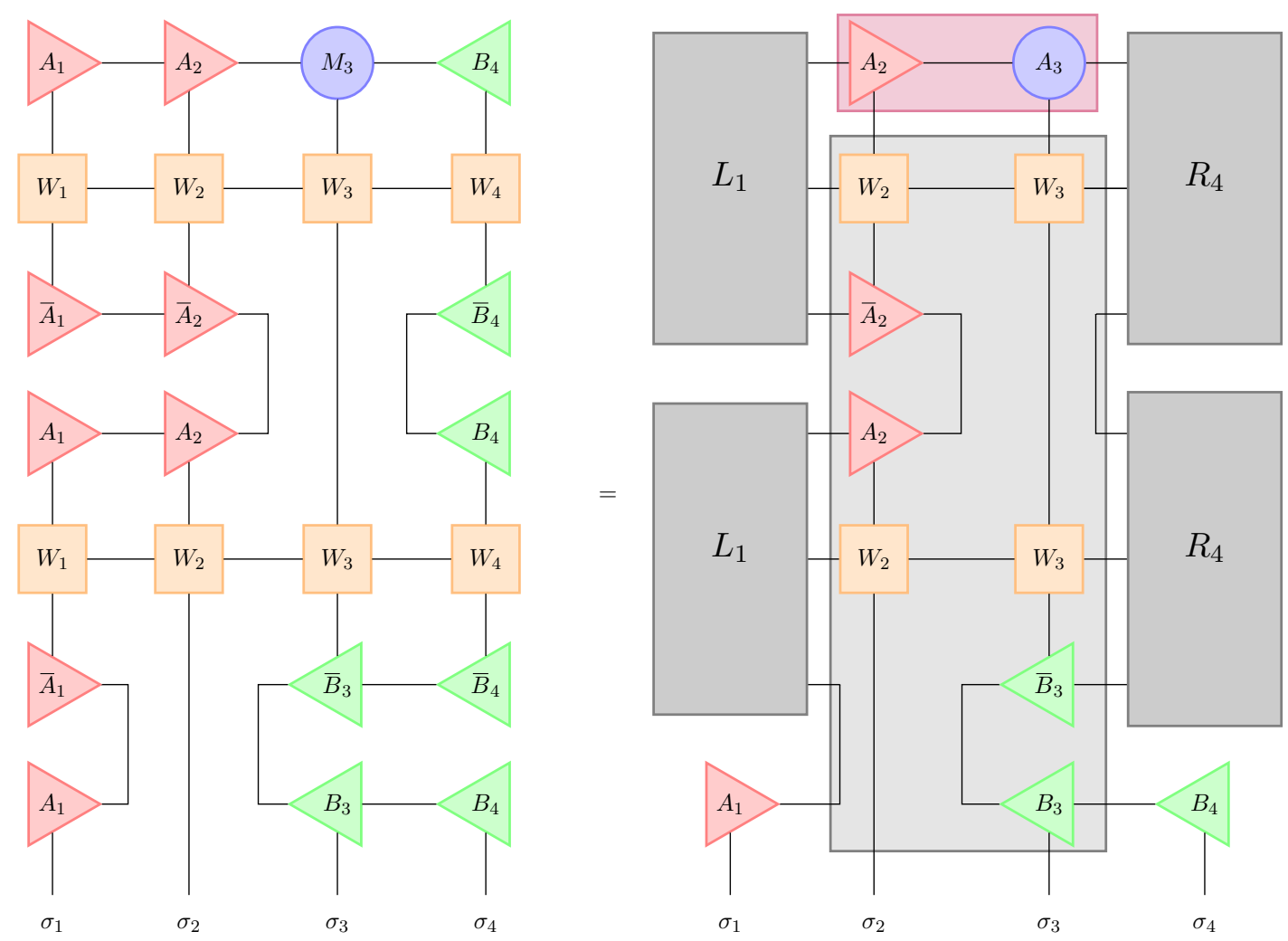

Figure 4.25: $\quad$ Evaluation of $\hat{H}^{\hat{\Pi}_{2}^{|\psi\rangle}} \hat{H}_{3}^{\hat{\Pi}_{3}^{|\psi\rangle}}\left|\psi^{3}(t)\right\rangle$ at the example of a four-site system. The tensors $L_{i} / R_{i}$ correspond to partially contracted MPS-MPO-MPS-networks. The burgundy-shaded rectangular area at the top encloses the reduced site tensor $\psi_{i, \ldots, j ; m_{i-1}, m_{j}}^{C ; \sigma_{i}, \ldots, \sigma_{j}}$. The commutator $\left[\hat{H}^{\hat{\Pi}_{2}^{|\psi\rangle}} \hat{H}^{\hat{\Pi}_{3}^{|\psi\rangle}}\right]\left|\psi^{3}(t)\right\rangle$ requires also the calculation of $\hat{H}_{3}^{\hat{\Pi}_{3}^{|\psi\rangle}} \hat{H}^{\hat{\Pi}_{2}^{|\psi\rangle}}\left|\psi^{3}(t)\right\rangle$, which is obtained from vertically flipping the tensors covered by the central rectangular grey area between the sites $(i, j)$, i.e., $(2,3)$ in the presented example.

is the standard truncation error incurred during the SVD to split the merged two-site tensors again while truncating to the desired bond dimension. This error can be measured and observed throughout the calculation and is much the same as in the other methods.

The third error is due to the approximation in Eq. (4.147). This projection error is difficult to measure and strongly depends on the initial state. If the initial state has a reasonably large bond dimension and the Hamiltonian has reasonably short-range interactions, this error will be very small. The longer the interactions in the Hamiltonian, the larger the state has to be. In the two-site method, nearest-neighbor interactions can be handled at all bond dimensions, in the single-site variant, only on-site interactions are error-free at small bond dimensions. The projection error is in particular problematic when globally quenching from a product state with a very non-local Hamiltonian (e.g. resulting from a $2 \mathrm{D} \rightarrow 1 \mathrm{D}$ map). When calculating equilibrium Green's functions for short-range Hamiltonians, this error is quite negligible.

Finally, there is an error due to the sequential solution of the local TDSE as resulting from the Lie-Trotter decomposition. This error can be quantified, but doing so requires some additional work, which will follow now: We continue from the Taylor expansion Eq. (4.131). We emphasize

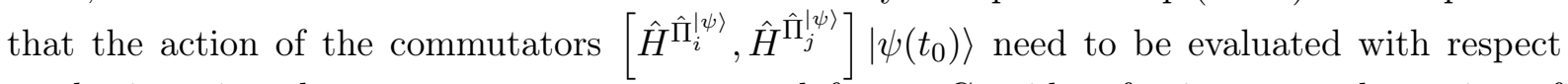
to the iteration the commutators are generated from. Consider, for instance, the action of $\hat{H}^{\hat{\Pi}_{2}^{|\psi\rangle}} \hat{H}^{\hat{\Pi}_{3}^{|\psi\rangle}}|\psi(t)\rangle$, which is generated from the first-order contribution $\hat{H}_{3}^{\hat{\Pi}_{3}^{|\psi\rangle}}\left|\psi^{3}\right\rangle$ and subsequent 
application of $\hat{H}^{\hat{\Pi}_{2}^{|\psi\rangle}}$. Thus the commutator also needs to be evaluated considering the partial solution $\left|\psi^{j}\right\rangle$, so that in general we have for $i<j$

$$
\left[\hat{H}^{\hat{\Pi}_{i}^{|\psi\rangle}}, \hat{H}^{\hat{\Pi}_{j}^{|\psi\rangle}}\right]|\psi(t)\rangle=\left[\hat{H}_{i}^{\hat{\Pi}_{i}^{|\psi\rangle}}, \hat{H}^{\hat{\Pi}_{j}^{|\psi\rangle}}\right]\left|\psi^{j}(t)\right\rangle .
$$

In Fig. 4.25 the action of $\hat{H}^{\hat{\Pi}_{i}^{|\psi\rangle}} \hat{H}^{\hat{\Pi}_{j}^{|\psi\rangle}}\left|\psi^{j}(t)\right\rangle$ is demonstrated in case of a four-site system with $i=2, j=3$ by performing most of the contractions graphically. In order to obtain the matrix element with an arbitrary state $|\phi\rangle$ we will introduce a compact notation for contractions of MPS and MPO site-tensors with the boundary tensors (partially contracted MPS-MPO-MPS-networks $\left.L_{j} / R_{j}\right)$

$$
\begin{aligned}
L_{j-1} E_{j} & \equiv L_{j-1} \bar{M}_{j} W_{j} M_{j}=\sum_{m_{j-1}, \sigma_{j}^{\prime}}\left(\sum_{w_{j-1}, \sigma_{j}}\left(\sum_{\bar{m}_{j-1}} L_{j-1 ; m_{j-1}}^{\bar{m}_{j-1}, w_{j-1}} \bar{M}_{j ; \bar{m}_{j-1}, \bar{m}_{j}}^{\sigma_{j}}\right) W_{j, w_{j-1}, w_{j}}^{\sigma_{j}, \sigma_{j}^{\prime}}\right) M_{j, m_{j-1}, m_{j}}^{\sigma_{j}^{\prime}} \\
E_{j} R_{j+1} & \equiv \bar{M}_{j} W_{j} M_{j} R_{j+1}=\sum_{\bar{m}_{j}, \sigma_{j}} \bar{M}_{j ; \bar{m}_{j-1}, \bar{m}_{j}}^{\sigma_{j}}\left(\sum_{w_{j}, \sigma_{j}^{\prime}} W_{j ; w_{j-1}, w_{j}}^{\sigma_{j}, \sigma_{j}^{\prime}}\left(\sum_{m_{j}} M_{j ; m_{j-1}, m_{j}}^{\sigma_{j}^{\prime}} R_{j+1 ; m_{j}}^{\bar{m}_{j}, w_{j}}\right)\right)
\end{aligned}
$$

with the transfer tensors $E_{j}=\bar{M}_{j} W_{j} M_{j}$. We also need transfer tensors with the target state, which we define by $E_{j}^{\phi}=\bar{M}_{j}^{\phi} W_{j} M_{j}$. Finally, there will be open bonds at sites $i$ and $j$ that correspond to the contractions originating from the "brace"-contractions in the projectors $\hat{P}_{i}^{L,|\psi\rangle}$ and $\hat{P}_{i}^{R,|\psi\rangle}$ as well as $\hat{P}_{j+1}^{L,|\psi\rangle}$ and $\hat{P}_{j+1}^{R,|\psi\rangle}$, and we will label these bonds explicitly. Considering for instance the first summand of the commutator at $i=2, j=3$, that is $\left\langle\phi\left|\hat{H}^{\hat{\Pi}_{2}^{|\psi\rangle}} \hat{H}^{\hat{\Pi}_{3}^{|\psi\rangle}}\right| \psi^{3}(t)\right\rangle$ (c.f. Fig. 4.25) the left part of the contractions can be written as

$$
\sum_{m_{2}, \bar{m}_{2}}\left(L_{1} E_{2}\right)_{\bar{m}_{2}} \otimes\left(L_{1} E_{2}^{\phi}\right)_{m_{2}} \delta_{\bar{m}_{2}, m_{2}} \equiv \sum_{m_{2}, \bar{m}_{2}}\left(L_{1} \otimes L_{1}\right)\left(E_{2} \otimes E_{2}^{\phi}\right)_{\bar{m}_{2}, m_{2}} \delta_{\bar{m}_{2}, m_{2}} .
$$

To obtain a compact notation for the right part we introduce the "dangling" transfer tensors $D_{j}=W_{j} M_{j}$ if only the ket site-tensor of the state is included and $\bar{D}_{j}=\bar{M}_{j} W_{j}$ if only the bra site-tensor is considered. In the same manner as for the left part we can now write for the right contractions compactly

$$
\sum_{m_{3}, \bar{m}_{3}}\left(D_{3} R_{4}\right)_{\bar{m}_{3}} \otimes\left(\bar{D}_{3}^{\phi} R_{4}\right)_{m_{3}} \delta_{\bar{m}_{3}, m_{3}} \equiv \sum_{m_{3}, \bar{m}_{3}}\left(D_{3} \otimes \bar{D}_{3}^{\phi}\right)\left(R_{4} \otimes R_{4}\right)_{\bar{m}_{3}, m_{3}} \delta_{\bar{m}_{3}, m_{3}} .
$$

In general we obtain for the matrix element of the commutator with the target state $|\phi\rangle$ (suppressing the Kronecker- $\delta$ for brevity)

$$
\begin{aligned}
& \langle\phi|\left[\hat{H}^{\mid \hat{\Pi}_{i}^{|\psi\rangle}}, \hat{H}^{\left.\hat{\Pi}_{j}^{|\psi\rangle}\right]}\left|\psi^{j}(t)\right\rangle\right. \\
& =\sum_{\substack{m_{j-1}, \bar{m}_{j-1} \\
m_{j}, \bar{m}_{j}}}\left(L_{i-1} \otimes L_{i-1}\right)\left(E_{i} \cdots E_{j-1} \otimes E_{i}^{\phi} \cdots E_{j-1}^{\phi}\right)_{\bar{m}_{j-1}, m_{j-1}}\left(D_{j} \otimes \bar{D}_{j}^{\phi}\right)\left(R_{j+1} \otimes R_{j+1}\right)_{\bar{m}_{j}, m_{j}}+\cdots \\
& -\sum_{\substack{m_{i-1}, \bar{m}_{i-1} \\
m_{i}, \bar{m}_{i}}}\left(L_{i-1} \otimes L_{i-1}\right)_{\bar{m}_{i-1}, m_{i-1}}\left(D_{i} \otimes \bar{D}_{i}^{\phi}\right)\left(E_{i+1} \cdots E_{j} \otimes E_{i+1}^{\phi} \cdots E_{j}^{\phi}\right)_{\bar{m}_{i}, m_{i}}\left(R_{j+1} \otimes R_{j+1}\right) .
\end{aligned}
$$


It becomes immediately clear that only those terms in the Hamiltonian contribute to the commutator that cross the bond $(j-1, j)$ or $(i, i+1)$. For the current purpose it suffices to find a general estimate for the incurred error $\propto \delta^{2}$ in the Taylor expansion. Thus, we will only consider the contributions from nearest-neighbor interactions and hence set $i=j-1$ so that

$$
\begin{aligned}
& \left\langle\phi\left|\left[\hat{H}^{\hat{\Pi}_{j-1}^{|\psi\rangle}}, \hat{H}^{\hat{\Pi}_{j}^{|\psi\rangle}}\right]\right| \psi^{j}(t)\right\rangle \\
& =\sum_{\substack{m_{j-1}, \bar{m}_{j-1} \\
m_{j}, \bar{m}_{j}}}\left(L_{j-2} \otimes L_{j-2}\right)\left(E_{j-1} \otimes E_{j-1}^{\phi}\right)_{\bar{m}_{j-1}, m_{j-1}}\left(D_{j} \otimes \bar{D}_{j}^{\phi}\right)\left(R_{j+1} \otimes R_{j-1}\right)_{\bar{m}_{j}, m_{j}}+\cdots \\
& -\sum_{\substack{m_{j-2}, \bar{m}_{j-2} \\
m_{j-1}, \bar{m}_{j-1}}}\left(L_{j-2} \otimes L_{j-2}\right)_{\bar{m}_{j-2}, m_{j-2}}\left(D_{j-1} \otimes \bar{D}_{j-1}^{\phi}\right)\left(E_{j} \otimes E_{j}^{\phi}\right)_{\bar{m}_{j-1}, m_{j-1}}\left(R_{j+1} \otimes R_{j+1}\right) .
\end{aligned}
$$

The crucial observation here is that for each "open" bond index pair we can treat the combined MPS-MPS and MPO-MPO tensor-contractions over the bond $(j-1, j)$ as scalar product between the left and right part of the system. We can thus write for the first summand for each open index pair

$$
\begin{aligned}
& \underbrace{\left(L_{j-2} \otimes L_{j-2}\right)\left(E_{j-1} \otimes E_{j-1}^{\phi}\right)_{\bar{m}_{j-1}, m_{j-1}}}_{\equiv\left\langle e_{j-1}^{L} \otimes e_{j-1}^{L, \phi}\right|} \underbrace{\left(D_{j} \otimes \bar{D}_{j}^{\phi}\right)\left(R_{j+1} \otimes R_{j-1}\right)_{\bar{m}_{j}, m_{j}}}_{\equiv\left|d_{j}^{R} \otimes \bar{d}_{j}^{R, \phi}\right\rangle} \\
& \left.=\frac{1}{2}\left(|| e_{j-1}^{L} \otimes e_{j-1}^{L, \phi}\right\rangle+\left.\left|d_{j}^{R} \otimes \bar{d}_{j}^{R, \phi}\right\rangle\right|^{2}-\left\langle e_{j-1}^{L} \otimes e_{j-1}^{L, \phi} \mid e_{j-1}^{L} \otimes e_{j-1}^{L, \phi}\right\rangle-\left\langle d_{j}^{R} \otimes \bar{d}_{j}^{R, \phi} \mid d_{j}^{R} \otimes \bar{d}_{j}^{R, \phi}\right\rangle\right)
\end{aligned}
$$

and for the second summand

$$
\begin{aligned}
& \underbrace{\left(L_{j-2} \otimes L_{j-2}\right)_{\bar{m}_{j-2}, m_{j-2}}\left(D_{j-1} \otimes \bar{D}_{j-1}^{\phi}\right)}_{\equiv\left\langle d_{j-1}^{L} \otimes \bar{d}_{j-1}^{L, \phi}\right|} \underbrace{\left(E_{j} \otimes E_{j}^{\phi}\right)_{\bar{m}_{j-1}, m_{j-1}}\left(R_{j+1} \otimes R_{j+1}\right)}_{\equiv\left|e_{j}^{R} \otimes e_{j}^{R, \phi}\right\rangle} \\
& =-\frac{1}{2}\left(\left|d_{j-1}^{L} \otimes \bar{d}_{j-1}^{L, \phi}\right\rangle-\left.\left|e_{j}^{R} \otimes e_{j}^{R, \phi}\right\rangle\right|^{2}-\left\langle d_{j-1}^{L} \otimes \bar{d}_{j-1}^{L, \phi} \mid d_{j-1}^{L} \otimes \bar{d}_{j-1}^{L, \phi}\right\rangle-\left\langle e_{j}^{R} \otimes e_{j}^{R, \phi} \mid e_{j}^{R} \otimes e_{j}^{R, \phi}\right\rangle\right) .
\end{aligned}
$$

We can bound the sums over the open bonds for the scalar products in terms of absolute values 
of expectation values of effective Hamiltonians $\hat{h}_{j}^{L / R \text {,eff }}$ at site $j$

$$
\begin{aligned}
& \left.\sum_{\substack{m_{j-1} \\
\bar{m}_{j-1}}}\left\langle e_{j-1}^{L} \otimes e_{j-1}^{L, \phi} \mid e_{j-1}^{L} \otimes e_{j-1}^{L, \phi}\right\rangle \leq \sum_{\substack{m_{j-1} \\
\bar{m}_{j-1}}}|| e_{j-1}^{L} \otimes e_{j-1}^{L, \phi}\right\rangle\left.\right|^{2}=\left|\bar{\psi}_{j-1}^{\mathrm{eff}} \hat{h}_{j-1}^{L, \mathrm{eff}} \psi_{j-1}^{\mathrm{eff}} \cdot \bar{\phi}_{j-1}^{\mathrm{eff}} \hat{h}_{j-1}^{L, \mathrm{eff}} \psi_{j-1}^{\mathrm{eff}}\right| \\
& \left.\sum_{\substack{m_{j} \\
\bar{m}_{j}}}\left\langle d_{j}^{R} \otimes \bar{d}_{j}^{R, \phi} \mid d_{j}^{R} \otimes \bar{d}_{j}^{R, \phi}\right\rangle \leq \sum_{\substack{m_{j} \\
\bar{m}_{j}}}|| d_{j}^{R} \otimes \bar{d}_{j}^{R, \phi}\right\rangle\left.\right|^{2}=\left|\bar{\phi}_{j}^{\mathrm{eff}}\left[\hat{h}_{j}^{R, \mathrm{eff}}\right]^{2} \psi_{j}^{\mathrm{eff}}\right| \\
& \left.\sum_{\substack{m_{j-2} \\
\bar{m}_{j-2}}}\left\langle d_{j-1}^{L} \otimes \bar{d}_{j-1}^{L, \phi} \mid d_{j-1}^{L} \otimes \bar{d}_{j-1}^{L, \phi}\right\rangle \leq \sum_{\substack{m_{j-2} \\
\bar{m}_{j-2}}}|| d_{j-1}^{L} \otimes \bar{d}_{j-1}^{L, \phi}\right\rangle\left.\right|^{2}=\left|\bar{\phi}_{j-1}^{\mathrm{eff}}\left[\hat{h}_{j-1}^{L, \mathrm{eff}}\right]^{2} \psi_{j-1}^{\mathrm{eff}}\right| \\
& \left.\sum_{\substack{m_{j-1} \\
\bar{m}_{j-1}}}\left\langle e_{j}^{R} \otimes e_{j}^{R, \phi} \mid e_{j}^{R} \otimes e_{j}^{R, \phi}\right\rangle \leq \sum_{\substack{m_{j-1} \\
\bar{m}_{j-1}}}|| e_{j}^{R} \otimes e_{j}^{R, \phi}\right\rangle\left.\right|^{2}=\left|\bar{\psi}_{j}^{\mathrm{eff}} \hat{h}_{j}^{R, \mathrm{eff}} \psi_{j}^{\mathrm{eff}} \cdot \bar{\phi}_{j}^{\mathrm{eff}} \hat{h}_{j}^{R, \mathrm{eff}} \psi_{j}^{\mathrm{eff}}\right|
\end{aligned}
$$

where we defined

$$
\hat{h}_{j}^{L, \text { eff }}=L_{j-1} W_{j} \quad \text { and } \quad\left[\hat{h}_{j}^{L, \text { eff }}\right]^{2}=\left(L_{j-1} \otimes L_{j-1}\right)\left(W_{j} \otimes W_{j}\right)
$$

and in a similiar way $\hat{h}_{j}^{R \text {,eff }}$ replacing $L_{j-1} \rightarrow R_{j+1}$. The formal absolute values "|." on the right side can be estimated by replacing the $L_{j} / R_{j}$ tensors with fractions of the overall energy expectation value. This is a valid approximation as long as there are no interactions connecting the left/right contracted MPS-MPO-MPS tensor networks with sites to the right/left of them, which was exactly the condition for non-vanishing contributions to the commutator. We hence compare only squares of single-site expectation values with either one or two MPO site tensors sandwiched between the effective site tensors at sites $j-1, j$. For a large system with smoothly varying site tensors the differences at neighboring sites are negligible so that the commutator can be estimated to

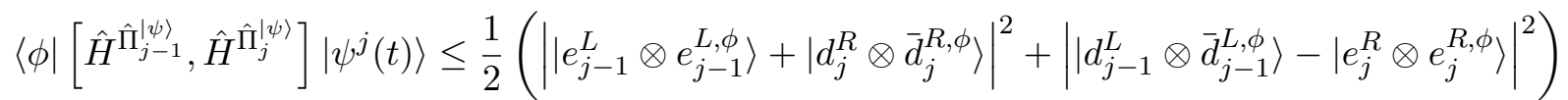

$$
\begin{aligned}
& \left.\left.\leq\left.\frac{1}{2}\left(|| e_{j-1}^{L} \otimes e_{j-1}^{L, \phi}\right\rangle\right|^{2}+|| d_{j}^{R} \otimes \bar{d}_{j}^{R, \phi}\right\rangle\left.\right|^{2}\right) .
\end{aligned}
$$

The last expression can be estimated easily by the coupling strength of the nearest-neighbor interaction term, which we denote by $\Gamma$. The contributions from the boundary tensors $L_{j} / R_{j}$ are bounded by the square of the system size and cancel with the prefactors $1 / L$ of each projected Hamiltonian. We hence conclude this analysis by the somewhat straightforward statement that in general the error at second order in $\delta$ scales with $L \cdot \Gamma^{2}$. However, there is one special situation that simplifies the preceeding arguments drastically namely if we set $|\phi\rangle=\left|\psi^{j}(t)\right\rangle$. Then the commutator compares only local overlaps between site tensors sandwiched between either one or two MPO tensors on neighboring sites, which scales as $1 / L$. Therefore equal-time observables are evolved with very high precision $\sim 1 / L^{2}$ and the contribution due to nearest-neighbor interactions is strongly suppressed. 


\section{Time-dependent variational principle (TDVP) $\mid 4.5 .4$}

There is an alternative to the Lie-Trotter decomposition introduced in the previous Sec. 4.5.3, which also results in a series of local problems: the time-dependent variational principle $\left[\mathrm{HCO}^{+} 11\right.$, $\mathrm{HLO}^{+} 16$. The motivation of this approach is quite different though: its primary aim is to constrain the time evolution to a specific manifold of MPS of a given initial bond dimension. To do so, it projects the action of the Hamiltonian into the tangent space to this manifold at $|\psi(t)\rangle$ and then solves the TDSE solely within the manifold. While TDVP is originally formulated and ideally used in its single-site variant, the two-site variant allows for flexibility in the bond dimension just as in the case of the local Krylov.

There is also another point of view that closely relates the time-dependent variational principle to the local Krylov described above. In the latter method a projection of the ansatz states is required to render the derivative in the decoupled problems to act only locally on the active site-tensor. The projection manifests itself by solving local problems for transformed site tensors $Q_{j-1}^{L} M_{j} Q_{j}^{R}$ where the basis transformations $Q_{j}^{L / R}$ are complete only without truncation. From a conceptual point of view this is an ad-hoc construction and to the best of our knowledge there is no general notion of how controlled it is if truncation errors are present. It is this point where the projection into the tanget space enters the game allowing for an optimal approximation of the dynamics in a well-defined subspace.

\section{Tangent space and matrix-product states}

The foundation of the TDVP splitting of the time-dependent Schrödinger equation is a representation of the projection operator $\hat{P}_{T_{|\psi\rangle}}$ to the tangent space of the manifold of MPS with a fixed bond dimension using gauge-fixing projectors as introduced in Sec. 4.5.3. The general idea is to interpret matrix-product states with a fixed bond dimension $m$ as a smooth submanifold $\mathcal{M}$ of the full many-body Hilbert space, which is parametrized by the $L \sigma \mathrm{m}^{2}$ coefficients of the site tensors $M_{j ; m_{j-1}, m_{j}}^{\sigma_{j}}$. Clearly, $\mathcal{M}$ is non-linear because the addition of two MPS in $\mathcal{M}$ in general does not have bond dimension $m$ and by the same reasoning, linear operators on $\mathcal{H}$ in general are non-linear on $\mathcal{M}$, too $^{17}$. Therefore, it is desirable to linearize the manifold and we begin by expanding variations with respect to a parameter $s$ to first order

$$
\begin{aligned}
\left|\psi\left[\left\{M_{j}\right\}\right]\left(s_{0}+\delta s\right)\right\rangle & \approx\left|\psi\left[\left\{M_{j}\right\}\right]\left(s_{0}\right)\right\rangle+\left.\delta s \sum_{j} \underbrace{\left[\frac{\partial}{\partial s} M_{j}(s)\right]}_{:=B_{j}(s)} \underbrace{\frac{\partial}{\partial M_{j}}\left|\psi\left[\left\{M_{j}\right\}\right]\right\rangle}_{:=\left|\partial_{j} \psi\left[\left\{M_{j}\right\}\right]\right\rangle}\right|_{s_{0}} \\
& \equiv\left|\psi\left[\left\{M_{j}\right\}\right]\left(s_{0}\right)\right\rangle+\delta s \sum_{j} B_{j}\left(s_{0}\right)\left|\partial_{j} \psi\left[\left\{M_{j}\right\}\right]\right\rangle,
\end{aligned}
$$

where we introduced the shorthand notation $\left|\psi\left[\left\{M_{j}\right\}\right]\right\rangle \equiv\left|\psi\left[\left\{M_{1}, \ldots, M_{L}\right\}\right]\right\rangle$ for the MPS parametrization of the state $|\psi\rangle$ in the manifold $\mathcal{M}$. Without loss of generality in each summand

\footnotetext{
${ }^{17}$ We need to be a bit more careful here. Actually, it is not the bond dimension $m$ defining $\mathcal{M}$ but the rank vector $\mathbf{r}=\left(\begin{array}{llll}r_{1} & r_{2} & \cdots & r_{L-1}\end{array}\right)$ of the site tensors in their left canonical representations where $r_{j} \leq m$. It is then clear that states that are linearly dependent up to a phase factor will leave the resulting state in $\mathcal{M}$. Apart from these special situations in general the addition of two states will create a state exhibiting a different rank vector $\mathbf{r}^{\prime} \neq \mathbf{r}$ and the state will not be in $\mathcal{M}$. In the main text we will use the maximal bond dimension $m$ of a state synonymously for the upper bound of the rank vector and assume all site tensors to have full rank upto $r_{j}=m$.
} 
let $B_{j}\left|\partial_{j} \psi\left[\left\{M_{j}\right\}\right]\right\rangle$ be in a mixed canonical representation with center of orthogonality at site $j$ and define the subspace $\mathcal{V}_{j} \subset \mathcal{H}$

$$
\mathcal{V}_{j}=\left\{\delta B_{j}\left|\partial_{j} \psi\left[\left\{M_{j}\right\}\right]\right\rangle \in \mathcal{H} \mid \delta B_{j} \in \mathbb{V}^{\sigma \times m \times m}, \sum_{\sigma_{j}} \bar{M}^{\sigma_{j}} \delta B^{\sigma_{j}}=\mathbf{0}_{j}\right\},
$$

i.e., the coefficient tensors $\delta B_{j}$ fulfill the gauge constraints $\sum_{\sigma_{j}} \bar{M}^{\sigma_{j}} \delta B^{\sigma_{j}}=\mathbf{0}_{j}$. Note that reshaping the tensors into matrices $\delta \mathbf{B}_{j}=\left(B_{j}^{1} \cdots B_{j}^{\sigma}\right)^{t}$ the gauge constraints are equivalent to force $\delta \mathbf{B}_{j}$ to lie in the null space of $\overline{\mathbf{M}}_{j}$, which we will denote compactly as $\overline{\mathbf{M}}_{j} \cdot \delta \mathbf{B}_{j}=\mathbf{0}_{j}$. Then, it has been shown [HRS12] that the single-site tangent space $T_{|\psi\rangle} \mathcal{M}$ at $|\psi\rangle$ orthogonally decomposes as

$$
T_{|\psi\rangle} \mathcal{M}=\mathcal{V}_{1} \oplus \mathcal{V}_{2} \oplus \cdots \oplus \mathcal{V}_{L}
$$

In fact, by using the argument graphically shown in Fig. 4.21 and the additional gauge conditions $\overline{\mathbf{M}}_{j} \cdot \delta \mathbf{B}_{j}=\mathbf{0}_{j}$ it is easy to see that $\left\langle X_{i}, X_{j}\right\rangle=\delta_{i, j}$ for $X_{i, j} \in \mathcal{V}_{i, j}$. The orthogonal decomposition of the tangent space is the key to decouple the time-dependent Schrödinger equation by a LieTrotter splitting into strictly local problems. We, hence, need a representation of the projector to the tangent space $\hat{P}_{T_{|\psi\rangle}}$ and we give a sketch of its derivation motivated by Lubich et al. [LOV15]. Consider a state $|\phi\rangle \in \mathcal{H}$ and its projection $|\delta \phi\rangle$ into the tangent space $T_{|\psi\rangle} \mathcal{M}$ at $|\psi\rangle \in \mathcal{H}$. Geometrically, the projection $|\delta \phi\rangle \in T_{|\psi\rangle} \mathcal{M}$ has to obey the following equality

$$
\langle\phi \mid \delta \psi\rangle=\langle\delta \phi \mid \delta \psi\rangle
$$

and from Eq. (4.172) it follows that the overlaps decompose into the orthogonal subspaces so that the condition reduces to

$$
\left\langle\phi \mid \delta \psi_{j}\right\rangle=\left\langle\delta \phi_{j} \mid \delta \psi_{j}\right\rangle \equiv\left\langle\hat{P}_{j ; T_{|\psi\rangle}} \phi \mid \delta \psi_{j}\right\rangle,
$$

where we introduced the short hand notation $\left|\delta \psi_{j}\right\rangle=\delta M_{j}\left|\partial_{j} \psi\left[\left\{M_{j}\right\}\right]\right\rangle$ for tangent vectors $\left|\delta \psi_{j}\right\rangle \in \mathcal{V}_{j}$. Since $\left|\delta \psi_{j}\right\rangle$ is in a mixed canonical representation with left-/right-canonical site tensors $A_{k<j}, B_{k>j}$ drawn from $|\psi\rangle$ we can use Eq. (4.138) and insert a projection $\hat{\Pi}_{j}^{|\psi\rangle}$ in the left hand site

$$
\left\langle\phi \mid \hat{\Pi}_{j}^{|\psi\rangle} \delta \psi_{j}\right\rangle=\left\langle\hat{\Pi}_{j}^{|\psi\rangle} \phi \mid \delta \psi_{j}\right\rangle .
$$

Refering to Eq. (4.146) we find that the state $\left|\hat{\Pi}_{j}^{|\psi\rangle} \phi\right\rangle$ is now also in a mixed-canonical representation and the active site tensor is transformed as $M_{j}^{\phi} \rightarrow Q_{j-1}^{L} M_{j}^{\phi} Q_{j}^{R}:=\tilde{M}_{j}^{\phi}$. Now, the transformed and reshaped site tensor $\tilde{\mathbf{M}}_{j}^{\phi}$ in general does not need to obey the gauge constraint for tangent vectors, that is, $\overline{\mathbf{M}}_{j}^{\psi} \cdot \tilde{\mathbf{M}}_{j}^{\phi} \neq \mathbf{0}_{j}$. But this is easy to account for by using multi-linearity of the MPS representation and inserting a suitable projector

$$
\tilde{\mathbf{M}}_{j}^{\phi} \rightarrow \underbrace{\left(\hat{\mathbf{1}}_{j}-C \cdot \mathbf{M}_{j}^{\psi} \overline{\mathbf{M}}_{j}^{\psi}\right)}_{\hat{P}_{M_{j}^{\psi}}^{\perp}} \cdot \tilde{\mathbf{M}}_{j}^{\phi}
$$

where $C$ is a proper normalization constant so that $\left[\hat{P}_{M_{j}^{\psi}}^{\perp}\right]^{2}=\hat{P}_{M_{j}^{\psi}}^{\perp}$. Normalization is achieved by constructing $\hat{P}_{M_{j}^{\psi}}^{\perp}$ from the left-canonical site-tensor $A_{j}^{\psi}$ obtained from shifting the center of 


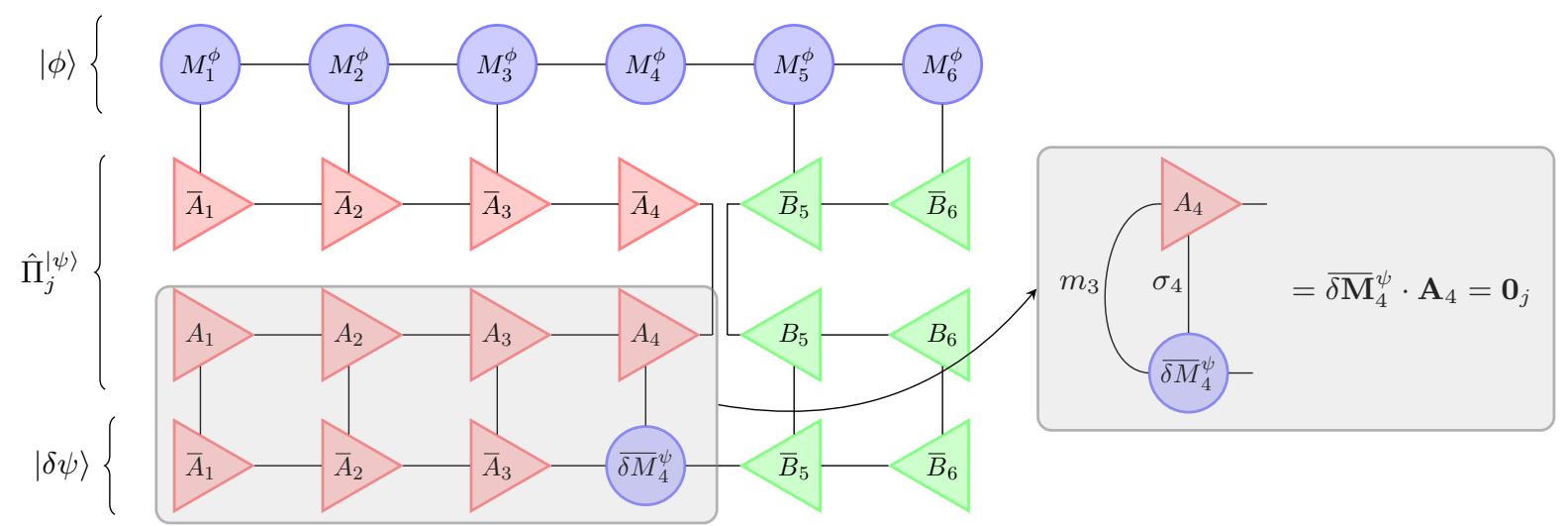

Figure 4.26: Evaluation of the action of the gauge-constraint restoring projector in the overlap $\left\langle\left(\hat{P}_{j}^{L,|\psi\rangle} \otimes \hat{P}_{j+1}^{R,|\psi\rangle}\right) \phi \mid \delta \psi_{j}\right\rangle$ at the example of a system with $L=6$ and center of orthogonality at site $j=4$. Contracting the gray shaded box on the left yields the gauge-constraint for tangent space vectors $\bar{\delta} \mathbf{M}_{j}^{\psi} \cdot \mathbf{A}_{4}=\mathbf{0}_{j}$ so that the whole scalar product vanishes.

orthogonality of $|\psi\rangle$ to the next site $j+1$ so that we obtain

$$
\hat{P}_{M_{j}^{\psi}}^{\perp}=\hat{\mathbf{1}}_{j}-\mathbf{A}_{j}^{\psi} \overline{\mathbf{A}}_{j}^{\psi} .
$$

Note that the scalar product $\left\langle\hat{\Pi}_{j}^{|\psi\rangle} \phi \mid \delta \psi_{j}\right\rangle$ is invariant under insertion of a projector to the null space of $\overline{\mathbf{M}}_{j}^{\psi}$. This can be seen most easily by expanding the additional term $\mathbf{A}_{j}^{\psi} \overline{\mathbf{A}}_{j}^{\psi}$ in the representation of $\hat{\Pi}_{j}^{|\psi\rangle}$

$$
\left\langle\hat{\Pi}_{j}^{|\psi\rangle} \phi \mid \delta \psi_{j}\right\rangle \rightarrow\left\langle\hat{\Pi}_{j}^{|\psi\rangle} \phi \mid \delta \psi_{j}\right\rangle-\left\langle\left(\hat{P}_{j}^{L,|\psi\rangle} \otimes \hat{P}_{j+1}^{R,|\psi\rangle}\right) \phi \mid \delta \psi_{j}\right\rangle
$$

Evalulation of the second scalar product reveals the appearance of the gauge constraint for the tangent vector $\left|\delta \psi_{j}\right\rangle$ (see also Fig. 4.26) so that $\left\langle\left(\hat{P}_{j}^{L,|\psi\rangle} \otimes \hat{P}_{j+1}^{R,|\psi\rangle}\right) \phi \mid \delta \psi_{j}\right\rangle \equiv 0$. Also note that at $j=L$ the action of the gauge-constraint restoring projection is trivial in the sense that it only projects to the last dummy index and it is usually dropped $\hat{P}_{L}^{L,|\psi\rangle} \otimes \hat{P}_{L+1}^{R,|\psi\rangle} \equiv 0$. Therefore, the first equality in Eq. (4.174) is fulfilled if we insert the projector $\hat{\Pi}_{j}^{|\psi\rangle}$ enhanced by the projection $\hat{P}_{j}^{L,|\psi\rangle} \otimes \hat{P}_{j+1}^{R,|\psi\rangle}$ to fulfill the gauge-constraints for tangent vectors

$$
\left\langle\delta \phi_{j} \mid \delta \psi_{j}\right\rangle=\left\langle\phi\left|\hat{P}_{j-1}^{L,|\psi\rangle} \otimes \hat{\mathbf{1}}_{j} \otimes \hat{P}_{j+1}^{R,|\psi\rangle}-\hat{P}_{j}^{L,|\psi\rangle} \otimes \hat{P}_{j+1}^{R,|\psi\rangle}\right| \delta \psi_{j}\right\rangle
$$

and thus, from Eq. (4.172) we deduce that the projector to the tangent space $T_{|\psi\rangle} \mathcal{M}$ is given by

$$
\hat{P}_{T_{|\psi\rangle}}=\sum_{j} \hat{P}_{j ; T|\psi\rangle}=\sum_{j=1}^{L} \hat{P}_{j-1}^{L,|\psi\rangle} \otimes \hat{\mathbf{1}}_{j} \otimes \hat{P}_{j+1}^{R,|\psi\rangle}-\sum_{j=1}^{L-1} \hat{P}_{j}^{L,|\psi\rangle} \otimes \hat{P}_{j+1}^{R,|\psi\rangle} .
$$

If we take a step back and have a look at the very result of this discussion we find a remarkable connection to the projection explored in the derivation of the local Krylov method Sec. 4.5.3. The operator $\hat{\Pi}_{j}^{|\psi\rangle}$ introduced therein is only corrected by the gauge-constraint restoring contribution $\sum_{j=1}^{L-1} \hat{P}_{j}^{L,|\psi\rangle} \otimes \hat{P}_{j+1}^{R,|\psi\rangle}$, which takes the projected states to the tangent space. But we can also put it the other way: The projection appearing in the local Krylov method lacks a gauge-fixing 
constraint to project the time evolution into a proper subspace. As we noted at the beginning of this section this means that linearity of the projected time-dependent Schrödinger equation in the submanifold $\mathcal{M}$ is not restored in case of the local Krylov method. Here, we can connect to the error analysis in Sec. 4.5.3, which revealed that equal-time expectation values of time-evolved states can be evaluated very exactly because effects of the unconstraint projection happened to cancel each other. But this is not the case for a general expectation value as we illustrate in the following. Historically, using the time-step targeting tDMRG with the projection as described in Sec. 4.5.3 turned out to be very challenging when evaluating time-dependent correlation functions of the form $\left\langle\psi\left|\hat{O}_{i}^{\dagger}(t) \hat{O}_{j}(0)\right| \psi\right\rangle$. It is only reasonable to assume that this can be addressed to the fact that in each time step evolved under the local Krylov the states pick up uncontrolled contributions in the orthogonal complement of $T_{|\psi\rangle} \mathcal{M}$. These contributions may, for instance, destroy the phase coherence of the states, which is very important for the correct evaluation of time-dependent correlation functions.

\section{Decomposing the time-dependent Schrödinger equation}

The main difference between the TDVP and the local Krylov method is in the derivation of the series of the local time-dependent Schrödinger equations. Here, the starting point is to consider the submanifold $\mathcal{M} \subset \mathcal{H}$ of MPS $\left|\psi\left\{M_{j}\right\}\right\rangle \in \mathcal{M}$ parametrized by site tensors $M_{j}$ with a fixed bond dimension $m$. The time-derivative of a state in $\mathcal{M}$ is then an element of the tangent space at that point

$$
\frac{d}{d t}\left|\psi\left\{M_{j}(t)\right\}\right\rangle \in T_{|\psi(t)\rangle} \mathcal{M}
$$

On the other hand, the action of a generic Hamiltonian $\hat{H}$ in general takes the state out of the manifold $\hat{H}\left|\psi\left\{M_{j}(t)\right\}\right\rangle \notin \mathcal{M}$. But we can formulate a variational principle by demanding that we want to approximate the exact time-evolved state $|\psi(t+\delta)\rangle_{\text {exact }} \in \mathcal{H}$ by a state $|\psi(t+\delta)\rangle \in \mathcal{M}$ in an optimal way, that is, by minimizing the distance

$$
|\psi(t+\delta)\rangle=\min _{\phi \in \mathcal{M}} \||\psi(t+\delta)\rangle_{\text {exact }}-|\phi\rangle \|^{2} .
$$

From elementary differential geometry it follows that this is equivalent to projecting the TDSE into the tangent space $T_{|\psi\rangle} \mathcal{M}$ so that by inserting the projector $\hat{P}_{|\psi\rangle}$ we obtain

$$
\begin{aligned}
-\mathrm{i} \frac{d}{d t}|\psi(t)\rangle & =\hat{P}_{T_{|\psi\rangle}} \hat{H}|\psi\rangle=\sum_{j} \hat{P}_{j ; T|\psi\rangle} \hat{H}|\psi\rangle \\
& =\sum_{j=1}^{L} \hat{P}_{j-1}^{L,|\psi\rangle} \otimes \hat{\mathbf{1}}_{j} \otimes \hat{P}_{j+1}^{R,|\psi\rangle} \hat{H}|\psi\rangle-\sum_{j=1}^{L-1} \hat{P}_{j}^{L,|\psi\rangle} \otimes \hat{P}_{j+1}^{R,|\psi\rangle} \hat{H}|\psi\rangle .
\end{aligned}
$$

Then, the same considerations as in Sec. 4.5.3 can be employed to construct a first order integration scheme in the interval $\left[t_{0}, t_{0}+\delta\right]$ by solving $2 L-1$ problems which are coupled through there initial value conditions:

$$
\begin{aligned}
-\mathrm{i} \frac{d}{d t}\left|\psi^{j, f}(t)\right\rangle & =\left[\hat{P}_{j-1}^{L,|\psi\rangle} \otimes \hat{\mathbf{1}}_{j} \otimes \hat{P}_{j+1}^{R,|\psi\rangle}\right] \hat{H}\left|\psi^{j, f}(t)\right\rangle \quad \text { (forward evolution) } \\
\text { with } \quad\left|\psi^{j, f}\left(t_{0}\right)\right\rangle & =\left|\psi^{j-1, b}\left(t_{0}+\delta\right)\right\rangle,
\end{aligned}
$$

and for $j<L$

$$
\begin{aligned}
\mathrm{i} \frac{d}{d t}\left|\psi^{j, b}(t)\right\rangle & =\left[\hat{P}_{j}^{L,|\psi\rangle} \otimes \hat{P}_{j+1}^{R,|\psi\rangle}\right] \hat{H}\left|\psi^{j, b}(t)\right\rangle \quad \text { (backward evolution) } \\
\text { with } \quad\left|\psi^{j, b}\left(t_{0}\right)\right\rangle & =\left|\psi^{j, f}\left(t_{0}+\delta\right)\right\rangle
\end{aligned}
$$




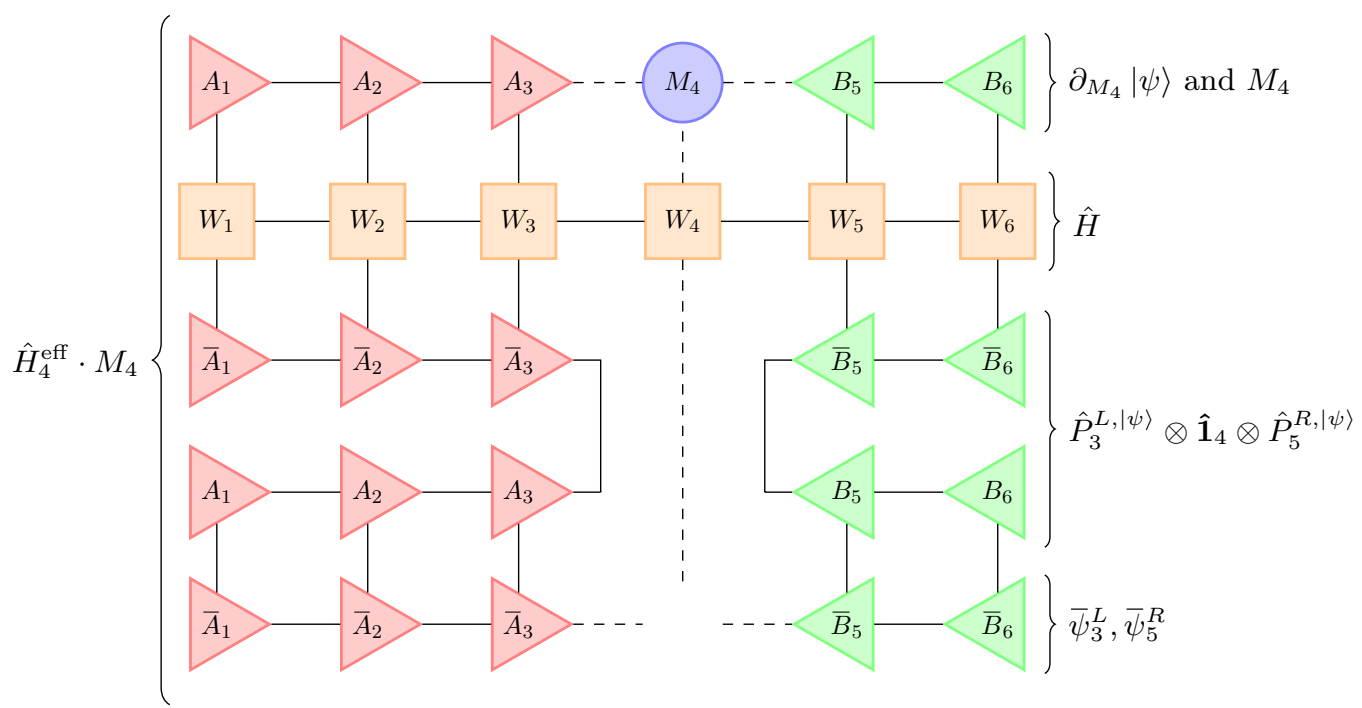

Figure 4.27: Right-hand side of the effective single-site forwards-evolving Schrödinger equation (with $j=4)$. The effective Hamiltonian $\hat{H}_{j}^{\text {eff }}$ is given by the cornered green, orange and red tensors. The effective state is given by the blue circled tensor $M_{j}$. During the calculation, the connected dashed lines are contracted, resulting in a new tensor with three legs (the three open dashed lines).

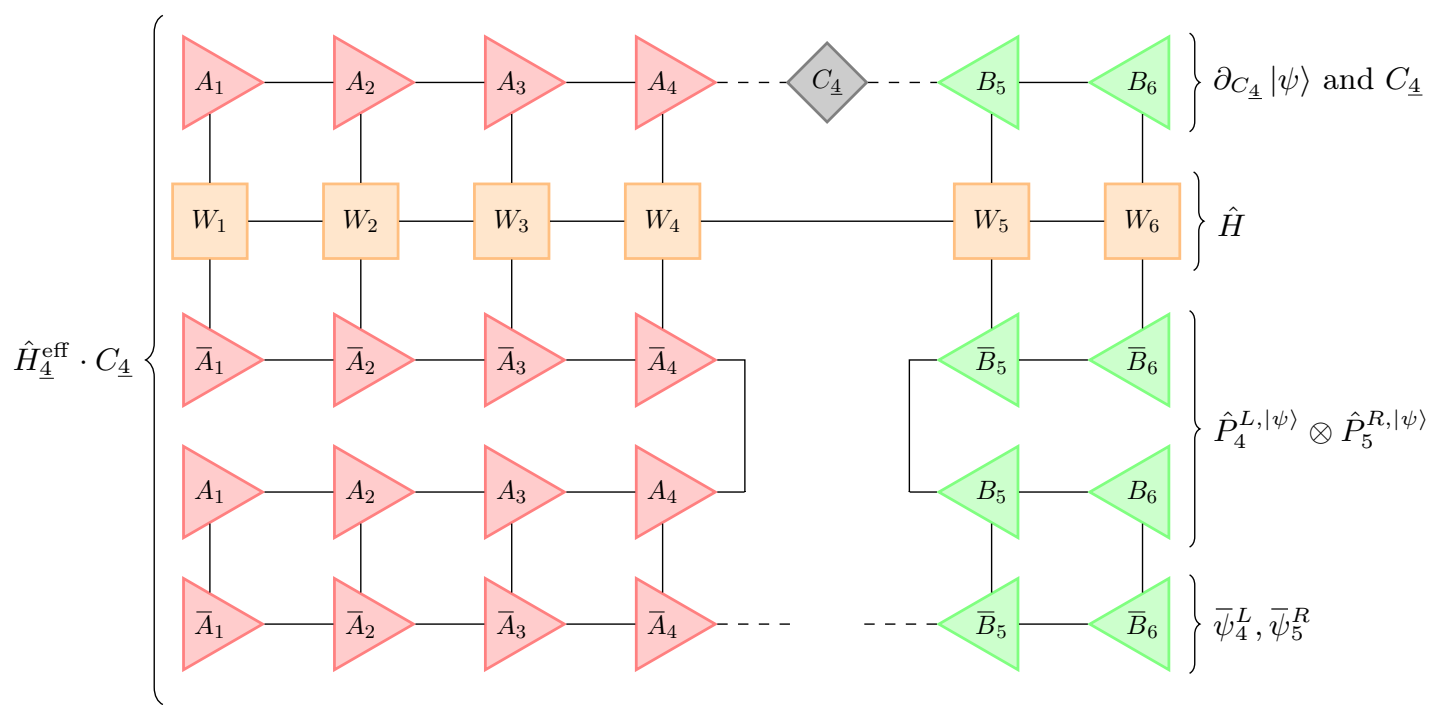

Figure 4.28: Right-hand side of the effective center matrix backward-evolving Schrödinger equation with $\underline{j}=4$. The effective state over the bond between sites $j$ and $j+1$ is given by the grey diamond $C_{\underline{j}}$. During the calculation, the connected dashed lines are contracted, resulting in a new tensor with two legs (the two open dashed lines). 
Here, we identify $\left|\psi^{1, f}\left(t_{0}\right)\right\rangle \equiv\left|\psi\left(t_{0}\right)\right\rangle$ and the final time-evolved state $\left|\psi\left(t_{0}+\delta\right)\right\rangle \equiv\left|\psi^{L, f}\left(t_{0}+\delta\right)\right\rangle$. Due to the orthogonal decomposition of $T_{|\psi\rangle} \mathcal{M}$ (c.f. Eq. (4.172)) the time derivative in the $j$ th problem acts non-trivially only in the subspace $\mathcal{V}_{j}$. Additionally, in [LOV15] it has been shown that updating the projectors in a sweep from the left to the right according to

$$
\hat{P}_{j-1}^{L,|\psi\rangle}=\sum_{\substack{\sigma_{1}, \ldots, \sigma_{j-1} \\ \sigma_{1}^{\prime}, \ldots, \sigma_{j-1}^{\prime} \\ m_{j-1}}} \psi_{j-1 ; m_{j-1}}^{L ; j-1, b}\left(t_{0}+\delta\right) \bar{\psi}_{j-1 ; m_{j-1}}^{L ; j-1, b}\left(t_{0}+\delta\right)\left|\sigma_{1}, \ldots, \sigma_{j-1}\right\rangle\left\langle\sigma_{1}^{\prime}, \ldots, \sigma_{j-1}^{\prime}\right| \otimes \hat{\mathbf{1}}_{j}^{R}
$$

when solving the $j$ th forward evolution and

$$
\hat{P}_{j}^{L,|\psi\rangle}=\sum_{\substack{\sigma_{1}, \ldots, \sigma_{j} \\ \sigma_{1}^{\prime}, \ldots, \sigma_{j}^{\prime} \\ m_{j}}} \psi_{j ; m_{j}}^{L ; j, f}\left(t_{0}+\delta\right) \bar{\psi}_{j ; m_{j}}^{L ; j, f}\left(t_{0}+\delta\right)\left|\sigma_{1}, \ldots, \sigma_{j}\right\rangle\left\langle\sigma_{1}^{\prime}, \ldots, \sigma_{j}^{\prime}\right| \otimes \hat{\mathbf{1}}_{j+1}^{R}
$$

when solving the $j$ th backwards evolution, the time derivatives act non-trivially only on the active site tensor $M_{j}$ (forward evolution, see also Fig. 4.27) or the center matrix $C_{j}$ (backward evolution, see also Fig. 4.28). Note that we have put additional (upper) site labels and letters to the bond maps to indicate the state they are generated from, for instance, the bond map $\psi_{j-1 ; m_{j-1}}^{L ; j-1, b}\left(t_{0}+\delta\right)$ is generated from the left-canonical site-tensors of the solution $\left|\psi^{j-1, b}\left(t_{0}+\delta\right)\right\rangle$ of the previous backwards evolution. We then multiply each individual equation in Eqs. (4.184) and (4.185) by the single-site map $\bar{\psi}_{j-1}^{L ; j-1, b}\left(t_{0}+\delta\right) \otimes \bar{\psi}_{j+1}^{R}$ or the center-bond map $\bar{\psi}_{j}^{L ; j, f} \otimes \bar{\psi}_{j+1}^{R}$, respectively. As a result, instead of having to work with the full MPS $|\psi\rangle$, we can work with the effective single-site and effective center matrix tensors and associated local Schrödinger equations directly:

$$
\begin{aligned}
\frac{\partial}{\partial t} M_{j} & =-\mathrm{i} \hat{H}_{j}^{\mathrm{eff}} M_{j} \\
\frac{\partial}{\partial t} C_{\underline{j}} & =+\mathrm{i} \hat{H}_{\underline{j}}^{\mathrm{eff}} C_{\underline{j}} .
\end{aligned}
$$

The tensor contraction in the RHS of Eq. (4.188) is graphically represented in Fig. 4.27, while the RHS of Eq. (4.189) is shown in Fig. 4.28. Each of these equations can be solved with a local application of the Krylov method much as in DMRG or the local Krylov method of the previous section.

Sweeping right-to-left (rather than left-to-right) through the system results in solving the equations in reverse order. This turns the initial first-order integrator into a second-order integrator, reducing the time step error (as described in Sec. 4.5.4) from $O(\delta)$ to $O\left(\delta^{2}\right)$ if both sweeps are done with halved time steps $\delta / 2$.

An interesting property of the single-site TDVP variant (1TDVP) is that it can be shown that symmetries are conserved by making explicit use of the metric tensor. To be more precise, 1TDVP conserves energy exactly and every other symmetry that is generated from a sum of single-site operators [VHV19]. This is in contrast to the local Krylov method, where the basis transformation generated by the $Q^{L / R}$ tensors (as described at the end of Sec. 4.5.3) continuously introduces errors scaling with the discarded weight and the system size. Alternatively, it is straightforward to extend the mechanism to a two-site variant. This 2TDVP forward-evolves a local tensor $M_{(j, j+1)}$ that needs to be split into two separate site tensors again following the evolution. The advantage is that the bond dimension of the state can be adapted on the fly. However, norm and energy are now no longer conserved exactly if a truncation of the evolved bond is necessary. 


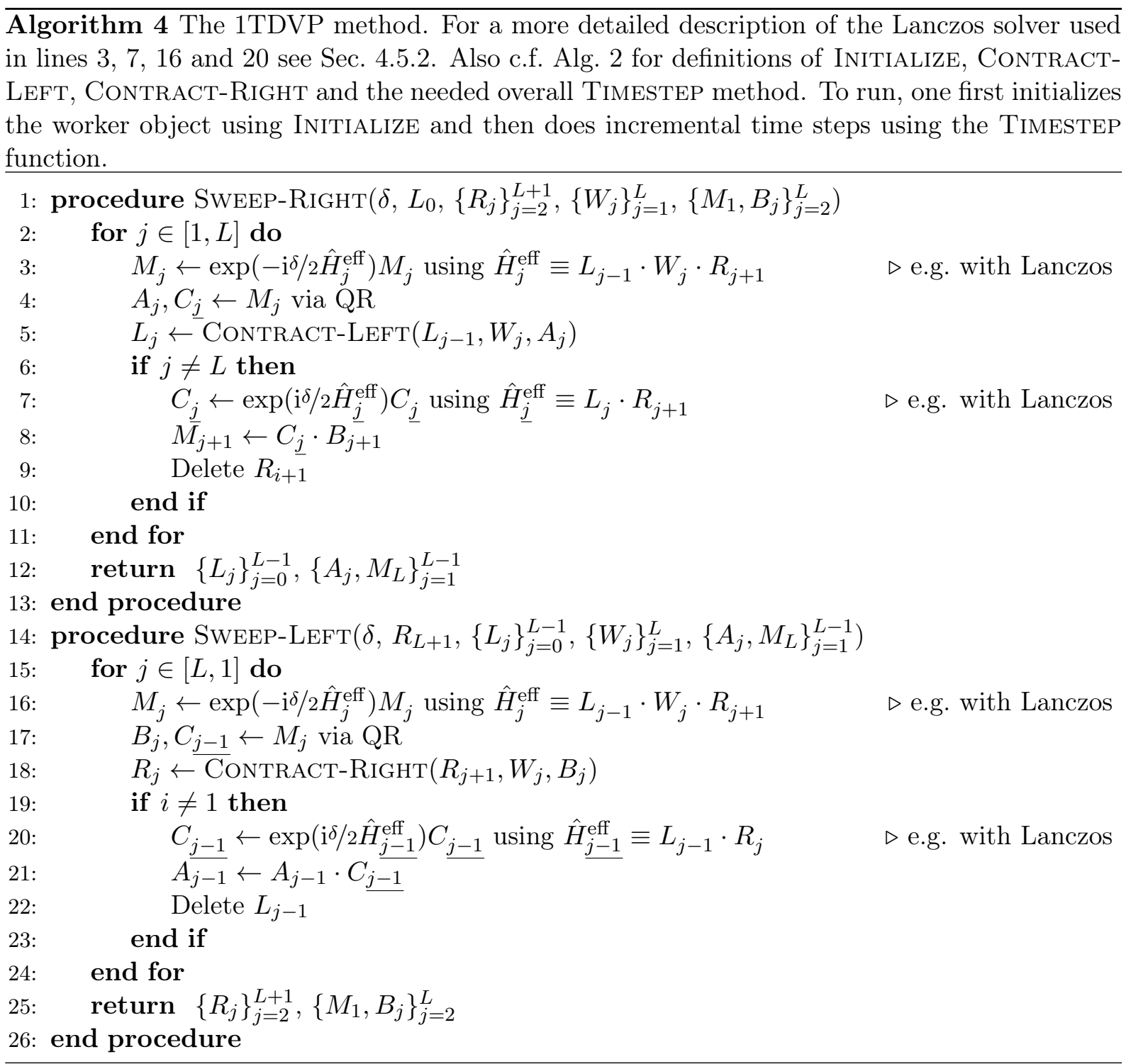

\section{Algorithm}

In practice, the implementation of the $1 / 2$ TDVP method is quite similar to the $1 / 2 \mathrm{DMRG}$ method and nearly identical to the local Krylov method. Compared to ground state DMRG, one of course has to replace the local eigensolver by a local exponentiation $e^{-\mathrm{i} \hat{\delta} H_{j}^{\text {eff }}}$ or $e^{-\mathrm{i} \hat{\delta} H_{j, j+1}^{\text {eff }}}$ for 1TDVP or 2TDVP. Compared to both the local Krylov and the DMRG methods, we also need an additional backwards evolution step either on each bond (1TDVP) or the second site of a two-site evolution (2TDVP). This replacement of the explicit basis transformation done by the local Krylov method with a properly motivated backwards evolution will result in smaller errors in each step. The 1TDVP algorithm is described in detail in Alg. 4, the 2TDVP algorithm in Alg. 5.

\section{Errors}

The TDVP has four sources of errors: firstly, there is a projection error due to the projection of the full time-dependent Schrödinger equation (TDSE) onto the MPS manifold of limited bond 


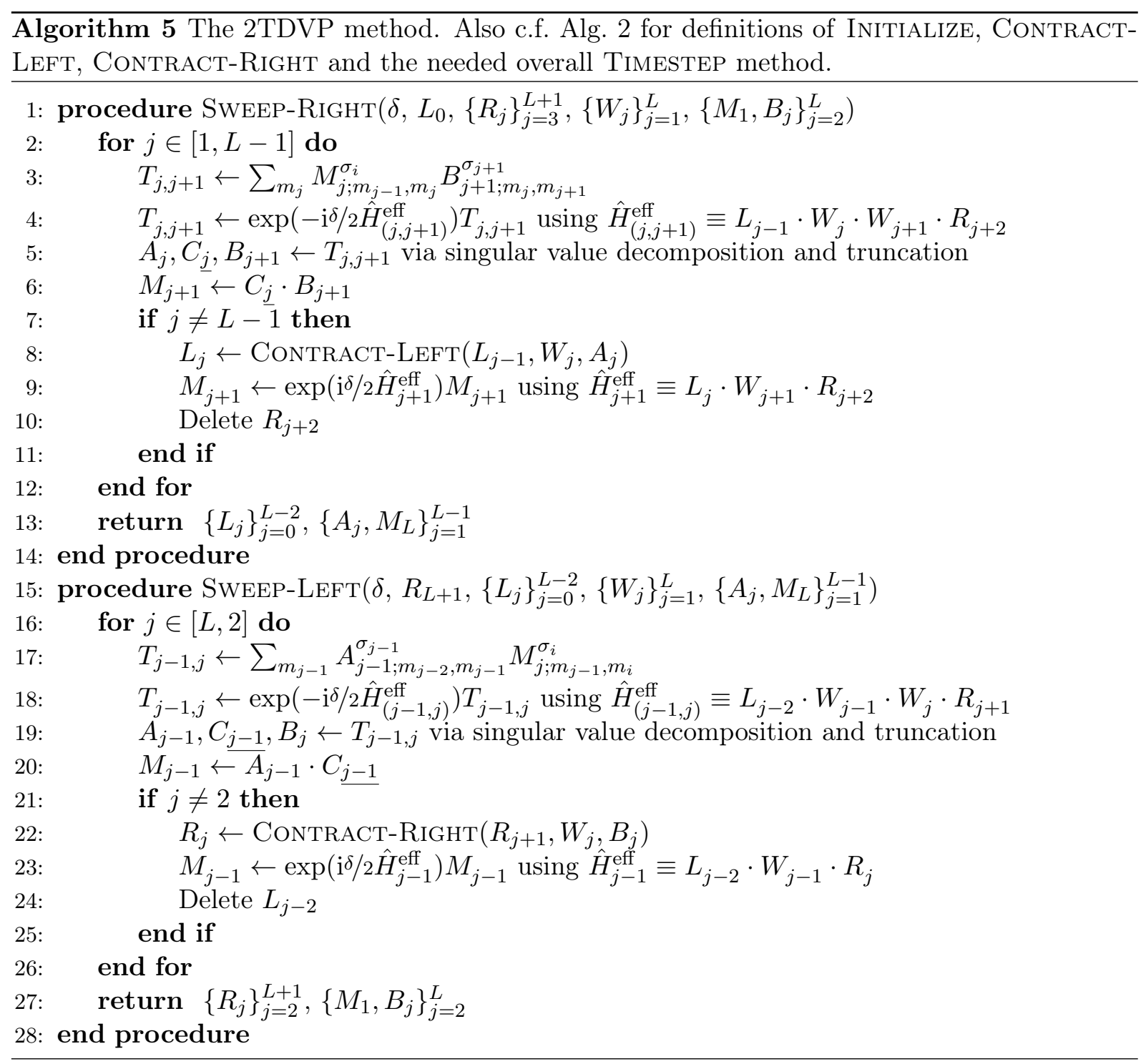

dimension. This error is particularly severe if the MPS in question has a small bond dimension, but it is exactly zero if the MPS has maximal (exponentially growing) bond dimension. However, the projection error occurs during the projection of the TDSE onto the relevant subspace, i.e., before the time evolution. As such, it cannot lead to a violation of energy conservation or change the norm of the time-evolved state (during real-time evolution). Using a two- or multisite variance[HHS18] it is possible to estimate this projection error. If the $n$-site variance of the state is large, the $(n-1)$ TDVP will provide inadequate results. Vice versa, if the up-to- $n$-site variance of a state is small, the $n$ TDVP will consider this state an eigenstate of the Hamiltonian and the time evolution will only add a global phase to the state. As a corollary, the 2TDVP can evolve Hamiltonians with only nearest-neighbor interactions without incurring a projection error.

Second, the chain of forwards and backwards evolutions can be considered a sequential solution of a series of coupled TDSE (which are the result of the projection above), each describing the evolution of any particular site tensor. Except in the special case that all these evolutions describe exactly the same dynamics (due to the state having maximal bond dimension), there is a finite time-step error of order $O\left(\delta^{3}\right)$ per time step and order $O\left(\delta^{2}\right)$ per unit time. In practice, 
the prefactor of this error is often much smaller than, e.g., in a TEBD calculation, in particular if the bond dimension of the input state is reasonably large. If the bond dimension is very small, the time-step error will be relatively large.

Third, the 2TDVP contains a SVD to split the evolved two-site tensor into two separate tensors again. During this SVD, a truncation is typically unavoidable, leading to a measurable truncation error. Careful analysis of this truncation error is necessary as always, but also proceeds in much the same way as always. In 1TDVP, this error is exactly zero.

The fourth source of error lies in the inexact solution of the local equations. Using sufficiently many Krylov vectors locally, it is very easy to make this error small. Therefore, one should always use sufficiently many vectors such that the obtained error is at least smaller than the truncation error in the previous step.

Note that changing the time-step size $\delta$ in the TDVP affects the four errors differently: the projection and truncation error affect each time step relatively independently of the size of that step. Hence, increasing the number of time steps during a fixed total time evolution increases the projection and truncation errors. The finite time-step error and the error from the inexact local solution, on the other hand, decrease when increasing the number of time steps and the total time is kept fixed. As such, choosing a smaller $\delta$ decreases the time-step error but increases the projection and truncation error. It is hence typically useful to take some care when choosing, e.g., the truncation threshold and the time-step size such as to approximately balance the induced errors.

Additionally, the energy, norm and most symmetries of the state are conserved exactly within the 1TDVP and only affected by the truncation error in the 2TDVP. This exact conservation may extend to those quantities that are contained within the Hamiltonian[LPB ${ }^{+} 17$, GD19]. While such energy conservation is certainly very helpful to obtain long-time hydrodynamic observables such as diffusion constants, care has to be taken when using only 1TDVP during the calculation as shown in Ref. [KLR18]. Specifically, one has to take great care to ensure that the obtained data is completely converged in the bond dimension of the state at all times.

\section{Exploiting $U(1)$ symmetries $\mid 4.6$}

The algorithms described in the previous section are founded on evaluations of dense matrixmatrix/vector products and additions as well as matrix factorizations. There are certain exceptions where sparse matrices are constructed, for instance, in the Lanczos method [L50, CW85] but in general there is no way around these numerically expensive operations. However, physical problems often come with certain conserved quantities corresponding to the invariance under global symmetries. From elementary group theory [Zee16] it is well-known that under the action of these symmetries the Hilbert space decomposes into irreducible representations. In principle these can be exploited during numerical calculations, particularly if the considered degrees of freedom are represented by tensors that transform well behaved under the global symmetries. It was clear from the very beginning that the formulation of DMRG allows for taking benefit of global symmetries, both abelean and non-abelean [NW93, RPK ${ }^{+} 96$, MG02] even though exploiting non-abelean symmetries such as the conservation of global $S U(2)$ symmetries turns out to be more challenging [MG02]. In fact, decomposing the reduced density matrices into irreducible representations by introducing a flow of quantum numbers (which may or may not be degenerated) the reduced block sizes speed up calculations drastically, as matrix-matrix contractions and matrix factorizations asymptotically scale with the matrix dimension cubed. Clearly, these ideas can be translated to general tensor networks [SPV10, SPV11, Wei12, Hub17] so that they are in 
particular applicable to speed up calculations using MPS. In the following we will introduce the concepts used to build MPS algorithms taking benefit of conserved global $U(1)$ symmetries as they were implemented in the SYMMPS toolkit developed by the author and T. Köhler in the scope of this thesis.

\section{Reducing computational complexity exploiting symmetries $\mid 4.6 .1$}

The idea of exploiting symmetries in order to reduce the computational complexity may be illustrated best by a particular example. Let $\mathcal{H}=\mathcal{H}_{\sigma}^{\otimes L}$ be a tensor-product Hilbert space describing $L \in \mathbb{N}$ local $\sigma$-dimensional degrees of freedom. Let us consider a Hamiltonian $\hat{H}$ acting on $\mathcal{H}$ that commutes with a global observable $\hat{N}$. Let us further assume that $\hat{N}$ can be decomposed into local operators $\hat{N}=\sum_{j} \hat{n}_{j}$ with $\hat{n}_{j}: \mathcal{H}_{\sigma} \longrightarrow \mathcal{H}_{\sigma}$ acting only on a single lattice site (local operators) and let each of them commute with all the others $\left[\hat{n}_{j}, \hat{n}_{k}\right]=0$. Also, in the following we set their eigenvalues to $n_{j} \equiv(j-1)$ so that the spectrum of the $\hat{n}_{j}$ 's is non-degenerated. ${ }^{18}$ Since $[\hat{H}, \hat{N}]=0$ we can diagonalize both operators $\hat{H}, \hat{N}$ in one and the same basis $|N\rangle$ with $\hat{N}|N\rangle=N|N\rangle$ as well as $\left\langle N \mid N^{\prime}\right\rangle=\delta_{N, N^{\prime}}$ and we will call $N$ the global quantum number of the state $|N\rangle$. Usually, the basis labeled by the eigenstates of $\hat{N}$ is degenerated extensively. In our example, we can specify this degeneracy by making use of the fact that $\hat{N}=\sum_{j} \hat{n}_{j}$, that is, since $\hat{N}$ is conserved and $\left[\hat{n}_{j}, \hat{n}_{k}\right]=0$ we can work in a basis set spanned by the eigenstates of th $\hat{n}_{j}$, i.e.,

$$
\mathcal{H}=\operatorname{span}\left\{\left|n_{1}, n_{2}, \ldots, n_{L}\right\rangle\right\}_{n_{j=1, \ldots, \sigma}} .
$$

Writing $\hat{H}$ in this basis we obtain a matrix $\left\langle n_{1}, \ldots, n_{L}|\hat{H}| n_{1}^{\prime}, \ldots, n_{L}^{\prime}\right\rangle \equiv H$ with dimension $\sigma^{L} \times \sigma^{L}$. But since each basis state $\left|n_{1}, \ldots, n_{L}\right\rangle$ can be identified with a global quantum number $N=\sum_{j} n_{j}$ sorting the rows and columns according their values of $N$ we find that $H$ exhibits a block structure where each block is labeled by $N$. Therefore, all possible combinations of the local occupations $n_{j}$ yielding a certain global quantum number $N$ determines the degeneracy of the corresponding eigenstate $|N\rangle$ of $\hat{H}$. In summary, expressing the Hamiltonian in a basis whose overall occupation $N$ is conserved by $\hat{H}$ we can easily construct a decomposition of $H$ into smaller blocks, which, for instance, can be diagonalized independently. The reduction of computational complexity due to this sparse matrix representation depends on the particular global quantum number sector $N$, i.e., the subblock of $H$ under consideration. Its dimension is given by counting the number of possibilities to occupy $L$ degrees of freedom $n_{j}$ from $0, \ldots, \sigma-1$ so that $\sum_{j} n_{j}=N$.

The given example was, of course, chosen with a certain care. In fact, it corresponds to a representation of the unitary group $U(1)$ on $\mathcal{H}$. The connection to the global symmetry of $\hat{H}$ is found by noting that from $[\hat{H}, \hat{N}]=0$ it follows that eigenstates $|E, N\rangle$ of $\hat{H}$ are invariant under the gauge transformation $|E, N\rangle \rightarrow e^{\mathrm{i} \vartheta \hat{N}}|E, N\rangle$. The fact that $e^{\mathrm{i} \vartheta \hat{N}}$ generates a representation of $U(1)$ is now due to the property that all of the local densities $\hat{n}_{j}$ commute with each other

$$
e^{\mathrm{i} \vartheta_{1} \hat{N}} e^{\mathrm{i} \vartheta_{2} \hat{N}}=e^{\mathrm{i} \vartheta_{1} \sum_{j} \hat{n}_{j}} e^{\mathrm{i} \vartheta_{2} \sum_{j} \hat{n}_{j}}=e^{\mathrm{i}\left(\vartheta_{1}+\vartheta_{2}\right) \sum_{j} \hat{n}_{j}}=e^{\mathrm{i}\left(\vartheta_{1}+\vartheta_{2}\right) \hat{N}} .
$$

There is a lot more to say about representations of symmetry groups (see for instance [Zee16]) but for the current purpose this short detour shall be sufficient. All we need to know is that

\footnotetext{
${ }^{18}$ If the spectrum is degenerate the discussion is merely the same but we have to be a bit more careful expanding operators.
} 
(a)

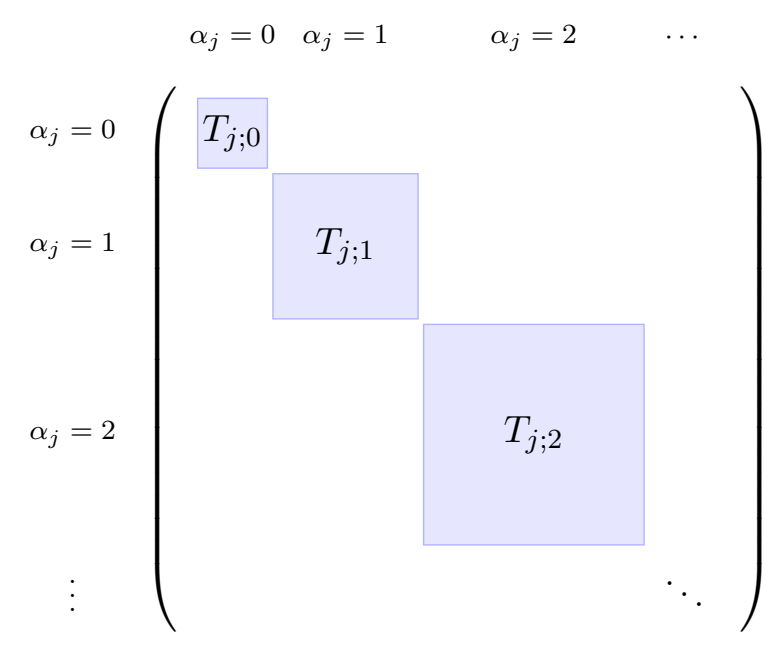

(b)

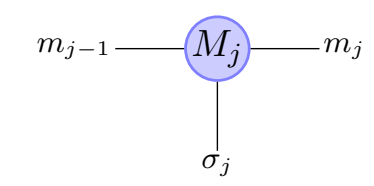

$$
U(1) \downarrow
$$

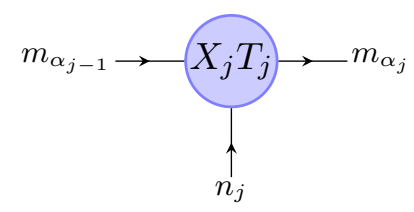

Figure 4.29: In (a) the block diagonal representation $T^{\left(n_{1}, \ldots, n_{j}\right),\left(n_{j+1}, \ldots, n_{L}\right)}=\bigoplus_{\alpha_{j}} Y_{N ; j ; \alpha_{j}} T_{j ; \alpha_{j}}$ of symmetry adapted tensor $T^{n_{1}, \ldots, n_{L}}$ is shown. Each block corresponds to an irreducible representations of the global $U(1)$ symmetry generated by $\hat{N}$. The decomposition of a site tensor under the action of a global $U(1)$ symmetry is shown in (b). Arrows are indicating the flow of local quantum numbers $\alpha_{j-1}, \alpha_{j}, n_{j}$, which is specified by the sign in the conservation laws contained in the structure tensor $X_{j}$.

given a Hamiltonian $\hat{H}$, if there is a global symmetry $e^{\mathrm{i} \vartheta \hat{N}}$ whose generator decomposes into local operators $\hat{N}=\sum_{j} \hat{n}_{j}$, which all commute with each other, we have found a global $U(1)$ symmetry of $\hat{H}$ that can be exploited numerically. Next we are going to elaborate how such a $U(1)$ symmetry can be exploited to construct sparse tensors that are both more memory efficient and computationally advantageous. But before doing so, we want to emphasize that we have not accidentally changed the notation to label the local degrees of freedom from $\sigma_{j}$ to $n_{j}$. In fact, whenever we are working in a basis of an irreducible representation of some global $U(1)$ symmetry we will label the corresponding local basis states by the $n_{j}$ 's to unambigously distinguish them from the general local basis sets used in the previous sections. We will call the eigenvalues $n_{j}$ of $\hat{n}_{j}$ in the irreducible representations local quantum numbers.

\section{Decomposition of general tensor networks}

Let $\hat{H}$ be a Hamiltonian with a global $U(1)$ symmetry generated by $\hat{N}=\sum_{j} \hat{n}_{j}$ as introduced in the example above. Consider a rank- $L$ tensor $T^{n_{1}, \ldots, n_{L}}$ whose elements specify the coefficients of a state $|\psi\rangle \in \mathcal{H}$ in a tensor-product Hilbert space represented in the basis of irreducible representations of the $\hat{n}_{j}$. Finally, let $|\psi\rangle$ be an eigenstate of $\hat{N}$ with global quantum number $N$. Since the global quantum number decomposes as $N=\sum_{j} n_{j}$ the coefficients of $|\psi\rangle$ in the basis of irreducible representations $\left|n_{1}, \ldots, n_{L}\right\rangle$ are constraint by a conservation law

$$
\begin{aligned}
|\psi\rangle & =\sum_{n_{1}, \ldots, n_{L}}\left|n_{1}, \ldots, n_{L}\right\rangle\left\langle n_{1}, \ldots, n_{L} \mid \psi\right\rangle \delta\left(n_{1}+\cdots+n_{L}-N\right) \\
& =\sum_{n_{1}, \ldots, n_{L}} T^{n_{1}, \ldots, n_{L}} \delta\left(n_{1}+\cdots+n_{L}-N\right)\left|n_{1}, \ldots, n_{L}\right\rangle .
\end{aligned}
$$


Now we consider a bipartion $\mathcal{H}=\mathcal{H}_{j}^{L} \otimes \mathcal{H}_{j+1}^{R}$. We can formally factorize the $\delta$ function ensuring the decomposition of the global quantum number $N$ into local ones $n_{j}$

$$
\delta\left(n_{1}+\cdots+n_{L}-N\right)=\sum_{\alpha_{j}} \delta\left(n_{1}+\cdots+n_{j}-\alpha_{j}\right) \delta\left(\alpha_{j}+n_{j+1}+\cdots+n_{L}-N\right) .
$$

Note that for fixed local quantum numbers there is only one value of $\alpha_{j}$ so that the factorization is non-vanishing. However, if we allow for varying local quantum numbers $n_{1}, \ldots, n_{j}$, then $\alpha_{j}$ can have different values corresponding to the possible combinations fulfilling $n_{1}+\cdots+n_{j}=$ $N-\left(n_{j+1}+\cdots+n_{L}\right)$. This yields a decomposition into the left and right basis sets with two conservation laws

$$
\begin{aligned}
|\psi\rangle=\sum_{n_{1}, \ldots, n_{j}} T^{n_{1}, \ldots, n_{L}} \sum_{\alpha_{j}} & \delta\left(n_{1}+\cdots+n_{j}-\alpha_{j}\right)\left|n_{1}, \ldots, n_{j}\right\rangle \times \\
& \delta\left(\alpha_{j}+n_{j+1}+\cdots+n_{L}-N\right)\left|n_{j+1}, \ldots, n_{L}\right\rangle .
\end{aligned}
$$

We can now reshape the tensor $T$ into a matrix w.r.t. the chosen bipartition of the Hibert space

$$
T^{n_{1}, \ldots, n_{L}} \rightarrow T^{\left(n_{1}, \ldots, n_{j}\right),\left(n_{j+1}, \ldots, n_{L}\right)} .
$$

Reordering the rows and columns of $T^{\left(n_{1}, \ldots, n_{j}\right),\left(n_{j+1}, \ldots, n_{L}\right)}$ so that

- rows with the same value $\alpha_{j}=\sum_{k \leq j} n_{k}$ are grouped together

- columns with the same value $\alpha_{j}=N-\sum_{k>j} n_{k}$ are grouped together

we find that the conservation laws enforce a block structure in the reshaped tensor so that it can be decomposed as

$$
\begin{aligned}
T & =T^{\left(n_{1}, \ldots, n_{j}\right),\left(n_{j+1}, \ldots, n_{L}\right)} \\
& =\bigoplus_{\alpha_{j}} \underbrace{\delta\left(n_{1}+\cdots+n_{L}-N\right)}_{:=X_{j ; \alpha_{j}}^{n_{1}, \ldots, n_{j}} X_{j ; N}^{\alpha_{j}, n_{j}+1, \ldots, n_{L}} \equiv Y_{j ; N, \alpha_{j}}^{n_{1}, \ldots, n_{L}}} T_{j ; \alpha_{j}}^{\left(n_{1}, \ldots, n_{j}\right),\left(n_{j+1}, \ldots, n_{L}\right)} \\
& =\bigoplus_{\alpha_{j}} Y_{N ; j ; \alpha_{j}} T_{j ; \alpha_{j}} .
\end{aligned}
$$

Here, we introduced structure tensors $Y_{N ; j ; \alpha}=X_{j ; \alpha_{j}}^{n_{1}, \ldots, n_{j}} X_{j ; N}^{\alpha_{j}, n_{j+1}, \ldots, n_{L}}$ that fix the decomposition into the block structure and block tensors $T_{j ; \alpha_{j}}$ containing the decomposed matrix entries of $T_{j ; \alpha_{j}}^{\left(n_{1}, \ldots, n_{j}\right),\left(n_{j+1}, \ldots, n_{L}\right)}$. The reshaped block-diagonal matrix representation of $T$ is shown in Fig. 4.29a. Now we can use a matrix factorization routine to split the tensor exploiting the decomposition generated by the structure tensor

$$
\begin{aligned}
T & =\bigoplus_{\alpha_{j}} Y_{N ; j ; \alpha_{j}} T_{j ; \alpha_{j}}=\bigoplus_{\alpha_{j}} Y_{N ; j ; \alpha_{j}} \sum_{m_{\alpha_{j}}} T_{m_{\alpha_{j}}}^{n_{1}, \ldots, n_{j}} T_{j ; m_{\alpha_{j}}}^{n_{j+1}, \ldots, n_{L}} \\
& =\sum_{\alpha_{j}} \sum_{m_{\alpha_{j}}} X_{j ; \alpha_{j}}^{n_{1}, \ldots, n_{j}} T_{m_{\alpha_{j}}}^{n_{1}, \ldots, n_{j}} X_{j ; N}^{\alpha_{j}, n_{j+1}, \ldots, n_{L}} T_{j ; m_{\alpha_{j}}}^{n_{j+1}, \ldots, n_{L}}
\end{aligned}
$$

where in the last line we replaced the direct sum for matrices by the general sum over tensor indices. Note the formal similarity of the described procedure to the decomposition of a state $|\psi\rangle \in \mathcal{H}$ into its MPS representation. The only difference is that the index $m_{j}$ gains an additional 
structure in terms of the label $\alpha_{j}$. In fact, we can repeat this procedure for any bipartition of the Hilbert space so that any tensor transforming under a global $U(1)$ symmetry can be decomposed uniquely into a loop-free network of symmetry adapted tensors exhibiting a block structure that is fixed by the structure tensors $X_{j ; \alpha_{j}}$. It is important to note that the dependency on the global quantum number sector $N$ can be unwrapped into the last structure tensor (or any other) so that $\alpha_{j+1} \equiv N$. Therefore, the described decomposition introduces an orientation in the decomposed tensor network. In case of MPS the structure tensors are given by

$$
X_{j ; \alpha_{j}}^{n_{j}, \alpha_{j-1}}=\delta\left(n_{j}+\alpha_{j-1}-\alpha_{j}\right), \quad \alpha_{0} \equiv 0, \quad \alpha_{L+1} \equiv N .
$$

Note the additional condition $\alpha_{0} \equiv 0$.

In the graphical representation the orientation introduced by the structure tensors is indicated by attaching arrows to the tensor legs. As shown in Fig. $4.29 \mathrm{~b}$ we add an incoming arrow to a leg if the sign of the index of the structure tensor decomposing the leg is positive and negative in the other case. This graphical representation motivates an intuitive rule: The structure tensors decompose tensor legs into symmetry sectors that are labeled by local quantum numbers. These quantum numbers need to fulfill a local conservation law, for instance, $n_{j}+\alpha_{j-1}=\alpha_{j}$ in case of an MPS. The conservation of these local quantum numbers is indicated by the arrow directions. Note that this construction is not restricted to invariance of the Hamiltonian under a single global $U(1)$ symmetry. The generalization to $K \in \mathbb{N} U(1)$ symmetries is straightforward. We only have to replace the single local quantum number labels by $K$ labels $\alpha_{j}^{k}(k=1, \ldots, K)$ and construct structure tensors enforcing $K$ conservation laws for ingoing and outgoing local quantum numbers, independently.

We can also formulate the above decomposition in the context of group theory. Therefore recall that tensors are multi-linear maps between vector spaces. To proceed we assign a meaning to the position of the indices, namely distinguishing between vector and dual spaces. In the following upper indices belong to a vector space and lower indices belong to a dual space, for instance

$$
T_{a, b}^{c, d}: \mathbb{V}_{a} \otimes \mathbb{V}_{b} \longrightarrow \mathbb{V}_{c} \otimes \mathbb{V}_{d}
$$

so that a contracted tensor-network representing an MPS is consistently written as a vector in a tensor-product Hilbert space with only upper indices. Next we define local operators $\hat{n}_{j}$ generating a unitary representation of $U(1)$ at each local Hilbert space, which we represent now as $\mathcal{H}_{\sigma}=\mathbb{V}_{\mathbb{C}}^{\sigma \times \sigma}$. We define a tensor to be $U(1)$-invariant if it transforms symmetrically under the combined action of all generators. To clarify this definition we return to the above example $T_{a, b}^{c, d}$ and see how it transforms (we identify $n_{a} \equiv a, n_{b} \equiv b, n_{c} \equiv c, n_{d} \equiv d$ )

$$
T_{n_{a}, n_{b}}^{n_{c}, n_{d}} \longrightarrow\left(e^{\mathrm{i} \vartheta \hat{n}_{a}}\right)^{\dagger}\left(e^{\mathrm{i} \vartheta \hat{n}_{b}}\right)^{\dagger} e^{\mathrm{i} \vartheta \hat{n}_{c}} e^{\mathrm{i} \vartheta \hat{n}_{d}} T_{n_{a}, n_{b}}^{n_{c}, n_{d}}=e^{\mathrm{i} \vartheta\left(n_{a}+n_{b}-n_{c}-n_{d}\right)} T_{n_{a}, n_{b}}^{n_{c}, n_{d}} .
$$

Clearly, if $T_{n_{a}, n_{b}}^{n_{c}, n_{d}}$ decomposes as $T_{n_{a}, n_{b}}^{n_{c}, n_{d}}=T_{n_{a}, n_{b}}^{n_{c}, n_{d}} \delta\left(n_{c}+n_{d}-n_{a}-n_{b}\right)$ it is invariant under the action of the gauge transformation $\left(e^{\mathrm{i} \vartheta \hat{n}_{a}}\right)^{\dagger}\left(e^{\mathrm{i} \vartheta \hat{n}_{b}}\right)^{\dagger} e^{\mathrm{i} \vartheta \hat{n}_{c}} e^{\mathrm{i} \vartheta \hat{n}_{d}}$, which justifies the above definition. Additionally, if $T_{n_{a}, n_{b}}^{n_{c}, n_{d}}$ transforms symmetrically under a global $U(1)$ symmetry the factorization

$$
T_{n_{a}, n_{b}}^{n_{c}, n_{d}}=Q_{m}^{n_{c}, n_{d}} R_{n_{a}, n_{b}}^{m},
$$

has to transform symmetrically, too. For the intermediate vector space $\mathbb{V}_{m}$ we can introduce another local operator $\hat{n}_{m}$ generating a unitary representation of $U(1)$ on $\mathbb{V}_{m}$ by means of the unitary transformation $e^{\mathrm{i} \vartheta \hat{n}_{m}}$. Then, if $T_{n_{a}, n_{b}}^{n_{c}, n_{d}}$ transforms symmetrically we can always insert an identity $e^{\mathrm{i} \vartheta \hat{n}_{m}} e^{-\mathrm{i} \vartheta \hat{n}_{m}}$

$$
\begin{aligned}
e^{-\mathrm{i} \vartheta \hat{n}_{a}} e^{-\mathrm{i} \vartheta \hat{n}_{b}} e^{\mathrm{i} \vartheta \hat{n}_{c}} e^{\mathrm{i} \vartheta \hat{n}_{d}} T_{n_{a}, n_{b}}^{n_{c}, n_{d}} & =e^{-\mathrm{i} \vartheta \hat{n}_{a}} e^{\mathrm{i} \vartheta \hat{n}_{b}} e^{\mathrm{i} \vartheta \hat{n}_{m}} R_{n_{a}, n_{b}}^{m} e^{\mathrm{i} \vartheta \hat{n}_{c}} e^{\mathrm{i} \vartheta \hat{n}_{d}} e^{-\mathrm{i} \vartheta \hat{n}_{m}} Q_{m}^{n_{c}, n_{d}} \\
& \stackrel{!}{=} Q_{m}^{n_{c}, n_{d}} R_{n_{a}, n_{b}}^{m},
\end{aligned}
$$


which implies $Q_{m}^{n_{c}, n_{d}}=Q_{m}^{n_{c}, n_{d}} \delta\left(n_{c}+n_{d}-m\right)$ and $R_{n_{a}, n_{b}}^{m}=R_{n_{a}, n_{b}}^{m} \delta\left(m-n_{a}-n_{b}\right)$. Hence, we can decompose a $U(1)$ symmetric tensor into a network of $U(1)$ symmetric tensors with oriented legs indicating if the corresponding index transforms as an element of a vector or dual space.

At this point we have a look back at the decomposition of $U(1)$ invariant tensors representing states in the many-body Hilbert space (see for instance Eq. (4.198)). The attentative reader may have noted the slight change in the distribution of upper and lower indices in the structure tensors $X_{j ; \alpha_{j}}$. This is due to the fact that the structure tensors determine the transformation under the action of $U(1)$. Hence, these tensors introduce the orientation of the legs, which is why the upper/lower-index notation to indicate the transformation behavior is used only in their context.

\section{\begin{tabular}{l|l} 
Implementation & 4.6 .3
\end{tabular}}

The effect of the decomposition of dense tensors into sparse tensors $T$ becomes very clear if we write sparse tensors as collection of tensor blocks. Let us consider a general dense rank- $r$ tensor

$$
M^{a_{1}, \ldots, a_{s}, b_{1}, \ldots, b_{t}}: \mathbb{V}_{a_{1}} \otimes \cdots \otimes \mathbb{V}_{a_{s}} \longrightarrow \mathbb{V}_{b_{1}} \otimes \cdots \otimes \mathbb{V}_{b_{t}},
$$

with $r=s+t$. Generically $M$ has complex numbers as elements $M^{a_{1}, \ldots, a_{s}, b_{1}, \ldots, b_{t}} \in \mathbb{C}$. We call $M$ a dense tensor because typically a numerial implementation would form a matrix $M^{\left(a_{1}, \ldots, a_{s}\right),\left(b_{1}, \ldots, b_{t}\right)} \in$ $\mathbb{V}_{\mathbb{C}}^{n \times m}$ out of the fused vector and dual space indices with dimensions $n=\prod_{j=1}^{s} \operatorname{dim} a_{j}$ and $m=\prod_{j=1}^{t} \operatorname{dim} b_{j}$. Now assume that $M$ transforms symmetrically under the action of $U(1)$ and can be decomposed into symmetry blocks

$$
M^{a_{1}, \ldots, a_{s}, b_{1}, \ldots, b_{t}}=\bigoplus_{\substack{\alpha_{1}, \ldots, \alpha_{s} \\ \beta_{1}, \ldots, \beta_{t}}} X_{\alpha_{1}, \ldots, \alpha_{s}}^{\beta_{1}, \ldots, \beta_{r}} T^{a_{\alpha_{1}}, \ldots, a_{\alpha_{s}}, b_{\beta_{1}}, \ldots, b_{\beta_{r}}}
$$

where each index of $M$ is decomposed into a set of indices. We can have this in a more compact notation by only writing the labels determining the local quantum numbers

$$
M=\bigoplus_{\substack{\alpha_{1}, \ldots, \alpha_{s} \\ \beta_{1}, \ldots, \beta_{t}}} X_{\alpha_{1}, \ldots, \alpha_{s}}^{\beta_{1}, \ldots, \beta_{r}} T^{\alpha_{1}, \ldots, \alpha_{s}, \beta_{1}, \ldots, \beta_{r}}
$$

This way, the elements of the sparse tensor representation of $M$ are again dense tensors, namely the non-vanishing symmetry blocks $T^{\alpha_{1}, \ldots, \alpha_{s}, \beta_{1}, \ldots, \beta_{r}}$. In a numerical implementation each symmetry block is now stored by fusing the vector and dual space indices so that the elements of $M$ are given by complex matrices: $M^{\alpha_{1}, \ldots, \alpha_{s}, \beta_{1}, \ldots, \beta_{t}} \in \mathbb{V}_{\mathbb{C}}^{n_{\alpha} \times m_{\beta}}$ with dimensions $n_{\alpha}=\prod_{j=1}^{s} \operatorname{dim} \alpha_{j}$ and $m_{\beta}=\prod_{j=1}^{t} \operatorname{dim} \beta_{j}$.

The most important and also expensive operations in MPS algorithms are contractions over shared indices of two tensors, which we will embark on now. Let $M, N$ be two tensors with ranks $r_{M}=s_{M}+t_{M}, r_{N}=s_{N}+t_{N}$ represented as multi-linear maps between vector spaces

$$
\begin{gathered}
M: \mathbb{V}_{a_{1}} \otimes \cdots \otimes \mathbb{V}_{a_{s_{M}}} \longrightarrow \mathbb{V}_{b_{1}} \otimes \cdots \otimes \mathbb{V}_{b_{t_{M}}} \\
N: \mathbb{V}_{b_{1}^{\prime}} \otimes \cdots \otimes \mathbb{V}_{b_{s_{N}}^{\prime}} \longrightarrow \mathbb{V}_{c_{1}} \otimes \cdots \otimes \mathbb{V}_{c_{t_{N}}}
\end{gathered}
$$

Let $\mathbb{V}_{b_{j}}=\mathbb{V}_{b_{k}^{\prime}} \equiv \mathbb{V}_{b}$ then we consider a contraction over the respective indices $b_{j} \equiv b_{k}^{\prime}$

$$
M \cdot N=\sum_{b} M^{a_{1}, \ldots, a_{s_{M}}, b_{1}, \ldots, b, \ldots, b_{t_{M}}} N^{b_{1}^{\prime}, \ldots, b, \ldots, b_{s_{N}}^{\prime}, c, \ldots, c_{t_{N}}} .
$$


The general idea is to reshape the tensors into matrices to exploit effective contraction routines that asymtptically scale as $m^{\alpha}$ where $\alpha<3$ [STR69, blans] and $m$ the (geometrically averaged) matrix dimension. In the case of dense tensors after reshaping we would then have a single matrix contraction

$$
M \cdot N=\sum_{b} M^{\left(a_{1}, \ldots, a_{s_{M}}, b_{1}, \ldots, b_{t_{M}}\right),(b)} N^{(b),\left(b_{1}^{\prime}, \ldots, \ldots, b_{s_{N}}^{\prime}, c, \ldots, c_{t_{N}}\right)} \equiv \mathbf{M}_{(b)} \cdot \mathbf{N}^{(b)}
$$

where we introduced a shorthand notation for the reshaped matrices in the last equality. Let us denote furthermore the dimensions of the reshaped matrices as

$$
\begin{array}{lll}
n_{M}^{(b)}=\prod_{l=1}^{s_{M}} \operatorname{dim} a_{l} \prod_{l \neq j=1}^{t_{M}} \operatorname{dim} b_{l} & \text { and } & m_{M}^{(b)}=\operatorname{dim} b \equiv|b| \\
n_{N}^{(b)}=\operatorname{dim} b \equiv|b| & \text { and } & m_{N}^{(b)}=\prod_{l \neq j=1}^{s_{N}} \operatorname{dim} b_{l}^{\prime} \prod_{l=1}^{t_{N}} \operatorname{dim} c_{l} .
\end{array}
$$

Depending on the used linear algebra library the contraction scales as $\left(n_{M}^{(b)} m_{N}^{(b)}|b|\right)^{\alpha / 3}$. Now if $M, N$ transform symmetrically under $U(1)$ the calculation procedure is similar but we pickup an additional summation. The contraction of the reshaped tensors now reads

$$
\begin{aligned}
M \cdot N= & \bigoplus_{\beta_{j}} X_{M ; \beta_{j}}^{\alpha_{1}, \ldots, \alpha_{s_{M}}, \beta_{1}, \ldots, \beta_{t_{M}}} X_{N ; \beta_{1}^{\prime}, \ldots, \beta_{s_{N}}^{\prime}, \gamma_{1}, \ldots, \gamma_{t_{N}}}^{\beta^{\prime}} \delta_{\beta_{j}, \beta_{k}^{\prime}} \times \\
& \left.\sum_{b_{\beta_{j}}} T_{M}^{\left(a_{\alpha_{1}}, \ldots, a_{\alpha_{s_{M}}}, b_{\beta_{1}}, \ldots, b_{\beta_{t_{M}}}\right),\left(b_{\beta_{j}}\right)} T_{N}^{\left(b_{\beta_{j}}\right),\left(b_{\beta_{1}^{\prime}}^{\prime}, \ldots, b_{\beta_{S_{N}}^{\prime}}^{\prime}, c\right.}{ }_{\gamma_{1}}, \ldots, c_{\gamma_{t_{N}}}\right) \\
\equiv & \bigoplus_{\beta} X_{M}^{(\beta)} X_{N ;(\beta)} \mathbf{T}_{M}^{\left(b_{\beta}\right)} \cdot \mathbf{T}_{N}^{\left(b_{\beta}\right)}
\end{aligned}
$$

where in the last equation we set $\beta_{j}=\beta_{k}^{\prime} \equiv \beta$. Note that the decomposition of the local quantum numbers must not be unique with respect to $\beta$. In general, there are several ways to combine indices $\left(\alpha_{1}, \ldots, \alpha_{s_{M}}, \beta_{1}, \ldots, \beta_{t_{M}}\right)$ or $\left(\beta_{1}^{\prime}, \ldots, \beta_{s_{N}}^{\prime}, \gamma_{1}, \ldots, \gamma_{t_{N}}\right)$ to obtain a single value of $\beta$. We will denote this additional degeneracy in the direct summation above by $d_{M ;(\beta)}$ and $d_{N ;(\beta)}$. The important observation is that instead of one contraction there are $d_{M ;(\beta)} d_{N ;(\beta)}|\beta|=$ $d_{M ;(\beta)} d_{N ;(\beta)}\left|\left\{\beta_{j}\right\} \cap\left\{\beta_{k}^{\prime}\right\}\right|$ contractions $\mathbf{T}_{M}^{\left(b_{\beta}\right)} \cdot \mathbf{T}_{N}^{\left(b_{\beta}\right)}$, each of which scales as $\left(n_{M}^{\left(b_{\beta}\right)} m_{N}^{\left(b_{\beta}\right)}\left|b_{\beta}\right|\right)^{\alpha / 3}$ where we expanded the above introduced notation to account for the different blocks labeled by $\beta$. Therefore, the speedup gained by reducing the contraction volume strongly depends on the distribution of the block sizes. For instance, if the index $b$ decomposes into $n$ equally sized blocks so that $\left|b_{\beta}\right|=\frac{|b|}{n}$ the contractions become cheaper by a factor of $n^{\alpha / 3}$. Additionally, there are also factors arising from the decomposition of the other indices. On the other hand we have to perform $d_{M ;(\beta)} d_{N ;(\beta)} n$ contractions instead of one. These can be parallelized and in the situation of equally distributed block sizes such a parallelization can furthermore yield a speed up. However, there is also the other extreme in which there is one large block $\tilde{\beta}$ dominating the decomposition so that $\left|b_{\tilde{\beta}}\right| \gg\left|b_{\beta \neq \tilde{\beta}}\right|$. In this case there is only a very small speed up in the decomposed contractions and also a parallelization could not improve the situation since the overall contraction time will be dominated by the calculation of the very expensive matrix-matrix $\operatorname{product}(\mathrm{s}) \mathbf{T}_{M}^{\left(b_{\tilde{\beta}}\right)} \cdot \mathbf{T}_{N}^{\left(b_{\tilde{\beta}}\right)}$. 
For MPS (recall Eq. (4.198)) the situation is typically that there are few large blocks with nearly equal bond dimensions and several blocks with smaller bond dimension in the decomposition of the auxiliary indices $m_{j} \rightarrow m_{\alpha_{j}}$. The speed-up that one usually obtains is $\sim \mathcal{O}(10)-$ $\mathcal{O}(100)$ and depends also on the number of $U(1)$ symmetries. Note that if we exploit global symmetries being generated from local operators $\hat{n}_{j}$ that have a non-degenerated spectrum, then these (physical) indices always decompose into one-dimensional representations, i.e., $\operatorname{dim} n_{j}=1$. This information can be used to speed-up an implementation, for instance, such indices can be fused and contracted in combination with other indices without additional computational costs. 


\section{$U(1)$ symmetric matrix-product operators and finite-state machines}

The problem of constructing MPO representations of operators is a surprisingly rich one [CB08, PMCV10, HMS17, PKM17]. It has been noted in [CB08] that the low-rank decomposition of operators can be related to the local structure of the coupling terms appearing in these operators. This revealed a surprising connection to the field of automata theory, which can be used to construct a uniform framework to obtain MPO representation in terms of FSMs. Importantly, within this framework the construction of the MPO matrices can be derived from a purely diagrammatic representation. As a consequence, no arithmetics and thereby necessary compressions of the matrices is required rendering the produced MPO representations exact. Clearly, this does not have to be an advantage in every situation. As an example one may think of multi-orbital Hamiltonians from quantum chemistry where in a mapping to a one-dimensional chain all sites are coupled with each other and there is no obvious way to factorize the coupling constants whatsoever [KDTR15, $\left.\mathrm{CKN}^{+} 16, \mathrm{HMS17}\right]$. In such a situation one cannot expect to obtain a compact and exact low-rank representation as there is no notion of locality in the couplings. Nevertheless, typical condensed matter systems come with a local coupling structure, which can be represented efficiently by FSMs.

In the following we will introduce the notion of finite-state machines as a unifiying framework to create MPO representations. We will derive the $U(1)$ symmetric representation of the elementary building blocks in the FSM representation. Then, we put the pieces together and give an automatized construction scheme for $U(1)$ symmetric MPO representations of global operators. These sections are all based on the publication [PKM17] wich was written by the author and in collaboration with Thomas Köhler and Salvatore Manmana. Finally we will comment on further developements, which are also published in [PKM17] but of minor relevance for this thesis so that they are not discussed in detail, and summarize the implementation of the presented framework in the SYMMPS toolkit.

\section{\begin{tabular}{l|l} 
MPO construction from FSMs & 5.1
\end{tabular}}

As discussed in [CB08, CDV08], the MPO representation of an operator on a tensor-product space can be obtained from the transition amplitudes of FSMs. In the following, the underlying graph structure of these FSMs is used extensively. Thus, we first give a brief review on how to identify FSMs with MPO representations, following [CB08]. This requires some additional notational conventions so we begin by setting up the stage for the main act. We say an operator $\hat{K}: \mathcal{H} \longrightarrow \mathcal{H}$ is represented by an operator string, if it is given by a tensor product of local operators $\hat{k}: \mathcal{H}_{\sigma} \longrightarrow \mathcal{H}_{\sigma}$ only. That is, let $\mathcal{K}$ be a complete representation of the algebra of local operators, then there is a constant $c \in \mathbb{C}$ so that

$$
\hat{K}=c \bigotimes_{j=1}^{L} \hat{k}_{j}, \quad \hat{k}_{j} \in \mathcal{K}
$$


For instance, each local operator $\hat{K}^{(j)}$ acting on the many-particle Hilbert space is given by an operator string, i.e. there is an $\hat{k} \in \mathcal{K}$ so that

$$
\hat{K}^{(j)}=\underbrace{\hat{\mathbf{1}} \otimes \cdots \otimes \hat{\mathbf{1}}}_{j-1 \text { times }} \otimes \hat{k} \otimes \underbrace{\hat{\mathbf{1}} \otimes \cdots \otimes \hat{\mathbf{1}}}_{L-j \text { times }} .
$$

We call an operator $\hat{K}^{\left(j_{1}\right), \ldots,\left(j_{n}\right)}$ a $n$-site gate $\left(n \in \mathbb{N}, j_{k} \in\{1, \ldots, L\}\right)$ if it has a representation as an operator string and all operators at positions $j \notin\left\{j_{1}, \ldots, j_{n}\right\}$ are identities on the local Hilbert spaces. Clearly, we can immediately give the general MPO representation of any $n$-site gate by constructing site tensors $W_{j}^{\sigma_{j}, \sigma_{j}^{\prime}}=\left\langle\sigma_{j}\left|\hat{k}_{j}\right| \sigma_{j}^{\prime}\right\rangle$ for $\hat{k}_{j} \in \mathcal{K}$. Note that for convenience we will casually switch between the general representation of a local operator $\hat{K}^{(j)}$ and its representation as an operator string $\propto \hat{\mathbf{1}}^{\otimes j-1} \otimes \hat{k} \otimes \hat{\mathbf{1}}^{\otimes L-j}$. Also, we put round brackets around indices labeling lattice sites whenever there may be confusion about the meaning of the indices.

Importantly, any operator acting on a tensor product Hilbert space can be represented by a sum of operator strings

$$
\hat{K}=\sum_{\nu, r} \hat{K}_{\nu, r}=\sum_{\nu, r} \sum_{j} \hat{K}_{\nu, r}^{(j)}
$$

with $\hat{K}_{\nu, r}^{(j)}=f_{\nu_{1} \ldots \nu_{n}}^{r} \hat{K}_{\nu_{1}}^{(j)} \cdots \hat{K}_{\nu_{n}}^{(j+r)}$ being $n$-site gates consisting of local operators $\hat{k}_{\nu} \in \mathcal{K}$. Each such operator string couples lattice sites in the range from site $j$ to $j+r(r+1 \geq n)$ with amplitude $f_{\nu_{1} \ldots \nu_{n}}^{r} \in \mathbb{C}$. Note that we abbreviated the index set $\nu=\left(\nu_{1} \ldots \nu_{n}\right)$. For later convenience we will call $\hat{K}_{\nu, r}^{(j)}$ lattice ordered $n$-point $r+1$-ranged operator strings. The set of all lattice-ordered $n$-point $r+1$-ranged operator strings $\Sigma=\left\{\hat{K}_{\nu, r}^{(j)}\right\}_{\nu, r}$ defines a language of a FSM, i.e., there is a set of states $\mathcal{L}$ and a transition function $\delta: \mathcal{L} \times \mathcal{K} \longrightarrow \mathcal{L}$ so that with a proper initial and final state $I, F \in \mathcal{L}$, the $\mathrm{FSM} M(\mathcal{K}, \mathcal{L}, \delta, I, F)$ is obtained with a generated language $\Sigma$. The corresponding graph is then a representation of $\hat{H}$ and is denoted by $\Lambda(\hat{H})$.

Figure 5.1a sketches a general FSM and Fig. 5.1b shows the generated MPO bulk tensor, in which the rows and columns are labeled by the states of the FSM. We can construct the MPO representation, i.e., the operator-valued matrices $\hat{W}^{n_{j} n_{j}^{\prime}}$, by assigning matrices $W_{j ; \tau_{j-1} \tau_{j}}^{n_{j} n_{j}^{\prime}}$ of dimension $|\mathcal{L}| \times|\mathcal{L}|$ for all non-vanishing elements $\left(n_{j}, n_{j}^{\prime}, \tau_{j-1}, \tau_{j}\right)$ (i.e., those with at least one local operator block $\left.k_{j ; \tau_{j-1} \tau_{j}}^{n_{j} n_{j}^{\prime}} \neq 0\right)$. The corresponding boundary tensors are obtained by projecting out (a) the transition from the initial state into the bulk for $j=1$ and (b) the transitions from the bulk into the final state for $j=L$. We emphasize that the site-dependent coefficients $c_{a b}^{(j)} \in \mathbb{C}$ are free parameters and therefore can be chosen independently for every site.

An example for the graph representation of the XX model is given in Fig. 5.2a and in the following, we shortly explain how to obtain this graph. At first, we have to rewrite the global operator in terms of lattice-ordered $n$-point $r+1$-ranged operator strings ${ }^{1}$

$$
\hat{H}_{X X}=\sum_{j} \hat{S}_{(j)}^{+} \hat{S}_{(j+1)}^{-}+\sum_{j} \hat{S}_{(j)}^{-} \hat{S}_{(j+1)}^{+} \equiv \sum_{j} \hat{H}_{(j)}^{+-}+\sum_{j} \hat{H}_{(j)}^{-+}
$$

i.e., we have two distinct 2-point 2-ranged operator strings. Then, we define a default set of states $\mathcal{L}_{0}=\{I, F\}$ with $I, F$ being the initial and final state of the FSM, respectively. For convenience, we will highlight them in the corresponding graphs with green $(I)$ and red $(F)$ background. In general, FSMs are capable to generate sequences of symbols $\hat{k} \in \mathcal{K}$ by transitioning between

\footnotetext{
${ }^{1}$ For convenience we moved the site labels of the local operators to the bottom to avoid overloaded upper labels. The author deeply regrets this notational inconsistency.
} 
(a)

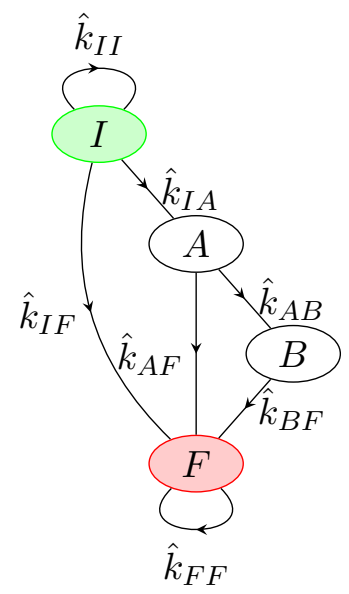

(b)

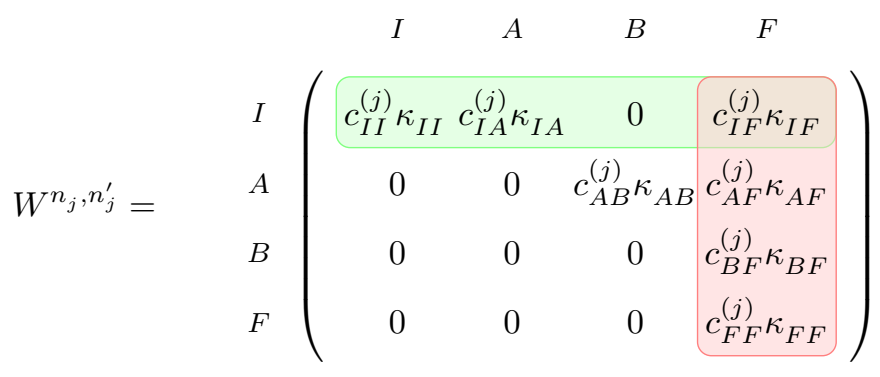

with local operator blocks $\kappa_{a b}=\left\langle n_{j}, n_{j}^{\prime}\left|\hat{k}_{a b}^{n_{j}, n_{j}^{\prime}}\right| n_{j}, n_{j}^{\prime}\right\rangle$

Figure 5.1: (a) FSM defined on states $\mathcal{L}=\{I, A, B, F\}$ with transition amplitudes $\hat{k}_{a b} \in \mathcal{K}$. The initial and the final state are highlighted in green or red, respectively. Transitions between states are denoted by arrows with the corresponding transition amplitudes $\hat{k}_{a b}$. (b) Bulk MPO site-tensor block $W_{j ; \tau_{j-1} \tau_{j}}^{n_{j} n_{j}^{\prime}}$, obtained from the FSM in (a). The initial and the final site tensor are marked by a green or red background, respectively. The coefficients $c_{a b}^{(j)}$ are site-dependent weight functions, which can be used to introduce position-dependent transition amplitudes. This graphic is adopted from the author's publication [PKM17].

(a)

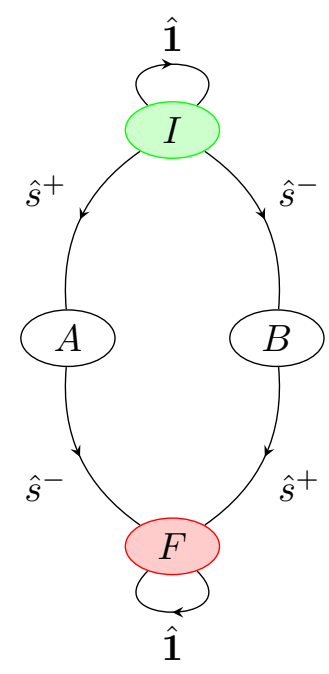

(b)

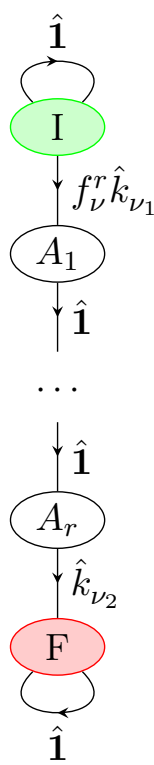

Figure 5.2: (a) Graph representation $\Lambda\left(\hat{H}_{X X}\right)$ of the XX model $\hat{H}_{X X}=\sum_{j} \hat{S}_{(j)}^{+} \hat{S}_{(j+1)}^{-}+$h.c.. (b) Graph representation of an operator with only one 2-point interaction term $f_{\nu_{1} \nu_{2}}^{r} \hat{O}_{\nu_{1}}^{(j)} \hat{o}_{\nu_{2}}^{(j+r)}$ of range $r$. Note that the initial and final states are highlighted with a green and red background, respectively while intermediate states $A, B, A_{1} \ldots A_{r}$ are left white. This graphic is adopted from the author's publication [PKM17]. 
states $a \in \mathcal{L}$ via allowed transitions, i.e., those with non-vanishing transition function $\delta(\hat{k}, a)$. We therefore add $r=1$ intermediate states (here only one state $A_{1} \equiv A$ ) to the set of states $\mathcal{L}=\mathcal{L}_{0} \cup\{A\}$ and define transitions $\delta\left(I, \hat{s}^{+}\right)=A$ and $\delta\left(A, \hat{s}^{-}\right)=F$. This way, the FSM can move from the initial state (after placing an arbitrary number of identities) into state $A$ by appending an operator $\hat{s}^{+}$onto the so far constructed operator string. Being in state $A$, the FSM has no other choice than to transition into state $F$ by appending a subsequent operator $\hat{s}^{-}$. A generalization of the construction of such local operator strings is shown in Fig. $5.2 \mathrm{~b}$ for the case of a general 2-point $r$-ranged operator string. From this point on, we will call graphs that generate only one distinct type of $n$-point $r+1$-ranged operator string single-branched graphs and identify them with the corresponding contribution in the global operator $\hat{H}_{\nu, r}$. Returning to the XX model, we can complete the construction of the FSM by adding another single-branched graph transitioning from the initial state into an additional state $B$ via a local operator $\hat{s}^{-}$and a transition into the final state via a local operator $\hat{s}^{+}$.

It is easy to check that the operator valued matrices indeed create the desired representation of the $X X$ model by explicitely contracting the obtaine matrix representations. We will consider the situation of a system with $L=3$ lattice sites yielding the following MPO representation in terms of operator-valued matrices

$$
\begin{aligned}
\hat{W}_{1} \hat{W}_{2} \hat{W}_{3} & =\left(\begin{array}{llll}
\hat{\mathbf{1}} & \hat{s}^{+} & \hat{s}^{-} & 0
\end{array}\right) \otimes\left(\begin{array}{cccc}
\hat{\mathbf{1}} & \hat{s}^{+} & \hat{s}^{-} & 0 \\
0 & 0 & 0 & \hat{s}^{-} \\
0 & 0 & 0 & \hat{s}^{+} \\
0 & 0 & 0 & \hat{\mathbf{1}}
\end{array}\right) \otimes\left(\begin{array}{c}
0 \\
\hat{s}^{-} \\
\hat{s}^{+} \\
\hat{\mathbf{1}}^{\prime}
\end{array}\right) \\
& =\left(\begin{array}{llll}
\hat{\mathbf{1}} & \hat{s}^{+} & \hat{s}^{-} & 0
\end{array}\right) \otimes\left(\begin{array}{c}
\hat{s}^{+} \otimes \hat{s}^{-}+\hat{s}^{-} \otimes \hat{s}^{+} \\
\hat{s}^{-} \otimes \hat{\mathbf{1}} \\
\hat{s}^{+} \otimes \hat{\mathbf{1}} \\
\hat{\mathbf{1}} \otimes \hat{\mathbf{1}}^{-}
\end{array}\right) \\
& =\hat{\mathbf{1}} \otimes \hat{s}^{+} \otimes \hat{s}^{-}+\hat{\mathbf{1}} \otimes \hat{s}^{-} \otimes \hat{s}^{+}+\hat{s}^{+} \otimes \hat{s}^{-} \otimes \hat{\mathbf{1}}+\hat{s}^{-} \otimes \hat{s}^{+} \otimes \hat{\mathbf{1}} .
\end{aligned}
$$

\section{Two-site gates as building blocks for $U(1)$ symmetric MPOs

In order to derive a construction scheme for MPO representations transforming symmetrically under a global $U(1)$ symmetry that can be automatized we consider the elementary building blocks of any such operators. Therefore, we will work in the basis of the tensor product of eigenstates of local operators $\hat{n}_{j}$ generating a global $U(1)$ symmetry $e^{\mathrm{i} \vartheta \hat{N}}=e^{\mathrm{i} \vartheta \sum_{j} \hat{n}_{j}}$ as described in Sec. 4.6. Note that if an operator transforms symmetrically under a global $U(1)$ symmetry so must the operator strings it can be decomposed into. Thus, we take a more elaborate look at the action of a two-site gate on a state $|\psi\rangle$, which transforms under $U(1)$, ignoring the common framework of irreducible representations and block diagonal structures for a moment (later we generalize the considerations to arbitrary operator expressions). Introducing local operators $\hat{A}^{(i)}, \hat{B}^{(j)}$ acting only on the sites $i, j$, we start from a generic $U(1)$-invariant expression of the form

$$
\begin{gathered}
\left\langle n_{1}, \ldots, n_{L}\left|\hat{A}^{(i)} \hat{B}^{(j)}\right| \psi\right\rangle \equiv \sum_{n_{i}^{\prime}, n_{j}^{\prime}} T^{n_{1}} \cdots A^{n_{i} n_{i}^{\prime}} T^{n_{i}} \cdots B^{n_{j} n_{j}^{\prime}} T^{n_{j}} \cdots T^{n_{L}} \\
\times \delta\left(n_{i}+n_{j}-\left(n_{i}^{\prime}+n_{j}^{\prime}\right)\right),
\end{gathered}
$$


where the $\delta$ function ensures that the global quantum number $\hat{N}$ is conserved when applying the operator. Note that for brevity we have surpressed the structure tensors in the MPS representation of the state $|\psi\rangle$. A dummy index $\tau$ with $-(d-1) \leq \tau \leq(d-1)$ is introduced to factorize the $\delta$ function so that we obtain

$$
\begin{array}{r}
\left\langle n_{1}, \ldots, n_{L}\left|\hat{A}^{(i)} \hat{B}^{(j)}\right| \psi\right\rangle=\sum_{n_{i}^{\prime}, n_{j}^{\prime}}\left\{\cdots T ^ { n _ { i - 1 } } \left[\sum_{\tau} \hat{A}^{n_{i} n_{i}^{\prime}} T^{n_{i}} \delta\left(\tau-\left(n_{i}-n_{i}^{\prime}\right)\right) T^{n_{i+1}} \times\right.\right. \\
\left.\left.\hat{B}^{n_{j} n_{j}^{\prime}} T^{n_{j}} \delta\left(\tau-\left(n_{j}^{\prime}-n_{j}\right)\right)\right] T^{n_{j+1}} \cdots\right\},
\end{array}
$$

which nearly restores the factorized shape of generic MPS, even though the physical sites $i, j$ are still connected by the sum over the dummy index $\tau$. It is desirable to restore a tensor-network form so that the action of an operator pair can be written as contraction of tensors acting on the local Hilbert spaces so that we can read-off the $U(1)$ symmetric MPO representation. Therefore, we need to take a closer look at the matrix elements $A_{j ; \gamma_{i-1}, \gamma_{i}}^{n_{i}, n_{i}^{\prime}}, B_{j ; \gamma_{j-1}, \gamma_{j}}^{n_{j}, n_{j}^{\prime}}$ of the local operators $\hat{A}^{(i)}, \hat{B}^{(j)}$. If the total operator expression is $U(1)$-invariant, then the local operators themselves have to be one-dimensional representations in the physical indices (acting on the local Hilbert space) so that each operator carries a unique mapping between states $\left|n_{i}\right\rangle \rightarrow\left|n_{i}^{\prime}\right\rangle$. In other words, for $U(1)$-invariant operator pairs each local operator is non-vanishing only for a certain value of the dummy index $\tau^{\prime}=\Delta$. For instance, in case of spin-ladder operators $\left(\hat{S}^{ \pm}\right)^{(i)}$ the total value of $S^{z}$ is locally changed by \pm 1 . Hence,

$$
\left(\hat{S}^{ \pm}\right)^{n_{i}, n_{i}^{\prime}} \neq 0 \Leftrightarrow n_{i}-n_{i}^{\prime}= \pm 1 \equiv \Delta
$$

where we introduced the change of local quantum numbers $\Delta$. Having this in mind, block-index conservation laws for the local operators can be realized via

$$
\begin{aligned}
& A_{i} \equiv A_{i ; \tau_{i-1} \tau_{i}}^{n_{i} n_{i}^{\prime}} \delta\left(\Delta-\left(n_{i}-n_{i}^{\prime}\right)\right) \delta\left(\left(n_{i}+\tau_{i-1}\right)-\left(n_{i}^{\prime}+\tau_{i}\right)\right) \\
& B_{j} \equiv B_{j ; \tau_{j-1} \tau_{j}}^{n_{j} n_{j}^{\prime}} \delta\left(\Delta-\left(n_{j}^{\prime}-n_{j}\right)\right) \delta\left(\left(n_{j}+\tau_{j-1}\right)-\left(n_{j}^{\prime}+\tau_{j}\right)\right) .
\end{aligned}
$$

The case of two-site gates is obtained by setting $\tau_{i-1}=\tau_{j} \equiv 0$ as well as $\tau_{i}=\tau_{j-1} \equiv \tau$; the nonvanishing operator blocks $A_{i ; \tau_{i-1} \tau_{i}}^{n_{i} n_{i}^{\prime}}, B_{j ; \tau_{j-1} \tau_{j}}^{n_{j} n_{j}^{\prime}} \in \mathbb{V}_{\mathbb{C}}^{d_{\tau_{i-1, j-1}} \times d_{\tau_{i, j}}}$ now contain the reduced operator matrix elements acting on the local basis states $\left|n_{i, j}^{\prime}\right\rangle$. The contraction of the block index $\tau$ over the intermediate site tensors $T^{n_{k}}, i<k<j$ can be recast into a matrix contraction using the matrix identity

$$
\left(A_{1}, \cdots, A_{n}\right)\left(\begin{array}{ccc}
D & \cdots & 0 \\
\vdots & \ddots & \vdots \\
0 & \cdots & D
\end{array}\right)\left(\begin{array}{c}
B_{1} \\
\vdots \\
B_{n}
\end{array}\right)=\sum_{i=1}^{n} A_{i} D B_{i}
$$

Therefore, the application of the above two-site gate is factorized completely if we define intermediate shift tensors that act on the sites $k$ with $i<k<j$ as identity, but are contracted over auxiliary bond indices $\tau_{k}$,

$$
R_{j ; \Delta}=R_{j ; \tau_{j-1} \tau_{k}}^{n_{j} n_{j}^{\prime}}(\Delta) \delta\left(\left(n_{j}+\tau_{j-1}\right)-\left(n_{j}^{\prime}+\tau_{j}\right)\right) \equiv \delta\left(n_{j}-n_{j}^{\prime}\right) \delta\left(\tau_{j-1}-\Delta\right) \delta\left(\Delta-\tau_{j}\right) .
$$


(a)

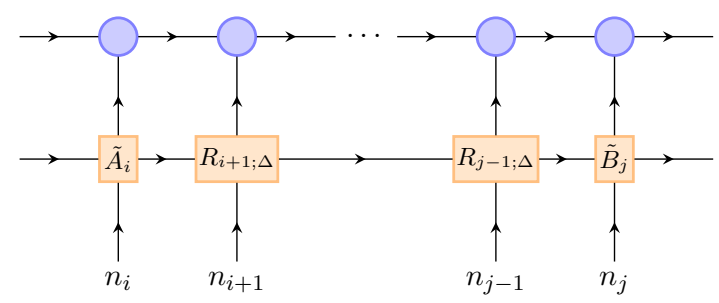

(b)

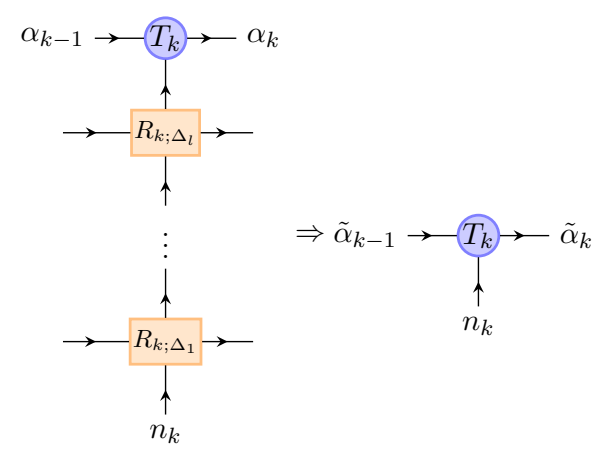

Figure 5.3: (a) MPO representation of the $U(1)$-invariant operator expression $\hat{A}^{(i)} \hat{B}^{(j)}$ with shift tensors $R_{k ; \Delta}$ mediating the shift in the auxiliary-bond quantum numbers. (b) Network of various shift tensors applied to the $U(1)$-invariant site tensor $T_{k}$ before and after performing the contractions, respectively. After contraction, the auxiliary block indices are given via $\tilde{\alpha}_{k-1, k}=\alpha_{k-1, k}+\Delta_{1}+\cdots+\Delta_{l}$. This graphic is adopted from the author's publication [PKM17].

Hence, the action of the $U(1)$-invariant operator pair can conveniently be written as tensor network,

$$
\hat{A}^{(i)} \hat{B}^{(j)}|\psi\rangle=\sum_{n_{1} \cdots n_{L}} T^{n_{1}} \cdots T^{n_{i-1}} \tilde{A}^{n_{i}} P^{n_{i+1}} \cdots P^{n_{i-1}} \tilde{B}^{n_{j}} T^{n_{j+1}} \cdots T^{n_{L}}\left|n_{1} \cdots n_{L}\right\rangle
$$

with the definitions

$$
\begin{aligned}
\tilde{A}^{n_{i}} & =\sum_{n_{i}^{\prime}} A_{i ; \tau_{i-1} \tau_{i}}^{n_{i} n_{i}^{\prime}} \delta\left(\Delta-\left(n_{i}-n_{i}^{\prime}\right)\right) \delta\left(\left(n_{i}+\tau_{i-1}\right)-\left(n_{i}^{\prime}+\tau_{i}\right)\right) T^{n_{i}^{\prime}} \\
P^{n_{k}} & =\sum_{n_{k}^{\prime}} R_{k ; \tau_{k-1} \tau_{k}}^{n_{k} n_{k}^{\prime}}(\Delta) \delta\left(\left(n_{k}+\tau_{k-1}\right)-\left(n_{k}^{\prime}+\tau_{k}\right)\right) T^{n_{k}^{\prime}} \\
\tilde{B}^{n_{j}} & =\sum_{n_{j}^{\prime}} R_{j ; \tau_{j-1} \tau_{j}}^{n_{j} n_{j}^{\prime}} B_{j ; \tau_{j-1} \tau_{j}}^{n_{j} n_{j}^{\prime}} \delta\left(\Delta-\left(n_{j}^{\prime}-n_{j}\right)\right) \delta\left(\left(n_{j}+\tau_{j-1}\right)-\left(n_{j}^{\prime}+\tau_{j}\right)\right) T^{n_{j}^{\prime}} .
\end{aligned}
$$

When further local operators to the left and right of $\hat{A}^{(i)}, \hat{B}^{(i)}$ are absent, the auxiliary indices are shrunk to dummy indices $\tau_{i-1}=\tau_{j} \equiv 0$. Thus, the sum over all remaining auxiliary block indices $\tau_{i \leq l \leq j}$ restores the global conservation law. Note that we have implicitly fixed a gauge freedom carried by the auxiliary indices $\tau_{i}$ to $\tau_{0}=\tau_{L} \equiv 0$ (we can even go further and permit any global change $\delta$ of the overall quantum number $\hat{N}$ by setting $\tau_{L}=\delta$ ).

Even though these expressions look a bit tedious, they can be represented compactly in form of a tensor network, which also reveals how useful this decoupling turns out to be (see Fig. 5.3a). For example, it is possible to identify the well-known transformation law for $U(1)$-invariant MPO site tensors in the expressions above [SPV11], yet the block indices $\tau_{j}$ are related to the change of the quantum number, captured by the shift tensors $R_{j ; \Delta}$.

Generally, contractions over physical bond indices $n_{j}, n_{j}^{\prime}$ of such shift tensors correspond to a mapping from one block $a$ of an irreducible representation $U_{j}(\sigma)=\bigoplus_{a} U_{j ; a}(\sigma)$ on a site $j$ to another block $a+\Delta$. Hence, given a decomposition into the different quantum-number sectors of an $U(1)$-invariant MPS or MPO tensor

$$
T_{j}=\bigoplus_{a} T_{j ; a}
$$


the contraction along a chain of $l$ shift tensors over physical indices (with $\Delta_{1} \ldots \Delta_{l}$ the changes in the local quantum numbers) is given via

$$
T_{j ; a+\Delta_{1}+\cdots+\Delta_{l}}=R_{j ; \Delta_{1}} \ldots R_{j ; \Delta_{l}} T_{j ; a} .
$$

From this point on it is clear how to generalize the considerations to arbitrary strings of local operators. In a given expression of local operators, we need to identify pairs of operators that conserve the global $U(1)$ quantum number but locally change the on-site quantum numbers $n_{i_{1}}^{\prime} \rightarrow n_{i_{1}}, n_{i_{2}}^{\prime} \rightarrow n_{i_{2}}$. For each pair, shift tensors then have to be inserted acting on the intermediate sites $i_{1}<k \leq i_{2}$. Note that in a code there is no need to explicitly implement the shift tensors. It suffices to implement only their action on the virtual bonds of either the MPS or of another MPO, i.e., to collect all vertical strings of shift tensors $\left\{R_{k ; \Delta_{r}}\right\}_{1 \leq r \leq l}$ in the network and to apply the shifting in the auxiliary block indices $\alpha_{k-1, k} \rightarrow \tilde{\alpha}_{k-1, k}=\alpha_{k-1, k}+\Delta_{1}+\cdots+\Delta_{l}$ (see Fig. 5.3b).

\section{\begin{tabular}{l|l}
$U(1)$-invariant MPO representation from FSMs & 5.3
\end{tabular}}

As already mentioned, every global operator on $\mathcal{H}$ can be formulated as a sum over lattice-ordered $n$-point $r+1$-ranged operator strings

$$
\hat{K}=\sum_{\nu, r} \hat{K}_{\nu, r}
$$

Note that the graph representation via FSM is not unique; for every operator $\hat{K}$, there is a set of corresponding FSMs $\{\Lambda(\hat{K})\}_{\Lambda}$. Therefore, we are free to choose one representation $\Lambda(\hat{K})$, which makes it easier to perform operator arithmetics and then switch to another representation $\tilde{\Lambda}(\hat{K})$ to find the most compact MPO.

Referring to Eq. (5.19), a natural translation to the graph representations of $\hat{K}$ can be obtained by introducing a commutative map $\oplus$ between graph representations of sums of operators $\hat{K}_{1}, \hat{K}_{2}$ via

$$
\oplus: \quad \Lambda\left(\hat{K}_{1}+\hat{K}_{2}\right)=\Lambda\left(\hat{K}_{1}\right) \oplus \Lambda\left(\hat{K}_{2}\right) .
$$

The realization of $\oplus$ in terms of graphs is obtained by taking the graph representations of the operators $\hat{K}_{1}, \hat{K}_{2}$ and by merging the initial and final states as depicted in Fig. 5.4. Next, we can define the notion of a maximally branched graph representation, which is given by the graph $\Lambda_{\max }(\hat{K})$ satisfying the conditions: a) the initial state $I$ is the only state with more than one child state, b) the final state $F$ is the only state with more than one parent state. $\Lambda_{\max }(\hat{K})$ satisfies the equation

$$
\Lambda_{\max }(\hat{K})=\bigoplus_{\nu, r} \Lambda\left(\hat{K}_{\nu, r}\right)
$$

This representation has several advantages; most importantly for our discussion, any local transformation of the operator can be mapped one-to-one to the transitions of the graph representation. Here local transformations are those transformations that map a lattice-ordered $n$-point $r+1$-ranged operator string into another lattice-ordered $m$-point $r+1$-ranged operator string $\hat{K}_{\nu, r} \rightarrow \hat{\tilde{K}}_{\tilde{\nu}, r}$ without changing $r$. To clarify what is meant by this mapping we emphasize that each branch $\hat{K}_{\nu, r}$ generates exactly one type of local-operator string $\hat{K}_{\nu_{1}} \ldots \hat{K}_{\nu_{r}}$ and therefore the tensor representation of these strings factorizes on the local Hilbert spaces. Hence, if we can give a factorization of the local transformation $\hat{U}$ in terms of tensors acting on the local Hilbert 

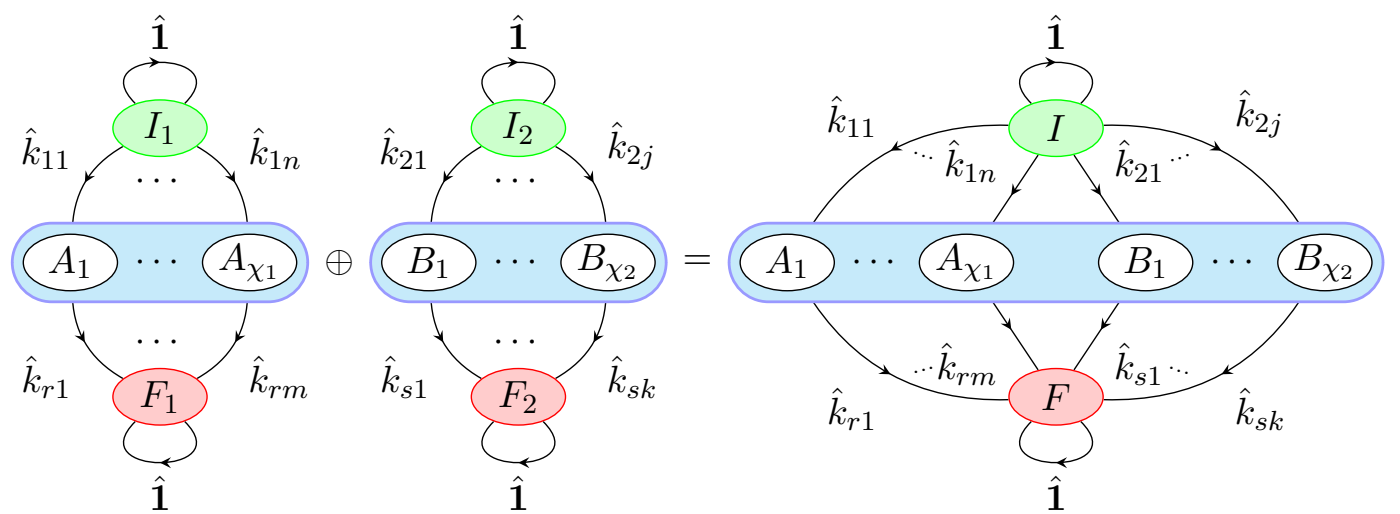

Figure 5.4: Realization of the operator sum $\hat{K}_{1}+\hat{K}_{2}$ in terms of graph representations $\Lambda\left(\hat{K}_{1}+\hat{K}_{2}\right)=$ $\Lambda\left(\hat{K}_{1}\right) \oplus \Lambda\left(\hat{K}_{2}\right)$. Graph representations of operators $\hat{K}_{1,2}$ are illustrated by transitions from the initial state into the graph's bulk $\left(\hat{k}_{11} \ldots \hat{k}_{1 n}\right.$ and $\left.\hat{k}_{21} \ldots \hat{k}_{2 j}\right)$ and from the graph's bulk to the final state $\left(\left(\hat{k}_{r 1} \ldots \hat{k}_{r m}\right.\right.$ and $\left.\left.\hat{k}_{s 1} \ldots \hat{k}_{s k}\right)\right)$. Blue boxes denote the bulk of the graph representations $\Lambda\left(\hat{K}_{1,2}\right)$ and $\Lambda\left(\hat{K}_{1}+\hat{K}_{2}\right)$. This graphic is adopted from the author's publication [PKM17].

(a)

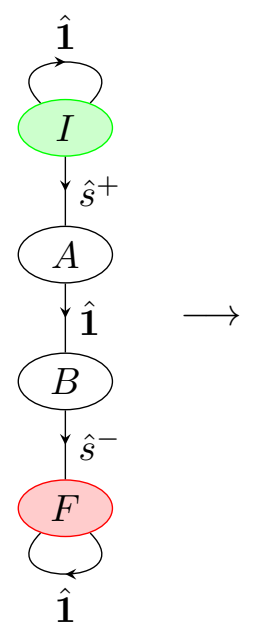

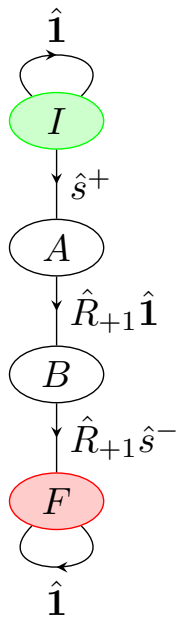

(b)

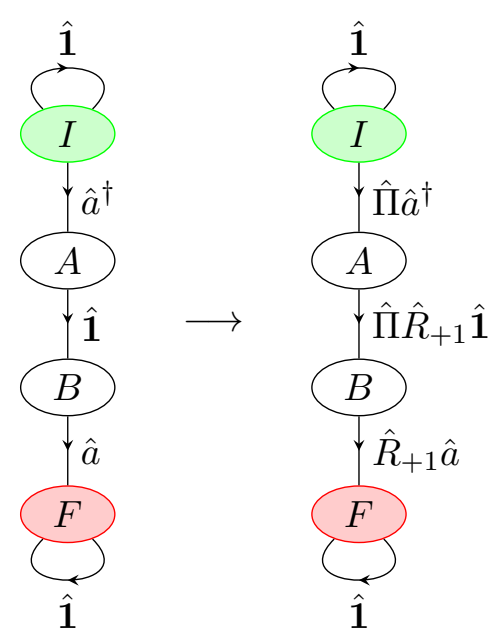

Figure 5.5: (a) Transformation of local operators $\hat{s}^{ \pm}, \hat{\mathbf{1}}$ to conserve $U(1)$ quantum numbers in the graph representation $\Lambda\left(\hat{K}_{1}\right)$ for a single-branch string operator $\hat{K}_{1}=\sum_{j} \hat{s}_{(j)}^{+} \hat{s}_{(j+2)}^{-}$. (b) Transformation of local operators $\hat{a}, \hat{a}^{\dagger}, \hat{\mathbf{1}}$ to conserve $U(1)$ quantum numbers in the graph representation $\Lambda\left(\hat{K}_{2}\right)$ for a singlebranch string operator, which describes fermionic next-to-nearest-neighbor hopping $\hat{K}_{2}=\sum_{j} \hat{f}_{(j)}^{\dagger} \hat{f}_{(j+2)}$. This graphic is adopted from the author's publication [PKM17].

spaces (e.g., $\hat{u}_{\nu_{1}} \ldots \hat{u}_{\nu_{r}}$ ), then we can represent the transformation directly by contracting the transformation tensors with the local operators over their physical indices

$$
\hat{k}_{\nu_{1}} \ldots \hat{k}_{\nu_{r}} \longrightarrow\left[\hat{u}_{\nu_{1}} \hat{k}_{\nu_{1}}\right] \ldots\left[\hat{u}_{\nu_{r}} \hat{k}_{\nu_{r}}\right] \text {. }
$$

Note that the transformations in Eq. (5.13) forcing conservation of $U(1)$ quantum numbers for two-site gates are of exactly this kind and so is their generalization to arbitrary strings of local operators. Thus, conservation of $U(1)$ quantum numbers can be implemented by transforming the graph representation of an operator into its maximally branched representation. The local 
operator strings in each branch are transformed by applying shift tensors $\hat{R}_{\Delta}$. For example, let us consider the transformation for a next-to-nearest-neighbor spin-flip term

$$
\hat{s}^{+} \otimes \hat{\mathbf{1}} \otimes \hat{s}^{-} \quad \longrightarrow \quad \hat{s}^{+} \otimes\left[\hat{R}_{+1} \hat{\mathbf{1}}\right] \otimes\left[\hat{R}_{+1} \hat{s}^{-}\right]
$$

with $\hat{s}^{ \pm}$being the usual angular-momentum ladder operators. The transformed graph representation is given in Fig. 5.5a.

Conveniently, these rules can be extended to anticommuting operators by applying a JordanWigner transformation ${ }^{2}$. For $U(1)$-invariant operators, the Jordan-Wigner transformation is also local, as there has to be a corresponding annihilation operator for every fermionic creation operator appearing in a string operator - and vice versa - because of quantum-number conservation. The Jordan-Wigner transformation can be implemented as a product of parity operators $\hat{\Pi}(j)$ via

$$
\hat{a}_{(j)} \rightarrow \hat{a}_{(j)} \mathrm{e}^{\mathrm{i} \pi \sum_{k<j} \hat{n}_{k}}=\prod_{k<j} \hat{\Pi}^{(k)} \hat{a}_{(j)}
$$

with $\hat{a}_{(j)}^{(\dagger)}$ annihilation (creation) operators for hard-core bosons at site $j$. Then we find that, for any $U(1)$-conserving product of fermionic creation and annihilation operators, the transformations act only within the operator strings. For instance, for a next-to-nearest-neighbor-hopping term we find

$$
\hat{a}^{\dagger} \otimes \hat{\mathbf{1}} \otimes \hat{a} \quad \longrightarrow \quad\left[\hat{\Pi} \hat{a}^{\dagger}\right] \otimes\left[\hat{\Pi} \hat{R}_{+1} \hat{\mathbf{1}}\right] \otimes\left[\hat{R}_{+1} \hat{a}\right] .
$$

Again, these transformations have a simple graph representation, see Fig. 5.5b.

To sum up, we have derived a construction scheme for MPO representations of generic $U(1)$ invariant operators that takes a FSM as input. Specifying the phase factor $e^{\mathrm{i} \phi}$ for the commutation relations of the local operators (e.g., $\phi=2 \pi$ for bosons, $\pi$ for fermions), the scheme automatizes the construction of the MPO site tensors so that we can identify the graph representation of the FSM with the MPO.

\section{MPO compression, graph arithmetics and implementation

The framework derived in the previous sections was the foundation to implement an interface in the SYMMPS toolkit to input arbitrary operators. The implementation permits for sitedependent transition amplitudes so that also non-homogenous operators can be created easily rendering the interface very flexible. We want to stress again that no MPO arithmetics is required to construct the desired MPO representation. Also, the framework is independent of local basis systems because it works on a completely abstract level without even knowing about the underlying algebraic structure (for instance, in the SYMMPS toolkit there are also mixedbasis systems available combining fermionic and bosonic lattice sites).

However, there are also further developements, which are not discussed in the context of this thesis but can be found in [PKM17] and are also presented in great detail in [Köh19]. One of these aspects is the possibility to compress the MPO representation already on the graph level. This procedure exploits the independence of the represented operator $\hat{K}$ on the particular graph representation $\Lambda(\hat{K})$. It is easy to see that branches in the graph corresponding to the same sequence of transitions in most situations can be fused into one and the same branch. The

\footnotetext{
${ }^{2}$ Note that this argument also holds in higher dimensions, because in MPS approaches a 1D path is used to sweep through the system, and the Jordan-Wigner transformation is also applicable in the presence of long-range interactions.
} 
fact that by this operation nodes are discarded corresponds to a reduction of the number of states required to represent $\hat{K}$ and thereby reduces the resulting MPO bond dimension. These compression methods are called delinearization and deparallelization in [HMS17] where they are introduced on the basis of MPO arithmetics. In [Köh19] the corresponding graph transformations are discussed. In the implementation of the SYMMPS toolkit we also included the possibility to truncate the constructed MPO representations by means of a rescaled SVD [HMS17] so that the most compact MPO representation can be achieved.

Another point is the generalization of the already introduced concept of operator arithmetics by means of the map $\oplus$ to also formulate multiplication of operators in a graph representation. To achieve this more work has to be done but the payoff is a representation of operator products that comes completely without subsequent compressions during the multiplication, which are required if the same operation is done using MPO arithmetics. We demonstrated the gain in the precision of the resulting MPO representations by evaluating the variance of a MPS approximation of a ground state of the Spin-1 Heisenberg chain in [PKM17]. The improvement is not only due to avoiding compression errors but also based on the fact that taylored graph representations can be constructed to reduce the effect of catastrophic cancellation.

Finally, we want to comment on the general advantage of having the possibility to create MPO representations by means of FSM. In fact, avoiding MPO arithmetics, the created matrices have an upper block-triangular structure. This structure can be used to decompose the MPO for later purposes such as an efficient parallelization exploiting the fact that in this representation we can deduce the contributions of the current site tensor to operator strings connecting the left and right system and those that open or close local operator strings (c.f. Eq. (4.10)). Notably, knowing about this structure is crucial to construct the $W^{\mathrm{I}, \mathrm{II}}$ time-evolution operator representation discussed in Sec. 4.5.1. Therefore, in order to have a generic implementation of the $W^{\mathrm{I}, \mathrm{II}}$ method an input in terms of FSMs is absolutely necessary. 


\section{Projected purifications to restore $U(1)$ symmetries}

In Secs. 4.6 and 5.3 we discussed how to exploit the conservation of $U(1)$ symmetries to reduce the numerical costs in MPS algorithms. However, there are many fundamental lattice models that do not support conservation of global symmetries, for instance, the transverse field Ising model, the Kitaev chain or the Holstein model. Additional complications arise if the dimension of the local Hilbert space is large. Then, without using a symmetry adapted basis in which the local operators are one-dimensional representations the contractions become extremly expensive. For instance, during the discussed ground-state search algorithm (c.f. Sec. 4.4.2) applying the effective two-site Hamiltonian scales as $\mathcal{O}\left(m^{3} w \sigma+m^{3} w \sigma^{2}+m^{2} w^{2} \sigma^{3}\right)$ and thus cubic in the local dimension $\sigma$ (quadratic in a single-site algorithm). Hence, while in the case of a small local Hilbert space $\sigma=2,3,4$ the dominating numerical expenses are due to the maximal bond dimension $m$ the situation may change drastically if we treat bosonic degrees of freedom where typically local dimensions of $\sigma>10$ are required. The large contribution of the local dimension to the numerical expenses reduces the accessible range of bond dimensions that can be treated and thereby impacts the quality of the approximated states drastically. This is even worse if the time evolution of some system is calculated as in these situation the entanglement growth demands large maximal bond dimensions to capture the dynamics faithfully. The paradox is that in many situations these additional degrees of freedom are not even occupied during the simulations. In order to resolve such situations, schemes to compress the local basis size were invented, which diagonalize the single-site reduced density matrix and truncate its eigenvalue spectrum [ZJW98, GWvDV12, BDV $\left.{ }^{+} 15\right]$. Such a local basis optimization has been applied successfully, for instance, to study the dynamics of a Holstein polaron $\left[\mathrm{BDV}^{+} 15\right]$ or the melting of a charge-density wave state in the Holstein model out-of equilibrium [ $\left.\mathrm{SHD}^{+} 20\right]$.

In the following we will present a different approach to overcome such restrictions, which we called projected purification (PP). However, in contrast to a local basis optimization that truncates the local dimension by means of a diagonalization of the single-site reduced density matrix we developed a method, which purifies the system to restore global $U(1)$ symmetries and then projects the purified Hamiltonian into a subspace of the same dimension as the original Hilbert space. Note that because of the projection we are not introducing additional degrees of freedom into the system. Since the MPO and MPS tensors decompose under the restored $U(1)$ symmetry the local dimension per block is immediately shrunk to one, yielding a significant speed-up. Additionally, having restored a global $U(1)$-symmetry we can exploit the usual truncation schemes, which enable us to discard non-occupied blocks by their contribution to the Schmidt decomposition, which gives a well-controlled approximation. Finally, we want to point out that our method is merely based on a reformulation of the Hamiltonian and thus no additional implementations are necessary and, hence, can be used in any existing code without further modifications.

We tested the developed method by comparing ground-state calculations of the Holstein model against results obtained from a code exploiting local basis optimization [SHD $\left.{ }^{+} 20\right]$. This code was provided and the corresponding calculations were performed by Jan Stolpp in the group of Fabian Heidrich-Meisner at the Georg-August-Universität Göttingen.

Furthermore, we also used this new method to study a proximity-coupled nanowire in the Coulomb blockade regime coupled to one or two normal leads as discussed in Chap. 7. 


\section{\begin{tabular}{l|l} 
Projected purified operators & 6.1
\end{tabular}}

Let $\hat{O}$ be an operator acting on a tensor product Hilbert space over $L$ sites, which we will refer to from now on as the physical Hilbert space $\mathcal{H}_{P}=\mathcal{H}_{P, \sigma}^{\otimes L}$ and $\hat{N}_{P}=\sum_{j} \hat{n}_{j}^{P}$ an operator acting on $\mathcal{H}_{P}$ with local operators $\hat{n}_{j}^{P}: \mathcal{H}_{P, \sigma} \longmapsto \mathcal{H}_{P, \sigma}$ fulfilling the commutation relations $\left[\hat{n}_{j}^{P}, \hat{n}_{k}^{P}\right]=0$. We denote the ladder operators spanning the algebra of local operators by $\hat{c}_{P, j} / \hat{c}_{P, j}^{\dagger}$ that obey canonical commutation relations $\left[\hat{c}_{j}, \hat{c}_{k}^{\dagger}\right]_{\epsilon}=\delta_{j, k}$ and $\epsilon= \pm$ distinguishes between the commutator or anticommutator. Without loss of generality, we choose the spectrum of the local operators $\hat{n}_{j}^{P / B}$ to be $n_{j}^{P / B} \in\{0,1, \ldots, \sigma-1\}$. Let us assume furthermore that $\hat{O} \equiv \hat{O}_{P}$ contains summands with ladder operators $\hat{c}_{P ; j} / \hat{c}_{P ; j}^{\dagger}$ that are not paired up with their hermitian conjugates breaking the global $U(1)$ symmetry generated by $\hat{N}_{P}$. For instance, in the Kitaev chain such contributions are given by the $p$-wave like pairing

$$
\hat{O}=-\sum_{j}\left(\hat{c}_{P ; j} \hat{c}_{P ; j+1}+\hat{c}_{P ; j+1}^{\dagger} \hat{c}_{P ; j}^{\dagger}\right) \Rightarrow\left[\hat{N}_{P}, \hat{O}\right] \neq 0 .
$$

Next, we introduce a bath system $\mathcal{H}_{B}=\mathcal{H}_{B, d}^{\otimes L}$ as an exact copy of $\mathcal{H}_{P}$ and an operator $\hat{N}_{B}=$ $\sum_{j} \hat{n}_{j}^{B}$ where the $\hat{n}_{j}^{B}$ 's have exactly the same properties as the density operators $\hat{n}_{j}^{P}$ introduced before but are acting only on the local Hilbert spaces $\mathcal{H}_{B, \sigma}$ of the bath system. In particular, the basis states $\left|n_{1}^{B}\right\rangle \otimes \cdots \otimes\left|n_{L}^{B}\right\rangle \equiv\left|n_{1}^{B} \cdots n_{L}^{B}\right\rangle$ span a complete orthonormal basis of $\mathcal{H}_{B}$. Then, we consider the subspace $\mathcal{P} \subset \mathcal{H}_{P B}$ of the purified system that is spanned by all

$$
\begin{aligned}
\left.\mid n_{1}, \ldots, n_{L}\right) & =\left|n_{1}, \ldots, n_{L}\right\rangle_{P} \otimes\left|g\left(n_{1}\right), \ldots, g\left(n_{L}\right)\right\rangle_{B} \\
& =\left|n_{1}, \ldots, n_{L}, g\left(n_{1}\right), \ldots, g\left(n_{L}\right)\right\rangle_{P B}
\end{aligned}
$$

with $n_{i} \in[0, \sigma-1]$ and $g(x)=\sigma-1-x$. Note that for convenience we have labeled the kets in the physical and bath system by subscripts and introduced rounded kets to indicate states in the subspace $\mathcal{P} \subset \mathcal{H}_{P B}$, which depend only on a reduced number of coefficients $n_{1}, \ldots, n_{L}$. It is easy to see that this subspace is contained in the subspace with $N_{P}+N_{B}=(\sigma-1) \cdot L$, i.e.,

$$
\left.\left.\left(\hat{N}_{P}+\hat{N}_{B}\right) \mid n_{1}, \ldots, n_{L}\right)=(\sigma-1) \cdot L \mid n_{1}, \ldots, n_{L}\right),
$$

so that all states in the subspace $\mathcal{P}$ transform symmetrically under the action of the global $U(1)$ symmetry generated by $\hat{N}_{P}+\hat{N}_{B}$. Furthermore note that by simply counting the number of basis states spanning $\mathcal{P}$ it follows that $\operatorname{dim} \mathcal{H}_{P}=\operatorname{dim} \mathcal{P}$.

Now, denote by $\mathcal{H}$ the tensor product Hilbert space on which $\hat{O}$ was defined, originally. We define the map

$$
\begin{aligned}
M: \mathcal{P} & \longrightarrow \mathcal{H} \\
\mid \psi) & \longmapsto|\psi\rangle
\end{aligned}
$$

identifying states $|\psi\rangle \in \mathcal{P}$ in the subspace of the purified system with states $|\psi\rangle \in \mathcal{H}$ in the original Hilbert space. Since $g(x)$ is invertible and $\operatorname{dim} \mathcal{P}=\operatorname{dim} \mathcal{H}_{P}=\operatorname{dim} \mathcal{H}$ it follows that $M$ is invertible. Now we define the projected purified operator $\hat{O}_{P P}: \mathcal{P} \longrightarrow \mathcal{P}$ by the following relation

$$
\hat{O}=M \hat{O}_{P P} M^{-1} .
$$


Assuming $\hat{O}_{P P}$ exists, this definition implies in particular that

$$
\left\langle n_{1}, \ldots, n_{L}|\hat{O}| n_{1}^{\prime}, \ldots, n_{L}^{\prime}\right\rangle=\left(n_{1}, \ldots, n_{L}\left|\hat{O}_{P P}\right| n_{1}^{\prime}, \ldots, n_{L}^{\prime}\right),
$$

that is, the matrix representations of $\hat{O}$ and $\hat{O}_{P P}$ in the local basis sets $\left\{\left|n_{1}, \ldots, n_{L}\right\rangle\right\}$ and $\left.\left\{\mid n_{1}, \ldots, n_{L}\right)\right\}$ are identical. We can, hence, work with $\hat{O}_{P P}$ in the subspace $\mathcal{P}$ instead of $\hat{O}$.

In order to show that $\hat{O}_{P P}$ always exists we construct it explicitely. Therefore, we note that the above definition of $\mathcal{P}$ is equivalent to

$$
\left.\left.|\psi| \in \mathcal{P} \Leftrightarrow\left(\hat{n}_{j}^{P}+\hat{n}_{j}^{B}\right) \mid \psi\right)=(\sigma-1) \mid \psi\right) \quad \text { for all } j \in\{1, \ldots, L\} .
$$

But this means that each operator $\hat{O}_{P P}$ has to satisfy

$$
\left[\hat{O}_{P P}, \hat{n}_{j}^{P}+\hat{n}_{j}^{B}\right]=0 \quad \text { for all } j \in\{1, \ldots, L\} .
$$

This motivates us to define balancing operators $\hat{\beta}_{B ; j} / \hat{\beta}_{B ; j}^{\dagger}: \mathcal{H}_{B, \sigma} \longrightarrow \mathcal{H}_{B, \sigma}$

$$
\begin{aligned}
{ }_{B}\left\langle\tilde{n}_{j}\left|\hat{\beta}_{B ; j}\right| n_{j}\right\rangle_{B} & =\delta_{\tilde{n}_{j}, n_{j}+1} \\
{ }_{B}\left\langle\tilde{n}_{j}\left|\hat{\beta}_{B ; j}^{\dagger}\right| n_{j}\right\rangle_{B} & =\delta_{\tilde{n}_{j}, n_{j}-1} \\
{\left[\hat{\beta}_{B ; j}, \hat{\beta}_{B ; k}^{\dagger}\right] } & =\delta_{j, k}
\end{aligned}
$$

Since every operator $\hat{O}_{P} \otimes \hat{\mathbf{1}}_{B}$ acting non-trivially on $\hat{H}_{P}$ only can be expressed as function of a product of ladder operators $\hat{c}_{P, j}^{[\dagger]}$ we can thus map it to $\mathcal{P}$ by the following transformations

$$
\hat{c}_{P ; j}^{\dagger} \longrightarrow \hat{c}_{P ; j}^{\dagger} \hat{\beta}_{B ; j} \text { and } \hat{c}_{P ; j} \longrightarrow \hat{c}_{P ; j} \hat{\beta}_{B ; j}^{\dagger} \text {. }
$$

It can be checked easily that by means of this transformation the local conservation laws $\left[\hat{O}_{P P}, \hat{n}_{j}^{P}+\hat{n}_{j}^{B}\right]=0$ are fulfilled for all sites $j$. As example we consider again the Kitaev chain (setting the chemical potential to $\mu \equiv 0$ ) and construct the projected purified operator exemplary:

$$
\begin{aligned}
\hat{H} & =-\sum_{j}\left(t \hat{c}_{j}^{\dagger} \hat{c}_{j+1}+\Delta \hat{c}_{j} \hat{c}_{j+1}+\text { h.c. }\right) \\
\Rightarrow \hat{H}_{P P} & =-\sum_{j}\left(t \hat{c}_{P ; j}^{\dagger} \hat{\beta}_{B ; j} \hat{c}_{P ; j+1} \hat{\beta}_{B ; j+1}^{\dagger}+\Delta \hat{c}_{P ; j} \hat{\beta}_{B ; j}^{\dagger} \hat{c}_{P ; j+1} \hat{\beta}_{B ; j+1}^{\dagger}+\text { h.c. }\right) .
\end{aligned}
$$

There is also another way to introduce projected purified operators. We can define the projection operator

$$
\left.\hat{P}=\sum_{\left\{n_{j}\right\}} \mid n_{1} \cdots n_{L}\right)\left(n_{1} \cdots n_{L} \mid\right.
$$

and look for operators satisfying $\hat{P} \hat{O}_{P P} \hat{P}=\hat{O}_{P P}$. Those operators are manifestly invariant under a projection into $\mathcal{P}$ and therefore, ignoring zero elements, have the same matrix elements in both $\mathcal{H}$ and $\mathcal{P}$. Here the important observation is that restricting the ansatz class of MPS states $|\psi\rangle_{P B} \in \mathcal{H}_{P B}$ to $\mathcal{P}$ we have found a one-to one mapping between $\mathcal{H}$ and $\mathcal{H}_{P B}$, and the states $|\psi\rangle=\hat{P}|\psi\rangle_{P B}$ have a representation in terms of $U(1)$-invariant MPS. In the following, we will explicitely derive this representation of states in $\mathcal{P}$ and demonstrate the capability of the introduced $U(1)$-symmetrization to improve the numerical efficiency of MPS calculations. 


\section{Projected purified MPS}

The introduced mapping from an operator breaking a global $U(1)$ symmetry to a $U(1)$ symmetry conserving one can be exploited to effciently reduce the matrix sizes of MPS representations. The key observation is that, while purified states in general have a huge redundancy that comes with additional gauge degrees of freedom, the projection into $\mathcal{P}$ fixes all these gauge degrees of freedom by the $L$ local gauge conditions given in Eq. (6.8). Here, we will discuss the implications on the projection of purified MPS into $\mathcal{P}$ and derive an important connection between the Schmidt decomposition of the purified states and the single-site reduced density matrix.

Let $|\psi\rangle \in \mathcal{H}$ and consider its single-site representation

$$
|\psi\rangle=\sum_{\bar{\alpha}, \beta, n_{j}} M_{j ; \bar{\alpha}, \beta}^{n_{j}}|\bar{\alpha}\rangle \otimes\left|n_{j}\right\rangle \otimes|\beta\rangle,
$$

with $\left\langle\bar{\alpha} \mid \bar{\alpha}^{\prime}\right\rangle=\delta_{\bar{\alpha}, \bar{\alpha}^{\prime}}$ and $\left\langle\beta \mid \beta^{\prime}\right\rangle=\delta_{\beta, \beta^{\prime}}$. Following the previous considerations we take this state representation into the subspace $\mathcal{P}$ of the enlarged Hilbert space $\mathcal{H}_{P} \otimes \mathcal{H}_{B}$ with $N_{P}+N_{B}=$ $(\sigma-1) \cdot L$ by mapping $\left.\left|n_{j}\right\rangle \stackrel{M^{-1}}{\longmapsto}\left|n_{j}, g\left(n_{j}\right)\right\rangle_{P B}=\mid n_{j}\right)$. Then, the single-site representation of $|\psi\rangle$ can be interpreted as a two-site representation in $\mathcal{H}_{P} \otimes \mathcal{H}_{B}$

$$
|\psi\rangle_{P B}=\sum_{\substack{\bar{\alpha}, \alpha \\ n_{j}^{P}, n_{j}^{B}}} M_{j ; \bar{\alpha}, \beta}^{n_{j}^{P}, n_{j}^{B}} \delta_{n_{j}^{B}, g\left(n_{j}^{P}\right)}|\bar{\alpha}\rangle_{P} \otimes\left|n_{j}^{P}, n_{j}^{B}\right\rangle_{P B} \otimes|\beta\rangle_{P}
$$

Pursuing this prescription at all sites $j \in\{1, \ldots, L\}$ the resulting state representation is in the subspace $\mathcal{P}$ of the enlarged Hilbert space and the site tensors decompose under the global $U(1)$-symmetry as

$$
M^{n_{j}^{P}, n_{j}^{B}}=\sum_{\bar{\alpha}, \beta} T_{j ; \bar{\alpha}, \beta}^{n_{j}^{P}, n_{j}^{B}} \delta\left(n_{j}^{P}+n_{j}^{B}+\bar{\alpha}-\beta\right)
$$

A matrix factorization of the decomposed site tensors $M^{n_{j}^{P}, n_{j}^{B}}=\bigoplus_{N_{j}} T_{N_{j}}^{n_{j}^{P}, n_{j}^{B}}$ in each symmetry block $N_{j}$ yields the MPS representation of $|\psi\rangle_{P}$ in the subspace $\mathcal{P}$ of the enlarged Hilbert space

$$
\begin{aligned}
|\psi\rangle_{P B}= & \sum_{\bar{\alpha}, \gamma, n_{j}^{P}} T_{j ; \bar{\alpha}, \gamma}^{n_{j}^{P}} \delta\left(n_{j}^{P}+\bar{\alpha}-\gamma\right)|\bar{\alpha}\rangle \otimes\left|n_{j}^{P}\right\rangle_{P} \times \cdots \\
& \sum_{\beta, n_{j}^{B}} T_{j ; \bar{\gamma}, \beta}^{n_{j}^{B}} \delta\left(n_{j}^{B}+\bar{\gamma}-\beta\right) \delta_{n_{j}^{B}, g\left(n_{j}^{P}\right)}\left|n_{j}^{B}\right\rangle \otimes|\beta\rangle .
\end{aligned}
$$

The MPS constructed in this way is shown in Fig. 6.1 and consists of alternating physical and bath sites, which are labeled by the physical and bath degrees of freedom $n_{j}^{P}$ and $n_{j}^{B}$, respectively. The delta-function $\delta_{n_{j}^{B}, g\left(n_{j}^{P}\right)}$ in the last line of Eq. (6.19) is just a manifestation of the $L$ gaugefixing conditions imposed in Eq. (6.8). It motivates the introduction of auxiliary bond labels $\eta$ enumerating the irreducible representations of each local conserved quantity between the physical and bath sites so that the bond label $\gamma$ can be decomposed into labels $\gamma \rightarrow\left(\alpha^{\prime} \eta\right)$ that need to fulfill $\alpha^{\prime}+\eta=\bar{\alpha}+n_{j}^{P}$. From the local conservation laws and the gauge fixing we can furthermore conclude that the bond label $\bar{\alpha}$ has only one non-vanishing block with respect to the global $U(1)$ symmetry, which is characterized by a quantum number $(j-1) \cdot(\sigma-1) \equiv \bar{\alpha}$. Accordingly, 


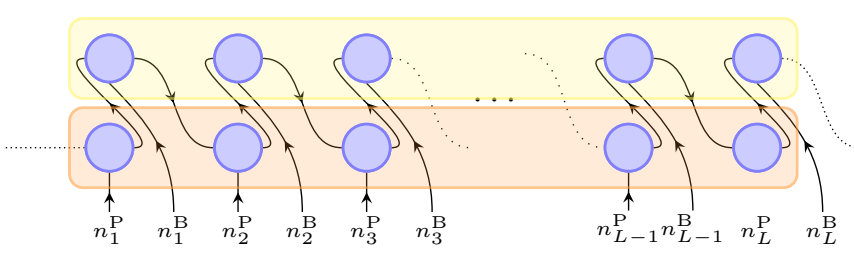

Figure 6.1: $\quad$ MPS representation in enlarged Hilbert space with each physical site accompanied by a bath site.

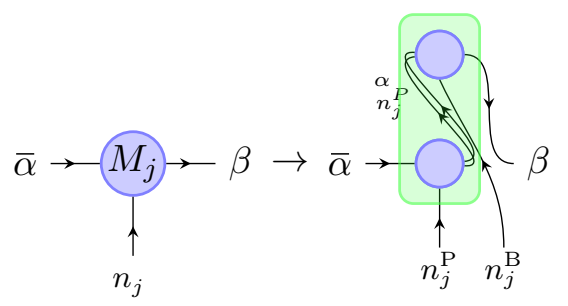

Figure 6.2: Decomposition of general MPS tensor into $U(1)$-invariant physical and bath site tensors. Decomposition of the introduced auxiliary index $\alpha_{j}^{\prime}$ into irreducible representation of the local conservation law generated by the gauge-fixing $\hat{n}_{j}^{P}+\hat{n}_{j}^{B} \equiv \sigma-1$ is sketched by double bonds $\alpha_{j}^{\prime} \rightarrow\left(\alpha_{j} n_{j}^{P}\right)$.

there is only one non-vanishing block $\beta$ to the right of the bath site, which is characterized by a quantum number $j \cdot(\sigma-1) \equiv \beta$. In tensor notation this can be expressed by a reformulation of the local conservation laws at every site, introducing for brevity $N_{j}=(\sigma-1) \cdot(j-1)$

$$
\sum_{\gamma} T_{j ; \bar{\alpha}, \gamma}^{n_{j}^{P}} T_{j ; \bar{\gamma}, \beta}^{n_{j}^{B}} \delta_{n_{j}^{B}, g\left(n_{j}^{P}\right)}=\sum_{\alpha^{\prime}, \eta} T_{j ; \bar{\alpha}, \alpha^{\prime} \eta}^{n_{j}^{P}} \delta\left(N_{j}-\alpha\right) T_{j ; \bar{\alpha}^{\prime} \eta, \beta}^{n_{j}^{B}} \delta\left(N_{j+1}-\beta\right) .
$$

Therefore, we arrive at the important observation that there is a unique decomposition of the auxiliary bond label $\gamma=\left(\alpha^{\prime}, \eta\right)$ given by identifying $\eta \equiv n_{j}^{P}$ and thus also $\alpha^{\prime} \equiv \alpha$. This can be summarized by decomposing the site tensors as

$$
\sum_{\gamma} T_{j ; \bar{\alpha}, \gamma}^{n_{j}^{P}} T_{j ; \bar{\gamma}, \beta}^{n_{j}^{B}}=\bigoplus_{n_{j}^{P}} \sum_{\alpha} T_{j ; \bar{\alpha},\left(\alpha n_{j}^{P}\right)}^{n_{P}^{P}} T_{j ;\left(\bar{\alpha} \bar{n}_{j}^{P}\right), \beta}^{n_{j}^{B}},
$$

which is exemplary shown in Fig. 6.2.

Now, we consider the expectation value $\left\langle\hat{n}_{j}^{P}\right\rangle$ in the single-site representation

$$
\begin{aligned}
\left\langle\hat{n}_{j}^{P}\right\rangle & =\sum_{n_{j}^{P}} \sum_{\alpha}\left(T_{j ; \bar{\alpha},\left(\alpha n_{j}^{P}\right)}^{n^{P}}\right)^{*} T_{j ; \bar{\alpha},\left(\alpha n_{j}^{P}\right)}^{n_{j}^{P}} n_{j}^{P} \\
& =\sum_{n_{j}^{P}} n_{j}^{P} \sum_{\alpha}\left|T_{j ; \bar{\alpha},\left(\alpha n_{j}^{P}\right)}^{n_{j}^{P}}\right|^{2} \equiv \sum_{n_{j}^{P}} n_{j}^{P} \rho_{n_{j}^{P}}
\end{aligned}
$$

where we made use of the fact that the local symmetry generators $\hat{n}_{j}^{P}$ are one-dimensional representations of the global $U(1)$ symmetry. In the last expression we can identify the expectation value in terms of the single-site reduced density matrix $\hat{\rho}_{j}=\operatorname{Tr}_{k \neq j} \hat{\rho}$

$$
\operatorname{Tr}_{j}\left\langle\hat{\rho}_{j} \hat{n}_{j}^{P}\right\rangle=\sum_{n_{j}^{P}}\left\langle n_{j}^{P}\left|\rho_{n_{j}^{P}, n_{j}^{P}} \hat{n}_{j}^{P}\right| n_{j}^{P}\right\rangle \equiv \sum_{n_{j}^{P}} \rho_{n_{j}^{P}} n_{j}^{P}
$$




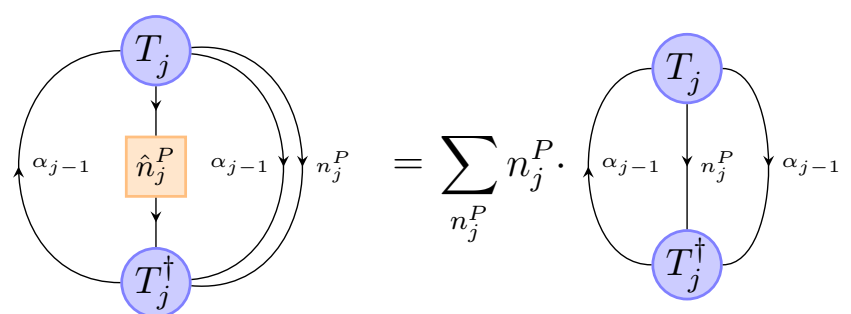

Figure 6.3: Expectation value of the local density $\hat{n}_{j}^{P}$, which by Eq. (6.24) can be directly related to the diagonal elements of the single-site reduced density matrix in the eigenbasis $\hat{n}_{j}^{P}$.

so that

$$
\left\langle n_{j}^{P}\left|\hat{\rho}_{j}\right| n_{j}^{P}\right\rangle=\rho_{n_{j}^{P}}=\sum_{\alpha}\left|T_{j ; \bar{\alpha},\left(\alpha n_{j}^{P}\right)}^{n_{P}^{P}}\right|^{2} \equiv\left|T^{n_{j}^{P}}\right|^{2}
$$

which is also shown in Fig. 6.3.

This last equation reveals a very intimate relation between the physical quantity $\hat{\rho}_{j}$ and the singular values of the factorized site matrices between the physical and bath sites. If we consider the Schmidt decomposition of a state $|\psi\rangle$ at the auxiliary bond $\gamma_{j-1}=\left(\alpha_{j-1} n_{j}^{P}\right)$ by factorizing each block

$$
T_{j ; \bar{\alpha}_{j-1}, \gamma_{j-1}}^{n_{j}^{P}}=U_{j ; \bar{\alpha}_{j-1}}^{n_{j}^{P}} \Lambda^{n_{j}^{P}} V_{j ; \gamma_{j-1}}^{n_{j}^{P}}
$$

where $\Lambda^{n_{j}^{P}}=\operatorname{diag}\left(\Lambda_{j ; 0}^{n_{j}^{P}}, \cdots \Lambda_{j ; m_{j ; n_{j}^{P}}}^{n_{P}^{P}}\right)$ we can identify the sum over the squared singular values in each block with the occupation $\rho_{n_{j}^{P}}$ of the single-site reduced density matrix

$$
\sum_{\tau}\left(\Lambda_{j ; \tau}^{n_{j}^{P}}\right)^{2}=\rho_{n_{j}^{P}}
$$

Hence, truncating the singular values according to a certain threshold $0<\delta \ll 1$, so that $\sum_{n_{j}^{P}} \sum_{\tau}\left(\Lambda_{j ; \tau}^{n_{j}^{P}}\right)^{2}<1-\delta$, this implies a rescaling of the diagonal elements of the single-site reduced density matrix $\left\langle n_{j}^{P}\left|\hat{\rho}_{j}\right| n_{j}^{P}\right\rangle$ that is governed by the decay of the singular values $\Lambda_{j, \tau}^{n_{j}^{P}}$ in each block.

Here, we can also make the connection to the local basis optimization [ZJW98, BDV $\left.{ }^{+} 15\right]$. Therefore we note that summing Eq. (6.26) over all basis states $n_{j}^{P}$ is invariant under unitary basis transformations of the local basis states

$$
\sum_{n_{j}^{P}} \sum_{\tau}\left(\Lambda_{j ; \tau}^{n_{j}^{P}}\right)^{2}=\left\langle n_{j}^{P}\left|\hat{\rho}_{j}\right| n_{j}^{P}\right\rangle=\left\langle\hat{U} n_{j}^{P}\left|\hat{U} \hat{\rho}_{j} \hat{U}^{\dagger}\right| \hat{U} n_{j}^{P}\right\rangle \equiv\left\langle\tilde{n}_{j}^{P}\left|\hat{\tilde{\rho}}_{j}\right| \tilde{n}_{j}^{P}\right\rangle
$$

However, we can insert the same unitary transformation in the explicit $U(1)$ symmetric decomposition of the physical site tensor

$$
\bigoplus_{n_{j}^{P}} \sum_{\alpha} T_{j ; \bar{\alpha},\left(\alpha n_{j}^{P}\right)}^{n_{j}^{P}} \stackrel{U}{\longrightarrow} \sum_{n_{j}^{P}} U_{\tilde{n}_{j}^{P}, n_{j}^{P}} \bigoplus_{n_{j}^{P}} \sum_{\alpha} T_{j ; \bar{\alpha},\left(\alpha n_{j}^{P}\right)}^{n^{P}} .
$$


Note that the action of the unitary transformation is a relabeling of the block decomposition destroying the $U(1)$ symmetric form of the tensor. But we can use the gauge degree of freedom of MPS to insert the transformation $\left(\hat{\mathbf{1}}_{\alpha} \otimes U^{\dagger}\right)\left(\hat{\mathbf{1}}_{\alpha} \otimes U\right)$ on the bond between the physical and bath site tensor. Then, the physical site tensor is transformed as

$$
\begin{aligned}
\sum_{n_{j}^{P}} U_{\tilde{n}_{j}^{P}, n_{j}^{P}} \bigoplus_{n_{j}^{P}} \sum_{\alpha} T_{j ; \bar{\alpha},\left(\alpha n_{j}^{P}\right)}^{n_{j}^{P}} \stackrel{\stackrel{\hat{\mathbf{1}} \otimes U^{\dagger}}{\longrightarrow} \sum_{\tilde{n}_{j}^{\prime}, n_{j}^{P}} \underbrace{U_{\tilde{n}_{j}^{\prime} P, n_{j}^{P}}^{*} U_{\tilde{n}_{j}^{P}, n_{j}^{P}}}_{\delta_{\tilde{n}_{j}^{P}, \tilde{n}_{j}^{\prime} P}} \bigoplus_{n_{j}^{P}} \sum_{\alpha} T_{j ; \bar{\alpha},\left(\alpha \tilde{n}_{j}^{\prime}\right)}^{\left.\tilde{n}_{j}^{\prime P}\right)}}{ } \\
=\bigoplus_{\tilde{n}_{j}^{P}} \sum_{\alpha} T_{j ; \bar{\alpha},\left(\alpha \tilde{n}_{j}^{P}\right)}^{\tilde{n}_{j}^{P}} .
\end{aligned}
$$

If we do the same transformation on the bath-site tensor we find that the block-diagonal representation is restored and therefore the site tensors transform symmetrically under the local $U(1)$ symmetry generated by $\hat{U}\left(\hat{n}_{j}^{P}+\hat{n}_{j}^{B}\right) \hat{U}^{\dagger}$. More importantly, this representation is manifestly diagonal in the transformed physical indices $\tilde{n}_{j}^{P}$. Comparing this equation to Eq. (6.26) and remembering that the SVD also decomposes in the physical indices we find that the summed squared singular values in each block are given by the eigenvalues of the single-site reduced density matrix. Thus, truncating the bond index by means of the usual MPS truncation routine is completely equivalent to the truncation occuring in the local basis optimization. Even more, performing the truncation in the projected purified representation automatically picks those eigenvalues of $\hat{\rho}_{j}$ that have the largest weight without the necessity of constructing the single-site reduced density matrix at all. This is an important improvement as it prevents us from repeated constructions of $\hat{\rho}_{j}$ in contrast to the local basis optimization.

\section{\begin{tabular}{l|l} 
Characterization of numerical expenses & 6.2 .1
\end{tabular}}

The previous considerations enable us to compare the numerical complexity of typical tensor contractions arising from the MPS representation of states $|\psi\rangle \in \mathcal{P}$ with those of MPS representations without the expansion of the Hilbert space. Note that due to Eq. (6.26) and the previously discussed connection between the singular values and the eigenvalues of the single-site reduced density matrix we will consider $\hat{\rho}_{j}$ to be represented in its eigenbasis so that by $\rho_{n_{j}^{P}}$ we will refer to the eigenvalues of $\hat{\rho}_{j}$. At first we point out again that due to the local conservation laws and gauge fixing the bond labels $\alpha, \beta$ of the MPS site tensors $T_{j ; \bar{\alpha}, \gamma}^{n_{j}^{P}}$ and $T_{j ; ;}^{n_{j}^{B}}$ have only one non-vanishing entry, each of which is given by $\alpha=N_{j}, \beta=N_{j+1}$ with $N_{j}$ as defined above. Therefore, without truncation the bond dimensions $m_{j-1}, m_{j}$ are identical to those of the site tensors $M_{j ; \bar{\alpha}, \beta}^{n_{j}}$ representing the same state in the physical Hilbert space only. There is no additional complexity arising from the representation of $|\psi\rangle \in \mathcal{P}$ on these indices. Furthermore, without truncation the effective bond dimensions on the $\gamma$-bonds are given by $m_{j ; \gamma}=d \cdot \min \left(m_{j-1}, m_{j}\right)$. In what follows, we will analyze two truncation schemes on these bonds for states in the enlarged Hilbert space. Thereafter, we will discuss in which situations these yield a reduced numerical complexity of the most expensive operation during ground state calculations, i.e., the application of an MPO to a state.

A physically motivated truncation can be defined by exploiting Eq. (6.26) and discarding all single-site occupations of $\hat{\rho}_{j}$ whose sum is below a given threshold $\delta>0$. More precisely, let $\mathcal{D} \subset\{0, \cdots, \sigma-1\}$ be a set, for which $\sum_{n_{j}^{P} \in \mathcal{D}} \rho_{n_{j}^{P}}<1-\delta$. Since $\hat{\rho}_{j}$ is a reduced density matrix its trace is normalized and by sorting the diagonal elements such a set can always be defined. 


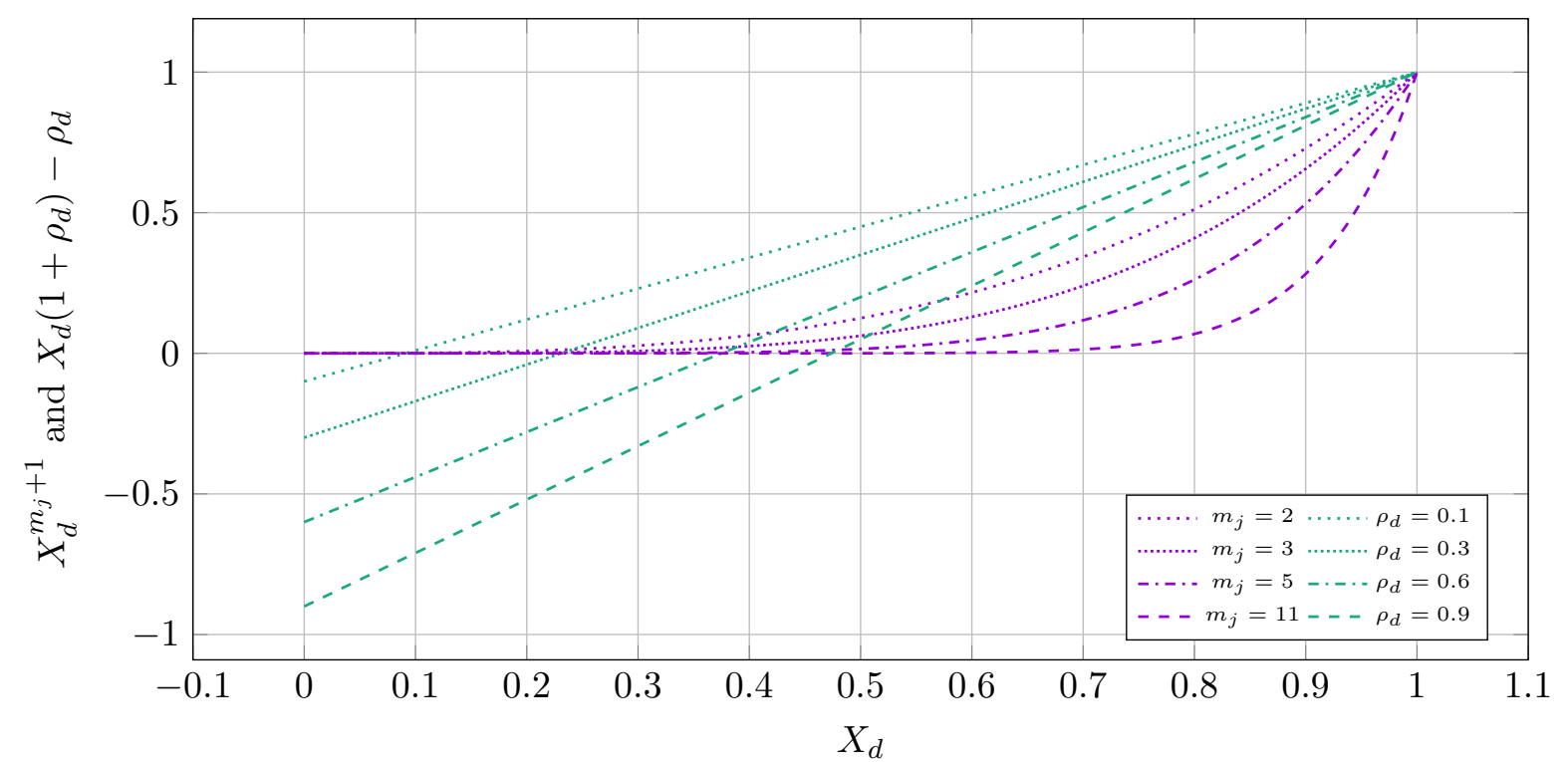

Figure 6.4: Left (purple) and right (green) hand sides of Eq. (6.32), $X_{d}$ values at intersections are solutions for distinct pairs of $\left(\rho_{d}, m_{j}\right)$.

Then, all tensor blocks $T_{j ; \bar{\alpha}, \alpha n_{j}^{P}}^{n_{p}^{P}}$ with $n_{j}^{P} \notin \mathcal{D}$ are discarded so that the total number of kept states on the auxiliary bond is bounded by $m_{j ; \gamma} \leq|\mathcal{D}| \min \left(m_{j-1}, m_{j}\right)$. The physical interpretation is straight forward: all tensor blocks $T^{n_{j}^{P}}$ that have a negligible single-site occupation $\left|T^{n_{j}^{P}}\right|^{2}=\hat{\rho}_{n_{j}^{P}}$ are discarded, i.e., empty modes do not contribute to the physics. However, we can give a tighter estimate by considering the explicit distribution of the singular values in each block. Motivated by the observation that in ground states of one-dimensional gapped systems the singular values typically decay exponentially [VC06], we assume such a decay in each block $T_{j ; \bar{\alpha}, \alpha d}^{d}(d \in \mathcal{D})$

$$
\Lambda_{j ; \tau}^{d}=e^{-a_{d} \tau}, \quad \sum_{\tau=1}^{m_{j}} e^{-2 a_{d} \tau}=\rho_{d},
$$

for some $a_{d}>0$ and we abbreviated $m_{j} \equiv \min \left(m_{j-1}, m_{j}\right)$. Normalization to the single-site occupation yields

$$
\rho_{d}=e^{-2 a_{d}} \sum_{\tau=0}^{m_{j}-1}\left(e^{-2 a_{d}}\right)^{\tau}=\frac{e^{-2 a_{d}}-e^{-2 a_{d}\left(m_{j}+1\right)}}{1-e^{-2 a_{d}}} .
$$

Defining $a_{d}=-\frac{1}{2} \log X_{d}$ with $0<X_{d}<1$ we can rewrite Eq. (6.31) into

$$
X_{d}^{m_{j}+1}=X_{d}\left(1+\rho_{d}\right)-\rho_{d} .
$$

Since $\delta \leq \rho_{d} \leq 1$ and $m_{j} \geq 1$ this equation has only one solution for $X_{d}$ in the given domain even though there is no closed expression (see Fig. 6.4 for graphical solution at distinct pairs $\left(\rho_{d}, m_{j}\right)$ ). Therefore, we consider two limiting cases that yield upper and lower bounds on the decay of the singular values in each tensor block. The lower bound $X_{d, \min }$ is obtained by the intersection of the right hand side with the horizontal axis and can be related to the limit $m_{j} \gg 1$ :

$$
\begin{aligned}
0 & =X_{d, \min }\left(1+\rho_{d}\right)-\rho_{d} \\
\Rightarrow X_{d} & \geq X_{d, \text { min }}=\frac{\rho_{d}}{1+\rho_{d}} .
\end{aligned}
$$


An upper bound $X_{d, \max }$ can be established if the right hand side of Eq. (6.32) is tangential to the left hand side

$$
\begin{aligned}
& \left.\frac{d}{d X_{d}} X_{d}^{m_{j}+1}\right|_{X_{d, \max }} \stackrel{!}{=} 1+\rho_{d} \\
& \Rightarrow X_{d} \leq X_{d, \max }=\left(\frac{1+\rho_{d}}{1+m_{j}}\right)^{1 / m_{j}} .
\end{aligned}
$$

Combining both bounds we find

$$
-\frac{1}{2 m_{d}} \log \frac{1+\rho_{d}}{1+m_{j}} \leq a_{d} \leq-\frac{1}{2} \log \frac{\rho_{d}}{1+\rho_{d}},
$$

which, introducing normalization constants $A_{d, \max / \min }$, limits the decay of the singular values

$$
\sqrt{A_{d, \min }\left[X_{d, \min }\right]^{\tau}} \leq \Lambda_{j ; \tau}^{d} \leq \sqrt{A_{d, \max }\left[X_{d, \max }\right]^{\tau}}
$$

and thus can be used to fix an upper and lower bound for the matrix dimensions required on the auxiliary bonds between physical and bath site. The normalization constants are determined from

$$
\begin{gathered}
\rho_{d}=A_{d, \eta} \sum_{\tau=1}^{m_{j}}\left[X_{d, \eta}\right]^{\tau}=A_{d, \eta} X_{d, \eta} \frac{1-\left[X_{d, \eta}\right]^{m_{j}}}{1-X_{d, \eta}} \\
\Rightarrow A_{d, \eta}=\frac{1-X_{d, \eta}}{X_{d, \eta}} \frac{\rho_{d}}{1-\left[X_{d, \eta}\right]^{m_{j}}}
\end{gathered}
$$

with $\eta=\min$, max. We introduce for each block a truncation threshold $\delta_{d}^{\prime}$ so that for singular values with $\tau \leq m_{d, \eta}^{\prime} \leq m_{j}$ we have

$$
\begin{gathered}
\rho_{d}-\delta_{d}^{\prime} \geq A_{d, \eta} \sum_{\tau=1}^{m_{d, \eta}^{\prime}}\left[X_{d, \eta}\right]^{\tau}=\rho_{d} \frac{1-\left[X_{d, \eta}\right]^{m_{d, \eta}^{\prime}}}{1-\left[X_{d, \eta}\right]^{m_{j}}} \\
\Rightarrow\left[X_{d, \eta}\right]^{m_{d, \eta}^{\prime}} \geq 1-\left(1-\frac{\delta_{d}^{\prime}}{\rho_{d}}\right)\left(1-\left[X_{d, \eta}\right]^{m_{j}}\right) .
\end{gathered}
$$

For this inequality to hold we necessarily need $\rho_{d}-\delta_{d}^{\prime} \geq 0$ because $A_{d, \eta}, X_{d, \eta}>0$. It is ensured by taking $d \in \mathcal{D}$ and choosing $\delta_{d}^{\prime}=\max \left(\frac{\delta}{|\mathcal{D}|}, \min _{d \in \mathcal{D}} \rho_{d}\right)$ as truncation scheme. Then, taking the logarithm of both sides and solving for $m_{d, \eta}^{\prime}$ we divide by $\log X_{d, \eta}<0$ so that

$$
m_{d, \eta}^{\prime} \leq \frac{\log \left\{1-\left(1-R_{d}\right)\left(1-\left[X_{d, \eta}\right]^{m_{j}}\right)\right\}}{\log X_{d, \eta}}
$$

where we defined the truncation ratio $R_{d}=\frac{\delta_{d}^{\prime}}{\rho_{d}} \leq 1$. Imposing equality between the left and right side we finally obtain an estimation for the upper and lower bound of the required bond dimension $m_{d, \eta}^{\prime}$ in each block. Introducing the relative change of the number of kept states $F_{\eta}\left(m_{j}, \rho_{d}\right)=\frac{m_{d, \eta}^{\prime}}{m_{j}}$ we show the bounds in Fig. 6.5 for varying $m_{j}$ and $\rho_{d}$. For the upper bound there are two regimes: in the limit of small truncation ratio $R_{d} \ll 1$ we have $F_{d, \max }\left(m_{j}, \rho_{d}\right) \approx 1$ while on the other hand for $R_{d} \rightarrow 1$ there is a sharp drop towards zero. The transition regime between both asymptotics is governed by the physical bond dimension $m_{j}$ and shifts towards 


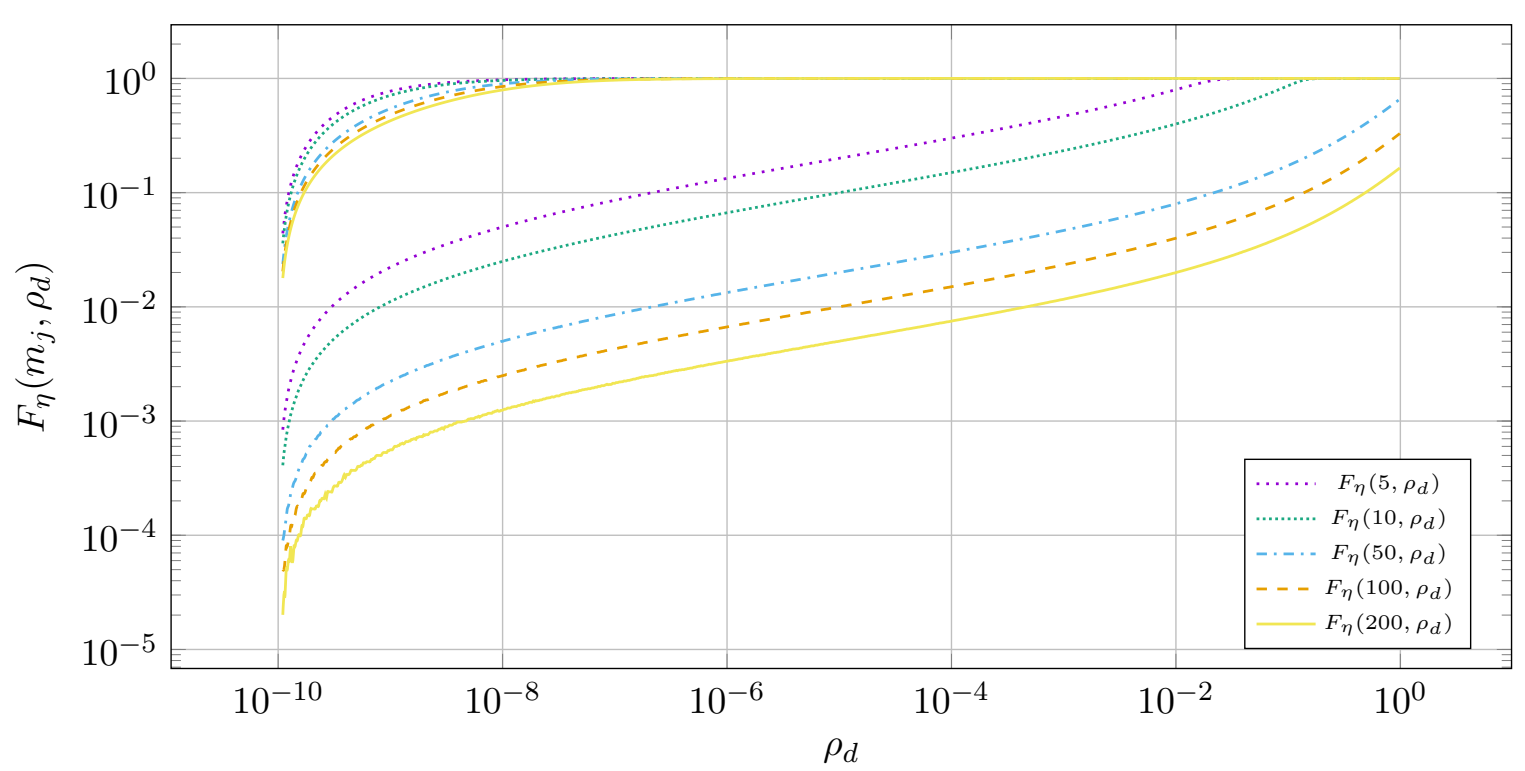

Figure 6.5: Upper and lower bounds $F_{\max / \min }\left(m_{j}, \rho_{d}\right)$ for relative change in bond dimension $\frac{m_{d}^{\prime}}{m_{j}}$ per tensor block on bond between physical and auxiliary sites derived from Eq. (6.32).

larger values of $\rho_{d}$ as $m_{j}$ increases. The lower bound exhibits a power law decay over several magnitudes of $\rho_{d}$ and saturates towards one if $m_{j}$ is small (c.f. Fig. 6.5). Finally, from Fig. 6.4 we can deduce that if $m_{j} \gg 1$ the lower bound becomes an increasingly better approximation for the bond dimension $m_{d, j}^{\prime}$.

In summary we found that for small physical bond dimension $m_{j}$ characterizing the approximation of the state without bath sites, the bond dimension $m_{j, n_{j}^{P}}^{\prime}$ between physical and auxiliary sites is of the order of $\left|\mathcal{D}^{\prime}\right| m_{j}$ if $m_{j}$ is small $(\sim \mathcal{O}(1))$ and $\mathcal{D}^{\prime}=\left\{n_{j}^{P} \mid \rho_{n_{j}^{P}}>m_{j} \delta\right\}$. However, if $m_{j} \gg 1$ the relative value of the bond dimension $m_{j, n_{j}^{P}}^{\prime}$ per tensor block compared to $m_{j}$ mostly follows a power law in $\rho_{n_{j}^{P}}$ and quickly decays to zero. In this situation the state can be efficiently approximated in the enlarged Hilbert space with a moderate growth of the bond dimension, given that the occupations of the single-site reduced density matrix $\rho_{n_{j}^{P}}$ decay fast enough. In physical problems one is often faced with exponentially decaying occupations of $\rho_{n_{j}^{P}}$. Exemplary, we consider a typical, physical bond dimension $m_{j}=100$ and assume $\rho_{n_{j}^{P}} \propto e^{-2 n_{j}^{P}}$ with a truncation threshold of $\delta=10^{-14}$ and take into consideration a local dimension of $d=21$ (i.e., permit for 20 occupied states). We use the derived lower bound and obtain $m_{j}^{\prime} \approx m_{j}$. It needs to be kept in mind that this estimation relies on the assumption of strictly exponentially decaying singular values in each tensor block, which not necessarily needs to be the case in actual calculations. However, a relative growth in the overall bond dimension of $\mathcal{O}(1)$ was also found in our test calculations. Finally we note that due to the rapid decrease of the lower bound derived above the total local dimension $d$ is not a limiting factor in first place, as long as $m_{j}$ is large enough. In turn, the decay of the single-site reduced density matrix occupation strongly dictates the numerical expenses.

We close this section by discussing the numerical benefits of the above introduced enlargement of the Hilbert space and projection into the subspace $\mathcal{P}$ by considering the scaling of the most expensive calculation in a DMRG two-site groundstate search. However, we must note that in order to exploit the optimal speed-up the contractions shall be parallelized by a proper decision 
scheme (c.f. Sec. 4.6.3). As discussed in Sec. 4.4.2 the asymptotic scaling is $\mathcal{O}\left(m^{3} w \sigma^{2}+m^{2} w^{2} \sigma^{3}\right)$ and for $m \gg \sigma, m \gg w$ the first term dominates the calculation time. Assuming a typical growth factor 2 between the physical and bath sites this operation is 8 times more expensive on these bonds than on the original bond between physical sites only. Therefore, in order to benefit from the introduction of $U(1)$-invariant state representations in first place we need to have a reasonable large local dimension $d>\sqrt{8}$, assuming that all local generators can be choosen as one-dimensional representations. Thus, $d \geq 3$ already speeds up this contraction and the benefits will grow with larger $d$ quadratically. Additionally, the second contribution in the scaling $\sim m^{2} w^{2} \sigma^{3}$ becomes negligible if $\sigma$ is decomposed into a one-dimensional representation. We may also consider a decomposition of the MPO bond dimension $w_{j}$ due to the $U(1)$-symmetry, which typically is of the order of $2-3$ and thereby also generates an additional speed-up. Finally, we note that the system size is doubled, which we may also incorporate into our estimations. But this is only a constant factor of two and can be compensated easily by the quadratically growing speed-up in the local dimension or the decomposition of the MPO bond dimension under the global symmetry.

\section{Numerical testcase: Groundstate of the Holstein model}

In order to demonstrate the applicability of the derived representation scheme we performed numerical ground-state calculations of the 1D Holstein model with spinless fermions [BMH98, CSC05]. The model is defined by a set of spinless fermions with creation/annihilation operators $\hat{c}_{j}^{[\dagger]}$ on a lattice, each of which is coupled to a phonon (bosonic degree of freedom) with creation/annihilation operators $\hat{b}_{j}^{[\dagger]}$

$$
\hat{H}=-t \sum_{j}\left(\hat{c}_{j}^{\dagger} \hat{c}_{j+1}+\text { h.c. }\right)+\omega_{0} \sum_{j} \hat{b}_{j}^{\dagger} \hat{b}_{j}+\gamma \sum_{j} \hat{n}_{j}\left(\hat{b}_{j}^{\dagger}+\hat{b}_{j}\right), \quad \hat{n}_{j}=\hat{c}_{j}^{\dagger} \hat{c}_{j} .
$$

The ground-state phase-diagram of this model exhibits a transition from a Luttinger liquid metallic phase into a CDW insulating phase when increasing the ratio $\gamma / t$. We investigated two points being either deep in the Luttinger liquid phase (P.1) or in the Luttinger liquid phase poised close to the phase transition (P.2). For the calculations we chose the following sets of parameters

$$
\begin{aligned}
& L=8, \quad N=4, \quad \omega=1, \quad t=0.5, \quad \gamma=0.5, \\
& L=8, \quad \quad N=4, \quad \omega=1, \quad t=1.0, \quad \gamma=1.0 \text {. }
\end{aligned}
$$

The calculated ground state observables using the projected purification with the SYMMPS toolkit and a two-site solver (2DMRG-PP) were compared to results obtained from a code $\left[\mathrm{SHD}^{+} 20\right]$ implementing a DMRG3S algorithm (c.f. Sec. 4.4.3) with local basis optimization (DMRG3SLBO). The latter calculations were performed by Jan Stolpp in the group of Fabian HeidrichMeisner at the Georg-August-Universität Göttingen. We configured the 2DMRG-PP solver (c.f. Sec. 4.4.2) to return if the relative energy gain per sweep is below a threshold $\delta=10^{-13}$ and allowed for a maximal number of sweeeps $N=50$. The local escape criterion for the Krylov solver in each sweep step was set to $\delta \lambda=10^{-14}$ and the largest allowed Krylov space dimension was $K=7$. We performed calculations for maximum bond dimensions $m \in[10,1000]$ and set the maximally discarded weight to $\varepsilon^{2}=10^{-14}$. For the DMRG3S-LBO solver the same discarded weight was chosen but no escape criterion was defined so that the algorithm always performed the maximum number of sweeps $N=1000$. 


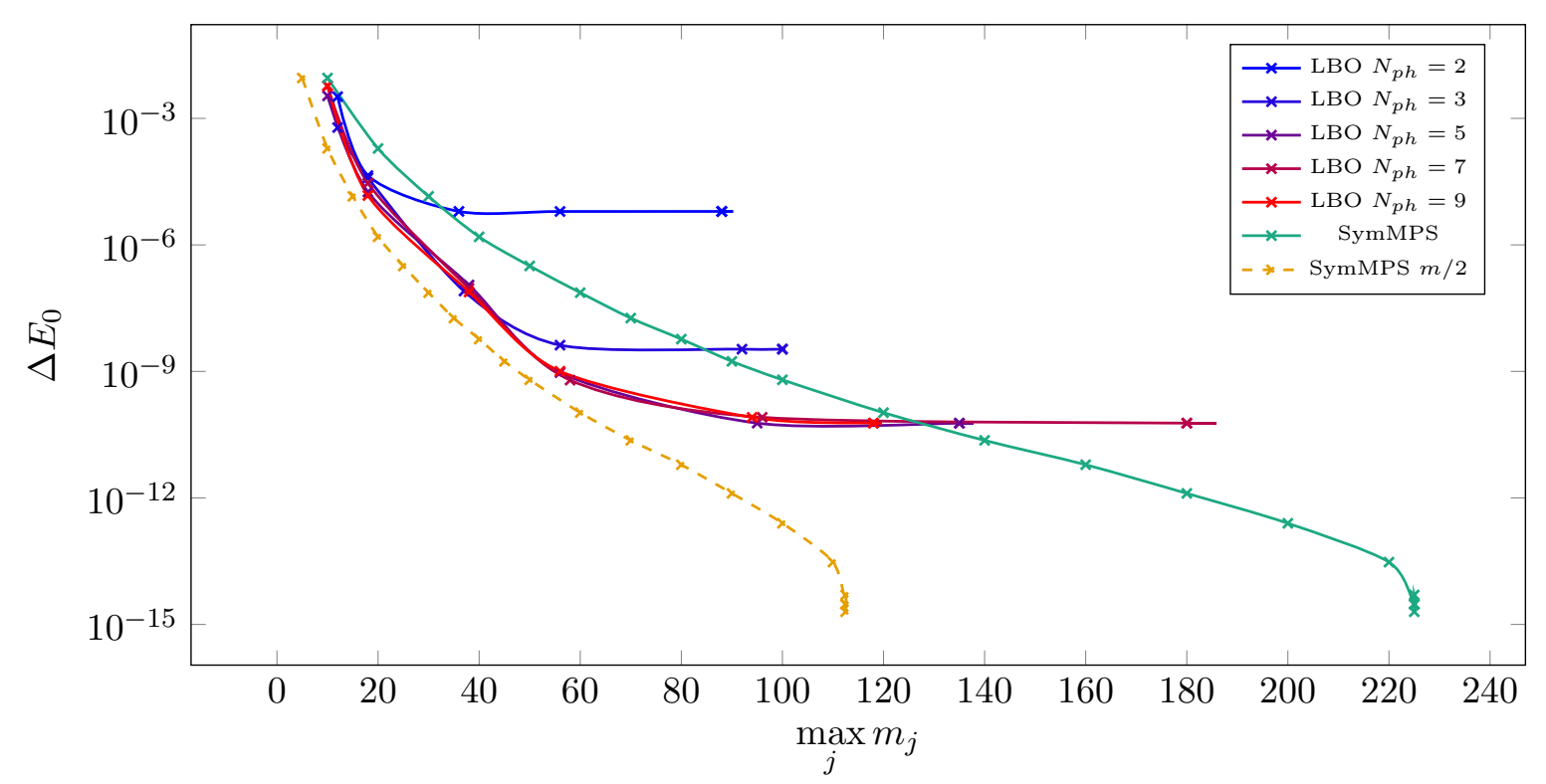

Figure 6.6: Distance to minimal found ground state energy $E_{0}$ over maximal bond dimension (max. discarded weight $\varepsilon^{2}=10^{-14}$ ) of the Holstein model with $t=0.5, \omega=1.0$ and $g=0.5$. The number of maximally allowed phonon modes per site is $N_{p h}=10$ in the 2DMRG-PP calculations and was varied for the DMRG3S-LBO implementation.

Due to the different configurations of the solver comparing the runtimes is not possible, yet. However, we can compare the quality of the approximations of the obtained ground states. Therefore, we looked for the lowest overall reached energy $E_{0}$ in both methods and evaluated for each calculation the distance of the ground state energy to the best approximation $E_{0}$. This distance is then analyzed as a function of the maximum bond dimension $m=\max _{j} m_{j}$ of the ground state after truncation to the desired maximal discarded weight $\varepsilon^{2}=10^{-14}$

$$
\Delta E_{0}(m)=E(m)-E_{0} .
$$

\section{Results}

We performed ground state calculations using the 2DMRG-PP solver with a maximally allowed number of phonon states $N_{p h}=10$ for the parameter set (P.1) and $N_{p h}=20$ for the parameter set (P.2). The calculations using the DMRG3S-LBO solver were repeated with different cutoffs $N_{p h}=2,3,5,7,9$ for the optimal modes, which correspond to the number of kept eigenstates of the single-site reduced density matrix. The lowest ground state energy found for (P.1) was $E_{0}=$ $-3.1245192963396(8)$ and obtained by 2DMRG-PP with a maximum bond dimension $m=225$ after truncation. The lowest ground state energy found for (P.2) was $E_{0}=-7.4660871303235(0)$ and obtained by 2DMRG-PP with a maximum bond dimension $m=552$ after truncation. The distances $\Delta E_{0}(m)$ as a function of the maximum bond dimension after truncation are shown in Figs. 6.6 and 6.7 for the two implementations. We find that the DMRG3S-LBO solver saturates at a distance $\sim 10^{-10}$ to the lowest found ground state energy for both parameter sets. A cutoff in the number of maximally allowed phonon modes of $N_{p h}=10$ and $N_{p h}=20$ for parameter sets (P.1) and (P.2) ensures convergence of both solvers. Note that rescaling the maximum bond dimension after truncation of the 2DMRG-PP data by a factor of $1 / 2$ the convergence behavior 


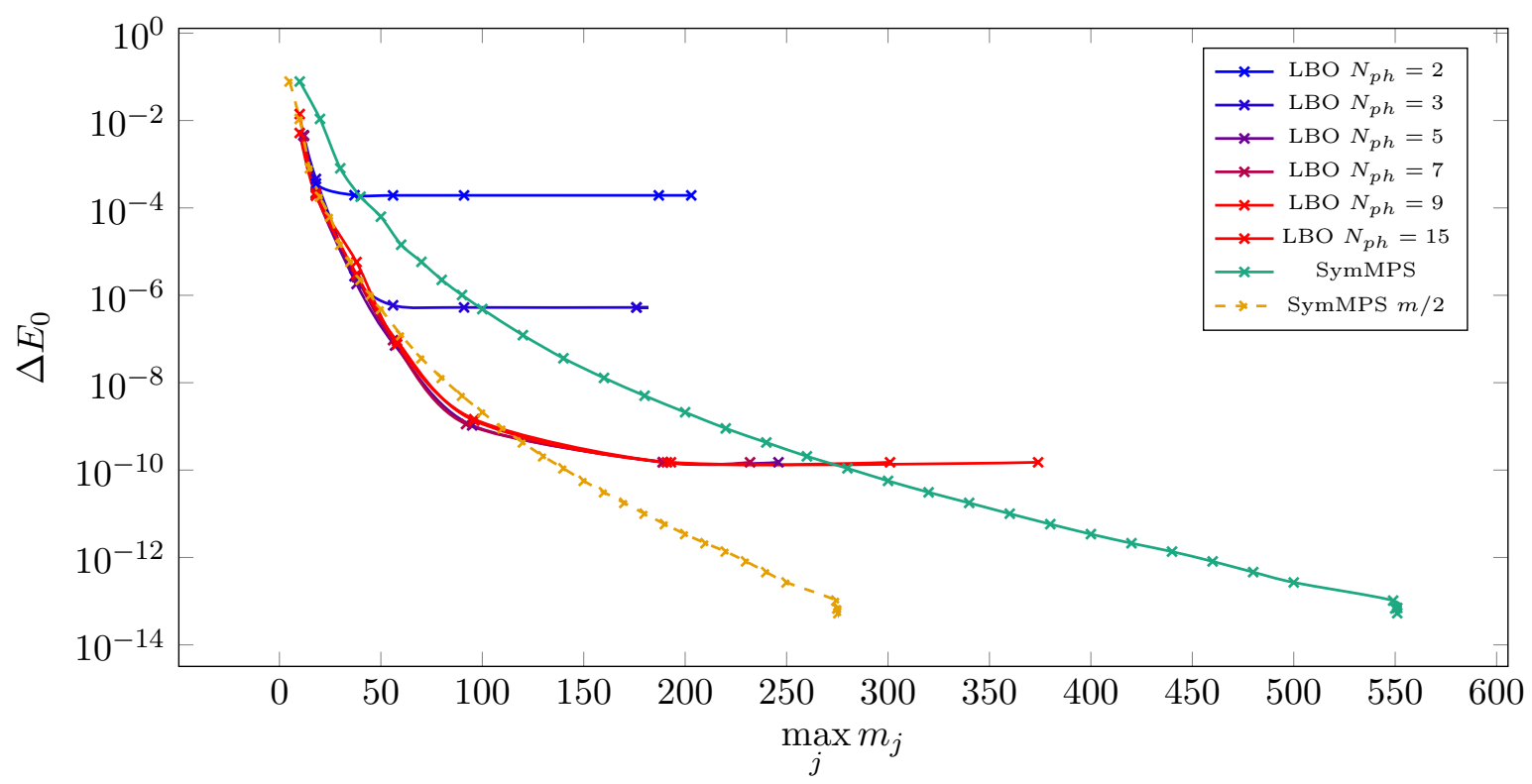

Figure 6.7: Distance to minimal found ground state energy $E_{0}$ over maximal bond dimension (max. discarded weight $\varepsilon^{2}=10^{-14}$ ) of the Holstein model with $t=1.0, \omega=1.0$ and $g=1.0$. The number of maximally allowed phonon modes per site is $N_{p h}=20$ in the 2DMRG-PP calculations and was varied for the DMRG3S-LBO implementation.

of the DMRG3S-LBO solver is nearly recovered except for the saturation of the distance. We address this saturation to a particular implementation detail of the DMRG3S-LBO algorithm. In fact, the basis expansion in the single-site solver requires an enrichment step, in which artificially noise is added to the system. As discussed in [HMSW15] this causes the ground state energy to increase after truncation and it appears that the criterion to completely suppress the introduced subspace expansion could not be fulfilled.

In summary, we have demonstrated that 2DMRG-PP for MPS provides an alternative efficient approach to study 1D systems with a large local dimension. The ground state energies obtained with DMRG3S-LBO are 4 orders of magnitudes above those found by 2DMRG-PP at the investigated points in the phase diagram. Clearly, there is much more benchmarking required to make a statement about which method is more suitable, in which limiting cases of the considered system. However, from the shown equivalence of the truncation procedures in 2DMRG-PP and DMRG3S-LBO we believe that 2DMRG-PP can be considered a valuable and competitive tool. We also want to point out that only by restoring the global $U(1)$ symmetry and thus having a one-dimensional representation of local operators in the above example we were able to use 2DMRG efficiently. This approach was succesfully applied to the problems studied in Chap. 7 where bosonic sites with a large local Hilbert space had to be dealt with. 

Systems 



\section{\begin{tabular}{r|r} 
Coulomb blockaded topological & \\
superconductivity & 7
\end{tabular}}

An ultimative goal of the experimental studies of topological superconductivity is the ability to create and control Majorana zero modes that can be used to realize fault-tolerant qubits. A first significant step is the unambiguous experimental proof of the existence of Majorana edge modes in a reproducible experimental setup. In what follows, we will concentrate on a type of structures suggested by Lutchyn et al. [LSDS10, ORvO10]. They showed that a one-dimensional semiconductor with strong spin-orbit coupling (Rashba wire) proximitized to an s-wave superconductor exhibits a phase transition into a topologically non-trivial phase when tuning a magnetic field along the wire. In such systems the existence of Majorana fermions creates specific zero-bias signals in the conductance which can be detected experimentally $\left[\mathrm{MZF}^{+} 12, \mathrm{DYH}^{+} 12, \mathrm{DRM}^{+} 12\right]$. However, these footprints need to be distinguished from other mid-gap states. One possibility is to directly measure the exponential protection of Majorana edge modes by studying the magnetic field dependency of the conductance peaks [DSSS12]. Here, the number of charges in the combined system of superconductor and nanowire typically is locked by a weak coupling to leads via tunnel contacts. An experimental realization of such a measurement extends the setup by a gate electrode that enables to control the number of electrons on the proximitized nanowire [Fu10]. In this so-called Coulomb blockade regime the exponential protection of the Majarana edge modes was studied experimentally by Albrecht et al. $\left[\mathrm{AHM}^{+} 16\right]$. They reported the finding of the theoretically predicted exponential suppression of the conductance peak-position oscillations upon increasing the sample size. Nevertheless, in detail the found behavior leaves open question. In particular their analysis was founded on theories that are valid only in the limit of a nanowire being isolated.

We will use DMRG as a numerically unbiased method to study ground-state properties far away from the regimes accessible by perturbation theory. To the best of our knowledge this is the first study taking into account the whole microscopic details modeling the experimental setup by Albrecht et al. The question we are trying to address in this section are therefore twofold. At first we have to demonstrate and benchmark DMRG with analytically available results in the limit of an isolated and weakly coupled island. We do this by studying exhaustively the properties of an island being coupled only to one normal lead which permits for very precise calculations keeping the discarded weight below a threshold of $\epsilon^{2}=10^{-10}$. In this setup we could easily test the weak tunneling limit and compare to previous works [HPAG05, LFG16]. After confirming the analytically expected behaviours we then moved on to the question of the influence of one or two normal leads being coupled to the nanowire in the intermediate and strong tunneling regime. Here, the goal was to reproduce the experimental setup by Albrecht et al. as best as possible. Even though this part of our research is still work in-progress we already find evidence that the naive application of the picture of an isolated nanowire cannot be carried over to the experimental situation with two leads being coupled to the island. However, there are good reasons to assume that the proximity-coupled Coulomb blockaded nanowire is an excellent plattform for experimentally proving the existence of Majorana edge modes. We hope that this unbiased study of the effects of the leads on the characteristic experimental signatures gives rise to more elaborated analysis schemes unveiling the exponential protection of Majorana edge modes. 


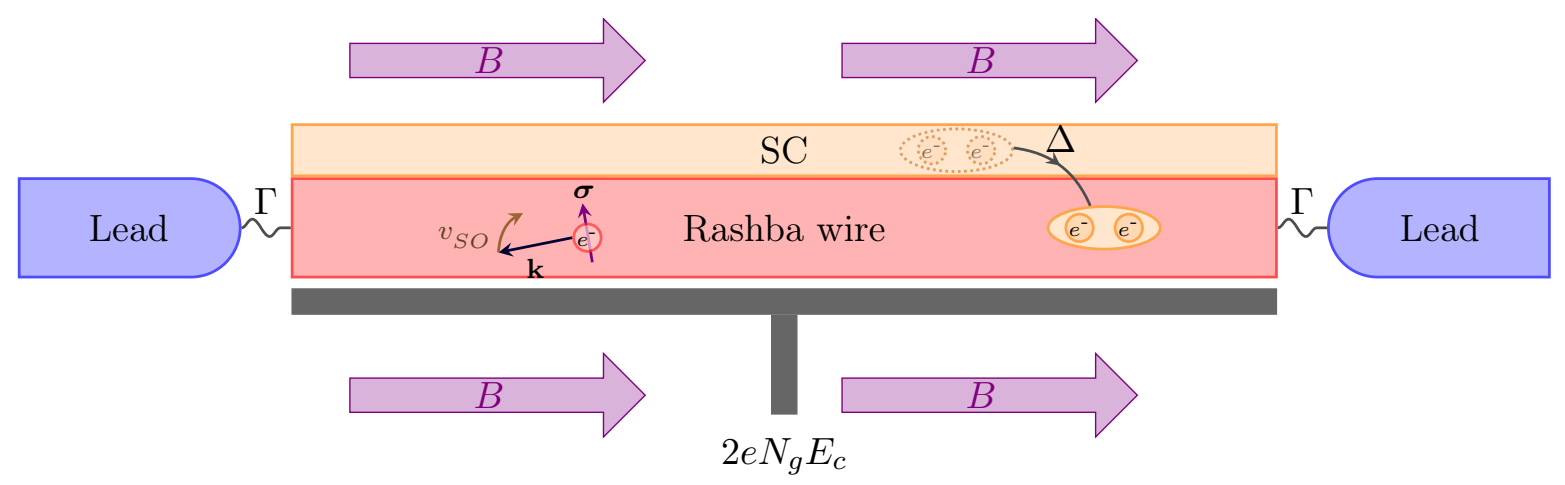

Figure 7.1: Schematic setup of leads (blue) coupled to an island consisting of a Rashba wire (red) proximity-coupled to an $s$-wave superconductor (orange) and subject to a charging energy induced by a gate electrode (gray).

\section{Proximity-coupled isolated superconducting island}

We consider a one-dimensional nanowire with strong spin-orbit coupling $v_{S O}$, which is proximitycoupled to an $s$-wave superconductor and capacitively charged by a gate voltage $V_{g}$

$$
\hat{H}_{I}=\hat{H}_{R W}(\hat{\varphi})+\hat{H}_{c},
$$

with $\hat{H}_{R W}(\hat{\varphi})$ and $\hat{H}_{c}$ specified below. A sketch of the setup is shown in Fig. 7.1 where the island is already coupled to normal leads but the isolated case can be recovered setting $\Gamma \equiv 0$. The tunneling amplitude to the leads is chosen to be the same for both leads in our studies. ${ }^{1}$ Cooper-pair tunneling from the $s$-wave superconductor into the nanowire is taken into account by an effective $s$-wave pairing term $\Delta \mathrm{e}^{\mathrm{i}} \hat{\varphi}_{\hat{c}_{j, \uparrow}} c_{j, \downarrow}$ with $\Delta$ being the induced superconducting gap [CMM97]. The canonically conjugated, anti-commuting operators $\hat{c}_{j, \sigma}^{(\dagger)}$ annihilate (create) an electron with spin projection $\sigma$ into the $z$-direction at lattice site $j$. Note, that $\mathrm{e}^{\mathrm{i} \hat{\varphi}}$ is the operator which adds a Cooper pair to the superconductor. The radius $R$ of the nanowire is chosen to the Fermi wavelength $R<\lambda_{F}$ yielding an effective 1D description. In a more realistic model this requires to take into account a hand full of bands but for this principle investigation we restrict to the case of only one band. Taking into account Zeeman splitting due to an external magnetic field $B$ perpendicular to the spin-orbit direction the proximity-coupled nanowire is described by the Rashba Hamiltonian

$$
\begin{aligned}
\hat{H}_{R W}(\hat{\varphi})= & -\sum_{j, \sigma, \sigma^{\prime}}\left(\hat{c}_{j, \sigma}^{\dagger}\left(t_{I, h} \delta_{\sigma, \sigma^{\prime}}+\mathrm{i} v_{S O} \sigma_{\sigma, \sigma^{\prime}}^{y}\right) \hat{c}_{j+1, \sigma^{\prime}}+\text { h.c. }\right)-\Delta \sum_{j}\left(\mathrm{e}^{-\mathrm{i} \hat{\varphi}} \hat{c}_{j, \uparrow}^{\dagger} \hat{c}_{j, \downarrow}^{\dagger}+\text { h.c. }\right) \\
& -\sum_{j, \sigma} \hat{c}_{j, \sigma}^{\dagger}\left(\mu-2 t_{I, h}+B \sigma_{\sigma, \sigma}^{z}\right) \hat{c}_{j, \sigma}
\end{aligned}
$$

with hopping amplitude $t_{I, h}$ and $\mu$ being the chemical potential. Here, we shifted the origin of the chemical potential towards the bottom of the non-interacting band $\mu \rightarrow \mu-2 t_{I, h}$. The

\footnotetext{
${ }^{1}$ Of course, in the modelling of the Hamiltonian we are free to study also different tunneling amplitudes for the left and right leads as there is no symmetry restriction from DMRG. We will investigate such situations in future studies.
} 
charging energy which is controlled by the applied gate voltage $V_{g}=\frac{e N_{g}}{C}$ is accounted for with an additional contribution to the system's energy

$$
\hat{H}_{c}=E_{c}\left(2 e \hat{N}_{c}+e \hat{N}_{f}-N_{g}\right)^{2}, \quad \hat{N}_{f}=\sum_{j, \sigma} \hat{c}_{j, \sigma}^{\dagger} \hat{c}_{j, \sigma} .
$$

Herein, $\hat{N}_{f}$ is the number operator for electrons in the nanowire while $\hat{N}_{c}$ counts Cooper pairs in the superconductor and $E_{c}=\frac{\mathrm{e}^{2}}{2 C}$.

\section{Gauge transformation}

The overall number of electrons on the isolated island counted in both the superconductor and the nanowire is a conserved quantity. In fact, the particle number operator of the Cooper pairs fulfills

$$
\left[\hat{N}_{c}, \mathrm{e}^{ \pm \mathrm{i} \hat{\varphi}}\right]=\mp \mathrm{e}^{ \pm \mathrm{i} \hat{\varphi}} \Rightarrow\left[2 \hat{N}_{c}+\hat{N}_{f}, \hat{H}_{c}\right]=0
$$

Due to this conservation the dependence on the superconducting phase $\hat{\varphi}$ can be gauged away by the transformation $\hat{U}(\hat{\varphi})=\mathrm{e}^{-\mathrm{i} \hat{\varphi} \hat{N}_{f} / 2}[\mathrm{KMvHB19}]$ :

$$
\begin{aligned}
\hat{c}_{j, \sigma} & \longmapsto \hat{U}^{\dagger}(\hat{\varphi}) \hat{c}_{j, \sigma} \\
\hat{H}_{I}(\varphi) & \longmapsto \hat{H}_{I}=\hat{U}(\hat{\varphi}) \hat{H}_{I}(\hat{\varphi}) \hat{U}^{\dagger}(\hat{\varphi}) .
\end{aligned}
$$

In particular, the charging energy is transformed as

$$
\begin{aligned}
\mathrm{e}^{-\mathrm{i} \hat{\varphi} \hat{N}_{f} / 2}\left(2 e \hat{N}_{c}+e \hat{N}_{f}-N_{g}\right)^{2} \mathrm{e}^{\mathrm{i} \hat{\varphi} \hat{N}_{f} / 2} & =\left(2 e \mathrm{e}^{-\mathrm{i} \hat{\varphi} \hat{N}_{f} / 2} \hat{N}_{c} \mathrm{e}^{\mathrm{i} \hat{\varphi} \hat{N}_{f} / 2}+e \hat{N}_{f}-N_{g}\right)^{2} \\
& =\left(2 e\left(\hat{N}_{c}-\frac{\hat{N}_{f}}{2}\right)+e \hat{N}_{f}-N_{g}\right)^{2} \equiv\left(e \hat{Q}-N_{g}\right)^{2}
\end{aligned}
$$

and identifies $2 e \hat{N}_{c}+e \hat{N}_{f} \mapsto e \hat{Q}$, i.e. the total number of fermions in the combined system of nanowire and superconductor. In the last equality we used the representation $\hat{N}_{c}=-\mathrm{i} \frac{\partial}{\partial \hat{\varphi}}$ of the particle number operator of the $s$-wave superconductor in the phase basis.

Since for the isolated island $\hat{Q}$ is a conserved quantity the charging energy reduces only to a constant. However, the action of the transformation onto the wave function $|\psi\rangle$ is a bit subtle but important for the MPS calculations which is why we are going to discuss it in detail. Expanding the superconducting, bosonic part of the wavefunction in terms of the phase basis and evaluating the action of $\hat{U}(\hat{\varphi})$ we find

$$
\begin{aligned}
\hat{U}(\hat{\varphi})|\psi\rangle & =\int_{0}^{2 \pi} \frac{d \varphi}{2 \pi} \sum_{n_{1}, \ldots, n_{L}} \psi\left(\varphi, n_{1}, \ldots, n_{L}\right) \hat{U}(\hat{\varphi})\left|\varphi, n_{1}, \ldots, n_{L}\right\rangle \\
& =\int_{0}^{2 \pi} \frac{d \varphi}{2 \pi} \sum_{n_{1}, \ldots, n_{L}} \psi\left(\varphi, n_{1}, \ldots, n_{L}\right) \mathrm{e}^{-\mathrm{i} \frac{\varphi}{2} \sum_{j} n_{j}}\left|\varphi, n_{1}, \ldots, n_{L}\right\rangle .
\end{aligned}
$$

Transforming back into the basis of occupations of Cooper pairs this yields

$$
\hat{U}(\hat{\varphi})|\psi\rangle=\sum_{n_{1}, \ldots, n_{L}} \int_{0}^{2 \pi} \sum_{n_{c}} \psi\left(\varphi, n_{1}, \ldots, n_{L}\right) \mathrm{e}^{\mathrm{i} \frac{\varphi}{2}\left(2 n_{c}-\sum_{j} n_{j}\right)}\left|n_{c}, n_{1}, \ldots, n_{L}\right\rangle .
$$


Since $\hat{Q}$ is a conserved quantity eigenstates of $\hat{H}_{I}(\hat{\varphi})$ have a well-defined parity $\hat{P}_{Q}|\psi\rangle=$ $\mathrm{e}^{\mathrm{i} \pi \hat{Q}}|\psi\rangle= \pm|\psi\rangle$. The parity of the gauged state is then

$$
\begin{aligned}
\hat{P}_{Q} \hat{U}(\hat{\varphi})|\psi\rangle & =\sum_{n_{1}, \ldots, n_{L}} \int_{0}^{2 \pi} \sum_{n_{c}} \frac{d \varphi}{2 \pi} \psi\left(\varphi, n_{1}, \ldots, n_{L}\right) \mathrm{e}^{\mathrm{i}\left(\pi+\frac{\varphi}{2}\right)\left(2 n_{c}-\sum_{j} n_{j}\right)}\left|n_{c}, n_{1}, \ldots, n_{L}\right\rangle \\
& =\sum_{n_{1}, \ldots, n_{L}} \mathrm{e}^{\mathrm{i} \pi \sum_{j} n_{j}} \int_{0}^{2 \pi} \underbrace{\sum_{n_{c}} \frac{d \varphi}{2 \pi} \psi\left(\varphi, n_{1}, \ldots, n_{L}\right) \mathrm{e}^{\mathrm{i} \frac{\varphi}{2}\left(2 n_{c}-\sum_{j} n_{j}\right)}\left|n_{c}, n_{1}, \ldots, n_{L}\right\rangle}_{=\hat{U}(\hat{\varphi})\left|\varphi, n_{1}, \ldots, n_{L}\right\rangle},
\end{aligned}
$$

where we used the $2 \pi$ periodicity of the wavefunction coefficients $\psi\left(\varphi, n_{1}, \ldots, n_{L}\right)$. The last equation now states that the action of $\hat{Q}$ creates a superposition of the gauged fermionic basis states $\left|n_{1}, \ldots, n_{L}\right\rangle$, where the coefficients are just the eigenvalues of the fermionic parity operator $\hat{P}_{f}$, and thus

$$
\hat{P}_{Q} \hat{U}(\hat{\varphi})|\psi\rangle=\hat{P}_{f} \hat{U}(\hat{\varphi})|\psi\rangle
$$

Hence, by choosing $N$ we fix the parity of the gauged electrons in the nanowire in the groundstate of $\hat{U}(\hat{\varphi}) \hat{H}_{I}(\hat{\varphi}) \hat{U}^{\dagger}(\hat{\varphi})$ to $P=\mathrm{e}^{\mathrm{i} \pi N}$.

A bit care has to be taken when working with the gauged representation. The good news is that since we are dealing with a quadratic model and the total number of electrons on the island is conserved, we can work with the gauged electrons in the Rashba wire and do not have to care about phase factors and exponentials. However, it should be kept in mind, that effectively we have coupled Cooper pairs and electrons by means of the total charge of the island being conserved under the action of the electronic ladder operators. The charge on the island can be modelled by a charge boson which counts the overall number of electrons on the island. Since in the gauged representation the condensate degrees of freedom are hidden we expanded the fermionic Hilbert space by a single bosonic site $|Q\rangle \in \mathcal{H}_{Q}$. Here, $\mathcal{H}_{Q}$ is a bosonic Hilbert space whose local dimension has to be chosen large enough so that no artificial truncation errors occur. Then, the charge operator $\hat{Q}$ only acts in $\mathcal{H}_{Q}$. Since for the isolated island $\hat{Q}$ is conserved we can simply replace it by its expectation value corresponding to the fixed, total charge sector. However, expanding the model including leads and tunnel contacts we have to act on $\mathcal{H}_{Q}$ with ladder operators whenever a tunneling process out of the island is described. Additionally note, that the above derived parity constraint Eq. (7.10) is independent on $\hat{Q}$ being conserved but is merely a result of the gauge transformation. In summary, the gauged Hamiltonian is given by

$$
\begin{aligned}
\hat{H}_{I}= & -\sum_{j, \sigma, \sigma^{\prime}}\left(\hat{c}_{j, \sigma}^{\dagger}\left(t_{I, h} \delta_{\sigma, \sigma^{\prime}}+\mathrm{i} v_{S O} \sigma_{\sigma, \sigma^{\prime}}^{y}\right) \hat{c}_{j+1, \sigma^{\prime}}+\text { h.c. }\right)-\sum_{j} \Delta\left(\hat{c}_{j, \uparrow}^{\dagger} \hat{c}_{j, \downarrow}^{\dagger}+\text { h.c. }\right) \\
& -\sum_{j, \sigma} \hat{c}_{j, \sigma}^{\dagger}\left(\mu-2 t_{I, h}+B \sigma_{\sigma, \sigma}^{z}\right) \hat{c}_{j, \sigma}+E_{c}\left(e \hat{Q}-N_{g}\right)^{2},
\end{aligned}
$$

where $\hat{Q}$ acts only in the artificially introduced bosonic Hilbert space $\mathcal{H}_{Q}$. 
(a)

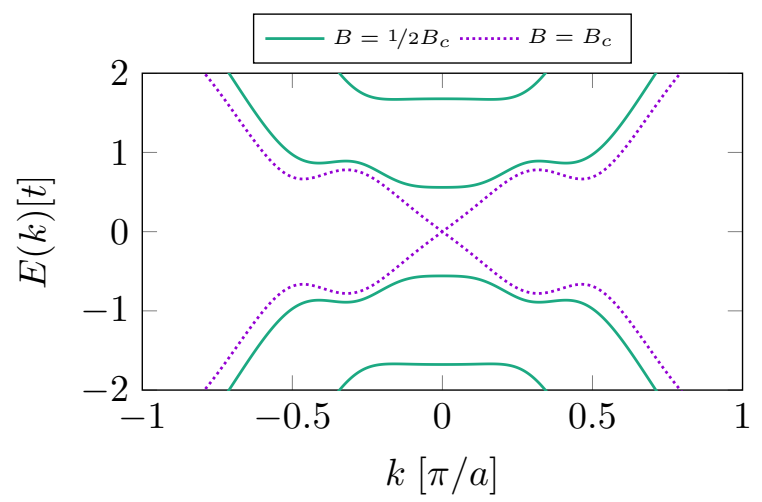

(b)

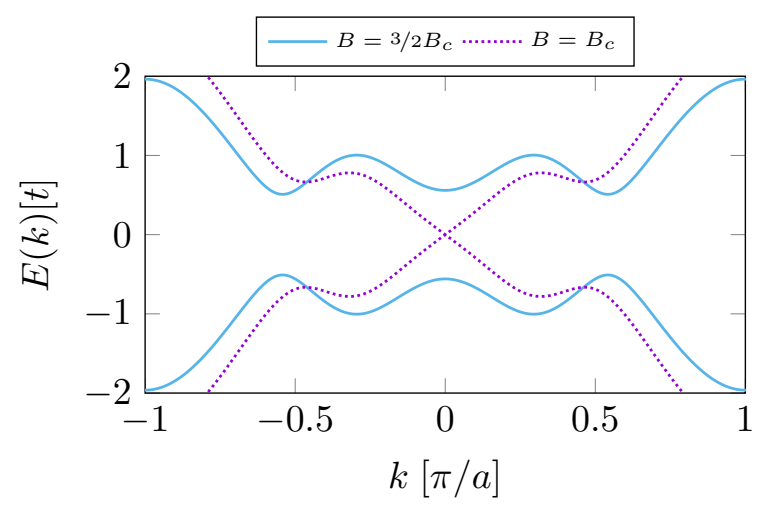

Figure 7.2: Band structure $E(k)$ of Rashba wire in trivial (a) and topological (b) phase. Parameters are set to $t_{I, h}=\Delta=1.0, v_{S O}=\mu=0.5$ with $B$ varying between trivial $\left(B=1 / 2 B_{c}\right)$ and topological phase $\left(B=3 / 2 B_{c}\right)$ as well as at the phase transition $B=B_{c}$.

\section{Topological and trivial phases}

The ground state phase diagram at fixed gate voltage $N_{g}$ and charge $\hat{Q}$ is obtained by bringing $\hat{H}$ into a BdG representation (c.f. Sec. 3.4.1) by introducing Nambu spinors

$$
\begin{aligned}
\hat{\mathbf{\Psi}}_{\sigma} & =\left(\begin{array}{lll}
\hat{c}_{1, \sigma} & \cdots & \hat{c}_{L, \sigma}
\end{array}\right), \quad \hat{\mathbf{\Psi}}=\left(\begin{array}{ll}
\hat{\mathbf{\Psi}}_{\uparrow} & \hat{\mathbf{\Psi}}_{\downarrow}
\end{array}\right) \\
\Rightarrow \hat{H}_{I} & =\frac{1}{2}\left(\begin{array}{ll}
\hat{\mathbf{\Psi}}^{\dagger} & \hat{\mathbf{\Psi}}
\end{array}\right)\left(\begin{array}{cc}
\mathbf{H} & -\overline{\boldsymbol{\Delta}} \\
\boldsymbol{\Delta} & -\overline{\mathbf{H}}
\end{array}\right)\left(\begin{array}{l}
\hat{\mathbf{\Psi}} \\
\hat{\mathbf{\Psi}}^{\dagger}
\end{array}\right)+\text { const. }
\end{aligned}
$$

where $\mathbf{H}, \boldsymbol{\Delta}$ are $2 L \times 2 L$ matrices. After a Fourier transformation imposing periodic boundary conditions $\hat{H}$ is in a block-diagonal form and can be written in the Nambu representation in $k$-space

$$
\hat{H}_{I}=\frac{1}{2} \sum_{k}\left(\begin{array}{cc}
\hat{\mathbf{\Psi}}_{k}^{\dagger} & \hat{\mathbf{\Psi}}_{-k}
\end{array}\right)\left(\begin{array}{cc}
\mathbf{H}(k) & -\boldsymbol{\Delta}(k) \\
\boldsymbol{\Delta}(k) & -\overline{\mathbf{H}}(-k)
\end{array}\right)\left(\begin{array}{c}
\hat{\mathbf{\Psi}}_{k} \\
\hat{\mathbf{\Psi}}_{-k}^{\dagger}
\end{array}\right),
$$

with $\hat{\mathbf{\Psi}}_{k}=\left(\hat{c}_{k, \uparrow} \hat{c}_{k, \downarrow}\right)$ and the usual definition of the Fourier transformed ladder operator $\hat{c}_{k, \sigma}=$ $\frac{1}{\sqrt{L}} \sum_{j} \hat{c}_{j, \sigma} \mathrm{e}^{\mathrm{i} k r_{j}}$. Note, that we have neglected the overall energy shift due to the charging energy for a moment. However, this is not as trivial as it may seem since from Eq. (7.10) it follows that the ground state parity is fixed by the overall choice of $\hat{Q}$. We will embark on this point in the next section.

The matrices $\mathbf{H}(k), \boldsymbol{\Delta}(k)$ are given by

$$
\begin{gathered}
\mathbf{H}(k)=\left(\begin{array}{cc}
-\left(2 t_{I, h} \cos (k a)+\mu-2 t_{I, h}\right)+B & 2 \mathrm{i} v_{S O} \sin (k a) \\
-2 \mathrm{i} v_{S O} \sin (k a) & -\left(2 t_{I, h} \cos (k a)+\mu-2 t_{I, h}\right)-B
\end{array}\right), \\
\boldsymbol{\Delta}(k)=\left(\begin{array}{cc}
0 & \Delta \\
-\Delta & 0
\end{array}\right) .
\end{gathered}
$$


(a)

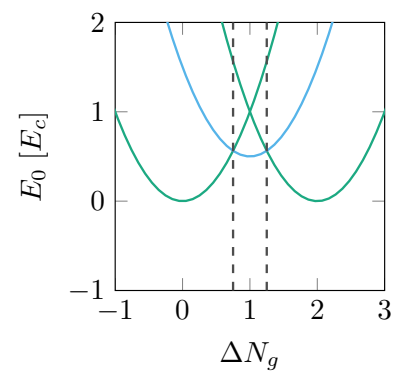

(b)

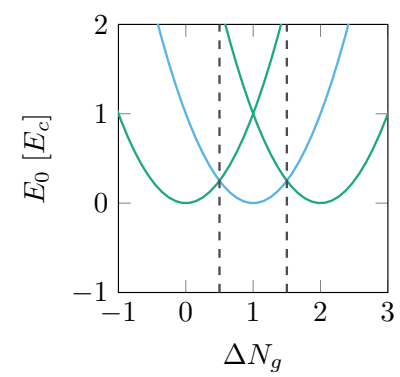

(c)

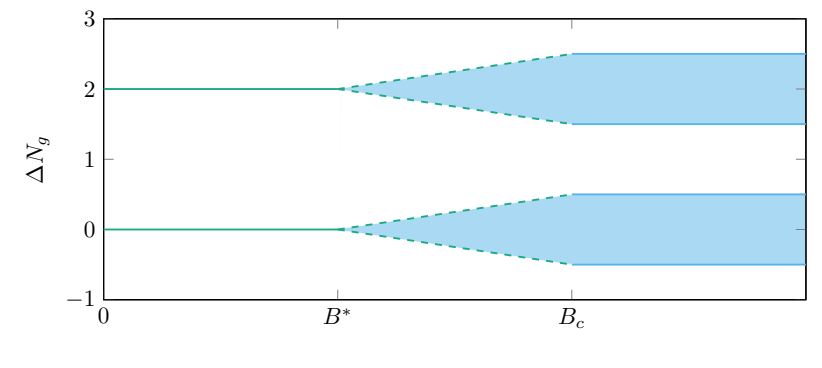

Figure 7.3: The shifting of the odd-charge parabolas in the regime $B>B^{*}$ is shown in (a) and (b). The position of the charge degeneracy points is marked by the dashed line. In (c) the splitting of the charge degeneracy points as a function of the in-plane magnetic field is shown for an isolated island.

At $k=0, \pi$ the eigenvalues can be determined easily and searching for a gap closing, i.e., $E(k)=0$, the critical field $B_{c}$ is

$$
\left|B_{c}\right|=\sqrt{\mu^{2}+\Delta^{2}}
$$

and the system is in the topological phase once $|B|>B_{c}$. The band structure around the Fermi level is shown for both, the trivial and topological phase in Fig. 7.2. Upon increasing $B$ the proximity-induced $s$-wave gap closes at the critical field $B_{c}$ and a $p$-wave gap $\Delta_{p}$ opens signaling the transition into the topological phase [LSDS10]. In total, away from the phase transition $|B| \gg B_{c}$ the important observation is that the low-energy physics can be mapped to the Kitaev chain and we can apply the considerations in Sec. 3.4. In the topological phase the system is a $p$-wave superconductor with Majorana edge states at zero energy that can feature odd parity, too. In this situation the ground state energy is insensitive to the applied magnetic field because the $s$-wave gap is closed. Then, even and odd parity ground states are degenerate with respect to a change in the overall charge $\hat{Q} \rightarrow \hat{Q} \pm 1$.

\section{Charging energy and Coulomb blockade $\mid 7.1 .3$}

The action of the charging energy is merely a global shift of the allowed ground state energy as long as the electronic charge on the island is kept fixed. Therefore, within the same parity sector one expects a quadratic dependence of the ground state energy $E_{0}\left(N_{g}\right) \sim\left(e Q-N_{g}\right)^{2}$ when tuning the gate voltage $N_{g}$ and fixing $\hat{Q} \equiv Q$. However, if we ask for the state with lowest energy with respect to all parameters then the situation changes. While varying the overall charge $Q \rightarrow Q+\Delta Q, \Delta Q \in \mathbb{Z}$ we find a series of parabola depending on the gate voltage $N_{g}$. Now, since in the trivial phase the ground state always exhibits even parity, we obtain a series of parabolas being periodic with period $2 e E_{c}$. On the other hand, in the topological phase the occurance of Majorana edge modes permits for the ground state to have odd parity so that we obtain a series of parabolas with period $1 e E_{c}$. Interestingly, we can study the closing of the $s$-wave gap $\Delta(B)$ when tuning $B$. In fact, in the trivial $s$-wave superconducting phase the lowest energy odd parity state is the first excited state and thereby the lowest possible energy is given by $E_{0}+\Delta(B)$. Calculating odd-parity parabola $E_{0}\left(N_{g}\right) \sim\left(2(2 Q+1)-N_{g}\right)^{2}, Q \in \mathbb{Z}$ we expect them to move towards the ground state energy in the topological phase [vHLG16] upon closing of the gap $\Delta(B)$. Therefore, there are two characteristic values for the magnetic field, one being the critical field at which the gap closes $\Delta\left(B_{c}\right)=0$ and the second one being defined by $E_{c}=\Delta\left(B^{*}\right)$ 


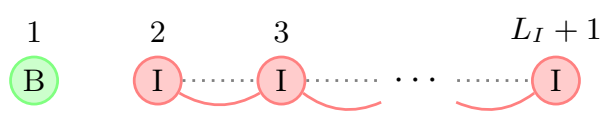

Figure 7.4: Chain geometry of isolated island with charge boson. Red circles denote lattice sites of the island and green site indicates position of the boson counting the island's charge. Labels adjacent to site symbols display numbering of the corresponding one-dimensional chain. Dotted lines between symbols represent the connectivity of the one-dimensional chain while solid, colored lines indicate the couplings between the lattice sites.

below which there are odd-parity ground states. This is shown in Figs. 7.3a and 7.3b. Having this in mind the charging energy acts as an effective chemical potential fixing the overall particle number on the island.

We implemented Eq. (7.1) as PP-MPO using projected purification to restore global charge $N_{f}$ and spin projection $S^{z}$ conservation as described in Sec. 6.1. Additionally, we added a bosonic site to account for the overall charge $\hat{Q}$ on the island which, however, is disconnected from the remaining system, yet. Its purpose is to fix the parity constraint Eq. (7.10) which we accounted for by introducing an additional term

$$
\hat{H}_{I} \rightarrow \hat{H}_{I}+\hat{H}_{P} \equiv \hat{H}_{I}+\alpha\left(\hat{P}_{Q}-\hat{P}_{f}\right)^{2} .
$$

The Langrange multiplier was chosen so that $\alpha>\Delta$ in order to avoid the algorithm to become trapped in excited states. We performed 2DMRG-PP ground state calculations to test the convergence properties of our implementation by studying the phase transition indicated by a change from $2 e$ to $1 e$ periodic parabolas in the ground state energy $E_{0}\left(N_{g}\right)$. We fixed the model parameters $t_{I, h}=1, \Delta=1.1, \mu=0.5, v_{S O}=2$ and chose a reference charge $Q_{0} \gg 2 L_{I}$ to avoid frustration effects. We calculated the ground states in each charge sector $Q=Q_{0}+\Delta Q$ for $\Delta Q=0, \pm 1$ tuning the magnetic field $B$ across the phase transition and varied the gate voltage $N_{g}$. During the calculations the maximum discarded weight was set to $\epsilon^{2}=10^{-10}$ which could be achieved setting the maximum bond dimension to $m=200$. The Zeeman shift causes a global shift in the obtained ground state energies. Therefore in each charge sector $Q$ we shifted the energy parabola $E_{0}\left(B, N_{g}\right)$ towards the minimum value found

$$
E_{0}(B) \rightarrow E_{0}(B)-\Delta E(B) \quad \text { with } \quad \Delta E(B)=\min _{N_{g}} E_{0}(B)-\min _{B^{\prime}, N_{g}} E_{0}\left(B^{\prime}, N_{g}\right) .
$$

The obtained values for the shifted groundstate energy as well as the splitting between different parity sectors

$$
\Delta E_{\min }(B)=E^{\Delta Q= \pm 1}-E_{\min }^{\Delta Q=0}(B)
$$

with $E_{\min }(B)=\min _{N_{g}} E_{0}\left(B, N_{g}\right)$ is shown in Fig. 7.5. We find the expected closing of the gap towards the critical field $B_{c}=\sqrt{\mu^{2}+\Delta^{2}} \approx 1.13$. Additionally, while the gap closes, we can clearly identify the transition from $2 e$-periodic parabolas towards $1 e$-periodic parabolas.

We also find the expected ground state degeneracies between even and odd charge sectors whenever even and odd parabola are intersecting in the regime $B_{c}>B>B^{*}$. If on the other hand $B<B^{*}$ there are degeneracy points only between even parity parabolas. In general, the charge degeneracy points $\Delta N_{g}^{d}$ closest to a reference gate voltage fixed by an even charge sector $Q_{0}$ and measured from the bottom of the parabola $N_{g}=e\left(Q_{0}+1\right)$ are given by

$$
\Delta N_{g}^{d}= \begin{cases} \pm e & \text { if } B<B^{*} \\ \pm e \frac{\Delta(B)+E_{c}}{2 E_{c}} & \text { if } B_{c}>B>B^{*}\end{cases}
$$




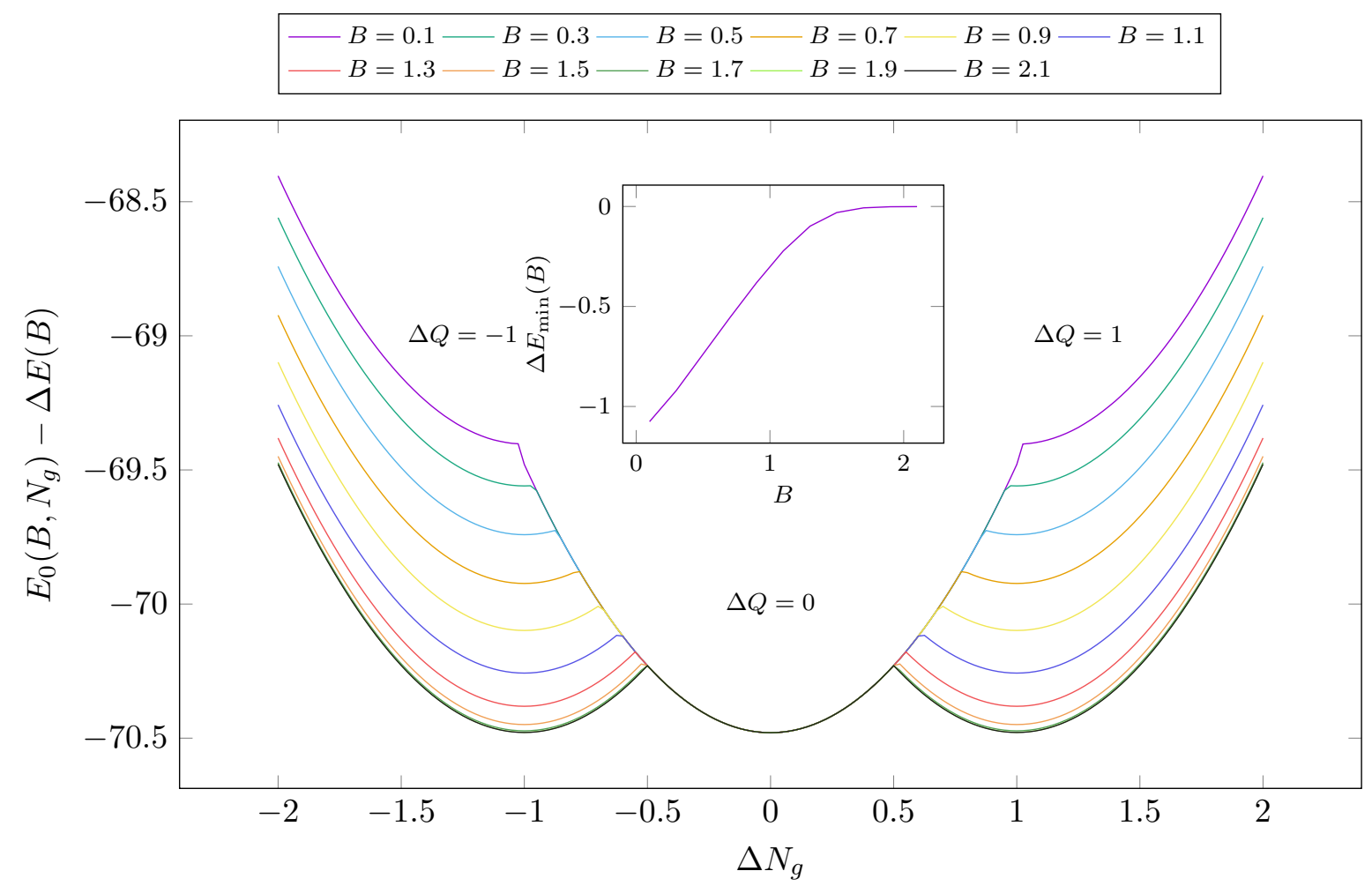

Figure 7.5: Shifted groundstate energies $E_{0}\left(B, N_{g}\right)-\Delta E(B)$ of an isolated island with $L_{I}=32$ sites and charge sectors $Q=Q_{0}+\Delta Q$ shifted w.r.t. to lowest energy as explained in the main text. The parameters of the island are chosen to be $\Delta=1.1, \mu=0.5$ as well as $v_{S O}=2.0$ and $t_{I, h}=E_{c}=1$ are kept fixed. The gate voltage is measured relative to the reference charge sector $\Delta N_{g}=Q_{0}-N_{g}$.

We show this dependency of the charge degeneracy points in Fig. 7.3c. Importantly, at the degeneracy points and zero temperature the conductance of the island is $\sim \frac{e^{2}}{h}$ and vanishes quickly as soon as the gate voltage is varied [vHLG16]. The appearance of such conductance peaks can be understood in a simple physical picture. The energy costs to add/remove charges to the island are $\propto E_{c}\left(e \hat{Q}-N_{g}\right)^{2}$ and therefore large $\left(\sim E_{c}\right)$ if $N_{g}$ is tuned away from integer multiples of the charge on the island. This suppresses transport to leading order, even though at higher order there can be, for instance, correlated tunneling. The situation changes at the charge degeneracy points where a charge unit can be added/removed without energy cost. Hence, the Coulomb interaction suppresses charge transport except for isolated points where the charge degeneracy of the ground state allows tunneling through the island. The crucial observation is, that after the transition into the topological phase the conductance is carried by the Majorana modes. Therefore, a transition from 2e-periodic conductance peaks [HGMS93] to 1e-periodic conductance peaks seen in experiment and driven by an external magnetic field while being robust against further perturbatios would be an evidence for the existence of Majorana zero modes. Unfortunately this is only part of the story. Practically, bringing the island in contact with normal leads can give rise to a plethora of bound states close to $E=0$. Additionally, a hybridization of the Majorana edge modes with lead states near the Fermi level smears out the sharp conductance peaks. Thus, it becomes necessary to have criteria to experimentally decide between signals generated by Majorana edge modes and other midgap states. For that purpose we now extend our studies taking into consideration a normal lead coupled to the island. 


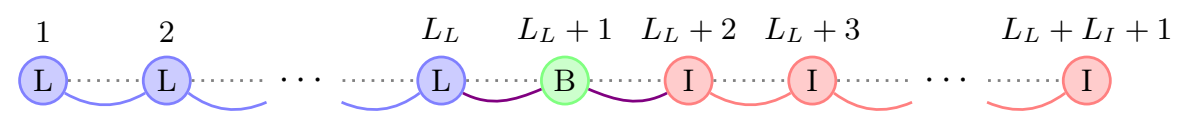

Figure 7.6: Mapping of island coupled to a single lead. Blue circles correspond to lattice sites of lead with contained labels $L$ indicating the position of the lead w.r.t the island. Red circles denote lattice sites of the island and green site indicates the position of the boson counting the island's charge. Labels adjacent to site symbols display numbering of the corresponding one-dimensional chain. Dotted lines between symbols represent the connectivity of the one-dimensional chain while solid, colored lines indicate the couplings between the lattice sites.

\section{Proximity-coupled superconducting island coupled to a normal lead}

We extend our studies by bringing the island in contact to a normal lead which we modeled by a tight-binding chain

$$
\hat{H}_{L}=-t_{h, L} \sum_{j, \sigma}\left[\left(\hat{c}_{j, \sigma}^{\dagger} \hat{c}_{j+1, \sigma}+\text { h.c. }\right)-\left(\mu_{L}-2 t_{L, h}\right) \hat{c}_{j, \sigma}^{\dagger} \hat{c}_{j, \sigma}\right]
$$

with $t_{h, L}$ and $\mu_{L}$ being the hopping amplitude and chemical potential in the lead. The overall Hamiltonian is then given by

$$
\hat{H}_{I L}=\hat{H}_{R W}+\hat{H}_{c}+\hat{H}_{P}+\hat{H}_{L}-\Gamma \sum_{\sigma}\left(\hat{c}_{L_{I}, \sigma}^{\dagger} c_{L_{I}+2, \sigma}+\text { h.c. }\right)
$$

where $L_{I}$ is the number of sites in the island. Here, we have introduced the tunneling amplitude $\Gamma$ (c.f. Fig. 7.1). For later purposes, we also introduce the conductance through the tunnel junction $g(\Gamma) \propto \Gamma^{2}$ which is a function of both, the density of states in the lead(s) and the nanowire [vHLG16]. The mapping to a chain geometry is shown in Fig. 7.6. Note, that in order to fulfill the gauge constraint on the parity Eq. (7.10), now we have couplings to the bosonic site $|Q\rangle$. Whenever an electron hops out of the island we have to reduce the occupation $|Q\rangle \rightarrow|Q-1\rangle$ whilst it has to be increased whenever electrons are entering into the chain $|Q\rangle \rightarrow|Q+1\rangle$.

We tested the convergence of our calculations by studying the softening of the degeneracy points of the energy parabolas while transitioning into the topological phase. We also tested a reduction of the eletron hopping amplitude $t_{h, L}$ in the lead to increase the density of states near the Fermi level and thereby reduce finite size effects. Note, that due to the hopping into and out of the island the overall charge $\hat{Q}$ is no longer conserved on the island. Additionally, in order to speedup convergence we found it useful to create a suitable initial state. Following the discussion in Sec. 4.4.2 for the island we chose an initial state obtained by randomly applying creation operators similar to Bogoliubov quasi-particles

$$
\hat{b}^{\dagger}=\prod_{k<k_{F}}\left(1+u_{k} \hat{c}_{k, \uparrow}^{\dagger} c_{-k, \downarrow}^{\dagger}\right)
$$

where $u_{k} \in(0,1)$ is a uniformly drawn random number. On the other hand we initalized the lead as an exact Fermi sea where $k_{F}$ is given by the chemical potential $\mu_{L}$ in the lead. During the calculations the maximum discarded weight was set to $\epsilon^{2}=10^{-10}$ which could be achieved setting the maximum bond dimension to $m=400$. The local dimension of the charge boson was set to $\operatorname{dim} \mathcal{H}_{B}=20$ enabling us to account for charge fluctuations on the island $\left|\langle\hat{Q}\rangle-Q_{0}\right| \leq 10$. 


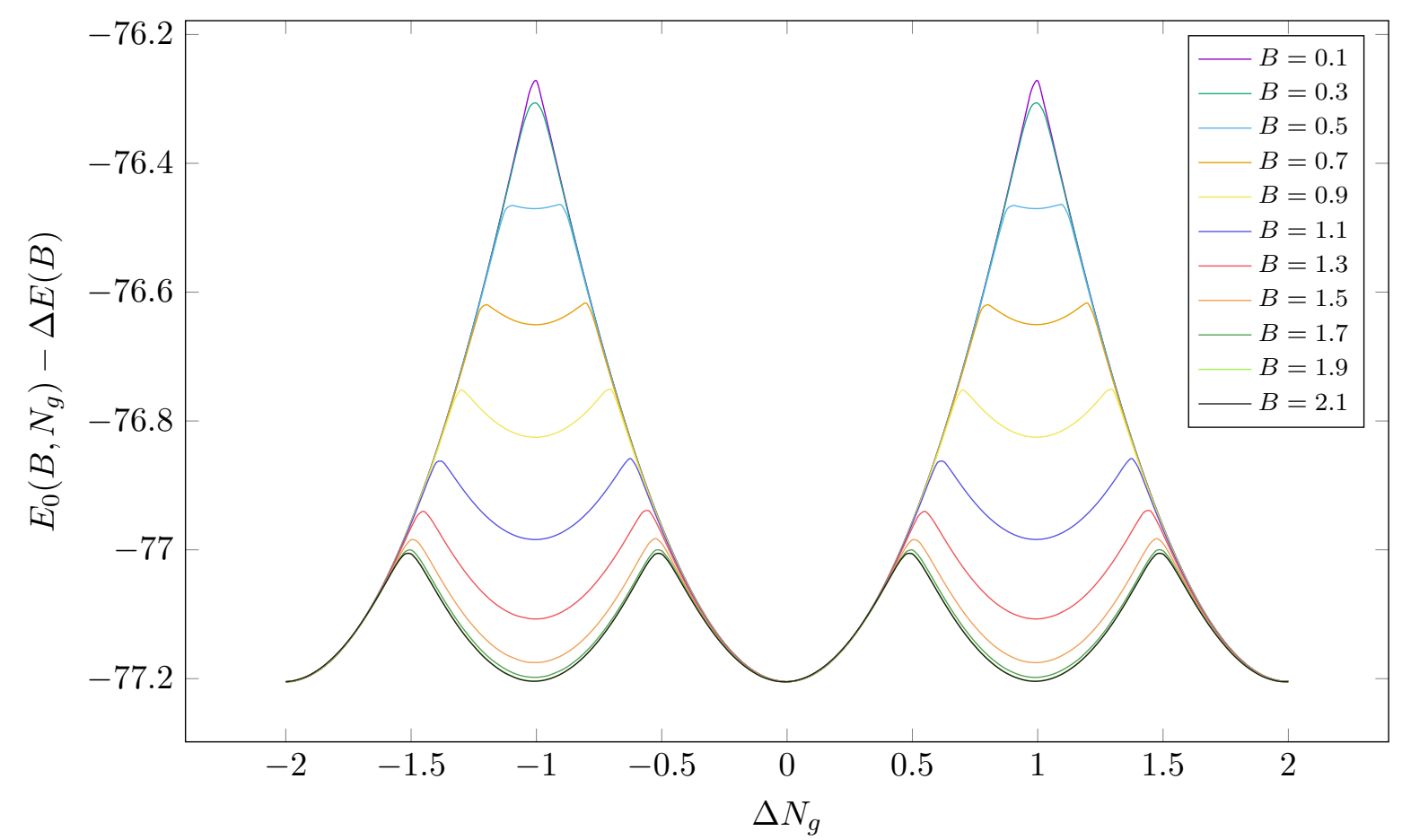

Figure 7.7: Shifted groundstate energies $E_{0}\left(B, N_{g}\right)-\Delta E(B)$ of an island with $L_{I}=32$ sites coupled to a lead with $L_{L}=32$ sites and shifted w.r.t. to lowest energy. The parameters of the island are chosen to $\Delta=1.1, \mu=0.5$ as well as $\Gamma=0.4, v_{S O}=2.0$ and $E_{c}=1$, in the lead we set $t_{h, L}=0.5, \mu_{L}=0.5$.

In order to ensure that no truncation errors are induced by this choice we evaluated the single-site reduced density matrix $\hat{\rho}_{q, q}, q \in[-10,10]$ in the strong-tunneling limit (c.f. Sec. 6.2). We found that for $|q|>5$ the occupations conistently fulfill $\hat{\rho}_{q, q}<\varepsilon^{2}$ so that our calculations are wellconverged in the charge-degree of freedom. In Fig. 7.7 results for a system with $L_{L}=L_{I}=32$ sites in both the lead and the island are shown. Further parameters of the island are chosen as in Sec. 7.1 and we set the chemical potential and hopping amplitude in the lead to $\mu_{L}=t_{h, L}=0.5$. Again, the critical field is given by $B_{c} \approx 1.13$. The tunneling amplitude between island and lead was set to $\Gamma=0.4$ so that we are in a regime with significant quantum fluctuations of the charge. We can clearly identify the transition from the trivial into the topological phase by the modification of the periodicity of the (softened) energy parabola from $2 e \rightarrow 1 e$. Also, we see the effect of the finite tunneling amplitude $\Gamma$ by smeared charge degeneracy points caused by hybridization with the lead states in both the trivial and topological phase.

\section{\begin{tabular}{l|l} 
Charge fluctuations & 7.2 .1
\end{tabular}}

The mechanisms behind these hybridizations are conceptually very different in the two phases and only certain limits are analytically accessible [HPAG05, LFG16]. Using MPS we can study the full microscopic problem within a well-defined approximation scheme. For that purpose, we are going to discuss the effects of the tunnel junction on the charge fluctuations of the island's charge $\langle\hat{Q}\rangle$ in more detail. In general, they are related to the ground-state energy expectation 


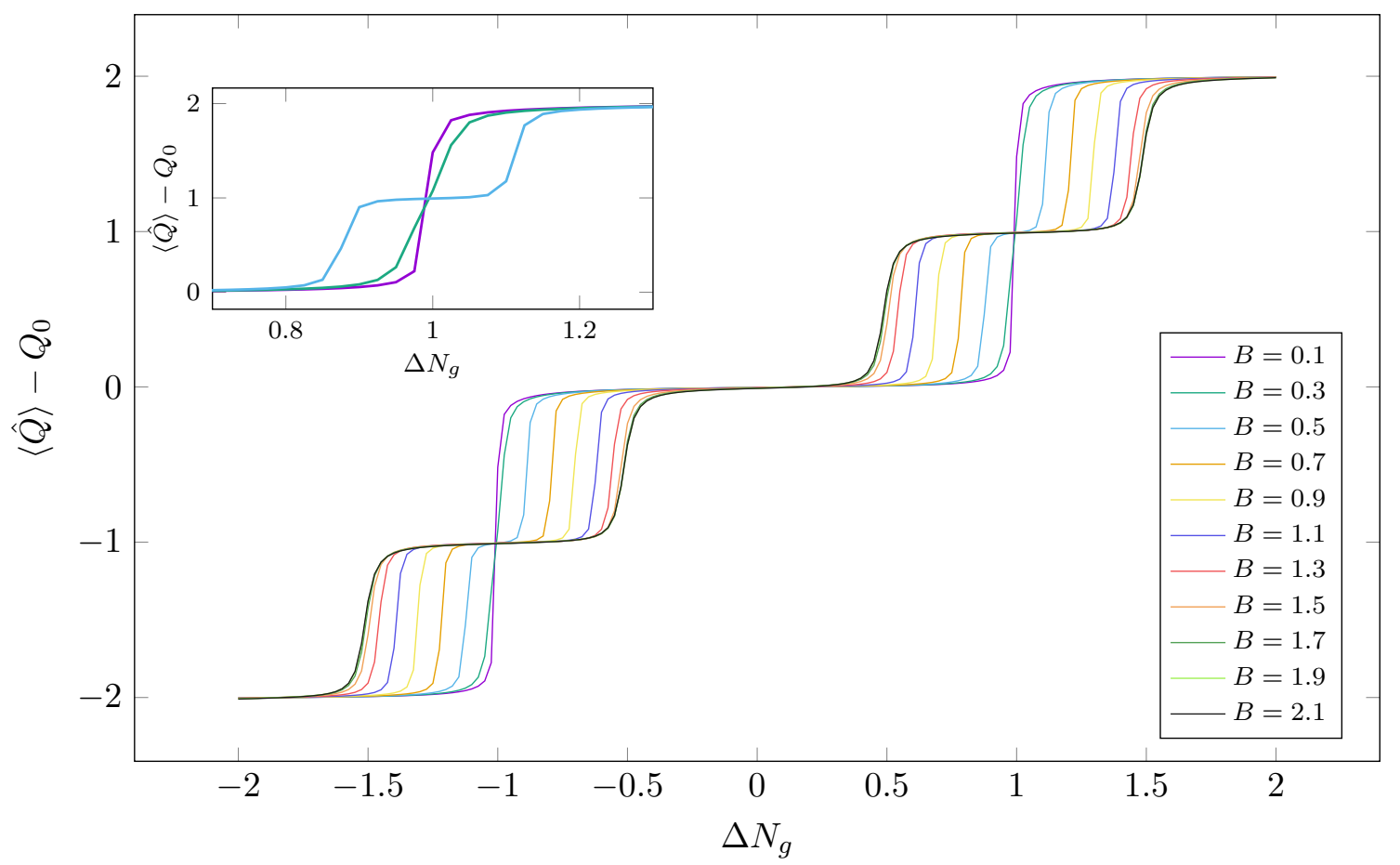

Figure 7.8: Expectation value of charge operator $\langle\hat{Q}\rangle-Q_{0}$ on the island as a function of the gate voltage for various magnetic fields $b$. The parameter in the island are chosen to $t_{I, h}=E_{c}=1, \Delta=1.1$, $\mu=0.5$ as well as $\Gamma=0.4, v_{S O}=2.0$. In the lead we set $t_{t, L}=0.5, \mu_{L}=0.5$. We fixed the reference charge $Q_{0} \gg 2 N_{I}$ to an even charge sector.

value via (c.f. Eq. (7.3))

$$
\langle\hat{Q}\rangle=N_{g}-\frac{1}{2 E_{c}} \frac{\partial E_{0}}{\partial N_{g}} .
$$

In Fig. 7.8 we plot the charge $\Delta Q \equiv\langle\hat{Q}\rangle-Q_{0}$ as a function of the gate voltage for various values of the magnetic field with even reference charge $Q_{0}$. In the trivial phase with $B<B^{*}$ there are plateaus at even values of the total island's charge of width $2 e E_{c}$ separated by charge steps. For an $s$-wave superconductor and large gap this can be expected by noting that hybridizations with odd-parity states would require an energy cost $\sim \Delta$. In the regime $B_{c}>B>B^{*}$ plateaus with an odd value of the overal charge open as soon as the closing of the $s$-wave gap permits for odd-parity ground states. Note, that avoided level crossing between the even and odd parity states increases the effective value of the field $B^{*}{ }^{2}$ This can be seen in particular in the inset where at $B=0.3$ there is still no opening of the odd-parity plateau as would be the case for the isolated island (also c.f. Fig. 7.5). Finally we can identify charge fluctuations away from the charge degeneracy points between island and lead giving rise to an analytic behaviour of the charge steps. Interestingly, these fluctuations are growing upon an increase of the magnetic field and are most pronounced in the topological phase. Even deep in the Coulomb valley, i.e., far away from the charge degeneracy points we find a finite slope of the island's charge as a function of the gate voltage.

We can use analytical results from perturbation theory in the weak-tunneling limit by Lutchyn et al. [LFG16] to check the concistency of our calculations for small $\Gamma$ in the topological phase. For

\footnotetext{
${ }^{2}$ Actually, $B^{*}$ is well-defined only for the isolated island. However, we can still formally take $B^{*}$ as the field, at which the $s$-wave gap crosses the energy maximum at the charge degeneracy points.
} 
(a)

\begin{tabular}{|c|c|c|c|c|c|c|c|c|c|}
\hline$B$ & $\Gamma$ & $\Delta_{p}$ & $a$ & $g(\Gamma)$ & $B$ & $\Gamma$ & $\Delta_{p}$ & $a$ & $g(\Gamma)$ \\
\hline 1.7 & 0.2 & 0.62 & 2.9 & 0.55 & 1.7 & 0.4 & 0.62 & 0.89 & 1.81 \\
\hline 1.9 & 0.2 & 0.78 & 2.77 & 0.46 & 1.9 & 0.4 & 0.78 & 0.85 & 1.5 \\
\hline 2.1 & 0.2 & 0.87 & 2.73 & 0.42 & 2.1 & 0.4 & 0.87 & 0.84 & 1.37 \\
\hline
\end{tabular}

Table 7.1: Fit data of half-integer charge steps in the topological phase fitted with the analytic expression Eq. (7.27) for a system of an island coupled to a lead with both $L_{L}=L_{I}=32$ sites. (a) and (b) show the results for $\Gamma=0.2$ and $\Gamma=0.4$, respectively. The remaining parameters were set to $t_{I, h}=E_{c}=1.0, \Delta=1.1, \mu_{I}=0.5, v_{S O}=2.0$ in the island and $t_{L, h}=0.5, \mu_{L}=0.5$ in the lead.

large charging energies $\frac{g \Delta_{p}}{16 \pi} \ll E_{c}$ the half integer charge steps in the interval $\Delta N_{g} \in[-1 / 2,1 / 2]$ can be captured by (setting $e \equiv 1$ )

$$
\Delta Q\left(N_{g}\right)=\frac{1}{\pi} \arctan \frac{E_{c}\left(\Delta N_{g}+1 / 2\right)}{g \Delta_{p} / 16 \pi}+1 / 2
$$

with $\Delta_{p}$ being the induced $p$-wave gap in the island and $g \equiv g(\Gamma)$ the conductance of the tunnel contact. We calculated the latter by exact diagonalization for the isolated island at the magnetic fields of interest using Eq. (7.14). Therefrom we can estimate the conductance which in general should be related to the tunneling amplitude via $g(\Gamma) \sim \Gamma^{2}$. The results are shown in Figs. 7.9a and $7.9 \mathrm{~b}$ where the dotted lines show the obtained fits with a free parameter $a$ of

$$
\Delta Q\left(N_{g}\right)=\frac{1}{\pi} \arctan \left(16 \pi \cdot a \cdot E_{c}\left(\Delta N_{g}+1 / 2\right)\right)+1 / 2 .
$$

The conductance can be extracted via

$$
g(\Gamma)=\frac{1}{a \cdot \Delta_{p}(B)} .
$$

The resulting fit data for tunneling amplitudes $\Gamma=0.2,0.4$ is summarized in tables $7.1 \mathrm{a}$ and $7.1 \mathrm{~b}$ and also plotted in Figs. 7.9a and 7.9b. For $\Gamma=0.2$ we find very good agreement with Eq. (7.26) with the extracted conductances approximately fulfilling $g(\Gamma) \ll 1$. Note, that the conductance decreases when increasing the magnetic field. On the one hand this is related to the observation by Heck et al. that the broadening of the Majorana states induced by the lead is proportional to the effective $p$-wave gap $\Delta_{p}(B)$ [vHLG16]. This way more scattering channels into occupied states of the lead have to be taken into account upon increasing $B$ reducing the conductance. However, we find an additional reduction due to the decrease of the fitting parameter $a$ as a function of $B$ (c.f. table 7.1a). This may be related to the finite system size and thus hybridization between the Majorana edge states. However, since the extracted values are not indicating a strong suppression of the conductance we did not pursue this question systematically, leaving it open for future works. In the intermediate tunneling regime $\Gamma=0.4$ we find good agreement with the fitted dependency, too. On the other hand, having a look at the extracted conductances in table 7.1b we find $g(\Gamma)$ to be significantly larger than one. This is an interesting observation, because the arctan behavior could imply that the perturbative approach is approximately capturing the charge fluctuations in the intermediate tunneling regime, though with renormalized parameters. However, as there are also other mechanism that could give rise to a similar functional dependency further investigations are required. 
(a)

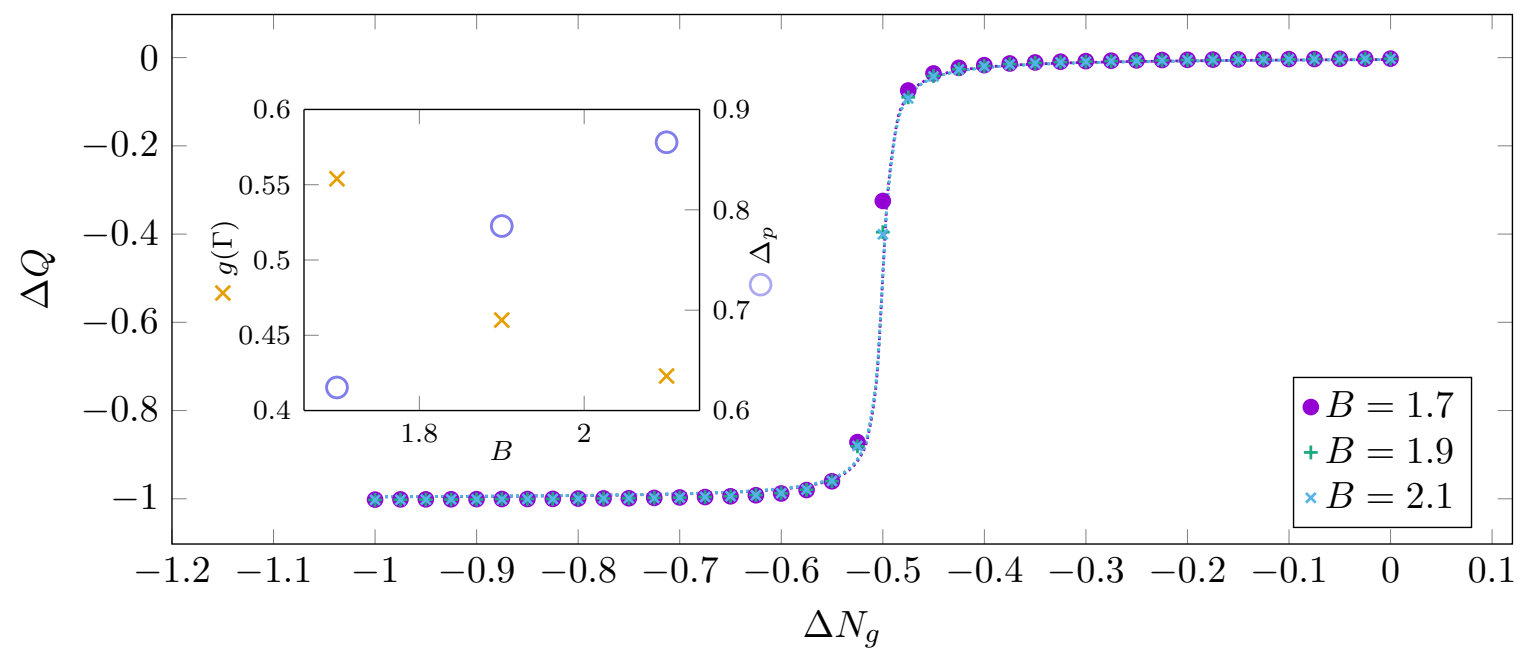

(b)

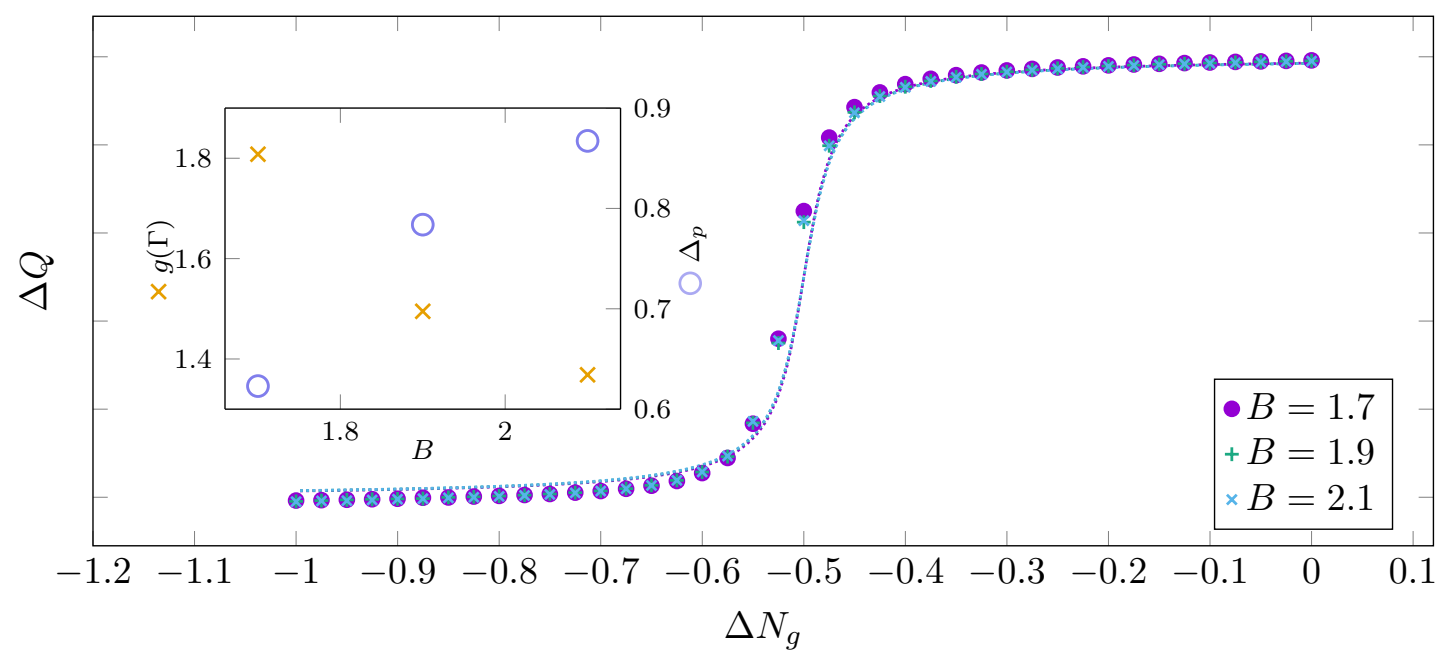

Figure 7.9: Fit data of half-integer charge steps in the topological phase fitted with the analytic expression Eq. (7.27) for a system of an island coupled to a lead with both $L_{L}=L_{I}=32$ sites. (a) and (b) show the results for $\Gamma=0.2$ and $\Gamma=0.4$, respectively. The remaining parameters were set to $t_{I, h}=E_{c}=1.0, \Delta=1.1, \mu_{I}=0.5, v_{S O}=2.0$ in the island and $t_{L, h}=0.5, \mu_{L}=0.5$ in the lead. The extracted conductance of the junction $g(\Gamma)$ is shown in the insets for the different values of the magnetic field $B$ and the corresponding p-wave gaps $\Delta_{p}$. 
(a)

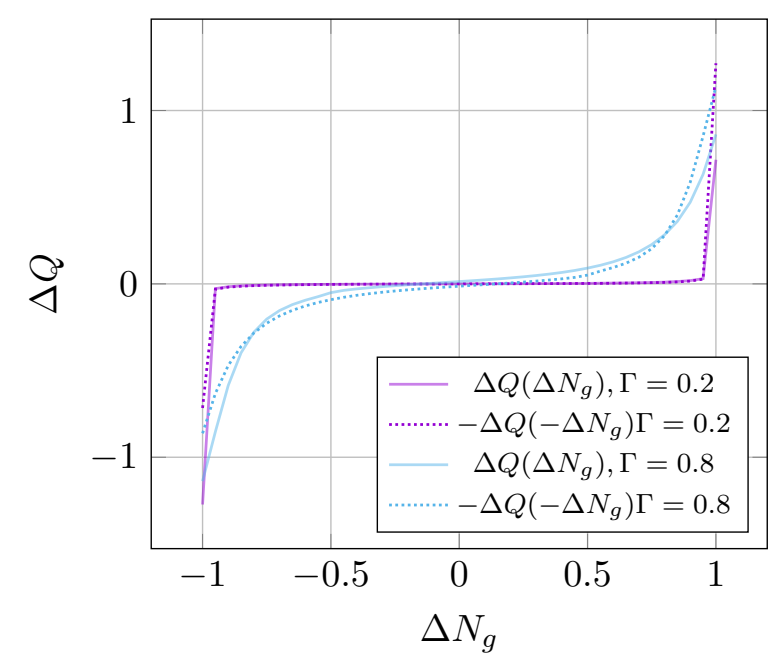

(b)

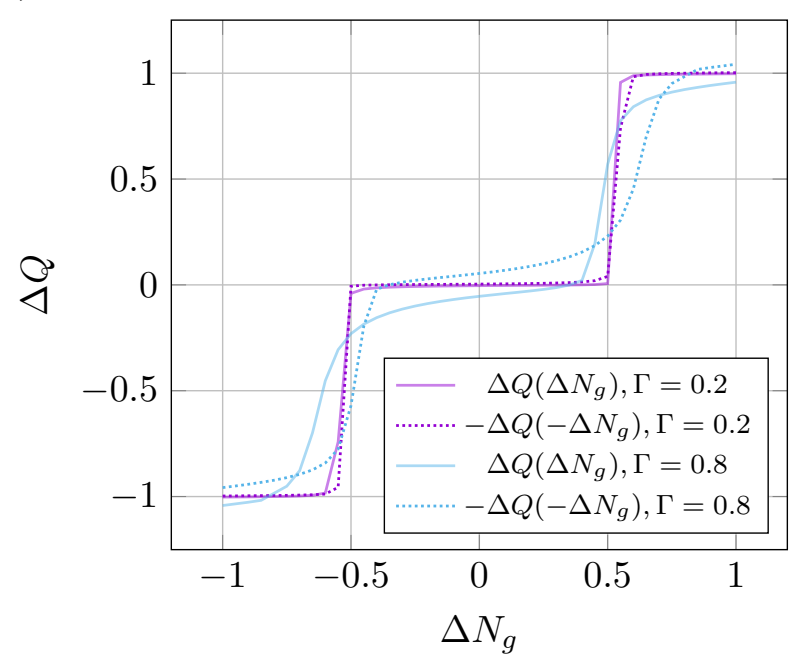

Figure 7.10: Charge steps and origin inverted charge steps $\pm \Delta Q\left( \pm \Delta N_{g}\right)$. Data is evaluated in trivial $(B=0.0,(\mathrm{a}))$ and topological phase $(B=3.0,(\mathrm{~b}))$ with further parameters $t_{I, h}=E_{c}=1.0, \Delta=1.1$ as well as $v_{S O}=0.1, \mu_{I}=0.5$ in the island and $t_{h, L}=0.5, \mu_{L}=0.5$ in the island. System sizes are set to $L_{I}=16, L_{L}=32$.

Taking a closer look at Fig. $7.9 \mathrm{~b}$ we find a mismatch of the fitted arctan-dependency on the gate voltage. Since the perturbative calculation [LFG16] predicts a symmetric charge step we checked our data for an asymmetry causing this mismatch. In Figs. 7.10a and 7.10b the charge steps in the trivial and the topological phase are compared to their mirror images. We observe a charge depletion on the island when approaching the charge degeneracy points while decreasing the gate voltages in both phases. Note, that we can define the charge degeneracy points $\Delta N_{g, \Gamma}^{d}$ by demanding $\left|\Delta Q\left(\Delta N_{g, \Gamma}^{d}\right)\right|=1$ in the trivial and $\left|\Delta Q\left(\Delta N_{g, \Gamma}^{d}\right)\right|=1 / 2$ in the topological phase. Then, the charge depletion corresponds to a shift of the charge degeneracy points towards smaller gate voltages compared to those of the isolated island

$$
\Delta N_{g, 0}^{d}>\Delta N_{g, \Gamma}^{d}, \quad \text { if } \Gamma>0 .
$$

Moreover, while in the trivial phase the charge steps are nearly symmetric around the center of the Coulomb valley where $\Delta Q(0)=0$ in the topological phase there is a clear shift towards less charges in the island. We checked that upon varying the chemical potential in the lead $\mu_{L}$ this shift in the topological phase is not due to the finite level-spacing in the lead.

We investigated the charge depletion more systematically by evaluating the absolute deviation from symmetric charge steps

$$
\xi\left(\Delta N_{g}\right)=\left|\Delta Q\left(\Delta N_{g}\right)+\Delta Q\left(-\Delta N_{g}\right)\right|
$$

in both the trivial and the topological phase. Here, the asymmetry around the charge steps are manifested by the peaks near the degeneracy points $\Delta N_{g}= \pm 1, \pm 1 / 2$. On the other hand, the overall shift generates plateaus deep in the Coulomb valley around $\Delta N_{g}=0$. We performed a two-parameter fit on the mean-square distance between the charge on the island and its origininverted

$$
\left(a_{0}, b_{0}\right)=\min _{a, b} \sum_{\Delta N_{g}}\left(\Delta Q\left(\Delta N_{g}-a\right)-\Delta Q\left(-\Delta N_{g}+a\right)+2 b\right)^{2} .
$$


(a)

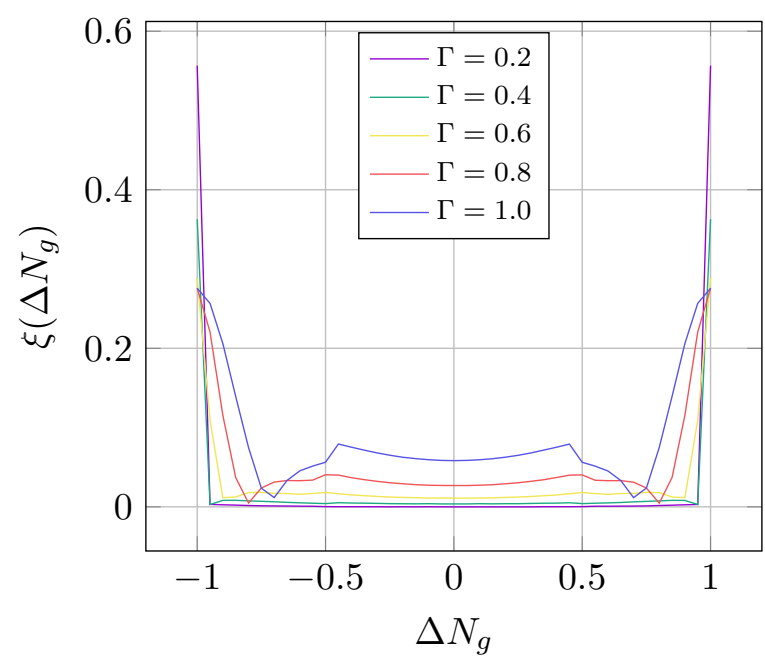

(b)

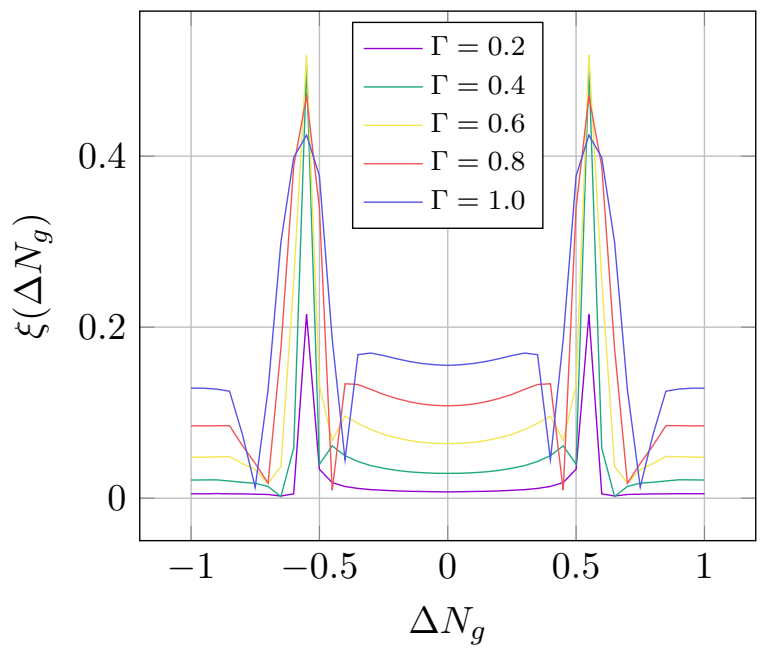

Figure 7.11: Plots display absolute value of deviations from symmetric charge steps around the origin $\xi\left(\Delta N_{g}\right)$ as defined in Eq. (7.30). Data is evaluated in trivial $(B=0.0,(\mathrm{a}))$ and topological phase $(B=3.0,(\mathrm{~b}))$ with further parameters $t_{I, h}=E_{c}=1.0, \Delta=1.1, v_{S O}=0.1, \mu_{I}=0.5$ in the island and $t_{h, L}=0.5, \mu_{L}=0.5$ in the island. System sizes are set to $L_{I}=16, L_{L}=32$.

The resulting set of parameters $\left(a_{0}, b_{0}\right)$ over the tunneling amplitude $\Gamma$ for various magnetic fields $B$ are shown in Figs. 7.12a and 7.12b. There is a clear transition in both offsets from the trivial into the topological phase indicated by a sign change. We also find a strong dependence on the tunneling amplitude $\Gamma$, so that even deep in the Coulomb valley the energy parabola's shifts become significant with larger values of $\Gamma$. Plotting the extracted parameter as a function of the tunnel contact's conductance $\Gamma^{2} \sim g(\Gamma)$ we find in very good approximation a linear dependence, away from the critical field $B_{c}$ as shown in Figs. 7.13a and 7.13b. Here, we see clearly that the systematic charge depletion $\left(a_{0}\right)$ and shifts in the Coulomb valley $\left(b_{0}\right)$ are more pronounced in the topological phase. Their linear dependency on $\Gamma^{2}$ is an interesting observation. We speculate that these effects could still be described by a perturbative treatment in the tunnel contact's conductance to first order. In [HPAG05] such a perturbation theory was constructed for the trivial phase and shown to be related to bound electron-hole states near the junction. Such electron-hole pairs are favored in the odd-parity parabolas since they already come with an additional electron above the ground state. This could give a possible explanation for the charge depletion, at least in the trivial phase: The odd-parity ground state with particle-hole excitations is energetically favorable in the even-parity region close to the charge degeneracy point. However, further examinations are required to test this hypothesis and rule out other possible effects, for instance, rescalings of the charge degeneracy points locations due to the finite hybridization of the Majorana edge modes.

The consequences of these findings are twofold. First of all we note, that even deep in the Coulomb valley near $\Delta N_{g}=0$ the influence of the tunnel contact is not negligible in the intermediate and strong tunneling regime. Interestingly, while in the trivial phase the charge is increased $(\Delta Q(0, \Gamma>0)>\Delta Q(0, \Gamma=0))$ in the topological phase the situation is reverted $(\Delta Q(0, \Gamma>0)<\Delta Q(0, \Gamma=0))$. Second, there is a significant asymmetry in the charge steps with increasing tunneling amplitude. While in the trivial phase the resulting shift of the degeneracy points $N_{g, \Gamma>0}^{*} \neq 0$ is periodic under translations $N_{g} \rightarrow N_{g} \pm 2 e E_{c}$ in the topological phase 
(a)

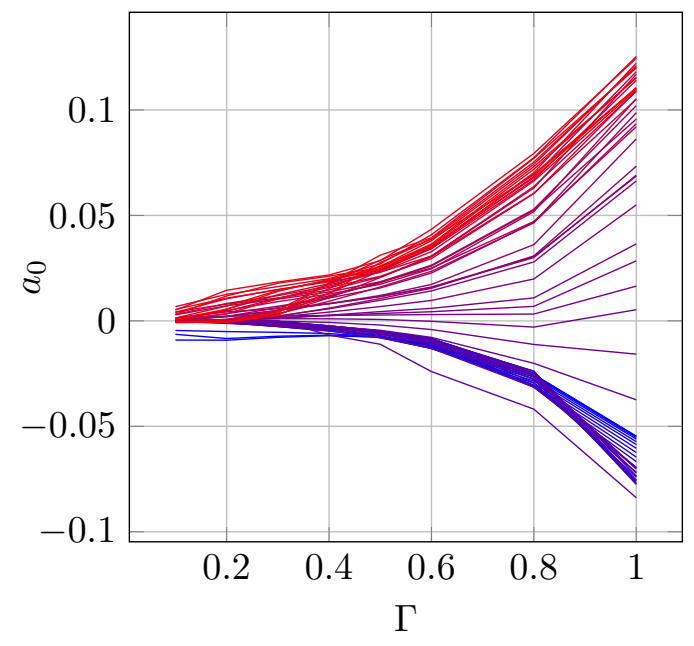

(b)

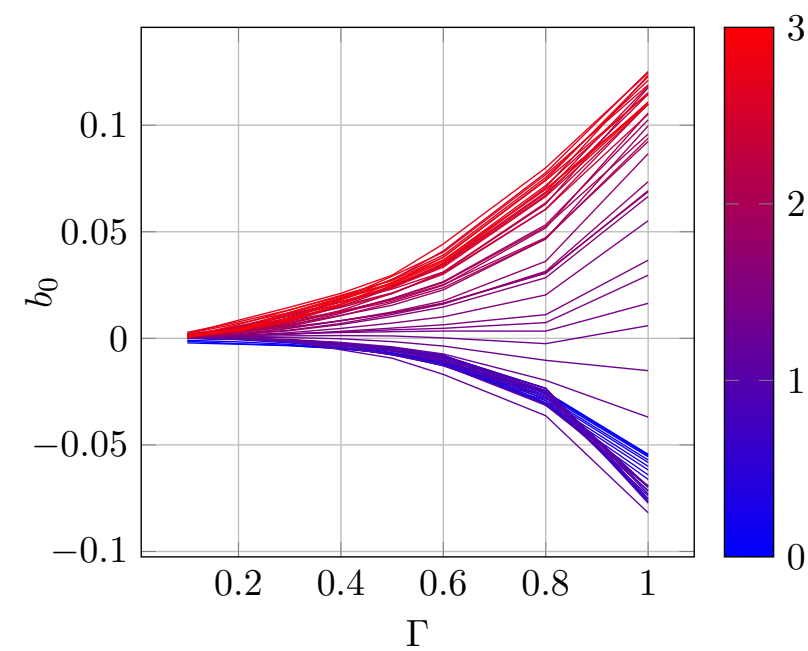

Figure 7.12: Optimization parameter $\left(a_{0}, b_{0}\right)$ minimizing the mean-square distance between charge steps $\Delta Q\left(\Delta N_{g}\right)$ and their origin-inverted as a function of the tunneling amplitude $\Gamma$ and magnetic field $B$. Each curve corresponds to one value of the applied magnetic field $B \in[0,3.0]$. Further parameters are set to $t_{I, h}=E_{c}=1.0, \Delta=1.1, v_{S O}=0.1, \mu_{I}=0.5$ in the island and $t_{h, L}=0.5, \mu_{L}=0.5$ in the lead. System sizes are set to $L_{I}=16, L_{L}=32$.

(a)

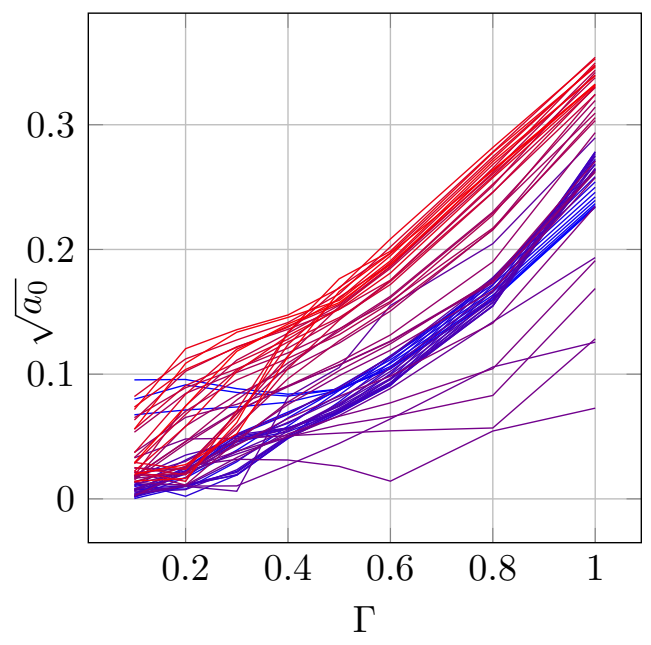

(b)

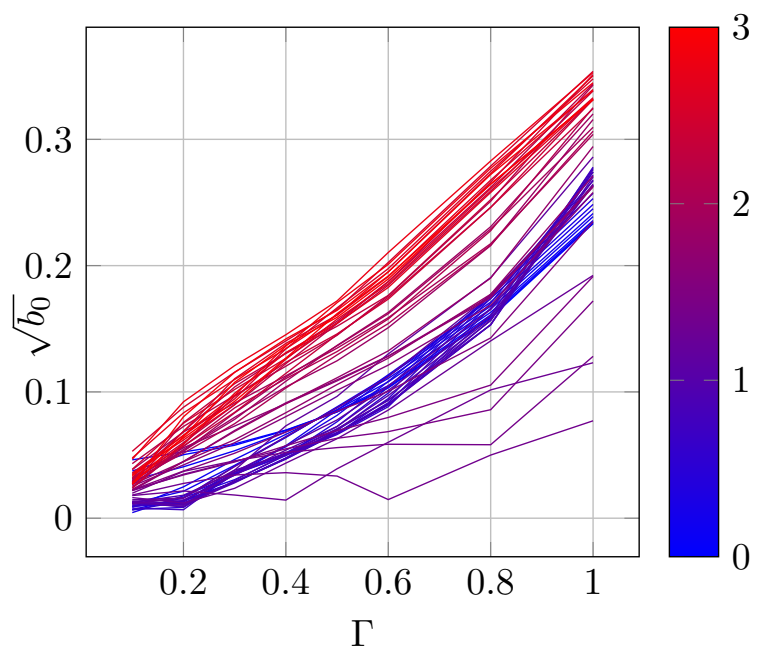

Figure 7.13: Square root of optimization parameter $\left(a_{0}, b_{0}\right)$ minimizing the mean-square distance between charge steps $\Delta Q\left(\Delta N_{g}\right)$ and their origin-inverted as a function of tunneling amplitude $\Gamma$ and magnetic field $B$. Each curve corresponds to one value of the applied magnetic field $B \in[0,3.0]$. Further parameters are set to $t_{I, h}=E_{c}=1.0, \Delta=1.1, v_{S O}=0.1, \mu_{I}=0.5$ in the island and $t_{h, L}=0.5, \mu_{L}=0.5$ in the lead. System sizes are set to $L_{I}=16, L_{L}=32$. 
the $1 e$ symmetry is broken. This will become important in the next section.

\section{Charge-degeneracy points oscillations}

In order to connect to recent experiments $\left[\mathrm{AHM}^{+} 16\right]$ we are now studying the position of the charge degeneracy points as function of the applied magnetic field $B$ and for tunneling amplitudes ranging from the weak to the strong tunneling regime. We resort to Eq. (7.25) so that finding charge degeneracy points is equivalent to searching for the maxima of the energy parabola in the interval $\Delta N_{g} \in[-1,1]$. In the topological phase and in finite systems the Majorana edge modes are not exact zero-energy states and exhibit a hybridization which for the case of the nanowire gives rise to a splitting in the ground state energy [DSSS12] (c.f. Sec. 3.4.6)

$$
\Delta E \propto k_{F, e f f} e^{-2 L / \xi} \cos \left(k_{F, e f f} L\right)
$$

with $k_{F, \text { eff }}$ being the effective Fermi wave vector of the Majorana edge modes. In the limit of small spin-orbit coupling $v_{S O}$ the latter depends on the applied magnetic field $B \sim \sqrt{B}$ in the topological phase. For the isolated island the condition to get charge degeneracy points is therefore modified to $\left(B>B^{*}\right)$

$$
\begin{aligned}
\left(e \Delta Q-\Delta N_{g}\right)^{2} & \stackrel{!}{=}\left(e \Delta Q+\left(e-\Delta N_{g}\right)\right)^{2}+\frac{\Delta E+\Delta(B)}{E_{c}} \\
\Rightarrow \Delta N_{g}^{d} & =\frac{e}{2}\left(1+\frac{\Delta E+\Delta(B)}{E_{c}}\right) .
\end{aligned}
$$

Albrecht et al. $\left[\mathrm{AHM}^{+} 16\right]$ studied the dependence of the zero-bias conductance for InAs nanowires with epitaxial $\mathrm{Al}$ and reported a transition from $2 e$ to $1 e$ periodic conductance peaks (in units of $\left.E_{c}\right)$ upon increasing an in-plane magnetic field in the Coulomb blockade regime. They also reported characteristic field-dependent oscillations of the peak positions in the 1e-periodic regime. They extracted the amplitude of the first oscillation for samples with different lengths exhibiting an exponential decay that was interpreted as experimental signature of exponentially localized Majorana edge modes (see fig. $2 \mathrm{~d}$ in $\left[\mathrm{AHM}^{+} 16\right]$ ). However, as mentioned in Sec. 3.4.6 analytical calculations for the isolated island showed that the oscillations in the topological phase are proportional to $k_{F, e f f}$ which depends on the field and a non-linearly increasing envelope is expected [DSSS12]. Assuming such an envelope also exists in the case where the island is coupled to leads and noting that the oscillations periodicity also depends on the in-plane magnetic field the exact shape of the envelope would be required in order to extract the coherence length $\xi$ via an exponential fit.

To get a better overview on the effects a lead may have when contacted to a proximity-coupled nanowire in the following we are going to study the position of the charge degeneracy points for various tunneling amplitudes as a function of the applied magnetic field. We measure the gate voltage from the bottom of the Coulomb valley, i.e., $N_{0}=e\left(Q_{0}+1\right)$. Then, from Eq. (7.33) we can identify the energy splitting $\Delta E+\Delta(B)$ with the gate voltages at which the charge degeneracy points are located

$$
\Delta N_{g}^{d}=e \frac{\Delta E+\Delta(B)}{2 E_{c}} .
$$

Using Eq. (7.11) for the isolated island we can calculate $\Delta N_{g}^{d}$ by means of exact diagonalization of $\hat{H}_{I}$ and evaluation of the energy difference between the ground state and first excited state. 


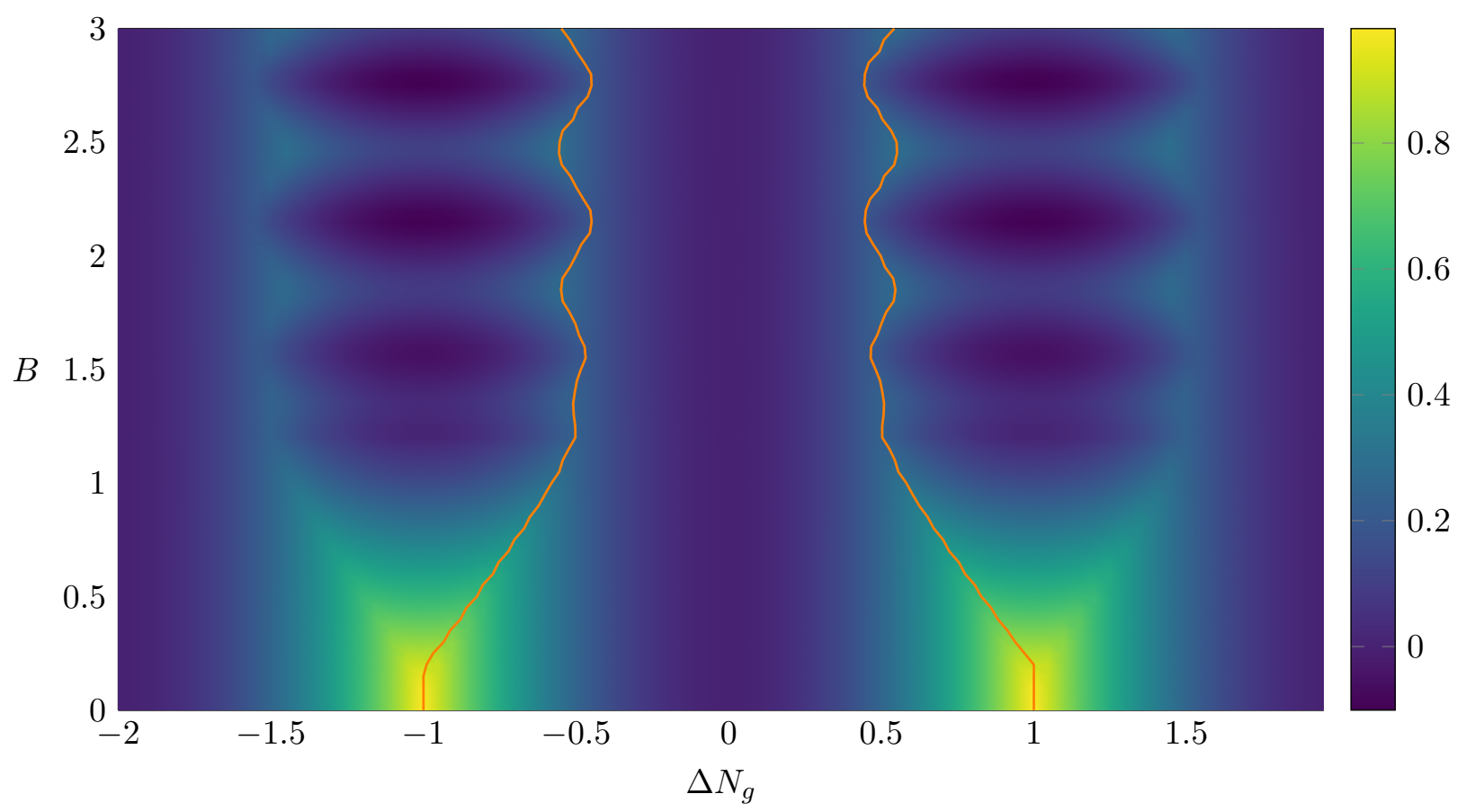

Figure 7.14: Ground-state energy measured from the center of the Coulomb valley $E_{0}\left(\Delta N_{g}, B\right)-$ $E_{0}(0, B)$ for an island $\left(L_{I}=16\right)$ coupled to a lead $\left(L_{L}=32\right)$ with tunneling amplitude $\Gamma=0.2$. Overlayed orange curves mark the extracted positions of the energy maxima corresponding to the charge degeneracy points. Further parameters are $t_{I, h}=E_{c}=1.0, \Delta=1.1, v_{S O}=0.1, \mu_{I}=0.5$ in the island and $t_{h, L}=0.5, \mu_{L}=0.5$ in the lead.

The resulting oscillations in $\Delta N_{g}^{d}$ are then used as reference values to study the influence of the lead. For the case of the island coupled to a lead we can extract $\Delta N_{g}^{d}$ from determining the gate voltages at which the nearest maxima in the ground state energy occur. We scanned a large region of the gate voltage $\Delta N_{g} \in[-1,1]$ in steps $\delta N_{g}=0.05$ and the magnetic field $B \in[0,3.0]$ in steps $\delta B=0.05$ determining the ground-state energies $E_{0}\left(\Delta N_{g}, B\right)$. In Fig. 7.14 the ground-state energies measured with respect to the bottom of the Coulomb valley $E_{0}\left(\Delta N_{g}, B\right)-E_{0}(0, B)$ as a function of the gate voltage and magnetic field are shown exemplary for a tunnelig amplitude $\Gamma=0.2$. The expected $2 e$ periodicity of the ground state energies was checked and used to extend the gate voltage range displayed in Fig. 7.14. Note, that a horizontal slice of constant magnetic field $B$ corresponds to one series of ground-state energies such as shown in Fig. 7.7. We also indicated the fitted position of the ground-state energy maxima from which we extracted $\Delta N_{g}^{d}(B)$. Here, the origin for the asymmetric charge steps can be seen clearly in the different energy landscapes of Coulomb valleys corresponding to even and odd parity parabolas $\left(\Delta N_{g} \in\right.$ $[-0.5,0.5]$ and $\left.\Delta N_{g} \in[-1.5,-0.5], \Delta N_{g} \in[0.5,1.5]\right)$. We emphasize, that the breakdown is not due to the chosen value of the charging energy $E_{c}$. In fact, for Eq. (7.26) to be valid it is assumed that the induced $p$-wave gap is small $\frac{g \Delta_{p}}{16 \pi} \ll E_{c}$. For the given parameter set we have extracted the induced $p$-wave gap of the isolated island which is $\Delta_{p} \sim 0.1$ for $B>1.4$.

Repeating the simulations with different values for the tunneling amplitude $\Gamma$ we extracted the oscillations of the charge degeneracy points $\Delta N_{g}^{d}$. In Fig. 7.15a the results are shown for the same island size $\left(L_{I}=16\right)$ where $\Gamma$ was varied between 0.2 and 1.0. Note, that we labeled the hybridized single-particle energies corresponding to the Majorana edge modes by $E_{I, 0 / 1}$. For small tunneling amplitudes $\Gamma=0.2,0.4$ the oscillations are comparable to those calculated from 
(a)

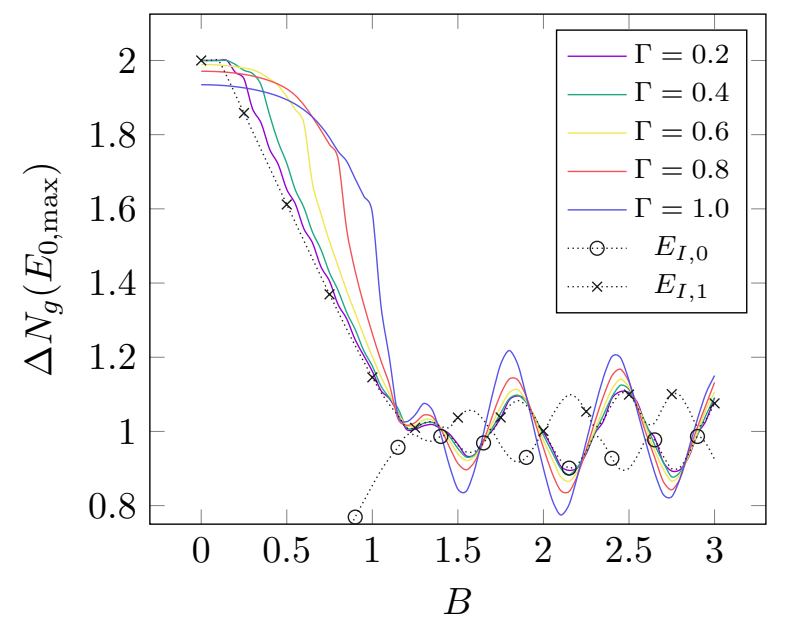

(b)

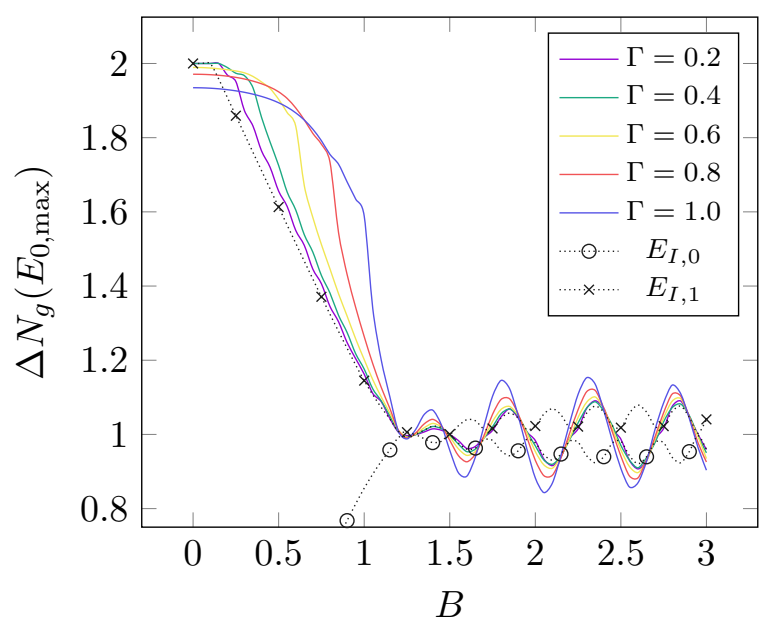

Figure 7.15: Extracted oscillations of gate voltage $\Delta N_{g}$ separating energy maxima $E_{0, \max }=$ $\max _{N_{g}} E_{0}\left(N_{g}, B\right)$ compared with the corresponding ground state splittings obtained from diagonalizing $\hat{H}_{I}$, c.f. Eq. (7.11). (a) shows results for a system with $L_{I}=16$ sites on the island while data in (b) is obtained for system with $L_{I}=20$ island sites. Further parameters are $t_{I, h}=E_{c}=1.0, \Delta=1.1$ as well as $v_{S O}=0.1, \mu_{I}=0.5$ in the island and $t_{h, L}=0.5, \mu_{L}=0.5$ in the lead.

the single-particle eigenstates of Eq. (7.11) using Eq. (7.34). Interestingly, the asymmetry in the Coulomb valleys does not modify the oscillations significantly and we can identify the $\sqrt{B}$ envelope. The situation changes if we further increase $\Gamma$ where we see two distinct effects arising. First of all, the position of the maxima is shifted towards smaller values of the magnetic field upon increasing $\Gamma$. Second, the field-dependent magnitude of the oscillations is increased and the $\sqrt{B}$-envelope is altered so that in the considered field ranges we can identify a monotony-change of the envelope. We repeated the calculations for a larger island with $L_{I}=20$ sites where the observed deviations are confirmed as shown in Fig. 7.15b. Note, that comparing the oscillations for the considered island sizes $L_{I}=16$ and $L_{I}=20$ in Fig. 7.15 we find nevertheless a decrease of the envelope's magnitude as expected for localized Majorana edge modes.

We can see the reason for the found modifications of the oscillations in Fig. 7.16 where the ground-state energies measured from the bottom of the Coulomb valley are shown for an island with $L_{I}=16$ sites coupled to a lead with tunneling amplitude $\Gamma=1.0$ as function of the magnetic field and gate voltage. Comparing to Fig. 7.14 we find on the one hand, that the transition into the topological phase which is indicated by the opening of the odd-parity parabola is smeared out over the entire range of gate voltages $\Delta N_{g} \in[-1.5,-0.5]$ as well as $\Delta N_{g} \in[-1.5,-0.5]$. Notably, while $B^{*}>B>B_{c}$ there is only one energy maximum per $2 e$-interval of the gate voltage which moves towards larger values of $\Delta N_{g}$ upon increasing $B$. It is only near the phase transition, that a second energy maximum evolves giving rise to the emergence of a second charge step which is indicated by the jump in the extracted position of the energy maxima near $B=1.0$ for $\Delta N_{g}>0$. However, the important feature with respect to the oscillations of $\Delta N_{g}^{d}$ in the topological phase is the strong perturbation of the energy maxima in the odd-parity regime. There we find significant modifications in the energy landscape with pronounced oscillations as function of the magnetic field and comparing to the even-parity regime there are regions where no clear Coulomb valley can be identified, (c.f. gate-voltages $\Delta N_{g} \in[-1.5,-0.5]$ and $\Delta N_{g} \in[0.5,1.5]$ near $B=1.8,2.4$ ). The extension of the energy maxima deep into the odd-parity regime increases the magnitude of 


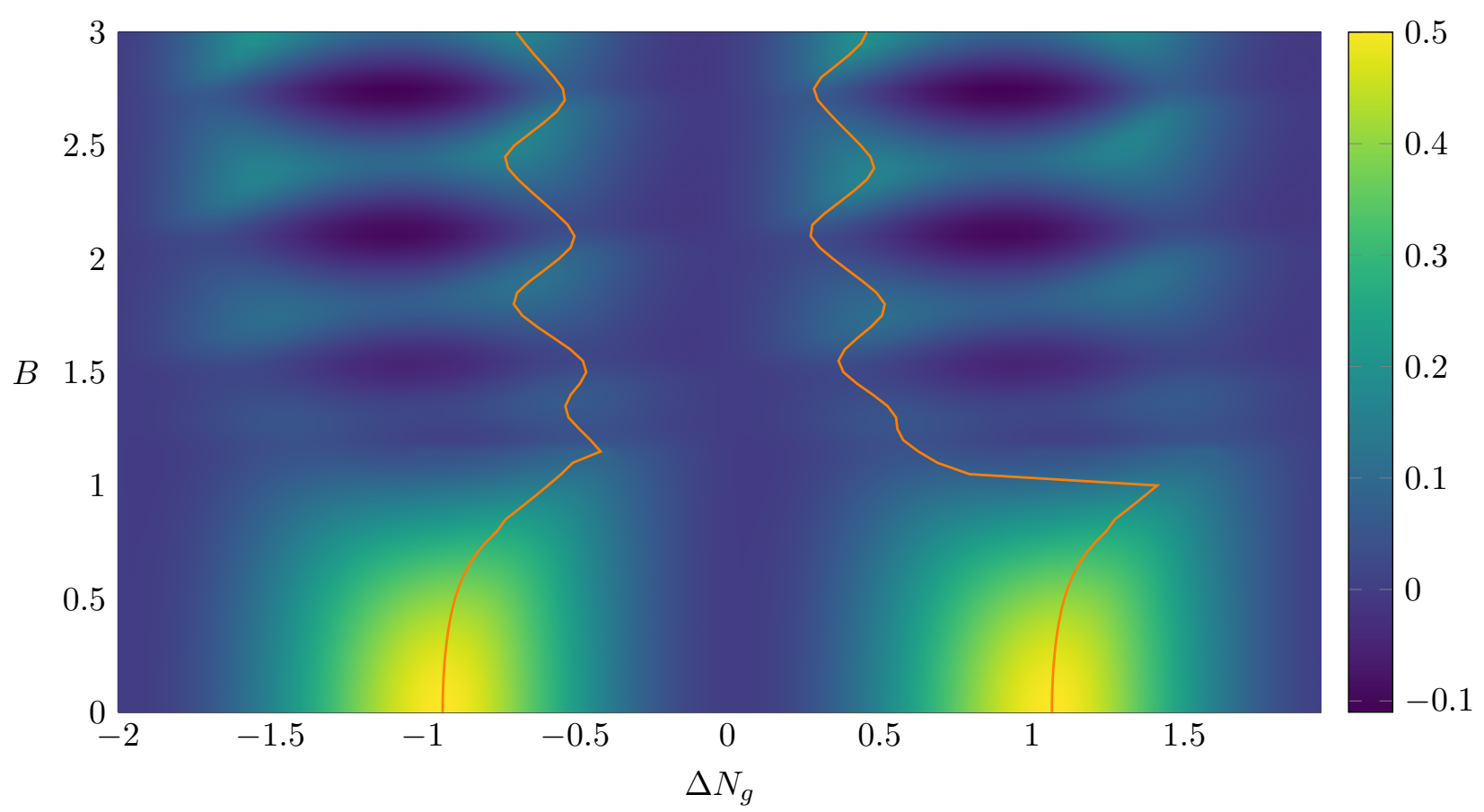

Figure 7.16: Ground-state energy measured from the center of the Coulomb valley $E_{0}\left(\Delta N_{g}, B\right)-$ $E_{0}(0, B)$ for an island $\left(L_{I}=16\right)$ coupled to a lead $\left(L_{L}=32\right)$ with tunneling amplitude $\Gamma=1.0$. Overlayed orange curves mark the extracted positions of the energy maxima corresponding to the charge degeneracy points. Further parameters are $t_{I, h}=E_{c}=1.0, \Delta=1.1, v_{S O}=0.1, \mu_{I}=0.5$ in the island and $t_{h, L}=0.5, \mu_{L}=0.5$ in the lead.

oscillations of the charge degeneracy points. Additionally, we find a shift of the overall smeared energy-parabolas towards smaller gate voltages. This shift causes the found shift in the charge steps in Sec. 7.2.1. We also see that in the odd-parity region inversion symmetry of the groundstate energy with respect to the center of the Coulomb valley $\Delta N_{g}= \pm 1$ is broken.

To sum it up, there are major modifications in the odd-parity regime of the ground-state energy if the tunneling amplitude is large $\Gamma>0.4$. They give rise to a significant change of the oscillations of the charge-degeneracy points concerning both, their position as well as their magnitude and envelope. Nevertheless, in the topological phase we are still able to identify well-defined energymaxima and thereby charge-degeneracy points. The strong changes due to the lead are also present in the trivial phase (for instance there is a suppressed splitting of the energy maxima for small fields in Fig. 7.16). However, while Coulomb-blockade effects in the trivial phase with $B^{*}>B>B_{c}$ are partially hidden by the influences of the lead, they reemerge in the topological phase. These observations suggest, that the formation of Majorana edge modes stabilizes the Coulomb-blockaded regime. We speculate, that for this effect the excited bulk-electron in the odd-parity regime in the trivial phase is crucial. While the trivial superconducting ground state is partially protected by its parity-restriction the additional electron can hybridize with states in the lead and thereby compensate the charging energy contribution. The lack of this bulk electron in the topological phase where both, even- and odd-parity states are nearly-degenerated ground states, restores the blocking effect of the charging energy. 


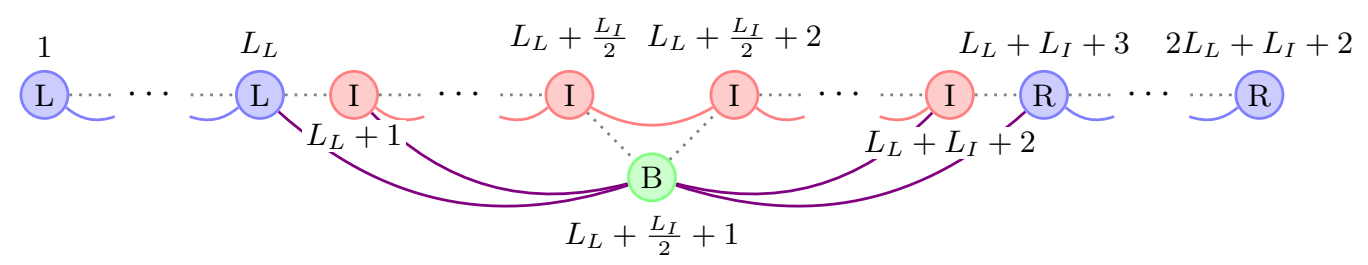

Figure 7.17: Mapping of the island coupled to two leads using only one bosonic site. Color and style encoding is the same as in Fig. 7.6

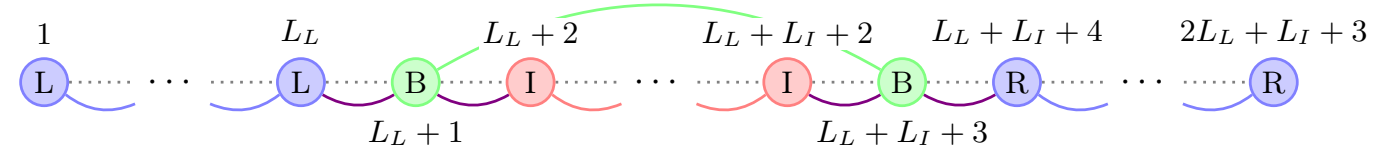

Figure 7.18: Mapping of the island coupled to two leads using two bosonic sites. Color and style encoding is the same as in Fig. 7.6

\section{Proximity-coupled superconducting island coupled to two normal leads

The following discussion is part of on-going research where we extended the setup to account for a realistic experimental realizations. However, the analysis and interpretation of the data is in an early stage so far. Therefore, we are going to present only the main results of our investigations together with some technical details on the numerical techniques. We will make no attempt to equip the results with physical interpretations as they will be highly speculative. Nevertheless, the established data demonstrates the capability of ground-state DMRG to study complicated, mesoscopic Hamiltonians in parameter regions far from what could be reached using perturbative approaches. The found results are consistent to our expectations in the limit of small tunneling amplitudes and rather surprising if we go to the intermediate and strong tunneling regime. We therefore believe that even without a well-founded physical interpretation the data itself is interesting enough to be presented within this thesis.

We are going to model the experimental situation in the Albrecht experiment $\left[\mathrm{AHM}^{+} 16\right]$ using a realistic model in which we treat the full microscopic couplings without further approximations than the well-controlled truncation error of the MPS representation. Therefore, the superconducting island is coupled to two leads with the same lengths $L_{L, l e f t}=L_{L, \text { right }}=L_{L}$. This situation renders the numerical simulation more complicated as for the naive mapping to a onedimensional chain there are long-ranged interactions or hoppings from/to the charge boson(s). The Hamiltonian without taking into account the charge boson(s) now reads

$$
\begin{aligned}
\hat{H}_{L I L}= & \hat{H}_{R W}+\hat{H}_{c}+H_{P}+\hat{H}_{L, l}+\hat{H}_{L, r} \\
& -\Gamma \sum_{\sigma}\left(\hat{c}_{L_{L}, \sigma}^{\dagger} \hat{c}_{L_{L}+1, \sigma}+\hat{c}_{L_{L}+L_{I}+1, \sigma}^{\dagger} \hat{c}_{L_{L}+L_{I}+2, \sigma}+\text { h.c. }\right),
\end{aligned}
$$


with

$$
\begin{aligned}
& \hat{H}_{L, l}=-t_{L, h} \sum_{\sigma} \sum_{j=1}^{L_{L}}\left[\left(\hat{c}_{j, \sigma}^{\dagger} \hat{c}_{j+1, \sigma}+\text { h.c. }\right)-\left(\mu_{L}-2 t_{L, h}\right) \hat{c}_{j, \sigma}^{\dagger} \hat{c}_{j, \sigma}\right] \\
& \hat{H}_{L, r}=-t_{L, h} \sum_{\sigma} \sum_{j=L_{L}+L_{I}+1}^{2 L_{L}+L_{I}}\left[\left(\hat{c}_{j, \sigma}^{\dagger} \hat{c}_{j+1, \sigma}+\text { h.c. }\right)-\left(\mu_{L}-2 t_{L, h}\right) \hat{c}_{j, \sigma}^{\dagger} \hat{c}_{j, \sigma}\right],
\end{aligned}
$$

and we set the same values for the chemical potential and hopping amplitude in both leads. Figure 7.1 provides a sketch of Eq. (7.37). In order to account for the charging energy in the gauged representation we have to introduce bosonic degrees of freedom, again. There are various ways to do so which we tested for their applicability to our problem. One possibility is to place the charge boson right in the center of the system as shown in Fig. 7.17. This keeps the charging energy local $\hat{H}_{c}=E_{c}\left(e \hat{Q}-N_{g}\right)^{2}$ but involves long-ranged hoppings from the junctions, i.e.

$$
\begin{aligned}
\text { left junction: } & \Gamma \sum_{\sigma} \hat{c}_{L_{L}, \sigma}^{\dagger} c_{L_{L}+1, \sigma} \hat{b}_{L_{b}}+\text { h.c. } \\
\text { right junction: } & \Gamma \sum_{\sigma} \hat{c}_{L_{L}+L_{I}+2, \sigma}^{\dagger} \hat{c}_{L_{L}+L_{I}+3} \hat{b}_{L_{b}}^{\dagger}+\text { h.c. }
\end{aligned}
$$

where $L_{b}=L_{L}+\frac{L_{L}+L_{I}}{2}$ is the place of the boson site with operators $\hat{b}_{L_{b}}^{[\dagger]}$ decreasing(increasing) the boson occupation. Note, that we have shifted the site labels at the right junction to account for the additional bosonic site.

Another possibility would be to symmetrically split the boson $\hat{Q} \rightarrow \hat{Q}_{l}+\hat{Q}_{r}$ measuring the charge flow at the left and right junction independently, as shown in Fig. 7.18. This keeps the hoppings to the bosonic sites local

$$
\begin{aligned}
\text { left junction: } & \Gamma \sum_{\sigma} \hat{c}_{L_{L}, \sigma}^{\dagger} \hat{b}_{L_{L}+1} c_{L_{L}+2, \sigma}+\text { h.c. } \\
\text { right junction: } & \Gamma \sum_{\sigma} \hat{c}_{L_{L}+L_{I}+2, \sigma}^{\dagger} \hat{b}_{L_{L}+L_{I}+3}^{\dagger} \hat{c}_{L_{L}+L_{I}+4}+\text { h.c. }
\end{aligned}
$$

In turn, long-ranged interactions emerge in the charging energy $\hat{H}_{c}=E_{c}\left(e \hat{Q}_{l}+e \hat{Q}_{r}+N_{g}\right)^{2}$.

We tested both approaches by performing ground-state searches for the parameter regimes of interest. Unfortunately, we found that in both cases the long-ranged nature prevents the 2DMRG solver from reliably converged to the global minimum. In the first setup, where we kept the interactions local, the energy-gain, mediated by the long-ranged hopping from the junction's electrons into the boson was merely truncated away yielding very poor convergence. Eventually the algorithm got stuck in an asymmetric charge distribution on the island. In the second setup, the situation was comparable even though now the interaction between the bosons was truncated away. This led to the odd situation, that the two bosons where equipped with different charge occupations, i.e., for odd parity states the algorithm was unable to place "half" of an electron at the two bosons, properly. To sum it up, a straight-forward mapping to a one-dimensional chain turned out to be unsuited for the numerical calculations so that we created another mapping which we describe in the following.

\section{Mapping to a ladder geometry $\quad 7.3 .1$}

In order to resolve the convergence issues when coupling the island to two leads we mapped the system onto a ladder as shown in Fig. 7.19. Employing this mapping we were able to 


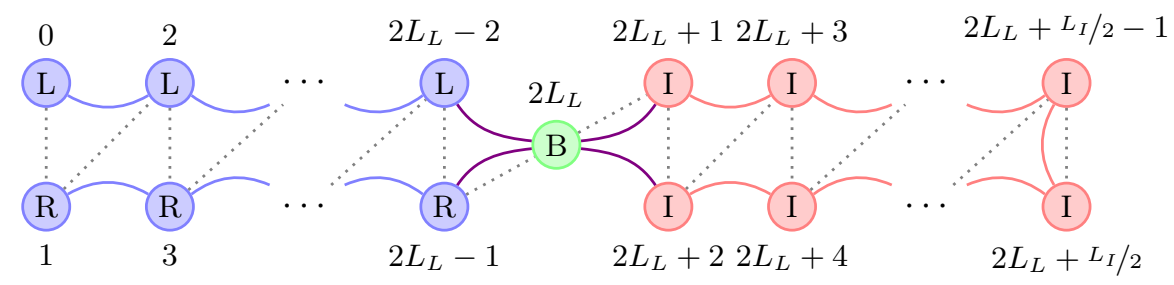

Figure 7.19: Mapping of Island coupled to two leads from linear setup to a ladder setup. Blue circles correspond to lattice sites of leads with contained labels $l / r$ indicating the position of the leads w.r.t. to the island. Red circles denote lattice sites of the island and green site indicates position of the boson counting the island's charge. Labels adjacent to site symbols display numbering of the corresponding one-dimensional chain. Dotted lines between symbols represent the connectivity of the one-dimensional chain while solid, colored lines indicate the couplings between the lattice sites.

keep the charging energy local while having only short-ranged hoppings to the bosonic site. However, nearest neighbor interactions and hoppings between adjacent lattice sites are now mapped to next-nearest neighbors. Therefore, the required bond dimension to faithfully represent the system's ground state becomes significantly larger in particular in the leads. This can be understood by noting that we are dealing with spinfull normal leads. It is well-known, that for non-interacting spinless fermions the half-chain entanglement entropy scales logarithmically in the system size $S_{v N} \sim \log L$ [CC04]. In our ladder geometry in the leads we have four chains of non-interacting spinless fermions stacked on top of each, two for each spinfull lead. At this point we would like to mention that already for only a single lead coupled to the island the maximally required bond dimension was merely set to $m=400$ to faithfully capture the critical correlations in the normal lead. Due to the intertwined structure of two normal leads we therefore had to double the maximally required bond dimension compared to the situation of only a single lead to achieve the same precision. Nevertheless, the achieved convergence during the 2DMRG groundstate search is superior to the other exploited lattice geometries. Notably, this mapping can be generalized to incorporate even more leads while keeping the charging energy strictly local. Thus, a system with $n$ leads can be mapped to an $n$-leg ladder with nearest-neighbor interactions and hoppings being transformed to $n$ th-nearest neighbors.

To account for the intertwining of the leads in this representation, we increased the bond dimension in our simulations to $m=700$ in contrast to $m=400$ which was used for the single-leg ladder of only one lead being coupled to the island. Thus, for the ground-state calculations we had to increase the maximum discarded weight to $\epsilon^{2}=10^{-8}$ to achieve the desired precision with the chosen maximum bond dimension. The local dimension of the charge boson was set to $\operatorname{dim} \mathcal{H}_{B}=20$ again permitting to capture charge fluctuations on the island $\left|\langle\hat{Q}\rangle-Q_{0}\right| \leq 10$. Following the procedure described in Sec. 7.2 we checked that our calculations are well converged in the charge degree of freedom.

\section{Charge-degeneracy points oscillations $\mid 7.3 .2$}

We performed a series of ground-state calculations for an island with $L_{I}=16$ sites using the same parameters as in Sec. 7.2.2. The island was coupled to two leads each of which consists of $L=16$ lattice sites and we applied periodic boundary conditions closing the system to a ring. In the leads we chose $\mu_{L}=0.5$ and $t_{L, h}=0.5$ in order to increase the density of states near the Fermi level. Using this setup we evaluated the oscillations of the charge degeneracy 


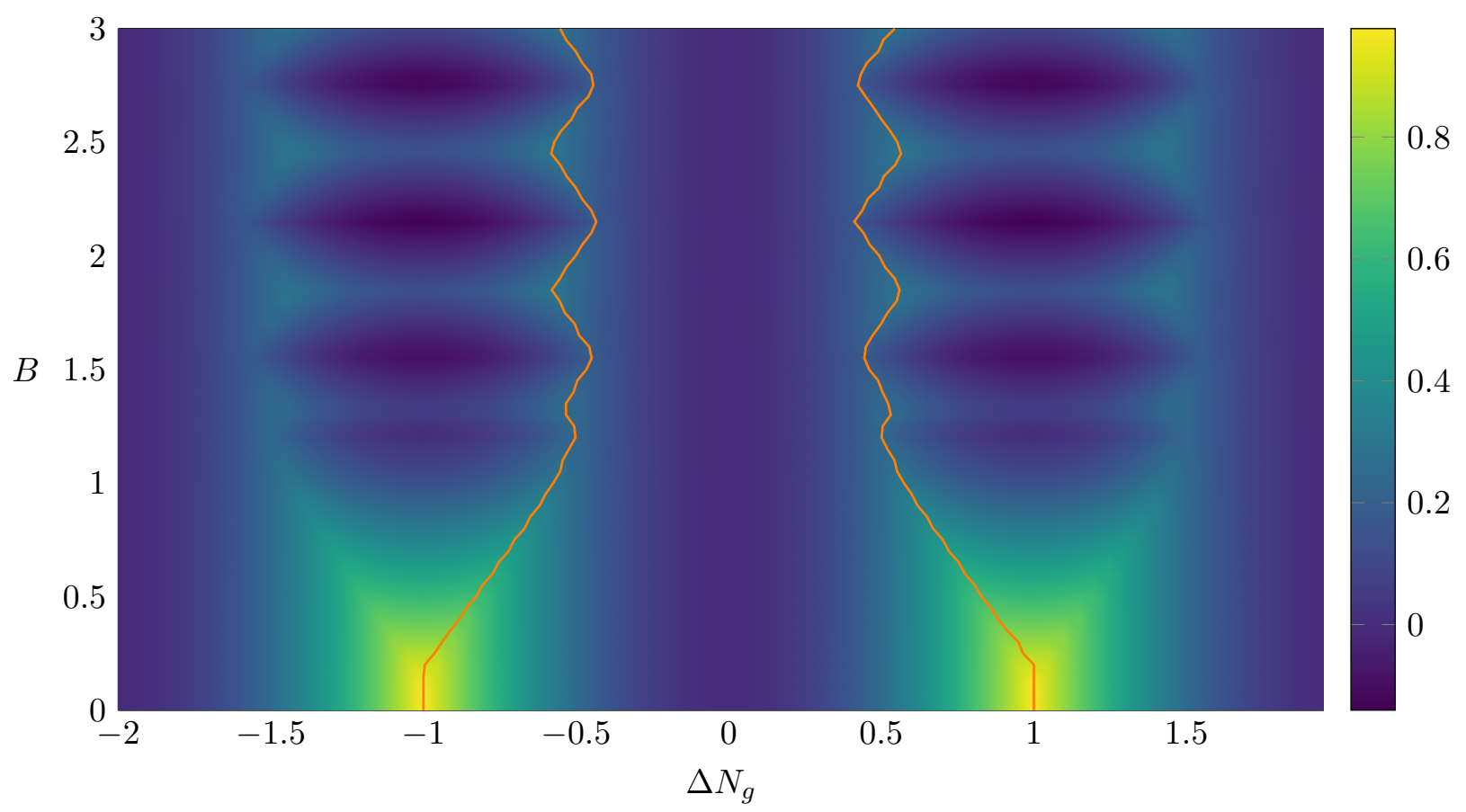

Figure 7.20: Ground-state energy measured from the center of the Coulomb valley $E_{0}\left(\Delta N_{g}, B\right)-$ $E_{0}(0, B)$ for an island $\left(L_{I}=16\right)$ coupled to two leads $\left(L_{L}=16\right)$ with tunneling amplitude $\Gamma=0.2$ and periodic boundary conditions. Overlayed orange curves mark the extracted positions of the energy maxima corresponding to the charge degeneracy points. Further parameters are $t_{I, h}=E_{c}=1.0, \Delta=$ $1.1, v_{S O}=0.1, \mu_{I}=0.5$ in the island and $t_{h, L}=0.5, \mu_{L}=0.5$ in the lead.

points $N_{g}^{d}$ as in Sec. 7.3.2 to study the effect of two leads coupled to the island. In Fig. 7.20 the ground-state energies measured from the bottom of the even-parity Coulomb valley $E_{0}(0, B)$ are shown as function of the gate voltage and the magnetic field for a tunneling amplitude $\Gamma=0.2$ and we have marked the extracted positions of the energy maxima. Similar to what we found in Sec. 7.2.2 in the odd-parity regime there are strong modifications of the ground state energies compared to the isolated island case breaking the $1 e$-symmetry with respect to the gate voltage. These effects are more pronounced than what we found in Fig. 7.14 where only one lead was coupled to the island. Here, a hexagonal pattern of the ground-state energie maxima can be observed developing, for instance, in the region $\Delta N_{g} \in[-1.5,-0.5]$ and $B \in[1.8,2.5]$.

We evaluated the position of the ground-state energy maxima varying the tunneling amplitude between $\Gamma=0.2$ and $\Gamma=1.0$. The resulting oscillations in the ground-state energy splitting is shown in Fig. 7.21a and compared to the values extracted from a diagonalization of the isolated island (c.f. Eq. (7.11) and Sec. 7.2.2). Here, in the topological phase $B>B_{c}$ we observe qualitatively similar effects as in the case of an island coupled only to a single lead (c.f. Fig. 7.15). However, the increased amplitude of the oscillations as well as the modified envelope and the shift of the oscillations is already present for only weak tunneling amplitudes $\Gamma=0.2$. In order to compare the differences between the situation of one and two leads in Fig. 7.21b we plot together the extracted oscillations for the same parameters in the island and the lead(s). We find that the oscillations with tunneling amplitude $\Gamma=0.8$ for only a single lead are following nicely the oscillations with tunneling amplitude $\Gamma=0.4$ for two leads coupled to the island even though at the local extrema there are deviations. The same behaviour is found comparing the oscillations with tunneling amplitudes $\Gamma=0.4$ and $\Gamma=0.2$ for a single and two leads coupled to the island, 
(a)

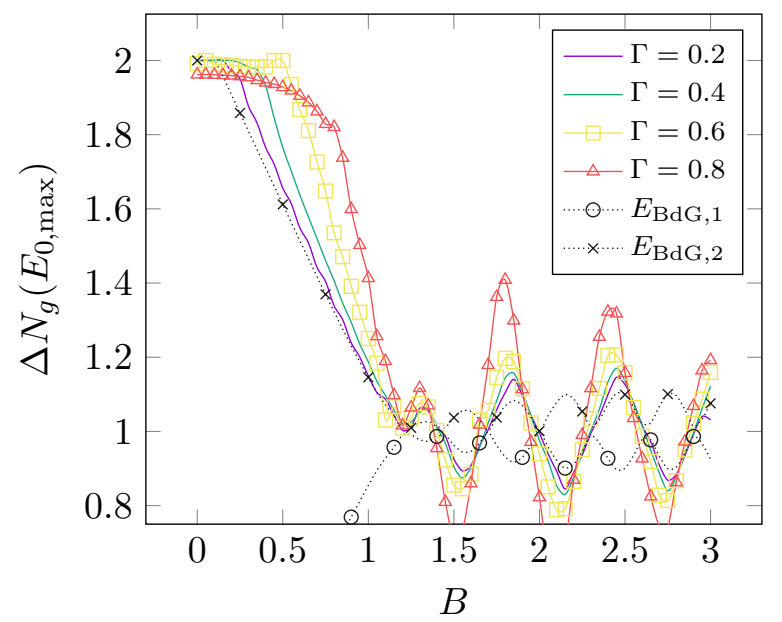

(b)

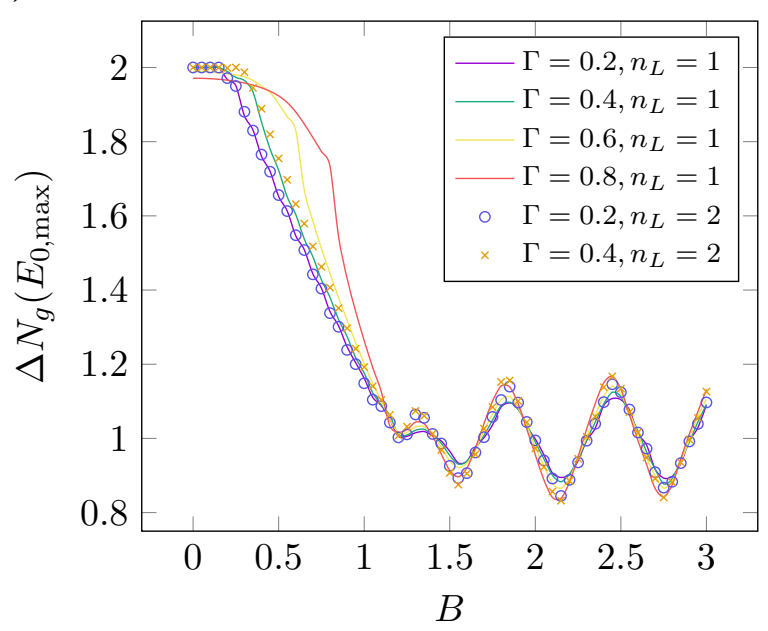

Figure 7.21: Extracted oscillations of gate voltage $\Delta N_{g}$ separating energy maxima $E_{0, \max }=$ $\max _{N_{g}} E_{0}\left(N_{g}, B\right)$ compared with the corresponding ground state splittings obtained from diagonalizing $\hat{H}_{I}$, c.f. Eq. (7.11). (a) shows results for a system with $L_{I}=16$ sites coupled to two leads with $L_{L}=16$ sites in each lead and periodic boundary conditions. (b) compares results for the island coupled to $n_{L}=1$ lead using open boundary conditions and $n_{L}=2$ leads with periodic conditions. Further parameters are $t_{I, h}=E_{c}=1.0, \Delta=1.1$ as well as $v_{S O}=0.1, \mu_{I}=0.5$ in the island and $t_{h, L}=0.5, \mu_{L}=0.5$ in the lead(s).

respectively. The observed deviations near the extreme points of the oscillations are related to the strong deformation of the energy parabola in the odd-parity regime. In detail, the positions of the maxima were extracted from interpolating the ground-state energies as a function of the gate voltage for fixed magnetic field. Due to the finite step-width in the gate voltage (which was chosen to be $\delta N_{g}=0.05$ ) the used cubic interpolation of the discretized energy parabola is most error-prone at the local maxima. In principle, this problem could be resolved easily by reducing the step width $\delta N_{g}$. More importantly, the oscillations for $\Gamma=0.8$ in Fig. 7.21a show a sudden increase in their magnitude compared to those at $\Gamma=0.6$. The reason for this jump is the strong deformation of the odd-parity regime ground-state energies which show a crossover-behaviour for $\Gamma>0.6$. In Figs. 7.22a and 7.22b we show the ground-state energies measured from the center of the even-parity Coulomb valleys $E_{0}(0, B)$ for tunneling amplitudes $\Gamma=0.8,1.0$. In the regime $B^{*}<B<B_{c}$ there is no clear energy maximum evolving corresponding to the formation of an odd-parity ground state. In turn, we find nearly linear translations of the ground-state energy maximum in the odd-parity regime which are more pronounced for $\Gamma=1.0$ and indicated in the figures by orange lines. These translations of the ground-state energy maxima are separated by gate voltages $\Delta N_{g}=2 e E_{c}$. We can also identify small Coulomb blockaded regions which, however, are shifted towards smaller values of $\Delta N_{g}$ upon increasing the magnetic field. The found behaviour is characterized by a competition between the magnetic field and the charging energy. The $2 e$-periodicity of the ground-state energy maxima indicates the addition of two electrons in a singlet state to the ground state once the charging energy becomes more costly then the Zeeman energy of the singlet. We thus find that the transition into the topological phase is suppressed at magnetic fields $B>B_{c}$ in the strong tunneling regime. 
(a)

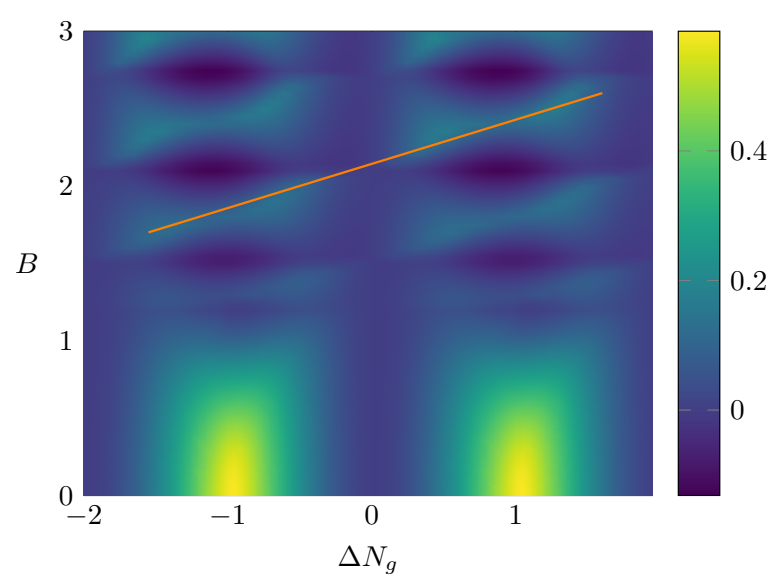

(b)

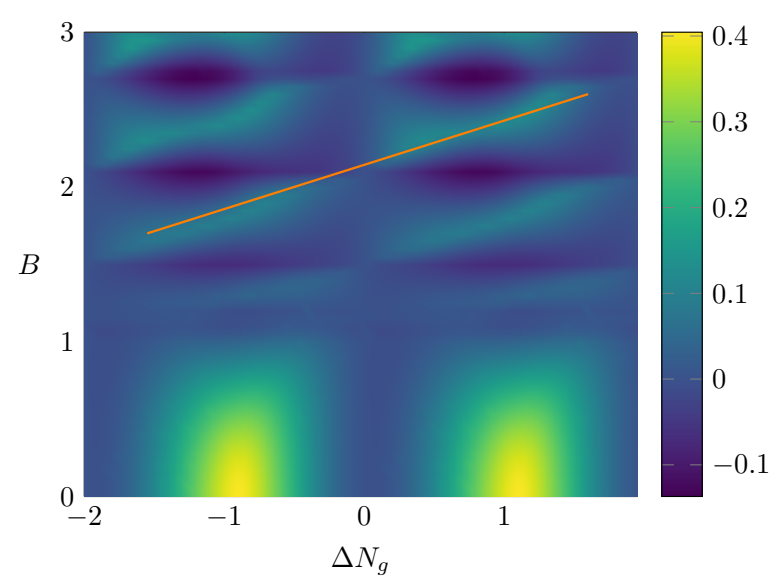

Figure 7.22: Ground-state energy measured from the center of the Coulomb valley $E_{0}\left(\Delta N_{g}, B\right)-$ $E_{0}(0, B)$ for an island $\left(L_{I}=16\right)$ coupled to two leads $\left(L_{L}=16\right)$ with (a) tunneling amplitude $\Gamma=0.8$ and (b) tunneling amplitude $\Gamma=1.0$ with periodic boundary conditions. Linear translations of groundstate energy maxima are indicated by an orange line. Further parameters are $t_{I, h}=E_{c}=1.0, \Delta=$ $1.1, v_{S O}=0.1, \mu_{I}=0.5$ in the island and $t_{h, L}=0.5, \mu_{L}=0.5$ in the lead.

\section{\begin{tabular}{l|l} 
Permanent ring current and conductance & 7.3 .3
\end{tabular}}

The quantity measured in the Albrecht experiment $\left[\mathrm{AHM}^{+} 16\right]$ was the zero-bias conductance $\left.\frac{d I}{d V}\right|_{V=0}$. However, using the linear response theory each value of the conductance would require to perform a time evolution measuring time-dependent current-current correlation functions ${ }^{3}$. Since the bond dimensions needed to represent the ground states faithfully are already comparably large this is not the method of choice. Instead, we can make use of periodic boundary conditions to induce a permanent current in the ground state. It has been shown in [Sus01] that penetrating a ring which posseses a spatially localized potential ${ }^{4} V(x)$ with a magnetic flux $\Phi=\varphi \Phi_{0}$ (with the elementary flux quantum $\Phi_{0}=\frac{\hbar}{e}$ ), the transmission amplitude $T$ through the potential is related to the current induced in the ring via

$$
T=\left|\frac{j(\varphi=0.5)}{j_{0}(\varphi=0.5)}\right|^{2} .
$$

Here, $j(\varphi)$ and $j_{0}(\varphi)$ are the induced currents in the ring with and in a reference system without potential barrier $V \equiv 0$. Given the transmission amplitude, the zero-bias conductance is then obtained from

$$
G=\frac{2 e^{2}}{h} T=G_{0}|j(\varphi=0.5)|^{2} .
$$

The magnetic flux is incorporated using Peierl's substitution [Pei33] that is adding a complex phase-factor to the hopping amplitudes

$$
\hat{c}_{j, \sigma}^{\dagger} \hat{c}_{j+1, \sigma} \longrightarrow e^{i \pi \varphi_{j}} \hat{c}_{j, \sigma}^{\dagger} \hat{c}_{j+1, \sigma} .
$$

\footnotetext{
${ }^{3}$ We have not discussed linear response theory in this thesis but there is a variety of excellent literature available (see for instance [DVC13])

${ }^{4}$ The potential needs to be symmetric w.r.t. to some point $x_{0}$, i.e. $V\left(x-x_{0}\right)=V\left(\left|x-x_{0}\right|\right)$ which is the case for the proximitized island
} 
(a)

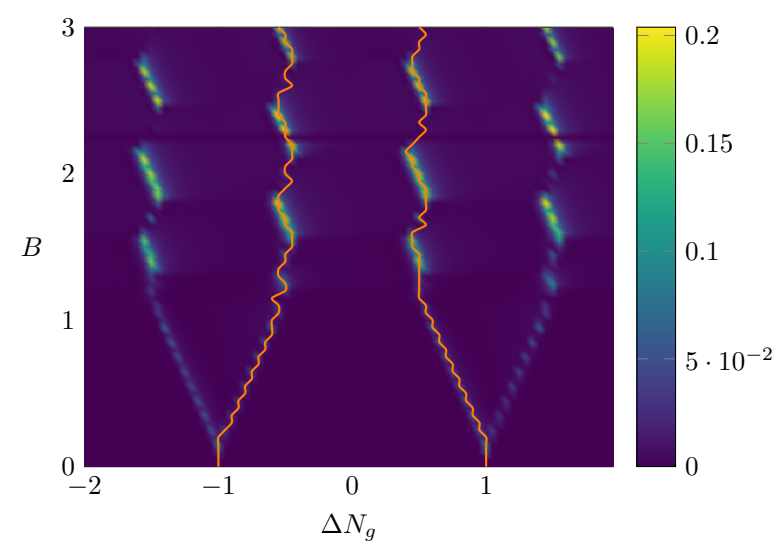

(b)

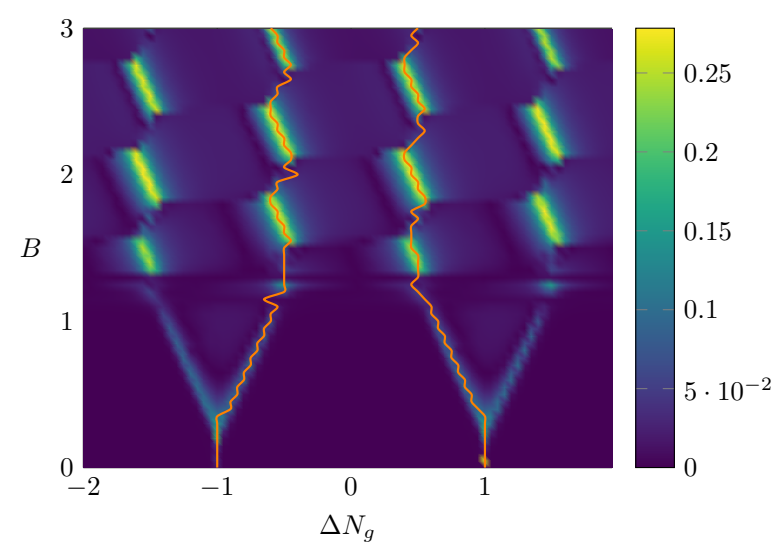

Figure 7.23: Oscillations of the absolute value $|j(0.5)|$ for an island $\left(L_{I}=16\right)$ coupled to two leads $\left(L_{L}=16\right)$ with (a) tunneling amplitude $\Gamma=0.2$ and (b) tunneling amplitude $\Gamma=0.4$ with periodic boundary conditions and penetrated by a flux $\Phi=0.5 \Phi_{0}$. Further parameters are $t_{I, h}=E_{c}=1.0, \Delta=1.1$ as well as $v_{S O}=0.1, \mu_{I}=0.5$ in the island and $t_{h, L}=0.5, \mu_{L}=0.5$ in the lead.

We gauged the vector potential so that the flux is non-vanishing only at the single bond in the lead where we close the ring $\varphi_{j}=\delta_{j, 0} \varphi$. Note, that there is no additional magnetic field added to the system, i.e. the vector potential generating the magnetic flux does not give rise to magnetic fields at the lattice sites.

We determined the induced ring currents in the ground states by calculating the expectation value with the current operator

$$
j(\varphi)=-\Im\left[\left\langle G S\left|\sum_{j}^{\prime} \sum_{\sigma} e^{\mathrm{i} \pi \varphi_{j}} \hat{c}_{j, \sigma}^{\dagger} \hat{c}_{j+1, \sigma}\right| G S\right\rangle\right]
$$

where the primed sum indicates, that the current was measured only in the leads. Indeed, the correlation function $\left\langle e^{\mathrm{i} \pi \varphi_{j}} \hat{c}_{j, \sigma}^{\dagger} \hat{c}_{j+1, \sigma}\right\rangle$ can only exhibit a non-vanishing imaginary part if $\varphi \neq 2 n \pi$ $(n \in \mathbb{Z})$. In the chosen gauge this happens if $j=0$ and therefore only at the bond at which we close the ring, i.e., in the leads. In Figs. 7.23a and 7.23b the absolute values of the persistent ringcurrents induced in the ground states are shown for tunneling amplitudes $\Gamma=0.2$ and $\Gamma=0.4$, respectively. The extracted position of the current peaks in the interval $\Delta N_{g} \in[-1,1]$ are overlayed. We find peculiar periodic suppressions in the measured currents along the expected oscillations for both cases. The origin of the suppressed currents remains unclear so far but could be related to the flux-dependence of the induced ring currents prefering a particular direction upon tuning the Zeeman field strength.

In the plots we indicated the extracted positions of the current maxima. Clearly, the suppressed currents make it difficult to unambiguously identify the current maxima oscillations. Nevertheless, we can try to display the oscillations and the results are shown in Fig. 7.24a. We find a surprisingly good agreement with the oscillations extracted from oscillations of the separations of the charge degeneracy points $\Delta N_{g}^{d}$ which are also shown. We can clearly identify the larger amplitude in the oscillations, compared to the case of an isolated island as already found in Sec. 7.3.2. Note, that we have not shown data for larger tunneling amplitudes since the effects of the crossover regime observed in the ground-state energies in Sec. 7.3.2 is even more pronounced in the induced ring currents. To illustrate this we plot the calculated ring currents for a tunneling 
(a)

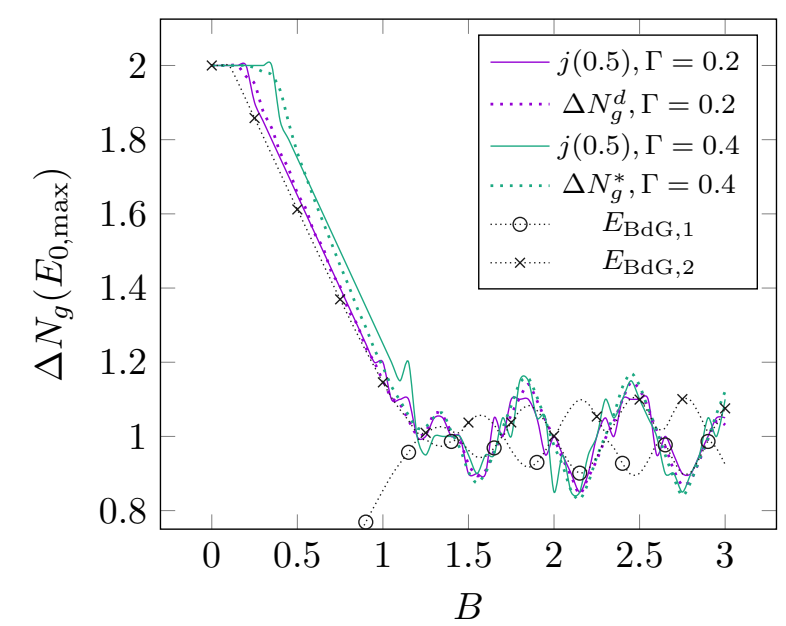

(b)

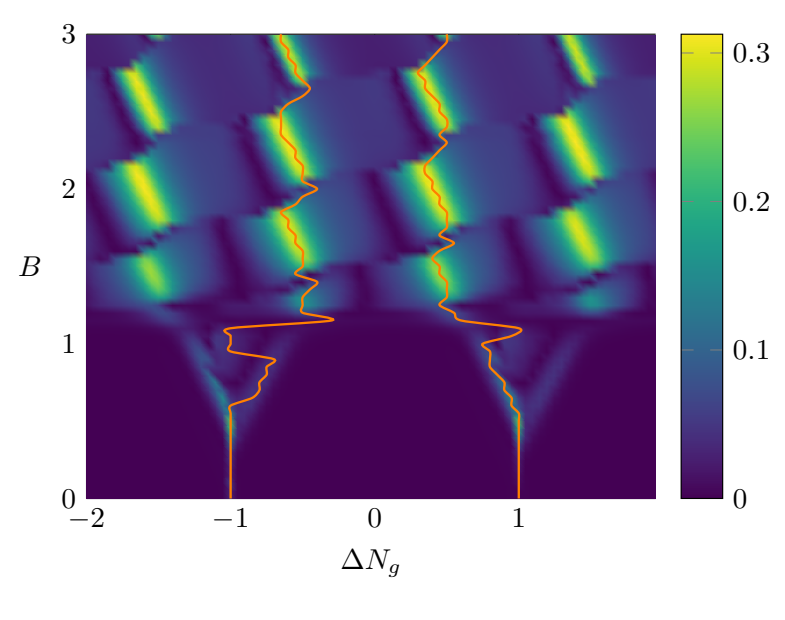

Figure 7.24: (a) shows extracted oscillations of induced ring-current $j(0.5)$ compared with the oscillations of charge degeneracy point separations $\Delta N_{g}^{d}$ for a system with $L_{I}=16$ sites coupled to two leads with $L_{L}=16$ sites in each lead and periodic boundary conditions. In (b) the induced currents $j(0.5)$ for a tunneling amplitude $\Gamma=0.6$ is shown with the extracted current maxima being overlayed. Further parameters are $t_{I, h}=E_{c}=1.0, \Delta=1.1$ as well as $v_{S O}=0.1, \mu_{I}=0.5$ in the island and $t_{h, L}=0.5, \mu_{L}=0.5$ in the lead(s).

amplitude $\Gamma=0.6$ in Fig. 7.24b. The tilted and asymmetric expanded regions of finite currents are a clear indicator for the transition into the crossover regime discussed in Sec. 7.3.2 where the topological phase is suppressed and the physics is governed by a competition between charging energy and magnetic field.

\section{\begin{tabular}{l|l} 
Summary and Outlook & 7.4
\end{tabular}}

We studied a mesoscopic model for a Coulomb blockaded Rashba nanowire proximity-coupled to an $s$-wave superconductor and contacted to a single or two leads. Being exposed to an inplane magnetic field this system is a promising candidate for the experimental realization of a topological qubit exploiting exponentially localized Majorana edge modes. Using a gauge transformation the isolated island without leads can be mapped to a quadratic fermion model and diagonalized numerically. We used the extracted spectral properties as benchmark-tools to study the effects when coupling the island to one or two leads. In detail, a strong charging energy blocks charge transport through the island except for charge-degeneracy points where the island conducts current via coherent electron transport. This transport is carried either by two electrons (Cooper-pair) in the trivial phase or a single electron in the topological phase. The corresponding ground-state quantity to study is the overall charge on the island which in the isolated case changes step-wise whenever the gate voltage at the island passes a charge-degeneracy point. Using DMRG ground-state searches we were able to tune the tunneling amplitudes and magnetic field into regions that are out-of scope for perturbative treatments.

For the single-lead setup and in the weak-tunneling regime we found good agreements of the smeared charge-steps with results from perturbation theory [HPAG05, LFG16]. For intermediate tunneling amplitudes this changes drastically. We found clear evidences for an arising 
asymmetry in the charge steps which is not predicted by perturbation theory. This is acompanied by a shift of the position of the charge steps and happens even in regimes where in the isolated case the charging energy is expected to be the dominating energy scale. We also observe significant modifications of the oscillations of the charge-degeneracy points as a function of the magnetic field. These oscillations are a unique feature of the finite hybridization of Majorana edge modes in finite systems and have been studied experimentally $\left[\mathrm{AHM}^{+} 16\right]$. Importantly, the amplitudes of these oscillations decay exponentially upon increasing the system size. However, in our simulations we found that the amplitudes are altered by the hybridization with a single lead in the strong tunneling regime. For the isolated island the oscillations exhibit an envelope $\propto \sqrt{B}$ and in order to extract the exponential decay accounting for this envelope is crucial. Our results suggest, that the envelope is modified upon increasing the tunneling amplitude when the island is coupled to a single lead. Therefore, in future works the dependency of the oscillations on the island's system size should be investigated. This would give valuable insights into the question if the exponential localization of Majorana edge modes can be confirmed in experiments.

Coupling the island to two leads confirms the observed effects but they appear at smaller tunneling amplitude, i.e., in the intermediate tunneling regime. At strong tunneling here we found a crossover regime in which the transition into the topological phase is suppressed and instead the physics is governed by a competition between charging energy and magnetic field. Imposing periodic boundary conditions on the system we could qualitatively calculate the zero-bias conductance threading the system with a finite magnetic flux. This enables us to evaluate the experimentally acessible quantity directly. In the weak tunneling limit we found the same modified oscillations in the position of the conductance peaks as for the charge-degeneracy points in the intermediate and strong tunneling regime. For the intermediate tunneling amplitudes we found a clean indication of the transition into the crossover regime already identified when studying the charge-degeneracy points and ground-state energies. Here, sharp conductance peaks and their oscillations could no longer be identified, unambiguously. However, there are also many open questions concerning the method of calculating the conductance out of persistent ring currents being induced by a threaded magnetic flux. For instance, the dependency of the induced currents on the flux needs to be understood in more detail. Additionally, the origin of the suppressed currents requires further investigation. Therefore, in order to make a clean connection to the experimental results $\left[\mathrm{AHM}^{+} 16\right]$ the methodical peculiarities have to be studied in more detail. 



\section{\begin{tabular}{r|r} 
Detecting Superconductivity out-of & \\
Equilibrium & 8
\end{tabular}}

This chapter is based on the authors publication $\left[\mathrm{PFO}^{+} 19\right]$ with an augmented discussion at several points.

$$
\begin{array}{l|l} 
& \\
\text { Introduction } & 8.1
\end{array}
$$

Superconductivity (SC) is one of the hallmarks of condensed-matter systems and has inspired researchers since its discovery in 1911, and later by the advent of high-temperature SC in cuprate materials [BM86, $\mathrm{WAT}^{+}$87, Dag94, LNW06]. While, in particular for the latter class of materials, many questions are subject of ongoing research, the basic characteristics of the SC phase are by now well established as long as the system is in equilibrium. However, recent experiments (e.g., $\left[\mathrm{FFK}^{+} 14, \mathrm{MSF}^{+} 14, \mathrm{HKN}^{+} 14, \mathrm{HNK}^{+} 16, \mathrm{MCN}^{+} 16\right]$ on copper oxides, or on $\mathrm{K}_{3} \mathrm{C}_{60}$ ) report the observation of possible photo-induced transient $\mathrm{SC}$ phases, which can exist at elevated temperatures, even above the equilibrium-critical temperature $\mathrm{T}_{c}\left[\mathrm{FMK}^{+} 11, \mathrm{FTD}^{+} 11, \mathrm{KHN}^{+} 14\right]$. In these investigations, ultrashort $\mathrm{THz}$ pulses excite single phonon modes, which decay very slowly compared to the typical time scale of the electron dynamics and thereby offer the possibility to control the interaction parameters of the electronic system $\left[\mathrm{SCK}^{+} 15\right]$. Subsequently, the $\omega-$ dependent optical conductivity is determined as a function of time via reflectivity measurements using a probe pulse, and SC correlations are identified by the emergence or enhancement of a signal at $\omega \rightarrow 0$. This is by now a standard experimental procedure, which, however, leaves many questions open, in particular concerning the characterization of the state induced by the pump excitation (see, e.g., Refs. EKW10, KWRM17, WCMD17, BTKM19). Recently, non-equilibrium Higgs oscillations have been suggested as a probe for the existence of a SC condensate [FST $\left.{ }^{+} 17\right]$. In the following, we address this issue regarding further experimental measures to probe $\mathrm{SC}$ in such non-equilibrium setups. For the sake of simplicity, we focus on one-dimensional (1D) systems using the SYMMPS toolkit. We argue that it does not suffice to study only the optical conductivity, since the pump as well as the probe pulse can induce currents, which can modify the low-frequency behavior, without being a direct proof for SC. Nevertheless, we are able to provide evidence for the emergence of SC in the course of time by studying the time evolution of spectral functions, which are accessible to time-resolved ARPES (tr-ARPES) experiments [DHS03, Dam04, LO05, EK08, FKP09, $\left.\mathrm{LAGcv}^{+} 18, \mathrm{WCP}^{+} 18\right]$. We propose to study the usual single-particle and a pairing spectral function, which we introduce below. We find particularly in the latter quantity clear signatures for the accumulation of weight at $k=0$, indicative for the (quasi-)condensation of pairs, realizing a transient SC state with qLRO. While our results indicate the persistence of qLRO, non-equilibrium situations can be beyond the realm of validity of the Mermin-Wagner-Hohenberg theorem [MW66a, MW66b, Hoh67], which inhibits the formation of true LRO in 1D systems. The scope of this chapter is, therefore, three-fold:

1. We demonstrate that the time evolution of the optical conductivity does not suffice to unambiguously establish transient SC order 
(a)

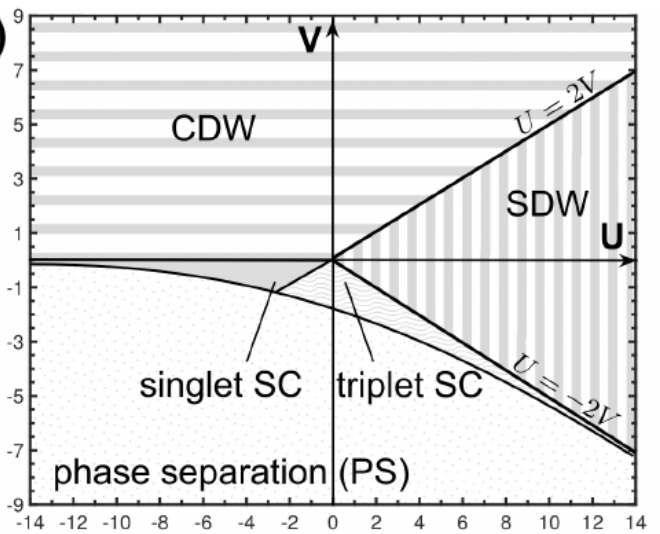

(b)

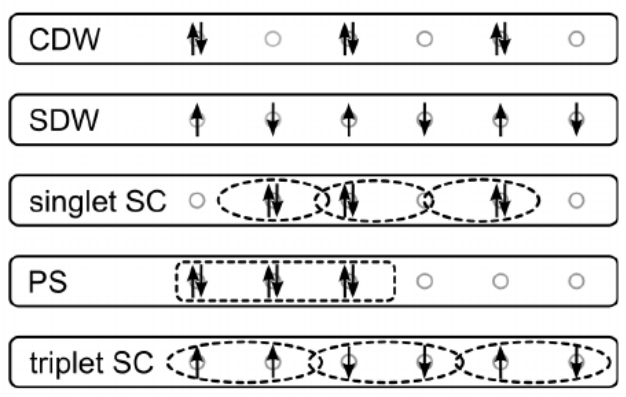

Figure 8.1: Phase diagram of the extended Hubbard model at zero temperature in the $U-V$ plane. Image extracted from [Bit17].

2. We present spectral functions as a more reliable probe

3. We test the possible realization of LRO in 1D out-of-equilibrium systems by investigating correlation matrices and their natural orbitals

The general validity of our findings is supported by comparing the extended Hubbard model [Voi95, Voi92, Jec02, TF02, SBC04, EN07, HP12] and a variant of the 1D $t-J$ model [CSO77, And87, ZR88, Dag94, MMM11, GMC ${ }^{+} 11$, MMGH17] where the latter is part of the author's publication $\left[\mathrm{PFO}^{+} 19\right]$ but not a subject of this thesis.

\section{Model}

We studied the time evolution of Hubbard chains [Hub63, Gut63, Kan63, $\mathrm{EFG}^{+}$05] following a quantum quench [EFG15], which is characterized by a sudden change of one or more parameters of the Hamiltonian. Recent experiments $\left[\mathrm{FTD}^{+} 11, \mathrm{HKN}^{+} 14, \mathrm{HNK}^{+} 16, \mathrm{MCN}^{+} 16\right]$ on high- $\mathrm{T}_{c}$ superconductors suggest that if there are preformed double occupancies, e.g., in the normal state slightly above $\mathrm{T}_{c}$, pumping particular phonon modes induces charge coherences, which drive the system into a transient superconducting state. Therefore, our starting point was to assume that lattice distortions modify the strength of the couplings $\left[\mathrm{FMK}^{+} 11, \mathrm{SCK}^{+} 15\right]$ (c.f. Secs. 2.3 and 3.3.3). Due to the long lifetime of phononic excitations compared to the electron dynamics we considered a global quench in the 1D extended Hubbard model

$$
\hat{H}=-t_{h} \sum_{j, \sigma}\left(\hat{c}_{j, \sigma}^{\dagger} \hat{c}_{j+1, \sigma}+\text { h.c. }\right)+U \sum_{j} \hat{n}_{j, \uparrow} \hat{n}_{j, \downarrow}+V \sum_{j} \hat{n}_{j} \hat{n}_{j+1},
$$

which was introduced in Sec. 2.3. Therein, $\hat{c}_{j, \sigma}^{[\dagger]}$ are $S-1 / 2$ fermionic ladder operators, which obey the canonical anticommutation relations

$$
\left\{\hat{c}_{i, \sigma}, \hat{c}_{j, \sigma^{\prime}}^{\dagger}\right\}=\delta_{i, j} \delta_{\sigma, \sigma^{\prime}}, \quad\left\{\hat{c}_{i, \sigma}, \hat{c}_{j, \sigma^{\prime}}\right\}=\left\{\hat{c}_{i, \sigma}, \hat{c}_{j, \sigma^{\prime}}\right\}=0
$$

and we denote by $\hat{n}_{j}=\hat{n}_{j, \uparrow}+\hat{n}_{j, \downarrow}$ the total electron occupation at site $j$. For later convenience, we also define doublon ladder operators $\hat{d}_{j} \equiv \hat{c}_{j, \uparrow} \hat{c}_{j, \downarrow}$. As motivated above, we started in a chargeordered state (see Fig. 8.1), which favors double occupancies [BTKM19, Bit17]. We performed 
a sudden quench in the nearest-neighbor interaction into the s-wave superconducting phase at zero temperature, keeping the local Hubbard interaction fixed. In detail, the quench is given by

$$
U / t_{h}=-4, V / t_{h}=1 / 4 \longrightarrow U / t_{h}=-4, V / t_{h}=-1 / 4 .
$$

We then calculated the real-time evolution using a combined single- and two-site TDVP scheme in the MPS formulation of the DMRG for lattices (c.f. Sec. 4.5.4) with up to $L=80$ sites, open boundary conditions, and a maximal bond dimension of $m_{\max }=1000$ states. Subsequently, in order to investigate the formation and stability of transient SC, we studied the differential optical conductivity after a probe pulse [LcvevGcvBcvPcv14, STLL16], spectral functions, and the correlation matrices [PO56, RM04] of single- and two-particle excitations [DHS03, Dam04, LO05, EK08, FKP09, $\mathrm{LAGcv}^{+}$18]. In order to demonstrate that the found behavior is not caused by a mere increase of the electron mobility we repeated the calculations for a quench within the charge-ordered phase only (see Fig. 8.1)

$$
U / t_{h}=-4, V / t_{h}=1 / 4 \longrightarrow U / t_{h}=-2, V / t_{h}=1 / 4 .
$$

We choose our energy and time units by setting $t_{h} \equiv 1$ and $\hbar \equiv 1$.

\section{Signatures of superconductivity out-of equilibrium

In the following we discuss two experimental techniques to detect signatures of superconductivity out of equilibrium: time dependent optical conductivity and tr-ARPES. While measurements of the optical conductivity are a well developed tool, tr-ARPES setups probing the two-particle channel are currently under development. For instance, a promising approach is to study noise correlations as demonstrated by Stahl et al. [SE19]. Nevertheless, we hope to convince the reader that in particular having access to tr-ARPES spectra of two-particle excitations provides valuable insight into the formation of a superconducting condensate.

\section{Time-dependent optical conductivity}

The experimental setups $\left[\mathrm{FTD}^{+} 11, \mathrm{HKN}^{+} 14, \mathrm{HNK}^{+} 16, \mathrm{MCN}^{+} 16\right]$ we refer to typically measure the reflectivity after pump-probe excitations, from which the optical conductivity $\sigma(\omega)$ is extracted. The optical conductivity is introduced by the linear current-response of a solid to an external electric field $\mathbf{E}$ giving rise to a current density $\mathbf{j}$

$$
\mathbf{j}(\omega)=\sigma(\omega) \mathbf{E}(\omega) .
$$

From this definition it is clear that in general $\sigma(\omega)$ is a rank-2 tensor. However, since we are dealing with one-dimensional systems only we treat $\sigma(\omega)$ as a scalar concentrating on the longitudinal response of the system, only. As is usually done, we decompose the optical conductivity into its real and imaginary part

$$
\sigma(\omega)=\sigma_{1}(\omega)+\mathrm{i} \sigma_{2}(\omega)
$$

For free charge carriers the Drude theory yields a Lorenzian $\delta(\omega)$-peak for $\sigma_{1}(\omega)$ which is broadened by the scattering time while $\sigma_{2}(\omega)$ diverges as $1 / \omega$, the so-called Drude peak. For BCS 
superconductors (see for instance [Tin04]) a similar behavior is expected at low frequencies near $\omega=0$ for the real part $\sigma_{1}(\omega)$. Breaking Cooper pairs costs an excitation energy of $2 \Delta$ so that an absorption edge in the imaginary part $\sigma_{2}(\omega)$ is expected at frequencies $\omega \approx 2 \Delta$. For smaller frequencies there should be a $1 / \omega$ dependency as can be obtained from the dissipationless limit of Drude theory.

In equilibrium one typically employs linear response theory and evaluates the time-dependent current-current correlation function $C_{\hat{j}}(t)=\langle\hat{j}(t) \hat{j}(0)\rangle$ from which, after a Fourier transformation, the optical conductivity is obtained. However, in a non-equilibrium setup such timedependent correlation functions become very costly quantities. This can be understood by noting that the time-dependent current-density operator in second quantization is given by

$$
\hat{j}(t)=-\frac{1}{L} \sum_{j, \sigma} \Im\left[e^{\mathrm{i} \varphi_{j}(t)} \hat{c}_{j, \sigma}^{\dagger} \hat{c}_{j+1, \sigma}\right] .
$$

Here, we explicitely added a phase dependency $e^{\mathrm{i} \varphi_{j}(t)}$ to the ladder operators which will become important in the following. The important observation is that $\hat{j}(t)$ is a global operator. Therefore, calculating the time-dependent correlation function $C_{\hat{j}}(t)$ requires to apply a global operator to the initial state. In contrast to the application of local operators, here, the entanglement entropy growth due to the excitation is not bounded and can be extensive in the system size $L$. Now the point is that already the initial state of the time-evolution is not an eigenstate of $\hat{H}$ any more and therefore we are faced with a continuous growth of the entanglement in the scope of our simulations. In order to not render the situation even worse, it is therefore desirable to avoid explicit evaluations of $C_{\hat{j}}(t)$. Therefore, we follow [LcvcvGcvBcvPcv14, STLL16] and compute the time-depenent differential optical conductivity from

$$
\sigma(\omega, \Delta t)=-\frac{j(\omega, \Delta t)}{\mathrm{i}(\omega+\mathrm{i} \eta) A(\omega, \Delta t)} \equiv \sigma_{1}(\omega, \Delta t)+\mathrm{i} \sigma_{2}(\omega, \Delta t),
$$

after the quench at $t=0$. The idea behind this approach is basically a generalization of Eq. (8.3) to non-equilibrium situations. The system is pushed out-of equilbirium by the global quench rendering the time evolution of the initial state $|\psi(0)\rangle$ non-trivial. A probe pulse $A(t, \Delta t)$ is applied centered around $\Delta t+4 \tau$ and given by a vector potential

$$
A(t, \Delta t)=A_{0} e^{-\frac{(t-(\Delta t+4 \tau))^{2}}{2 \tau^{2}}} \cos \left(\omega_{0} t\right)
$$

so that the Hamiltonian becomes time dependent while $A(t, \Delta t)$ is finite. Motivated by recent studies [BTKM19, Bit17] we chose $A_{0}=0.5, \omega_{0}=2.38$ and $\tau=0.05$. We incorporated the pulse using Peierls substitution [Hof76] so that the hopping terms of $\hat{H}$ pickup a time-dependent phase factor

$$
\hat{c}_{j, \sigma}^{\dagger} \hat{c}_{j+1, \sigma} \longrightarrow e^{-\mathrm{i} A(t, \Delta t)} \hat{c}_{j, \sigma}^{\dagger} \hat{c}_{j+1, \sigma}
$$

Now, the basic assumption is that the applied probe pulse is weak enough so that we only have to consider the response current up to linear order in the perturbation strength $A_{0}$

$$
\begin{aligned}
& j_{\text {probe }}(t, \Delta t)=j_{0}(t)+\underbrace{\int_{\Delta t}^{t+\Delta t} d \tau \sigma(t+\Delta t, \tau) E(\tau, \Delta t)}_{:=j(t, \Delta t)}+\mathcal{O}\left(A_{0}^{2}\right) \\
& \Rightarrow j(t, \Delta t) \approx j_{\text {probe }}(t, \Delta t)-j_{0}(t),
\end{aligned}
$$


(a)

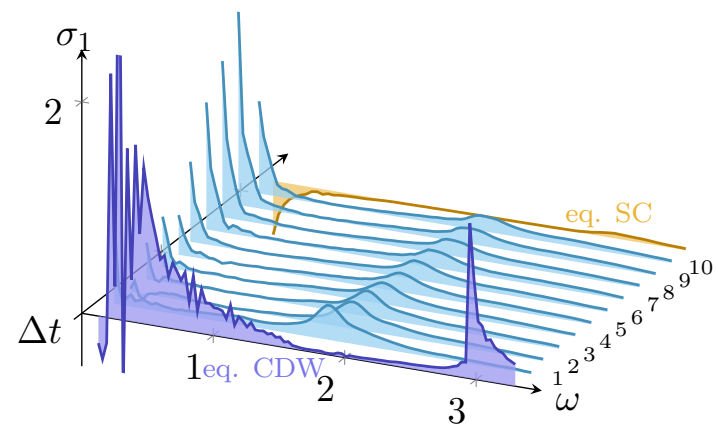

(b)

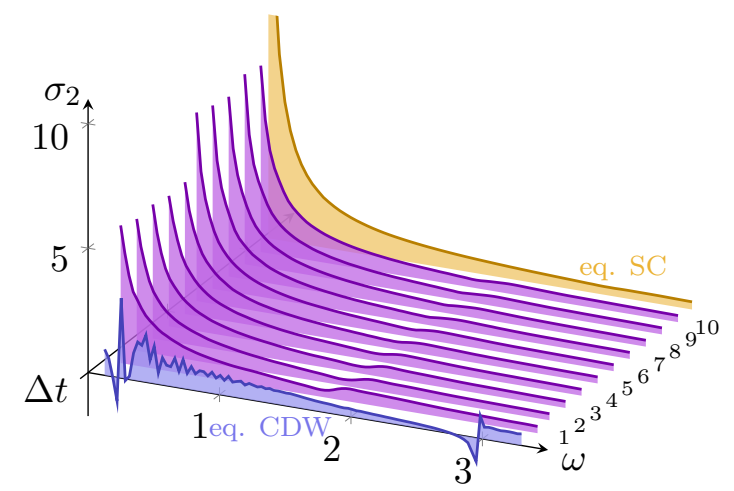

Figure 8.2: Real (a) and imaginary (b) part of the optical conductivity $\sigma_{1,2}(\omega, \Delta t)$ with probe pulses applied at different time delays $\Delta t$ after the quench (Q.1).

where $j_{0}(t)=\left\langle\psi\left|\hat{j}_{0}(t)\right| \psi\right\rangle$ is the current measured during the time evolution without the probe pulse (i.e., $A_{0}=0$ ) while $j_{\text {probe }}(t, \Delta t)=\langle\psi|\hat{j}(t+\Delta t)| \psi\rangle$ is the current measured when incorporating the probe pulse. Note that the integral in Eq. (8.9) is a convolution so that it factorizes upon Fourier transforming

$$
j(\omega, \Delta t)=\sigma(\omega, \Delta t) E(\omega, \Delta t) .
$$

The Fourier transformed time-dependent response caused by the probe pulse is given by

$$
j(\omega, \Delta t)=\int_{0}^{T} d t j(t, \Delta t) e^{\mathrm{i} \omega t} .
$$

Then, Eq. (8.6) is recovered by replacing the Fourier transform of $E(t, \Delta t)$

$$
E(\omega, \Delta t)=-\frac{\partial}{\partial t} \int_{0}^{T} A(t, \Delta t) e^{\mathrm{i}(\omega+\mathrm{i} \eta)}
$$

where a damping factor $\eta=0.01$ was introduced. For the numerical realization of the timedependent Hamiltonian $\hat{H}(t+\Delta t)$ with probe pulse we discretized the time axis chosing time steps $\delta_{\text {pulse }}=0.01$ and approximated the Hamiltonian to be constant throughout each time step. Note that this procedure involves to reconstruct the MPO representing $\hat{H}(t+\Delta t)$ before evolving the state by $\delta_{\text {pulse }}$. This small time-step was used to propagate the state by a unit time $|\psi(t+\Delta)\rangle \longrightarrow|\psi(t+\Delta+1.0)\rangle$ after which the time step was increased to $\delta=0.05$. We evaluated the time evolution after applying the probe pulse up to times $T=25$. We also checked the validity of the approximation Eq. (8.9) by varying the amplitude $A_{0}$ of the probe pulse in a small interval around $A_{0}=0.5$. We found the expected linear scaling of the response current $j_{\text {probe }}(t, \Delta t)$.

We show the real (Fig. 8.2a) and imaginary (Fig. 8.2b) parts of $\sigma(\omega, \Delta t)$ for delays up to $\Delta t=10$ and a system with $L=64$ sites after the quench (Q.1). For the real part, we find a sudden transfer of spectral weight from the CDW signal at around $\omega \approx 3$ towards $\omega \approx 1.7$, which is due to the sudden change of the Hamiltonian. This insinuates that quasi-particles with twice the mass are seen in the response function, since their energy is (about) half of the original one $\left[\mathrm{GMC}^{+} 11\right]$. At the same time, in the imaginary part a peak forms near $\omega=0$. We compare this to the SC ground state and realize that, at the first glance, similar behavior is induced. However, in particular in $\sigma_{1}$, clear differences appear, and $\sigma_{2}$ shows additional features. Also, it is hard to tell whether $\sigma_{2}(\omega, \Delta t)$ is diverging as $1 / \omega$ for $\omega \rightarrow 0$, since we are limited in the frequency resolution. 
(a)

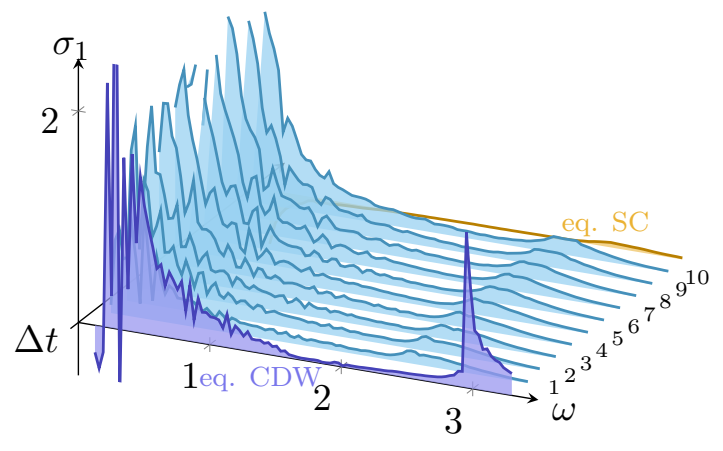

(b)

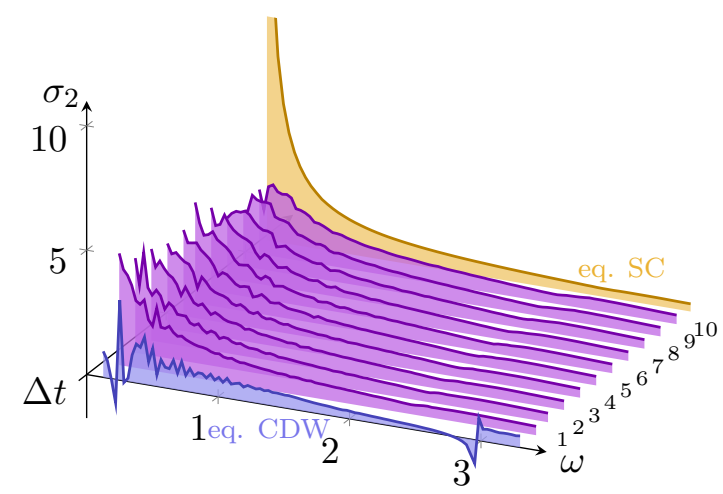

Figure 8.3: Real (a) and imaginary (b) part of the optical conductivity $\sigma_{1,2}(\omega, \Delta t)$ with probe pulses applied at different time delays $\Delta t$ after the quench (Q.2).

(a)

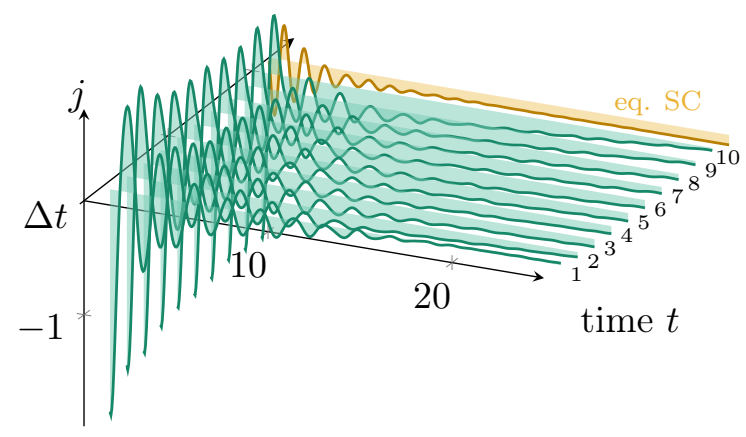

(b)

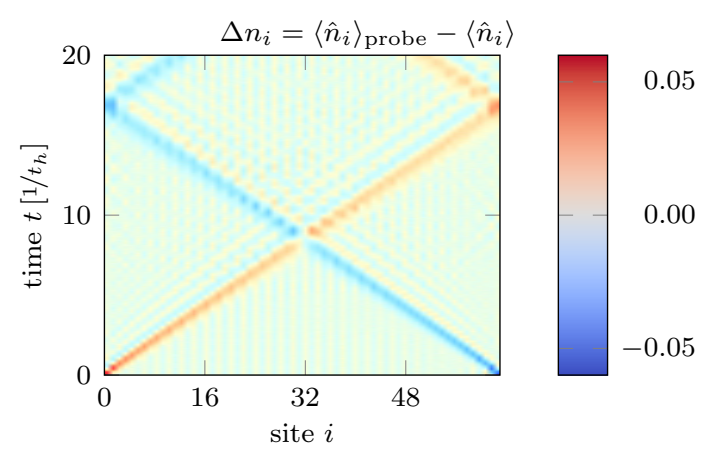

Figure 8.4: (a) shows real-time evolution of the response current $j(t-\Delta t, \Delta t)$ after application of a probe pulse at time delay $\Delta t$ following the quench (green) and in the SC ground state (orange). The charge flow after applying the probe pulse in the SC phase is shown in (b).

In Fig. 8.3 the optical conductivity after the quench within the CDW phase is shown (c.f. (Q.2)). The data is more noisy compared to the case of a quench into the SC phase, which is related to the observation that here the response current exhibits oscillations with larger amplitude and slower decay compared to a quench into the SC phase. We address this to the fact that in the insulating CDW phase there are various scattering processes between the particles. In order to properly resolve the frequecy dependencies of these processes the simulation time-range after applying the probe pulse needed to be increased way beyond what was computationally feasible. Figure 8.3a displays the real part of the optical conductivity where we find a weak transfer of spectral weight towards smaller frequencies. The imaginary part Fig. 8.3b also shows an increased spectral weight towards smaller frequencies compared to the equilibrium case. Therefore, without having a reference curve of the system being in the superconducting phase the only possible observation would be a redistribution of spectral weight towards smaller frequencies, which may also be interpreted as a sign for enhanced superconductivity. In particular $\sigma_{2}$ suggests that a $1 / \omega$ behavior can be realized after the quench if we also take into consideration that due to the finite simulation time $t=25 / t_{\text {hop }}$ after applying the probe pulse the minimally achieved frequency resolution is bounded by $2 \pi / 25 t_{\text {hop }}$. This misinterpretation of the data can be supported even 


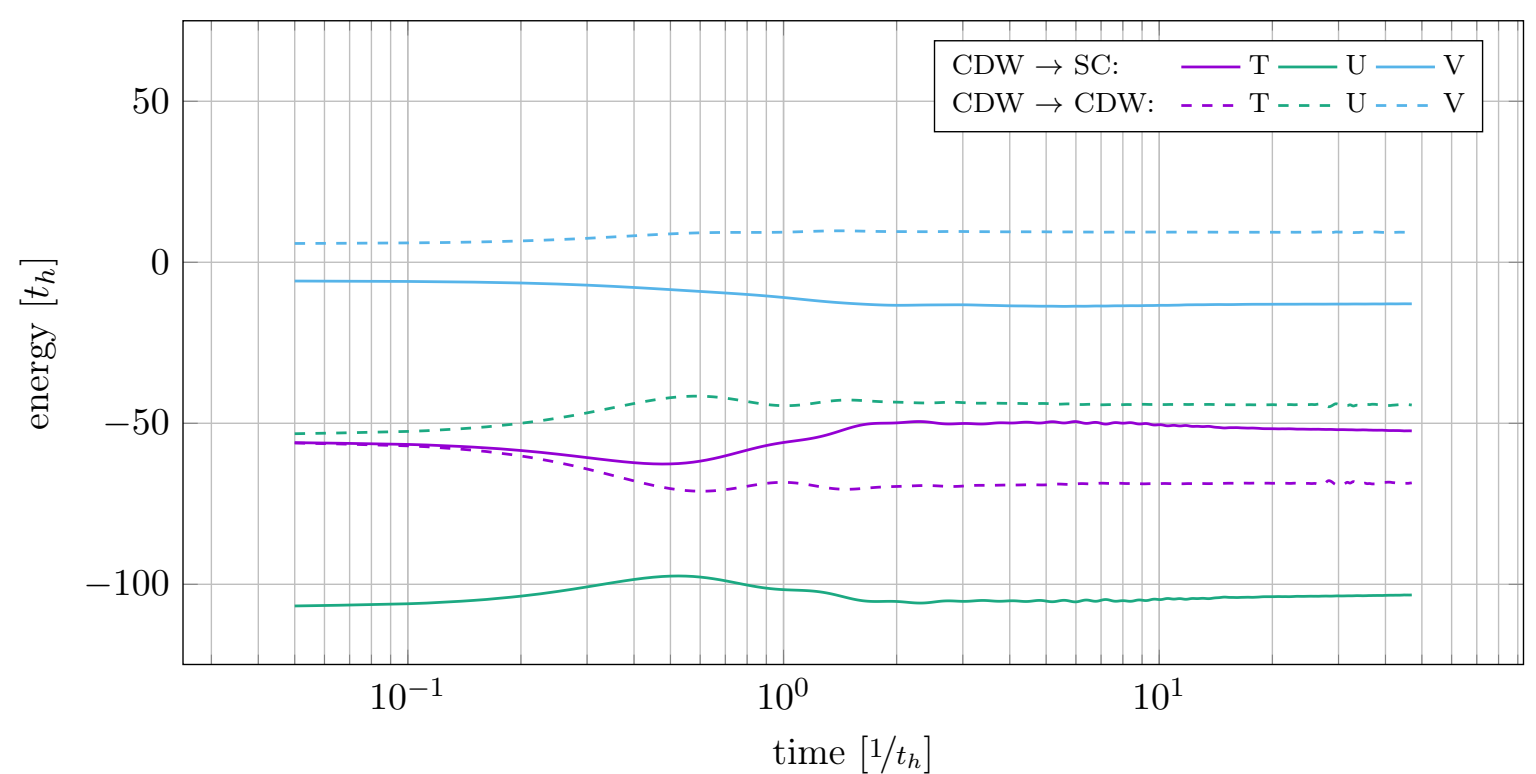

Figure 8.5: Kinetic energy $(\mathrm{T})$, on-site $(\mathrm{U})$ and nearest neighbor repulsion $(\mathrm{V})$ after quenching $V=1 / 4 \rightarrow V=-1 / 4(\mathrm{CDW} \rightarrow \mathrm{SC}$, solid lines, c.f. (Q.1)) and after quenching $U=-4.0 \rightarrow U=-2.0$ $(\mathrm{CDW} \rightarrow \mathrm{CDW}$, dashed lines, c.f. (Q.2)).

more by the supression of the CDW signal near $\omega \approx 3$ after the quench. We conclude that, as in the experiments, the question whether the accumulation of spectral weight near $\omega=0$ is due to induced SC or an enhanced metallicity after a pump pulse is hard to decide.

However, in contrast to the experimental situation we have direct access to the time dependence of the current induced by the probe pulse. The properties of this current are further shown in Fig. 8.4b, where we display the response electron density $\left\langle\hat{n}_{i}(t)\right\rangle_{\text {probe }}-\left\langle\hat{n}_{i}(t)\right\rangle_{0}$, which compares the time evolution of the local density in the SC phase with and without probe pulse. As can be seen, the effect of the probe pulse is to accumulate charge at the edges of the system. After passage of the probe pulse, this causes the measured current. In the SC phase the probe pulse induces a long-living DC-like current, while in non-equilibrium we find no clear evidence for a comparable response (see Fig. 8.4a). In turn, in our simulations the induced charge flow decays on time scales of at least $t \sim T / t_{h}$, which sets the scale of a low-frequency response $2 \pi / t \approx 0.25$ in the imaginary part $\sigma_{2}$. Thus, a strengthening of the response at $\omega \rightarrow 0$ alone, as observed here, does not suffice to demonstrate SC.

In the previous discussion we related our findings to an enhanced metalliticity of the system after quenching only within the CDW phase. In order to further demonstrate that in fact the postquench state exhibits more charge mobility we calculated the energy contributions of the kinetic energy, the on-site repulsion and the nearest-neighbor density-density interactions separately. As expected, in Fig. 8.5 we see that the contribution of the kinetic energy increases, while the on-site and the nearest neighbor repulsion contributions decrease for the quench within the CDW phase compared to the quench from the CDW phase into the SC phase.

\section{Spectral Functions}

From now on we consider postquench states at $\Delta t=15 / t_{\mathrm{hop}}$. This is justified, since for times $t>$ $4 / t_{h}$ a transient state is reached, as seen in the time evolution of the eigenvalues of the correlation 
matrix (see below), which we find to be non-thermal as described in Sec. 8.5. Motivated by trARPES, we consider the in- and out-of equilibrium time-dependent lesser Greens functions (see Sec. 3.2.1) for $t>\Delta t$ :

$$
C_{\hat{O}}(j, t, \Delta t)=\left\langle\psi(\Delta t)\left|\hat{O}_{j}^{\dagger}(t) \hat{O}_{L / 2}(0)\right| \psi(\Delta t)\right\rangle
$$

in the single- and two-particle channel, i.e., $\hat{O}_{j}=\hat{c}_{j, \uparrow}$ and $\hat{O}_{j}=\hat{d}_{j}=\hat{c}_{j, \uparrow} \hat{c}_{j, \downarrow}$, respectively, and we indicate the equilibrium case by setting $\Delta t \equiv 0$. We refer to the Fourier transform to momentum and frequency space

$$
S_{\hat{O}}(q, \omega, \Delta t)=\int_{-\infty}^{\infty} \frac{d t}{2 \pi} \sum_{j} e^{-\mathrm{i}\left(q r_{j}-(\omega+\mathrm{i} \eta) t\right)} C_{\hat{O}}(j, t, \Delta t),
$$

as the differential spectral function, where we have introduced a spectral broadening $\eta>$ $0\left[\mathrm{PKS}^{+} 19\right]$. Note that here we explicitly do not restrict ourselves to single-electron excitations, but also study processes that may excite double occupations, i.e., doublons. From our considerations in Sec. 3.3.2 we then expect to find signatures of a condensate in the superconducting ground state as a peak at momentum $k=0$ in the two-particle channel.

\section{Numerical evaluation}

Before we discuss the results, we briefly embark into some numerical pecularities concerning the explicit evaluation of Eq. (8.14). For brevity we write expectation values at time delay $\Delta t$ as $\langle\psi(\Delta t)|\cdots| \psi(\Delta t)\rangle \equiv\langle\cdots\rangle_{\Delta t}$ so that

$$
C_{\hat{O}}(q, t, \Delta t)=\left\langle\hat{O}_{q}^{\dagger}(t) \hat{O}_{q}(0)\right\rangle_{\Delta t}
$$

where we introduced $r_{i j}=r_{i}-r_{j}=(i-j) \cdot a$ in terms of the lattice spacing $a$. The $\hat{O}_{i}$ 's are operators obeying canonical (anti-)commutation relations $\left[\hat{O}_{i}, \hat{O}_{j}^{\dagger}\right]_{ \pm} \propto \delta_{i j}$ and so do their Fourier transformed counterparts

$$
\hat{O}_{q}=\frac{1}{\sqrt{L}} \sum_{r_{i}} e^{-\mathrm{i} q r_{i}} \hat{O}_{i}, \quad\left[\hat{O}_{q}, \hat{O}_{k}^{\dagger}\right]_{ \pm} \propto \delta_{q k}
$$

The differential spectral function is reformulated to simplify the numerical evaluation:

$$
\begin{aligned}
S_{\hat{O}}(q, \omega, \Delta t) & =\int_{0}^{\infty} \frac{d t}{2 \pi}\left[e^{\mathrm{i} \omega t} C_{\hat{O}}(q, t, \Delta t)+e^{-\mathrm{i} \omega t} C_{\hat{O}}(q,-t, \Delta t)\right] \\
& =\int_{0}^{\infty} \frac{d t}{2 \pi}\left[e^{\mathrm{i} \omega t} C_{\hat{O}}(q, t, \Delta t)+e^{-\mathrm{i} \omega t} C_{\hat{O}}^{*}(q, t, \Delta t)\right],
\end{aligned}
$$

where we assumed a (quasi-)steady state to exploit time translational invariance to shift the argument:

$$
\left\langle\hat{O}_{q}^{\dagger}(-t) \hat{O}_{q}(0)\right\rangle=\left\langle\hat{O}_{q}^{\dagger}(0) \hat{O}_{q}(t)\right\rangle=\left\langle\hat{O}_{q}^{\dagger}(t) \hat{O}_{q}(0)\right\rangle^{*} .
$$

Thus, we can evaluate the time integral by taking the real part of the Fourier transformation and restrict the integration domain to $t \geq 0$. Note that in order to discretize the Fourier transformation, we defined the limit as

$$
S_{\hat{O}}(q, \omega, \Delta t)=\lim _{T \rightarrow \infty} \frac{1}{T} \int_{-T / 2}^{T / 2} d t e^{\mathrm{i} \omega t} C_{\hat{O}}(q, t, \Delta t) .
$$


(a)

(b)
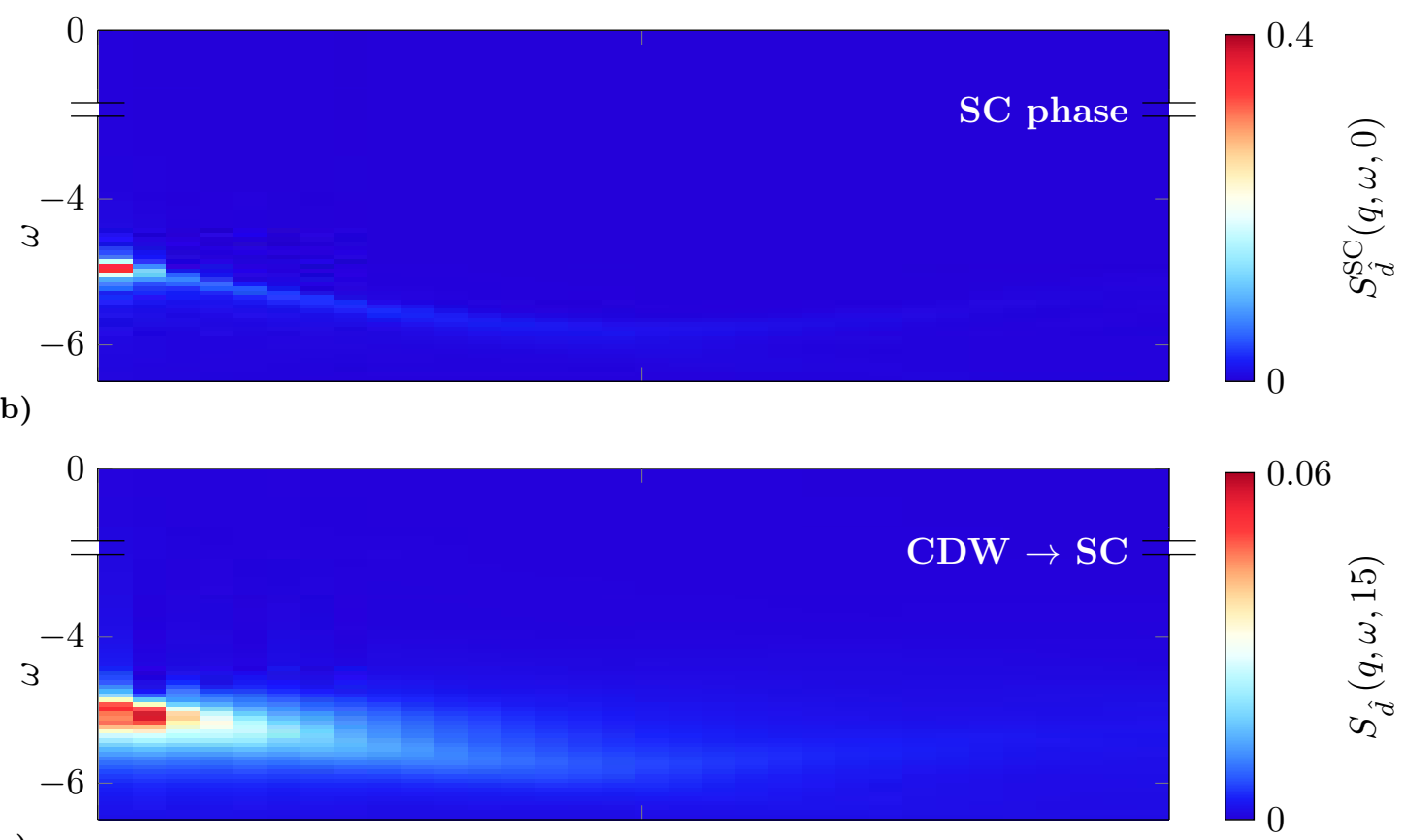

(c)

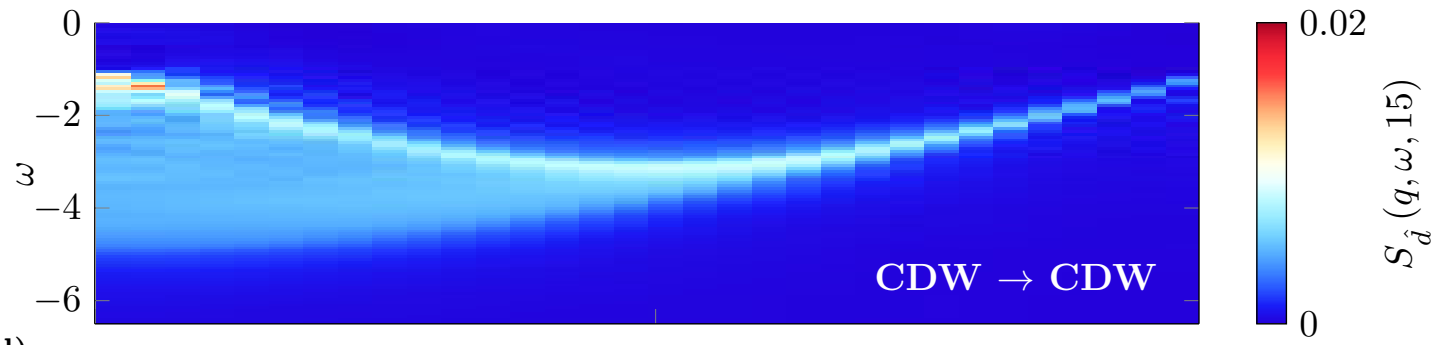

(d)

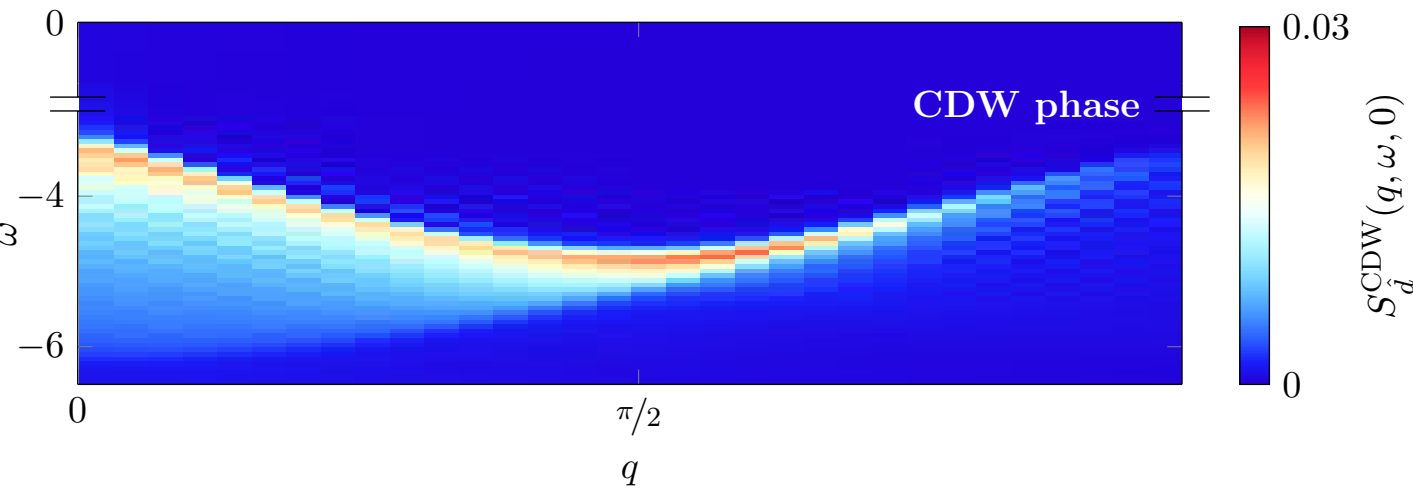

Figure 8.6: Spectral functions of two-particle excitations in equilibrium SC phase (a), CDW phase (d), in non-equilibrium after quenching from CDW ground state into SC phase (b) and in non-equilibrium after quenching in the CDW phase (c). Out-of-equilibrium spectral function are evaluated from time $t=15$ after the quench.

Using Eq. (8.17), we numerically evaluated the Fourier transformation via

$$
S_{\hat{O}}\left(q, \omega_{n}, \Delta t\right)=\frac{\delta}{T} \Re \sum_{m=0}^{N_{T}-1} e^{\mathrm{i} \omega_{n} t_{m}} C_{\hat{O}}\left(q, t_{m}, \Delta t\right),
$$


(a)

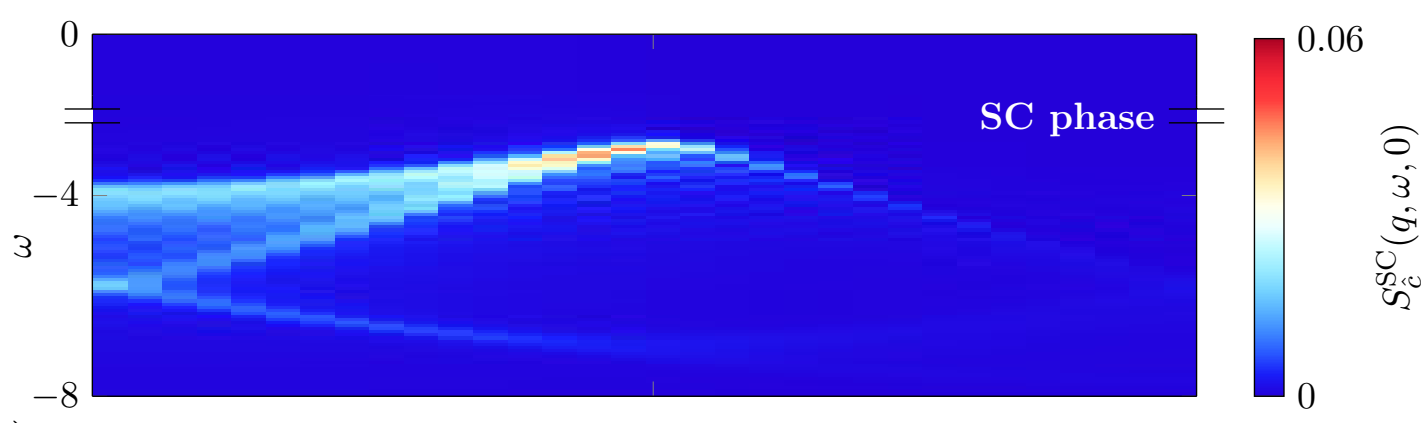

(b)

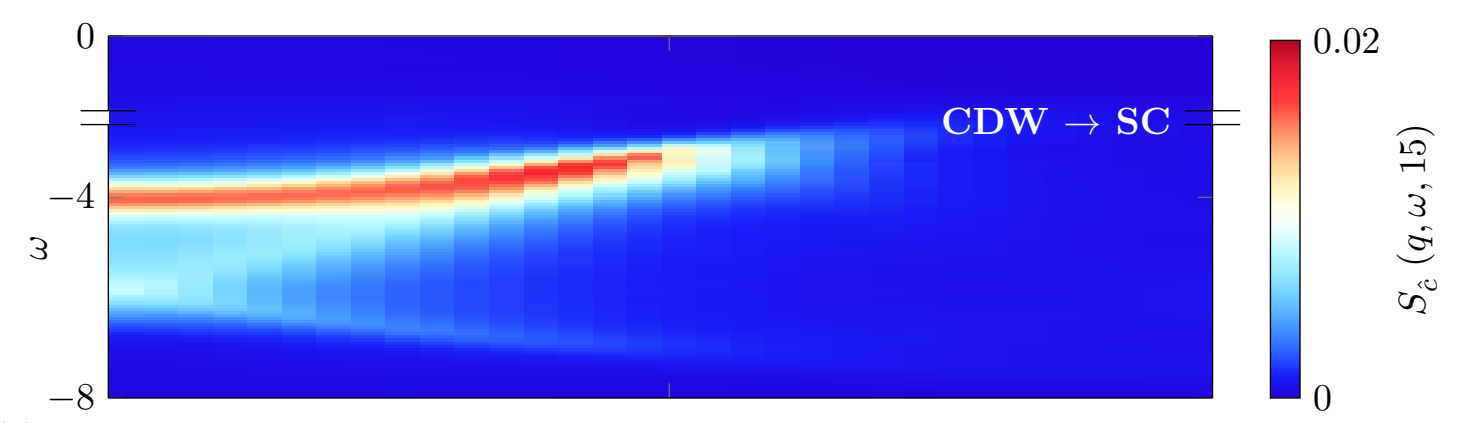

(c)

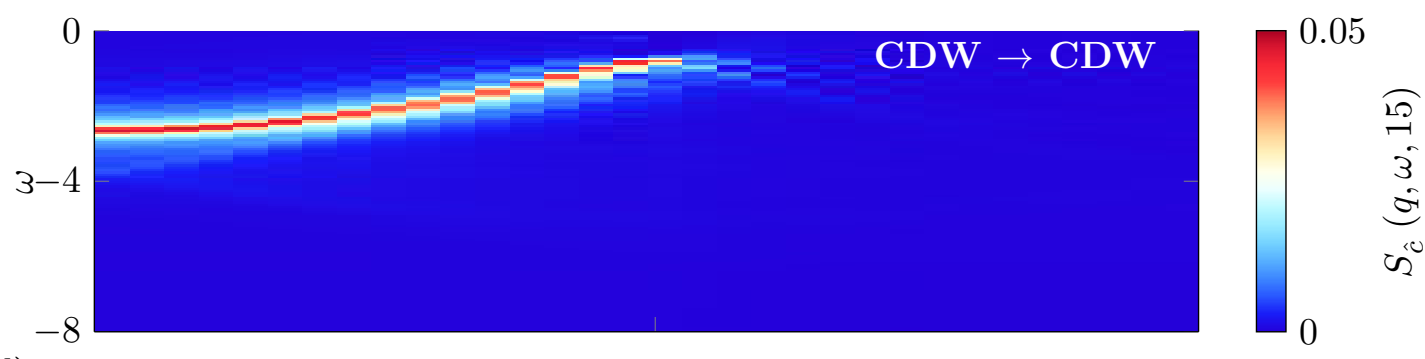

(d)

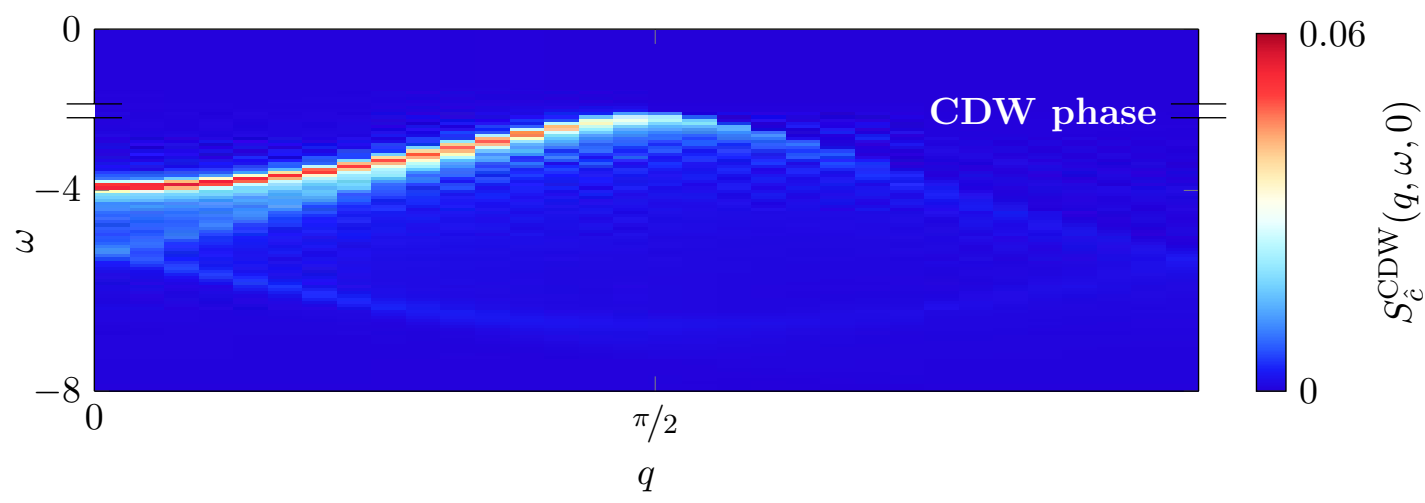

Figure 8.7: Spectral functions of single-particle excitations in equilibrium SC phase (a), CDW phase (d), in non-equilibrium after quenching from CDW ground state into SC phase (b) and in non-equilibrium after quenching in the CDW phase (c). Out-of-equilibrium spectral function are evaluated from time $t=15$ after the quench.

with discretized frequencies $\omega_{n}=n \frac{2 \pi}{T}\left(n=0, \ldots, N_{T}-1\right), t_{m}=m \delta$ and the summation range fixed by the time step $\delta=T / N_{T}$. 


\section{Results}

In contrast to the optical conductivity, in the spectral functions we find clearly distinguishable features belonging either to the elementary excitations of the SC or CDW phase. In Figs. 8.6 and 8.7 the spectral functions of two- and single-particle excitations are shown, respectively. For easier comparison, the ground states of the SC and the CDW phase, and the quenches into the SC (c.f.(Q.1)) and within the CDW phase (c.f.(Q.2)) are included in both figures. When quenching into the SC phase, in Fig. 8.6b we obtain a clear accumulation of weight at $q=0$ in the postquench state, which renders the result similar to the one of the SC ground state displayed in Fig. 8.6a. Our interpretation is that we observe the formation of a (quasi-)condensate of swave (Cooper-)pairs after the quench, which is clearly detectable in $S_{\hat{d}}(q, \omega, \Delta t)$. We emphasize that this coherence between the charges is dynamically created after the quench, as seen in the comparison with the equilibrium CDW spectral function in Fig. 8.6d, which shows dispersive, incoherent doublons. Figure 8.6c shows the two-particle spectral function when quenching only within the CDW phase. We observe a continuum of excitations at small frequencies, which is to be contrasted with the sharp excitations in the SC phase. Interestingly, we also find a weak transfer of spectral weight towards $k=0$ after the quench, which we relate to the enhanced metalliticity in the post-quench state. However, the formation of a condensate is suppressed by the continuum at smaller frequencies.

In summary, spectral weight is shifted due to the quench from the dispersive band in the CDW ground state towards $q=0$, indicating the formation of a (quasi-)condensate of bosonic quasiparticles, with a striking similarity to the spectral function in the SC ground state. We observe similar behavior in the corresponding pairing spectral function of the $t-J_{\perp}$ model $\left[\mathrm{PFO}^{+} 19\right]$, so that we expect this to be a generic feature, at least for quenches to SC phases.

Current tr-ARPES experiments usually investigate the time evolution of the spectral functions for single-electron excitations $S_{\hat{c}}(q, \omega, \Delta t)$, which we show in Fig. 8.7. The signatures to discriminate the SC phase from the CDW phase are not as prominent as for the double occupations. Nevertheless, we find that in the SC ground state (see Fig. 8.7a) there is a dominating spectral weight at $q=\pi / 2$. This is to be contrasted with the nearly equal distribution of spectral weight in the low-lying dispersive branch between $\omega \approx 1.3$ and $\omega \approx 2$ in case of the CDW ground state (see Fig. 8.7d). Comparing to the spectral function after the quench into the SC phase shown in Fig. 8.7b, a weak transfer of spectral weight from $q=0$ towards $q=\pi / 2$ can be identified. However, from the numerical data it is not possible to clearly identify this as a signal for a (transient) SC phase. On the other hand, in the single-particle spectral function shown in Fig. 8.7c after quenching within the CDW phase, the identification of the excitation spectrum with either the SC or the CDW phase is not possible, unambiguously. These findings are consistent with our suggestion that the two-particle spectral function may serve as more reliable indicator for enhanced superconductivity.

\section{\begin{tabular}{l|l} 
Convergence & 8.3 .3
\end{tabular}}

In order to check the results for convergence we performed simulations with reduced maximal bond dimension $\chi_{\max }=500$. In Figs. 8.8 and 8.9, the calculated spectral functions of two- and single-particle excitations for those bond dimensions are shown. The fidelity is very high, only small derivations around $q=0$ are visible, which we relate to an induced length scale due to the truncation errors, but are not relevant for the scope of our investigations. Those derivations are shown in more detail in Figs. 8.10a and 8.10b for modes near $q=0$. As expected the spectral 
(a)

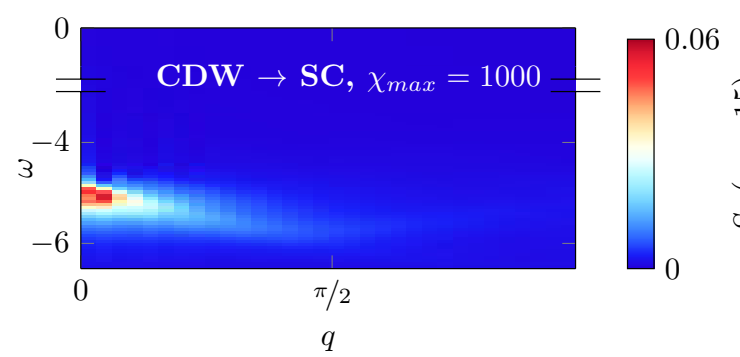

(b)

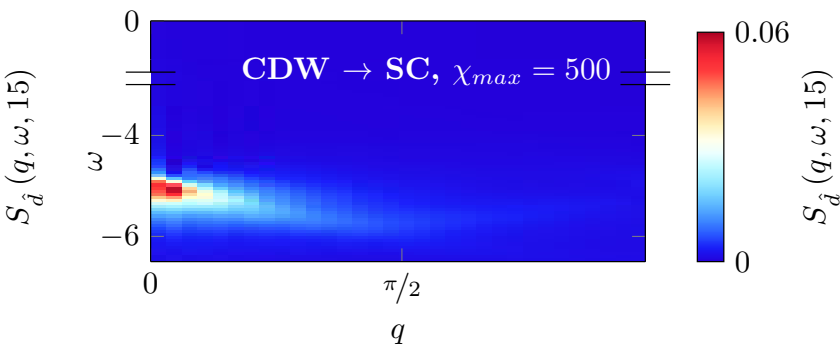

Figure 8.8: Spectral functions of two-particle excitations after quenching $V=0.25 \rightarrow V=-0.25$ with (a) $\chi_{\max }=1000$ and (b) $\chi_{\max }=500$.

(a)

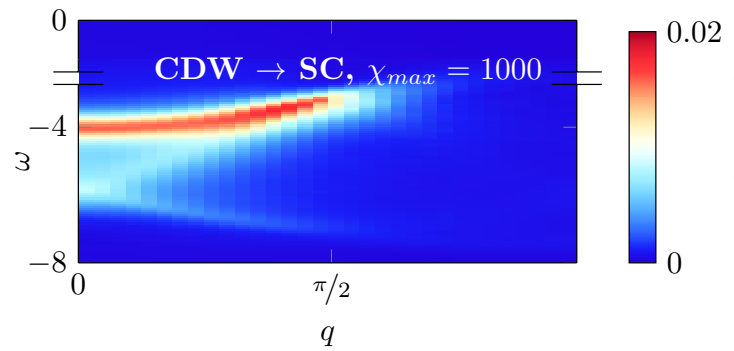

(b)

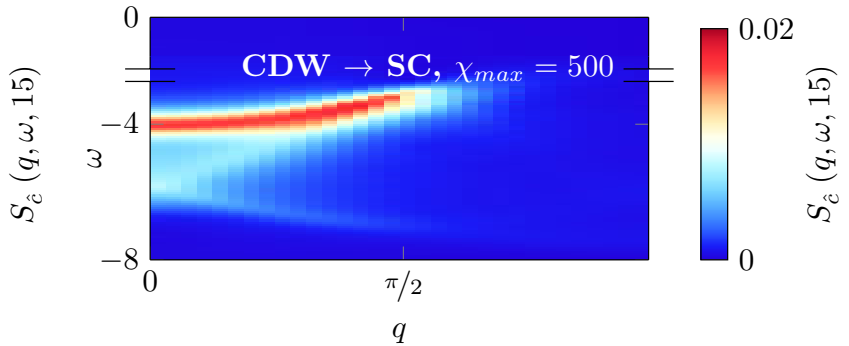

Figure 8.9: Spectral functions of single-particle excitations after quenching $V=0.25 \rightarrow V=-0.25$ with (a) $\chi_{\max }=1000$ and (b) $\chi_{\max }=500$.

(a)

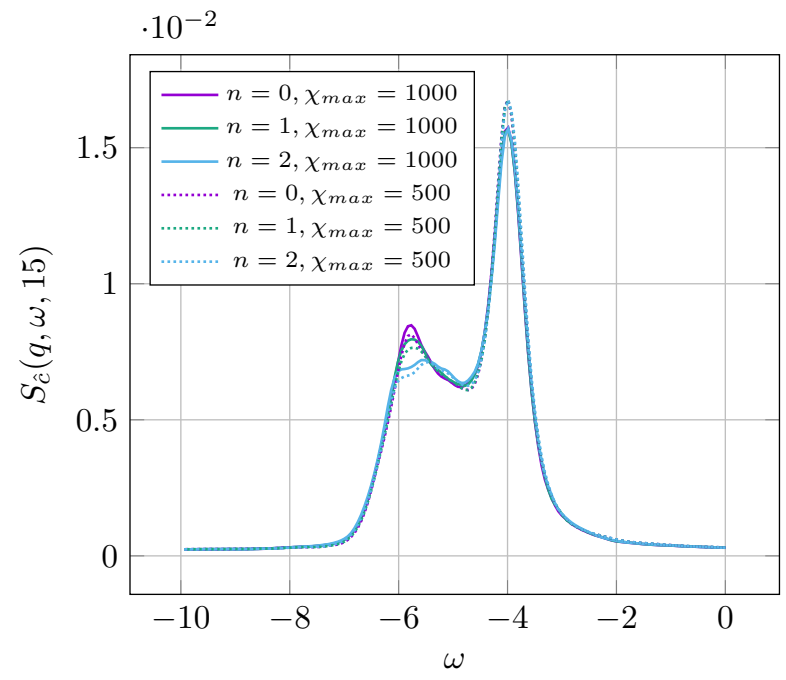

(b)

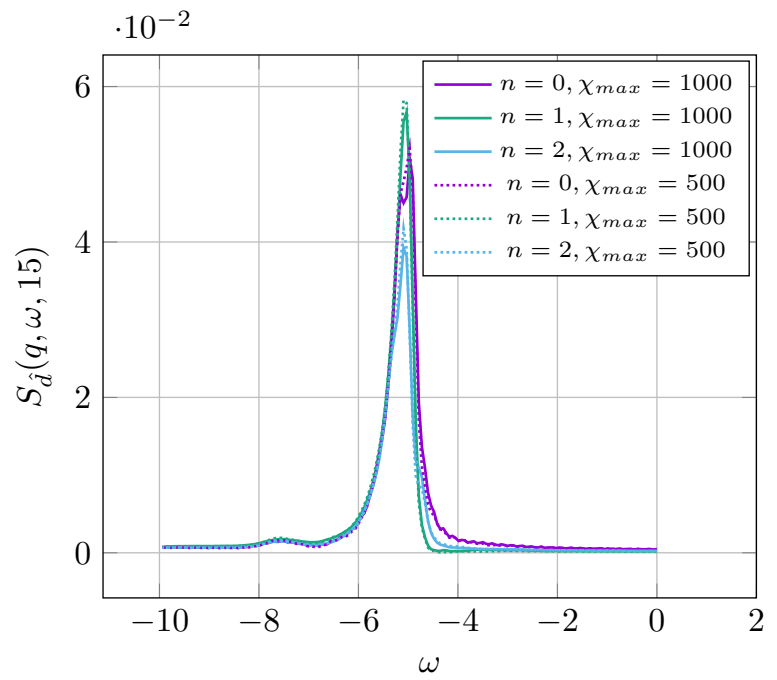

Figure 8.10: Comparison of single (a) and two-particle (b) spectral functions $S_{\hat{d}}(q, \omega, 15)$ after quenching $V=0.25 \rightarrow V=-0.25$ (c.f. (Q.1)) evaluated at fixed values of the wave vector $q_{n}=\frac{2 \pi}{L-1} n$ and for maximal bond dimensions $\chi_{\max }=500,1000$. 


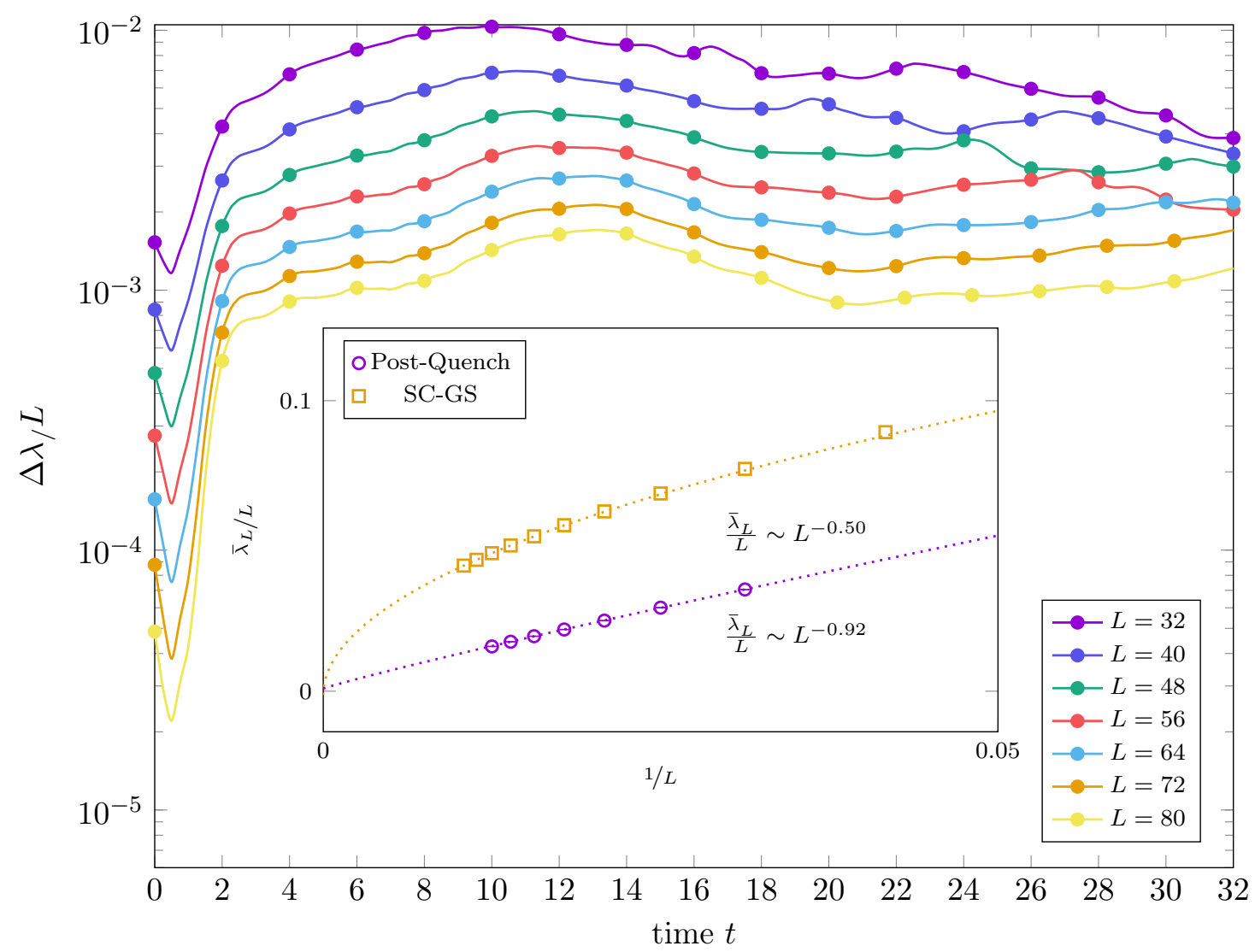

Figure 8.11: Separation of largest natural orbital occupation $\Delta \lambda / L=\frac{\lambda_{L}-\lambda_{L-1}}{L}$ during time evolution after quench for various system sizes. Inset shows extrapolation of the scaling of the dominating natural orbital with the system size.

functions obtained with higher maximal bond dimension resolve more features. The probably most interesting one can be found in the two-particle spectral functions Fig. 8.10b. Therein, an additional peak structure appears suggesting the existence of two different types of condensate states. This could be due to finite-size effects, however, it would be interesting to study them in future work.

\section{\begin{tabular}{l|l} 
Correlation matrices & 8.4
\end{tabular}}

Now we turn to the question whether there is true LRO forming after the quench. For this purpose we employ the framework developed in Sec. 3.1.2. There, the correlation matrix determines the order parameter if the dominating eigenvalue scales extensively in the system size

$$
\lim _{L \rightarrow \infty} \frac{\lambda_{L}}{L} \neq 0
$$

which also implies an extensive separation of the dominating eigenvalue $\lambda_{L}$ from the bulk. Due to the Mermin-Wagner-Hohenberg theorem [MW66a, MW66b, Hoh67], in 1D and in equilibrium, only qLRO can be realized (see also Sec. 3.1.1), which translates to a SC order parameter vanishing in the thermodynamic limit. In order to test this in a non-equilibrium setup, we 
studied the time evolution of the correlation matrix

$$
\chi_{\hat{d}}(i, j)=\left\langle\psi(t)\left|\hat{d}_{i}^{\dagger} \hat{d}_{j}\right| \psi(t)\right\rangle
$$

where the Fourier transform of the ground state expectation value $\left\langle\hat{d}_{j}\right\rangle$ provides the SC order parameter, as discussed in Sec. 3.3.2. Figure 8.11 shows the difference between the two largest eigenvalues $\Delta \lambda / L=\frac{\lambda_{L}-\lambda_{L-1}}{L}$ as a function of time. At later times we find this separation to be about an order of magnitude larger than in the initial state. We have estimated the saturation values of the dominating eigenvalue by averaging over a window of the accessible time scales

$$
\bar{\lambda}_{L}=\frac{1}{t_{1}-t_{0}} \sum_{t=t_{0}}^{t_{1}} \lambda_{L}(t)
$$

with $t_{0}=10, t_{1}=32$. We assumed that in this time-window a quasi-stationary state is realized. In order to perform a finite-size scaling of the dominating natural orbital occupation we proceeded by extracting the algebraic exponents from fitting the dependency

$$
\frac{\bar{\lambda}_{L}}{L} \propto L^{-\gamma} .
$$

Using the exponent $\gamma$ we then estimated the saturation values $\bar{\lambda}_{L} / L$ towards the thermodynamic limit performing another fit with the ansatz

$$
\frac{\bar{\lambda}_{L}}{L}=\alpha L^{-\gamma}+\beta_{\infty},
$$

where $\beta_{\infty}$ is the desired saturation when $L \rightarrow \infty$. In the inset of Fig. 8.11, we compare the scaling behaviors. In the SC ground state we found the coefficients

$$
\gamma_{e q}=0.50, \quad \beta_{\infty, e q}=-1.3 \times 10^{-3} \approx 0 .
$$

The exponent $\gamma_{e q} \neq 1$ indicates quasi-long range order and thus the saturation $\beta_{\infty, e q}$ should vanish. Since the extracted value of $\beta_{\infty, e q}$ is negative we can take the absolute value as an error bar resulting from the extrapolation procedure. After the quench, the scaling is best described by fitting the asymptotic behavior with the coefficients

$$
\gamma_{n e q}=0.92, \quad \beta_{\infty, n e q}=9 \times 10^{-4} .
$$

In this case, the magnitude of the extrapolated saturation value is even smaller than the one obtained in equilibrium, and hence zero within the error bars of our scaling analysis. This scaling to zero indicates that no true LRO is obtained on the time scales investigated but cannot be excluded for later times [LM17, LM18]. However, the modified exponent is interesting, as it closely resembles the expected value of $\gamma_{\infty}=1$ for a state with true long-range order. It is not clear at the moment how this can be related to the vanishing order parameter and further theoretical anlysis is required.

\section{Comparison to finite temperature states $\quad 8.5$}

All our simulations were performed on pure states that are either ground states of the models under consideration or quenches from the latter. The question arises whether, following the 


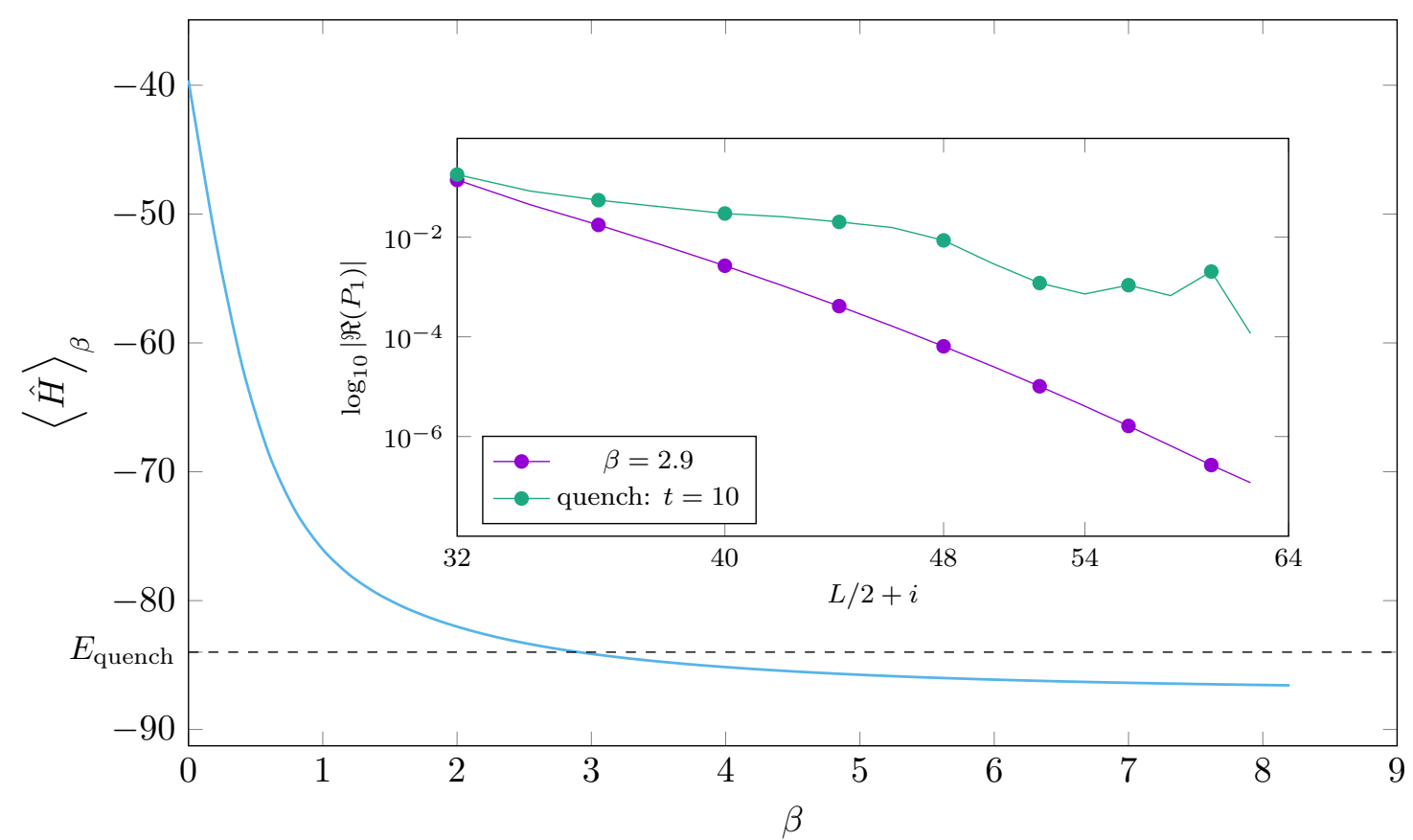

Figure 8.12: $\quad$ Energy $\langle\hat{H}\rangle_{\beta}$ during imaginary time evolution of a system with $L=32$ sites over imaginary time $\beta$. The inset shows the superconducting correlation function $P_{1}(L / 2)$ during the time evolution after a global quench from the CDW to the SC phase at time step $t=10$ (green), and in a thermal state with $\langle\hat{H}\rangle_{\beta}$ corresponding to the energy of the quenched system $E_{\text {quench }}$ (purple).

quench, the system reaches a state, in which local observables are thermalized towards their values in the canonical ensemble. To address this issue, we calculated the density matrix $\hat{\rho}(\beta)$ in the SC phase where we choose the inverse temperature $\beta \equiv 1 / T$ in a way that $\langle\hat{H}\rangle_{\beta_{Q}} \equiv\langle H\rangle_{0}$. Here, $\langle\cdots\rangle_{0}$ denotes the expectation value of $\hat{H}$ after the quench and $\langle\cdots\rangle_{\beta}$ is the expectation value of $\hat{H}$ with respect to a thermal state $\hat{\rho}(\beta)$ in the superconducting phase. In order to obtain the correct inverse temperature, we performed an imaginary time evolution on an infinitetemperature state $\hat{\rho}(0)$ and cooled down the system this way until the energy matched the one of the quenched state $\left[\mathrm{PKS}^{+} 19\right]$. In Fig. 8.12, the obtained relation between energy and inverse temperature is displayed with the energy of the quenched state marked as dashed line. Having found the respective density matrix $\hat{\rho}\left(\beta_{Q}\right)$, we exemplarily plot the expectation value of the superconducting correlation function

$$
\hat{P}_{1}(i, j)=\hat{c}_{i, \uparrow}^{\dagger} \hat{c}_{i, \downarrow}^{\dagger} \hat{c}_{j, \uparrow} \hat{c}_{j, \downarrow}
$$

in both the thermal state and the quenched state at $t=10$ in the inset of Fig. 8.12. As can be seen, we do not obtain a state that is characterized by a thermal density matrix with inverse temperature $\beta_{Q}$ chosen in a way such that its energy matches the one after the quench.

\section{Summary and Outlook $\mid 8.6$}

We studied the time evolution of the optical conductivity, pair- and single-particle spectral functions, and correlation matrices after a quantum quench in the extended Hubbard model. 
Our goal was to shed light onto the possible emergence of transient SC states in non-equilibrium setups and its experimental signatures. Our results for the differential optical conductivity in the extended Hubbard model after a quench indicate that the enhancement of $\sigma_{2}(\omega, \Delta t)$ in the low-frequency regime does not suffice to uniquely identify SC: The currents induced by a probe pulse cannot unambiguously be identified as supercurrents. In contrast, the pairing spectral function shows a clear accumulation of weights at $q \rightarrow 0$, which is absent in our initial state, but present in the SC ground state. This provides stronger evidence for the formation of a SC state than the reflectivity measurements.

However, the scaling to zero of the largest eigenvalue of the pairing correlation matrix shows that this transient state does not carry SC qLRO. Nevertheless, the algebraic exponent changed upon quenching the system into the SC phase from $\gamma_{e q}=0.50$ to $\gamma_{n e q}=0.92 \approx 1$ with the latter being the necessary scaling to allow for a finite order parameter in thermodynamic limit. This behavior poses several questions. First of all it has to be cleared out, if the non-equilibrium exponent is truly smaller than one or if this is an artefact arising from the fitting procedure and/or the finitesize scaling. Calculations including even larger system sizes could help to reduce the error bars in the fitting procedure and thereby may clear-out the latter point. Assuming an exponent $\gamma_{n e q}=1$ the question arises, if the extrapolated condensate fraction $\beta_{\infty, n e q}$ is just within the error bars. Again, this question can be answered most easily by increasing the system sizes. However, it has to be taken into account that both approaches may require larger bond dimensions so that the computational costs may increase very quickly for only a few more data points.

In summary, our study indicates that reflectivity measurements need to be complemented by trARPES-type experiments in future investigations. Readily available tr-ARPES setups measure the single-particle spectral function, which, however, are not suitable to unambiguously identify enhanced superconducting correlations. However, according to our results the time evolution of the pairing spectral function provide a clear evidence for the formation of a transient SC state. 


\section{\begin{tabular}{l|l} 
& \\
Conclusion & 9
\end{tabular}}

In this thesis, we studied unconventional superconductivity in one-dimensional systems using state-of the art matrix-product-state methods. In order to treat these challenging problems, a $\mathrm{C}++-$ based MPS-code was implemented from scratch based on [PKM17]. This resulted in the SYMMPS-toolkit, which is publicly available [SP]. It comes with several new developments such as a generic interface to create arbitrary $U(1)$-invariant MPO representations where the construction scheme is an improvement of the existing framework of finite-states machines.

In order to study out-of equilibrium dynamics, we implemented several time-evolution schemes. During this process we formulated and implemented the time-step targeting tDMRG in terms of MPS and showed its close relationship to the recently developed TDVP algorithm for MPS by Verstraete et al. This development can improve the understanding of why time-step targeting tDMRG was so successful in describing equal-time observables out-of equilibrium but exhibits convergence problems when calculating spectral functions. All the implemented time-evolution schemes are available without restrictions due to the choice of the model. This is achieved by the development of a method to map generic symmetry-breaking lattice models to symmetry conserving ones, the projected purification. Using this framework we demonstrated at the example of the Holstein model that 2DMRG solvers are applicable to problems with large local Hilbert spaces and without a conservation of particle number. Compared to an existing code that implemented 1DMRG-LBO we showed that exploiting the convergence properties of 2DMRG can result in approximations of the ground states with orders of magnitudes higher precision by only a moderate growth of the bond dimension.

We used the SYMMPS toolkit to investigate two problems of recent research: the experimental verification of Majorana edge modes in topological superconductors and emergent superconductivity out-of equilibrium. Regarding the first problem, in Chap. 7 we studied the fully interacting microscopic model of a proximity-coupled Rashba wire in contact with normal leads in the Coulomb blockade regime. As this is the first time MPS was applied to this particular problem, we carefully benchmarked and tested our implementation by studying the isolated island first. Subsequently, we coupled the island to a normal lead and investigated the hybridization of the Majorana edge modes into the lead.

This is an important problem since in experimental transport measurements midgap states naturally appear at interfaces between superconductors and normal leads. These can give rise to conductance peaks similar to what is expected for localized Majorana edge modes, in particular since the well-defined charge degeneracies in the isolated island are washed-out when coupled to leads. In turn, in finite systems the hybridization between Majorana edge modes gives rise to characteristic oscillations of the charge degeneracies upon varying the external magnetic field. Therefore, we studied the evolution of these oscillations for a wide parameter range and various tunneling amplitudes between island and lead. The resulting strong dependence on the tunneling amplitude is surprising and needs to be understood in future work as it seems to overlay the expected envelope of the oscillations.

Additionally, we found an anisotropic shift in the location of the charge degeneracies evolving as a function of the tunneling amplitude. This is somewhat surprising as the underlying model is completely symmetric and a symmetry of observables around the Coulomb valley is expected at 
zero temperature. Finally, we closed the setup to a ring adding another tunnel contact to the island. We found an enhancement of the described features, which supports our conclusion that they are caused by the hybridization of the Majorana edge modes between the leads and the island. Within this ring geometry we are also able to study the zero-bias conductance directly by penetrating the system with a finite magnetic flux. This enables us to extract a foremost dynamic quantity by means of pure ground-state calculations. The results obtained so far support our conclusions from studying only the behavior of the charge degeneracy points but there are many open questions about the detailed structure of the conductance.

While for the first problem we could focus on ground state properties only, for the second problem (emergent superconductivity out-of equilibrium) we needed to treat the full time dependency explicitly. We did this on the example of the extended Hubbard model. This serves as a minimal model for the recently reported enhancement of superconducting correlations following an optical excitation. In the experiments the imaginary part of the optical conductivity exhibited a significant increase near frequencies $\omega=0$, which was interpreted as a precursor of the Meißner effect, i.e., a $1 / \omega$ divergence. This poses several questions, which we have addressed in the scope of this thesis. The most prominent is whether there is long-ranged order out-of equilibrium and if so, could it be induced by an optical excitation.

Clearly, in a one-dimensional setup we cannot expect to answer this question in its full complexity but we can study the evolution of typical order parameters after the excitation. We modeled the excitation by a global quench, which we believe reflects the long relaxation time scales of the excitations predominantly being phonon modes. Studying the evolution of the correlation functions corresponding to the CDW and SC phase we did not observe a significant increase. However, evaluating the natural orbital occupations after the quench the picture was completely different. There we found a significant increase in the natural orbital occupation of the superconducting order parameter, which after an initial transient behavior remains stable over the time scales reached in our simulations. This motivated us to study also the spectral functions generated by the time-dependent correlation functions of the respective order parameters. Using the gained insight into the time scales of the initially transient regime from the natural orbitals, we could evaluate the spectral functions after the system reached a (meta-)stable state after the quench. We found a drastic transformation of the obtained two-particle spectral functions w.r.t. the initial state with an accumulation of spectral weight around $q=0$ indicating the formation of a (quasi-)condensate. We also demonstrated that measuring only the optical conductivity does not unambiguously allow to discriminate enhanced superconducting correlations from an increased metalliticity of the electronic system. Summing up these observations we suggest to complement future measurements of the optical conductivity with two-particle ARPES where promising experimental realizations are developed, currently. Concerning our results we expect such experiments to give much more insight into the formation of superconducting correlations out-of equilibrium.

The problems addressed in the scope of this thesis are at the edge of today's experimental means. Proving the existence of Majorana edge states experimentally would be an important milestone towards the realization of a topological qubit. Significant progress in creating superconductorsemiconductor heterostructures with hard gaps induced by the proximity effect has been made in the past years. Now, exploring the parameter regions where to look for unambiguous signatures of Majorana edge modes in these systems is of major importance. A more profound theoretical understanding of the hybridization effects between topological superconductors and normal leads would be one direction to look at and we tried to get a first glimpse into it.

Concerning the second problem, we believe that the extremely high level of control in today's trARPES experiments already sets the stage for the development of techniques that are able 
to measure two-particle excitation spectra. As we tried to demonstrate, having access to such quantities would enable the experimentalists to study the formation and decay of quasi-particle condensates. In the context of unconventional high- $T_{\mathrm{c}}$ superconductivity this would open the doors to study the mechanisms behind the enhanced superconducting correlations measured in certain systems so far. 



\section{References in Alphabetical Order of Labels}

[A.A57] A.A.Abrikosov. The magnetic properties of superconducting alloys. Journal of Physics and Chemistry of Solids, 2(3):199-208, 1957.

[AFL83] N. Andrei, K. Furuya, and J. H. Lowenstein. Solution of the kondo problem. Rev. Mod. Phys., 55:331-402, Apr 1983.

$\left[\mathrm{AHM}^{+} 16\right]$ S. M. Albrecht, A. P. Higginbotham, M. Madsen, F. Kuemmeth, T. S. Jespersen, J. Nygård, P. Krogstrup, and C. M. Marcus. Exponential protection of zero modes in majorana islands. Nature, 531(7593):206-209, 2016.

[AKLT87] Ian Affleck, Tom Kennedy, Elliott H. Lieb, and Hal Tasaki. Rigorous results on valence-bond ground states in antiferromagnets. Phys. Rev. Lett., 59:799-802, Aug 1987.

[AKLT88] Ian Affleck, Tom Kennedy, Elliott H Lieb, and Hal Tasaki. Valence bond ground states in isotropic quantum antiferromagnets. Communications in Mathematical Physics, 115:477, Sep 1988.

[AKLV13] Itai Arad, Alexei Kitaev, Zeph Landau, and Umesh Vazirani. An area law and sub-exponential algorithm for 1d systems, 2013.

[AM03] R. E. Allen and A. R. Mondragon. No spin-statistics connection in nonrelativistic quantum mechanics, 2003.

[And87] P. W. Anderson. The resonating valence bond state in la2cuo4 and superconductivity. Science, 235(4793):1196-1198, 1987.

$\left[\mathrm{AOR}^{+} 11\right]$ Jason Alicea, Yuval Oreg, Gil Refael, Felix von Oppen, and Matthew P. A. Fisher. Non-abelian statistics and topological quantum information processing in 1d wire networks. Nature Physics, 7(5):412-417, 2011.

[AW62] B. J. Alder and T. E. Wainwright. Phase transition in elastic disks. Phys. Rev., 127:359-361, Jul 1962.

[Bax68] R. J. Baxter. Dimers on a rectangular lattice. Journal of Mathematical Physics, 9(4):650-654, 1968.

[BCS57] J. Bardeen, L. N. Cooper, and J. R. Schrieffer. Theory of superconductivity. Phys. Rev., 108:1175-1204, Dec 1957.

$\left[\mathrm{BDV}^{+} 15\right]$ C. Brockt, F. Dorfner, L. Vidmar, F. Heidrich-Meisner, and E. Jeckelmann. Matrix-product-state method with a dynamical local basis optimization for bosonic systems out of equilibrium. Phys. Rev. B, 92:241106, Dec 2015.

[Bee13] C.W.J. Beenakker. Search for majorana fermions in superconductors. Annual Review of Condensed Matter Physics, 4(1):113-136, 2013.

[Ber72] VL Berezinsky. Destruction of long-range order in one-dimensional and two-dimensional systems possessing a continuous symmetry group. ii. quantum systems. Zh. Eksp. Teor. Fiz., 61:610, 1972.

[Bit17] Nikolaj Bittner. Novel Non-Equilibrium Dynamics in Superconductors: 
Induced Superconductivity and Higgs Modes. PhD thesis, Freie Universität Berlin, 2017.

[blans] Basic Linear Algebra $\mathbf{S}$ ubprograms, BLAS, Fortran libraries providing basic matrix and vector operations.

[Blo29] Felix Bloch. Über die quantenmechanik der elektronen in kristallgittern. Zeitschrift für Physik, 52(7):555-600, Jul 1929.

[BM86] J. G. Bednorz and K. A. Müller. Possible high-t $t_{c}$ superconductivity in the ba-la-cu-o system. Zeitschrift für Physik B Condensed Matter, 64:189-193, 1986.

[BMH98] Robert J. Bursill, Ross H. McKenzie, and Chris J. Hamer. Phase diagram of the one-dimensional holstein model of spinless fermions. Phys. Rev. Lett., 80:5607-5610, Jun 1998.

[BO27] M. Born and R. Oppenheimer. Zur quantentheorie der molekeln. Annalen der Physik, 389(20):457-484, 1927.

[Bog58] N. N. Bogoljubov. On a new method in the theory of superconductivity. Il Nuovo Cimento (1955-1965), 7(6):794-805, Mar 1958.

[Bog62] N. N. Bogoliubov. Physik. Abhandl. Sowjetunion, 6(113):229, 1962.

[BSW09] Thomas Barthel, Ulrich Schollwöck, and Steven R. White. Spectral functions in one-dimensional quantum systems at finite temperature using the density matrix renormalization group. Phys. Rev. B, 79(24):245101, Jun 2009.

[BTKM19] Nikolaj Bittner, Takami Tohyama, Stefan Kaiser, and Dirk Manske. Possible light-induced superconductivity in a strongly correlated electron system. Journal of the Physical Society of Japan, 88(4):044704, 2019.

[BZ06] H. Burzlaff and H. Zimmermann. Point-group symbols, chapter 12.1, pages 818-820. American Cancer Society, 2006.

$\left[\mathrm{BZT}^{+} 17\right]$ Daniel Bauernfeind, Manuel Zingl, Robert Triebl, Markus Aichhorn, and Hans Gerd Evertz. Fork tensor-product states: Efficient multiorbital realtime dmft solver. Phys. Rev. X, 7:031013, Jul 2017.

$\left[\mathrm{CAC}^{+}\right.$09] Y. L. Chen, J. G. Analytis, J.-H. Chu, Z. K. Liu, S.-K. Mo, X. L. Qi, H. J. Zhang, D. H. Lu, X. Dai, Z. Fang, S. C. Zhang, I. R. Fisher, Z. Hussain, and Z.-X. Shen. Experimental realization of a three-dimensional topological insulator, bi2te3. Science, 325(5937):178-181, 2009.

[Car10] Lincoln Carr. Understanding quantum phase transitions. CRC press, 2010.

[Cav18] Andrea Cavalleri. Photo-induced superconductivity. Contemporary Physics, 59(1):31-46, 2018.

[CB08] Gregory M. Crosswhite and Dave Bacon. Finite automata for caching in matrix product algorithms. Phys. Rev. A, 78:012356, Jul 2008.

[CC04] Pasquale Calabrese and John Cardy. Entanglement entropy and quantum field theory. J. Stat. Mech.: Theor. and Exp., (06):P06002, 2004.

[CDV08] Gregory M. Crosswhite, A. C. Doherty, and Guifré Vidal. Applying matrix product operators to model systems with long-range interactions. Phys. Rev. B, 78:035116, Jul 2008.

[CEP07] M. Cramer, J. Eisert, and M. B. Plenio. Statistics dependence of the entanglement entropy. Phys. Rev. Lett., 98:220603, May 2007. 
[Chi18] Gennady Y. Chitov. Local and nonlocal order parameters in the kitaev chain. Physical Review B, 97(8), Feb 2018.

$\left[\mathrm{CKN}^{+} 16\right]$ Garnet Kin-Lic Chan, Anna Keselman, Naoki Nakatani, Zhendong Li, and Steven R. White. Matrix product operators, matrix product states, and ab initio density matrix renormalization group algorithms. The Journal of Chemical Physics, 145(1):014102, 2016.

[CMM97] A. Chrestin, T. Matsuyama, and U. Merkt. Evidence for a proximityinduced energy gap in nb/inas/nb junctions. Phys. Rev. B, 55:8457-8465, Apr 1997.

[Coo56] Leon N. Cooper. Bound electron pairs in a degenerate fermi gas. Phys. Rev., 104:1189-1190, Nov 1956.

[CSC05] C. E. Creffield, G. Sangiovanni, and M. Capone. Phonon softening and dispersion in the $1 \mathrm{~d}$ holstein model of spinless fermions. The European Physical Journal B - Condensed Matter and Complex Systems, 44(2):175181, Mar 2005.

[CSO77] K A Chao, J Spalek, and A M Oles. Kinetic exchange interaction in a narrow s-band. Journal of Physics C: Solid State Physics, 10(10):L271, 1977.

[CW51] Herbert B. Callen and Theodore A. Welton. Irreversibility and generalized noise. Phys. Rev., 83:34-40, Jul 1951.

[CW85] Jane K. Cullum and Ralph A. Willoughby. Lanczos algorithms for large symmetric eigenvalue computations, volume 1. Progress in Scientific Computing, 1985.

[Dag94] Elbio Dagotto. Correlated electrons in high-temperature superconductors. Rev. Mod. Phys., 66(3):763, 1994.

[Dam04] Andrea Damascelli. Probing the electronic structure of complex systems by ARPES. Physica Scripta, T109:61, 2004.

[Dav93] Ernest R. Davidson. Monster matrices: Their eigenvalues and eigenvectors. Computers in Physics, 7(5), Sep./Oct. 1993.

[DBMB $\left.{ }^{+} 17\right]$ A. Di Bernardo, O. Millo, M. Barbone, H. Alpern, Y. Kalcheim, U. Sassi, A. K. Ott, D. De Fazio, D. Yoon, M. Amado, A. C. Ferrari, J. Linder, and J. W. A. Robinson. p-wave triggered superconductivity in single-layer graphene on an electron-doped oxide superconductor. Nature Communications, 8(1):14024, 2017.

[DHS03] Andrea Damascelli, Zahid Hussain, and Zhi-Xun Shen. Angle-resolved photoemission studies of the cuprate superconductors. Rev. Mod. Phys., 75:473-541, Apr 2003.

[DKSV04] A. J. Daley, C. Kollath, U. Schollwöck, and G. Vidal. Time-dependent density-matrix renormalization-group using adaptive effective hilbert spaces. Journal of Statistical Mechanics: Theory and Experiment, 2004(04):P04005, 2004.

[DMDNS98] J. Dukelsky, M. A. Martín-Delgado, T. Nishino, and G. Sierra. Equivalence of the variational matrix product method and the density matrix renormalization group applied to spin chains. EPL (Europhysics Letters), 43(4):457, 1998.

[Don02] Jack Dongarra. Preface: Basic Linear Algebra Subprograms Technical 
(Blast) Forum Standard I. The International Journal of High Performance Computing Applications, 16(1), Spring 2002.

$\left[\mathrm{DRM}^{+} 12\right]$ Anindya Das, Yuval Ronen, Yonatan Most, Yuval Oreg, Moty Heiblum, and Hadas Shtrikman. Zero-bias peaks and splitting in an al-inas nanowire topological superconductor as a signature of majorana fermions. Nature Physics, 8(12):887-895, 2012.

$\left[\mathrm{DRN}^{+} 08\right]$ H. Ding, P. Richard, K. Nakayama, K. Sugawara, T. Arakane, Y. Sekiba, A. Takayama, S. Souma, T. Sato, T. Takahashi, Z. Wang, X. Dai, Z. Fang, G. F. Chen, J. L. Luo, and N. L. Wang. Observation of fermisurface-dependent nodeless superconducting gaps in ba0.6k0.4fe2as2. EPL (Europhysics Letters), 83(4):47001, jul 2008.

[DSSS12] S. Das Sarma, Jay D. Sau, and Tudor D. Stanescu. Splitting of the zerobias conductance peak as smoking gun evidence for the existence of the majorana mode in a superconductor-semiconductor nanowire. Physical Review B, 86(22), Dec 2012.

[DVC13] Jozef T Devreese and Piet Van Camp. Electronic Structure, Dynamics, and Quantum Structural Properties of Condensed Matter, volume 121. Springer Science \& Business Media, 2013.

$\left[\mathrm{DYH}^{+} 12\right]$ M. T. Deng, C. L. Yu, G. Y. Huang, M. Larsson, P. Caroff, and H. Q. Xu. Anomalous zero-bias conductance peak in a nb-insb nanowire-nb hybrid device. Nano Letters, 12(12):6414-6419, 2012. PMID: 23181691.

[EBN16] Dominic V. Else, Bela Bauer, and Chetan Nayak. Floquet time crystals. Phys. Rev. Lett., 117:090402, Aug 2016.

[EBN17] Dominic V. Else, Bela Bauer, and Chetan Nayak. Prethermal phases of matter protected by time-translation symmetry. Phys. Rev. X, 7:011026, Mar 2017.

[ECP10] J. Eisert, M. Cramer, and M. B. Plenio. Colloquium: Area laws for the entanglement entropy. Rev. Mod. Phys., 82(1):277-306, Feb 2010.

[EFG $\left.{ }^{+} 05\right]$ Fabian H. L. Essler, Holger Frahm, Frank Göhmann, Andreas Klümper, and Vladimir E. Korepin. The One-Dimensional Hubbard Model. Cambridge University Press, Cambridge, 2005.

[EFG15] J. Eisert, M. Friesdorf, and C. Gogolin. Quantum many-body systems out of equilibrium. Nature Physics, 11:124-, February 2015.

[EG11] Andrew M. Essin and Victor Gurarie. Bulk-boundary correspondence of topological insulators from their respective green's functions. Phys. Rev. $B, 84: 125132$, Sep 2011.

[EHK15] Mike Espig, Wolfgang Hackbusch, and Aram Khachatryan. On the convergence of alternating least squares optimisation in tensor format representations. 052015.

[Eis13] J. Eisert. Entanglement and tensor network states, 2013.

[EK08] Martin Eckstein and Marcus Kollar. Measuring correlated electron dynamics with time-resolved photoemission spectroscopy. Phys. Rev. B, 78:245113, Dec 2008.

[EKW10] Martin Eckstein, Marcus Kollar, and Philipp Werner. Interaction quench in the hubbard model: Relaxation of the spectral function and the optical conductivity. Phys. Rev. B, 81:115131, Mar 2010. 
[EN07] Satoshi Ejima and Satoshi Nishimoto. Phase diagram of the onedimensional half-filled extended hubbard model. Phys. Rev. Lett., 99:216403, Nov 2007.

[EWN17] G. Ehlers, S. R. White, and R. M. Noack. Hybrid-space density matrix renormalization group study of the doped two-dimensional hubbard model. Physical Review B, 95(12), Mar 2017.

$\left[\mathrm{FFK}^{+} 14\right]$ M. Först, A. Frano, S. Kaiser, R. Mankowsky, C. R. Hunt, J. J. Turner, G. L. Dakovski, M. P. Minitti, J. Robinson, T. Loew, M. Le Tacon, B. Keimer, J. P. Hill, A. Cavalleri, and S. S. Dhesi. Femtosecond x rays link melting of charge-density wave correlations and light-enhanced coherent transport in $\mathrm{YBa}_{2} \mathrm{Cu}_{3} \mathrm{O}_{6.6}$. Phys. Rev. B, 90:184514, Nov 2014.

[FK08] Liang Fu and C. L. Kane. Superconducting proximity effect and majorana fermions at the surface of a topological insulator. Physical Review Letters, 100(9), Mar 2008.

[FKP09] J. K. Freericks, H. R. Krishnamurthy, and Th. Pruschke. Theoretical description of time-resolved photoemission spectroscopy: Application to pump-probe experiments. Phys. Rev. Lett., 102:136401, Mar 2009.

$\left[\mathrm{FMK}^{+} 11\right]$ M. Först, C. Manzoni, S. Kaiser, Y. Tomioka, Y. Tokura, R. Merlin, and A. Cavalleri. Nonlinear phononics as an ultrafast route to lattice control. Nature Physics, 7:854-, August 2011.

$\left[\mathrm{FNP}^{+}{ }^{14}\right]$ Mark H Fischer, Titus Neupert, Christian Platt, Andreas P Schnyder, Werner Hanke, Jun Goryo, Ronny Thomale, and Manfred Sigrist. Chiral d-wave superconductivity in srptas. Physical Review B, 89(2):020509, 2014.

[FNW92] M. Fannes, B. Nachtergaele, and R. Werner. Finitely correlated states on quantum spin chains. Communications in Mathematical Physics, 144:443490, 1992.

$\left[\right.$ FST $^{+}$17] B. Fauseweh, L. Schwarz, N. Tsuji, N. Cheng, N. Bittner, H. Krull, M. Berciu, G. S. Uhrig, A. P. Schnyder, S. Kaiser, and D. Manske. Higgs spectroscopy of superconductors in nonequilibrium. arXiv e-prints, page arXiv:1712.07989, Dec 2017.

$\left[\mathrm{FTD}^{+} 11\right]$ D. Fausti, R. I. Tobey, N. Dean, S. Kaiser, A. Dienst, M. C. Hoffmann, S. Pyon, T. Takayama, H. Takagi, and A. Cavalleri. Light-induced superconductivity in a stripe-ordered cuprate. Science, 331(6014):189-191, 2011.

[Fu10] Liang Fu. Electron teleportation via majorana bound states in a mesoscopic superconductor. Physical Review Letters, 104(5), Feb 2010.

[FW05] Adrian E. Feiguin and Steven R. White. Time-step targeting methods for real-time dynamics using the density matrix renormalization group. Phys. Rev. B, 72(2):020404(R), 2005.

[GD19] Shimpei Goto and Ippei Danshita. Performance of the time-dependent variational principle for matrix product states in the long-time evolution of a pure state. Phys. Rev. B, 99:054307, Feb 2019.

[GKKR96] Antoine Georges, Gabriel Kotliar, Werner Krauth, and Marcelo J. Rozenberg. Dynamical mean-field theory of strongly correlated fermion systems and the limit of infinite dimensions. Rev. Mod. Phys., 68(1):13, 1996.

[GL50] V. L. Ginzburg and L. D. Landau. J. Exptl. Theoret. Physo (U.S.S.R.), 
20(1064), 1950.

$\left[\mathrm{GMC}^{+} 11\right]$ Alexey V. Gorshkov, Salvatore R. Manmana, Gang Chen, Jun Ye, Eugene Demler, Mikhail D. Lukin, and Ana Maria Rey. Tunable superfluidity and quantum magnetism with ultracold polar molecules. Phys. Rev. Lett., 107:115301, Sep 2011.

[GME $\left.{ }^{+} 02\right]$ Markus Greiner, Olaf Mandel, T. Esslinger, Theodor W. Hänsch, and Immanuel Bloch. Quantum phase transition from a superfluid to a mott insulator in a gas of ultracold atoms. Nature, 415:39-44, 2002.

[Gor59] Lev Petrovich Gor'kov. Microscopic derivation of the ginzburg-landau equations in the theory of superconductivity. Sov. Phys. JETP, 9(6):1364$1367,1959$.

[GR06] Juan José García-Ripoll. Time evolution of matrix product states. New Journal of Physics, 8(12):305, 2006.

[Gri64] Robert B. Griffiths. Peierls proof of spontaneous magnetization in a twodimensional ising ferromagnet. Phys. Rev., 136:A437-A439, Oct 1964.

[Gri04] David J. Griffiths. Introduction to Quantum Mechanics (2nd Edition). Pearson Prentice Hall, 2nd edition, April 2004.

[Gut63] Martin C. Gutzwiller. Effect of correlation on the ferromagnetism of transition metals. Phys. Rev. Lett., 10:159-162, Mar 1963.

[GWvDV12] Cheng Guo, Andreas Weichselbaum, Jan von Delft, and Matthias Vojta. Critical and strong-coupling phases in one- and two-bath spin-boson models. Phys. Rev. Lett., 108:160401, Apr 2012.

[Hal18] Bertrand I. Halperin. On the hohenberg-mermin-wagner theorem and its limitations. Journal of Statistical Physics, 175(3-4):521-529, Dec 2018.

[Has07a] M B Hastings. An area law for one-dimensional quantum systems. Journal of Statistical Mechanics: Theory and Experiment, 2007(08):P08024, 2007.

[Has07b] M. B. Hastings. Entropy and entanglement in quantum ground states. Phys. Rev. B, 76:035114, Jul 2007.

$\left[\mathrm{HCO}^{+} 11\right]$ Jutho Haegeman, J. Ignacio Cirac, Tobias J. Osborne, Iztok Pižorn, Henri Verschelde, and Frank Verstraete. Time-dependent variational principle for quantum lattices. Phys. Rev. Lett., 107:070601, Aug 2011.

[HGMS93] F. W. J. Hekking, L. I. Glazman, K. A. Matveev, and R. I. Shekhter. Coulomb blockade of two-electron tunneling. Phys. Rev. Lett., 70:41384141, Jun 1993.

[HHS18] C. Hubig, J. Haegeman, and U. Schollwöck. Error estimates for extrapolations with matrix-product states. Phys. Rev. B, 97:045125, Jan 2018.

[HHZ05] Peter Heinzner, Alan Huckleberry, and M.R. Zirnbauer. Symmetry classes of disordered fermions. Communications in Mathematical Physics, 257:725-771, 082005.

[HK64] Pierre Hohenberg and Walter Kohn. Inhomogeneous electron gas. Phys. Rev., 136:B864, 1964.

$\left[\mathrm{HKN}^{+} 14\right]$ W. Hu, S. Kaiser, D. Nicoletti, C. R. Hunt, I. Gierz, M. C. Hoffmann, M. Le Tacon, T. Loew, B. Keimer, and A. Cavalleri. Optically enhanced coherent transport in yba2cu3o6.5 by ultrafast redistribution of interlayer coupling. Nature Materials, 13(7):705-711, Jul 2014.

[HL97] Marlis. Hochbruck and Christian. Lubich. On krylov subspace approxi- 
mations to the matrix exponential operator. SIAM Journal on Numerical Analysis, 34(5):1911-1925, 1997.

$\left[\mathrm{HLO}^{+} 16\right]$ Jutho Haegeman, Christian Lubich, Ivan Oseledets, Bart Vandereycken, and Frank Verstraete. Unifying time evolution and optimization with matrix product states. Phys. Rev. B, 94:165116, Oct 2016.

[HMOV14] Jutho Haegeman, Michaël Mariën, Tobias J. Osborne, and Frank Verstraete. Geometry of matrix product states: Metric, parallel transport, and curvature. Journal of Mathematical Physics, 55(2):021902, 2014.

[HMS17] C. Hubig, I. P. McCulloch, and U. Schollwöck. Generic construction of efficient matrix product operators. Phys. Rev. B, 95:035129, Jan 2017.

[HMSW15] C. Hubig, I. P. McCulloch, U. Schollwöck, and F. A. Wolf. Strictly singlesite dmrg algorithm with subspace expansion. Phys. Rev. B, 91:155115, Apr 2015.

$\left[\mathrm{HNK}^{+} 16\right]$ C. R. Hunt, D. Nicoletti, S. Kaiser, D. Pröpper, T. Loew, J. Porras, B. Keimer, and A. Cavalleri. Dynamical decoherence of the light induced interlayer coupling in $\mathrm{YBa}_{2} \mathrm{Cu}_{3} \mathrm{O}_{6+\delta}$. Phys. Rev. B, 94:224303, Dec 2016.

[HOC19] R. Haghshenas, Matthew J. O'Rourke, and Garnet Kin-Lic Chan. Canonicalization of projected entangled pair states. 2019.

[Hof76] Douglas R. Hofstadter. Energy levels and wave functions of bloch electrons in rational and irrational magnetic fields. Phys. Rev. B, 14:2239-2249, Sep 1976.

[Hoh67] P. C. Hohenberg. Existence of long-range order in one and two dimensions. Phys. Rev., 158:383-386, Jun 1967.

[HOV13] Jutho Haegeman, Tobias J. Osborne, and Frank Verstraete. Post-matrix product state methods: To tangent space and beyond. Phys. Rev. B, 88:075133, Aug 2013.

[HP12] Felix Hofmann and Michael Potthoff. Doublon dynamics in the extended fermi-hubbard model. Phys. Rev. B, 85:205127, May 2012.

[HPAG05] M. Houzet, D. A. Pesin, A. V. Andreev, and L. I. Glazman. Quantum charge fluctuations in a superconducting grain. Phys. Rev. B, 72:104507, Sep 2005.

[HRS12] Sebastian Holtz, Thorsten Rohwedder, and Reinhold Schneider. On manifolds of tensors of fixed tt-rank. Numerische Mathematik, 120(4):701-731, Apr 2012.

[Hub63] J. Hubbard. Electron correlations in narrow energy bands. Proceedings of the Royal Society of London A: Mathematical, Physical and Engineering Sciences, 276(1365):238-257, 1963.

[Hub17] Claudius Hubig. Symmetry-protected tensor networks. PhD thesis, Ludwig-Maximilians-Universität München, Oktober 2017.

$\left[\mathrm{HWC}^{+} 10\right]$ Y. S. Hor, A. J. Williams, J. G. Checkelsky, P. Roushan, J. Seo, Q. Xu, H. W. Zandbergen, A. Yazdani, N. P. Ong, and R. J. Cava. Superconductivity in $\mathrm{cu}_{x} \mathrm{bi}_{2} \mathrm{Se}_{3}$ and its implications for pairing in the undoped topological insulator. Phys. Rev. Lett., 104:057001, Feb 2010.

[Jec02] Eric Jeckelmann. Ground-state phase diagram of a half-filled onedimensional extended hubbard model. Phys. Rev. Lett., 89:236401, Nov 2002. 
[JHA99] Martin Jourdan, Michael Huth, and Hermann Adrian. Superconductivity mediated by spin fluctuations in the heavy-fermion compound upd 2 al 3. Nature, 398(6722):47, 1999.

[Kad66] Leo P. Kadanoff. Scaling laws for ising models near $T_{c}$. Physics Physique Fizika, 2:263-272, Jun 1966.

[Kan63] Junjiro Kanamori. Electron correlation and ferromagnetism of transition metals. Progress of Theoretical Physics, 30(3):275-289, 1963.

[KDTR15] Sebastian Keller, Michele Dolfi, Matthias Troyer, and Markus Reiher. An efficient matrix product operator representation of the quantum chemical hamiltonian. The Journal of Chemical Physics, 143(24):244118, 2015.

$\left[\mathrm{KHN}^{+} 14\right]$ S. Kaiser, C. R. Hunt, D. Nicoletti, W. Hu, I. Gierz, H. Y. Liu, M. Le Tacon, T. Loew, D. Haug, B. Keimer, and A. Cavalleri. Optically induced coherent transport far above $T_{c}$ in underdoped $\mathrm{yba}_{2} \mathrm{Cu}_{3} \mathrm{O}_{6+\delta}$. Phys. Rev. B, 89:184516, May 2014.

[Kit01] A Yu Kitaev. Unpaired majorana fermions in quantum wires. PhysicsUspekhi, 44(10S):131-136, oct 2001.

[Kit03] A.Yu. Kitaev. Fault-tolerant quantum computation by anyons. Annals of Physics, 303(1):2-30, Jan 2003.

[Kit09] Alexei Kitaev. Periodic table for topological insulators and superconductors. AIP Conference Proceedings, 1134(1):22-30, 2009.

[KLMS16] Vedika Khemani, Achilleas Lazarides, Roderich Moessner, and S. L. Sondhi. Phase structure of driven quantum systems. Phys. Rev. Lett., 116:250401, Jun 2016.

[KLR18] Benedikt Kloss, Yevgeny Bar Lev, and David Reichman. Time-dependent variational principle in matrix-product state manifolds: Pitfalls and potential. Phys. Rev. B, 97:024307, Jan 2018.

[KM05] C. L. Kane and E. J. Mele. $Z_{2}$ topological order and the quantum spin hall effect. Phys. Rev. Lett., 95:146802, Sep 2005.

[KMB12] G. Kells, D. Meidan, and P. W. Brouwer. Near-zero-energy end states in topologically trivial spin-orbit coupled superconducting nanowires with a smooth confinement. Phys. Rev. B, 86:100503, Sep 2012.

[KMvHB19] Anna Keselman, Chaitanya Murthy, Bernard van Heck, and Bela Bauer. Spectral response of josephson junctions with low-energy quasiparticles. SciPost Physics, 7(4), Oct 2019.

[Köh19] Thomas Köhler. Photoexcitations of Model Manganite Systems using Matrix-Product States. PhD thesis, Universität Göttingen, 2019.

[Kon64] Jun Kondo. Resistance Minimum in Dilute Magnetic Alloys. Progress of Theoretical Physics, 32(1):37-49, 071964.

[KPM18] Thomas Köhler, Sebastian Paeckel, and Salvatore R. Manmana. Charge density patterns in spin-selectively photoexcited interacting fermions. arXiv e-prints, page arXiv:1808.02138, Aug 2018.

[KRS $\left.{ }^{+} 18\right]$ Thomas Köhler, Sangeeta Rajpurohit, Ole Schumann, Sebastian Paeckel, Fabian R. A. Biebl, Mohsen Sotoudeh, Stephan C. Kramer, Peter E. Blöchl, Stefan Kehrein, and Salvatore R. Manmana. Relaxation of photoexcitations in polaron-induced magnetic microstructures. Physical Review B, 97:235120, Jun 2018. 
[KS65] Walter Kohn and Lu J. Sham. Self-consistent equations including exchange and correlation effects. Phys. Rev., 140:A1133, 1965.

[KSY03] H.-J. Kwon, K. Sengupta, and V. M. Yakovenko. Fractional ac josephson effect in p- and d-wave superconductors. The European Physical Journal B - Condensed Matter, 37(3):349-361, Feb 2003.

[KT73] John Michael Kosterlitz and David James Thouless. Ordering, metastability and phase transitions in two-dimensional systems. Journal of Physics C: Solid State Physics, 6(7):1181, 1973.

[Kub66] R Kubo. The fluctuation-dissipation theorem. Reports on Progress in Physics, 29(1):255-284, jan 1966.

[KVKS17] Vedika Khemani, CW Von Keyserlingk, and Shivaji Lal Sondhi. Defining time crystals via representation theory. Physical Review B, 96(11):115127, 2017.

[KW41] H. A. Kramers and G. H. Wannier. Statistics of the two-dimensional ferromagnet. part ii. Phys. Rev., 60:263-276, Aug 1941.

[KWRM17] D. M. Kennes, E. Y. Wilner, D. R. Reichman, and A. J. Millis. Nonequilibrium optical conductivity: General theory and application to transient phases. Phys. Rev. B, 96:054506, Aug 2017.

[L50] C. Lánczos. An iterative method for the solution of the eigenvalue problem of linear differential and integral. J. Res. Natl. Bur. Stand, 45:225, 1950.

$\left[\mathrm{LAGcv}^{+}\right.$18] M. Ligges, I. Avigo, D. Golež, H. U. R. Strand, Y. Beyazit, K. Hanff, F. Diekmann, L. Stojchevska, M. Kalläne, P. Zhou, K. Rossnagel, M. Eckstein, P. Werner, and U. Bovensiepen. Ultrafast doublon dynamics in photoexcited 1t-tas 2 . Phys. Rev. Lett., 120:166401, Apr 2018.

[Lan37a] LD Landau. On the theory of phase transitions, part i. Sov Phys JETP, 7:19ff, 1937.

[Lan37b] Lev Davidovich Landau. On the theory of phase transitions. II. Phys. Z. Sowjet., 11:545, 1937.

[Lan50] C. Lanczos. Electromagnetic fields and dielectric response in empirical tight-binding theory. J. Res. Nat. Bur. Standards, 45:255-282, 1950.

[Lau83] R. B. Laughlin. Anomalous quantum hall effect: An incompressible quantum fluid with fractionally charged excitations. Phys. Rev. Lett., 50:13951398, May 1983.

[LcvcvGcvBcvPcv14] Zala Lenarčič, Denis Golež, Janez Bonča, and Peter Prelovšek. Optical response of highly excited particles in a strongly correlated system. Phys. Rev. B, 89:125123, Mar 2014.

[LF12] Martin Leijnse and Karsten Flensberg. Introduction to topological superconductivity and majorana fermions. Semiconductor Science and Technology, 27(12):124003, nov 2012.

[LFG16] Roman M. Lutchyn, Karsten Flensberg, and Leonid I. Glazman. Quantum charge fluctuations of a proximitized nanowire. Phys. Rev. B, 94:125407, Sep 2016.

[LLL35] F. London, H. London, and Frederick Alexander Lindemann. The electromagnetic equations of the supraconductor. Proceedings of the Royal Society of London. Series A - Mathematical and Physical Sciences, 149(866):7188, 1935. 
[LM17] Yonah Lemonik and Aditi Mitra. Time-resolved spectral density of interacting fermions following a quench to a superconducting critical point. Phys. Rev. B, 96:104506, Sep 2017.

[LM18] Yonah Lemonik and Aditi Mitra. Model predictions for time-resolved transport measurements made near the superfluid critical points of cold atoms and $\mathrm{k}_{3} \mathrm{c}_{60}$ films. Phys. Rev. Lett., 121:067001, Aug 2018.

[LNW06] Patrick A. Lee, Naoto Nagaosa, and Xiao-Gang Wen. Doping a mott insulator: Physics of high-temperature superconductivity. Rev. Mod. Phys., 78:17-85, Jan 2006.

[LO05] D.W. Lynch and C.G. Olson. Photoemission Studies of High-Temperature Superconductors. Cambridge Studies in Low Temperature Physics. Cambridge University Press, 2005.

[LOV15] Christian. Lubich, Ivan V. Oseledets, and Bart. Vandereycken. Time integration of tensor trains. SIAM Journal on Numerical Analysis, 53(2):917941, 2015.

$\left[\mathrm{LPB}^{+} 17\right]$ Eyal Leviatan, Frank Pollmann, Jens H. Bardarson, David A. Huse, and Ehud Altman. Quantum thermalization dynamics with matrix-product states, 2017.

[LSDS10] Roman M. Lutchyn, Jay D. Sau, and S. Das Sarma. Majorana fermions and a topological phase transition in semiconductor-superconductor heterostructures. Phys. Rev. Lett., 105:077001, Aug 2010.

[McC07] Ian P McCulloch. From density-matrix renormalization group to matrix product states. Journal of Statistical Mechanics: Theory and Experiment, 2007(10):P10014, 2007.

$\left[\mathrm{MCN}^{+} 16\right]$ M. Mitrano, A. Cantaluppi, D. Nicoletti, S. Kaiser, A. Perucchi, S. Lupi, P. Di Pietro, D. Pontiroli, M. Riccò, S. R. Clark, D. Jaksch, and A. Cavalleri. Possible light-induced superconductivity in k3c60 at high temperature. Nature, 530:461-, February 2016.

[MG02] I. P. McCulloch and M. Gulácsi. The non-abelian density matrix renormalization group algorithm. EPL (Europhysics Letters), 57(6):852, 2002.

[MMGH17] Salvatore R. Manmana, Marcel Möller, Riccardo Gezzi, and Kaden R. A. Hazzard. Correlations and enlarged superconducting phase of $t-J_{\perp}$ chains of ultracold molecules on optical lattices. Phys. Rev. A, 96:043618, Oct 2017.

[MMM11] Alexander Moreno, Alejandro Muramatsu, and Salvatore R. Manmana. Ground-state phase diagram of the one-dimensional $t-j$ model. Phys. Rev. B, 83(20):205113, May 2011.

[MMN05] Salvatore R. Manmana, Alejandro Muramatsu, and Reinhard M. Noack. Time evolution of one-dimensional quantum many body systems. AIP Conf. Proc., 789:269-278, 2005.

[MO33] W. Meissner and R. Ochsenfeld. Ein neuer effekt bei eintritt der supraleitfähigkeit. Naturwissenschaften, 21(44):787-788, 1933.

[Moh13] Martin J. Mohlenkamp. Musings on multilinear fitting. Linear Algebra and its Applications, 438(2):834 - 852, 2013. Tensors and Multilinear Algebra.

[MRGF09] S. Montangero, M. Rizzi, V. Giovannetti, and Rosario Fazio. Critical exponents with a multiscale entanglement renormalization ansatz channel. 
Phys. Rev. B, 80:113103, Sep 2009.

[MS11] Roger S. K. Mong and Vasudha Shivamoggi. Edge states and the bulkboundary correspondence in dirac hamiltonians. Phys. Rev. B, 83:125109, Mar 2011.

[MSF ${ }^{+14}$ ] R. Mankowsky, A. Subedi, M. Först, S. O. Mariager, M. Chollet, H. T. Lemke, J. S. Robinson, J. M. Glownia, M. P. Minitti, A. Frano, M. Fechner, N. A. Spaldin, T. Loew, B. Keimer, A. Georges, and A. Cavalleri. Nonlinear lattice dynamics as a basis for enhanced superconductivity in yba2cu3o6.5. Nature, 516:71-, December 2014.

[MSRV86] K. Miyake, S. Schmitt-Rink, and C. M. Varma. Spin-fluctuation-mediated even-parity pairing in heavy-fermion superconductors. Phys. Rev. B, 34:6554-6556, Nov 1986.

[MW66a] N. D. Mermin and H. Wagner. Absence of ferromagnetism or antiferromagnetism in one- or two-dimensional isotropic heisenberg models. Phys. Rev. Lett., 17:1133-1136, Nov 1966.

[MW66b] N. D. Mermin and H. Wagner. Absence of ferromagnetism or antiferromagnetism in one- or two-dimensional isotropic heisenberg models. Phys. Rev. Lett., 17:1307-1307, Dec 1966.

$\left[\mathrm{MZF}^{+} 12\right]$ V. Mourik, K. Zuo, S. M. Frolov, S. R. Plissard, E. P. A. M. Bakkers, and L. P. Kouwenhoven. Signatures of majorana fermions in hybrid superconductor-semiconductor nanowire devices. Science, 336(6084):10031007, 2012.

[MZMP16] Johannes Motruk, Michael P. Zaletel, Roger S. K. Mong, and Frank Pollmann. Density matrix renormalization group on a cylinder in mixed real and momentum space. Physical Review B, 93(15), Apr 2016.

[NMML04] K. D. Nelson, Z. Q. Mao, Y. Maeno, and Y. Liu. Odd-parity superconductivity in sr2ruo4. Science, 306(5699):1151-1154, 2004.

$\left[\mathrm{NSS}^{+} 08\right]$ Chetan Nayak, Steven H. Simon, Ady Stern, Michael Freedman, and Sankar Das Sarma. Non-abelian anyons and topological quantum computation. Reviews of Modern Physics, 80(3):1083-1159, Sep 2008.

[NW93] R. M. Noack and S. R. White. Real-space quantum renormalization group and anderson localization. Phys. Rev. B, 47:9243-9248, Apr 1993.

[NW99] Reinhard M. Noack and Steven R. White. The density matrix renormalization group. In Ingo Peschel, Xiaoqun Wang, Matthias Kaulke, and Karen Hallberg, editors, Density Matrix Renormalization - A New Numerical Method in Physics, pages 27-66. Springer Verlag, 1999.

[OR00] J. M. Ortega and W. C. Rheinboldt. Iterative Solution of Nonlinear Equations in Several Variables. Society for Industrial and Applied Mathematics, 2000.

[ORR $\left.{ }^{+} 84\right]$ HR Ott, H Rudigier, TM Rice, K Ueda, Z Fisk, and JL Smith. p-wave superconductivity in ube 13. In Ten Years of Superconductivity: 19801990, pages 156-159. Springer, 1984.

[ORvO10] Yuval Oreg, Gil Refael, and Felix von Oppen. Helical liquids and majorana bound states in quantum wires. Physical Review Letters, 105(17), Oct 2010.

[PAD03] M. Potthoff, M. Aichhorn, and C. Dahnken. Variational cluster ap- 
proach to correlated electron systems in low dimensions. Phys. Rev. Lett., 91:206402, Nov 2003.

[Par69] R.D. Parks. Superconductivity: Part 1 (In Two Parts). Superconductivity. Taylor \& Francis, 1969.

[PEDC05] M. B. Plenio, J. Eisert, J. Dreißig, and M. Cramer. Entropy, entanglement, and area: Analytical results for harmonic lattice systems. Phys. Rev. Lett., 94:060503, Feb 2005.

[Pei33] Rudolf Ernst Peierls. On the Theory of the Diamagnetism of Conduction Electrons. Z.Phys., 80:763-791, 1933.

[Pei36] R. Peierls. On ising's model of ferromagnetism. Mathematical Proceedings of the Cambridge Philosophical Society, 32(03):477-481, 1936.

$\left[\mathrm{PFO}^{+} 19\right]$ Sebastian Paeckel, Benedikt Fauseweh, Alexander Osterkorn, Thomas Köhler, Dirk Manske, and Salvatore R. Manmana. Detecting superconductivity out-of-equilibrium. arXiv e-prints, 1905.08638:arXiv:1905.08638, May 2019.

[PKM17] Sebastian Paeckel, Thomas Köhler, and Salvatore R. Manmana. Automated construction of $u(1)$-invariant matrix-product operators from graph representations. SciPost Phys., 3:035, 2017.

[PKS $\left.{ }^{+} 19\right]$ Sebastian Paeckel, Thomas Köhler, Andreas Swoboda, Salvatore R. Manmana, Ulrich Schollwöck, and Claudius Hubig. Time-evolution methods for matrix-product states. Annals of Physics, 411:167998, Dec 2019.

[PLL $\left.{ }^{+} 06\right]$ L. Perfetti, P. A. Loukakos, M. Lisowski, U. Bovensiepen, H. Berger, S. Biermann, P. S. Cornaglia, A. Georges, and M. Wolf. Time evolution of the electronic structure of $1 t-$ tas $_{2}$ through the insulator-metal transition. Phys. Rev. Lett., 97:067402, Aug 2006.

[PMCV10] B Pirvu, V Murg, J I Cirac, and F Verstraete. Matrix product operator representations. New Journal of Physics, 12(2):025012, 2010.

[PO56] Oliver Penrose and Lars Onsager. Bose-einstein condensation and liquid helium. Phys. Rev., 104(3):576-584, Nov 1956.

[PWKH99] Ingo Peschel, Xiaoqun Wang, Matthias Kaulke, and Karen Hallberg, editors. Density Matrix Renormalization - A New Numerical Method in Physics. Springer Verlag, Berlin, 1999.

[QHZ08] Xiao-Liang Qi, Taylor L. Hughes, and Shou-Cheng Zhang. Topological field theory of time-reversal invariant insulators. Phys. Rev. B, 78:195424, Nov 2008.

$\left[\mathrm{RHW}^{+}{ }^{11}\right]$ Timm Rohwer, Stefan Hellmann, Martin Wiesenmayer, Christian Sohrt, Ankatrin Stange, Bartosz Slomski, Adra Carr, Yanwei Liu, Luis Miaja Avila, Matthias Kalläne, Stefan Mathias, Lutz Kipp, Kai Rossnagel, and Michael Bauer. Collapse of long-range charge order tracked by timeresolved photoemission at high momenta. Nature, 471:490, Mar 2011.

[RLJHC17] Enrico Ronca, Zhendong Li, Carlos A. Jimenez-Hoyos, and Garnet Kin-Lic Chan. Time-step targeting time-dependent and dynamical density matrix renormalization group algorithms with ab initio hamiltonians. Journal of Chemical Theory and Computation, 13(11):5560-5571, 2017. PMID: 28953377.

[RM04] Marcos Rigol and Alejandro Muramatsu. Universal properties of hard-core 
bosons confined on one-dimensional lattices. Phys. Rev. A, 70(3):031603, 2004.

[RM05] Marcos Rigol and Alejandro Muramatsu. Ground-state properties of hardcore bosons confined on one-dimensional optical lattices. Phys. Rev. A, 72(1):013604, 2005.

[RMR $\left.{ }^{+} 06\right]$ K. Rodriguez, S. R. Manmana, M. Rigol, R. M. Noack, and A. Muramatsu. Coherent matter waves emerging from mott-insulators. New Journal of Physics, 8(8):169, 2006.

[RMS $\left.{ }^{+} 10\right]$ M Rizzi, S Montangero, P Silvi, V Giovannetti, and Rosario Fazio. Homogeneous multiscale entanglement renormalization ansatz tensor networks for quantum critical systems. New Journal of Physics, 12(7):075018, jul 2010.

[Ros72] II. The Doctor's Dissertation (Text and Translation)**[See Introduction, sect. 2.]. In L. Rosenfeld and J. Rud Nielsen, editors, EARLY WORK (1905-1911), volume 1 of Niels Bohr Collected Works, pages 163 - 393. Elsevier, 1972.

$\left[\mathrm{RPK}^{+} 96\right]$ S. Ramasesha, Swapan K. Pati, H. R. Krishnamurthy, Z. Shuai, and J. L. Brédas. Symmetrized density-matrix renormalization-group method for excited states of hubbard models. Phys. Rev. B, 54:7598-7601, Sep 1996.

[RS95] T M Rice and M Sigrist. Sr2ruo4: an electronic analogue of3he? Journal of Physics: Condensed Matter, 7(47):L643-L648, nov 1995.

[RSN51] C. A. Reynolds, B. Serin, and L. B. Nesbitt. The isotope effect in superconductivity. i. mercury. Phys. Rev., 84:691-694, Nov 1951.

[SA17] Masatoshi Sato and Yoichi Ando. Topological superconductors: a review. Reports on Progress in Physics, 80(7):076501, may 2017.

[Sac99] S. Sachdev. Quantum Phase Transitions. Cambridge University Press, Cambridge, 1999.

[Sak94] J. J. Sakurai. Modern Quantum Mechanics, revised edition. AddisonWesley, 1994.

[SASF11] E. M. Stoudenmire, Jason Alicea, Oleg A. Starykh, and Matthew P.A. Fisher. Interaction effects in topological superconducting wires supporting majorana fermions. Phys. Rev. B, 84:014503, Jul 2011.

[SBC04] Anders W. Sandvik, Leon Balents, and David K. Campbell. Ground state phases of the half-filled one-dimensional extended hubbard model. Phys. Rev. Lett., 92:236401, Jun 2004.

$\left[\mathrm{SBP}^{+} 19\right]$ Oskar Schnaack, Niklas Bölter, Sebastian Paeckel, Salvatore R. Manmana, Stefan Kehrein, and Markus Schmitt. Tripartite information, scrambling, and the role of hilbert space partitioning in quantum lattice models. Physical Review B, 100:224302, Dec 2019.

[Sch26] E. Schrödinger. An undulatory theory of the mechanics of atoms and molecules. Phys. Rev., 28:1049-1070, Dec 1926.

[Sch05] U. Schollwöck. The density-matrix renormalization group. Rev. Mod. Phys., 77:259-315, Apr 2005.

[Sch06] F. Schwabl. Statistische Mechanik. Springer-Lehrbuch. Springer-Verlag Berlin Heidelberg, 2006.

[Sch11] Ulrich Schollwöck. The density-matrix renormalization group in the age of 
matrix product states. Annals of Physics, 326(1):96 - 192, 2011. January 2011 Special Issue.

[SCK $\left.{ }^{+} 15\right]$ R. Singla, G. Cotugno, S. Kaiser, M. Först, M. Mitrano, H. Y. Liu, A. Cartella, C. Manzoni, H. Okamoto, T. Hasegawa, S. R. Clark, D. Jaksch, and A. Cavalleri. Thz-frequency modulation of the hubbard $u$ in an organic mott insulator. Phys. Rev. Lett., 115:187401, Oct 2015.

[SDV06] Y.-Y. Shi, L.-M. Duan, and G. Vidal. Classical simulation of quantum many-body systems with a tree tensor network. Phys. Rev. A, 74(2):022320, Aug 2006.

[SE19] Christopher Stahl and Martin Eckstein. Noise correlations in time- and angle-resolved photoemission spectroscopy. Phys. Rev. B, 99:241111, Jun 2019 .

[Sén03] D. Sénéchal. A cluster method for spectral properties of correlated electrons. In D. Sénéchal, editor, High Performance Computing Systems and Applications and OSCAR symposium. NRC Press, Ottawa, NRC Press, Ottawa, 2003.

[SFN15] Sankar Das Sarma, Michael Freedman, and Chetan Nayak. Majorana zero modes and topological quantum computation. npj Quantum Information, 1(1):15001, 2015.

$\left[\mathrm{SHD}^{+} 20\right]$ Jan Stolpp, Jacek Herbrych, Florian Dorfner, Elbio Dagotto, and Fabian Heidrich-Meisner. Charge-density-wave melting in the one-dimensional holstein model. Physical Review B, 101(3), Jan 2020.

[SLMT05] David Sénéchal, P.-L. Lavertu, M.-A. Marois, and A.-M. S. Tremblay. Competition between antiferromagnetism and superconductivity in high$T_{c}$ cuprates. Phys. Rev. Lett., 94:156404, Apr 2005.

[SP] Thomas Köhler Sebastian Paeckel. Symmps. https://www. symmps.eu. Accessed: 2019-12-29.

[SPV10] Sukhwinder Singh, Robert N. C. Pfeifer, and Guifré Vidal. Tensor network decompositions in the presence of a global symmetry. Phys. Rev. A, 82:050301, Nov 2010.

[SPV11] Sukhwinder Singh, Robert N. C. Pfeifer, and Guifre Vidal. Tensor network states and algorithms in the presence of a global u(1) symmetry. Phys. Rev. B, 83:115125, Mar 2011.

[SRFL08] Andreas P. Schnyder, Shinsei Ryu, Akira Furusaki, and Andreas W. W. Ludwig. Classification of topological insulators and superconductors in three spatial dimensions. Phys. Rev. B, 78:195125, Nov 2008.

[SRN50] B. Serin, C. A. Reynolds, and L. B. Nesbitt. Superconductivity of isotopes of mercury. Phys. Rev., 78:813-814, Jun 1950.

[SS14] Ken Shiozaki and Masatoshi Sato. Topology of crystalline insulators and superconductors. Physical Review B, 90(16), Oct 2014.

[STLL16] Can Shao, Takami Tohyama, Hong-Gang Luo, and Hantao Lu. Numerical method to compute optical conductivity based on pump-probe simulations. Phys. Rev. B, 93:195144, May 2016.

[STR69] V. STRASSEN. Gaussian elimination is not optimal. Numerische Mathematik, 13:354-356, 1969.

[Sus01] O. Sushkov. Conductance anomalies in a one-dimensional quantum con- 
tact. Physical Review B, 64(15), Sep 2001.

[Suz76] M. Suzuki. Generalized Trotter's Formula and Systematic Approximants of Exponential Operators and Inner Derivations with Applications to Many Body Problems. Commun. Math. Phys., 51:183-190, 1976.

[Suz90] Masuo Suzuki. Fractal decomposition of exponential operators with applications to many-body theories and monte carlo simulations. Physics Letters A, 146(6):319 - 323, 1990.

[SvdV96] G. L. G. Sleijpen and H. A. van der Vorst. A jacobi-davidson iteration method for linear eigenvalue problems. SIAM J. Matrix Anal. Appl., 17:401, 1996.

[SW78] R.F. Streater and A.S. Wightman. PCT, spin and statistics, and all that. The mathematical physics monograph series. Benjamin/Cummings Publ. Co, 1978.

[SW10] E M Stoudenmire and Steven R White. Minimally entangled typical thermal state algorithms. New Journal of Physics, 12(5):055026, 2010.

[SWSH06] D. N. Sheng, Z. Y. Weng, L. Sheng, and F. D. M. Haldane. Quantum spin-hall effect and topologically invariant chern numbers. Phys. Rev. Lett., 97:036808, Jul 2006.

[SWVC07] Norbert Schuch, Michael M. Wolf, Frank Verstraete, and J. Ignacio Cirac. Computational complexity of projected entangled pair states. Phys. Rev. Lett., 98:140506, Apr 2007.

[TF02] M Tsuchiizu and Akira Furusaki. Phase diagram of the one-dimensional extended hubbard model at half filling. Physical review letters, 88:056402, 032002.

[TH13] Jeffrey C. Y. Teo and Taylor L. Hughes. Existence of majorana-fermion bound states on disclinations and the classification of topological crystalline superconductors in two dimensions. Physical Review Letters, 111(4), Jul 2013.

[Tin04] Michael Tinkham. Introduction to superconductivity. Courier Corporation, 2004.

$\left[\mathrm{TIO}^{+} 19\right]$ E. F. Talantsev, K. Iida, T. Ohmura, T. Matsumoto, W. P. Crump, N. M. Strickland, S. C. Wimbush, and H. Ikuta. p-wave superconductivity in iron-based superconductors. Scientific Reports, 9(1), Oct 2019.

[TMB61] David James Thouless, H S W Massey, and Keith A Brueckner. The quantum mechanics of many-body systems; 1st ed. Academic Press, New York, NY, 1961.

[TSG82] D. C. Tsui, H. L. Stormer, and A. C. Gossard. Two-dimensional magnetotransport in the extreme quantum limit. Phys. Rev. Lett., 48:1559-1562, May 1982.

[UYTS13] Yuji Ueno, Ai Yamakage, Yukio Tanaka, and Masatoshi Sato. Symmetryprotected majorana fermions in topological crystalline superconductors: Theory and application tosr2ruo4. Physical Review Letters, 111(8), Aug 2013.

[VB58] J. G. Valatin and D. Butler. On the collective properties of a boson system. Il Nuovo Cimento (1955-1965), 10(1):37-54, 1958.

[VC06] F. Verstraete and J. I. Cirac. Matrix product states represent ground 
states faithfully. Phys. Rev. B, 73:094423, Mar 2006.

[vHLG16] B. van Heck, R. M. Lutchyn, and L. I. Glazman. Conductance of a proximitized nanowire in the coulomb blockade regime. Phys. Rev. B, 93:235431, Jun 2016.

[VHV19] Laurens Vanderstraeten, Jutho Haegeman, and Frank Verstraete. Tangentspace methods for uniform matrix product states. SciPost Phys. Lect. Notes, page 7, 2019.

[Vid03] Guifre Vidal. Efficient classical simulation of slightly entangled quantum computations. Phys. Rev. Lett., 91(14):147902, 2003.

[Vid08] G. Vidal. Class of quantum many-body states that can be efficiently simulated. Phys. Rev. Lett., 101(11):110501, Sep 2008.

[Voi92] Johannes Voit. Phase diagram and correlation functions of the half-filled extended hubbard model in one dimension. Phys. Rev. B, 45:4027-4042, Feb 1992.

[Voi95] Johannes Voit. One-dimensional fermi liquids. Rep. Prog. Phys., 58:9771116, 1995.

[VWPGC06] F. Verstraete, M. M. Wolf, D. Perez-Garcia, and J. I. Cirac. Criticality, the area law, and the computational power of projected entangled pair states. Phys. Rev. Lett., 96:220601, Jun 2006.

[WA08] Steven R. White and Ian Affleck. Spectral function for the $s=1$ heisenberg antiferromagetic chain. Phys. Rev. B, 77(13):134437, Apr 2008.

[WAT $\left.{ }^{+} 87\right]$ M. K. Wu, J. R. Ashburn, C. J. Torng, P. H. Hor, R. L. Meng, L. Gao, Z. J. Huang, Y. Q. Wang, and C. W. Chu. Superconductivity at $93 \mathrm{k}$ in a new mixed-phase y-ba-cu-o compound system at ambient pressure. Phys. Rev. Lett., 58:908-910, Mar 1987.

[WCMD17] Yao Wang, Martin Claassen, B. Moritz, and T. P. Devereaux. Producing coherent excitations in pumped mott antiferromagnetic insulators. Phys. Rev. B, 96:235142, Dec 2017.

$\left[\mathrm{WCP}^{+} 18\right]$ Yao Wang, Martin Claassen, Chaitanya Das Pemmaraju, Chunjing Jia, Brian Moritz, and Thomas P Devereaux. Theoretical understanding of photon spectroscopies in correlated materials in and out of equilibrium. Nature Reviews Materials, page 1, 2018.

[Wei12] Andreas Weichselbaum. Non-abelian symmetries in tensor networks: A quantum symmetry space approach. Annals of Physics, 327(12):2972 3047, 2012.

[WF04] Steven R. White and Adrian E. Feiguin. Real-time evolution using the density matrix renormalization group. Phys. Rev. Lett., 93:076401, Aug 2004.

[Whi92] Steven R. White. Density matrix formulation for quantum renormalization groups. Phys. Rev. Lett., 69(19):2863-2866, Nov 1992.

[Whi93] Steven R. White. Density-matrix algorithms for quantum renormalization groups. Phys. Rev. B, 48:10345-10356, Oct 1993.

[Whi05] Steven R. White. Density matrix renormalization group algorithms with a single center site. Phys. Rev. B, 72:180403, Nov 2005.

[Wil75] Kenneth G. Wilson. The renormalization group: Critical phenomena and the kondo problem. Rev. Mod. Phys., 47(4):773, 1975. 
[Wil90] Frank Wilczek. Fractional statistics and anyon superconductivity, volume 5. World scientific, 1990.

[WK17] Rukhsan-Ul-Haq Wani and Louis Kauffman. Z/2z topological order and majorana doubling in kitaev chain. 042017.

[WWZ89] X. G. Wen, Frank Wilczek, and A. Zee. Chiral spin states and superconductivity. Phys. Rev. B, 39:11413-11423, Jun 1989.

[WZS $\left.{ }^{+} 14\right]$ Endong Wang, Qing Zhang, Bo Shen, Guangyong Zhang, Xiaowei Lu, Qing Wu, and Yajuan Wang. Intel Math Kernel Library, pages 167-188. Springer International Publishing, Cham, 2014.

[XB18] Cenke Xu and Leon Balents. Topological superconductivity in twisted multilayer graphene. Phys. Rev. Lett., 121:087001, Aug 2018.

[YA70] G. Yuval and P. W. Anderson. Exact results for the kondo problem: Onebody theory and extension to finite temperature. Phys. Rev. B, 1:15221528, Feb 1970.

[Yan62] C. N. Yang. Concept of off-diagonal long-range order and the quantum phases of liquid he and of superconductors. Rev. Mod. Phys., 34:694-704, Oct 1962.

[Zee16] A. Zee. Group Theory in a Nutshell for Physicists. In a Nutshell. Princeton University Press, 2016.

$\left[Z_{H}^{+} 17\right]$ J. Zhang, P. W. Hess, A. Kyprianidis, P. Becker, A. Lee, J. Smith, G. Pagano, I.-D. Potirniche, A. C. Potter, A. Vishwanath, N. Y. Yao, and C. Monroe. Observation of a discrete time crystal. Nature, 543:217, Mar 2017.

[ZJW98] Chunli Zhang, Eric Jeckelmann, and Steven R. White. Density matrix approach to local hilbert space reduction. Phys. Rev. Lett., 80:2661-2664, Mar 1998.

$\left[\mathrm{ZMK}^{+} 15\right]$ Michael P. Zaletel, Roger S. K. Mong, Christoph Karrasch, Joel E. Moore, and Frank Pollmann. Time-evolving a matrix product state with longranged interactions. Phys. Rev. B, 91:165112, Apr 2015.

[ZR88] F. C. Zhang and T. M. Rice. Effective hamiltonian for the superconducting cu oxides. Phys. Rev. B, 37:3759-3761, Mar 1988. 